\author{
Mon ograph \\ urn:lsid:zoobank.org:pub:6384213C-8966-4349-A695-225C5CA0BC2F
}

\title{
Atlas of European millipedes 3: Order Chordeumatida (Class Diplopoda)
}

\author{
Richard Desmond $\mathrm{KIME}^{1}$ \& Henrik ENGHOFF ${ }^{2, *}$ \\ ${ }^{1}$ La Fontaine, La-Chapelle-Montmoreau, 24300 Nontron, France. \\ ${ }^{2}$ Natural History Museum of Denmark, University of Copenhagen, Universitetsparken 25, \\ 2100 Copenhagen Ø, Denmark. \\ *Corresponding author: henghoff@snm.ku.dk \\ 1Email: deskime2@aol.com \\ ${ }^{1}$ urn:Isid:zoobank.org:author:847CC68F-00BF-4DAB-8E53-B7A3384D66C1
${ }^{2}$ urn:Isid:zoobank.org:author:FB09A817-000D-43C3-BCC4-2BC1E5373635
}

Abstract. For each of the 534 species of the millipede order Chordeumatida known from Europe, available information on taxonomy, distribution and habitat is summarized, and the distribution in $50 \times$ $50 \mathrm{~km}$ UTM/MGRS squares is shown on a map. Comparisons between Chordeumatida and the equallysized order Julida are made with respect to distribution patterns and history of exploration.

Keywords. Distribution, map, UTM, MGRS, faunistics, taxonomy.

Kime R.D. \& Enghoff H. 2021. Atlas of European millipedes 3: Order Chordeumatida (Class Diplopoda). European Journal of Taxonomy 769: 1-244. https://doi.org/10.5852/ejt.2021.769.1497

\section{Contents}

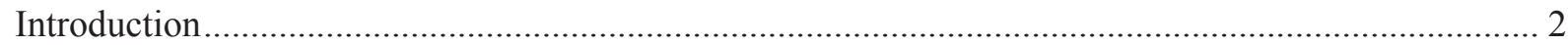

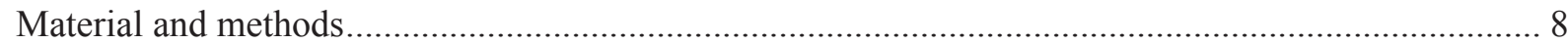

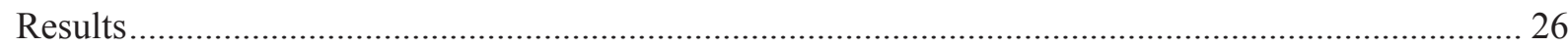

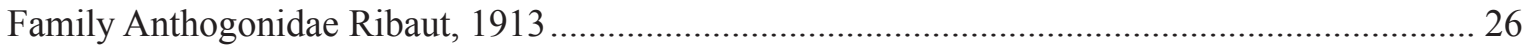

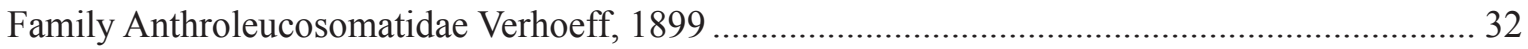

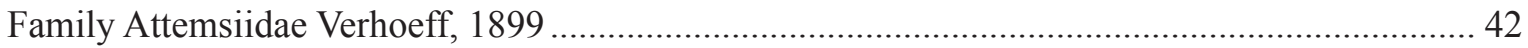

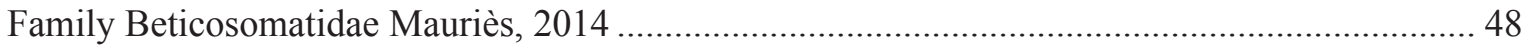

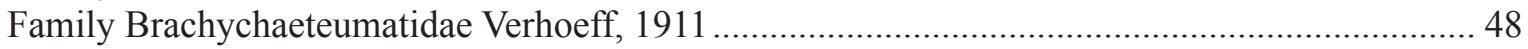

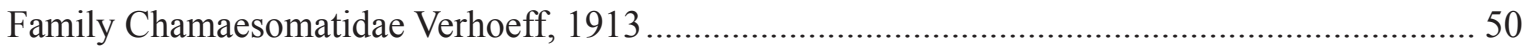

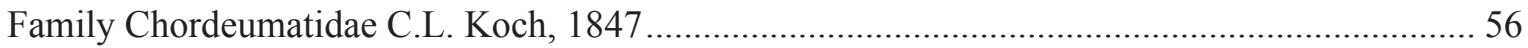

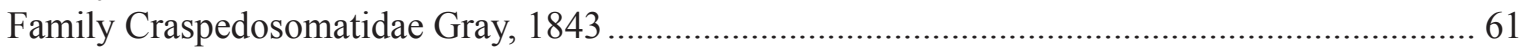

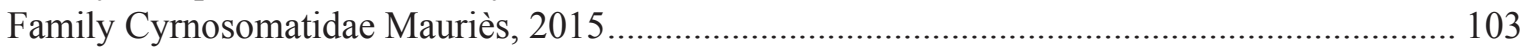

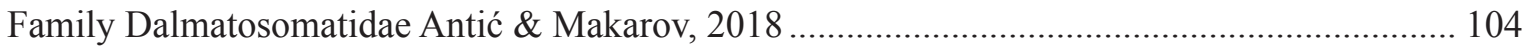

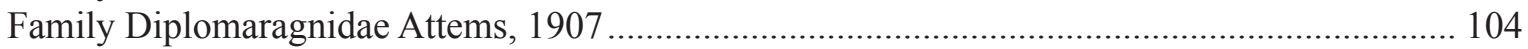

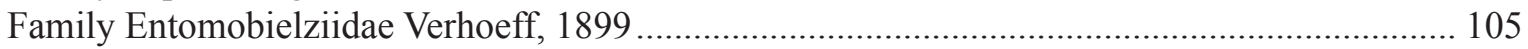




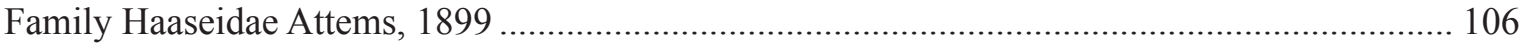

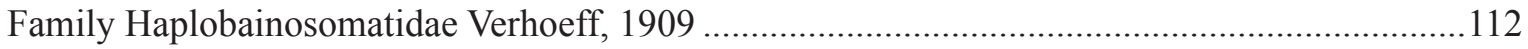

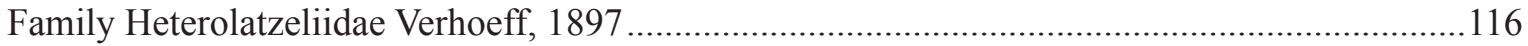

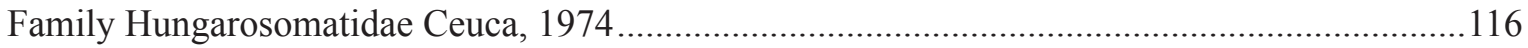

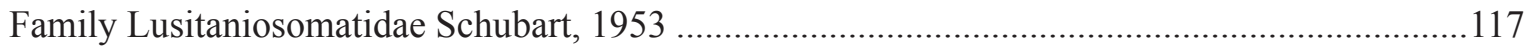

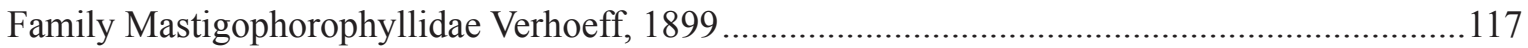

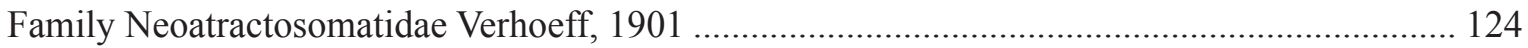

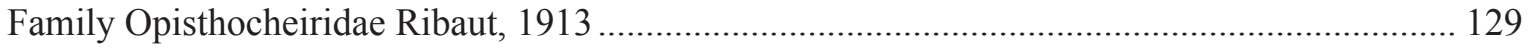

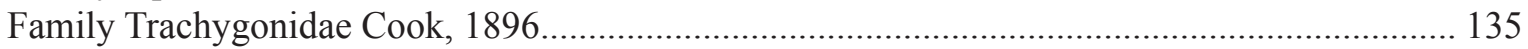

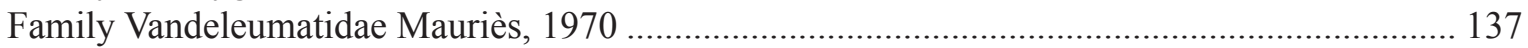

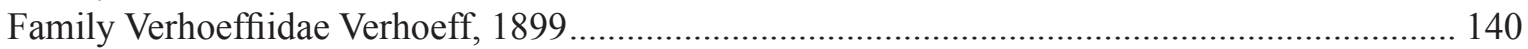

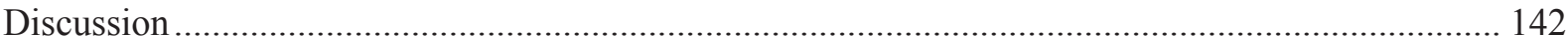

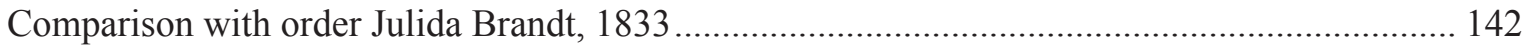

The European millipede fauna - updates on other orders ……….............................................. 142

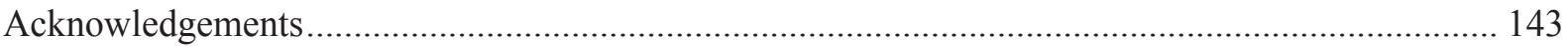

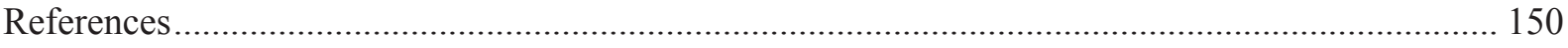

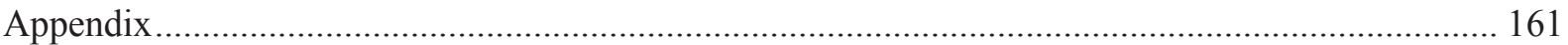

\section{Introduction}

The primary purpose of this atlas is to provide maps showing the known distribution of all European millipedes. The present volume (the last of three) deals with the 534 species belonging to the order Chordeumatida C.L. Koch, 1847, which are currently known from Europe. The other seven orders were treated by Kime \& Enghoff $(2011,2017)$.

The Chordeumatida is the second-largest order of millipedes in Europe, only surpassed by the Julida Brandt, 1833. Representative European species of Chordeumatida are shown in Figs 1-4.

All in all, well over 1600 species of millipedes are currently known from Europe in the sense of this atlas (Fig. 5), but the European millipede fauna is by no means sufficiently known. Not only are new species being discovered and described virtually every year, but species concepts and delimitations are under continuous revisions - certain apparently well-known species become split, while others get synonymized, see for example Antić \& Akkari (2020). In no other order is this as evident as in the Chordeumatida where such genera as Craspedosoma Leach, 1814, Pyrgocyphosoma Verhoeff, 1910 and Rhymogona Cook, 1896 apparently offer endless problems for taxonomists.

Taxonomic problems in Chordeumatida are not restricted to the species level; the higher classification within the order is extremely convoluted. Antic et al. (2018c: 299) described the situation well: "Of some 50 families within the order Chordeumatida, 19 contain only one genus, while even seven are monospecific. This fact suggests that it may be a matter of too many families, if we take into account that the number of species within the order is "only" a little more than 1100. On the other hand, it has been shown that some previously monotypic/monogeneric families were much later filled up with additional species or genera ... It is obvious that the real number of species of chordeumatidans is much greater than the current number. Many of them are characterized by a small body and cryptic life-style, are very rare, inhabit some extreme environments, or everything mentioned. There are numerous examples among chordeumatidans where only one or several individuals are known, on the basis of which a taxon is described, while repeated attempts to collect additional material from the type localities were unsuccessful". Enghoff et al. (2015) agreed that probably too many families are currently recognized in the order, and that multiple major changes are foreseen. Phylogenetic analyses involving molecular data will hopefully help, but so far there have been very few such. An exception is the analysis of Mock et al. (2016), and if their results are representative, major changes are indeed to be expected: in 

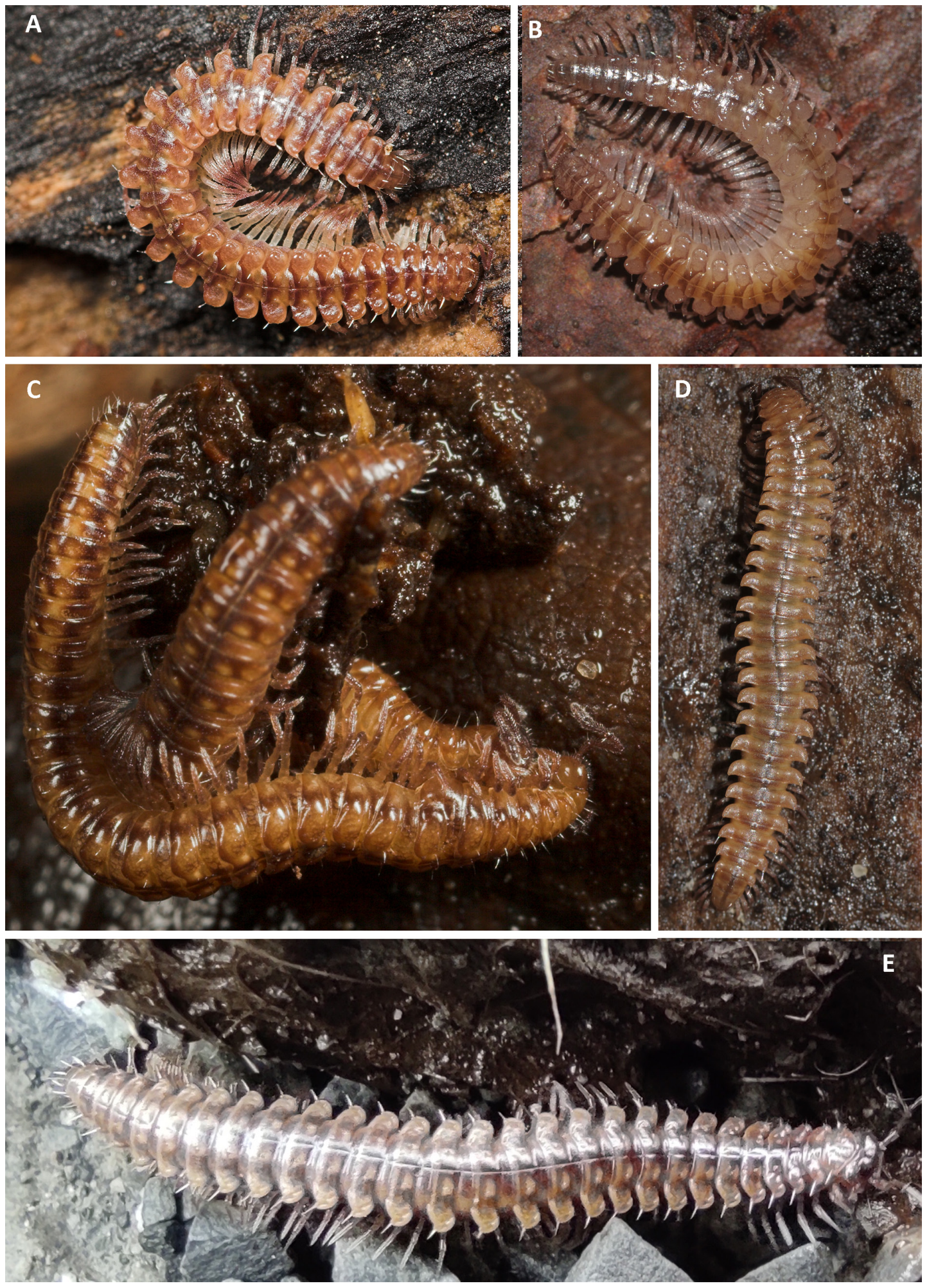

Fig. 1. A selection of European species of Chordeumatida C.L. Koch, 1847, family Craspedosomatidae Gray, 1843. A. Nanogona polydesmoides (Leach, 1814). B. Rhymogona verhoeffi (Bigler, 1913). C. Craspedosoma raulinsii Leach, 1814, in copula. D. Atractosoma meridionale Fanzago, 1876. E. Bergamosoma canestrinii (Fedrizzi, 1878), feeding on excrements of fox (Vulpes vulpes (Linnaeus, 1758)). Photo credits: Paul Richards, www.flickr.com/invertimages (A, C), Jörg Spelda (B, D), José Domingo Gilgado (E). 

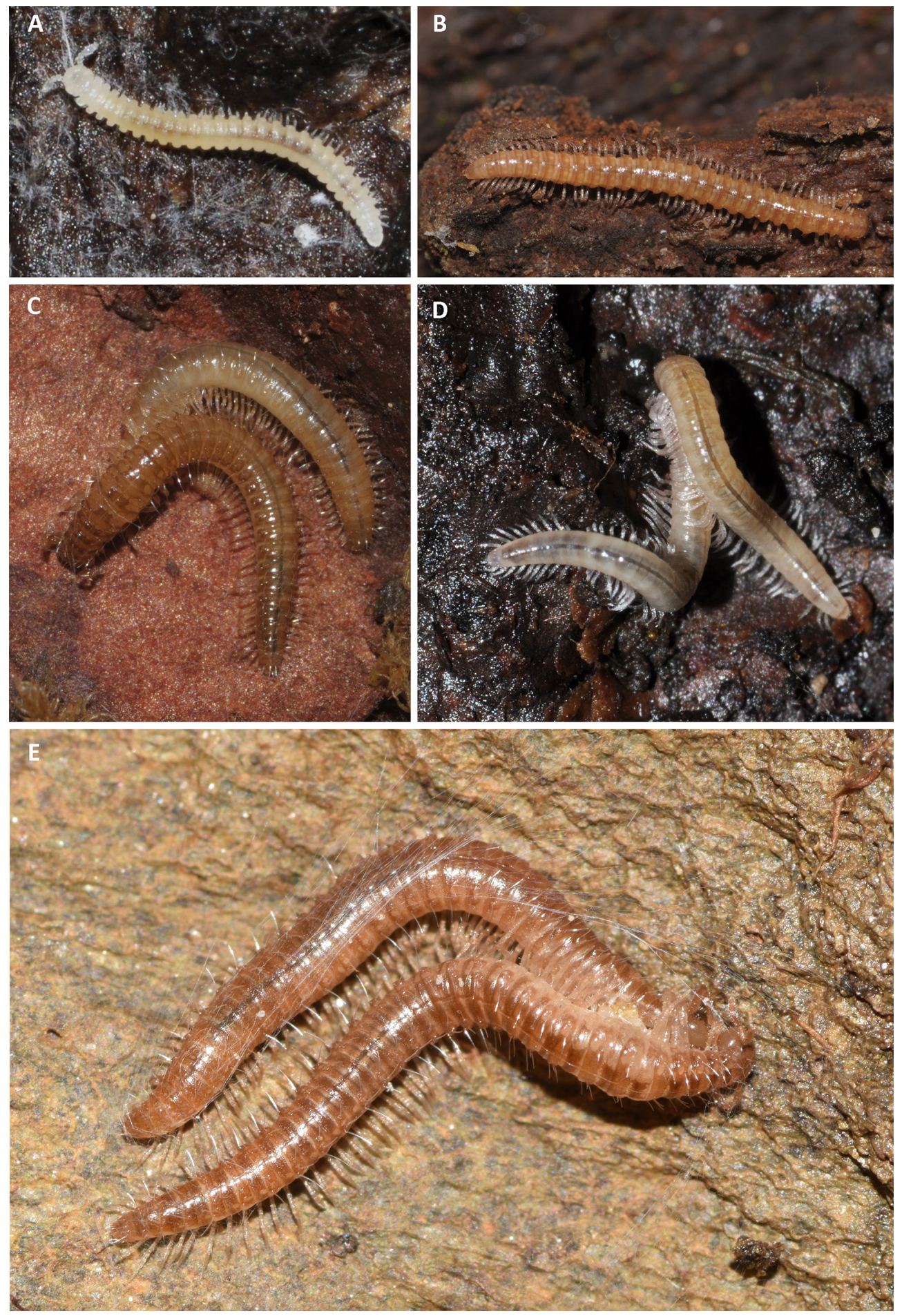

Fig. 2. A selection of European species of Chordeumatida C.L. Koch, 1847, various families. A. Halleinosoma noricum Verhoeff, 1913 (Trachygonidae Cook, 1896). B. Syngonopodium aceris Verhoeff, 1913 (Attemsiidae Verhoeff, 1899). C. Xylophageuma zschokkei Bigler, 1912 (Haaseidae Attems, 1899), in copula. D. Haplogona oculodistincta (Verhoeff, 1893) (Verhoeffiidae Verhoeff, 1899), in copula. E. Haasea fonticulorum (Verhoeff, 1910) (Haaseidae), in copula. Jörg Spelda has pointed out that on Figs $2 \mathrm{C}$ and 2E, both showing copulation in species of Haaseidae, the female is seen to be partially covered in a fine web (most evident in Fig. 2E), probably produced by the male's spinning glands and perhaps serving to 'strap down' the female to the ground. Photo credits: Jörg Spelda. 

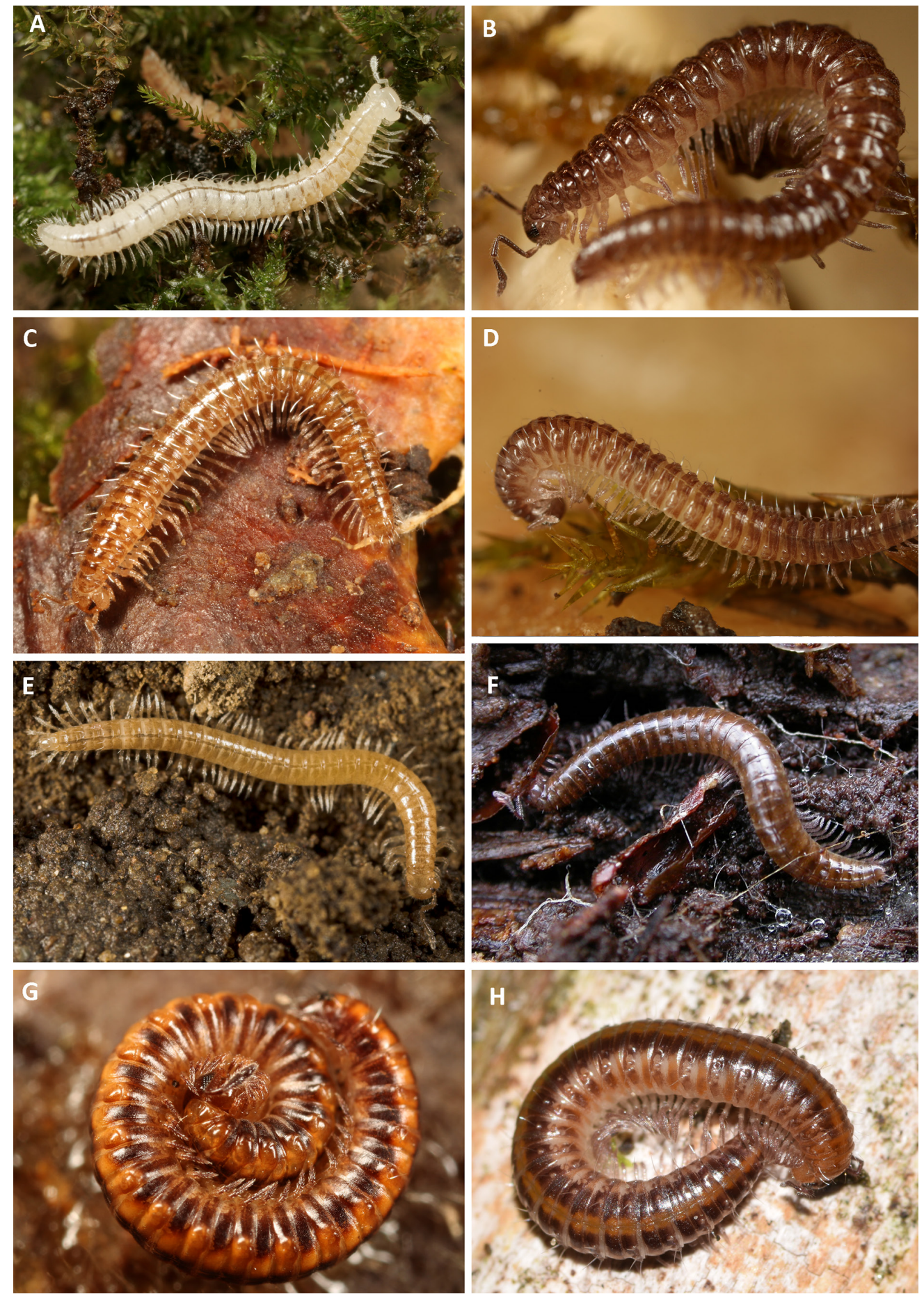

Fig. 3. A selection of European species of Chordeumatida C.L. Koch, 1847, various families. A. Psichrosoma cf. breuli (Mauriès, 1970) (Vandeleumatidae Mauriès, 1970). B. Turdulisoma cf. helenreadae Mauriès, 2015 (Haplobainosomatidae Verhoeff, 1909). C. Hylebainosoma nontronense Mauriès \& Kime, 1999 (Haaseidae Attems, 1899). D. Anamastigona pulchella (Silvestri, 1894) (Anthroleucosomatidae Verhoeff, 1899). E. Melogona gallica (Latzel, 1884) (Chordeumatidae C.L. Koch, 1847). F. Chordeuma proximum Ribaut, 1913 (Chordeumatidae). G. Ceratosphys amoena Ribaut, 1920 (Opisthocheiridae Ribaut, 1913). H. Mastigona mutabilis (Latzel, 1884) (Mastigophorophyllidae Verhoeff, 1899). Photo credits: Paul Richards, www.flickr.com/invertimages (A-E, G), Trevor and Dilys Pendleton (F), Jörg Spelda (H). 

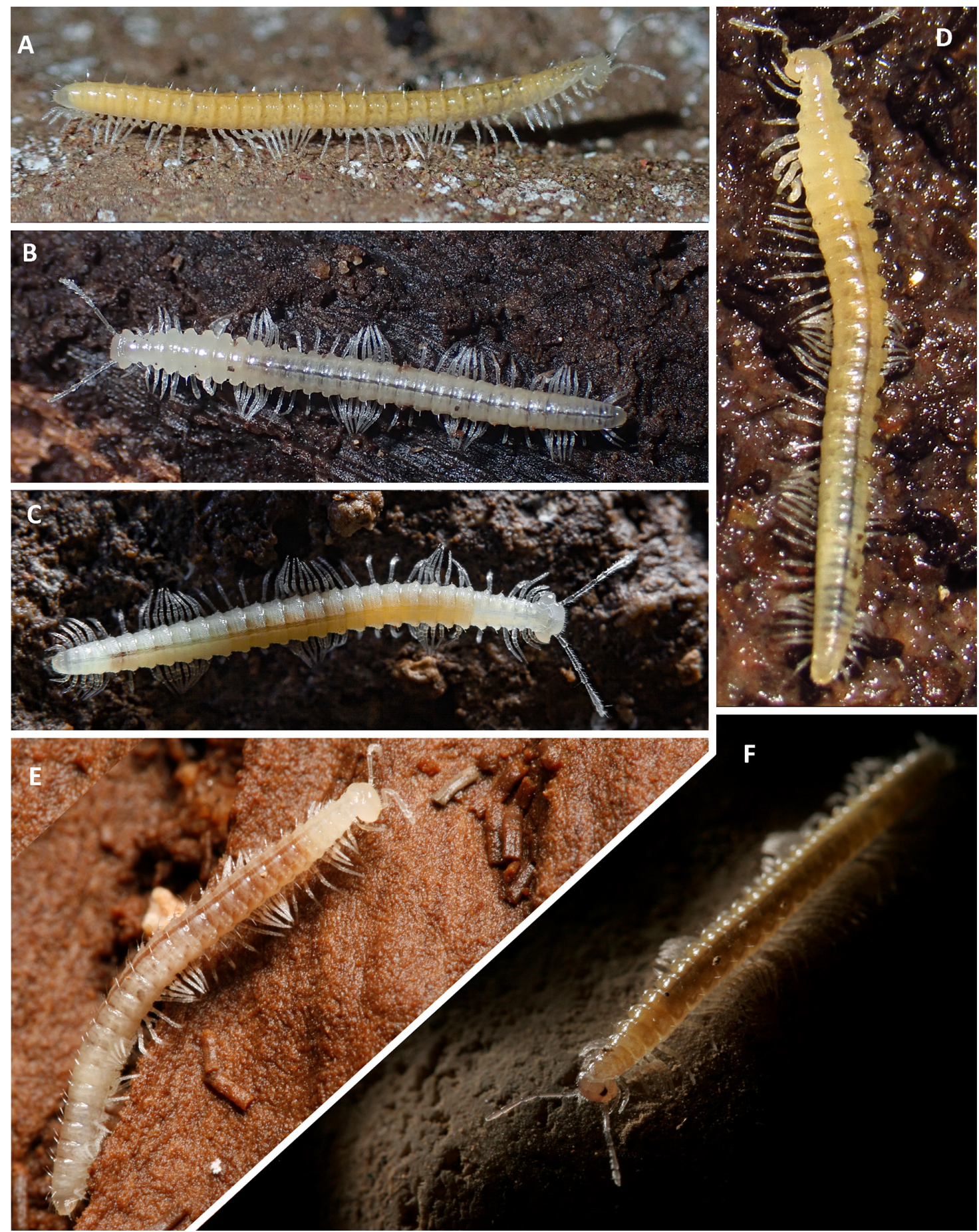

Fig. 4. A selection of European subterranean species of Chordeumatida C.L. Koch, 1847, various families. A. Schizmohetera curcici Makarov, 2001, topotype (Neoatractosomatidae Verhoeff, 1901). B. Serbosoma kucajense (Ćurčić \& Makarov, 1998), topotype (Anthroleucosomatidae Verhoeff, 1899). C. Egonpretneria vudutschaljdi Antić \& Dražina, 2015 (Anthogonidae Ribaut, 1913). D. Cornogonopus pavicevici Antić, 2020, the latest addition to the European chordeumatidan fauna, holotype (Anthroleucosomatidae) E. Haasia likana (Strasser, 1966) (Anthogonidae). F. Dyocerasoma intermedium Makarov, Lučić, Mitić \& Rađa, 2003 (Craspedosomatidae Gray, 1843). Photo credits: Marjan Komnenov (A), Dragan Antić (B, D), Tamara Čuković-Malenica (C), Tvrtko Dražina (E), Kazimir Muculinić (F). 
their tree, taxa currently assigned to Attemsiidae Verhoeff, 1899, Hungarosomatidae Ceuca, 1974 and Neoatractosomatidae Verhoeff, 1901 are nested within a clade otherwise composed of genera assigned to the large family Craspedosomatidae Gray, 1843.

All millipede orders have many more species in southern Europe than further north. This is particularly pronounced in the case of Chordeumatida where the vast majority of species are confined to the Iberian, Italian and Balkan peninsulas, and very few species occur north of Germany. Only one of the European species, Altajosoma golovatchi (Shear, 1990) in the family Diplomaragnidae Attems, 1907, occurs naturally inside as well as outside Europe as here defined. Similarly, just one species, Craspedosoma raulinsii Leach, 1814 in the family Craspedosomatidae, has been artificially introduced to another continent (N America). In contrast to the two other large orders, Julida and Polydesmida, no European species of Chordeumatida is confined to hothouses or other indoor habitats.

Restricted distribution also characterizes higher-level taxa. Under the current classification, all European families of Chordeumatida are endemic, with the following exceptions: Chamaesomatidae Verhoeff, 1913 and Opisthocheiridae Ribaut, 1913 have two or one species, respectively, in North Africa; Anthroleucosomatidae Verhoeff, 1899 has many species in the Caucasus, Middle East and temperate Asia, even one in North America, and Diplomaragnidae is a predominantly C and E Asian family.

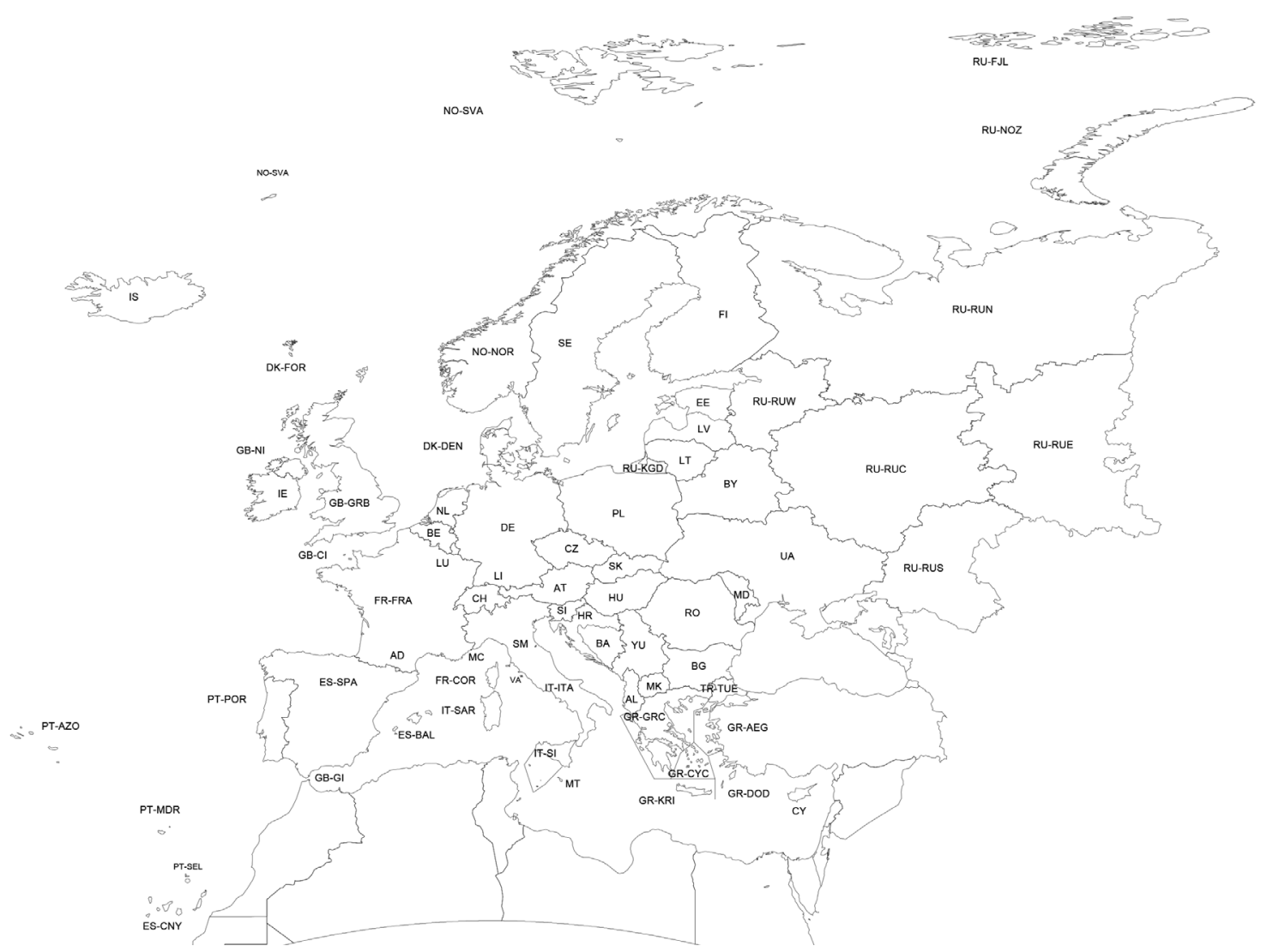

Fig. 5. Area codes as used in the atlas, from Fauna Europaea guidelines (de Jong et al. 2014: supplementary material 1), reproduced with permission. Note that MN (Montenegro) and SB (Serbia) are collectively shown as YU (Yugoslavia) on this map. Also note that CY (Cyprus) is not covered by the atlas. 
Our knowledge of the distribution of many species is very good, though for others it is extremely sketchy or inadequate. There are many regional and point endemics. The maps are published in the knowledge that some of them do not show the full distribution of the species concerned. It is left to present and future myriapodologists to fill in those gaps that remain and to complete the ecological information and other data that are missing. The rich southern faunas are most in need of investigation, especially in Iberia.

\section{Material and methods}

Families, genera and species are arranged alphabetically. See Table 1 for a list of families of Chordeumatida. Subfamilies, tribes, subgenera and subspecies are not considered.

At family level, we follow the classification in Enghoff et al. (2015), except where noted. Within each family, genera and species are as a rule delimited as in Fauna Europaea, https://fauna-eu.org (de Jong et al. 2014), but MilliBase (Sierwald \& Spelda 2020), has been particularly helpful for finding valid names and synonyms.

Subgenera have been suggested within many chordeumatidan genera. As a rule, names of subgenera are not considered here, unless the taxon in question has been regarded as a full genus at some point. Likewise, a large number of subspecies and varieties have been described for very many species of Chordeumatida, for example, in the genus Craspedosoma, see Hauser (2004a). Except in cases where a subspecific or varietal name has been employed at the full species level, such names are not included in the synonymies in the species descriptions. The interested reader is referred to MilliBase (Sierwald \& Spelda 2020), and Fauna Europaea (https://fauna-eu.org).

There has been some confusion about the gender of several genus names of chordeumatidans. Names ending in -gona are feminine, whereas names ending in -euma and -soma are neuter, cf. Jeekel (1970). This influences the spelling of the species epithet, which, if it is an adjective, must agree in gender with the genus name. For example, Anthogona variegata Ribaut, 1913 (feminine) has sometimes been cited as Anthogona variegatum (neuter) which is wrong. Alternative spellings of this type have not been included in the lists of synonyms.

For each species, the text is arranged as follows:

The valid name. A list of species covered in the present atlas is provided in Table 2.

The original combination, if different.

Other synonyms. Whereas the synonymy does not pretend to be complete, we have tried to include all synonyms which may cause confusion (see above concerning names of subspecies and varieties). Where a name is followed by "auct." rather than an author name, it means that some authors used this name/ combination, which is now not regarded as valid.

Distribution. The European distribution is given as a list of the geopolitical units from where the species has been reliably documented. The units and the abbreviations are, with one exception, the same as those used in Fauna Europaea, adopted and modified from the TDWG level 4 standard (Brummitt 2001), see Table 3 and Fig. 5. The exception concerns Serbia (SB) and Montenegro (MN) which in Fauna Europaea are treated as one unit ("Yugoslavia", YU). Despite recent political changes, records from Kosovo are ascribed to Serbia (SB), and records from Crimea are ascribed to Ukraine (UA). We have found several publications in which distributional data are erroneous with regard to present-day geopolitical units, including some which have been reiterated even as recently as 2012 in national inventories of species made after boundary changes. The Balkan countries have been particularly affected in this respect; this has complicated our work considerably and may have resulted in errors. 
Table 1 (continued on next page). Suborders, superfamilies and families of Chordeumatida C.L. Koch, 1847, according to Enghoff et al. (2015) with recent modifications:

1. Family Anthogonidae Ribaut, 1913 has been reinstated and added to superfamily Anthroleucosomatoidea; it includes Acherosomatidae Verhoeff, 1929 and Biokoviellidae Mršić, 1992 as junior synonyms (Antić et al. 2015a, 2016).

2. Family Dalmatosomatidae Antić \& Makarov, 2018, was described as new and placed in Branneroidea by Antić et al. (2018c).

3. Family Hungarosomatidae Ceuca,1974, was reinstated by Mock et al. (2016) but not assigned to a superfamily. Based on the discussion by Mock et al. (2016), it is here placed as Craspedosomatidea incertae sedis.

Families covered by the present volume are shown in bold and are followed by the number of known European species.

Order Chordeumatida Pocock, 1894

Suborder Chordeumatidea Pocock, 1894

Family Chordeumatidae C.L. Koch, 1847 (23)

Family Speophilosomatidae Takakuwa, 1949

Suborder Craspedosomatidea Cook, 1895

Superfamily Anthroleucosomatoidea Verhoeff, 1899

Family Anthogonidae Ribaut, 1913 (32)

Family Anthroleucosomatidae Verhoeff, 1899 (43)

Family Vandeleumatidae Mauriès, 1970 (15)

Superfamily Brannerioidea Cook, 1896

Family Beticosomatidae Mauriès, 2014 (1)

Family Brachychaeteumatidae Verhoeff, 1911 (8)

Family Branneriidae Cook, 1896

Family Chamaesomatidae Verhoeff, 1913 (33)

Family Dalmatosomatidae Antić \& Makarov, 2018 (1)

Family Golovatchiidae Shear, 1992

Family Heterolatzeliidae Verhoeff, 1897 (4)

Family Kashmireumatidae Mauriès, 1982

Family Macrochaeteumatidae Verhoeff, 1914

Family Microlympiidae Shear \& Leonard, 2003

Family Niponiosomatidae Verhoeff, 1941

Family Tingupidae Loomis, 1966

Family Trachygonidae Cook, 1896 (5)

Superfamily Cleidogonoidea Cook, 1896

Family Cleidogonidae Cook, 1896

Family Entomobielziidae Verhoeff, 1899 (4)

Family Lusitaniosomatidae Schubart, 1953 (1)

Family Opisthocheiridae Ribaut, 1913 (37)

Family Trichopetalidae Verhoeff, 1914

Superfamily Craspedosomatoidea Gray, 1843

Family Attemsiidae Verhoeff, 1899 (25)

Family Craspedosomatidae Gray, 1843 (190)

Family Haplobainosomatidae Verhoeff, 1909 (19) 
Table 1 (continued). Suborders, superfamilies and families of Chordeumatida, according to Enghoff et al. (2015) with recent modifications:

1. Family Anthogonidae Ribaut, 1913 has been reinstated and added to superfamily Anthroleucosomatoidea; it includes Acherosomatidae Verhoeff, 1929 and Biokoviellidae Mršić, 1992 as junior synonyms (Antić et al. 2015a, 2016).

2. Family Dalmatosomatidae Antić \& Makarov, 2018, was described as new and placed in Branneroidea by Antić et al. (2018c).

3. Family Hungarosomatidae Ceuca,1974, was reinstated by Mock et al. (2016) but not assigned to a superfamily. Based on the discussion by Mock et al. (2016), it is here placed as Craspedosomatidea incertae sedis.

Families covered by the present volume are shown in bold and are followed by the number of known European species.

Superfamily Haaseoidea Attems, 1899

Family Haaseidae Attems, 1899 (26)

Superfamily Neoatractosomatoidea Verhoeff, 1901

Family Cyrnosomatidae Mauriès, 2015 (3)

Family Guizhousomatidae Mauriès, 2005

Family Hoffmaneumatidae Golovatch, 1978

Family Kirkayakidae Özdikmen, 2008

Family Mastigophorophyllidae Verhoeff, 1899 (35)

Family Neoatractosomatidae Verhoeff, 1901 (22)

Superfamily Verhoeffioidea Verhoeff, 1899

Family Verhoeffiidae Verhoeff, 1899 (4)

Craspedosomatidea incertae sedis

Family Hungarosomatidae Ceuca, 1974 (2)

Suborder Heterochordeumatidea Shear, 2000

Superfamily Conotyloidea Cook, 1896

Family Adritylidae Shear, 1971

Family Conotylidae Cook, 1896

Superfamily Diplomaragnoidea Attems, 1907

Family Diplomaragnidae Attems, 1907 (1)

Superfamily Heterochordeumatoidea Pocock, 1894

Family Eudigonidae Verhoeff, 1914

Family Heterochordeumatidae Pocock, 1894

Family Megalotylidae Golovatch, 1978

Family Metopidiotrichidae Attems, 1907

Family Peterjohnsiidae Mauriès, 1987

Superfamily Pygmaeosomatoidea Carl, 1941

Family Lankasomatidae Mauriès, 1978

Family Pygmaeosomatidae Carl, 1941

Suborder Striariidea Cook, 1896

Superfamily Caseyoidea Verhoeff, 1909

Family Caseyidae Verhoeff, 1909

Family Urochordeumatidae Silvestri, 1909

Superfamily Striarioidea Bollman, 1893

Family Apterouridae Loomis, 1966

Family Buotidae Shear, 2009

Family Rhiscosomididae Silvestri, 1909

Family Striariidae Bollman, 1893 
Table 2 (continued on the next 11 pages). Species covered by the present atlas.

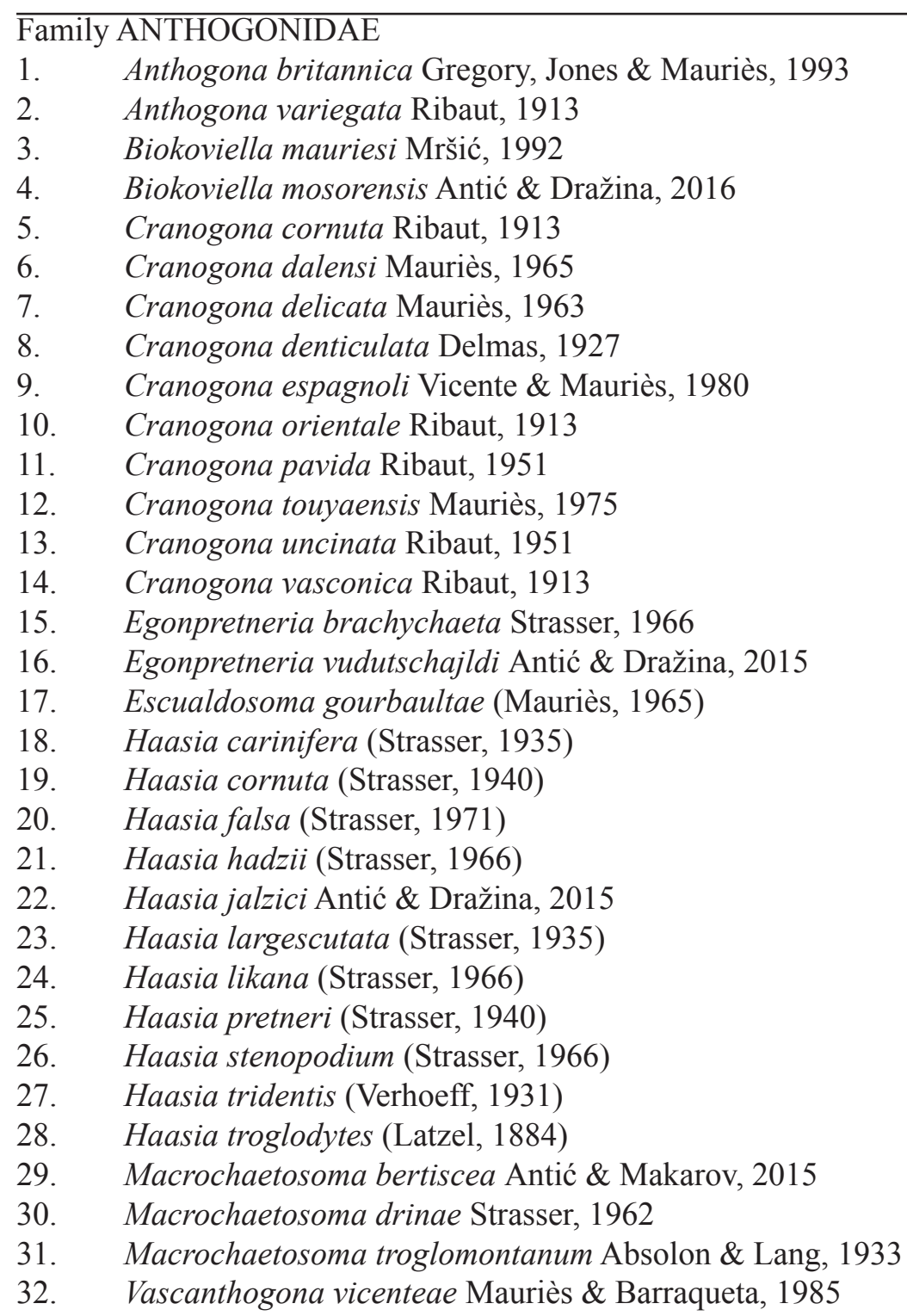

Family ANTHROLEUCOSOMATIDAE

33. Anamastigona alba (Strasser, 1960)

34. Anamastigona albanensis Mauriès, Golovatch \& Stoev, 1997

35. Anamastigona aspromontis (Strasser, 1970)

36. Anamastigona bilselii (Verhoeff, 1940)

37. Anamastigona delcevi (Strasser, 1973)

38. Anamastigona falcata (Gulička, 1967)

39. Anamastigona halophila (Verhoeff, 1940)

40. Anamastigona hauseri (Strasser,1974)

41. Anamastigona hispidula (Silvestri, 1894)

42. Anamastigona lepenicae (Strasser, 1975)

43. Anamastigona matsakisi Mauriès \& Karamouna, 1984

44. Anamastigona mediterranea Ćurčić, Makarov \& Lymberakis, 2001

45. Anamastigona meridionalis Silvestri, 1898

46. Anamastigona penicillata (Attems, 1902) 
Table 2 (continued). Species covered by the present atlas.

\begin{tabular}{ll}
\hline 47. & Anamastigona pentelicona (Verhoeff, 1925) \\
48. & Anamastigona pulchella (Silvestri, 1894) \\
49. & Anamastigona radmani Makarov, Rađa, Rađa, Tomić, Mitić \& Curcić, 2007 \\
50. & Anthroleucosoma banaticum Verhoeff, 1899 \\
51. & Anthroleucosoma spelaeum Ceuca, 1964 \\
52. & Banatosoma ocellatum (Tabacaru, 1967) \\
53. & Belbogosoma bloweri Ćurčić \& Makarov, 2008 \\
54. & Belbogosoma stribogi Antić \& Makarov, 2014 \\
55. & Bulgardicus bucarestensis Tabacaru \& Giurginca, 2006 \\
56. & Bulgardicus tranteevi Strasser, 1960 \\
57. & Bulgarosoma bureschi Verhoeff, 1926 \\
58. & Bulgarosoma superficiei Strasser, 1975 \\
59. & Camptogona delamarei Mauriès, 1969 \\
60. & Camptogona duboscqui (Brölemann, 1903) \\
61. & Cornogonopus pavicevici Antić, 2020 \\
62. & Dacosoma motasi Tabacaru, 1967 \\
63. & Dazbogosoma naissi Makarov \& Ćurčić, 2012 \\
64. & Krueperia nivalis Verhoeff, 1900 \\
65. & Perunosoma trojanicum Ćurčić \& Makarov, 2007 \\
66. & Rhodoposoma rhodopinum (Strasser, 1966) \\
67. & Serbosoma beljanicae (Ćurčić \& Makarov, 1998) \\
68. & Serbosoma crucis (Strasser, 1960) \\
69. & Serbosoma kucajense (Ćurčić \& Makarov, 1998) \\
70. & Serbosoma lazarevense (Ceuca, 1964) \\
71. & Serbosoma zagubicae (Ćurčić \& Makarov, 1998) \\
72. & Stygiosoma beroni Gulička, 1967 \\
73. & Svarogosoma bozidarcurcici Makarov, 2003 \\
74. & Troglodicus meridionalis (Tabacaru, 1967) \\
75. & Troglodicus tridentifer Gulička, 1967
\end{tabular}

\section{Family ATTEMSIIDAE}

76. Allorhiscosoma sphinx (Verhoeff, 1907)

77. Attemsia coniuncta Strasser, 1939

78. Attemsia dolinensis Verhoeff, 1909

79. Attemsia falcifera Verhoeff, 1899

80. Attemsia likana Strasser, 1966

81. Attemsia stygia (Latzel, 1884)

82. Coelogonium cavernarum Strasser, 1937

83. Dendromonomeron oribates (Latzel, 1884)

84. Dimastosternum franzi Attems, 1949

85. Dimastosternum holdhausi Attems, 1927

86. Eurygonium alticola (Strasser, 1937)

87. Glomogonium karawankarum Strasser, 1965

88. Grassographia makolensis Mršić, 1987

89. Julialpium alabardatum (Strasser, 1937)

90. Mecogonopodium bohiniense Strasser, 1933

91. Mecogonopodium carpathicum Mock \& Tajovský, 2008

92. Mecogonopodium zirianum Mršić, 1987 
Table 2 (continued). Species covered by the present atlas.

93. Polyphematia moniliformis (Latzel, 1884)

94. Schubartia lohmanderi Verhoeff, 1927

95. Stiphrogonium attemsi Strasser, 1937

96. Symphyosphys serkoi Strasser, 1939

97. Syngonopodium aceris Verhoeff, 1913

98. Syngonopodium cornutum Verhoeff, 1929

99. Tylogonium hoelzeli Strasser, 1959

100. Tylogonium nivifidele Strasser, 1937

Family BETICOSOMATIDAE

101. Beticosoma longipenis Mauriès, 1990

Family BRACHYCHAETEUMATIDAE

102. Brachychaeteuma bagnalli Verhoeff, 1911

103. Brachychaeteuma bradeae (Brölemann, H.K. Brade-Birks \& S.G. Brade-Birks, 1917)

104. Brachychaeteuma cadurcense Mauriès, 1967

105. Brachychaeteuma furcatum Ribaut, 1956

106. Brachychaeteuma melanops H.K. Brade-Birks \& S.G. Brade-Birks, 1919

107. Brachychaeteuma peniculatum Ribaut, 1948

108. Brachychaeteuma plumosum Ribaut, 1947

109. Brachychaeteuma provinciale Ribaut, 1956

Family CHAMAESOMATIDAE

110. Asturasoma chapmani Mauriès, 1981

111. Asturasoma fowleri Mauriès, 1981

112. Chamaesoma broelemanni Ribaut \& Verhoeff, 1913

113. Coiffaiteuma turdetanorum Mauriès, 1964

114. Krauseuma viscaianum Mauriès \& Barraqueta, 1985

115. Marboreuma brouquissei Mauriès, 1988

116. Origmatogona catalonica Ribaut, 1913

117. Origmatogona jacetanorum Mauriès, 1964

118. Origmatogona kimeorum Mauriès, 1990

119. Origmatogona tinauti Mauriès, 1990

120. Origmatogona toniperezi Mauriès, 2014

121. Scutogona alba Schubart, 1958

122. Scutogona ferrolensis Mauriès, 2015

123. Scutogona jeanneli Ribaut, 1913

124. Scutogona minor Enghoff \& Reboleira, 2013

125. Scutogona mutica Ribaut, 1913

126. Scutogona oculinigra Mauriès \& Vicente, 1977

127. Scutogona suboculinigra Mauriès, 2015

128. Scutogona vivesi Mauriès \& Vicente, 1977

129. Vascosoma coiffaiti Mauriès, 1966

130. Vascosoma duprei Mauriès, 1990

131. Verhoeffeuma minellii Mauriès, 1990

132. Verhoeffeuma spinosum Strasser, 1937

133. Xystrosoma beatense Ribaut, 1927

134. Xystrosoma cassagnaui Mauriès, 1965 
Table 2 (continued). Species covered by the present atlas.

135. Xystrosoma catalonicum Ribaut, 1927

136. Xystrosoma coiffaiti Mauriès, 1964

137. Xystrosoma lusitanicum Mauriès, 2015

138. Xystrosoma murinum Ribaut, 1927

139. Xystrosoma pyrenaicum Ribaut, 1927

140. Xystrosoma santllorence Serra \& Mauriès, 2018

141. Xystrosoma tectosagum Ribaut, 1927

142. Xystrosoma vasconicum Mauriès \& Barraqueta, 1985

Family CHORDEUMATIDAE

143. Chordeuma consoranense Ribaut, 1956

144. Chordeuma iluronense Ribaut, 1913

145. Chordeuma inornatum Ribaut, 1913

146. Chordeuma intermedium Ribaut, 1913

147. Chordeuma montanum Ribaut, 1956

148. Chordeuma muticum Ribaut, 1913

149. Chordeuma proximum Ribaut, 1913

150. Chordeuma reflexum Brolemann, 1927

151. Chordeuma sylvestre C.L.Koch, 1847

152. Chordeuma trifidum Ribaut, 1913

153. Chordeuma utriculosum Ribaut, 1913

154. Chordeuma vasconicum Ribaut, 1913

155. Melogona broelemanni (Verhoeff, 1897)

156. Melogona gallica (Latzel, 1884)

157. Melogona scutellare (Ribaut, 1913)

158. Melogona transsilvanica (Verhoeff, 1897)

159. Melogona voigti (Verhoeff, 1899)

160. Mycogona germanica (Verhoeff, 1892)

161. Orthochordeumella fulva (Rothenbühler, 1899)

162. Orthochordeumella leclerci Mauriès, 1985

163. Orthochordeumella pallida (Rothenbühler, 1899)

164. Orthochordeumella pyrenaica Mauriès, 1965

165. Parachordeuma broelemanni Ribaut, 1912

Family CRASPEDOSOMATIDAE

166. Aspromontia ruffoi Strasser, 1970

167. Atractosoma abnorme Verhoeff, 1900

168. Atractosoma blechnorum Verhoeff, 1936

169. Atractosoma cavannae Silvestri, 1898

170. Atractosoma cecconii Silvestri, 1898

171. Atractosoma confine Berlese, 1895

172. Atractosoma divaricatum Strasser, 1981

173. Atractosoma ghidinii Manfredi, 1935

174. Atractosoma gibberosum Verhoeff, 1900

175. Atractosoma marinense Verhoeff, 1932

176. Atractosoma meridionale Fanzago, 1876

177. Atractosoma paolettii (Strasser, 1977)

178. Atractosoma ruffoi Manfredi, 1940 
Table 2 (continued). Species covered by the present atlas.

179. Atractosoma tellinense Brölemann, 1892

180. Atractosoma troglobium Manfredi, 1930

181. Autaretia aliciae Geoffroy \& Mauriès, 2017

182. Autaretia osellai Strasser, 1978

183. Basigona athesina (Fedrizzi, 1877)

184. Bergamosoma bergomatium (Verhoeff, 1925)

185. Bergamosoma canestrinii (Fedrizzi, 1877)

186. Bergamosoma grottoloi (Strasser, 1973)

187. Bergamosoma hessei (Verhoeff, 1931)

188. Bergamosoma plavis (Strasser, 1960)

189. Bergamosoma sevini (Verhoeff, 1931)

190. Bomogona helvetica (Verhoeff, 1894)

191. Bomogona lombardica (Brölemann, 1892)

192. Brentosoma nivale Verhoeff, 1932

193. Broelemanneuma furcatum Ribaut, 1913

194. Broelemanneuma gayi Demange, 1968

195. Broelemanneuma gineti Ribaut, 1954

196. Broelemanneuma palmatum (Brölemann, 1902)

197. Broelemanneuma pectiniger (Brölemann, 1902)

198. Carniosoma verhoeffi (Attems, 1927)

199. Chelogona carpathicum (Latzel, 1882)

200. Corsicosoma legeri (Brölemann, 1903)

201. Craspedosoma blaniulides Latzel, 1900

202. Craspedosoma brentanum Verhoeff, 1926

203. Craspedosoma doranum Verhoeff, 1932

204. Craspedosoma fontanellum Attems, 1927

205. Craspedosoma furculigerum Verhoeff, 1936

206. Craspedosoma italicum Silvestri, 1903

207. Craspedosoma levicanum Fedrizzi, 1876

208. Craspedosoma montenigrinum Mršić, 1987

209. Craspedosoma oropense Verhoeff, 1936

210. Craspedosoma raulinsii Leach, 1814

211. Craspedosoma ruborum Verhoeff, 1930

212. Craspedosoma slavum Attems, 1929

213. Craspedosoma taurinorum Silvestri, 1898

214. Craspedosoma trilobum Silvestri, 1903

215. Crossosoma brolemanni Strasser, 1975

216. Crossosoma casalei Strasser, 1979

217. Crossosoma cavernicola (Manfredi, 1951)

218. Crossosoma falciferum Strasser, 1975

219. Crossosoma fossum Strasser, 1979

220. Crossosoma mauriesi Strasser, 1970

221. Crossosoma parvum Strasser, 1979

222. Crossosoma peyerimhoffi (Brölemann, 1902)

223. Crossosoma phantasma Strasser, 1970

224. Crossosoma semipes (Strasser, 1958)

225. Dactylophorosoma albocarinatum Manfredi, 1940 
Table 2 (continued). Species covered by the present atlas.

226. Dactylophorosoma nivisatelles Verhoeff, 1900

227. Dyocerasoma biokovense Mršić, 1986

228. Dyocerasoma drimicum Mršić, 1985

229. Dyocerasoma furcilliferum (Verhoeff, 1897)

230. Dyocerasoma insulanum Attems, 1951

231. Dyocerasoma intermedium Makarov, Lučić, Mitić \& Rađa, 2003

232. Dyocerasoma lignivorum (Verhoeff, 1899)

233. Dyocerasoma narentanum (Verhoeff, 1901)

234. Dyocerasoma nivisatelles (Verhoeff, 1897)

235. Helvetiosoma blanci (Faës, 1902)

236. Helvetiosoma helveticum (Verhoeff, 1900)

237. Helvetiosoma montemorense Faës, 1905

238. Iulogona apenninorum (Verhoeff, 1913)

239. Iulogona hamuligera (Verhoeff, 1913)

240. Iulogona ligurina (Verhoeff, 1910)

241. Iulogona tirolensis (Verhoeff, 1894)

242. Janetschekella valesiaca (Faës, 1902)

243. Kelempekia martensi Strasser, 1974

244. Listrocheiritium bohemicum (Rosický, 1876)

245. Listrocheiritium cervinum Verhoeff, 1925

246. Listrocheiritium noricum Verhoeff, 1913

247. Listrocheiritium nubium Verhoeff, 1915

248. Listrocheiritium septentrionale Gulićka, 1965

249. Listrocheiritium styricum Verhoeff, 1915

250. Listrocheiritium susurrinum Attems, 1926

251. Litogona hyalops (Latzel, 1889)

252. Litogona mirabilis (Manfredi, 1948)

253. Manfredia aemiliana (Manfredi,1932)

254. Manfredia apuana Strasser, 1971

255. Manfredia concii Manfredi, 1953

256. Manfredia guareschii Manfredi, 1950

257. Manfredia lanzai Manfredi, 1948

258. Nanogona balazuci (Schubart, 1958)

259. Nanogona cebennica (Ribaut, 1947)

260. Nanogona davidi (Demange, 1966)

261. Nanogona digitata (Ribaut, 1913)

262. Nanogona polydesmoides (Leach, 1814)

263. Nanogona uncinata (Ribaut, 1913)

264. Ochogona apfelbecki (Verhoeff, 1897)

265. Ochogona attemsi (Verhoeff, 1907)

266. Ochogona brentana (Verhoeff, 1927)

267. Ochogona caroli (Rothenbühler, 1900)

268. Ochogona cervina (Verhoeff, 1899)

269. Ochogona condylocoxa (Attems, 1899)

270. Ochogona elaphron (Attems, 1895)

271. Ochogona euganeorum (Verhoeff, 1927)

272. Ochogona friulana (Strasser, 1937) 
Table 2 (continued). Species covered by the present atlas.

273. Ochogona gallitarum (Brölemann, 1900)

274. Ochogona hanfi (Attems, 1926)

275. Ochogona holdhausi (Attems, 1926)

276. Ochogona jankowskii (Jawłowski, 1938)

277. Ochogona latzeli (Attems, 1927)

278. Ochogona manfredii (Strasser, 1942)

279. Ochogona phyllophaga (Attems, 1899)

280. Ochogona pusilla (Verhoeff, 1893)

281. Ochogona regalis (Verhoeff, 1913)

282. Ochogona triaina (Attems, 1895)

283. Oroposoma catascaphium Verhoeff, 1936

284. Oroposoma emiliae Manfredi, 1953

285. Oroposoma fagorum Verhoeff, 1936

286. Oroposoma granitivagum Verhoeff, 1936

287. Oroposoma nivale (Faës, 1902)

288. Oroposoma ticinense Manfredi, 1957

289. Oroposoma varallense Verhoeff, 1936

290. Paradactylophorosoma insulanum (Attems, 1908)

291. Pedemontia delmastroi Mauriès, 1994

292. Plectogona angusta (Latzel, 1887)

293. Plectogona bonzanoi (Strasser, 1975)

294. Plectogona franciscoloi (Manfredi, 1953)

295. Plectogona morisii (Strasser, 1975)

296. Plectogona sanfillipoi (Manfredi, 1956)

297. Plectogona vignai (Strasser, 1970)

298. Pterygophorosoma alticolum (Verhoeff, 1894)

299. Pterygophorosoma cornuigerum (Verhoeff, 1900)

300. Pyrgocyphosoma armigerum Verhoeff, 1925

301. Pyrgocyphosoma arvernum (Ribaut \& Brolemann, 1932)

302. Pyrgocyphosoma aspidiorum Verhoeff, 1931

303. Pyrgocyphosoma balazuci Mauriès \& Kime, 1999

304. Pyrgocyphosoma bidentatum (Verhoeff, 1900)

305. Pyrgocyphosoma brembanum Verhoeff, 1931

306. Pyrgocyphosoma brunatense (Verhoeff, 1910)

307. Pyrgocyphosoma centrale (Silvestri, 1898)

308. Pyrgocyphosoma dalmazzense Verhoeff, 1930

309. Pyrgocyphosoma dentatum (Brölemann, 1892)

310. Pyrgocyphosoma doriae (Silvestri, 1898)

311. Pyrgocyphosoma edrinum Verhoeff, 1934

312. Pyrgocyphosoma florentinum (Silvestri, 1903)

313. Pyrgocyphosoma fonticuli Verhoeff, 1936

314. Pyrgocyphosoma gattii (Silvestri, 1898)

315. Pyrgocyphosoma grassii (Silvestri, 1898)

316. Pyrgocyphosoma jucundum (Brolemann, 1935)

317. Pyrgocyphosoma ligusticum (Silvestri, 1898)

318. Pyrgocyphosoma longilamellatum Verhoeff, 1931

319. Pyrgocyphosoma marmorense Verhoeff, 1932 
Table 2 (continued). Species covered by the present atlas.

320. Pyrgocyphosoma marrucinum Manfredi, 1950

321. Pyrgocyphosoma mevaniense (Silvestri, 1894)

322. Pyrgocyphosoma oppidicola (Silvestri, 1898)

323. Pyrgocyphosoma ormeanum Verhoeff, 1930

324. Pyrgocyphosoma picenum Manfredi, 1953

325. Pyrgocyphosoma pontremolense Verhoeff, 1936

326. Pyrgocyphosoma pracchiense Verhoeff, 1932

327. Pyrgocyphosoma quercuum Verhoeff, 1936

328. Pyrgocyphosoma ravinense Verhoeff, 1936

329. Pyrgocyphosoma reatinum Strasser, 1977

330. Pyrgocyphosoma renanum Verhoeff, 1932

331. Pyrgocyphosoma roccavionense Verhoeff, 1937

332. Pyrgocyphosoma savonense (Verhoeff, 1910)

333. Pyrgocyphosoma serianum Verhoeff, 1937

334. Pyrgocyphosoma serpentinum Verhoeff, 1932

335. Pyrgocyphosoma serravallense Verhoeff, 1936

336. Pyrgocyphosoma tendanum Verhoeff, 1930

337. Pyrgocyphosoma terminilli Strasser, 1977

338. Pyrgocyphosoma titianum (Verhoeff, 1910)

339. Pyrgocyphosoma tridentinum (Silvestri, 1898)

340. Pyrgocyphosoma vallicola (Silvestri, 1898)

341. Pyrgocyphosoma vallombrosae (Silvestri, 1898)

342. Pyrgocyphosoma zangherii Manfredi, 1951

343. Rhymogona hessei (Ravoux, 1935)

344. Rhymogona montivaga (Verhoeff, 1894)

345. Rhymogona serrata (Bigler, 1912)

346. Rhymogona verhoeffi (Bigler, 1913)

347. Rhymogona wehrana (Verhoeff, 1910)

348. Rothenbuehleria minima (Rothenbühler, 1899)

349. Rothenbuehleria tirolensis Verhoeff, 1900

350. Sardosoma franchettii Manfredi, 1956

351. Semiosoma bordei Ribaut, 1913

352. Semiosoma devillei (Brölemann, 1901)

353. Semiosoma minutum (Berlese, 1894)

354. Synischiosoma argentarium Attems, 1927

355. Synischiosoma murorum (Silvestri, 1902)

Family CYRNOSOMATIDAE

356. Cyrnosoma beroni (Mauriès, 1969)

357. Cyrnosoma coineaui (Mauriès, 1969)

358. Cyrnosoma strasseri (Mauriès, 1969)

Family DALMATOSOMATIDAE

359. Dalmatosoma agaricum Antić \& Makarov, 2018

Family DIPLOMARAGNIDAE

360. Altajosoma golovatchi (Shear, 1990)

Family ENTOMOBIELZIIDAE

361. Entomobielzia getica Ceuca, 1964

362. Entomobielzia kimakowizii (Verhoeff, 1897) 
Table 2 (continued). Species covered by the present atlas.

\begin{tabular}{|c|c|}
\hline $\begin{array}{l}363 . \\
364 .\end{array}$ & $\begin{array}{l}\text { Entomobielzia varvarai Ceuca, } 1985 \\
\text { Pseudoclis octocera Attems, } 1899\end{array}$ \\
\hline \multicolumn{2}{|c|}{ Family HAASEIDAE } \\
\hline 365. & Haasea cyanopida (Attems, 1903) \\
\hline 366. & Haasea faucium (Verhoeff, 1931) \\
\hline 367. & Haasea filicis (Verhoeff, 1929) \\
\hline 368. & Haasea flavescens (Latzel, 1884) \\
\hline 369. & Haasea fonticulorum (Verhoeff, 1910) \\
\hline 370. & Haasea germanica (Verhoeff, 1901) \\
\hline 371. & Haasea gruberi Antić \& Akkari, 2020 \\
\hline 372. & Haasea hungarica (Verhoeff, 1928) \\
\hline 373. & Haasea inflata (Verhoeff, 1907) \\
\hline 374. & Haasea intermedia Mršić, 1985 \\
\hline 375. & Haasea lacusnigri (Gulička, 1968) \\
\hline 376. & Haasea makarovi Antić \& Akkari, 2020 \\
\hline 377. & Haasea microcorna (Strasser, 1971) \\
\hline 378. & Haasea musimontium (Strasser, 1937) \\
\hline 379. & Haasea plasana (Verhoeff, 1899) \\
\hline 380. & Haasea pretneri (Strasser, 1966) \\
\hline 381. & Haasea vidinensis (Strasser, 1973) \\
\hline 382. & Hylebainosoma birtei (Ceuca, 1967) \\
\hline 383. & Hylebainosoma cavernicola (Ceuca, 1967) \\
\hline 384. & Hylebainosoma gulickai Tajovský, Mock \& Papáč, 2014 \\
\hline 385. & Hylebainosoma nontronense Mauriès \& Kime, 1999 \\
\hline 386. & Hylebainosoma odici (Ceuca, 1967) \\
\hline 387. & Hylebainosoma oltenicum (Ceuca, 1967) \\
\hline 388. & Hylebainosoma tatranum Verhoeff, 1899 \\
\hline & Xylophageuma vomrathi Verhoeff, 1911 \\
\hline 390. & Xylophageuma zschokkei Bigler, 1912 \\
\hline
\end{tabular}

\section{Family HAPLOBAINOSOMATIDAE}

391. Cantabrosoma rogeri Mauriès, 1970

392. Cantabrosoma serrai Mauriès \& Vicente, 1977

393. Galicisoma biltoni Mauriès, 2015

394. Galicisoma desmondkimei Mauriès, 2015

395. Guadarramasoma ramosae Gilgado, Ledesma, Enghoff \& Mauriès, 2017

396. Haplobainosoma lusitanum Verhoeff, 1899

397. Pyreneosoma aranense Mauriès, 2010

398. Pyreneosoma barbieri (Mauriès, 1971)

399. Pyreneosoma bessoni Mauriès, 1974

400. Pyreneosoma birosense Mauriès, 2010

401. Pyreneosoma consoranense Mauriès, 2010

402. Pyreneosoma convenarense Mauriès, 2010

403. Pyreneosoma digitatum Mauriès, 1959

404. Pyreneosoma grandicoxae Mauriès, 2010

405. Pyreneosoma ribauti Mauriès, 1959

406. Pyreneosoma vicdessosense Mauriès, 2010

407. Turdulisoma galiciense Mauriès, 2015 
Table 2 (continued). Species covered by the present atlas.

408. Turdulisoma helenreadae Mauriès, 2015

409. Turdulisoma turdulorum Mauriès, 1964

Family HETEROLATZELIIDAE

410. Heterolatzelia durmitorensis Gulička, 1968

411. Heterolatzelia karlstrasseri Antić \& Makarov, 2015

412. Heterolatzelia nivalis Verhoeff, 1897

413. Massarilatzelia dugopoljica Makarov \& Rađa, 2011

Family HUNGAROSOMATIDAE

414. Hungarosoma bokori Verhoeff, 1928

415. Hungarosoma inexpectatum Ceuca, 1967

Family LUSITANIOSOMATIDAE

416. Lusitaniosoma machadoi Schubart, 1953

Family MASTIGOPHOROPHYLLIDAE

417. Bucovinosoma capusei Tabacaru, 1978

418. Haploporatia cervina Verhoeff, 1929

419. Haploporatia eremita Verhoeff, 1909

420. Haploporatia similis (Attems, 1895)

421. Heterobraueria karoli Verhoeff, 1897

422. Heterobraueria scopifera Verhoeff, 1898

423. Karpatophyllon banaticum Ceuca, 1989

424. Karpatophyllon carpaticum Ceuca, 1985

425. Karpatophyllon dacicum Ceuca, 1964

426. Karpatophyllon polinskii Jawłowski, 1928

427. Mastigona bosniensis (Verhoeff, 1897)

428. Mastigona mutabilis (Latzel, 1884)

429. Mastigona transsylvanica (Verhoeff, 1897)

430. Mastigophorophyllon aberratum Ceuca, 1985

431. Mastigophorophyllon alpivagum (Verhoeff, 1897)

432. Mastigophorophyllon banarescui Ceuca, 1976

433. Mastigophorophyllon bulgaricum Schubart, 1934

434. Mastigophorophyllon carpaticum Ceuca, 1976

435. Mastigophorophyllon cirriferum Verhoeff, 1899

436. Mastigophorophyllon crinitum Attems, 1926

437. Mastigophorophyllon deubeli Verhoeff, 1898

438. Mastigophorophyllon jickelii Verhoeff, 1900

439. Mastigophorophyllon moldavicum Ceuca, Crisan \& Olaru, 1996

440. Mastigophorophyllon parapenicilligerum Crisan \& Ceuca, 1998

441. Mastigophorophyllon penicilligerum Verhoeff, 1899

442. Mastigophorophyllon saxonicum Verhoeff, 1910

443. Mastigophorophyllon serrulatum Attems, 1926

444. Paraporatia racovitzai Ceuca, 1967

445. Taurinosoma graniticola Verhoeff, 1932

446. Tessinosoma caelebs Verhoeff, 1911

447. Thaumaporatia apenninorum Verhoeff, 1909

448. Thaumaporatia apuana Verhoeff, 1909 
Table 2 (continued). Species covered by the present atlas.

449. Thaumaporatia oropensis Verhoeff, 1936

450. Thaumaporatia plumigera (Verhoeff, 1900)

451. Thaumaporatia sorattina Verhoeff, 1951

Family NEOATRACTOSOMATIDAE

452. Epirosomella loebli Strasser, 1976

453. Fagina silvatica (Attems, 1904)

454. Microbrachysoma alpestre Verhoeff, 1897

455. Neoatractosoma herzegowinense Verhoeff, 1901

456. Neoatractosoma kleinenbergi Silvestri, 1898

457. Neoatractosoma strandi Attems, 1927

458. Osellasoma caoduroi Mauriès, 1984

459. Paeonisoma faucium Verhoeff, 1932

460. Pseudocraspedosoma alpivagum (Verhoeff, 1901)

461. Pseudocraspedosoma bensai (Manfredi, 1935)

462. Pseudocraspedosoma brentanum (Verhoeff, 1930)

463. Pseudocraspedosoma falteronense (Manfredi, 1951)

464. Pseudocraspedosoma grypischium (Rothenbühler, 1900)

465. Pseudocraspedosoma nemorense Silvestri, 1898

466. Pseudocraspedosoma peniculorum (Verhoeff, 1910)

467. Pseudocraspedosoma vestonense (Verhoeff, 1934)

468. Schizmohetera curcici Makarov, 2001

469. Schizmohetera olympica Mauriès, 2003

470. Schizmohetera sketi Mršić, 1987

471. Trimerophorella ornata (Faës, 1902)

472. Trimerophorella paradisia Meyer, 1983

473. Trimerophorella rhaetica (Rothenbühler, 1901)

Family OPISTHOCHEIRIDAE

474. Brachytropisoma giardinae Silvestri, 1898

475. Ceratosphys amoena Ribaut, 1920

476. Ceratosphys angelieri Mauriès, 1964

477. Ceratosphys bakeri Mauriès, 1990

478. Ceratosphys banyulensis Brolemann, 1926

479. Ceratosphys cryodeserti Gilgado, Mauriès \& Enghoff, 2015

480. Ceratosphys deharvengi Mauriès, 1978

481. Ceratosphys escolai Mauriès, 2013

482. Ceratosphys fernandoi Mauriès, 2014

483. Ceratosphys flammeola Mauriès, 2014

484. Ceratosphys geronensis Mauriès, 1963

485. Ceratosphys guttata Ribaut, 1956

486. Ceratosphys hispanica Ribaut, 1920

487. Ceratosphys jabaliensis Mauriès, 2013

488. Ceratosphys mariacristinae Mauriès, 2013

489. Ceratosphys nivium Ribaut, 1927

490. Ceratosphys nodipes (Attems, 1952)

491. Ceratosphys occidentalis Mauriès, 1976

492. Ceratosphys picta Ribaut, 1951 
Table 2 (continued). Species covered by the present atlas.

493. Ceratosphys poculifer (Brolemann, 1920)

494. Ceratosphys simoni Ribaut, 1920

495. Ceratosphys solanasi (Mauriès \& Vicente, 1978)

496. Ceratosphys soutadei Mauriès, 1969

497. Ceratosphys toniserrai Mauriès, 2013

498. Ceratosphys vandeli Mauriès, 1963

499. Ceratosphys vicenteae Mauriès, 1990

500. Hispaniosoma racovitzai Ribaut, 1913

501. Marquetiella auriculata (Ribaut, 1920)

502. Marquetiella lunata (Ribaut, 1920)

503. Marquetiella pyrenaica (Ribaut, 1905)

504. Opisthocheiron canayerensis Mauriès \& Geoffroy, 1982

505. Opisthocheiron cornutum Ribaut, 1922

506. Opisthocheiron elegans Ribaut, 1922

507. Opisthocheiron fallax Ribaut, 1922

508. Opisthocheiron lacazei Brolemann, 1932

509. Opisthocheiron penicillatum Ribaut, 1913

510. Sireuma nobile Reboleira \& Enghoff, 2014

Family TRACHYGONIDAE

511. Acrochordum evae Loksa, 1960

512. Acrochordum flagellatum Attems, 1899

513. Acrochordeum plitvicense (Verhoeff, 1929)

514. Halleinosoma noricum Verhoeff, 1913

515. Trachygona capito (Attems, 1894)

Family VANDELEUMATIDAE

516. Alavasoma muniesai Mauriès \& Vicente, 1977

517. Eopsychrosoma serrapradense Serra \& Mauriès, 2015

518. Guipuzcosoma comasi Vicente \& Mauriès, 1980

519. Hypnosoma exornatum Ribaut, 1952

520. Hypnosoma juberthieorum Mauriès, 1968

521. Hypnosoma pallidum Ribaut, 1952

522. Miniusoma litorea Mauriès, 2015

523. Niphatrogleuma wildbergeri Mauriès, 1986

524. Psichrosoma baeticaense Mauriès, 2013

525. Psichrosoma breuili (Mauriès, 1970)

526. Psichrosoma tarraconense Mauriès, 1970

527. Strangulogona lugoensis Mauriès, 2015

528. Typhlopsychrosoma fadriquei (Mauriès \& Vicente, 1977)

529. Vandeleuma hispanicum Ceuca, 1967

530. Vandeleuma vasconicum Mauriès, 1966

Family VERHOEFFIIDAE

531. Haplogona carynthiaca (Strasser, 1967)

532. Haplogona gestri (Silvestri, 1898)

533. Haplogona oculodistincta (Verhoeff, 1893)

534. Haplogona rothenbuehleri (Verhoeff, 1900) 
Table 3. Geopolitical units in Europe as used in the present atlas and in Fauna Europaea.

\begin{tabular}{|c|c|}
\hline $\mathrm{AD}$ & Andorra \\
\hline AL & Albania \\
\hline AT & Austria \\
\hline BA & Bosnia-Herzegovina \\
\hline $\mathrm{BE}$ & Belgium \\
\hline BG & Bulgaria \\
\hline BY & Belarus \\
\hline $\mathrm{CH}$ & Switzerland \\
\hline $\mathrm{CZ}$ & Czech Republic \\
\hline $\mathrm{DE}$ & Germany \\
\hline DK-DEN & Danish mainland \\
\hline DK-FOR & Faroe Islands \\
\hline EE & Estonia \\
\hline ES-BAL & Balearic Islands \\
\hline ES-CNY & Canary Islands \\
\hline ES-SPA & Spanish mainland \\
\hline FI & Finland \\
\hline FR-COR & Corsica \\
\hline FR-FRA & French mainland \\
\hline GB-CI & Channel Islands \\
\hline GB-GI & Gibraltar \\
\hline GB-GRB & Great Britain, including Shetlands, Orkneys, Hebrides and Isle of Man \\
\hline GB-NI & Northern Ireland \\
\hline GR-CYC & Cyclades Islands \\
\hline GR-DOD & Dodecanese Islands \\
\hline GR-GRC & Greek mainland, including Ionian Islands, Evia, Sporades and North Aegean Islands \\
\hline GR-KRI & Crete, including small adjacent islands \\
\hline $\mathrm{HR}$ & Croatia \\
\hline HU & Hungary \\
\hline IE & Republic of Ireland \\
\hline IS & Iceland \\
\hline IT-ITA & Italian mainland \\
\hline IT-SAR & Sardinia \\
\hline IT-SI & Sicily, including all neighbouring Italian islands \\
\hline LI & Liechtenstein \\
\hline LT & Lithuania \\
\hline LU & Luxembourg \\
\hline LV & Latvia \\
\hline $\mathrm{MC}$ & Monaco \\
\hline MD & Republic of Moldova \\
\hline $\mathrm{MN}$ & Montenegro \\
\hline MK & North Macedonia \\
\hline MT & Malta \\
\hline NL & The Netherlands \\
\hline NO-NOR & Norwegian mainland and inshore islands \\
\hline NO-SVA & Svalbard, Jan Mayen and Bear Island \\
\hline PL & Poland \\
\hline PT-AZO & Azores Islands \\
\hline PT-MDR & Madeira Islands \\
\hline PT-POR & Portuguese mainland \\
\hline PT-SEL & Selvagens Islands \\
\hline $\mathrm{RO}$ & Romania \\
\hline RU-FJL & Franz Josef Land \\
\hline RU-KGD & Kaliningrad Region \\
\hline RU-NOZ & Novaya Zemlya \\
\hline RU-RUC & Central European Russia \\
\hline RU-RUE & East Central Russia \\
\hline RU-RUN & North European Russia \\
\hline RU-RUS & South European Russia \\
\hline RU-RUW & Northwest Russia \\
\hline SB & Serbia \\
\hline SE & Sweden \\
\hline SI & Slovenia \\
\hline SK & Slovakia \\
\hline SM & San Marino \\
\hline TR-TUE & European Turkey, including Imroz I.-Gökceada, but not islands in the Sea of Marmara \\
\hline UA & Ukraine \\
\hline VA & Vatican City \\
\hline
\end{tabular}


Habitat. Ecological information is given if known. It has, however, not been possible to examine every paper that has been published throughout Europe to find if such details are given. The habitat sections for each species are thus highly heterogeneous. When a paper includes an important contribution to the knowledge of the ecology of a species, we have quoted it, otherwise the information is an amalgam of that found in the original description and subsequent publications, together with our own experience in the field and unpublished information provided by colleagues. Publications which were particularly useful with regard to habitat information include Ribaut (1913), Schubart (1934), Pedroli-Christen (1993), Lee (2006), Berg et al. (2008), Gruber (2009), Reip et al. (2012) and Hauser \& Voigtländer (2019).

A large number of chordeumatidan millipedes live in forests. For the forest types (or other vegetation types) we have in general used the Latin names of the trees etc. involved (e.g., Quercus-Carpinus forest) rather than, e.g., 'oak-hornbeam forest' or 'Querceto-Carpinetum'. The latter types of descriptive terms have, however, been included in some cases where this information is in the form of a citation of particular papers.

Many chordeumatidans are restricted to mountains, even above the tree line, and we have tried to include known altitude ranges, expressed as 'meters above sea level' ( $\mathrm{m}$ a.s.l.). The ranges given are simply a compilation of what we have been able to trace in the literature.

Very many chordeumatidan species live underground, either in caves (i.e., cavities big enough for human collectors to enter) or in smaller underground spaces, especially the so-called 'mesovoid shallow substratum' = 'meso-cavernous shallow stratum' = milieu superficiel souterrarine $=$ MSS (see Eusébio et al. 2021). Some are confined to such habitats and are known as troglobionts, others (troglophiles and trogloxenes) may also occur in surface habitats. See Sendra et al. (2020) for a review of the classification of underground habitats. We have avoided any attempt to classify the species according to their degree of 'subterraneousness' and simply have written 'Cave' under habitat where applicable.

Remarks. May include notes on taxonomy and distribution, and/or may refer to particular studies on, e.g., reproduction, life cycle etc. Again, we do not claim completeness of coverage.

\section{The maps}

The maps record the presence of the species in unit fields corresponding to $50 \times 50 \mathrm{~km}$ squares of the UTM grid, or more precisely the Military Grid Reference System, MGRS. It should be noted that some of the 'squares' are actually not square because they are truncated on the left or right side where the UTM/MGRS longitudinal projection zones meet. Henceforward, the unit fields will nevertheless be referred to as ' $50 \mathrm{~km}$ squares'. Four different base maps are used:

- all of Europe, for species occurring north of ca $48^{\circ} \mathrm{N}$ (and/or the Macaronesian Islands, just three species);

- southwestern Europe, for species confined to the Iberian Peninsula and southern to central France;

- south-central Europe, for species confined to Italy and neighbouring areas;

- southeastern Europe, for species confined to the Balkans and neighbouring areas.

For referring a locality to the correct $50 \mathrm{~km}$ square, physical UTM maps were used, as well as GISsurfer (https://mappingsupport.com/p2/gissurfer.php). Where a locality was given with longitude and latitude, Legal Land Converter (https://legallandconverter.com/p50.html) was used. For batch conversion of lat long coordinates, Earthpoint (https://Earthpoint.us) was used.

The maps were made by André J. van Loon (EIS Kenniscentrum Insecten, Leiden, Netherlands), based on lists of $50 \times 50 \mathrm{~km}$ MGRS coordinates, using the 'Klasse' software distributed by the Nederlandse Entomologische Vereniging (Netherlands Entomological Society). 
A map is presented for each species: each species name is preceded by a number, which refers to the number of the corresponding map. Squares in which precisely located records occur are shown by blue dots in the centre of the $50 \mathrm{~km}$ square concerned. Because a record may refer to a corner of a 50 $\mathrm{km}$ square the centre of which is in another country/region some species seem to occur in countries from where they have not been recorded. Some records referable only to, e.g., a province, occasionally a country, are shown using a blue circle located at the centre of the province or similar in question. There are particularly many circles in France, representing numerous département-level records for this country. No distinction is made between old and new records; this is mainly because the number of specialist recorders has been relatively small and because they have operated over different time periods in many of the countries concerned. There are few recent records from some countries and few old records from others. Under these circumstances, using different symbols for different time periods would give a false impression of distribution changes. Moreover, there is little evidence that millipede distributions have changed much over the past century, with the possible exception of a few introduced or pioneer species. The coverage map (Fig. 6) shows all the $50 \mathrm{~km}$ squares from which records included in this volume have been reported. Thus, while interpreting the distribution of the species indicated on the maps it should be borne in mind that the coverage has been far from uniform at the European scale and, indeed, within individual countries. While there are exceptions, there has been a marked tendency for collectors to concentrate on the more interesting mountainous areas, especially in areas of known endemism, and to pay scant attention to the less biodiverse lowland plains. Even on the basis of units of $50 \mathrm{~km} \times 50 \mathrm{~km}$ squares, many squares have no records at all, especially in the larger countries. The records derive from numerous publications, as well as from several colleagues who have generously provided information (for a list of these, see Kime \& Enghoff 2017: 173). Many records

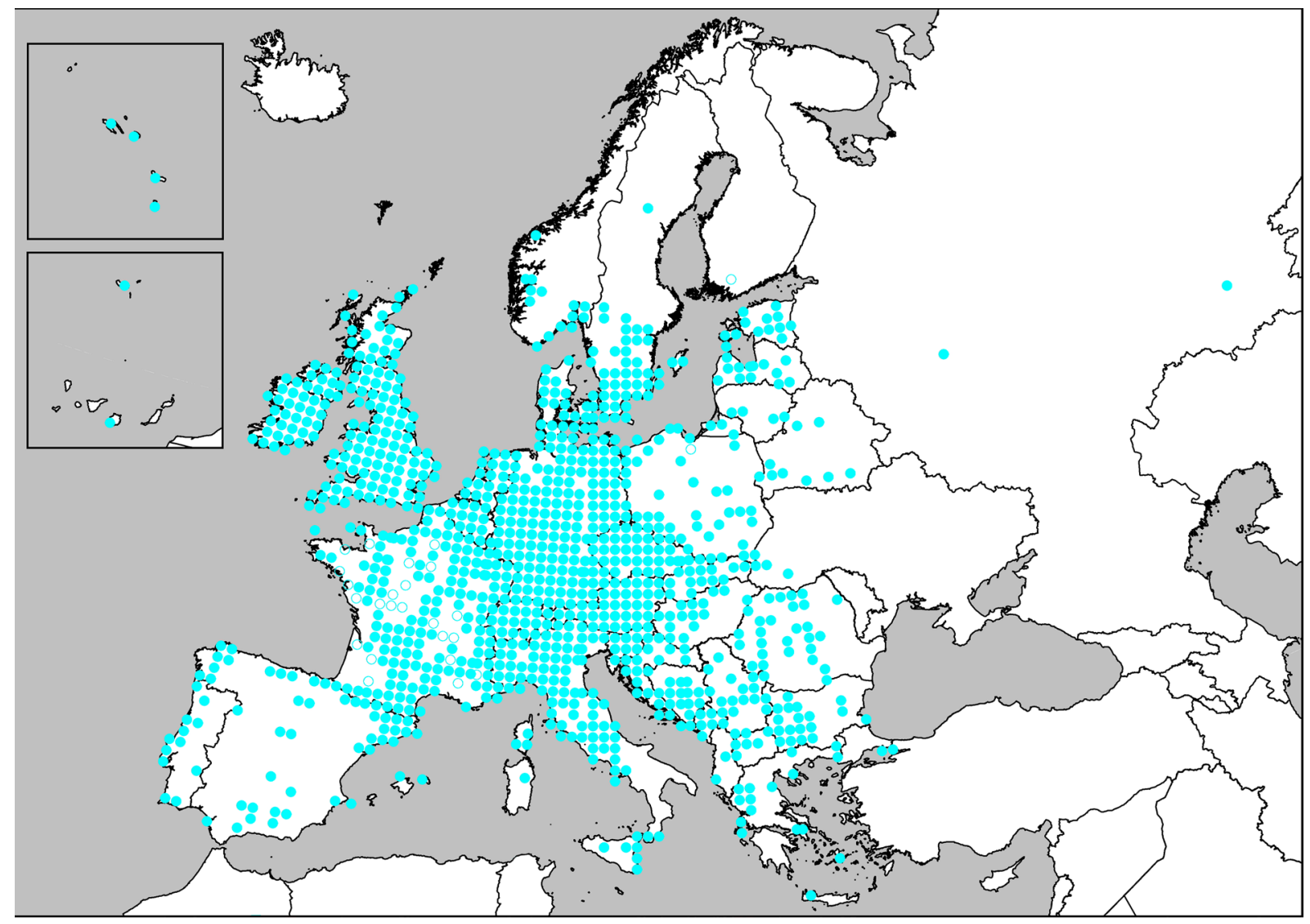

Fig. 6. Map showing all the $50 \mathrm{~km}$ squares which provided records in this volume. 
were extracted from Edaphobase (https://portal.edaphobase.org/), and some vouchered records were extracted from the Global Biodiversity Information Facility (www.gbif.org), but records based only on "Human observation" were not considered.

In most calcareous regions, speleologists have been very active; in some of these there are far more records of cavernicolous species than of species living in the vegetation, litter and soil. There are many true troglobionts, but, at the same time, many of the species that were first found in caves are strongly pigmented and are either known to occur, or most probably occur, on the surface.

In this atlas, 'Europe' extends from the Azores to the Ural Mountains in Russia: the European part of Russia is delimited as in Fauna Europaea (de Jong et al. 2014) which does not include the Caucasus area. In the Aegean area, this atlas covers all the islands belonging to Greece, while Cyprus and Asia Minor are not included. Only the European part of Turkey is covered. In the Atlantic, records from the Azores, Madeira and the Canary Islands are listed (just three species).

We have clearly not been able to find every published record and we have not been able to place some localities. Ambiguous or doubtful records have either been omitted or commented upon.

\section{Results}

Family Anthogonidae Ribaut, 1913

Including Acherosomatidae Verhoeff, 1929 (= Haasiidae Hoffman, 1980), Biokoviellidae Mršić, 1992, and Macrochaetosomatinae Mršić, 1992, following Antić et al. (2015a, 2016). All 32 species live in (S) $\mathrm{W}$ and SE Europe.

Genus Anthogona Ribaut, 1913

1. Anthogona britannica Gregory, Jones \& Mauriès, 1993

\section{Distribution}

GB-GRB.

\section{Habitat}

Collected from a variety of habitats including coastal cliffs, grassland, vegetated shingle and woodland, the majority of records from leaf litter samples below deciduous trees such as Quercus L., Fraxinus L. and Corylus L. or a ground cover of Hedera L.

\section{Anthogona variegata Ribaut, 1913}

\section{Distribution}

ES-SPA, FR-FRA.

\section{Habitat}

Collected from different habitats including woodland, leaf litter of deciduous forest dominated by Fagus L., Quercus and Carpinus L. Under moss at $1500 \mathrm{~m}$ a.s.1., at the upper forest limit. Accidentally in cave.

Genus Biokoviella Mršić, 1992

This genus was revised by Antić et al. (2016). 
3. Biokoviella mauriesi Mršić, 1992

\section{Distribution}

HR.

\section{Habitat}

Deep, cold caves at $1300 \mathrm{~m}$ a.s.l. and above.

4. Biokoviella mosorensis Antić \& Dražina, 2016

\section{Distribution}

HR.

\section{Habitat}

Cold caves.

Genus Cranogona Ribaut, 1913

5. Cranogona cornuta Ribaut, 1913

\section{Distribution}

ES-SPA.

\section{Habitat}

Cave.

6. Cranogona dalensi Mauriès, 1965

\section{Distribution}

FR-FRA, GB-GRB.

\section{Habitat}

In France, found in soil in an open habitat. In Wales, found at a colliery spoil heap.

7. Cranogona delicata Mauriès, 1963

\section{Distribution}

FR-FRA.

\section{Habitat}

A high mountain species.

8. Cranogona denticulata Delmas, 1927

\section{Distribution}

FR-FRA.

\section{Habitat}

Epigean. 


\section{Cranogona espagnoli Vicente \& Mauriès, 1980}

\section{Distribution}

ES-SPA.

Habitat

Cave.

10. Cranogona orientale Ribaut, 1913

\section{Distribution}

FR-FRA.

Habitat

Cave.

11. Cranogona pavida Ribaut, 1951

\section{Distribution}

FR-FRA.

\section{Habitat}

A high mountain species.

12. Cranogona touyaensis Mauriès, 1975

\section{Distribution}

FR-FRA.

\section{Habitat}

Cave.

13. Cranogona uncinata Ribaut, 1951

\section{Distribution}

FR-FRA.

\section{Habitat}

Among moss and leaf litter under Buxus L. and Corylus.

14. Cranogona vasconica Ribaut, 1913

\section{Distribution}

FR-FRA.

\section{Habitat}

Cave. 
KIME R.D. \& ENGHOFF H., Atlas of European millipedes 3: Order Chordeumatida

Genus Egonpretneria Strasser, 1966

15. Egonpretneria brachychaeta Strasser, 1966

\section{Distribution}

HR.

\section{Habitat}

Cave.

16. Egonpretneria vudutschajldi Antić \& Dražina, 2015

Fig. 4C

\section{Distribution}

HR.

\section{Habitat}

Cave.

Genus Escualdosoma Mauriès, 1965

Cranogona subgenus Escualdosoma Mauriès, 1965.

17. Escualdosoma gourbaultae (Mauriès, 1965)

Cranogona (Escualdosoma) gourbaultae Mauriès, 1965.

\section{Distribution}

FR-FRA.

\section{Habitat}

Cave.

Genus Haasia Bollman, 1893

Olotyphlops Silvestri, 1897.

Acherosoma Verhoeff, 1929.

18. Haasia carinifera (Strasser, 1935)

Acherosoma cariniferum Strasser, 1935.

\section{Distribution}

SI.

\section{Habitat}

Cave. 
19. Haasia cornuata (Strasser, 1940)

Acherosoma cornuatum Strasser, 1940.

\section{Distribution}

SI.

\section{Habitat}

Cave.

20. Haasia falsa (Strasser, 1971)

Achersoma falsum Strasser, 1971.

\section{Distribution}

SI.

\section{Habitat}

Cave.

21. Haasia hadzii (Strasser, 1966)

Acherosoma hadzii Strasser, 1966.

\section{Distribution}

SI.

\section{Habitat}

Cave.

22. Haasia jalzici Antić \& Dražina, 2015

\section{Distribution}

HR.

\section{Habitat}

Cave.

\section{Haasia largescutata (Strasser, 1935)}

Acherosoma largescutatum Strasser, 1935.

\section{Distribution}

SI.

\section{Habitat}

Cave. 
KIME R.D. \& ENGHOFF H., Atlas of European millipedes 3: Order Chordeumatida

24. Haasia likana (Strasser, 1966)

Fig. 4E

Acherosoma likanum Strasser, 1966.

\section{Distribution}

HR.

\section{Habitat}

Cave.

25. Haasia pretneri (Strasser, 1940)

Acherosoma pretneri Strasser, 1940.

\section{Distribution}

HR.

\section{Habitat}

Cave.

26. Haasia stenopodium (Strasser, 1966)

Acherosoma stenopodium Strasser, 1966.

\section{Distribution}

HR, SI.

\section{Habitat}

Cave.

27. Haasia tridentis (Verhoeff, 1931)

Acherosoma tridentis Verhoeff, 1931.

\section{Distribution}

SI.

\section{Habitat}

Cave.

28. Haasia troglodytes (Latzel, 1884)

Craspedosoma troglodytes Latzel, 1884.

Acherosoma verhoeffii Manfredi, 1935.

Acherosoma circoniense Strasser, 1935.

Olotyphlops troglodytes auct.

Scotherpes troglodytes auct. 


\section{Distribution}

SI.

\section{Habitat}

Cave.

Genus Macrochaetosoma Absolon \& Lang, 1933

29. Macrochaetosoma bertiscea Antić \& Makarov, 2015

\section{Distribution}

MN.

\section{Habitat}

Cave.

30. Macrochaetosoma drinae Strasser, 1962

\section{Distribution}

BA.

Habitat

Cave.

31. Macrochaetosoma troglomontanum Absolon \& Lang, 1933

Macrochaetosoma bifurcatum Ćurčić \& Makarov, 2001.

\section{Distribution}

AL, BA, HR, MN.

\section{Habitat}

Cave.

Genus Vascanthogona Mauriès \& Barraqueta, 1985

32. Vascanthogona vicenteae Mauriès \& Barraqueta, 1985

\section{Distribution}

ES-SPA.

\section{Habitat}

A stand of Pinus radiata D.Don.

Family Anthroleucosomatidae Verhoeff, 1899

This family is most diverse in the Balkans (Antic et al. 2020) and also in the Caucasus region where it is the only family of Chordeumatida (Antić \& Makarov 2016). A few among the 43 European species occur in Italy and Corsica, and one (Anamastigona pulchella (Silvestri, 1894)) is relatively widespread 
in W Europe and is one of the few chordeumatidans which have been introduced to Macaronesia, in casu Madeira. Further species are known from the Altai Mts, Siberia, and NW North America (Shear 1988; Shear \& Leonard 2004). This is the only European chordeumatidan family represented by an indigenous species in N America.

Genus Anamastigona Silvestri, 1898

Prodicus Attems, 1899.

Paraprodicus Verhoeff, 1940.

Antrodicus Gulička, 1967.

The largest and most widespread genus in the family, from Italy to Israel, and with a few outposts for Anamastigona pulchella. Reviewed by Vagalinski \& Golovatch (2016).

33. Anamastigona alba (Strasser, 1960)

Prodicus albus Strasser, 1960.

\section{Distribution}

BG.

Habitat

Cave.

34. Anamastigona albanensis Mauriès, Golovatch \& Stoev, 1997

\section{Distribution}

AL.

\section{Habitat}

Under stones among scrub and ruins.

35. Anamastigona aspromontis (Strasser, 1970)

Prodicus aspromontis Strasser, 1970.

\section{Distribution}

IT-ITA.

\section{Habitat}

320-1950 m a.s.1.

\section{Remarks}

Synonymized under A. meridionalis by Golovatch \& Makarov (2011) but resurrected by Vagalinski \& Golovatch (2016).

\section{Anamastigona bilselii (Verhoeff, 1940)}

Prodicus bilselii Verhoeff, 1940. 


\section{Distribution}

BG, GR-GRC, TR-TUE.

\section{Habitat}

Mixed broad-leaved forest, e.g., Fagus, Aesculus L. Tilia L., Acer L., Juglans L., Pinus L. forest; urbanized areas.

\section{Remarks}

Prodicus halophilus Verhoeff, 1940, is possibly a synonym of this species, see Vagalinski \& Golovatch (2016).

\section{Anamastigona delcevi (Strasser, 1973)}

Prodicus delcevi Strasser, 1973.

\section{Distribution}

BG.

38. Anamastigona falcata (Gulička, 1967)

Antrodicus falcatus Gulička, 1967.

Prodicus falcatus auct.

\section{Distribution}

BG.

\section{Habitat}

Cave.

39. Anamastigona halophila (Verhoeff, 1940)

Prodicus halophilus Verhoeff, 1940.

\section{Distribution}

TR-TUE.

\section{Habitat}

On the seacoast, at base of dry hill.

\section{Remarks}

Possibly a synonym of A. bilselii, see Golovatch \& Makarov (2011) and Vagalinski \& Golovatch (2016).

\section{Anamastigona hauseri (Strasser, 1974)}

Prodicus hauseri Strasser, 1974.

\section{Distribution}

GR-GRC.

\section{Habitat}

140 m a.s.l. 
Craspedosoma hispidulum Silvestri, 1894.

Prodicus napolitanus Attems, 1899.

Prodicus apenninorum Verhoeff, 1930.

Prodicus hiemalis Verhoeff, 1941.

Prodicus hispidulus auct.

\section{Distribution}

IT-ITA.

\section{Habitat}

Among small stones and dead leaves.

42. Anamastigona lepenicae (Strasser, 1975)

Prodicus lepenicae Strasser, 1975.

\section{Distribution}

BG.

\section{Habitat}

Cave.

43. Anamastigona matsakisi Mauriès \& Karamouna, 1984

\section{Distribution}

GR-CYC.

\section{Habitat}

Macquis with Juniperus phoenicea L.

44. Anamastigona mediterranea Ćurčić, Makarov \& Lymberakis, 2001

\section{Distribution}

GR-KRI.

\section{Habitat}

$800 \mathrm{~m}$ a.s.1.

\section{Anamastigona meridionalis Silvestri, 1898}

Prodicus meridionalis auct.

\section{Distribution}

IT-SI.

\section{Habitat}

$1500 \mathrm{~m}$ a.s.1. 
46. Anamastigona penicillata (Attems, 1902)

Prodicus penicillatus Attems, 1902.

Paraprodicus pennicillatus auct.

\section{Distribution}

GR-KRI.

\section{Habitat}

In leaf litter at the base of wild Olea L. trees.

47. Anamastigona pentelicona (Verhoeff, 1925)

Prodicus penteliconus Verhoeff, 1925.

\section{Distribution}

GR-GRC.

\section{Habitat}

$450 \mathrm{~m}$ a.s.1.

\section{Anamastigona pulchella (Silvestri, 1894)}

Fig. 3D

Craspedosoma pulchellum Silvestri, 1894.

Prodicus attemsii Verhoeff, 1900.

Anamastigona attemsi auct.

Prodicus macchiae Verhoeff, 1930.

\section{Distribution}

CH, DE, FR-FRA, GB-GRB, GB-NI, IE, IT-ITA, MC, PT-MDR.

\section{Habitat}

Among stones and leaf litter (Silvestri 1903). The finds from Northern Ireland are from woodland (Anderson 1996); those from Germany, Great Britain and France all seem to be synanthropic (Lindner et al. 2010; Gregory et al. 2015; Geoffroy in litt.). On Madeira, A. pulchella is common in the indigenous laurel forest (laurisilva) where it was mostly found under leaf litter, but also under stones, under moss on stones, under bark, in a bracket fungus, and in dead wood; at night it was observed crawling on tree trunks (HE pers. obs.).

49. Anamastigona radmani Makarov, Rađa, Rađa, Tomić, Mitić \& Ćurcić, 2007

\section{Distribution}

HR.

\section{Habitat}

Maquis, near a spring (Makarov pers. com.). Some specimens were found submerged. 
KIME R.D. \& ENGHOFF H., Atlas of European millipedes 3: Order Chordeumatida

Genus Anthroleucosoma Verhoeff, 1899

50. Anthroleucosoma banaticum Verhoeff, 1899

\section{Distribution}

RO.

\section{Habitat}

Deep inside caves on rotting vegetation.

\section{Anthroleucosoma spelaeum Ceuca, 1964}

\section{Distribution}

RO.

Habitat

Cave.

Genus Banatosoma Ćurčić \& Makarov, 2000

52. Banatosoma ocellatum (Tabacaru, 1967)

Bulgarosoma ocellatum Tabacaru, 1967.

\section{Distribution}

RO.

\section{Habitat}

Cave.

Genus Belbogosoma Ćurčić \& Makarov, 2008

53. Belbogosoma bloweri Ćurčić \& Makarov, 2008

\section{Distribution}

SB.

Habitat

Cave.

54. Belbogosoma stribogi Antić \& Makarov, 2014

\section{Distribution}

SB.

\section{Habitat}

Cave. 
Genus Bulgardicus Strasser, 1960

The assignment of this genus to Anthroleucosomatidae is dubious (Antić et al. 2020).

55. Bulgardicus bucarestensis Tabacaru \& Giurginca, 2006

\section{Distribution}

RO.

\section{Habitat}

Under pieces of tree-bark on the soil in a wet and shady clump of trees.

56. Bulgardicus tranteevi Strasser, 1960

\section{Distribution}

BG.

\section{Habitat}

Cave.

Genus Bulgarosoma Verhoeff, 1926

57. Bulgarosoma bureschi Verhoeff, 1926

\section{Distribution}

BG.

\section{Habitat}

Cave.

58. Bulgarosoma superficiei Strasser, 1975

\section{Distribution}

BG.

\section{Habitat}

Epigean (as the name implies); found at 1200-1950 m a.s.l. Bachvarova et al. (2017) reported it from the yard of a summer house, under plywood, and from leaf litter under scattered Pinus and deciduous trees. Dragan Antić (pers. com.) found several specimens on the buried surface of stones.

\section{Remarks}

This species may not belong in Bulgarosoma, but in a different genus (Antić et al. 2020).

Genus Camptogona Brolemann, 1935

The assignment of this genus to Anthroleucosomatidae is dubious (Antić et al. 2020). 


\section{Distribution}

FR-COR.

\section{Habitat}

Fagus forest at $1200 \mathrm{~m}$ a.s.l.

60. Camptogona duboscqui (Brölemann, 1903)

Ceratosoma dubosqui Brölemann, 1903.

Cranogona dubosqui auct.

\section{Distribution}

FR-COR.

Genus Cornogonopus Antić, 2020

61. Cornogonopus pavicevici Antić, 2020

Fig. 4D

\section{Distribution}

SB.

\section{Habitat}

Cave.

Genus Dacosoma Tabacaru, 1967

62. Dacosoma motasi Tabacaru, 1967

\section{Distribution}

RO.

\section{Habitat}

Cave.

Genus Dazbogosoma Makarov \& Ćurčić, 2012

63. Dazbogosoma naissi Makarov \& Ćurčić, 2012

\section{Distribution}

SB.

\section{Habitat}

Cave. 
Genus Krueperia Verhoeff, 1900

64. Krueperia nivalis Verhoeff, 1900

\section{Distribution}

GR-GRC.

\section{Habitat}

Close to a snowfield.

\section{Remarks}

Very poorly known. Described on the basis of a female.

Genus Perunosoma Ćurčić \& Makarov, 2007

65. Perunosoma trojanicum Ćurčić \& Makarov, 2007

[Protoserbosoma tuberculata Makarov, Mitić \& Tomić, 2005. Nomen nudum, see Remarks.]

\section{Distribution}

SB.

\section{Habitat}

Cave.

\section{Remarks}

Dragan Antić has called our attention to the strange case of "Protoserbosoma tuberculata Makarov, Mitić \& Tomić, 2005" which was recorded from Prekonoška Cave in a faunal list for caves and cavities in the northwestern part of the Svrljiške Mountains, East Serbia (Nešić et al. 2007). However, a genus and species of this name has never been described, and both names are obviously nomina nuda. Prekonoška Cave is the type locality for Perunosoma trojanicum, and the nomen nudum almost certainly refers to this species.

Genus Rhodoposoma Ćurčić \& Makarov, 2000

66. Rhodoposoma rhodopinum (Strasser, 1966)

Bulgarosoma rhodopinum Strasser, 1966.

\section{Distribution}

BG.

\section{Habitat}

Cave.

Genus Serbosoma Ćurčić \& Makarov, 2000

67. Serbosoma beljanicae (Ćurčić \& Makarov, 1998)

Bulgarosoma beljanicae Ćurčić \& Makarov, 1998. 


\section{Distribution}

SB.

\section{Habitat}

Cave.

68. Serbosoma crucis (Strasser, 1960)

Bulgarosoma crucis Strasser, 1960.

\section{Distribution}

SB.

\section{Habitat}

Cave.

69. Serbosoma kucajense (Ćurčić \& Makarov, 1998)

Fig. 4B

Bulgarosoma kucajensis Ćurčić \& Makarov, 1998.

\section{Distribution}

SB.

Habitat

Cave.

70. Serbosoma lazarevense (Ceuca, 1964)

Bulgarosoma crucis lazarevensis Ceuca, 1964.

\section{Distribution}

SB.

\section{Habitat}

Cave.

\section{Remarks}

Ćurčić \& Makarov (1997) described the post-embryonic development of this species.

71. Serbosoma zagubicae (Ćurčić \& Makarov, 1998)

Bulgarosoma zagubicae Ćurčić \& Makarov, 1998.

\section{Distribution}

SB.

\section{Habitat}

Cave. 
Genus Stygiosoma Gulička, 1967

72. Stygiosoma beroni Gulička, 1967

\section{Distribution}

BG.

Habitat

Cave.

Genus Svarogosoma Makarov, 2003

73. Svarogosoma bozidarcurcici Makarov, 2003

\section{Distribution}

SB.

\section{Habitat}

Cave.

Genus Troglodicus Gulička, 1967

74. Troglodicus meridionalis (Tabacaru, 1967)

Bulgarosoma meridionale Tabacaru, 1967.

\section{Distribution}

BG.

\section{Habitat}

Cave.

75. Troglodicus tridentifer Gulička, 1967

\section{Distribution}

BG.

\section{Habitat}

Cave.

Family Attemsiidae Verhoeff, 1899

Gruber (2009) provided habitat information on many species of this family which is endemic to a small area covering Austria, S Germany, Slovenia, N Croatia, NE Italy and Slovakia. It contains 25 species.

Genus Allorhiscosoma Verhoeff, 1907

76. Allorhiscosoma sphinx (Verhoeff, 1907)

Rhiscosoma sphinx Verhoeff, 1907.

\section{Distribution}

SK.

\section{Habitat}

Forest. 
77. Attemsia coniuncta Strasser, 1939

\section{Distribution}

HR.

\section{Habitat}

Cave.

78. Attemsia dolinensis Verhoeff, 1909

\section{Distribution}

IT-ITA, SI.

\section{Habitat}

Partly cavernicolous on the karst.

79. Attemsia falcifera Verhoeff, 1899

Attemsia pretneri Strasser, 1933.

\section{Distribution}

HR, SI.

\section{Habitat}

Partly cavernicolous on the karst.

80. Attemsia likana Strasser, 1966

Attemsia coninucta likana Strasser, 1966.

\section{Distribution}

HR.

\section{Habitat}

Cave in karst.

81. Attemsia stygia (Latzel, 1884)

Craspedosoma stygium Latzel, 1884.

Attemsia meerausi Verhoeff, 1931.

Attemsia wolfi Verhoeff, 1931.

Attemsia trevisioli Manfredi, 1940.

\section{Distribution}

SI.

\section{Habitat}

In caves and on the surface on karst. 
Genus Coelogonium Strasser, 1937

82. Coelogonium cavernarum Strasser, 1937

\section{Distribution}

SI.

\section{Habitat}

Cave, subalpine.

Genus Dendromonomeron Verhoeff, 1912

Heterohaasea Verhoeff, 1900.

83. Dendromonomeron oribates (Latzel, 1884)

Craspedosoma oribates Latzel, 1884.

Rhiscosoma alpestre Latzel, 1884 (?).

Heterohaasea (Dendromonomeron) lignivagum Verhoeff, 1912.

Dendromonomeron lignivagum auct.

\section{Distribution}

AT, DE.

\section{Habitat}

Submontane to alpine; altitude range 450-2400 m a.s.l.; from submontane-montane broad-leaved and mixed forests to subalpine and alpine biotopes.

\section{Remarks}

The taxonomy of $D$. oribates/lignivagum is complex, a revision of the type material is desirable (Thaler 1984; Mršić 1987a). As to the status of the name Rhiscosoma alpestre, see Gruber (2009).

Genus Dimastosternum Attems, 1926

84. Dimastosternum franzi Attems, 1949

\section{Distribution}

AT.

\section{Habitat}

Montane to high subalpine; altitudinal range up to $1950 \mathrm{~m}$ a.s.l.; Corylus and Alnus viridis (Chaix) DC. leaf litter, Rhododendron L., high perennial herbs.

\section{Dimastosternum holdhausi Attems, 1927}

\section{Distribution}

AT.

\section{Habitat}

Montane to high subalpine, $1800 \mathrm{~m}$ a.s.l. 
KIME R.D. \& ENGHOFF H., Atlas of European millipedes 3: Order Chordeumatida

Genus Eurygonium Strasser, 1937

86. Eurygonium alticola (Strasser, 1937)

Schubartia (Eurygonium) alticolum Strasser, 1937.

\section{Distribution}

SI.

\section{Habitat}

Alpine, epigeic.

Genus Glomogonium Strasser, 1965

87. Glomogonium karawankarum Strasser, 1965

\section{Distribution}

AT, SI.

\section{Habitat}

Montane, 1000-(?)1400 m a.s.l.; caves.

Genus Grassographia Mršić, 1987

88. Grassographia makolensis Mršić, 1987

\section{Distribution}

SI.

\section{Habitat}

Epigeic.

Genus Julialpium Strasser, 1937

89. Julialpium alabardatum (Strasser, 1937)

Attemsia (Julialpium) alabardatum Strasser, 1937.

\section{Distribution}

SI.

\section{Habitat}

Alpine, epigeic.

Genus Mecogonopodium Strasser, 1933

90. Mecogonopodium bohiniense Strasser, 1933

\section{Distribution}

SI.

\section{Habitat}

Cave. 
91. Mecogonopodium carpathicum Mock \& Tajovský, 2008

\section{Distribution}

SK.

\section{Habitat}

Cave.

92. Mecogonopodium zirianum Mršić, 1987

\section{Distribution}

SI.

\section{Habitat}

Cave.

Genus Polyphematia Verhoeff, 1912

Polyphemus Attems, 1899, pre-occupied name.

\section{Polyphematia moniliformis (Latzel, 1884)}

Craspedosoma moniliforme Latzel, 1884.

Polyphemus moniliformis auct.

Syngonopodium styricum Verhoeff, 1913.

Polyphematia bicornis Verhoeff, 1935.

Polyphematia dactylocoxa Strasser, 1939.

Polyphematia monilicorne Verhoeff, 1939.

Polyphematia antrobius Attems, 1949.

\section{Distribution}

AT, SI.

\section{Habitat}

Subalpine, partly caves. Submontane to alpine, 400-1600 m a.s.1., one cave find above $2300 \mathrm{~m}$ a.s.l. Mainly in caves, also in crevices, rarely on the surface. Most frequent cave millipede in Austria.

Genus Schubartia Verhoeff, 1927

Biplicogonium Strasser, 1939.

94. Schubartia lohmanderi Verhoeff, 1927

Schubartia verhoeffi Strasser, 1937.

\section{Disitribution}

HR, IT-ITA, SI.

\section{Habitat}

On the surface and in caves. 
95. Stiphrogonium attemsi Strasser, 1937

\section{Distribution}

SI.

\section{Habitat}

Subalpine, cave.

Genus Symphyosphys Strasser, 1939

96. Symphyosphys serkoi Strasser, 1939

\section{Distribution}

AT, SI.

\section{Habitat}

Subalpine, cave.

Genus Syngonopodium Verhoeff, 1913

97. Syngonopodium aceris Verhoeff, 1913

Fig. 2B

\section{Distribution}

AT.

\section{Habitat}

Submontane to montane, 550-1250 m a.s.1. Most finds from caves and cave entrances, but also on the surface, under thick Acer and Fagus litter between limestone boulders.

98. Syngonopodium cornutum Verhoeff, 1929

\section{Distribution}

AT.

\section{Habitat}

Submontane to alpine, 550-2000 m a.s.l. Most finds from caves but also, e.g., a montane Picea A.Dietr. forest at $1200 \mathrm{~m}$ a.s.l.

Genus Tylogonium Strasser, 1937

99. Tylogonium hoelzeli Strasser, 1959

\section{Distribution}

AT.

\section{Habitat}

Montane, 1100 m a.s.l.; on permafrost ground; in soil on calcareous gravel; cave, MSS. 
100. Tylogonium nivifidele Strasser, 1937

\section{Distribution}

AT, SI.

\section{Habitat}

Montane to subalpine, $1100-1700 \mathrm{~m}$ a.s.l. Caves, partly containing snow and ice; wet gravel at the edge of snow cover.

Family Beticosomatidae Mauriès, 2014

A monotypic family.

Genus Beticosoma Mauriès, 1990

Originally assigned to Chamaesomatidae (Mauriès 1990a), this monotypic genus was transferred to a new family by Mauriès (2014).

101. Beticosoma longipenis Mauriès, 1990

\section{Distribution}

ES-SPA.

Family Brachychaeteumatidae Verhoeff, 1911

A family with only one genus and eight species, endemic to W Europe with one (Brachychaeteuma bradeae) extending into C Europe.

Genus Brachychaeteuma Verhoeff, 1911

Iacksoneuma H.K. Brade-Birke \& S.G. Brade-Birks, 1917.

A difficult genus (like many other chordeumatidan genera), see Blower (1986).

102. Brachychaeteuma bagnalli Verhoeff, 1911

\section{Distribution}

BE, DE, FR-FRA, GB-GRB, GB-NI, IE.

\section{Habitat}

In Britain and Ireland an urban and suburban species living in the litter and upper soil layers. Also in caves in Germany and a limestone area in Belgium.

\section{Remarks}

Although this species and B. bradeae are usually well distinguishable, this is not always so (Blower 1986). The record of B. bagnalli from France (Dept Rhône) may be doubtful (J.-J. Geoffroy pers. com.). 
KIME R.D. \& ENGHOFF H., Atlas of European millipedes 3: Order Chordeumatida

103. Brachychaeteuma bradeae (Brölemann, H.K. Brade-Birks \& S.G. Brade-Birks, 1917)

Iacksoneuma bradeae Brölemann, H.K. Brade-Birks \& S.G. Brade-Birks, 1917.

Brachychaeteuma quartum Brade-Birks, 1918.

Brachychaeteuma bluncki Verhoeff, 1925.

Brachychaeteuma verhoeffi Schubart, 1930.

Brachychaeteuma herrioti Demange, 1962. Syn. nov., J.-P. Mauriès, see Remarks.

\section{Distribution}

AT, CH, CZ, DE, DK-DEN, FR-FRA, GB-GRB, NL, NO-NOR, RO, SE, SK.

\section{Habitat}

Woodlands, also on cultivated sites (synanthropic), up to $600 \mathrm{~m}$ a.s.l. in Switzerland. In caves in France.

\section{Remarks}

See Proudlove (2011) for a discussion of the authorship of this species and the generic name Iacksoneuma.

The distinction between this species and B. bagnalli is not always entirely clear (Blower 1986). Blower (1986) also pointed out the great similarity of $B$. herrioti Demange, 1962 with B. bradeae (ssp. hussoni Schubart \& Husson, 1936), and J.-P. Mauriès (unpublished) has confirmed the identity of the type specimens of $B$. herrioti with B. bradeae (J.-J. Geoffroy in litt.).

104. Brachychaeteuma cadurcense Mauriès, 1967

Brachychaeteuma caduriense auct. (misspelling).

\section{Distribution}

FR-FRA.

\section{Habitat}

Cave.

105. Brachychaeteuma furcatum Ribaut, 1956

\section{Distribution}

FR-FRA.

106. Brachychaeteuma melanops H.K. Brade-Birks \& S.G. Brade-Birks, 1918

\section{Distribution}

DE, FR-FRA, GB-GRB, IE.

\section{Habitat}

In most places strongly associated with cultivated sites in urban and suburban locations. Also in woodlands and cave entrances in France. 
107. Brachychaeteuma peniculatum Ribaut, 1948

\section{Distribution}

FR-FRA.

\section{Habitat}

Cave.

108. Brachychaeteuma plumosum Ribaut, 1947

\section{Distribution}

FR-FRA.

\section{Habitat}

Cave.

109. Brachychaeteuma provinciale Ribaut, 1956

\section{Distribution}

FR-FRA.

\section{Habitat}

In a garden.

Family Chamaesomatidae Verhoeff, 1913

Including Origmatogonidae Verhoeff, 1914. The convoluted history of this family was briefly reviewed by Enghoff \& Reboleira (2013). The vast majority of the 33 European species occurs in the SW, one (Chamaesoma broelemanni) is distributed over most of France, and the two species of Verhoeffeuma Strasser, 1937 occur in NE Italy and Slovenia. The family also has two species from North Africa: Meinerteuma lucasii (Silvestri, 1896) and Origmatogona strinatii Manfredi, 1956 (Manfredi 1956; Akkari et al. 2010).

Genus Asturasoma Mauriès, 1981

110. Asturasoma chapmani Mauriès, 1981

\section{Distribution}

ES-SPA.

\section{Habitat}

Cave.

\section{Distribution}

ES-SPA.

\section{Habitat}

Cave. 
KIME R.D. \& ENGHOFF H., Atlas of European millipedes 3: Order Chordeumatida

Genus Chamaesoma Ribaut \& Verhoeff, 1913

112. Chamaesoma broelemanni Ribaut \& Verhoeff, 1913

Chamaesoma brölemanni Ribaut \& Verhoeff, 1913.

Chamaesoma brolemanni auct.

\section{Distribution}

FR-FRA, LU.

\section{Habitat}

Under very wet leaf litter (Ribaut in Verhoeff 1913), Quercus forest with Carpinus (David 1989).

\section{Remarks}

According to the International Code of Zoological Nomenclature (ICZN 1999), Article 32.5.2.1 the spelling 'brölemanni' must be emended to 'broelemanni', not 'brolemanni'. David (1989) analyzed the postembryonic development and life cycle of C. broelemanni.

Genus Coiffaiteuma Mauriès, 1964

113. Coiffaiteuma turdetanorum Mauriès, 1964

\section{Distribution}

PT-POR.

Genus Krauseuma Mauriès \& Barraqueta, 1985

114. Krauseuma viscaianum Mauriès \& Barraqueta, 1985

\section{Distribution}

ES-SPA.

\section{Habitat}

A stand of Pinus radiata.

Genus Marboreuma Mauriès, 1988

115. Marboreuma brouquissei Mauriès, 1988

\section{Distribution}

ES-SPA.

\section{Habitat}

A cave at $2900 \mathrm{~m}$ a.s.1. 
Genus Origmatogona Ribaut, 1913

116. Origmatogona catalonica Ribaut, 1913

\section{Distribution}

ES-SPA.

\section{Habitat}

Cave.

117. Origmatogona jacetanorum Mauriès, 1964

\section{Distribution}

ES-SPA.

\section{Habitat}

Epigeic and troglophile.

\section{Origmatogona kimeorum Mauriès, 1990}

\section{Distribution}

FR-FRA.

\section{Habitat}

Found in leaf litter of a Quercus woodland.

\section{Remarks}

A depigmented species found after heavy rain at the end of a prolonged period of drought; probably cavernicolous.

119. Origmatogona tinauti Mauriès, 1990

\section{Distribution}

ES-SPA.

\section{Habitat}

Cave.

120. Origmatogona toniperezi Mauriès, 2014

\section{Distribution}

ES-SPA.

\section{Habitat}

Cave. 


\section{Distribution}

FR-FRA.

\section{Habitat}

Cave.

\section{Remarks}

A dubious species, based on a juvenile specimen and only tentatively referred to Scutogona. The holotype is probably a juvenile of the craspedosomatid genus Broelemanneuma (Mauriès 2015a).

\section{Scutogona ferrolensis Mauriès, 2015}

\section{Distribution}

ES-SPA.

\section{Habitat}

Soil between boulders.

123. Scutogona jeanneli Ribaut, 1913

\section{Distribution}

FR-FRA.

\section{Habitat}

Cave.

124. Scutogona minor Enghoff \& Reboleira, 2013

\section{Distribution}

PT-POR.

\section{Habitat}

Cave.

\section{Distribution}

ES-SPA, FR-FRA.

\section{Habitat}

Cave. 
Distribution

ES-SPA.

Habitat

Cave.

127. Scutogona suboculinigra Mauriès, 2015

\section{Distribution}

ES-SPA.

Habitat

Quercus forest.

128. Scutogona vivesi Mauriès \& Vicente, 1977

\section{Distribution}

ES-SPA.

\section{Habitat}

Cave.

Genus Vascosoma Mauriès, 1966

129. Vascosoma coiffaiti Mauriès, 1966

\section{Distribution}

ES-SPA, FR-FRA.

\section{Habitat}

Cave.

130. Vascosoma duprei Mauriès, 1990

\section{Distribution}

FR-FRA.

Habitat

Cave.

Genus Verhoeffeuma Strasser, 1937

131. Verhoeffeuma minellii Mauriès, 1990

\section{Distribution}

IT-ITA.

\section{Habitat}

Cave. 


\section{Distribution}

SI.

\section{Habitat}

Cave, on wet, decaying wood.

Genus Xystrosoma Ribaut, 1927

Speudosoma Ribaut, 1927.

133. Xystrosoma beatense Ribaut, 1927

\section{Distribution}

FR-FRA.

134. Xystrosoma cassagnaui Mauriès, 1965

\section{Distribution}

FR-FRA.

\section{Habitat}

Quercus-Castanea forest, in förna. Also in a small cave.

135. Xystrosoma catalonicum Ribaut, 1927

\section{Distribution}

FR-FRA.

136. Xystrosoma coiffaiti Mauriès, 1964

\section{Distribution}

ES-SPA.

137. Xystrosoma lusitanicum Mauriès, 2015

Distribution

PT-POR.

138. Xystrosoma murinum Ribaut, 1927

\section{Distribution}

FR-FRA.

139. Xystrosoma pyrenaicum Ribaut, 1927

\section{Distribution}

FR-FRA. 


\section{Xystrosoma santllorence Serra \& Mauriès, 2018}

\section{Distribution}

ES-SPA.

\section{Habitat}

A holm oak (Quercus ilex L.) forest with heliophilous species (Quercetum ilicis galloprovinciale arbutesotum).

\section{Remarks}

Serra \& Mauriès (2018) described the ecology and ontogenesis of this species.

141. Xystrosoma tectosagum Ribaut, 1927

\section{Distribution}

FR-FRA.

142. Xystrosoma vasconicum Mauriès \& Barraqueta, 1985

\section{Distribution}

ES-SPA.

\section{Habitat}

A stand of Pinus radiata.

Family Chordeumatidae C.L. Koch, 1847

The 23 species of this family pretty much cover all of the warmer parts of Europe, even extending into southern Scandinavia (although these occurrences may be due to introduction).

Genus Chordeuma C. L. Koch, 1847

143. Chordeuma consoranense Ribaut, 1956

\section{Distribution}

FR-FRA.

\section{Habitat}

1000 m a.s.l., among mosses.

144. Chordeuma iluronense Ribaut, 1913

\section{Distribution}

FR-FRA.

145. Chordeuma inornatum Ribaut, 1913

\section{Distribution}

FR-FRA. 


\section{Distribution}

FR-FRA.

\section{Habitat}

Fagus forest. Accidental in caves.

147. Chordeuma montanum Ribaut, 1956

\section{Distribution}

FR-FRA.

\section{Habitat}

$1500 \mathrm{~m}$ a.s.1., at the edge of a permanent snow field.

148. Chordeuma muticum Ribaut, 1913

\section{Distribution}

FR-FRA.

\section{Habitat}

Prefers high altitudes.

149. Chordeuma proximum Ribaut, 1913

Fig. 3F

\section{Distribution}

FR-FRA, GB-CI, GB-GRB, GB-NI, IE.

\section{Habitat}

A typical woodland animal, maybe with a preference for acidic soils. Most often found in leaf litter, e.g., Quercus, Carpinus, Fagus. An annual species in southern UK, maybe biannual further north (Blower 1985).

150. Chordeuma reflexum Brolemann, 1927

\section{Distribution}

FR-FRA.

\section{Remarks}

The species was based on a single male described from Aulus-les-Bains. It has never been found again and is probably a teratological form of Chordeuma montanum (Mauriès pers. com.).

\section{Chordeuma sylvestre C.L. Koch, 1847}

\section{Distribution}

AT, BE, CH, CZ, DE, FR-FRA, GB-GRB, HU, IE, IT-ITA, LU, NL, PL. 


\section{Habitat}

A hygrophilous species. Most types of forest and woodland, prefers chalky soils but also on acidic ones, waste ground and maritime habitats, typically in the leaf litter, often on rocky ground. 193-2500 m a.s.l. in Switzerland. In Calabria (S Italy) it is confined to woodland above $1200 \mathrm{~m}$ a.s.l.

\section{Remarks}

Most recently recorded from Ireland (University College Dublin campus) by Gaul \& Tighe (2021) who regarded it likely that this occurrence is due to recent introduction.

\section{Chordeuma trifidum Ribaut, 1913}

\section{Distribution}

FR-FRA.

\section{Habitat}

Forest.

\section{Chordeuma utriculosum Ribaut, 1913}

\section{Distribution}

FR-FRA.

\section{Habitat}

Forest in valleys as well as at higher altitudes.

154. Chordeuma vasconicum Ribaut, 1913

\section{Distribution}

ES-SPA, FR-FRA.

\section{Habitat}

Pinus radiata plantation. Cave.

Genus Melogona Cook, 1895

Microchordeuma Verhoeff, 1897.

Chordeumella Verhoeff, 1897.

155. Melogona broelemanni (Verhoeff, 1897)

Microchordeuma (Chordeumella) broelemanni Verhoeff, 1897.

Microchordeuma albanicum Verhoeff, 1901.

Chordeumella broelemanni auct.

\section{Distribution}

AL, AT, BA, BG, CZ, DE, GR-GRC, HR, HU, MK, RO, SB, SI.

\section{Habitat}

Wooded and bushy habitats, thermophile broad-leaved forests, mixed forests, moist alluvial floodplains in rural situations, 8-900 $\mathrm{m}$ a.s.l. 
Chordeuma gallicum Latzel, 1884.

Microchordeuma gallicum auct.

Chordeumella gallica auct.

\section{Distribution}

BE, CH, CZ, DE, DK-DEN, FR-FRA, GB-CI, GB-GRB, GB-NI, IE, LU, NL, NO-NOR.

\section{Habitat}

Broad-leaved forests, e.g., Quercus, Carpinus, Fagus, Betula L.; also coniferous forests, orchards, grassland, accidental in gardens and caves. Found from 380-530 m a.s.1. in Switzerland.

The life cycle is annual, at least in the southern part of the distribution area (David 1984), maybe biannual in Cheshire, UK (Blower 1985).

\section{Remarks}

Probably introduced in northern Europe.

157. Melogona scutellaris (Ribaut, 1913)

Chordeumella scutellare Ribaut, 1913.

Microchordeuma scutellare auct.

\section{Distribution}

BE, CH, FR-FRA, GB-GRB, GB-NI, IE, IT-ITA.

\section{Habitat}

No very strong link with any habitat type although most records are from woodland, and in the United Kingdom there is also a strong association with suburban sites. 360-2315 m a.s.l. in Switzerland.

\section{Remarks}

The Italian population in Piedmont was described as M. scutellare taurinorum Verhoeff, 1930.

\section{Melogona transsilvanica (Verhoeff, 1897)}

Microchordeuma transsilvanicum Verhoeff, 1897.

\section{Distribution}

AT, CZ, DE, HU, RO.

\section{Habitat}

Woodland, often with anthropogenic influence.

159. Melogona voigtii (Verhoeff, 1899)

Microchordeuma voigtii Verhoeff, 1899.

\section{Distribution}

AT, BE, CH, CZ, DE, DK-DEN, GB-GRB, NL, NO-NOR, PL, SE. 


\section{Habitat}

Broad-leaved forest, e.g., Fagus, Fraxinus, orchards, shrubland, grassland, gardens, occasionally coniferous forests. From 400-480 m a.s.1. in Switzerland. Most finds are from synanthropic sites.

\section{Remarks}

Probably introduced to the northern part of its distribution area. Tajovský (1996) described the life cycle of $M$. voigti in a suburban forest in South Bohemia.

\section{Genus Mycogona Cook, 1895}

Orthochordeuma Verhoeff, 1897.

160. Mycogona germanica (Verhoeff, 1892)

Chordeuma germanicum Verhoeff, 1892.

Orthochordeuma germanicum auct.

\section{Distribution}

AT, BE, CH, CZ, DE, FR-FRA, LU, NL, NO-NOR, PL.

\section{Habitat}

Broad-leaved forest incl. Fagus, Fraxinus, Acer, Quercus, montane Abies-Picea forest, Picea and Pinus plantations. In S Hessen (Germany) one of the few millipedes in Picea monocultures, raised bog, shrub, heathland, grassland, cave. Found from 620-780 m a.s.l. in Switzerland. One synanthropic find in Norway (2014, Kjell Magne Olsen leg. and det., HE conf.).

\section{Genus Orthochordeumella Verhoeff, 1900}

Allochordeuma Rothenbühler, 1900.

161. Orthochordeumella fulva (Rothenbühler, 1899)

Chordema pallidum fulvum Rothenbühler, 1899.

Allochordeumella pallidum fulvum auct.

\section{Distribution}

CH, DE, FR-FRA.

\section{Habitat}

Mainly in broad-leaved forests. Found from 320-1440 m a.s.1. in Switzerland.

162. Orthochordeumella leclerci Mauriès, 1985

\section{Distribution}

FR-FRA.

\section{Habitat}

A subterranean species, found along an underground river and in one other cave. 
163. Orthochordeumella pallida (Rothenbühler, 1899)

Chordeuma pallidum Rothenbühler, 1899.

Allochordeuma pallidum auct.

Orthochordeumella pallida cebennica Liechtenstein in Brolemann, 1935.

Orthochordeumella cebennica auct.

\section{Distribution}

AT, BE, CH, DE, FR-FRA, GB-GRB, LU, SE.

\section{Habitat}

Largely montane and also subalpine, up to $2740 \mathrm{~m}$ a.s.l. in Switzerland but found in lowland parts of Northeastern France and Belgium in particular. Generally in woodland. Synanthropic in northern Germany, Sweden (one find, 2018, Freddy Persson leg., HE det.) and Scotland (Davidson \& Weddle 2021)

\section{Remarks}

The species has a very unusual distribution. The taxon cebennica, regarded as a full species, e.g., by Mauriès (1985), has been found several times in the Cevennes region. It is troglophile - most of the records are from caves.

164. Orthochordeumella pyrenaica Mauriès, 1965

\section{Distribution}

FR-FRA.

Genus Parachordeuma Ribaut, 1912

Genus Parachordeuma Ribaut, 1912

165. Parachordeuma broelemanni Ribaut, 1912

\section{Distribution}

FR-FRA.

\section{Habitat}

Cave.

\section{Family Craspedosomatidae Gray, 1843}

By far the most species-rich family of Chordeumatida in Europe, and also with a particularly complicated taxonomy. Although much work has been done on some genera, several others are badly in need of revision, and several nominal species, of which only some have been included here, remain doubtful. The vast majority of the 189 species occur in Italy, especially N Italy, and adjoining countries, but several species occur further west, north and east. Craspedosoma raulinsii Leach, 1814 is the most widespread European species of the order, extending from France and the British Isles into southern Scandinavia, C and E Europe, with a few outliers (introductions?) in C Sweden and Russia. 
Genus Aspromontia Strasser, 1970

166. Aspromontia ruffoi Strasser, 1970

\section{Distribution}

IT-ITA.

\section{Habitat}

$1300 \mathrm{~m}$ a.s.l.

Genus Atractosoma Fanzago, 1876

Calatractosoma Verhoeff, 1900.

Paratractosoma Ceuca, 1973 (invalidly proposed).

Lessinosoma Strasser, 1977.

A genus very much in need of a revision.

167. Atractosoma abnorme Verhoeff, 1900

\section{Distribution}

IT-ITA.

\section{Habitat}

In a quarry.

Mesatractosoma blechnorum auct.

\section{Distribution}

IT-ITA.

\section{Habitat}

Forest with Castanea Mill., Alnus Mill., Rubus L. and ferns, 150-400 m a.s.l.

\section{Atractosoma cavannae Silvestri, 1898}

\section{Distribution}

IT-ITA.

\section{Atractosoma cecconii Silvestri, 1898}

Atractosoma berlesei Verhoeff, 1900.

\section{Distribution}

IT-ITA.

\section{Habitat}

Mixed forest, ravines, caves. 


\section{Distribution}

IT-ITA.

\section{Habitat}

Mountains, under stones.

172. Atractosoma divaricatum Strasser, 1981

\section{Distribution}

IT-ITA.

\section{Habitat}

Cave.

173. Atractosoma ghidinii Manfredi, 1935

Lessinosoma ghidinii auct.

\section{Distribution}

IT-ITA.

\section{Habitat}

Caves, also in a military shelter.

174. Atractosoma gibberosum Verhoeff, 1900

Paratractosoma chappuisi Ceuca, 1973.

Calatractosoma gibberosum auct.

\section{Distribution}

CH, IT-ITA.

\section{Habitat}

Mountains, under stones, also in caves.

\section{Distribution}

175. Atractosoma marinense Verhoeff, 1932

IT-ITA, SM.

\section{Habitat}

Mountains 450-700 m a.s.1.

\section{Remarks}

Regarded as synonymous with $A$. cecconii by Strasser (1981), but listed as a separate species by Strasser \& Minelli (1984). 


\section{Atractosoma meridionale Fanzago, 1876}

Fig. 1D

\section{Distribution}

CH, DE, HR, IT-ITA, SI.

\section{Habitat}

Forests, mainly cool ones; a pronounced mountain forest anmal, only above the timber line when wood debris is present; 1000-2600 m a.s.1. in Switzerland.

\section{Atractosoma paolettii (Strasser, 1977)}

Lessinosoma paolettii Strasser, 1977.

\section{Distribution}

IT-ITA.

\section{Habitat}

Cave.

178. Atractosoma ruffoi Manfredi, 1940

\section{Distribution}

IT-ITA.

\section{Habitat}

Caves, but also epigeically; broad-leaved forest 500-1000 m a.s.1.

\section{Atractosoma tellinense Brölemann, 1892}

\section{Distribution}

IT-ITA.

\section{Habitat}

Collected at $1820-2550 \mathrm{~m}$ a.s.l.

\section{Atractosoma troglobium Manfredi, 1930}

Paratractosoma jeannelli Ceuca, 1973.

\section{Distribution}

IT-ITA.

\section{Habitat}

Cave. 
KIME R.D. \& ENGHOFF H., Atlas of European millipedes 3: Order Chordeumatida

Genus Autaretia Strasser, 1978

181. Autaretia aliciae Geoffroy \& Mauriès, 2017

\section{Distribution}

FR-FRA.

\section{Habitat}

Cave.

182. Autaretia osellai Strasser, 1978

\section{Distribution}

IT-ITA.

\section{Habitat}

Epigean, 2500-2900 m a.s.1.

Genus Basigona Cook, 1895

183. Basigona athesina (Fedrizzi, 1877)

Megalosoma athesinum Fedirzzi, 1877.

Atractosoma athesina auct.

\section{Distribution}

CH?, IT-ITA.

\section{Habitat}

In very warm places (Fedrizzi 1877).

\section{Remarks}

The two records from $\mathrm{CH}$ are very doubtful and probably concern other species.

Genus Bergamosoma Hoffman, 1980

Megalosoma Fedrizzi, 1878.

Prionosoma Berlese, 1882.

184. Bergamosoma bergomatium (Verhoeff, 1925)

Prionosoma bergomatium Verhoeff, 1925.

Prionosoma pavani Manfredi, 1948.

\section{Distribution}

IT-ITA.

\section{Habitat}

Castenea growth with Corylus, under leaf litter and gravel. 
185. Bergamosoma canestrinii (Fedrizzi, 1877)

Fig. 1E

Megalosoma canestrinii Fedrizzi, 1877.

Prionosoma baldense Verhoeff, 1934.

Atractosoma canestrinii auct.

Craspedosoma canestrinii auct.

Prionosoma canestrinii auct.

\section{Distribution}

AT, CH, DE, IT-ITA.

\section{Habitat}

Rocky areas with shallow soil, alpine meadows with Dryas octopetala L. on south-facing slopes. Also subalpine, strictly calcicole and found from 1000-2460 m a.s.l. in Switzerland. Occasionally in caves, once in a botanic garden.

\section{Remarks}

Found feeding on fox (Vulpes vulpes (Linnaeus, 1758)) excrements in the Swiss National Park (J.D. Gilgado pers. com., see Fig. 1E).

\section{Bergamosoma grottoloi (Strasser, 1973)}

Prionosoma grottoloi Strasser, 1973.

\section{Distribution}

\section{IT-ITA.}

\section{Habitat}

Cave, 700 m a.s.1.

187. Bergamosoma hessei (Verhoeff, 1931)

Prionosoma hessei Verhoeff, 1931.

\section{Distribution}

IT-ITA.

\section{Habitat}

Among rubble in a ravine. The subspecies B. hessei lavonense (Strasser, 1973), listed as a separate species in MilliBase, was described from a cave.

188. Bergamosoma plavis (Strasser, 1960)

Prionosoma plavis Strasser, 1960.

\section{Distribution}

IT-ITA.

\section{Habitat}

Cave (accidentally?). 
Prionosoma sevini Verhoeff, 1931.

\section{Distribution}

IT-ITA.

\section{Habitat}

Among rubble, under cut tree branches.

Genus Bomogona Cook, 1895

Ornithogona Cook, 1896.

Limnalpium Verhoeff, 1921.

Dorasoma Verhoeff, 1932.

Revised by Pedroli-Christen \& Mauriès (1992).

190. Bomogona helvetica (Verhoeff, 1894)

Atractosoma helveticum Verhoeff, 1894.

Atractosoma dentatum Faës, 1902.

Ceratosoma (Limnalpium) verbani Verhoeff, 1910.

Dorasoma quercuum Verhoeff, 1932.

Dorasoma serratum Verhoeff, 1932.

Ornithogona helvetica auct.

Dorasoma helveticum auct.

\section{Distribution}

CH, IT-ITA.

\section{Habitat}

A wide variety of mainly damp habitats such as forests in gorges or sheltered ravines, principally in the montane zone, from 240-2100 m a.s.1. in Switzerland. One find from a mole's nest.

191. Bomogona lombardica (Brölemann, 1892)

Atractosoma lombardica Brölemann, 1892.

Ceratosoma (Limnalpium) luganense Verhoeff, 1921.

Ceratosoma ruscorum Vergoeff, 1921.

Ceratosoma larii Verhoeff, 1921.

Ceratosoma fontis Verhoeff, 1932.

\section{Distribution}

CH, IT-ITA.

\section{Habitat}

Warm forests, e.g., Fagus, and open waste land and subalpine meadows from 275-1600 m a.s.l. in Switzerland. 
Genus Brentosoma Verhoeff, 1932

192. Brentosoma nivale Verhoeff, 1932

\section{Distribution}

IT-ITA.

\section{Habitat}

$2350 \mathrm{~m}$ a.s.1.

Genus Broelemanneuma Verhoeff, 1905

Ribauteuma Verhoeff, 1929.

193. Broelemanneuma furcatum Ribaut, 1913

\section{Distribution}

FR-FRA.

\section{Habitat}

Cave.

194. Broelemanneuma gayi Demange, 1968

\section{Distribution}

FR-FRA.

\section{Habitat}

Cave.

195. Broelemanneuma gineti Ribaut, 1954

\section{Distribution}

FR-FRA.

\section{Habitat}

Cave.

196. Broelemanneuma palmatum (Brölemann, 1902)

Ceratosoma pectiniger var. palmata Brölemann, 1902.

\section{Distribution}

FR-FRA.

\section{Habitat}

Cave. 
Ceratosoma pectiniger Brölemann, 1902.

\section{Distribution}

FR-FRA.

\section{Habitat}

Caves, $900-1750 \mathrm{~m}$ a.s.1.

Genus Carniosoma Verhoeff, 1927

198. Carniosoma verhoeffi (Attems, 1927)

Ceratosoma verhoeffi Attems, 1927.

Carniosoma abietum Verhoeff, 1929.

Carniosoma fagorum Strasser, 1937.

Asandalum fagorum auct.

\section{Distribution}

HR, IT-ITA, SI.

\section{Habitat}

Under bark of Abies Mill., also in litter under ferns; Fagus forest at $1040 \mathrm{~m}$ a.s.1.

Genus Chelogona Cook, 1895

Tatrasoma Verhoeff, 1910.

199. Chelogona carpathica (Latzel, 1882)

Craspedosoma carpathicum Latzel, 1882.

\section{Distribution}

PL, SK.

\section{Habitat}

High mountains.

Genus Corsicosoma Brolemann, 1935

Corsiphilus Verhoeff, 1943.

\section{Corsicosoma legeri (Brölemann, 1903)}

Craspedosoma legeri Brölemann, 1903.

Corsiphilus legeri auct.

\section{Distribution}

FR-COR.

\section{Habitat}

2200 m a.s.l. 
Genus Craspedosoma Leach, 1814

201. Craspedosoma blaniulides Latzel, 1900

\section{Distribution}

FR-COR.

\section{Remarks}

A dubious species (Mauriès 1969), maybe a form of C. raulinsii (J.-J. Geoffroy pers. com.).

202. Craspedosoma brentanum Verhoeff, 1926

\section{Distribution}

IT-ITA.

\section{Habitat}

Thicket of Robinia L., Clematis L., Castanea and Rubus, gravel under litter.

203. Craspedosoma doranum Verhoeff, 1932

\section{Distribution}

IT-ITA.

\section{Habitat}

In Quercus and Populus L. litter, also in mole nests; up to 1900 m.a.s.1.

204. Craspedosoma fontanellum Attems, 1927

\section{Distribution}

IT-ITA.

205. Craspedosoma furculigerum Verhoeff, 1936

\section{Distribution}

IT-ITA.

\section{Habitat}

Rocky slopes, Castanea-Robinia forest.

206. Craspedosoma italicum Silvestri, 1903

Craspedosoma rawlinsii var. italicum Silvestri, 1903.

\section{Distribution}

IT-ITA. 
KIME R.D. \& ENGHOFF H., Atlas of European millipedes 3: Order Chordeumatida

207. Craspedosoma levicanum Fedrizzi, 1876

\section{Distribution}

IT-ITA.

\section{Remarks}

A dubious species, see Spelda (2008).

208. Craspedosoma montenigrinum Mršić, 1987

\section{Distribution}

MN.

209. Craspedosoma oropense Verhoeff, 1936

\section{Distribution}

IT-ITA.

\section{Habitat}

Under Aspidium Sw. and Rhododendron litter, 1750-1850 m a.s.1.

\section{Craspedosoma raulinsii Leach, 1814}

Fig. 1C

Craspedosoma raulinsii Leach, 1814.

Craspedosoma rawlinsii Leach, 1815.

Craspedosoma simile Verhoeff, 1891.

Craspedosoma transsilvanicum Verhoeff, 1897.

Craspedosoma alemannicum Verhoeff, 1910.

Craspedosoma suevicum Verhoeff, 1910.

Craspedosoma wehranum Verhoeff, 1910.

Craspedosoma simile vomrathi Verhoeff, 1910.

Craspedosoma simile germanicum Verhoeff, 1910.

Craspedosoma vomrathi auct.

Craspedosoma germanicum auct.

\section{Distribution}

AT, BA, BG, BE, BY, CH, CZ, DE, DK-DEN, FI, FR-COR?, FR-FRA, GB-GRB, GB-NI, HR, HU, IE, IT-ITA, LT, LU, LV, NL, NO-NOR, PL, RO, RU-KGD, RU-RUC, SB, SE, SI, SK. Introduced in North America.

\section{Habitat}

Mostly in woodland, e.g., Alnus swamps, prefers high humidity, often on sandy soils; sometimes in coniferous forests and more open habitats. A pioneer species in abandoned lignite (brown coal) mining areas (Hauser \& Voigtländer 2019). Its pioneering properties are also reflected in the isolated occurrences in Russia and North-Central Sweden. Up to $1540 \mathrm{~m}$ a.s.l. in Switzerland.

\section{Remarks}

This is the only species of Chordeumatida which has been introduced to another continent. See McAlpine \& Shear (2018) for a discussion of the occurrence in North America and the spelling of the species epithet (also see Dolejš \& Kocourek 2019 on the spelling issue). 
Craspedosoma raulinsii is highly variable, and a very large number of subspecies, varieties and subvarieties have been described. The taxa alemannicum Verhoeff, 1910, germanicum Verhoeff, 1910, vomrathi Verhoeff, 1910, and transsilvanicum have all been regarded as separate species by some authors, e.g., in the well-known handbook by Schubart (1934). Spelda (1991) and Hauser (2004a) analysed the extremely complex taxonomy of this group of taxa. As a result, Hauser (2004a) recognized a number of subspecies, regarded germanicum as a group of morphologically indistinguishable hybrids between various other subspecies, and provided extensive lists of synonyms. See also under C. blaniulides.

This species seems recently to have expanded its range in northeastern Europe. It had not been recorded from Estonia until 2010, but is now widespread in the country (Sammet et al. 2018). It is also a newcomer in the fauna of Latvia where it is now common in a variety of habitats (Spungis 2010). The first record from Russia outside the Kaliningrad region was from a park in Moscow and is probably due to a recent introduction (Golovatch \& Matyukhin 2011).

211. Craspedosoma ruborum Verhoeff, 1930

\section{Distribution}

IT-ITA.

\section{Habitat}

Under Rubus litter.

212. Craspedosoma slavum Attems, 1929

Craspedosoma aegonotum Attems, 1927.

Craspedosoma rawlinsii auct.

\section{Distribution}

AT, BA, HR, IT-ITA, SI.

\section{Habitat}

In valleys.

\section{Remarks}

The synonymy aegonotum = slavum was proposed by Mršić $(1987 b, 1994)$ who, however, used the younger, better known name slavum, as did Hauser (2004a), and so do we, awaiting a proper revision of C. aegonotum.

213. Craspedosoma taurinorum Silvestri, 1898

\section{Distribution}

AT, CH, DE, FR-FRA, IT-ITA.

\section{Habitat}

Most often low altitude forests, but up to $2900 \mathrm{~m}$ a.s.l. 


\section{Distribution}

IT-ITA

\section{Remarks}

A dubious species (Spelda 2008).

Genus Crossosoma Ribaut, 1913

Cryossoma Manfredi, 1951.

215. Crossosoma brolemanni Strasser, 1975

\section{Distribution}

FR-FRA.

\section{Habitat}

Cave at $1500 \mathrm{~m}$ a.s.1.

216. Crossosoma casalei Strasser, 1979

\section{Distribution}

IT-ITA.

\section{Habitat}

Cave.

\section{Crossosoma cavernicola (Manfredi, 1951)}

Cryossoma cavernicola Manfredi, 1951.

\section{Distribution}

FR-FRA, IT-ITA.

\section{Habitat}

Caves at 620-2435 m a.s.l.

218. Crossosoma falciferum Strasser, 1975

\section{Distribution}

IT-ITA.

\section{Habitat}

Caves at $1250-2005 \mathrm{~m}$ a.s.l. 


\section{Crossosoma fossum Strasser, 1979}

\section{Distribution}

IT-ITA.

\section{Habitat}

Fagus forest at $1200 \mathrm{~m}$ a.s.1., $50 \mathrm{~cm}$ below ground among rubble under leaf litter.

220. Crossosoma mauriesi Strasser, 1970

\section{Distribution}

FR-FRA, IT-ITA.

Habitat

Cave.

221. Crossosoma parvum Strasser, 1979

\section{Distribution}

IT-ITA.

Habitat

Cave.

222. Crossosoma peyerimhoffi (Brölemann, 1902)

Ceratosoma peyerimhoffi Brölemann, 1902.

\section{Distribution}

FR-FRA.

\section{Habitat}

Cave.

223. Crossosoma phantasma Strasser, 1970

\section{Distribution}

IT-ITA.

\section{Habitat}

Cave.

Antroherposoma semipes Strasser, 1958.

\section{Distribution}

IT-ITA.

\section{Habitat}

Cave. 
KIME R.D. \& ENGHOFF H., Atlas of European millipedes 3: Order Chordeumatida

Genus Dactylophorosoma Verhoeff, 1900

225. Dactylophorosoma albocarinatum Manfredi, 1940

\section{Distribution}

IT-ITA.

\section{Habitat}

Found in a cave - probably an accidental occurrence.

226. Dactylophorosoma nivisatelles Verhoeff, 1900

\section{Distribution}

CH, IT-ITA.

\section{Habitat}

Subalpine to subnival, altitude range 1300-3000 m a.s.1., exceptionally down to $700 \mathrm{~m}$ a.s.1.; subalpine coniferous forests, dwarf bush vegetation, Alnus viridis thickets, high alpine grass heaths, subnival grassland fragments and gravelly soils.

Genus Dyocerasoma Verhoeff, 1897

227. Dyocerasoma biokovense Mršić, 1986

\section{Distribution}

HR.

Habitat

Cave.

228. Dyocerasoma drimicum Mršić, 1985

\section{Distribution}

SB.

\section{Habitat}

At the source of the river Beli Drim.

229. Dyocerasoma furcilliferum (Verhoeff, 1897)

Polymicrodon furcilliferum Verhoeff, 1897.

\section{Distribution}

BA.

\section{Habitat}

Cave. 
230. Dyocerasoma insulanum Attems, 1951

\section{Distribution}

HR.

\section{Habitat}

Cave.

231. Dyocerasoma intermedium Makarov, Lučić, Mitić \& Rađa, 2003 Fig. $4 \mathrm{~F}$

\section{Distribution}

HR.

\section{Habitat}

Cave.

\section{Dyocerasoma lignivorum (Verhoeff, 1899)}

Polymicrodon lignivorum Verhoeff, 1899.

\section{Distribution}

BA, RO, SB.

\section{Habitat}

In decaying stumps of Fagus and Picea; also recorded from a cave in Romania.

\section{Dyocerasoma narentanum (Verhoeff, 1901)}

Polymicrodon narentanum Verhoeff, 1901.

\section{Distribution}

BA.

\section{Habitat}

Cave.

234. Dyocerasoma nivisatelles (Verhoeff, 1897)

Polymicrodon nivisatelles Verhoeff, 1897.

\section{Distribution}

BA.

\section{Habitat}

Caves, one record is from $2100 \mathrm{~m}$ a.s.l. 
KIME R.D. \& ENGHOFF H., Atlas of European millipedes 3: Order Chordeumatida

Genus Helvetiosoma Verhoeff, 1910

235. Helvetiosoma blanci (Faës, 1902)

Craspedosoma blanci Faës, 1902.

\section{Distribution}

$\mathrm{CH}$.

\section{Habitat}

A north-facing scree at $1960 \mathrm{~m}$ a.s.l.

\section{Remarks}

Possibly another synonym of $H$. helveticum.

\section{Helvetiosoma helveticum (Verhoeff, 1900)}

Craspedosoma helveticum Verhoeff, 1900.

Prionosoma helveticum auct.

Helvetiosoma alemannicum Verhoeff, 1911.

Helvetiosoma cornigerum Bigler, 1912.

Helvetiosoma jurassicum Verhoeff, 1911.

\section{Distribution}

$\mathrm{CH}, \mathrm{DE}, \mathrm{FR}-\mathrm{FRA}$.

\section{Habitat}

In gorges, rough valleys and screes, 400-2300 m a.s.1.; also broad-leaved forest at $330 \mathrm{~m}$ a.s.1.

\section{Remarks}

Pedroli-Christen (1993) provisionally included H. helveticum, H. jurassicum and H. cornigerum in her concept of $H$. alemannicum and noted that this complex may constitute one polytypic species. We adopt this view but follow Spelda (2008) in using the older name helveticum.

\section{Helvetiosoma montemorense (Faës, 1905)}

Craspedosoma montemorense Faës, 1905.

\section{Distribution}

$\mathrm{CH}$.

\section{Habitat}

Found at 2250-2600 m a.s.1., under rocks. 
Genus Iulogona Cook, 1896

Oxydactylon Verhoeff, 1897.

238. Iulogona apenninorum (Verhoeff, 1913)

Oxydactylon apenninorum Verhoeff, 1897.

\section{Distribution}

IT-ITA.

\section{Habitat}

Under decaying brackens (Pteridium Gled. ex Scop.) on limestone.

239. Iulogona hamuligera (Verhoeff, 1913)

Oxydactylon tirolense hamuligerum Verhoeff, 1913.

\section{Distribution}

IT-ITA.

\section{Habitat}

Under Castanea litter on sandstone.

\section{Iulogona ligurina (Verhoeff, 1910)}

Oxydactylon ligurinum Verhoeff, 1910.

\section{Distribution}

IT-ITA.

\section{Habitat}

Broad-leaved forest, under litter.

241. Iulogona tirolensis (Verhoeff, 1894)

Atractosoma tirolense Verhoeff, 1894.

Oxydactylon tirolense auct.

\section{Distribution}

AT, CH, DE, FR-FRA, IT-ITA.

\section{Habitat}

Broad-leaved and conifer forests and wooded pastures, occasionally in more open habitats, 275-2150 m a.s.1.

Genus Janetschekella Schubart, 1954

242. Janetschekella valesiaca (Faës, 1902)

Atractosoma valesiacum Faës, 1902.

Janetschekella nivalis Schubart, 1954. 


\section{Distribution}

CH, FR-FRA, IT-ITA.

\section{Habitat}

High-alpine, subnival to nival, 1600-3450 m a.s.l.

Genus Kelempekia Strasser, 1974

243. Kelempekia martensi Strasser, 1974

\section{Distribution}

GR-GRC.

\section{Habitat}

$900 \mathrm{~m}$ a.s.1.

Genus Listrocheiritium Verhoeff, 1913

244. Listrocheiritium bohemicum (Rosický, 1876)

Craspedosoma bohemicum Rosický, 1876.

\section{Distribution}

AT.

\section{Remarks}

A dubious species, possibly a synonym of Ochogona caroli.

245. Listrocheiritium cervinum Verhoeff, 1925

\section{Distribution}

AT, DE.

\section{Habitat}

(Montane-)high subalpine to alpine, up to $2700 \mathrm{~m}$ a.s.l. Under stones on sandy, raw soils, screes, also in mixed forests, rarely in dwarf bush vegetation.

\section{Remarks}

The status of this species vis-à-vis L. styricum and L. sussurinum needs clarification (Gruber 2009).

246. Listrocheiritium noricum Verhoeff, 1913

\section{Distribution}

AT.

\section{Habitat}

(Sub)montane, 700-1200 m a.s.1.; under Acer and Fagus leaf litter. 
247. Listrocheiritium nubium Verhoeff, 1915

\section{Distribution}

AT.

\section{Habitat}

(Colline-)submontane to low subalpine, 300-1400 (1600?) m a.s.l.; mainly in submontane-montane mixed forests with Acer, Carpinus, Fagus, Pinus, Tilia; also on an alp and in stunted woodland.

\section{Listrocheiritium septentrionale Gulička, 1965}

[Listricheiritium nibelungiacum Attems, 1949; nomen nudum.]

\section{Distribution}

AT, CZ.

\section{Habitat}

Picea forest, forested scree.

\section{Remarks}

See Spelda (1996) concering the name Listrocheiritium niebelungiacum.

249. Listrocheiritium styricum Verhoeff, 1915

Listrocheiritium styriacum Verhoeff, 1929.

\section{Distribution}

AT.

\section{Habitat}

Submontane to montane, 470-1260 m a.s.l.; montane coniferous, mixed and broad-leaved forests on calcareous soil.

\section{Remarks}

The status of this species vis-à-vis L. cervinum and L. sussurinum needs clarification (Gruber 2009). 250. Listrocheiritium susurrinum Attems, 1926

\section{Distribution}

AT.

\section{Habitat}

Montane to alpine. Once in a cave.

\section{Remarks}

The status of this species vis-à-vis L. styricum and L. cervinum needs clarification (Gruber 2009). 
Antroverhoeffia Strasser, 1970.

251. Litogona hyalops (Latzel, 1889)

Atractosoma hyalops Latzel, 1889.

Antroherposoma hyalops auct.

Ceratosoma hyalops auct.

\section{Distribution}

IT-ITA.

\section{Habitat}

Cave.

252. Litogona mirabilis (Manfredi, 1948)

Antroherposoma mirabile Manfredi, 1948.

Antroverhoeffia mirabilis auct.

\section{Distribution}

IT-ITA.

\section{Habitat}

Caves at 220-700 m a.s.1.

Genus Manfredia Verhoeff, 1940

253. Manfredia aemiliana (Manfredi, 1932)

Atractosoma aemilianum Manfredi, 1932.

\section{Distribution}

IT-ITA.

\section{Habitat}

Found in a cave, but probably epigean.

254. Manfredia apuana Strasser, 1971

\section{Distribution}

IT-ITA.

\section{Habitat}

Cave. 
255. Manfredia concii Manfredi, 1953

\section{Distribution}

IT-ITA.

\section{Habitat}

Cave.

256. Manfredia guareschii Manfredi, 1950

\section{Distribution}

IT-ITA.

\section{Habitat}

Found in a cave, but probably epigean.

257. Manfredia lanzai Manfredi, 1948

\section{Distribution}

IT-ITA.

\section{Habitat}

Cave.

Genus Nanogona Cook, 1895

Grypogona Cook, 1895.

Polymicrodon Verhoeff, 1897.

258. Nanogona balazuci (Schubart, 1958)

Polymicrodon balazuci Schubart, 1958.

\section{Distribution}

FR-FRA.

\section{Habitat}

Cave.

Polymicrodon cebennicus Ribaut, 1947.

\section{Distribution}

FR-FRA.

\section{Habitat}

A wide range of habitats including deciduous woodlands, caves and crevices. 
Polymicrodon davidi Demange, 1966.

\section{Distribution}

FR-FRA.

\section{Habitat}

Cave.

261. Nanogona digitata (Ribaut, 1913)

Polymicrodon digitatum Ribaut, 1913.

\section{Distribution}

FR-FRA.

\section{Habitat}

Cave.

\section{Nanogona polydesmoides (Leach, 1814)}

\section{Fig. 1A}

Craspedosoma polydesmoides Leach, 1814.

Atractosoma latzeli Verhoeff, 1891.

Polymicrodon polydesmoides auct.

Craspedosoma latzeli auct.

Grypogona latzeli auct.

\section{Distribution}

BE, DE, DK-DEN, FR-FRA, GB-GRB, GB-NI, IE, IT-ITA, NO-NOR, SE.

\section{Habitat}

A wide range of habitats including deciduous woodlands, caves, waste ground; very often caves and crevices in limestone and karstic areas; troglophile; also Picea plantation, grassland. Probably introduced in the northern part of the distribution area.

\section{Nanogona uncinata (Ribaut, 1913)}

Polymicrodon uncinatum Ribaut, 1913.

\section{Distribution}

FR-FRA.

\section{Habitat}

Cave. 
Genus Ochogona Cook, 1895

Ceratosoma Verhoeff, 1897.

Triakontazona Verhoeff, 1897.

Euceratosoma Verhoeff, 1899.

Beskidia Jawłowski, 1938.

Illyriosoma Strasser, 1942.

Asandalum Attems, 1959.

264. Ochogona apfelbecki (Verhoeff, 1897)

Ceratosoma apfelbecki Verhoeff, 1897.

Asandalum apfelbecki auct.

Triakontazona apfelbecki auct.

\section{Distribution}

BA.

\section{Habitat}

Forest, brushwood, under litter.

265. Ochogona attemsi (Verhoeff, 1907)

Ceratosoma attemsi Verhoeff, 1907.

Asandalum attemsi auct.

Triakontazona attemsi auct.

\section{Distribution}

SI.

\section{Habitat}

Picea-Fagus forest at $950 \mathrm{~m}$ a.s.1., under Picea bark.

\section{Ochogona brentana (Verhoeff, 1927)}

Ceratosoma brentanum Verhoeff, 1927.

Asandalum brentanum auct.

Triakontazona brentanum auct.

\section{Distribution}

AT, DE, IT-ITA.

\section{Habitat}

Limestone rock with Salix L. thicket; among moss and Quercus, Carpinus, Corylus, Castanea litter; low growth of Quercus, Castanea and Corylus, in soil and gravel.

\section{Ochogona caroli (Rothenbühler, 1900)}

Ceratosoma caroli Rothenbühler, 1900.

Asandalum caroli auct. 
Ceratosoma karoli auct.

Triakontazona caroli auct.

\section{Distribution}

AT, CH, CZ, DE, HU, IT-ITA, PL, SI, SK.

\section{Habitat}

Forests (Abies, Fagus, Fraxinus, Larix Mill., Picea, Pinus, Quercus); Alnus viridus thickets, dwarfscrub heath, xeric pastures, screes, once in a cave; 860-2740 m a.s.l. in Switzerland.

Meyer (1979) studied the ecology of $O$. caroli at $2050 \mathrm{~m}$ a.s.l. in Tyrol, Austria and found that the lifecycle spans three years.

\section{Remarks}

Craspedosoma bohemicum Rosický, 1876, now Listrocheiritium b., is possibly a senior synonym of O. caroli, see, e.g., Tajovský (2001), but it is listed separately in this atlas.

\section{Ochogona cervina (Verhoeff, 1899)}

Ceratosoma cervinum Verhoeff, 1899.

Asandalum cervinum auct.

Octeicosisoma cervinum auct.

Triakontazona cervinum auct.

\section{Distribution}

HR.

\section{Habitat}

Forest, under wet litter.

\section{Remarks}

See under Hungarosoma bokori (Hungarosomatidae).

269. Ochogona condylocoxa (Attems, 1899)

Atractosoma condylocoxa Attems, 1899.

Ceratosoma condylocoxa Attems, 1899.

Asandalum condylocoxa auct.

Triakontazona condylocoxa auct.

\section{Distribution}

AT, HR, SI.

Atractosoma elaphron Attems, 1895.

Ceratosoma elaphron auct.

Asandalum elaphron auct.

Triakontazona elaphron auct. 


\section{Distribution}

AT, HU.

\section{Habitat}

Colline to low subalpine, $200-1800 \mathrm{~m}$ a.s.l. Mainly broad-leaved mixed forests, montane mixed forests, Abies forests; lives in leaf litter, once in a cave.

\section{Ochogona euganeorum (Verhoeff, 1927)}

Ceratosoma euganeorum Verhoeff, 1927.

Asandalum euganeorum auct.

\section{Distribution}

IT-ITA.

\section{Habitat}

In Castanea coppice forest.

272. Ochogona friulana (Strasser, 1937)

Ceratosoma friulanum Strasser, 1937.

Asandalum friulana auct.

\section{Distribution}

IT-ITA.

\section{Habitat}

Mixed forest dominated by Castanea, under thick litter, 240-420 m a.s.1.

273. Ochogona gallitarum (Brölemann, 1900)

Ceratosoma gallitarum Brölemann, 1900.

\section{Distribution}

FR-FRA, IT-ITA.

\section{Habitat}

Montane and subalpine.

274. Ochogona hanfi (Attems, 1926)

Ceratosoma hanfi Attems, 1926.

Asandalum hanfi auct.

\section{Distribution}

AT.

\section{Habitat}

Montane to high subalpine, $1500-1800 \mathrm{~m}$ a.s.l.; one find possibly in a raised bog. 
Ceratosoma holdhausi Attems, 1926.

Asandalum holdhausi auct.

\section{Distribution}

AT.

\section{Habitat}

(Montane-)high subalpine to alpine, $1700 \mathrm{~m}$ a.s.1.; once in grassland (Caricetum curvulae).

276. Ochogona jankowskii (Jawłowski, 1938)

Beskidia jankowskii Jawłowski, 1938.

\section{Distribution}

PL, UA.

\section{Habitat}

Forests (Abies, Acer, Alnus, Fagus, Picea), 600-700 m a.s.1.

\section{Remarks}

This species was reviewed and redescribed by Wytwer \& Golovatch (2004).

277. Ochogona latzeli (Attems, 1927)

Ceratosoma latzeli Attems, 1927.

Asandalum latzeli auct.

Ornithogona latzeli auct.

\section{Distribution}

HR.

\section{Habitat}

In moist Fagus litter.

278. Ochogona manfredii (Strasser, 1942)

Ceratosoma manfredii Strasser, 1942.

Asandalum manfredii auct.

Triakontazona manfredii auct.

\section{Distribution}

HR.

\section{Habitat}

Fagus forest and naked slope at 1200-1273 m a.s.1. 
279. Ochogona phyllophaga (Attems, 1899)

Atractosoma phyllophagum Attems, 1899.

Asandalum phyllophagum auct.

Ceratosoma phyllophaghum auct.

\section{Distribution}

HU.

\section{Remarks}

A poorly known species, possibly a senior synonym of $O$. caroli, see Gruber (2009). The location of the type locality was recently clarified by Korsós \& Lazányi (2020).

280. Ochogona pusilla (Verhoeff, 1893)

Atractosoma pusillum Verhoeff, 1893.

Ceratosoma pusillum auct.

Triakontazona pusilla auct.

\section{Distribution}

AT, HR, IT-ITA, SI.

\section{Habitat}

Forests with Corylus, Fagus, Picea, Quercus, 370-900 m a.s.1.

281. Ochogona regalis (Verhoeff, 1913)

Ceratosoma regale Verhoeff, 1913.

Asandalum triaina regale auct.

\section{Distribution}

AT, DE.

\section{Habitat}

Mixed subalpine forest.

\section{Remarks}

The delimitation of this taxon vis-à-vis O. triaina requires clarification (Reip et al. 2016).

282. Ochogona triaina (Attems, 1895)

Atractosoma triaina Attems, 1895.

Asandalum triaina auct.

Ceratosoma triaina auct.

Triakontazona triaina auct.

\section{Distribution}

AT, DE, HR, HU. 


\section{Habitat}

Pasture, forest fringe.

\section{Remarks}

The delimitation of this taxon vis-à-vis $O$. regalis requires clarification (Reip et al. 2016).

$$
\text { Genus Oroposoma Verhoeff, } 1936
$$

283. Oroposoma catascaphium Verhoeff, 1936

\section{Distribution}

IT-ITA.

\section{Habitat}

Shady slope with ferns and leaf litter, $320 \mathrm{~m}$ a.s.1.

284. Oroposoma emiliae Manfredi, 1953

\section{Distribution}

IT-ITA.

\section{Habitat}

Cave.

285. Oroposoma fagorum Verhoeff, 1936

\section{Distribution}

IT-ITA.

\section{Habitat}

Forest (Fagus with Acer and conifers), 1000-1050 m a.s.l.

286. Oroposoma granitivagum Verhoeff, 1936

\section{Distribution}

CH, IT-ITA.

\section{Habitat}

Alpine and subalpine, (820-)1745-2580 m a.s.1., especially in litter of Vaccinium L.

\section{Remarks}

Pedroli-Christen (1993) did not distinguish O. granitivagum and O. nivale. 
287. Oroposoma nivale (Faës, 1902)

Atractosoma nivale Faës, 1902.

\section{Distribution}

$\mathrm{CH}$.

\section{Habitat}

Under stone in snow, $2450 \mathrm{~m}$ a.s.l. (the information under $O$. granitivagum may in part refer to $O$. nivale).

\section{Remarks}

See under $O$. granitivagum.

288. Oroposoma ticinense Manfredi, 1957

\section{Distribution}

$\mathrm{CH}$.

\section{Habitat}

Originally described from a cave, but also found from 353-1870 $\mathrm{m}$ a.s.l. in open and wooded habitats.

289. Oroposoma varallense Verhoeff, 1936

\section{Distribution}

IT-ITA.

\section{Habitat}

Shady slope among rubble with Castanea litter and Aspidium ferns.

Genus Paradactylophorosoma Attems, 1908

Dactylophorosoma subgenus Paradactylophorosoma Attems, 1908.

Paradactylosoma Verhoeff, 1930.

\section{Paradactylophorosoma insulanum (Attems, 1908)}

Dactylophorosoma (Paradactylophorosoma) insulanum Attems, 1908.

Paradactylosoma macchiae Verhoeff, 1930.

\section{Distribution}

IT-ITA.

\section{Habitat}

Dense macchia, under Pteridium and Aspidium ferns; in a hollow Castanea. 
KIME R.D. \& ENGHOFF H., Atlas of European millipedes 3: Order Chordeumatida

Genus Pedemontia Mauriès, 1994

291. Pedemontia delmastroi Mauriès, 1994

\section{Distribution}

IT-ITA.

\section{Habitat}

Forest, $250-335 \mathrm{~m}$ a.s.1.

Genus Plectogona Silvestri, 1897

Antroherposoma Verhoeff, 1898.

Antromanfredia Strasser, 1975.

292. Plectogona angusta (Latzel, 1887)

Atractosoma angustum Latzel, 1887.

Antroherposoma angustum auct.

\section{Distribution}

IT-ITA.

\section{Habitat}

Caves at 2230-2260 m a.s.1.

293. Plectogona bonzanoi (Strasser, 1975)

Antroherposoma bonzanoi Strasser, 1975.

\section{Distribution}

IT-ITA.

\section{Habitat}

Caves at $850-1350 \mathrm{~m}$ a.s.1.

294. Plectogona franciscoloi (Manfredi, 1953)

Antroherposoma franciscoloi Manfredi, 1953.

\section{Distribution}

IT-ITA.

\section{Habitat}

Cave. 
Antroherposoma morisiii Strasser, 1975.

\section{Distribution}

IT-ITA.

\section{Habitat}

Caves at $983-1045 \mathrm{~m}$ a.s.1.

296. Plectogona sanfillipoi (Manfredi, 1956)

Antroherposoma sanfillipoi Manfredi, 1956.

\section{Distribution}

IT-ITA.

\section{Habitat}

Caves at 525-836 m a.s.1.

\section{Plectogona vignai (Strasser, 1970)}

Antroherposoma vignai Strasser, 1970.

\section{Distribution}

IT-ITA.

\section{Habitat}

Cave at $770 \mathrm{~m}$ a.s.1.

Genus Pterygophorosoma Verhoeff, 1897

Orotrechosoma Verhoeff, 1900.

This genus was reviewed by Spelda (2001).

298. Pterygophorosoma alticolum (Verhoeff, 1894)

Atractosoma alticolum Verhoeff, 1894.

Craspedosoma alticolum auct.

Orotrechosoma alticolum auct.

Pterygophorosoma alticola auct.

\section{Distribution}

CH, IT-ITA.

\section{Habitat}

An alpine-nival species. Found from 2000-3090 m a.s.l., in waterlogged soil close to snowfields; also under stones and on a north-facing slope at the foot of a depression. 
Orotrechosoma cornuigerum Verhoeff, 1900.

\section{Distribution}

IT-ITA.

\section{Habitat}

High alpine, $2000-2600 \mathrm{~m}$.

\section{Genus Pyrgocyphosoma Verhoeff, 1910}

Eucharisoma Brolemann, 1935.

This large, predominantly Italian genus was reviewed by Spelda (2008). It is not clear in all cases whether a taxon is to be regarded as a species or a subspecies. All taxa with a separate entry in Spelda (2008) are here listed as species although some may turn out to be subspecies or synonyms of other species.

\section{Pyrgocyphosoma armigerum Verhoeff, 1925}

\section{Distribution}

IT-ITA.

\section{Habitat}

Castanea forest with Corylus, under litter and gravel.

\section{Pyrgocyphosoma arvernum (Ribaut \& Brolemann, 1932)}

Craspedosoma arvernum Ribaut \& Brolemann, 1932.

\section{Distribution}

FR-FRA.

\section{Pyrgocyphosoma aspidiorum Verhoeff, 1931}

Pyrgocyphosoma dentatum aspidiorum Verhoeff, 1931.

\section{Distribution}

IT-ITA.

\section{Habitat}

Brook ravine and slope, under Corylus, Fraxinus, Clematis and Aspidium; 420-710 m a.s.1.

\section{Pyrgocyphosoma balazuci Mauriès \& Kime, 1999}

\section{Distribution}

FR-FRA.

\section{Habitat}

Found in a cave, but also epigeically. 


\section{Pyrgocyphosoma bidentatum (Verhoeff, 1900)}

Craspedosoma oppidicola bidentatum Verhoeff, 1900.

\section{Distribution}

IT-ITA.

\section{Habitat}

Ravines, under litter and stones.

305. Pyrgocyphosoma brembanum Verhoeff, 1931

Pyrgocyphosoma armigerum brembanum Verhoeff, 1931.

\section{Distribution}

\section{IT-ITA.}

\section{Habitat}

Gravelly ground under Alnus and Tussilago L. litter; ravine, under limestone rocks and litter (Corylus, Fraxinus).

306. Pyrgocyphosoma brunatense (Verhoeff, 1910)

Craspedosoma brunatense Verhoeff, 1910.

\section{Distribution}

IT-ITA.

307. Pyrgocyphosoma centrale (Silvestri, 1898)

Craspedosoma centrale Silvestri, 1898.

\section{Distribution}

IT-ITA.

308. Pyrgocyphosoma dalmazzense Verhoeff, 1930

\section{Distribution}

FR-FRA, IT-ITA.

\section{Habitat}

Under decaying mushrooms.

\section{Pyrgocyphosoma dentatum (Brölemann, 1892)}

Craspedosoma dentatum Brölemann, 1892.

\section{Distribution}

CH, IT-ITA.

\section{Habitat}

Thermophile forest, 260-400 m a.s.1. and montane zone (1080 m a.s.1.) 
Craspedosoma doriae Silvestri, 1898.

\section{Distribution}

IT-ITA.

\section{Habitat}

Woodland soil.

\section{Remarks}

Also recorded from FR-FRA and MC, but these records are doubtful (J.-J. Geoffroy pers. com.)

311. Pyrgocyphosoma edrinum Verhoeff, 1934

Pyrgocyphosoma tridentinum edrinum Verhoeff, 1934.

\section{Distribution}

IT-ITA.

312. Pyrgocyphosoma florentinum (Silvestri, 1903)

Craspedosoma florentinum Silvestri, 1903.

\section{Distribution}

IT-ITA.

\section{Habitat}

600-650 m a.s.1. (Spelda 2008).

\section{Remarks}

Described from Florence, this species was re-collected at Monte Amiata, considerably further south, by Spelda (2008) who speculated that the type locality might not have been precise or that this species is widely distributed in the lower parts of Tuscany south of Florence.

\section{Pyrgocyphosoma fonticuli Verhoeff, 1936}

\section{Distribution}

IT-ITA.

\section{Habitat}

Brushwood with Corylus, Quercus, Crataegus L., Rubus, Pteridium.

314. Pyrgocyphosoma gattii (Silvestri, 1898)

Craspedosoma gattii Silvestri, 1898.

\section{Distribution}

IT-ITA. 
315. Pyrgocyphosoma grassii (Silvestri, 1898)

Craspedosoma grassii Silvestri, 1898.

\section{Distribution}

IT-ITA.

316. Pyrgocyphosoma jucundum (Brolemann, 1935)

Craspedosoma doriae jucumdum Brolemann, 1935.

\section{Distribution}

FR-FRA, MC.

\section{Habitat}

Woodland.

\section{Pyrgocyphosoma ligusticum (Silvestri, 1898)}

Craspedosoma ligusticum Silvestri, 1898.

Craspedosoma apenninorum Verhoeff, 1920.

\section{Distribution}

IT-ITA.

318. Pyrgocyphosoma longilamellatum Verhoeff, 1931

Pyrgocyphosoma dentatum longilamellatum Verhoeff, 1931.

\section{Distribution}

IT-ITA.

\section{Habitat}

Ravines under various types of litter, also among rubble; $225-300 \mathrm{~m}$ a.s.1.

319. Pyrgocyphosoma marmorense Verhoeff, 1932

Pyrgocyphosoma oppidicola marmorense Verhoeff, 1932.

\section{Distribution}

IT-ITA.

\section{Habitat}

Under rocks in moist ravine.

\section{Pyrgocyphosoma marrucinum Manfredi, 1950}

\section{Distribution}

IT-ITA.

\section{Habitat}

Epigeically at $700 \mathrm{~m}$ a.s.l.; in a cave at $1367 \mathrm{~m}$ a.s.l. 
Atractosoma mevaniense Silvestri, 1894.

Craspedosoma mevaniense auct.

\section{Distribution}

IT-ITA.

322. Pyrgocyphosoma oppidicola (Silvestri, 1898)

Craspedosoma oppidicola Silvestri, 1898.

\section{Distribution}

IT-ITA.

\section{Habitat}

Under leaf and twig litter.

323. Pyrgocyphosoma ormeanum Verhoeff, 1930

Pyrgocyphosoma dalmazzense ormeanum Verhoeff, 1930.

\section{Distribution}

IT-ITA.

\section{Habitat}

Castanea forest, $800-950 \mathrm{~m}$ a.s.1.

324. Pyrgocyphosoma picenum Manfredi, 1953

\section{Distribution}

IT-ITA.

\section{Habitat}

In submersed moss. Manfredi (1953) discussed this unusual habitat.

\section{Pyrgocyphosoma pontremolense Verhoeff, 1936}

\section{Distribution}

IT-ITA.

\section{Habitat}

Shady ravine with Alnus, Rubus and Castanea.

326. Pyrgocyphosoma pracchiense Verhoeff, 1932

\section{Distribution}

IT-ITA.

\section{Habitat}

Rocky ravine with leaf litter (Fagus, Carpinus, Fraxinus, Corylus, Alnus, Rubus). 


\section{Pyrgocyphosoma quercuum Verhoeff, 1936}

Pyrgocyphosoma fonticuli quercuum Verhoeff, 1936.

\section{Distribution}

IT-ITA.

\section{Habitat}

Brushwood with Quercus, Castanea, Rubus, Acer.

328. Pyrgocyphosoma ravinense Verhoeff, 1936

Pyrgocyphosoma tridentinum ravinense Verhoeff, 1936.

\section{Distribution}

IT-ITA.

\section{Habitat}

Ravine with Corylus and Alnus.

329. Pyrgocyphosoma reatinum Strasser, 1977

Pyrgocyphosoma oppidicola reatinum Strasser, 1977.

\section{Distribution}

IT-ITA.

\section{Habitat}

$720 \mathrm{~m}$ a.s.1.

330. Pyrgocyphosoma renanum Verhoeff, 1932

\section{Distribution}

IT-ITA.

\section{Habitat}

Ravines at 630-640 m a.s.1. in brushwood of Quercus, Castanea, Carpinus, Fraxinus, Rubus, Clematis.

331. Pyrgocyphosoma roccavionense Verhoeff, 1937

\section{Distribution}

IT-ITA.

\section{Habitat}

Ravines, under wet Castanea leaf litter.

\section{Pyrgocyphosoma savonense (Verhoeff, 1910)}

Craspedosoma savonense Verhoeff, 1910.

\section{Distribution}

IT-ITA. 
Pyrgocyphosoma dentatum serianum Verhoeff, 1937.

\section{Distribution}

IT-ITA.

\section{Habitat}

Ravines in karst.

334. Pyrgocyphosoma serpentinum Verhoeff, 1932

\section{Distribution}

IT-ITA.

\section{Habitat}

Small valley, among twigs, Alnus leaf litter and rubble covered with Rubus.

335. Pyrgocyphosoma serravallense Verhoeff, 1936

\section{Distribution}

IT-ITA.

\section{Habitat}

Deep ravine with brushwood of Robinia, Rubus, Crataegus and Euonymus L.

336. Pyrgocyphosoma tendanum Verhoeff, 1930

Craspedosoma doriae tendanum auct.

\section{Distribution}

IT-ITA.

\section{Habitat}

Under bark of decaying Castanea stump.

337. Pyrgocyphosoma terminilli Strasser, 1977

Pyrgocyphosoma oppidicola terminilli Strasser, 1977.

\section{Distribution}

IT-ITA.

\section{Habitat}

1500-1800 m a.s.l. 


\section{Pyrgocyphosoma titianum (Verhoeff, 1910)}

Craspedosoma titianum Verhoeff, 1910.

\section{Distribution}

DE.

\section{Habitat}

Seems to be associated with cool watercourses (Spelda 1991).

\section{Pyrgocyphosoma tridentinum (Silvestri, 1898)}

Craspedosoma tridentinum Silvestri, 1898.

\section{Distribution}

IT-ITA.

\section{Habitat}

Ravine with Corylus, Alnus, Equisetum L., etc.; 650 m a.s.1.

\section{Pyrgocyphosoma vallicola (Silvestri, 1898)}

Craspedosoma vallicola Silvestri, 1898.

\section{Distribution}

IT-ITA.

\section{Pyrgocyphosoma vallombrosae (Silvestri, 1898)}

Craspedosoma vallombrosae Silvestri, 1898.

Craspedosoma vittigerum Verhoeff, 1900.

Pyrgocyphosoma vittigerum auct.

\section{Distribution}

IT-ITA.

\section{Habitat}

Forest.

\section{Pyrgocyphosoma zangherii Manfredi, 1951}

\section{Distribution}

IT-ITA.

\section{Genus Rhymogona Cook, 1896}

Macheirophoron Verhoeff, 1897.

This genus epitomizes the difficulties with interpreting morphological variation in terms of a meaningful taxonomy. Rhymogona has been regarded as a monospecific genus or a "ring species" (Pedroli-Christen \& Scholl 1996a, 1996b), but we here follow Spelda (2005) and recognize five separate species. 
Macheirophoron montivagum hessei Ravoux, 1935.

\section{Distribution}

FR-FRA.

\section{Habitat}

Forest soils.

\section{Rhymogona montivaga (Verhoeff, 1894)}

Atractosoma montivaga Verhoeff, 1894.

Macheirophoron montivagum silvaticum Rothenbühler, 1910.

Macheirophoron alemannicum Verhoeff, 1910.

Macheirophoron cervinum Verhoeff, 1910.

Macheirophoron aelleni Schubart, 1960.

Macheirophoron montivagum auct.

Macheirophoron silvaticum auct.

\section{Distribution}

CH, DE, FR-FRA.

\section{Habitat}

Hygrophilous, mostly found in cool and especially humid forests (Abieti-Fagetum, at lower altitudes less frequent, in Abieti-Fagetum, Pruno-Fraxinetum and Aceri-Fagetum. Large altitudinal amplitude: 340-2314 m a.s.1.

\section{Remarks}

The synonymy list only includes taxa which at some point have been regarded as full species (cf. remarks on subspecies in the Introduction).

\section{Rhymogona serrata (Bigler, 1912)}

Macheirophoron serratum Bigler, 1912.

\section{Distribution}

CH, DE.

\section{Habitat}

Fagus woodland.

346. Rhymogona verhoeffi (Bigler, 1913)

Fig. 1B

Macheirophoron verhoeffi Bigler, 1913.

\section{Distribution}

DE.

\section{Habitat}

Abandoned garden; broad-leaved forest; forest dominated by Picea. 


\section{Rhymogona wehrana (Verhoeff, 1910)}

\section{Distribution}

DE.

\section{Habitat}

Same types as $R$. verhoeffi.

Genus Rothenbuehleria Verhoeff, 1900

Atractosoma tellinense Brölemann, 1892 (q.v.) was tentatively associated with Rothenbuehleria by Verhoeff (1900), but for the time being this species should be listed under its original genus (J. Spelda pers. com.).

\section{Rothenbuehleria minima (Rothenbühler, 1899)}

Atractosoma minimum Rothenbühler, 1899.

\section{Distribution}

AT, CH, IT-ITA.

\section{Habitat}

Rather xeric habitats; $1100-2400$ m a.s.l.

349. Rothenbuehleria tirolensis Verhoeff, 1900

Rothenbuehleria minimum var. tirolense Verhoeff, 1900.

\section{Distribution}

IT-ITA.

\section{Habitat}

Castanea forest, partly in decaying mushrooms.

Genus Sardosoma Manfredi, 1956

350. Sardosoma franchettii Manfredi, 1956

\section{Distribution}

IT-SAR.

\section{Habitat}

Cave.

Genus Semiosoma Ribaut, 1913

351. Semiosoma bordei Ribaut, 1913

\section{Distribution}

FR-FRA.

\section{Habitat}

Cave. 
Ceratosoma devillei Brölemann, 1901.

\section{Distribution}

FR-FRA.

\section{Habitat}

Cave.

353. Semiosoma minutum (Berlese, 1894)

Atractosoma minutum Berlese, 1894.

\section{Distribution}

IT-ITA.

\section{Habitat}

In soil and among mosses.

Genus Synischiosomaa Verhoeff, 1910

354. Synischiosoma argentarium Attems, 1927

\section{Distribution}

IT-ITA.

355. Synischiosoma murorum (Silvestri, 1902)

Prionosoma murorum Silvestri, 1902.

Synischiosoma buchneri Verhoeff, 1936.

Synischiosoma darwinii Verhoeff, 1943.

Synischiosoma romanum Strasser, 1958.

\section{Distribution}

IT-ITA.

\section{Habitat}

Castanea woodland with Hedera and Ruscus L.; garden.

Family Cyrnosomatidae Mauriès, 2015

A monogeneric family, endemic to Corsica, with three species. The name, invalidly proposed by Mauriès (2003), was validated by the same author (Mauriès 2015b)

\section{Genus Cyrnosoma Mauriès, 2015}

Neoatractosoma subgenus Cyrnosoma Mauriès, 1969, invalidly proposed.

The name of the genus was validated by Mauriès (2015b). 
356. Cyrnosoma beroni (Mauriès, 1969)

Neoatractosoma (Cyrnosoma) beroni Mauriès, 1969.

\section{Distribution}

FR-COR.

\section{Habitat}

Epigean, $2200 \mathrm{~m}$ a.s.l.

357. Cyrnosoma coineaui (Mauriès, 1969)

Neoatractosoma (Cyrnosoma) coineaui Mauriès, 1969.

\section{Distribution}

FR-COR.

\section{Habitat}

Cave.

358. Cyrnosoma strasseri (Mauriès, 1969)

Neoatractosoma (Cyrnosoma) strasseri Mauriès, 1969.

\section{Distribution}

FR-COR.

\section{Habitat}

Epigean, $700 \mathrm{~m}$ a.s.1.

Family Dalmatosomatidae Antić \& Makarov, 2018

A family with only one included species.

Genus Dalmatosoma Antić \& Makarov, 2018

359. Dalmatosoma agaricum Antić \& Makarov, 2018

\section{Distribution}

HR.

\section{Habitat}

Cave.

Family Diplomaragnidae Attems, 1907

A mainly Central and East Asian family, with only a marginal occurrence of one species within the area considered here (Shear 1990). 
Diplomaragna golovatchi Shear, 1990.

\section{Distribution}

RU-RUE.

\section{Habitat}

Found in leaf litter in Pinus, Betula and Ulmus forests (Shear 1990).

\section{Remarks}

This is the only chordeumatidan species which occurs inside the area covered by the atlas as well as outside. In addition to the 'European' sites, A. golovatchi also occurs further east, in western Siberia (Shear 1990).

Family Entomobielziidae Verhoeff, 1899

Endemic to Romania; four species.

Genus Entomobielzia Verhoeff, 1898

Bielzia Verhoeff, 1897, preoccupied.

Bielziana Strand, 1928.

361. Entomobielzia getica Ceuca, 1964

\section{Distribution}

RO.

\section{Habitat}

Forest.

362. Entomobielzia kimakowizii (Verhoeff, 1897)

Bielzia kimakowizii Verhoeff, 1897.

\section{Distribution}

RO.

363. Entomobielzia varvarai Ceuca, 1985

\section{Distribution}

RO.

\section{Habitat}

A mountain species preferring litter of coniferous and deciduous trees, sometimes mixed with gravel.

\section{Remarks}

Ceuca (1985) gave the type locality as "la forêt de Slătioara, située au nord de notre pays". There are several places of that name in northern Romania, but the type locality is tentatively interpreted as the village Slătioara in Strâmtura Commune, Maramures County. 
Genus Pseudoclis Attems, 1899

364. Pseudoclis octocera Attems, 1899

\section{Distribution}

RO.

Family Haaseidae Attems, 1899

Orobainosomidae Verhoeff, 1899.

Twenty-six species, all in C and E Europe, except Hylebainosoma nontronensis in the West.

Genus Haasea Verhoeff, 1895

Xiphogona Cook, 1895.

Orobainosoma Verhoeff, 1897.

Rhopalogona Silvestri, 1898.

Deuterohaasea Verhoeff, 1898.

Antić \& Akkari (2020) reviewed this genus taxonomically and faunistically, with the results that several older records, especially of the widespread H. flavescens, have been assigned to other species.

\section{Haasea cyanopida (Attems, 1903)}

Orobainosoma cyanopidum Attems, 1903.

Orobainosoma noricum Verhoeff, 1913.

Haasea norica auct.

\section{Distribution}

AT, DE.

\section{Habitat}

Submontane to low subalpine, 385-1700 (2000?) m a.s.1.; submontane-montane mixed and coniferous forests; under Fagus and Acer leaf litter, also under moss and bark on tree trunk, once in a cave.

366. Haasea faucium (Verhoeff, 1931)

Orobainosoma faucium Verhoeff, 1931.

\section{Distribution}

SI.

\section{Habitat}

Recorded from three pits, also found epigeically.

367. Haasea filicis (Verhoeff, 1929)

Orobainosoma filicis Verhoeff, 1929.

\section{Distribution}

AT. 


\section{Habitat}

Montane to low subalpine, $650-1500 \mathrm{~m}$ a.s.1.; coniferous forest, under dense cover of ferns.

368. Haasea flavescens (Latzel, 1884)

Craspedosoma flavescens Latzel, 1884.

Orobainosoma flavescens auct.

Orobainosma pinivagum Verhoeff, 1901.

Haasea pinivaga auct.

\section{Distribution}

AT, CH, CZ, DE, FR-FRA, HU, LU, PL, SK.

\section{Habitat}

A eurytopic species. Found in forests at 430-2250 m a.s.1. in Switzerland; most common in coniferous, broad-leaved and mixed forests, under bark, litter and stones; also in caves.

\section{Remarks}

Records of this species from BH, HR, IT-ITA and SI were referred to other species by Antić \& Akkari (2020). Records from LU, as well as some (but far from all) records from CZ are dubious (Antić \& Akkari 2020). Meyer \& Singer (1997) studied populations and phenology of this species.

\section{Haasea fonticulorum (Verhoeff, 1910)}

Fig. 2E

\section{Distribution}

AT, CH, HR, IT-ITA, SI.

\section{Habitat}

Verhoeff (1910) stated he found this species in a very wet, fibrous moss sprinkled with spring-water. A very unusual habitat for millipedes after which he named the new species (fonticulus $=$ little fountain or spring). Subsequently found in forests, dwarf bush vegetation grassland, screes and periglacial areas. From 240-2830 m a.s.l. in Switzerland. Meyer (1979) studied the ecology of H. fonticulorum at $2050 \mathrm{~m}$ a.s.l. in Tyrol, Austria and found that the lifecycle spans three years. See also caption to Fig. 2.

370. Haasea germanica (Verhoeff, 1901)

Orobainosoma germanicum Verhoeff, 1901.

\section{Distribution}

AT, CZ, DE, RO.

\section{Habitat}

Alnus and Picea forests, also in screes and caves, up to $950 \mathrm{~m}$ a.s.1.

\section{Remarks}

The records from RO, as well as some of those from $\mathrm{CZ}$ should be considered with caution (Antic \& Akkari 2020). 
371. Haasea gruberi Antić \& Akkari, 2020

\section{Distribution}

AT.

\section{Habitat}

One high alpine find; one in rather dry Fagus forest with Quercus pubescens Willd. (Antić \& Akkari 2020).

\section{Haasea hungarica Verhoeff, 1928}

Orobainosoma hungaricum Verhoeff, 1928.

\section{Distribution}

AT, HU, RO, SB, SI.

\section{Habitat}

Many finds are from caves, but some are epigean, from forest with, e.g., Castanea, Fagus, Quercus.

\section{Haasea inflata (Verhoeff, 1907)}

Orobainosoma inflatum Verhoeff, 1927.

\section{Distribution}

AT, HR, SI.

\section{Habitat}

Forests.

374. Haasea intermedia Mršić, 1985

Haasea lacusnigri intermedia Mršić, 1985.

\section{Distribution}

SB.

\section{Remarks}

The status of this species is questionable (Antić \& Akkari 2020).

375. Haasea lacusnigri (Gulička, 1968)

Orobainosoma lacusnigri Gulička, 1968.

\section{Distribution}

MN.

\section{Habitat}

Abies-Picea forest, $1450 \mathrm{~m}$ a.s.1. 


\section{Distribution}

SB.

\section{Habitat}

Cave.

377. Haasea microcorna (Strasser, 1971)

Orobainosoma lacusnigri microcornum Strasser, 1971.

\section{Distribution}

SB.

\section{Habitat}

Cave.

378. Haasea musimontium (Strasser, 1937)

Orobainosoma musimontium Strasser, 1937.

\section{Distribution}

IT-ITA.

\section{Habitat}

Fagus forest, in litter and on tree stump.

\section{Haasea plasana (Verhoeff, 1899)}

Orobainosoma plasanum Verhoeff, 1899.

\section{Distribution}

BA, SB.

\section{Habitat}

One find is from pitfall traps.

380. Haasea pretneri (Strasser, 1966)

Orobainosoma pretneri Strasser, 1966.

\section{Distribution}

SI.

\section{Habitat}

Cave. 
381. Haasea vidinensis (Strasser, 1973)

Orobainosoma vidinense Strasser, 1973.

Haasea guidononveilleri Makarov, 2008.

\section{Distribution}

BG, SB.

\section{Habitat}

Cave.

Genus Hylebainosoma Verhoeff, 1899

Romanosoma Ceuca, 1967 (invalidly proposed).

Romanosoma Mauriès, 2015.

This genus was revised by Tajovský et al. (2014).

382. Hylebainosoma birtei (Ceuca, 1967)

Romanosoma? birtei Ceuca, 1967.

\section{Distribution}

RO.

\section{Habitat}

Cave.

\section{Remarks}

A doubtful species, known only from a subadult male. Tajovský et al. (2014) regarded this specimen as most probably representing another species in the genus, or even a different genus. The allocation to Hylebainosoma is thus highly provisional.

\section{Hylebainosoma cavernicola (Ceuca, 1967)}

Romanosoma cavernicola Ceuca, 1967.

\section{Distribution}

RO.

\section{Habitat}

Cave at $800 \mathrm{~m}$ a.s.1.

384. Hylebainosoma gulickai Tajovský, Mock \& Papáč, 2014

\section{Distribution}

SK

\section{Habitat}

Caves with entrances at 460-770 m a.s.1., mostly on wood. 
Fig. 3C

\section{Distribution}

FR-FRA, GB-GBR.

\section{Habitat}

Forests and woodland on acidic soil in France (Castanea, Quercus, Betula, Pinus, Pteridium); in UK found in woodland (in Fagus leaf litter, under logs, heathland, and an unkempt cemetery).

\section{Remarks}

Telfer et al. (2015) discussed whether this species is native or introduced to the UK.

386. Hylebainosoma odici (Ceuca, 1979)

Romanosoma odici Ceuca, 1979.

\section{Distribution}

RO.

\section{Habitat}

In litter close to cave entrance at $800 \mathrm{~m}$ a.s.1.

\section{Hylebainosoma oltenicum (Ceuca, 1967)}

Romanosoma oltenica Ceuca, 1967.

\section{Distribution}

RO.

\section{Habitat}

Epigean, found under stones, $430 \mathrm{~m}$ a.s.1.

\section{Hylebainosoma tatranum Verhoeff, 1899}

\section{Distribution}

CZ, HU, PL, SK.

\section{Habitat}

Various surface and underground habitats on different bedrocks such as granite, limestone, basalt or flysh, 230-2300 m a.s.1., deciduous and coniferous forests, the dwarf Pinus zone above the timberline, alpine meadows and ridges with stony deposits and moraines; not in lowland or synanthropic habitats (Tajovský et al 2014). 
Genus Xylophageuma Verhoeff, 1911

389. Xylophageuma vomrathi Verhoeff, 1911

\section{Distribution}

DE.

\section{Habitat}

Humid forest ravines; also on decaying wood in a cave.

\section{Xylophageuma zschokkei Bigler, 1912}

Fig. 2C

\section{Distribution}

BE, $\mathrm{CH}, \mathrm{FR}-\mathrm{FRA}$.

\section{Habitat}

In cool ravines and shady, humid forests, especially under Abies bark, sometimes in cave entrances; from 410-1240 m a.s.l. in Switzerland.

\section{Remarks}

The record from Belgium (not shown on the map) refers to a single female found in a well (Kime 2004). See also caption to Fig. 2.

\section{Family Haplobainosomatidae Verhoeff, 1909}

All 19 species are endemic in the Iberian Peninsula or the Pyrenees; Haplobainosoma lusitanum is one of the few chordeumatidans which have been introduced to Macaronesia, in casu the Azores.

Genus Cantabrosoma Mauriès, 1970

391. Cantabrosoma rogeri Mauriès, 1970

\section{Distribution}

ES-SPA.

392. Cantabrosoma serrai Mauriès \& Vicente, 1977

\section{Distribution}

ES-SPA.

\section{Habitat}

Cave.

Genus Galicisoma Mauriès, 2015

393. Galicisoma biltoni Mauriès, 2015

\section{Distribution}

ES-SPA.

\section{Habitat}

Quercus forest. 
KIME R.D. \& ENGHOFF H., Atlas of European millipedes 3: Order Chordeumatida

394. Galicisoma desmondkimei Mauriès, 2015

\section{Distribution}

ES-SPA, PT-POR.

\section{Habitat}

Mixed forest.

Genus Guadarramasoma Gilgado, Ledesma, Enghoff \& Mauriès, 2017

395. Guadarramasoma ramosae Gilgado, Ledesma, Enghoff \& Mauriès, 2017

\section{Distribution}

ES-SPA.

\section{Habitat}

High altitude (1600-2200 m a.s.1.). Collected in traps set in the mesovoid shallow stratum (MSS); it is uncertain whether it also occurs on the surface (Gilgado et al. 2017).

Genus Haplobainosoma Verhoeff, 1899

396. Haplobainosoma lusitanum Verhoeff, 1899

\section{Distribution}

PT-AZO (Fayal, Pico, Terceira, San Miguel, Santa Maria), PT-POR.

\section{Habitat}

Abundant in MSS in central Portucal (Eusébio et al. 2021). Also in caves; epigean in the Azores.

Genus Pyreneosoma Mauriès, 1959

Aragosoma Mauriès, 1971.

An endemic Pyrenean genus found at high altitudes, reviewed by Mauriès (2010).

397. Pyreneosoma aranense Mauriès, 2010

\section{Distribution}

ES-SPA.

\section{Habitat}

Shelter under granitic rock.

398. Pyreneosoma barbieri (Mauriès, 1971)

Aragosoma barbieri Mauriès 1971.

\section{Distribution}

ES-SPA, FR-FRA.

\section{Habitat}

Caves. 
399. Pyreneosoma bessoni Mauriès, 1974

\section{Distribution}

FR-FRA.

Habitat

Caves.

400. Pyreneosoma birosense Mauriès, 2010

\section{Distribution}

FR-FRA.

Habitat

2300-2500 m a.s.1.

401. Pyreneosoma consoranense Mauriès, 2010

\section{Distribution}

FR-FRA.

\section{Habitat}

Scree at base of shale cliff, 2250-2300 m a.s.l.

402. Pyreneosoma convenarense Mauriès, 2010

\section{Distribution}

ES-SPA, FR-FRA.

\section{Habitat}

Under granitic rocks, 2140-2540 m a.s.l.

403. Pyreneosoma digitatum Mauriès, 1959

\section{Distribution}

FR-FRA.

\section{Habitat}

Most finds epigeic, one in a cave, one at $2500 \mathrm{~m}$ a.s.l.

404. Pyreneosoma grandicoxae Mauriès, 2010

\section{Distribution}

ES-SPA.

\section{Habitat}

Cave. 


\section{Distribution}

ES-SPA, FR-FRA.

\section{Habitat}

Epigeic, 2200-2400 m a.s.1.

406. Pyreneosoma vicdessosense Mauriès, 2010

\section{Distribution}

FR-FRA.

\section{Habitat}

Shale cliff, $2500 \mathrm{~m}$ a.s.1.

\section{Genus Turdulisoma Mauriès, 1964}

Fig. 3B

The genus was revised by Mauriès (2015a). In addition to the species listed below, two possibly undescribed species of Turdulisoma have recently been found in Wales, UK (Gregory 2018; Owen \& Gregory 2021).

407. Turdulisoma galiciense Mauriès, 2015

\section{Distribution}

ES-SPA.

\section{Habitat}

Quercus forest (?).

408. Turdulisoma helenreadae Mauriès, 2015

\section{Distribution}

ES-SPA, PT-POR.

\section{Habitat}

Forest (Alnus, Pinus), cultivated, irrigated woodland.

409. Turdulisoma turdulorum Mauriès, 1964

\section{Distribution}

PT-POR. 
Family Heterolatzeliidae Verhoeff, 1897

This small NW Balkan family (four species) was revised by Makarov et al. (2011), see also Antić et al. (2015c).

Genus Heterolatzelia Verhoeff, 1897

410. Heterolatzelia durmitorensis Gulička, 1968

Heterolatzelia cornutum Gulička, 1968.

\section{Distribution}

MN.

\section{Habitat}

Abies-Picea forest. 1450-2240 m a.s.1.

411. Heterolatzelia karlstrasseri Antić \& Makarov, 2015

\section{Distribution}

BA.

\section{Habitat}

Cave.

412. Heterolatzelia nivalis Verhoeff, 1897

\section{Distribution}

$\mathrm{BA}, \mathrm{MN}$.

\section{Habitat}

In dolines and pits at 1200-2100 m a.s.1.

Genus Massarilatzelia Makarov \& Rađa, 2011

413. Massarilatzelia dugopoljica Makarov \& Rađa, 2011

\section{Distribution}

HR.

\section{Habitat}

Cave.

Family Hungarosomatidae Ceuca, 1974

A monogeneric family of uncertain status with a circum-Pannonic distributon (Mock et al. 2016; Antić et al. 2018c). 
KIME R.D. \& ENGHOFF H., Atlas of European millipedes 3: Order Chordeumatida

Genus Hungarosoma Verhoeff, 1928

414. Hungarosoma bokori Verhoeff, 1928

\section{Distribution}

AT, CZ, HU, SK.

\section{Habitat}

Caves, as well as epigeically in forests of Tilia-Fraxinus, Quercus-Carpinus and Fagus.

\section{Remarks}

Males are rare, and parthenogenesis has been suggested for this species, supported by the presence of bacteria of Wolbachia Hertig, 1936 (Mock et al. 2016).

The status of this species, as that of the genus and the family, is uncertain: Antic et al. (2018c) pointed out that the gonopods of $\mathrm{H}$. bokori fully coincide with those of Ochogona (Octeicosisoma) cervina (Verhoeff, 1899), currently placed in Craspedosomatidae. The taxonomic consequences of this insight, however, still remain to be formalized.

415. Hungarosoma inexpectatum Ceuca, 1967

\section{Distribution}

RO.

\section{Habitat}

Collected in garden soil.

Family Lusitaniosomatidae Schubart, 1953

Another family with only one included species.

Genus Lusitaniosoma Schubart, 1953

416. Lusitaniosoma machadoi Schubart, 1953

\section{Distribution}

PT-POR.

Family Mastigophorophyllidae Verhoeff, 1899

Thirty-five species. Endemic in C and E Europe, with two species (Mastigona bosniensis and Matigophorophyllon saxonicum) extending north into the Baltic countries.

Genus Bucovinosoma Tabacaru, 1978

417. Bucovinosoma capusei Tabacaru, 1978

\section{Distribution}

RO. 
Genus Haploporatia Verhoeff, 1897

418. Haploporatia cervina Verhoeff, 1929

\section{Distribution}

AT.

\section{Habitat}

Submontane, $450-600 \mathrm{~m}$ a.s.1.

419. Haploporatia eremita Verhoeff, 1909

Heteroporatia macrodon Wernitzsch, 1910.

\section{Distribution}

AT, CZ, DE, HU, PL, SK.

\section{Habitat}

Forests, hygrophilous, petrophile, not above $1200 \mathrm{~m}$ a.s.1.

420. Haploporatia similis (Attems, 1895)

Craspedosoma simile Attems, 1895.

Heteroporatia carniolense Verhoeff, 1897.

Heteroporatia simile auct.

Haploporatia carniolensis auct.

\section{Distribution}

AT, BA, HR, IT-ITA, SI.

\section{Habitat}

Forests, e.g., Castanaea, Carpinus, Fagus, Fraxinus, Quercus, Tilia, Acer, Picea, up to 900 m a.s.1.

Genus Heterobraueria Verhoeff, 1897

421. Heterobraueria karoli Verhoeff, 1897

\section{Distribution}

RO.

\section{Habitat}

Under decaying wood at a forest stream.

\section{Heterobraueria scopifera Verhoeff, 1898}

Mastigophorophyllon scopiferum auct.

\section{Distribution}

RO.

\section{Habitat}

Alpine. 
KIME R.D. \& ENGHOFF H., Atlas of European millipedes 3: Order Chordeumatida

Genus Karpatophyllon Jawłowski, 1928

423. Karpatophyllon banaticum Ceuca, 1989

\section{Distribution}

RO.

\section{Habitat}

Under stones near broad-leaved forest.

424. Karpatophyllon carpaticum Ceuca, 1985

\section{Distribution}

RO.

\section{Habitat}

Forest at $500 \mathrm{~m}$ a.s.1.

425. Karpatophyllon dacicum Ceuca, 1964

\section{Distribution}

RO.

426. Karpatophyllon polinskii Jawłowski, 1928

\section{Distribution}

PL, RO, UA.

\section{Habitat}

Rocky riverbank, mixed forest, on felled Fagus trunk, 500-720 m a.s.1.

Genus Mastigona Cook, 1895

Poratia Verhoeff, 1895, preoccupied.

Macrotrichus Silvestri, 1896.

Heteroporatia Verhoeff, 1897.

427. Mastigona bosniensis (Verhoeff, 1897)

Heteroporatia bosniensis Verhoeff, 1897.

Heteroporatia mehelyi Verhoeff, 1897.

Heteroporatia bosniensis var. vihorlatica Attems, 1899.

Heteroporatia vihorlatica auct.

Mastogona vihorlatica auct.

Mastigona mehelyi auct.

\section{Distribution}

AT, BA, BG, BY, CZ, DE, HR, HU, IT-ITA, MD, PL, RO, RU-KGD, SB, SI, SK, UA. 


\section{Habitat}

Euryoecious: arable land, forests, quarries, along streams; up to at least $1000 \mathrm{~m}$ a.s.l.

\section{Remarks}

The variability and synonymy of this widespread species were addressed by Hauser (2004b) and Lazányi \& Korsós (2009).

\section{Mastigona mutabilis (Latzel, 1884)}

Fig. $3 \mathrm{H}$

Craspedosoma mutabile Latzel, 1884.

Heteroporatia alpestre Verhoeff, 1897.

Poratia mutabilis auct.

Heteroporatia mutabilis auct.

Macrotrichus mutabilis auct.

\section{Distribution}

AT, CH, CZ, DE, HR, HU, IT-ITA, SI.

\section{Habitat}

Forest, e.g., Larix, also alpine; from 1350-1570 m a.s.l. in Switzerland.

\section{Remarks}

The record of M. mutabilis from HU is based on a drawing by Sziráki (1966), who, however, provided no details of the find (E. Lazányi pers. com.).

429. Mastigona transsylvanica (Verhoeff, 1897)

Heteroporatia transsylvanica Verhoeff, 1897.

Mastigophorophyllon transsilvanicum Attems, 1900.

\section{Distribution}

BG, RO, HU.

\section{Habitat}

Forest edges, hedges, in leaf litter and decaying wood, once in a cave; $200-1500 \mathrm{~m}$ a.s.l.

Genus Mastigophorophyllon Verhoeff, 1897

430. Mastigophorophyllon aberratum Ceuca, 1985

\section{Distribution}

RO.

\section{Habitat}

Conifer forest litter. 
Heteroporatia alpivagum Verhoeff, 1897.

Mastogophorophyllon alpestre ssp. bohemicum Attems, 1900.

Mastogophorophyllon bohemicum auct.

\section{Distribution}

RO.

\section{Habitat}

Under stones and moss, $200 \mathrm{~m}$ a.s.1.

\section{Remarks}

The identity of the subspecies bohemicum, described from "Böhmen" without further information, remains uncertain (Tajovský 2001).

432. Mastigophorophyllon banarescui Ceuca, 1976

\section{Distribution}

RO.

\section{Habitat}

$1800 \mathrm{~m}$ a.s.l.

433. Mastigophorophyllon bulgaricum Schubart, 1934

\section{Distribution}

BG.

\section{Habitat}

Old Abies-Picea forest; $1500 \mathrm{~m}$ a.s.1.

434. Mastigophorophyllon carpaticum Ceuca, 1976

\section{Distribution}

RO.

435. Mastigophorophyllon cirriferum Verhoeff, 1899

\section{Distribution}

PL, SK.

\section{Habitat}

Forests, near streams, also alpine, 1800-2000 m a.s.l.

\section{Mastigophorophyllon crinitum Attems, 1926}

\section{Distribution}

RO, UA. 
437. Mastigophorophyllon deubeli Verhoeff, 1898

\section{Distribution}

RO.

438. Mastigophorophyllon jickelii Verhoeff, 1900

\section{Distribution}

RO.

439. Mastigophorophyllon moldavicum Ceuca, Crisan \& Olaru, 1996

\section{Distribution}

RO.

440. Mastigophorophyllon parapenicilligerum Crisan \& Ceuca, 1998

\section{Distribution}

RO.

441. Mastigophorophyllon penicilligerum Verhoeff, 1899

\section{Distribution}

RO.

\section{Habitat}

Forest, near small stream.

442. Mastigophorophyllon saxonicum Verhoeff, 1910

\section{Distribution}

BY, CZ, DE, EE, LT, LV, PL, RO, RU-KGD, UA.

\section{Habitat}

Humid broad-leaved forests, Alnus swamps, also meadows.

\section{Remarks}

Apparently a species in decline; thus, there are no newer finds from Estonia (Sammet et al. 2018), nor from Latvia (Spungóis 2010) or Germany (Reip et al. 2012).

443. Mastigophorophyllon serrulatum Attems, 1926

\section{Distribution}

RO, UA.

\section{Habitat}

Among Pinus and Alnus viridis. Ca 1550 m a.s.l. 
444. Paraporatia racovitzai Ceuca, 1967

\section{Distribution}

RO.

Genus Taurinosoma Verhoeff, 1932

445. Taurinosoma graniticola Verhoeff, 1932

\section{Distribution}

IT-ITA.

\section{Habitat}

In a deep gorge, under wet leaf litter, 700-750 $\mathrm{m}$ a.s.1.

Genus Tessinosoma Verhoeff, 1911

446. Tessinosoma caelebs Verhoeff, 1911

Tessinosoma ligurinum Verhoeff, 1930.

\section{Distribution}

CH, IT-ITA.

\section{Habitat}

Forest, e.g., Alnus, Castanea, Quercus, Corylus, Populus; from 193-800 m a.s.l.

\section{Remarks}

The lack of males from the northern part of the distribution area of this species was interpreted by Verhoeff (1921) as "klimatisch-geographisches Parthenogenese". This seems to have been the first time that the phenomenon "geographical parthenogenesis" was recognized (see Enghoff 1994).

Genus Thaumaporatia Verhoeff, 1900

447. Thaumaporatia apenninorum Verhoeff, 1909

\section{Distribution}

IT-ITA.

\section{Habitat}

Castanea forest, under leaf litter and wilted fruit shells.

448. Thaumaporatia apuana Verhoeff, 1909

\section{Distribution}

IT-ITA.

\section{Habitat}

Mixed broad-leaved forest, Alnus, under leaf litter. 
449. Thaumaporatia oropensis Verhoeff, 1936

\section{Distribution}

IT-ITA.

\section{Habitat}

1000-1050 m a.s.1., forested ravine with stream.

450. Thaumaporatia plumigera (Verhoeff, 1900)

Heteroporatia plumigerum Verhoeff, 1900.

\section{Distribution}

IT-ITA.

\section{Habitat}

Under moist moss cushions in open Picea forest.

451. Thaumaporatia sorattina Verhoeff, 1951

\section{Distribution}

IT-ITA.

Family Neoatractosomatidae Verhoeff, 1901

Including Faginidae Attems, 1926. Twenty-two species. Endemic in southern C Europe and the Balkans.

Genus Epirosomella Strasser, 1976

452. Epirosomella loebli Strasser, 1976

\section{Distribution}

GR-GRC.

\section{Habitat}

Sifted under Rhododendron.

Genus Fagina Attems, 1904

453. Fagina silvatica (Attems, 1904)

Heterolatzelia sylvatica Attems, 1904.

\section{Distribution}

BA.

\section{Habitat}

Fagus and Abies forest. 
KIME R.D. \& ENGHOFF H., Atlas of European millipedes 3: Order Chordeumatida

Genus Microbrachysoma Verhoeff, 1897

454. Microbrachysoma alpestre Verhoeff, 1897

\section{Distribution}

BA.

\section{Habitat}

$1650 \mathrm{~m}$ a.s.l., above the tree limit, under deeply embedded stones close to snow.

Genus Neoatractosoma Silvestri, 1898

455. Neoatractosoma herzegowinense Verhoeff, 1901

\section{Distribution}

BA, MN.

Habitat

Fagus forest.

456. Neoatractosoma kleinenbergi Silvestri, 1898

\section{Distribution}

IT-SI.

457. Neoatractosoma strandi Attems, 1927

\section{Distribution}

IT-ITA.

Genus Osellasoma Mauriès, 1984

458. Osellasoma caoduroi Mauriès, 1984

\section{Distribution}

IT-ITA.

\section{Habitat}

Cave, $1600 \mathrm{~m}$ a.s.1.

Genus Paeonisoma Verhoeff, 1932

459. Paeonisoma faucium Verhoeff, 1932

\section{Distribution}

MK.

\section{Habitat}

Probably cave. 
Genus Pseudocraspedosoma Silvestri, 1898

Trimerophoron Rothenbühler, 1900.

Mesotrimeron Verhoeff, 1912.

460. Pseudocraspedosoma alpivagum (Verhoeff, 1901)

Trimerophoron grypischium alpivagum Verhoeff, 1901.

Trimerophoron alpivagum auct.

\section{Distribution}

IT-ITA.

461. Pseudocraspedosoma bensai (Manfredi, 1935)

Trimerophoron bensai Manfredi, 1935.

\section{Distribution}

IT-ITA.

\section{Habitat}

Cave.

462. Pseudocraspedosoma brentanum (Verhoeff, 1930)

Trimerophoron brentanum Verhoeff, 1930.

\section{Distribution}

IT-ITA.

\section{Habitat}

Mixed forest, $950 \mathrm{~m}$ a.s.1., under moss.

\section{Pseudocraspedosoma falteronense (Manfredi, 1951)}

Trimerophoron falteronense Manfredi, 1951.

\section{Distribution}

IT-ITA.

464. Pseudocraspedosoma grypischium (Rothenbühler, 1900)

Trimerophoron grypischium Rothenbühler, 1900.

Trimerophoron grypischium germanicum Verhoeff, 1901.

Trimerophoron germanicum auct.

\section{Distribution}

AT, CH, DE, IT-ITA.

\section{Habitat}

From 540-2950 m a.s.1. in Switzerland, preferring the alpine and subapine levels. Forest and open vegetation including screes. 
KIME R.D. \& ENGHOFF H., Atlas of European millipedes 3: Order Chordeumatida

465. Pseudocraspedosoma nemorense Silvestri, 1898

Trimerophoron nemorense auct.

Distribution

IT-ITA.

\section{Habitat}

Cave.

466. Pseudocraspedosoma peniculorum (Verhoeff, 1910)

Trimerophoron peniculorum Verhoeff, 1910.

Mesotrimeron peniculorum auct.

\section{Distribution}

IT-ITA.

\section{Habitat}

Limestone canyon with bushes, 750 m a.s.1., in litter among limestone rubble (Verhoeff 1910).

\section{Pseudocraspedosoma vestonense (Verhoeff, 1934)}

Trimerophorom vestonense Verhoeff, 1934.

\section{Distribution}

IT-ITA.

\section{Habitat}

In dark humus.

Genus Schizmohetera Mršić, 1987

468. Schizmohetera curcici Makarov, 2001

Fig. 4A

\section{Distribution}

MK.

Habitat

Cave.

469. Schizmohetera olympica Mauriès, 2003

\section{Distribution}

GR-GRC.

\section{Habitat}

Epigean, 700-2100 m a.s.1. 
470. Schizmohetera sketi Mršić, 1987

\section{Distribution}

MK.

\section{Habitat}

Cave.

Genus Trimerophorella Verhoeff, 1902

471. Trimerophorella ornata (Faës, 1902)

Craspedosoma ornatum Faës, 1902.

\section{Distribution}

$\mathrm{CH}$.

\section{Habitat}

2200 m a.s.1

472. Trimerophorella paradisia Meyer, 1983

\section{Distribution}

IT-ITA.

\section{Habitat}

High alpine grassland, $2900 \mathrm{~m}$ a.s.l.

473. Trimerophorella rhaetica (Rothenbühler, 1901)

Trimerophoron rhaeticum Rothenbühler, 1901.

Trimerophorella nivicomes Verhoeff, 1902.

Trimerophorella glaciei Verhoeff, 1912.

\section{Distribution}

AT, CH, IT-ITA.

\section{Habitat}

Mostly found at alpine levels (grass heaths, rocky slopes, periglacial areas, subnival grassland fragments, Loiseleuria Desv. heaths), open coniferous forests. Altitude range 1100-2950 m a.s.1.

\section{Remarks}

Records from Italy are dubious (Gruber 2009). Meyer (1990) reported a four-year life cycle for this species. 
Family Opisthocheiridae Ribaut, 1913

In addition to the $37 \mathrm{SW}$ European species, this family includes Ceratosphys maroccana Mauriès, 1985, from North Africa (see Mauriès 1985). Ceratosphys poculifer is one the few chordeumatidans which have been introduced to Macaronesia, in casu the Canary Islands.

Genus Brachytropisoma Silvestri, 1898

474. Brachytropisoma giardinae Silvestri, 1898

\section{Distribution}

IT-SI.

Genus Ceratosphys Ribaut, 1920

Fuentea Brolemann, 1920.

Proceratosphys Mauriès \& Vicente, 1977.

Mauriès $(2012,2014)$ gave an overview of this genus and a key to its then known species.

475. Ceratosphys amoena Ribaut, 1920

Fig. 3G

Ceratosphys confusa Ribaut, 1955.

Ceratosphys dentata Ribaut, 1956.

Ceratosphys taurus Ribaut, 1956.

\section{Distribution}

BE, FR-FRA, GB-GBR.

\section{Habitat}

Largely montane, in woods which are wet and/or have deep litter. In Fagus woodland in Wales.

\section{Remarks}

Telfer et al. (2015) discussed whether this species is native or introduced to the UK. Five subspecies have been described from the Pyrenees and the Montagne Noire in France, but only C. amoena ssp. confusa has been found outside France: in Belgium and Wales (Telfer et al. 2015).

476. Ceratosphys angelieri Mauriès, 1964

\section{Distribution}

PT-POR.

477. Ceratosphys bakeri Mauriès, 1990

\section{Distribution}

PT-POR.

\section{Habitat}

Found in a shrubby area (Ulex densus Welw. ex Webb and Quercus coccifera L.). 
478. Ceratosphys banyulensis Brolemann, 1926

\section{Distribution}

FR-FRA.

\section{Habitat}

Close to the Mediterranean Sea.

479. Ceratosphys cryodeserti Gilgado, Mauriès \& Enghoff, 2015

\section{Distribution}

ES-SPA.

\section{Habitat}

Abundant in the mesovoid shallow stratum at 2500 to more than $3000 \mathrm{~m}$ a.s.l. in almost completely barren areas (cold deserts), but also on the surface (Gilgado et al. 2015b).

480. Ceratosphys deharvengi Mauriès, 1978

Ceratosphys deharvingi Mauriès, 1978

\section{Distribution}

ES-SPA.

\section{Habitat}

At $1500 \mathrm{~m}$ a.s.l.

\section{Remarks}

The species was named after L. Deharveng, hence the emendation of the species epithet (e.g., Mauriès $1990 \mathrm{~b}, 2012$ ) is justified.

481. Ceratosphys escolai Mauriès, 2013

\section{Distribution}

ES-BAL (Mallorca).

\section{Habitat}

Cave.

482. Ceratosphys fernandoi Mauriès, 2014

\section{Distribution}

ES-SPA.

\section{Habitat}

Cave. 
483. Ceratosphys flammeola Mauriès, 2014

\section{Distribution}

ES-SPA.

\section{Habitat}

Cave.

484. Ceratosphys geronensis Mauriès, 1963

\section{Distribution}

ES-SPA.

485. Ceratosphys guttata Ribaut, 1956

\section{Distribution}

ES-SPA, FR-FRA.

\section{Habitat}

Cold and humid soils above the tree line. $2350-2450 \mathrm{~m}$ a.s.l.

486. Ceratosphys hispanica Ribaut, 1920

\section{Distribution}

ES-SPA.

\section{Habitat}

Cave.

487. Ceratosphys jabaliensis Mauriès, 2013

\section{Distribution}

ES-SPA.

\section{Habitat}

Cave.

488. Ceratosphys mariacristinae Mauriès, 2013

Ceratosphys n. sp. - Enghoff \& Vicente 2000.

\section{Distribution}

ES-BAL (Menorca).

489. Ceratosphys nivium Ribaut, 1927

\section{Distribution}

ES-SPA, FR-FRA.

\section{Habitat}

Alpine slopes under snow cover for much of the year. At $2400 \mathrm{~m}$ a.s.l. in Spain. 
490. Ceratosphys nodipes (Attems, 1952)

Hispaniodesmus nodipes Attems, 1952.

\section{Distribution}

ES-SPA.

\section{Habitat}

Collected at $1400 \mathrm{~m}$ a.s.l. A similar form (Ceratosphys aff. nodipes) has been found in a cave (Mauriès 2013).

491. Ceratosphys occidentalis Mauriès, 1976

Ceratosphys nivium occidentalis Mauriès, 1976

\section{Distribution}

(ES-SPA?), FR-FRA.

\section{Habitat}

Lake shore at $2350 \mathrm{~m}$ a.s.1.

\section{Remarks}

Possibly also in Spain, Jaca region (Mauriès 1990b).

492. Ceratosphys picta Ribaut, 1951

\section{Distribution}

ES-SPA, FR-FRA.

\section{Habitat}

An accidental cavernicole.

493. Ceratosphys poculifer (Brolemann, 1920)

Fuentea poculifer Brolemann, 1920.

\section{Distribution}

ES-CAN (Gran Canaria), ES-SPA.

\section{Habitat}

On Gran Canaria, found on shady rock, under moss and in soil on gravelly slope, under Adenocarpus DC. bush, $1300-1450 \mathrm{~m}$ a.s.1.

\section{Ceratosphys simoni Ribaut, 1920}

\section{Distribution}

FR-FRA.

\section{Habitat}

2400-2800 m a.s.1. 
KIME R.D. \& ENGHOFF H., Atlas of European millipedes 3: Order Chordeumatida

495. Ceratosphys solanasi (Mauriès \& Vicente, 1977)

Proceratosphys solanasi Mauriès \& Vicente, 1977

\section{Distribution}

ES-SPA.

\section{Habitat}

Cave.

496. Ceratosphys soutadei Mauriès, 1969

\section{Distribution}

ES-SPA.

\section{Habitat}

A cryophilous species, collected at the surface of a snowfield at $2800 \mathrm{~m}$ a.s.1., and in the MSS at $2500 \mathrm{~m}$ a.s.l.

497. Ceratosphys toniserrai Mauriès, 2013

\section{Distribution}

ES-SPA.

\section{Habitat}

$1240 \mathrm{~m}$ a.s.1.

498. Ceratosphys vandeli Mauriès, 1963

\section{Distribution}

FR-FRA.

\section{Habitat}

$2400 \mathrm{~m}$ a.s.1.

499. Ceratosphys vicenteae Mauriès, 1990

\section{Distribution}

ES-SPA.

\section{Habitat}

$700 \mathrm{~m}$ a.s.1.

Genus Hispaniosoma Ribaut, 1913

500. Hispaniosoma racovitzai Ribaut, 1913

\section{Distribution}

ES-SPA, FR-FRA.

\section{Habitat}

Cave. 
Genus Marquetiella Jeekel, 1969

Marquetia Ribaut, 1905, preoccupied.

501. Marquetiella auriculata (Ribaut, 1920)

Marquetia auriculata Ribaut, 1920.

Distribution

FR-FRA.

\section{Habitat}

Leaf litter.

502. Marquetiella lunata (Ribaut, 1920)

Marquetia lunata Ribaut, 1920.

\section{Distribution}

ES-SPA, FR-FRA.

\section{Habitat}

Deciduous, mixed and coniferous forests, $950-1450 \mathrm{~m}$ a.s.1.

503. Marquetiella pyrenaica (Ribaut, 1905)

Marquetia pyrenaica Ribaut, 1905.

\section{Distribution}

FR-FRA.

\section{Habitat}

Leaf litter.

\section{Genus Opisthocheiron Ribaut, 1913}

504. Opisthocheiron canayerensis Mauriès \& Geoffroy, 1982

\section{Distribution}

FR-FRA.

\section{Habitat}

Deep caves, a long way from their entrances.

505. Opisthocheiron cornutum Ribaut, 1922

\section{Distribution}

FR-FRA.

\section{Habitat}

Under leaf litter and logs. 


\section{Distribution}

ES-SPA, FR-FRA.

\section{Habitat}

Woodland, mainly in decomposing leaf litter. Known to climb plants.

507. Opisthocheiron fallax Ribaut, 1922

\section{Distribution}

FR-FRA.

508. Opisthocheiron lacazei Brolemann, 1932

\section{Distribution}

FR-FRA.

\section{Habitat}

In a ravine.

509. Opisthocheiron penicillatum Ribaut, 1913

\section{Distribution}

ES-SPA, FR-FRA.

\section{Habitat}

Under leaf litter. Accidentally in caves.

Genus Sireuma Reboleira \& Enghoff, 2014

510. Sireuma nobile Reboleira \& Enghoff, 2014

\section{Distribution}

PT-POR.

\section{Habitat}

Cave, $350 \mathrm{~m}$ a.s.1.

Family Trachygonidae Cook, 1896

Endemic to a small area extending from Switzerland to Slovakia, Hungary and Croatia. Three genera, five species.

\section{Genus Acrochordum Attems, 1899}

Gottscheeosoma Verhoeff, 1927.

Heteracrochordum Loksa, 1960 (described as subgenus of Acrochordum). 
Heteracrochordum evae auct.

\section{Distribution}

HU, SK.

\section{Habitat}

Fagus forest on limestone; humus soil in bottom of sinkhole in limestone; litter and soil on forested slope of volcanic bedrock, $150-275 \mathrm{~m}$ a.s.l.

\section{Remarks}

Recently recorded from SK (Mock et al. 2019). The morphology and taxonomy of this species is subject of an ongoing study by A. Mock et al.

\section{Acrochordum flagellatum Attems, 1899}

Gottscheeosoma scabrum Verhoeff, 1927.

Acrochordeum tarnowanum Strasser, 1942.

\section{Distribution}

AT, BA, HR, SI.

\section{Habitat}

In Fagus-Picea forest at $1280 \mathrm{~m}$ a.s.1., under pieces of Abies bark on the ground.

\section{Acrochordum plitvicense (Verhoeff, 1929)}

Gottscheeosoma plitvicense Verhoeff, 1929.

\section{Distribution}

HR.

\section{Habitat}

On a resupinate fungus on a moist Fagus log.

Genus Halleinosoma Verhoeff. 1913

514. Halleinosoma noricum Verhoeff, 1913

Fig. 2A

\section{Distribution}

AT, CH, DE.

\section{Habitat}

Submontane to montane, altitude range 560-1480 m a.s.1.; mainly in mixed Fagus forests; found under deeply embedded stones, under logs and leaf litter (Fagus, Acer), under calcareous rocks.

\section{Remarks}

Possibly a synonym of Trachygona capito; see Gruber (2009). The only other species described in the genus Halleinosoma is possibly as well a synonym of Trachygona capito (q.v.). The records from Switzerland may refer to a different species (J. Spelda pers. com.). 
Trachysoma Attems, 1895, preoccupied.

515. Trachygona capito (Attems, 1894)

Trachysoma capito Attems, 1894.

? Halleinosoma styricum Verhoeff, 1929.

\section{Distribution}

AT.

\section{Habitat}

Submontane to subalpine; altitude range 350-1700 m a.s.1.; in Fagus leaf litter, under high herbs and Pinus mugo Turra., in Picea forest/plantation, in mixed deciduous forest.

\section{Remarks}

See Gruber (2009) concerning the status of Halleinosoma styricum.

Family Vandeleumatidae Mauriès, 1970

See Serra \& Mauriès (2015) for a discussion of this endemic SW European family. Fifteen species.

Genus Alavasoma Mauriès \& Vicente, 1977

The systematic position of this genus is unclear. Originally described in Anthogonidae (Mauriès \& Vicente 1977), it was listed in Vandeleumatidae by Spelda (2015). We provisionally keep it in this family although Mauriès (2014) did not include it in this list of vandeleumatid genera.

516. Alavasoma muniesai Mauriès \& Vicente, 1977

\section{Distribution}

ES-SPA.

\section{Habitat}

Cave.

Genus Eopsychrosoma Serra \& Mauriès, 2015

517. Eopsychrosoma serrapradense Serra \& Mauriès, 2015

\section{Distribution}

ES-SPA.

\section{Habitat}

Quercus woodland (Quercetum mediterraneo-montanum); mostly in the upper soil layers, only sparsely in pitfall traps. 
Genus Guipuzcosoma Vicente \& Mauriès, 1980

518. Guipuzcosoma comasi Vicente \& Mauriès, 1980

\section{Distribution}

ES-SPA.

Habitat

Cave.

Genus Hypnosoma Ribaut, 1952

Mauriès (1968) reviewed this genus, noting that species of Hypnosoma aren't rare in leaf litter and under stones in Quercus-Picea forest on the northern slopes of the central Pyrenees.

519. Hypnosoma exornatum Ribaut, 1952

\section{Distribution}

FR-FRA.

\section{Habitat}

Abies forest. Found in autumn in 'cocoons' attached to leaf axils of hypnaceous mosses.

520. Hypnosoma juberthieorum Mauriès, 1968

\section{Distribution}

FR-FRA.

521. Hypnosoma pallidum Ribaut, 1952

\section{Distribution}

ES-SPA, FR-FRA.

\section{Habitat}

In leaf litter in spring; in autumn enclosed in 'cocoons' attached to leaf axils of Hypnum Hedw. mosses.

Genus Miniusoma Mauriès, 2015

522. Miniusoma litorea Mauriès, 2015

\section{Distribution}

PT-POR.

\section{Habitat}

Coastal dune system.

Genus Niphatrogleuma Mauriès, 1986

The assignment of this genus to Vandeleumatidae is highly provisional (Mauriès 1986). 


\section{Distribution}

$\mathrm{CH}$.

\section{Habitat}

Cave at $2455 \mathrm{~m}$ a.s.1.

Genus Psichrosoma Mauriès, 2013

Psychrosoma Mauriès, 1970, preoccupied.

An unidentified, apparently undescribed species of Psichrosoma, has been found in Wales, UK (Gregory 2018; Owen \& Gregory 2021). See Fig. 3A.

524. Psichrosoma baeticaense Mauriès, 2013

Typhlopsychrosoma baeticaense auct.

\section{Distribution}

ES-SPA.

\section{Habitat}

Described from caves and subsequently found in the MSS (Gilgado et al. 2015c).

525. Psichrosoma breuili (Mauriès, 1970)

Psychrosoma breuili Mauriès, 1970.

Typhlopsychrosoma breuili auct.

\section{Distribution}

ES-SPA.

\section{Habitat}

Cave.

Psychrosoma tarraconense Mauriès, 1970.

Typhlopsychrosoma tarraconense auct.

\section{Distribution}

ES-SPA.

\section{Habitat}

Cave. 
Genus Strangulogona Mauriès, 2015

527. Strangulogona lugoensis Mauriès, 2015

\section{Distribution}

ES-SPA.

\section{Habitat}

Small woodland.

Genus Typhlopsychrosoma Mauriès, 1982

Psychrosoma subgen. Typhlopsychrosoma Mauriès, 1982, cf. Gilgado et al. (2015c) and Serra \& Mauriès (2015).

528. Typhlopsychrosoma fadriquei (Mauriès \& Vicente, 1977)

Psychrosoma fadriquei Mauriès \& Vicente, 1977.

\section{Distribution}

ES-SPA.

\section{Habitat}

Cave.

Genus Vandeleuma Mauriès, 1966

529. Vandeleuma hispanicum Ceuca, 1967

\section{Distribution}

ES-SPA.

\section{Habitat}

Cave.

530. Vandeleuma vasconicum Mauriès, 1966

\section{Distribution}

ES-SPA, FR-FRA.

\section{Habitat}

Cave.

Family Verhoeffiidae Verhoeff, 1899

A monotypic family endemic to south C Europe: one genus, four species.

Genus Haplogona Cook, 1895

Latzelia Verhoeff, 1895, preoccupied.

Verhoeffia Brölemann, 1895.

Protochordeuma Silvestri, 1898. 
Verhoeffia carynthiaca Strasser, 1967.

\section{Distribution}

AT.

\section{Habitat}

Montane, $1100 \mathrm{~m}$ a.s.1., in fern roots.

532. Haplogona gestri (Silvestri, 1898)

Protochordeuma gestri Silvestri, 1898.

Verhoeffia gestri auct.

Verhoeffia gestroi auct.

\section{Distribution}

IT-ITA.

\section{Habitat}

Laurus L. and Castanea litter.

533. Haplogona oculodistincta (Verhoeff, 1893)

Fig. 2D

Chordeuma oculodistinctum Verhoeff, 1893.

Verhoeffia oculodistincta auct.

Chordeuma gracense Attems, 1895.

Verhoeffia illyrica Verhoeff, 1899.

Latzelia illyrica auct.

\section{Distribution}

AT, CZ, HR, IT-ITA, SI, SK.

\section{Habitat}

In valleys and low highlands, in ravines, screes, gorges, in forest soil under litter and stones, e.g., Castanea, Fraxinus, Acer, Fagus, Picea. Synanthropic in Vienna. Regarded by Strasser (1959) as an expansive species which may occupy suboptimal habitats.

\section{Haplogona rothenbuehleri (Verhoeff, 1900)}

Verhoeffia rothenbuehleri Verhoeff, 1900.

\section{Distribution}

AT, IT-ITA, SI.

\section{Habitat}

Castanea forest, in moss cushions. 


\section{Discussion}

\section{Comparison with the order Julida}

With 534 European species, the Chordeumatida rival the order Julida which was treated by Kime \& Enghoff (2017) who included 593 European species of the order. A comparison between the two orders therefore invites itself. Table 4 compares the two orders with regard to the numbers of species, numbers of $50 \mathrm{~km}$ square records, number and proportion of species recorded from only one $50 \mathrm{~km}$ square, and number and proportion of purely subterranean species, i.e., species recorded only from caves or MSS. The additional species of Julida listed in Table 5 are not considered for this comparison.

An important difference between Julida and Chordeumatida is that the Julida includes very many (92 $15 \%$ of the total) species endemic to the Macaronesian archipelagos (Azores, Madeira, Canary Islands) whereas the Chordeumatida have no Macaronesian endemics (and very few Macaronesian records altogether). Table 4 includes a column for Julida without Macaronesian endemics (i.e., the genera Acipes Attems, 1937 (7 species, 12 records), Thalassisobates Verhoeff, 1908 (1 species, 1 record), Cylindroiulus Verhoeff, 1894 (32 species, 36 records) and Dolichoiulus Verhoeff, 1900 (52 species, 53 records). The numbers in this column are thus more comparable with those for Chordeumatida.

The data in Table 4 show that

- Chordeumatida is the largest family of millipedes in continental Europe, i.e., not counting the endemic Macaronesian julidans;

- there are much fewer $50 \mathrm{~km}$ square records of Chordeumatida than of Julida;

- the chordeumatidan species known from the highest number of $50 \mathrm{~km}$ squares (Craspedosoma raulinsii) has twice as high a share (12.2\%) of the total number of $50 \mathrm{~km}$ square records as the julidan species with the highest number of such records (Ommatoiulus sabulosus (Linnaeus, 1758), 6.1\%);

- no less than $53.7 \%$ of the chordeumatidan species have been recorded from only one $50 \mathrm{~km}$ square. For the non-Macaronesian julids, the corresponding percentage is $29.6 \%$;

- Chordeumatida are much more concentrated in southern Europe than Julida: only $6.4 \%$ of the chordeumatidan species occur $\mathrm{N}$ of ca $48^{\circ} \mathrm{N}$ vs $13.8 \%$ of the non-Macaronesian Julida;

- the percentage of species known only from caves or other underground habitats is much higher $(34.7 \%)$ in Chordeumatida than in Julida $(20.0 \%)$.

Given the much lower number of records of Chordeumatida, it is not surprising that the relative number of species known only from one $50 \mathrm{~km}$ square is higher than in the Julida. Nevertheless, it is clear that species of Chordeumatida as a rule are particularly localized. Figure 7 visualizes this: the frequency distribution of numbers of $50 \mathrm{~km}$ squares per species is much more concave for Chordeumatida than for Julida. The high percentage of underground species certainly contributes to this picture. Another factor that might play a role is that Chordeumatida are not so easy to find, and certainly not at all easy to work with taxonomically. This may be reflected in the graph shown in Fig. 8 where the distribution per decade of the year in which the valid name of each species was published. Although the two orders show very much the same pattern (a maximum around the year 1900, a low in the 1940s and 50s - probably an effect of the second World War -, another low around the year 2000, but luckily increased activity in the 2010s), the description of julidan species started much earlier than that of chordeumatidans: thirty-one julidan species had been given names described before 1870 , compared with only three chordeumatidan ones.

\section{The European millipede fauna - updates on other orders}

Volumes 1 and 2 of the atlas (Kime \& Enghoff 2011, 2017) covered 492 and 593 species, respectively. With the 534 species treated in the present, last volume, a total of 1619 European millipede species have 
Table 4. A numerical comparison of Chordeumatida and Julida. Data on Julida from Kime \& Enghoff (2017). Macaronesian endemics constitute a significant proportion (15\%) of the julidan species. As there are no Macaronesian endemics in the Chordeumatida, the numbers for Julida without Macaronesian endemics are more comparable to those for Chordeumatida. See main text.

\begin{tabular}{|c|c|c|c|}
\hline & Julida & $\begin{array}{c}\text { Julida without } \\
\text { Macaronesian endemics }\end{array}$ & Chordeumatida \\
\hline No. of species & 593 & 500 & 534 \\
\hline No. of $50 \mathrm{~km}$ squares records & 13229 & 13129 & 3600 \\
\hline $\begin{array}{l}\text { Average no. of } 50 \mathrm{~km} \\
\text { squares per species }\end{array}$ & 22.3 & 26.3 & 6.7 \\
\hline $\begin{array}{c}\text { Max. no. of } 50 \mathrm{~km} \text { squares } \\
\text { per species }\end{array}$ & $\begin{array}{c}796 \\
\text { Ommatoiulus sabulosus, } \\
6.0 \% \text { of all records }\end{array}$ & $\begin{array}{c}796 \\
\text { Ommatoiulus sabulosus, } \\
6.1 \% \text { of all records }\end{array}$ & $\begin{array}{c}438 \\
\text { Craspedosoma raulinsii, } \\
12.2 \% \text { of all records }\end{array}$ \\
\hline $\begin{array}{l}\text { No. and percentage of } \\
\text { species recorded from only } \\
\text { one } 50 \mathrm{~km} \text { square }\end{array}$ & $236(39.8 \%)$ & $148(29.6 \%)$ & $285(53.7 \%)$ \\
\hline $\begin{array}{l}\text { No. and percentage of } \\
\text { species recorded } \mathrm{N} \text { of ca } \\
48^{\circ} \mathrm{N}\end{array}$ & $69(11.6 \%)$ & $69(13.8 \%)$ & $34(6.4 \%)$ \\
\hline $\begin{array}{l}\text { No. of species with habitat } \\
\text { information }\end{array}$ & 528 & 436 & 461 \\
\hline $\begin{array}{l}\text { No. and percentage of } \\
\text { species recorded only from } \\
\text { caves/MSS (out of species } \\
\text { with habitat information) }\end{array}$ & $96(18.2 \%)$ & $87(20.0 \%)$ & $160(34.7 \%)$ \\
\hline
\end{tabular}

been mapped. Whereas the present volume claims to cover knowledge on European Chordeumatida up to and including 2020, the previous volumes are no longer completely up to date. Volumes 1 and 2 included information available by the end of 2007 (Kime \& Enghoff 2011) and by the end of 2014, respectively, but in an appendix Kime \& Enghoff (2017) gave some post-2014 updates concerning Julida. Table 5 summarizes these, as well as other additions and deletions. A different type of change concerns the transfer of a species from one genus to another (e.g., Vagalinski \& Lazányi 2018); such changes are not included in Table 5, nor are new records of a species to a particular geographical area.

Altogether, 67 species have been added, while seven have been deleted (including one of those which was added!), bringing the total number of known European millipede species up to 1679 .

\section{Acknowledgements}

The Atlas of European millipedes has very much been a team effort. The acknowledgements in the previous volumes (Kime \& Enghoff 2011, 2017) include names of the numerous persons who have contributed to the atlas in one way or another over the years. The support of these persons has continued to be important for the present volume as well.

Additional specific support for this volume has been generously given by several colleagues:

Records of species, information on taxonomy, distribution and habitat, as well as other useful comments were provided by Dragan Antić (who painstakingly read through all the species accounts and, together 


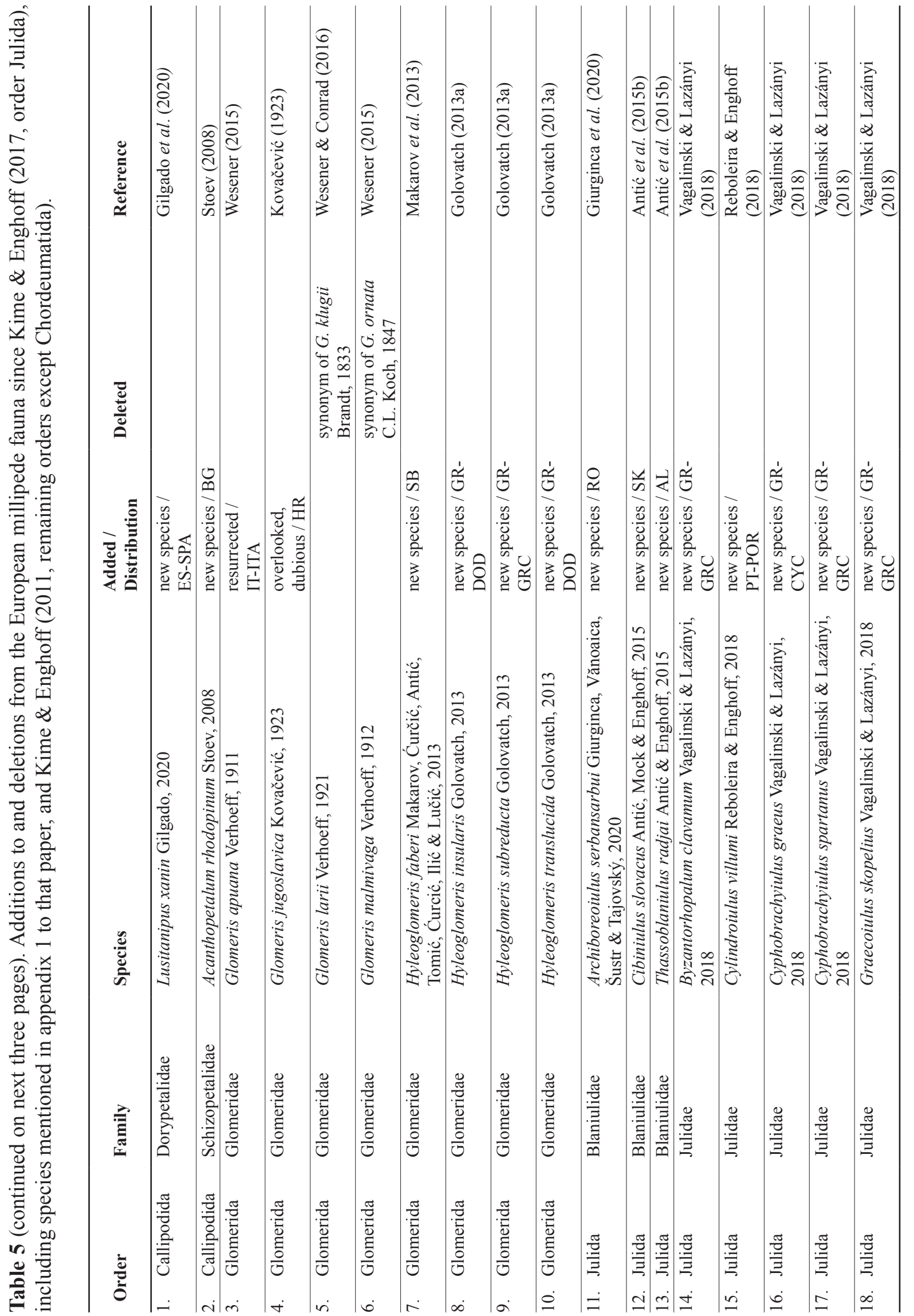




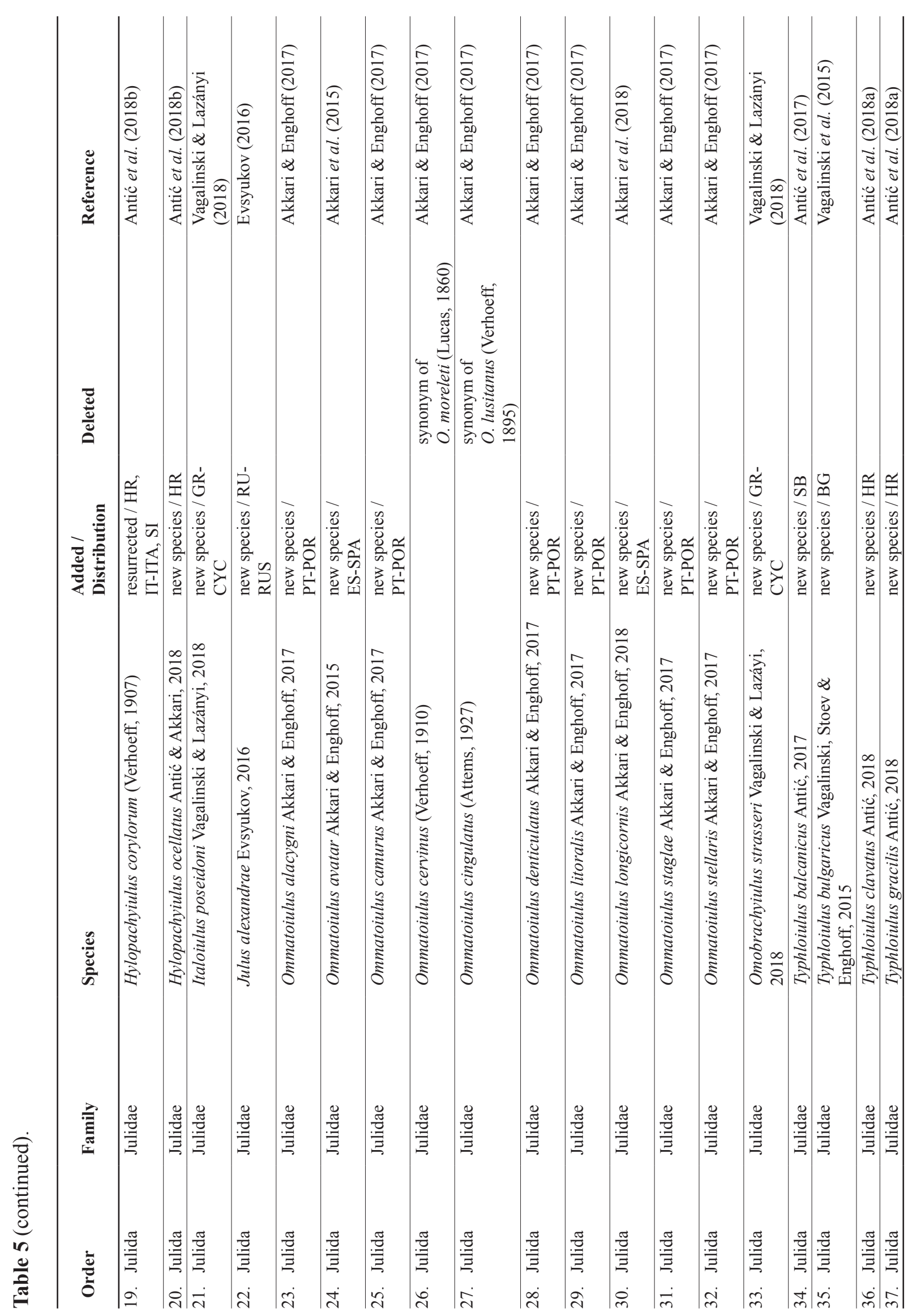




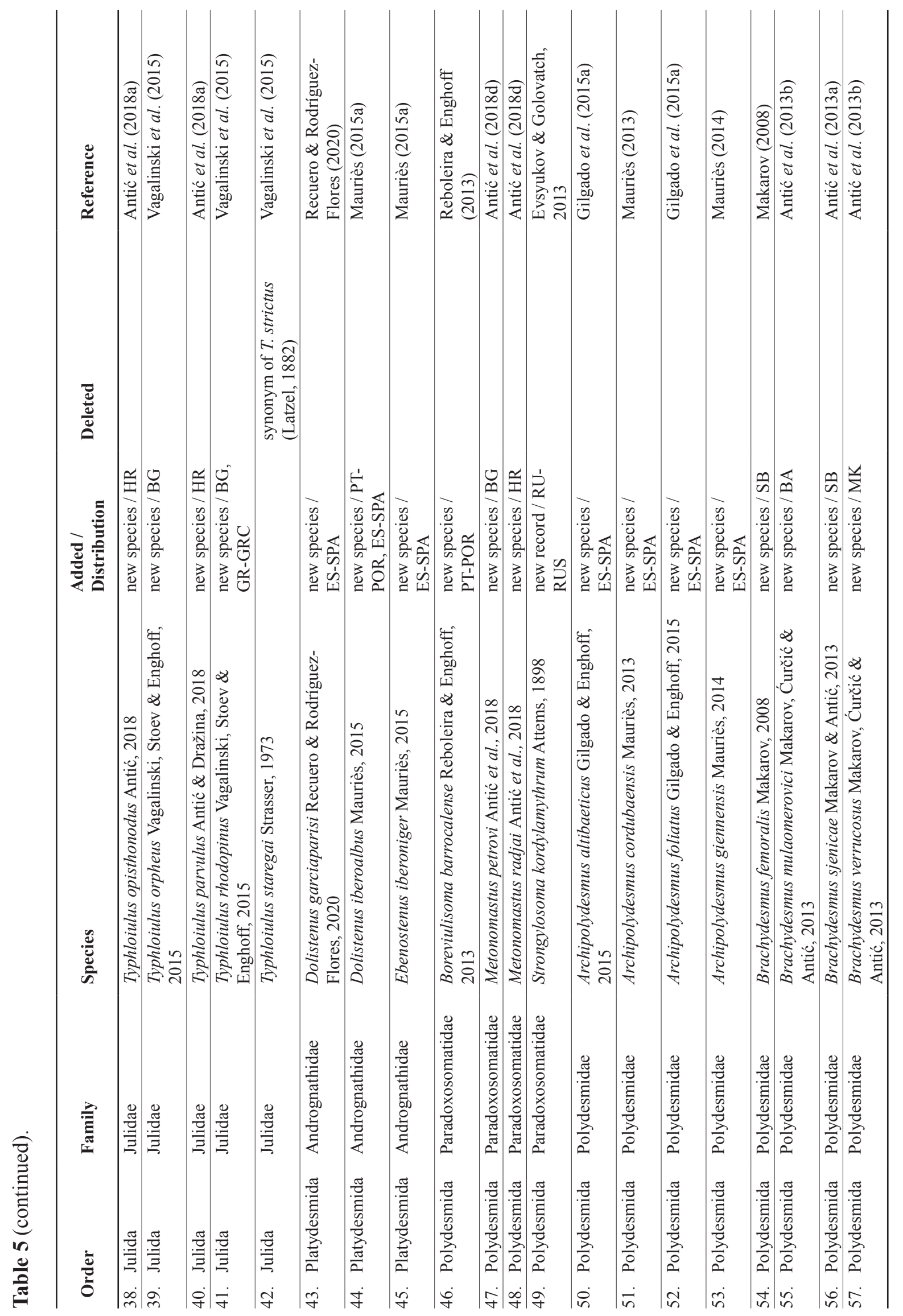




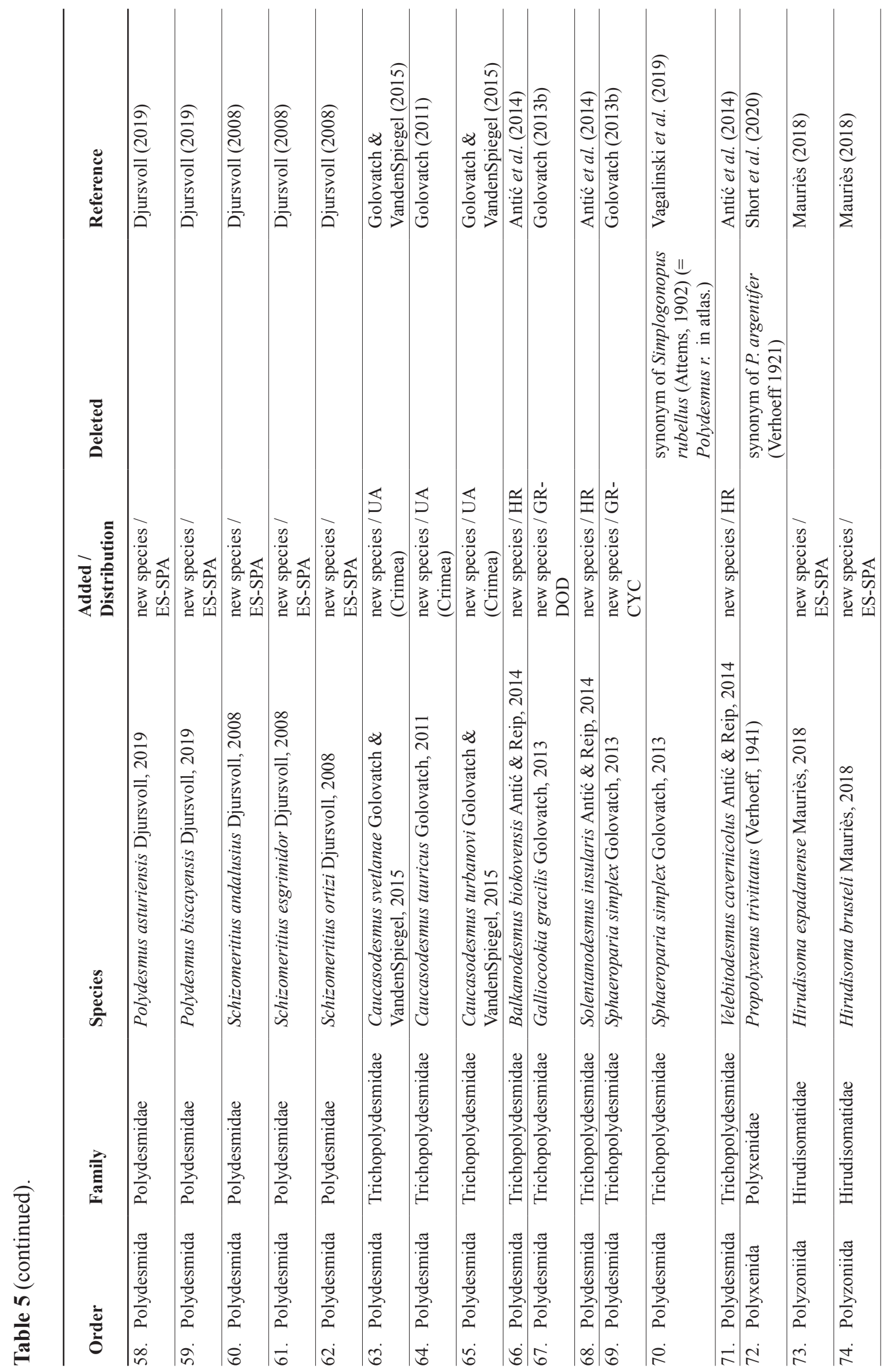


with Nesrine Akkari, placed their then unpublished distribution maps for species of Haasea at our disposal), Peter Decker (who extracted numerous records from Edaphobase), Jean-Jacques Geoffroy (including département-level distribution maps for all French species), José Domingo Gilgado, Eszter Lazányi, Slobodan Makarov, Jean-Paul Mauriès, Andrej Mock, Kjell Magne Olsen, Freddy Persson, Jörg Spelda, Karel Tajovský and Boyan Vagalinski.

Access to literature was greatly facilitated by Hans Reip. Help with nomenclatorial subtleties was provided by Verner Michelsen. Free access to the batch conversion functionality of Earthpoint.us was granted by Bill Clark.

Habitus photos of chordeumatidans were placed at our disposal by Dragan Antić, Tamara ČukovićMalenica, Tvrtko Dražina, José Domingo Gilgado, Marjan Komnenov, Kazimir Muculinić, Trevor and Dilys Pendleton, Paul Richards, and Jörg Spelda.

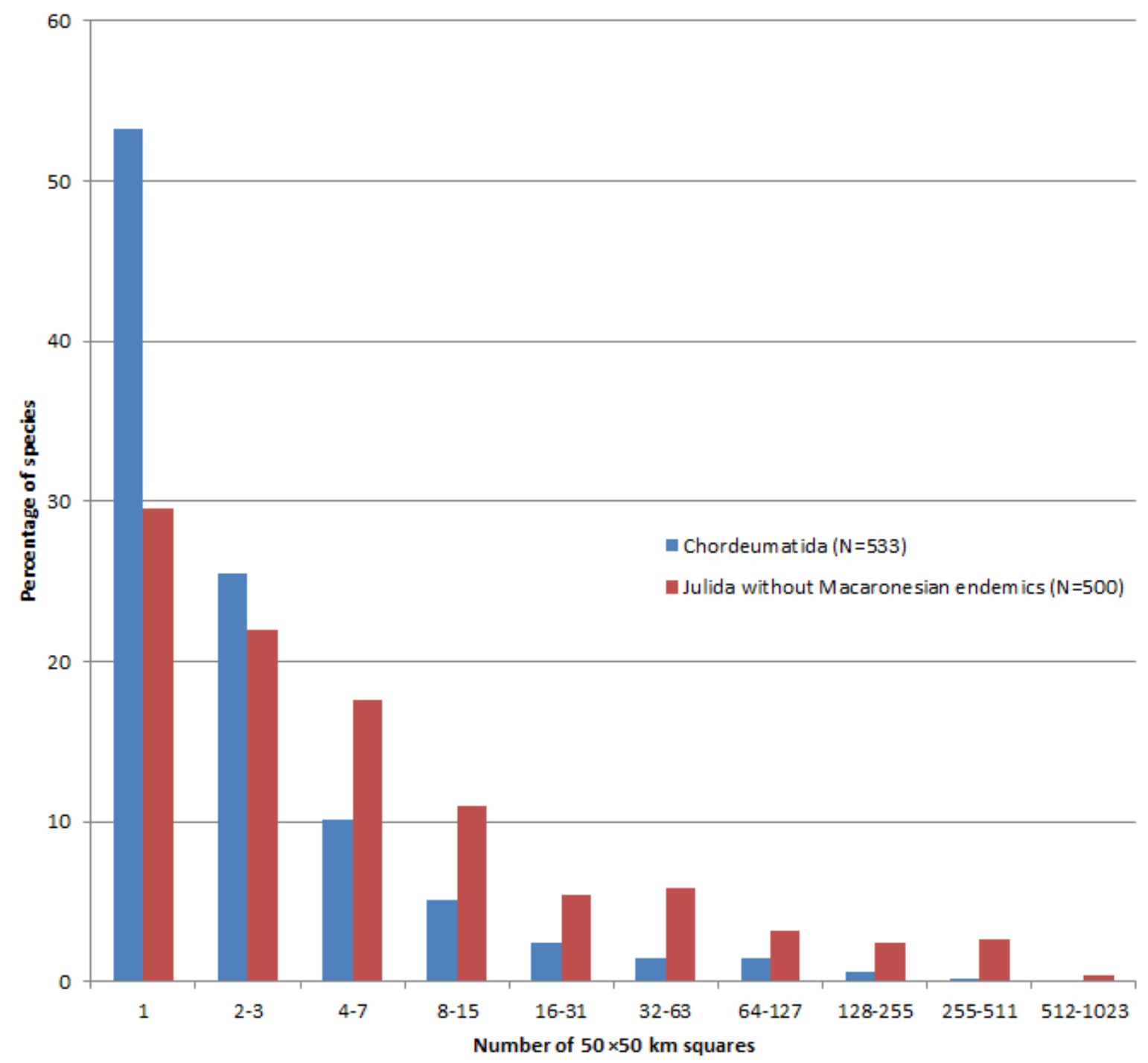

Fig. 7. Percentage of species recorded from 1, 2-3, 4-7 .. > 512-1023 $50 \mathrm{~km}$ squares of European Chordeumatida C.L. Koch, 1847 (blue columns) and Julida Brandt, 1833 without Macaronesian endemics (red columns, data from Kime \& Enghoff 2017). 
Dragan Antić and Jörg Spelda in addition bravely took it upon themselves to act as the editor's referees and in this capacity corrected several errors as well as made numerous constructive suggestions. Likewise, editor Nesrine Akkari suggested several important improvements. Kristiaan Hoedemakers provided exceptionally careful desk editing, and Radka Rosenbaumova helped with botanical nomenclature.

Last, but absolutely not least, the maps, without which the atlas would not be an atlas, were kindly produced by André J. van Loon (EIS Kenniscentrum Insecten, Leiden, Netherlands)

With the help of these persons, and the ones listed in previous volumes, the Atlas of European Millipedes has been completed - although, inevitably and fortunately, new species will continue to be discovered in Europe, new records will be made, errors will be corrected, and the status of more or less doubtful species will (hopefully) become resolved.

90

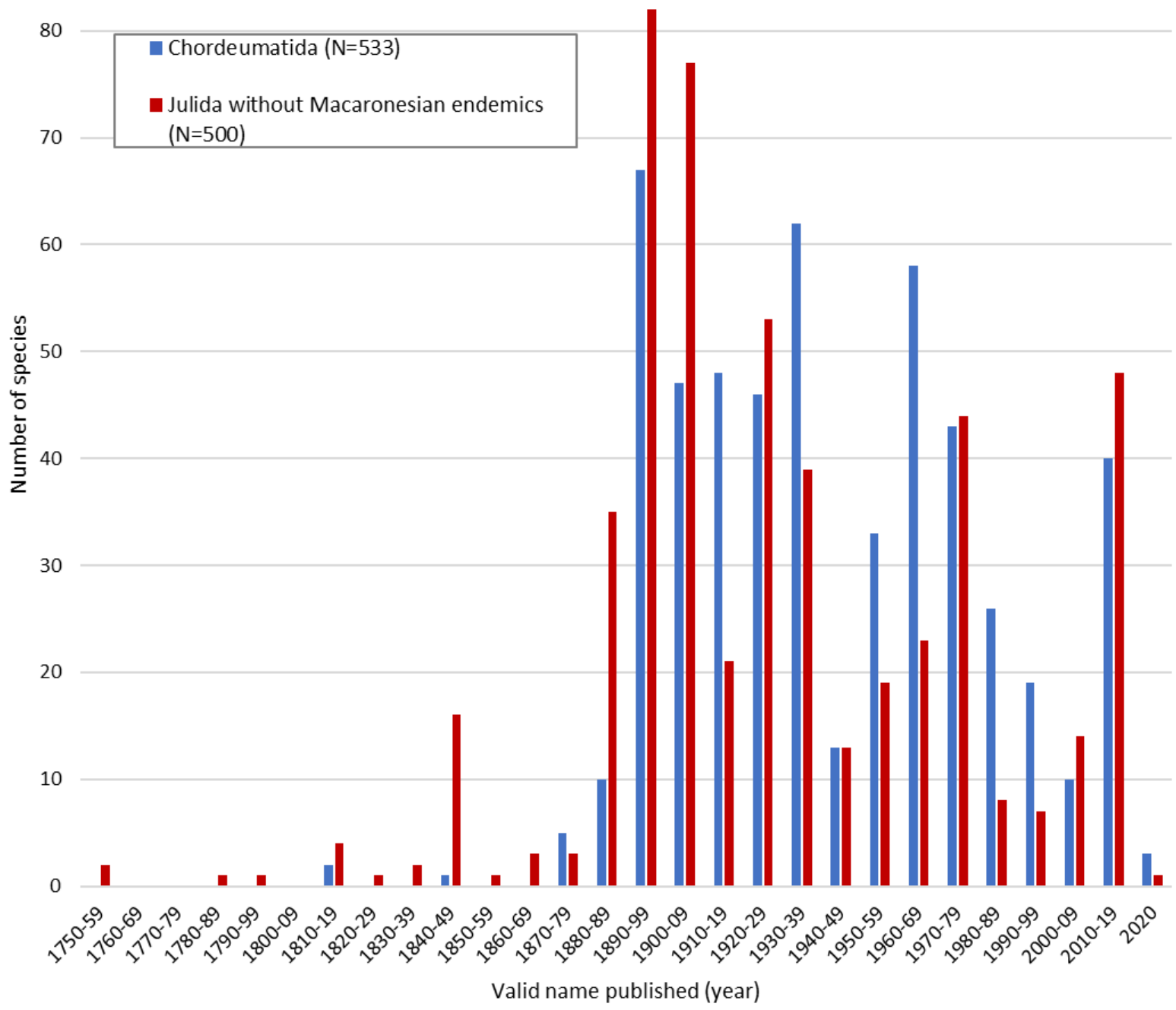

Fig. 8. Year (decade) of publication of valid names of European Chordeumatida C.L. Koch, 1847 (blue columns) and Julida Brandt, 1833 without Macaronesian endemics (red columns, data from Kime \& Enghoff 2017 and from Table 5 of the present work). 


\section{References}

Akkari N. \& Enghoff H. 2017. Revision of the genus Ommatoiulus Latzel, 1884 (Julida, Diplopoda) in Portugal, with description of six new species. European Journal of Taxonomy 295: 1-42. https://doi.org/10.5852/ejt.2017.295

Akkari N., Enghoff H., Stoev P. \& Mauriès J.-P. 2010. On the identity of Basigona lucasii Silvestri, 1896, a poorly known millipede from Tunisia, with notes on the North African Chordeumatida (Diplopoda: Chordeumatida: Chamaesomatidae). Zootaxa 2427: 64-68. https://doi.org/10.11646/zootaxa.2427.1.7

Akkari N., Enghoff H. \& Metscher B. 2015. A new dimension in documenting new species: high-detail imaging for myriapod taxonomy and first 3D cybertype of a new millipede species (Diplopoda, Julida, Julidae). PLoS ONE 10 (8): e0135243. https://doi.org/10.1371/journal.pone.0135243

Akkari N., Gilgado J.D., Ortuño V. \& Enghoff H. 2018. Out of the dark void: Ommatoiulus longicornis n. sp., a new julid from Spain (Julida, Diplopoda) with notes on troglobiomorphic traits in millipedes. Zootaxa 4420 (3): 415-429. https://doi.org/10.11646/zootaxa.4420.3.7

Anderson R. 1996. Anamastigona pulchellum Silvestri (Chordeumatida: Anthroleucosomatidae) an addition to the fauna of northern Europe, recorded from Ireland. Bulletin of the British Myriapod Group 12: $7-11$.

Antić D. \& Akkari N. 2020. Haasea Verhoeff, 1895 - a genus of tumultuous history and chaotic records - redefinition, revision of taxonomy and geographic distributions, with descriptions of two new species from Austria and Serbia (Diplopoda, Chordeumatida, Haaseidae). Zootaxa 4798 (1): 1-77. https://doi.org/10.11646/zootaxa.4798.1.1

Antić D.Ž. \& Makarov S.E. 2016. The Caucasus as a major hotspot of biodiversity: evidence from the millipede family Anthroleucosomatidae (Diplopoda, Chordeumatida). Zootaxa 4211 (1): 1-205. https://doi.org/10.11646/zootaxa.4211.1.1

Antić D.Ž., Ćurčić B.P.M., Mitić B.M., Tomić V.T., Lučić L.R., Dudić B.D., Stojanović D.Z. \& Makarov S.E. 2013a. A new cave diplopod of the genus Brachydesmus Heller, 1858 from southwest Serbia (Diplopoda: Polydesmida: Polydesmidae. Archives of Biological Science, Belgrade 65 (2): 745750. https://doi.org/10.2298/ABS1302745A

Antić D.Ž., Ćurčić B.P.M., Tomić V.T., Rađa T., Rađa B., Milinčić M.A. \& Makarov S.E. 2013b. Two new species of Brachydesmus Heller, 1858 from the Balkan Peninsula (Diplopoda: Polydesmida: Polydesmidae). Archives of Biological Science, Belgrade 65 (3): 1233-1243.

https://doi.org/10.2298/ABS1303233A

Antić D.Ž., Reip H.S., Dražina T., Rađa T. \& Makarov S.E. 2014. Three new monotypic genera of Trichopolydesmidae from Croatia, Balkan Peninsula (Diplopoda, Polydesmida). Zootaxa 3884 (2): 101-121. https://doi.org/10.11646/zootaxa.3884.2.1

Antić D., Dražina T., Rađa T., Tomić V.T. \& Makarov S.E. 2015a. Review of the family Anthogonidae (Diplopoda, Chordeumatida), with descriptions of three new species from the Balkan Peninsula. Zootaxa 3948 (2): 151-181. https://doi.org/10.11646/zootaxa.3948.2.1

Antić D.Ž., Mock A. \& Enghoff H. 2015b. Two new species of the millipede family Blaniulidae (Diplopoda, Julida) from caves in eastern and southeastern Europe. Zootaxa 3985 (4): 523-540. https://doi.org/10.11646/zootaxa.3985.4.3

Antić D.Ž, Tomić V.T., Rađa T., Lučić L.R., Dudić B.D. \& Makarov S.E. 2015c. A new species of the family Heterolatzeliidae from the Balkan Peninsula (Diplopoda, Chordeumatida). Zootaxa 3904 (1): 147-150. https://doi.org/10.11646/zootaxa.3904.1.20 
KIME R.D. \& ENGHOFF H., Atlas of European millipedes 3: Order Chordeumatida

Antić D.Ž., Dražina T., Rađa T., Lučić L.R. \& Makarov S.E. 2016. Taxonomic status of the family Biokoviellidae Mršić, 1992 (Diplopoda, Chordeumatida): reconsideration, with a description of one new species. European Journal of Taxonomy 205: 1-23. https://doi.org/10.5852/ejt.2016.205

Antić D.Ž., Dudić B.D., Gajić M.R. \& Lučić L.R. 2017. The first hydrophilous cave-dwelling millipede from Serbia - Typhloiulus balcanicus sp. nov. (Diplopoda, Julida, Julidae). Zootaxa 4226 (1): 137143. https://doi.org/10.11646/zootaxa.4226.1.8

Antić D.Ž., Dražina T., Rađa T. Lučić L.R., \& Makarov S.E. 2018a. Review of the genus Typhloiulus Latzel, 1884 in the Dinaric region, with a description of four new species and the first description of the male of Typhloiulus insularis Strasser, 1938 (Diplopoda, Julida, Julidae). Zootaxa 4455 (2): 258-294. https://doi.org/10.11646/zootaxa.4455.2.2

Antić D.Ž., Rađa T. \& Akkari N. 2018b. Revision of the genus Hylopachyiulus Attems, 1904, with the description of a new species from Croatia (Diplopoda, Julida, Julidae). Zootaxa 4531 (2): 225-241. https://doi.org/10.11646/zootaxa.4531.2.4

Antić D.Ž, Rađa T. \& Makarov S.E. 2018c. Dalmatosomatidae, a new monotypic family, and Dalmatosoma agaricum gen. et sp. nov. (Diplopoda: Chordeumatida: Craspedosomatidea) from Croatia, Balkan Peninsula. Zootaxa 4403 (2): 289-306. https://doi.org/10.11646/zootaxa.4403.2.4

Antić D.Ž., Vagalinski B., Stoev P. \& Golovatch S. 2018d. Two new species of the millipede genus Metonomastus Attems, 1937 from the Balkan Peninsula (Diplopoda, Polydesmida, Paradoxosomatidae). ZooKeys 786: 43-57. https://doi.org/10.3897/zookeys.786.28386

Antić D.Ž., Stojanović D.Z. \& Makarov S.E. 2020. Cornogonopus - a new monotypic cave-dwelling genus of the family Anthroleucosomatidae (Diplopoda, Chordeumatida) from Serbia, the Balkan Peninsula. Biologia Serbica 42 (1): 32-47. https://doi.org/10.5281/zenodo.4147289

Bachvarova D., Vagalinsky B., Doichninov A. \& Stoev P. 2017. New records of millipedes and centipedes from Bulgaria, with an annotated checklist of the Bulgarian myriapods. Zootaxa 4263 (3): 507-526. https://doi.org/10.11646/zootaxa.4263.3.4

Berg M.P., Soesbergen M., Tempelman D. \& Wijnhoven H. 2008. Verspreidingsatlas Nederlandse landpissebedden, duizendpoten en miljoenpoten. Stichting European Invertebrate Survey, Leiden and Vrije Universiteit, Afdeling Dierecologie, Amsterdam.

Blower J.G. 1985. Millipedes. Synopses of the British Fauna (New Series) 35: 1-242.

Blower J.G. 1986. Distribution and variation of the species of Brachychaeteuma occurring in Britain and Ireland. Bulletin of the British Myriapod Group 3: 43-48.

Brummitt R.K. 2001. World Geographic Scheme for Recording Plant Distributions. Edition 2. Published for the International Working Group on Taxonomic Databases for Plant Sciences (TDWG) by the Hunt Institute for Botanitcal Documentation, Carnegie Mellon University, Pittsburgh. Available from https://github.com/tdwg/wgsrpd/blob/master/109-488-1-ED/2nd\%20Edition/TDWG_geo2.pdf [accessed 10 Mar. 2021].

Ceuca T. 1985. Diplopodes nouveaux dans la faune de la Roumanie (Diplopoda - Ascospermophora). Studia Universitatis Babeş-Bolyai Biologia 30: 35-46.

Ćurčić B.P.M. \& Makarov S.E. 1997. Postembryonic development of Bulgarosoma lazarevensis a cave-dwelling millipede from Yugoslavia (Diplopoda, Chordeumatida: Anthroleucosomatidae). Entomologica scandinavica Supplement 51: 163-165.

David J.-F. 1984. Le cycle annuel du diplopode Microchordeuma gallicum (Latzel, 1884). Bulletin de la Société zoologique de France 104 (1): 61-70. 
David J.-F. 1989. Le cycle biologique de Chamaesoma brolemanni Ribaut \& Verhoeff, 1913 (Diplopoda, Craspedosomatida) en forêt d'Orléans (France). Bulletin du Museum national d'histoire naturelle, Paris, 4 série 11, section A 3: 639-647.

Davidson M.B. \& Weddle R.B. 2021. Cylindroiulus dahli Demange, 1970 (Julidae) \& Orthochordeumella pallida (Rothenbühler, 1899) (Chordeumatida): two millipedes new to Britain and Propolydesmus testaceus (C.L. Koch, 1847) (Polydesmidae) a millipede new to Scotland. Bulletin of the British Myriapod and Isopod Group 33: 19-26.

de Jong Y., Verbeek M., Michelsen V., de Place Bjørn P., Los W., Steeman F., Bailly N., Basire C., Chylarecki P., Stloukal E., Hagedorn G., Wetzel F.T., Glöckler F., Kroupa A., Korb G., Hoffmann A., Häuser C., KohlbeckerA., Müller A., Güntsch A., Stoev P. \& Penev L. 2014. Fauna Europaea-all European animal species on the web. Biodiversity Data Journal 2: e4034. https://doi.org/10.3897/BDJ.2.e4034

Djursvoll P. 2008. Revision of the Iberian millipede genus Schizomeritus Verhoeff, 1931 (Diplopoda: Polydesmidae), with the description of three new species. International Journal of Myriapodology 1: $111-122$.

Djursvoll P. 2019. Two new species of Polydesmus Latreille, 1802/1803 from northern Spain with reinstatements of two species, and a key to the Iberian Polydesmus species (Diplopoda, Polydesmida, Polydesmidae). ZooKeys 888: 51-65 https://doi.org/10.3897/zookeys.888.37816

Dolejš P. \& Kocourek P. 2019. Bohumil Němec and his millipede collection at the National Museum in Prague (Czechia), with notes on Craspedosoma rawlinsii simplex Němec, 1896. Schubartiana 8: 25-35.

Enghoff H. 1994. Geographical parthenogenesis in millipedes. Biogeographica 70 (1): 25-31.

Enghoff H. \& Reboleira A.S.P.S. 2013. A new cave-dwelling millipede of the genus Scutogona from central Portugal (Diplopoda, Chordeumatida, Chamaesomatidae). Zootaxa 3736 (2): 175-186. https://doi.org/10.11646/zootaxa.3736.2.5

Enghoff H. \& Vicente M. 2000. Millipedes of the Balearic Islands, including a revision of the species described by L. Koch in 1881 (Diplopoda). Steenstrupia 25: 195-200.

Enghoff H., Golovatch S., Short M., Stoev P. \& Wesener T. 2015. Diplopoda - Taxonomic overview. In: Minelli A. (ed.) The Myriapoda 2. Treatise on Zoology - Anatomy, Taxonomy, Biology: 363-453. Brill, Leiden and Boston. https://doi.org/10.1163/9789004188273_017

Eusébio R., Enghoff H., Solodovnikov A., Michelsen A., Barranco P., Sendra A. \& Reboleira A.S.P.S. 2021. Temporal and spatial dynamics of arthropod groups in terrestrial subsurface habitats in central Portugal. Zoology 147: 125931. https://doi.org/10.1016/j.zool.2021.125931

Evsyukov A.P. 2016. A new species of the millipede genus Julus from the Rostov-on-Don Region, southern Russia (Diplopoda: Julida: Julidae). Arthropoda Selecta 25 (3): 241-245. https://doi.org/10.15298/arthsel.25.3.02

Evsyukov A.P. \& Golovatch S.I. 2013. Millipedes (Diplopoda) of the Rostov-on-Don Region, southern Russia. Arthropoda Selecta 22 (3): 207-215. https://doi.org/10.15298/arthsel.22.3.03

Fedrizzi G. 1877. Miriapodi del Trentino raccolti e classificati. I. I Chilognathi. Annuario della Società dei Naturalisti in Modena, Serie 2 11: 80-110.

Gaul W. \& Tighe A.J. 2021. Morphological and genetic confirmation of the millipede Chordeuma sylvestre C. L. Koch, 1847 new to Ireland. Bulletin of the British Myriapod and Isopod Group 33: 52-62. https://doi.org/10.1101/2020.05.17.100263

Gilgado J.D., Enghoff H., Tinaut A. \& Ortuño V.M. 2015a. Hidden biodiversity in the Iberian mesovoid shallow substratum (MSS): new and poorly known species of the millipede genus 
Archipolydesmus Attems, 1898 (Diplopoda, Polydesmidae). Zoologischer Anzeiger 258: 13-38. https://doi.org/10.1016/j.jcz.2015.06.001

Gilgado J.D., Enghoff H., Tinaut A., Mauriès J.P. \& Ortuño V.M. 2015b. Sierra Nevada (Granada, Spain): a high-altitude biogeographical crossroads for millipedes (Diplopoda), with data on its MSS fauna and description of a new species of the genus Ceratosphys Ribaut, 1920 (Chordeumatida: Opisthocheiridae). Zootaxa 4044 (3): 391-410. https://doi.org/10.11646/zootaxa.4044.3.4

Gilgado J.D., Enghoff H. \& Ortuño V.M. 2015c. The hypogean Iberian genus Typhlopsychrosoma Mauriès, 1982 (Diplopoda, Chordeumatida, Vandeleumatidae): distribution map, key to species, first record in a Mesovoid Shallow Substratum (MSS) and detailed iconography of T. baeticaense (Mauriès 2013). Zootaxa 3927 (2): 337-346. https://doi.org/10.11646/zootaxa.3937.2.5

Gilgado J.D., Ledesma E., Enghoff H., Mauriès J.-P. \& Ortuño V.M. 2017. A new genus and species of Haplobainosomatidae (Diplopoda: Chordeumatida) from the MSS of the Sierra de Guadarrama National Park, central Spain. Zootaxa 4347 (3): 492-510. https://doi.org/10.11646/zootaxa.4347.3.4

Gilgado J.D., Martínez-Pillado V. \& Prieto C. 2020. A new green-coloured Lusitanipus Mauriès, 1978 from the Iberian Peninsula (Diplopoda: Callipodida: Dorypetalidae). European Journal of Taxonomy 714: 1-14. https://doi.org/10.5852/ejt.2020.714

Giurginca A., Vănoaica L., Šustr V. \& Tajovský K.2020 A new species of the genus Archiboreoiulus Brolemann, 1921 (Diplopoda, Julida) from Movile Cave (Southern Dobrogea, Romania). Zootaxa 4802 (3): 463-476. https://doi.org/10.11646/zootaxa.4802.3.4

Golovatch S. 2011. The millipede genus Caucasodesmus Golovatch, 1985, with the description of a new species from the Crimea, Ukraine (Polydesmida, Diplopoda, Trichopolydesmidae). ZooKeys 93: 1-8. https://doi.org/10.3897/zookeys.93.1159

Golovatch S. 2013a. Three new species of the millipede genus Hyleoglomeris Verhoeff, 1910 from the Aegean region of Greece (Diplopoda, Glomerida, Glomeridae). Biodiversity Data Journal 1: e1000. https://doi.org/10.3897/BDJ.1.e1000

Golovatch S.I. 2013b. A reclassification of the millipede superfamily Trichopolydesmoidea, with descriptions of two new species from the Aegean region (Diplopoda, Polydesmida). ZooKeys 340: 6378. https://doi.org/10.3897/zookeys.340.6295

Golovatch S.I. \& Makarov S.S.E. 2011. Two new, easternmost species of the millipede genus Anamastigona Silvestri, 1898 from Israel and the Caucasus (Diplopoda, Chordeumatida, Anthroleucosomatidae). Arthropoda Selecta 20 (1): 11-20. https://doi.org/10.15298/arthsel.20.1.02

Golovatch S.I. \& Matyukhin A.B. 2011. New records of millipedes (Diplopoda), mainly from bird nests, in European Russia. Arthropoda Selecta 20 (2): 115-116. https://doi.org/10.15298/arthsel.20.2.02

Golovatch S.I. \& VandenSpiegel D. 2015. Two new species of the millipede genus Caucasodesmus Golovatch, 1985 from the Crimea, Russia (Diplopoda, Polydesmida, Trichopolydesmidae). Russian Entomological Journal 24 (1): 1-6. https://doi.org/10.15298/rusentj.24.1.01

Gregory S. 2018. More sites for lumpy-bumpy millipedes in South Wales. British Myriapod and Isopod Group Newsletter 37: 4-5.

Gregory S.J., Davidson M., Owen C. \& Anderson R. 2015. Anamastigona pulchella (Silvestri, 1894) first British records for England, Scotland and Wales (Chordeumatida: Anthroleucosomatidae). Bulletin of the British Myriapod and Isopod Group 28: 31-37.

Gruber J. 2009. Diplopoda (Doppelfüsser). In: Rabitsch W. \& Essl F. (eds) Endemiten - Kostbarkeiten in Österreichs Pflanzen- und Tierwelt: 512-541. Naturwissenschaftlicher Verein für Kärnten und Umweltbundesamt GmbH, Klagenfurt und Wien. 
Hauser H. 2004a. Untersuchungen zur Systematik und Biogeografie der Craspedosoma rawlinsii LeachGruppe (Diplopoda: Chordeumatida: Craspedosomatidae). Entomologische Nachrichten und Berichte Beiheft 9: 1-33.

Hauser H. 2004b. Zur Taxonomie und Systematik von Mastigona bosniensis (Verhoeff, 1897) und Mastigona vihorlatica (Attems, 1899) (Diplopoda, Chordeumatida, Mastigophorophyllidae). Entomologische Nachrichten und Berichte 48 (2-3): 215-218.

Hauser H. \& Voigtländer K. 2019. Doppelfüßer (Diplopoda) Deutschlands. Verhalten Ökologie Verbreitung Lebendbestimmung. DJN, Deutscher Jugendbund für Naturbeobachtung, Göttingen.

ICZN. 1999. International Code of Zoological Nomenclature. Fourth Edition. The International Trust for Zoological Nomenclature, London, UK.

Jeekel C.A.W. 1970. Nomenclator generum et familiarum Diplopodorum. A list of the genus and family-group names in the Class Diplopoda from the $10^{\text {th }}$ edition of Linnaeus, 1758 , to the end of 1957. Monografieën van de Nederlandse Entomologische Verenigung 5: i-xii + 1-412.

Kime R.D. 2004. The Belgian millipede fauna (Diplopoda). Bulletin de l'Institut royal des Sciences naturelles de Belgique, Entomologie 74: 35-68.

Kime R.D. \& Enghoff H. 2011. Atlas of European millipedes (Class Diplopoda). Volume 1. Orders Polyxenida, Glomerida, Platydesmida, Siphonocryptidae, Polyzoniida, Callipodida, Polydesmida. Fauna Europaea Evertebrata 3. Pensoft, Sofia and Moscow.

Kime R.D. \& Enghoff H. 2017. Atlas of European millipedes 2: Order Julida (Class Diplopoda). European Journal of Taxonomy 346: 1-299. https://doi.org/10.5852/ejt.2017.346

Korsós Z. \& Lazányi E. 2020. Present status of the millipede fauna of Hungary, with a review of three species of Brachyiulus Berlése, 1884 (Diplopoda). Opuscula Zoologica Budapest 51 (Supplementum 2): 87-103. https://doi.org/10.18348/opzool.2020.S2.87

Kovačević Ž. 1923. Prinos poznavanju Glomerida Hrvatske. Glasnik Hrvatskog Prirodoslovnog Društva 35: $566-577$.

Lazányi E. \& Korsós Z. 2009. Millipedes (Diplopoda) of the Aggtelek National Park, Northeast Hungary. Opuscula Zoologica Budapest 40 (1): 35-46.

Lee P. 2006. Atlas of the millipedes (Diplopoda) of Britain and Ireland. Series Faunistica 59. Pensoft, Sofia.

Lindner E.N., Reip H.S. \& Spelda J. 2010. Anamastigona pulchella (Silvestri, 1898) (Diplopoda: Chordeumatida: Anthroleucosomatidae) - ein für Deutschland neuer Tausendfüsser. Schubartiana 4: $1-8$.

Makarov S.E. 2008. A new species of the diplopod subgenus Absurdodesmus Mršić, 1988 from a cave in the Tara Mountain, West Serbia (Diplopoda, Polydesmidae). In: Pavićević D. \& Perreau M. (eds) Advances in the Studies of the Fauna of the Balkan Peninsula. Papers Dedicated to the Memory of Guido Nonveiller: 81-84. Institute for Nature Conservation of Serbia, Belgrade.

Makarov S.E., Ćurčić B.P.M., Tomić V.T., Rađa T., Rađa B., Ćurčić S.B., Mitić B.M. \& Lučić L.R. 2011. Revision of the family Heterolatzeliidae (Diplopoda, Chordeumatida). Zootaxa 2994: 33-44. https://doi.org/10.11646/zootaxa.2994.1.3

Makarov S.E., Ćurčić B.P.M., Antić D.Ž, Tomić V.T., Ćurcić S.B., Ilić B. \& Lučić L.R. 2013. A new cave species of the genus Hyleoglomeris Verhoeff, 1910, from the Balkan Peninsula (Diplopoda: Glomerida: Glomeridae). Archives of Biological Science, Belgrade 65 (1): 341-344. https://doi.org/10.2298/ABS1301341M 
KIME R.D. \& ENGHOFF H., Atlas of European millipedes 3: Order Chordeumatida

Manfredi P. 1953. Miriapodi Italiani - VIII contribute. Atti della Società italiana di scienze naturali, e del Museo civile di storia naturale 92: 117-122.

Manfredi P. 1956. Miriapodi cavernicoli del Marocco, delle Sardegna e del Piemonte. Atti della Società Italiana di Scienze Naturali 95: 197-222.

Mauriès J.-P. 1968. Notes sur les Diplopodes Pyrénéens: IV. Le genre endémique Hypnosoma Ribaut, 1952. Bulletin de la Société d'Histoire naturelle de Toulouse 104: 399-404.

Mauriès J.-P. 1969. Contribution à la faune épigée et cavernicole de Corse: Diplopodes récoltés par P. Beron. Annales de Spéléologie 24: 505-527.

Mauriès J.-P. 1985. Polydesmide et Craspedosomides cavernicoles nouveaux de France et du Maroc (Myriapoda - Diplopoda). International Journal of Speleology 14: 51-62. https://doi.org/10.5038/1827-806X.14.1.6

Mauriès J.-P. 1986. Un Diplopode cavernicole relictuel des Alpes calcaires suisses: Niphatrogleuma wildbergeri, n. g., n. sp. (Craspedosomida, Cleidogonoidea). Revue suisse de Zoologie 93 (1): 249-256. https://doi.org/10.5962/bhl.part.79693

Mauriès J.-P. 1990a. Révision des Origmatogonidae. Taxons nouveaux de France et d'Espagne (Diplopoda, Craspedosomida, Chamaesomidae, Opisthocheiridae). Bulletin de la Société d'Histoire naturelle de Toulouse 148: 47-57.

Mauriès J.-P. 1990b. Diplopodes de la Péninsule Ibérique: Deux espèces nouvelles du genre Ceratosphys Ribaut, 1920 (Diplopoda, Craspedosomida, Opisthocheiridae). Miscellània zoológica Barcelona 14: $115-123$.

Mauriès J.-P. 2003. Schizmohetera olympica sp.n. from Greece, with a reclassification of the superfamily Neoatractosomatoidea (Diplopoda: Chordeumatidae). Arthropoda Selecta 12: 9-16.

Mauriès J.-P. 2010. Révision du genre endémique Pyreneosoma Mauriès, 1959: historique, nouveautés (Diplopoda, Craspedosomatida, Haplobainosomatidae). Bulletin de la Société d'Histoire naturelle de Toulouse 146: 21-46.

Mauriès J.-P. 2012. Le genre Ceratosphys Ribaut, 1920: trois nouveaux taxa de Catalogne et des Îles Baléares (Diplopoda, Craspedosomatida, Opisthocheiridae). Bulletin de la Société d'Histoire naturelle de Toulouse 148: 47-57.

Mauriès J.-P. 2013. Trois espèces nouvelles de diplopodes cavernicoles de l'Andalousie (Espagne) (Diplopoda: Polydesmida: Polydesmidae; Chordeumatida: Vandeleumatidae, Opisthocheiridae). Athropoda Selecta 22 (2): 97-112. https://doi.org/10.15298/arthsel.22.2.01

Mauriès J.-P. 2014. Quatre espèces nouvelles de Diplopodes cavernicoles de l'Andalousie (Espagne) (Diplopoda: Polydesmida: Polydesmidae; Chordeumatida: Chamaesomatidae, Opisthocheiridae). Arthropoda Selecta 23 (1): 33-50. https://doi.org/10.15298/arthsel.23.1.03

Mauriès J.-P. 2015a. Diplopodes platydesmides et polyzonides collectés dans le Nord-ouest de la Péninsule Ibérique par les missions britanniques de 1993 et 2004. Russian Entomological Journal 24 (4): 325-341. https://doi.org/10.15298/rusentj.24.4.08

Mauriès J.-P. 2015b. Taxa nouveaux de Diplopodes Craspedosomatides collectés dans le nord-ouest de la Péninsule ibérique par les missions britanniques de 1993 et 2004 (Diplopoda, Craspedosomatida). Bulletin de la Société d'Histoire naturelle de Toulouse 150: 27-57.

Mauriès J.-P. 2018. Le genre Hirudisoma Fanzago, 1881 dans la péninsule Ibérique: description de deux espèces atypiques, Hirudisoma espadanensis n. sp. et H. brusteli n. sp. du Levant d'Espagne. 
(Diplopoda, Polyzonida, Hirudisomatidae). Bulletin de la Société d'Histoire naturelle de Toulouse 154: $41-55$.

Mauriès J.-P. \& Vicente M.C. 1977. Diplópodos cavernícolas nuevos y poco conocidos de España recoletados par A. Lagar. Descripción de tres géneros nuevos. Miscellania Zoologica 4 (1): 109-134.

McAlpine D.F. \& Shear W.A. 2018. The millipede Craspedosoma raulinsii Leach, 1814 (Chordeumatida: Craspedosomatidae) in North America with comments on the derivation of its binomial name. Zootaxa 4455 (2): 389-394. https://doi.org/10.11646/zootaxa.4455.2.8

Meyer E. 1979. Life cycles and ecology of high alpine Nematophora. In: Camatini M. (ed.) Myriapod Biology: 295-306 Academic Press, London.

Meyer E. 1990. Altitude-related changes of life histories of Chordeumatida in the Central Alps (Tyrol, Austria). In: Minelli A. (ed.) Proceedings of the $7^{\text {th }}$ International Congress of Myriapodology: 311-322. E.J. Brill, Leiden.

Meyer E. \& Singer A. 1997. Verteilung, Aktivität und Besiedlungsdichte von Diplopoden in Wäldern Vorarlsbergs (Österreich). Berichte des naturwissenschaftlich-medizinischen Vereins Innsbruck 84: 287-306.

Mock A., Tajovský K., Žurovcová M., Jarošová A., Kocourek P., Gruber J., Angyal D. \& Spelda J. 2016. Hungarosoma bokori Verhoeff, 1928 (Diplopoda: Chordeumatida): new insights into its taxonomy, systematics, molecular genetics, biogeography and ecology. Zootaxa 4178 (2): 234-256. https://doi.org/10.11646/zootaxa.4178.2.4

Mock A., Hal'ková B. \& Tajovský K. 2019. Unique external morphology of millipedes of the family Trachygonidae (Diplopoda, Chordeumatida): case study on Heteracrochordum evae (Loksa, 1960). In: Dányi L., Korsós Z. \& Lazányi E. (eds) $18^{\text {th }}$ International Congress of Myriapodology, 25-31 August 2019, Budapest, Hungary. Program and Abstracts: 46. Hungarian Natural History Museum and Hungarian Biological Society, Budapest.

Mršić N. 1987a. Attemsiidae (Diplopoda) of Yugoslavia. Razprave IV. Razreda Sazu 27: 101-168.

Mršić N. 1987b. Genus Ochogona Cook 1895 (Craspedosomatidae, Diplopoda) of Yugoslavia. Bulletin du Muséum d'Histoire naturelle de Belgrade, Série B, Sciences biologiques 42: 51-74.

Mršić N. 1994. The Diplopoda (Myriapoda) of Croatia. Razprave IV. Razreda Sazu 35 (12): 219-296.

Nešić C., Pavićević D. \& Belij S. 2007. Results of the complex speleological research of northwest part of the Svrljiške Mountains. Protection of Nature: Journal of The Institute for Nature Conservation of Serbia 57 (1-2): 47-62. [In Serbian.]

Owen C. \& Gregory S. 2021. Three apparently undescribed silk millipedes (Diplopoda: Chordeumatida) recorded from south Wales. Bulletin of the British Myriapod and Isopod Group 33: 9-18.

Pedroli-Christen A. 1993. Faunistique des mille-pattes de Suisse (Diplopoda). Documenta Faunistica Helvetiae 14: 1-245.

Pedroli-Christen A. \& Mauriès J.-P. 1992. Un genre oublié d'Atractosomatinae des Alpes italo-suisses, Bomogona Cook, 1895 - statut, contenu, chorologie (Diplopoda, Craspedosomatida). Bulletin du Muséum national d'histoire naturelle, 4 Série, Section A, Zoologie 14: 457-472.

Pedroli-Christen A. \& Scholl A. 1996a: The taxa of Rhymogona (Diplopoda, Craspedosomatidae): a ring species. Part one: genetic analysis of population structure. Mémoires du Muséum national d'histoire naturelle, Paris 169: 45-51. 
KIME R.D. \& ENGHOFF H., Atlas of European millipedes 3: Order Chordeumatida

Pedroli-Christen A. \& Scholl A. 1996b: Rhymogona (Diplopoda, Craspedosomatidae), un genre monospécifique. Deuxième Partie: Révision basée sur les résultats morphologiques, génétiques et faunistiques. Mémoires du Muséum national d'histoire naturelle, Paris 169: 53-60.

Proudlove G.S. 2011. Notes on authorship, type material and current systematic position of the diplopod taxa described by Hilda K. Brade-Birks and S. Graham Brade-Birks. Bulletin of the British Myriapod and Isopod Group 25: 2-13.

Reboleira A.S.P.S. \& Enghoff H. 2013. The genus Boreviulisoma Brolemann, 1928 - an Iberian-N African outlier of a mainly tropical tribe of millipedes (Diplopoda: Polydesmida: Paradoxosomatidae). Zootaxa 3646 (5): 516-528. https://doi.org/10.11646/zootaxa.3646.5.2

Reboleira A.S.P.S. \& Enghoff H. 2018. First continental troglobiont Cylindroiulus millipede (Diplopoda, Julida, Julidae). ZooKeys 795: 93-103. https://doi.org/10.3897/zookeys.795.27619

Recuero E. \& Rodríguez-Flores P.C. 2020. A new Mediterranean species of Dolistenus (Diplopoda, Platydesmida, Andrognathidae), with an updated key for the genus and the first contribution for a barcode database of European Platydesmida. Zootaxa 4718 (1): 123-133.

https://doi.org/10.11646/zootaxa.4718.1.10

Reip H., Decker P., Voigtländer K., Lindner E.N., Hannig K. \& Spelda J. 2012. Seltene Myriapoden Deutschlands (Diplopoda, Chilopoda). Scubartiana 5: 49-112.

Reip H.S., Spelda J., Voigtländer K., Decker P. \& Lindner E.N. 2016. Rote Liste und Gesamtartenliste der Doppelfüßer (Myriapoda: Diplopoda) Deutschlands. In: Gruttke H., Balzer S., Binot-Hafke M., Haupt H., Hofbauer N., Ludwig G., Matzke-Hajek G. \& Ries M. (eds) Rote Liste der gefährdeten Tiere, Pflanzen und Pilze Deutschlands. Band 4: Wirbellose Tiere (Teil 2). Naturschutz und Biologische Vielfalt 70 (4): 301-324. Bundesamt für Naturschutz, Bonn.

Ribaut H. 1913. Biospeologica XXVIII Ascospermophora (Myriopodes) (Première Série). Archives de Zoologie expérimentale et générale 5e Série 10: 399-478.

Sammet K., Ivask M. \& Kurina O. 2018. A synopsis of Estonian myriapod fauna (Myriapoda: Chilopoda, Diplopoda, Symphyla and Pauropoda). ZooKeys 793: 63-96. https://doi.org/10.3897/zookeys.793.28050

Schubart O. 1934. Tausendfüssler oder Myriapoda I: Diplopoda. Die Tierwelt Deutschlands 28. Fischer, Jena.

Sendra A., Antić D., Barranco P., Borko Š., Christian E., Delić T., Fadrique F., Faille A., Galli L., Gasparo F., Georgiev D., Giachino P.M. Kováć L., Lukić M., Marcia P., Miculinić K., Nicolosi G., Palero F., Paragamian K., Pérez T., Polak S., Prieto C.E., Turbanov I., Vailati D. \& Reboleira A.S.P.S. 2020. Flourishing in subterranean ecosystems: Euro-Mediterranean Plusiocampinae and tachycampoids (Diplura,Campodeidae).European Journalof Taxonomy 591:1-138.https://doi.org/10.5852/ejt.2020.591

Serra A. \& Mauriès J.P. 2015. Ecologie, ontogenèse et description d'un Diplopode édaphique de Catalogne: Eopsychrosoma serrapradense n. g., n. sp. (Diplopoda, Chordeumatida, Craspedosomatidea, Vandeleumatidae). Bulletin de la Société d'Histoire naturelle de Toulouse 151: 15-28.

Serra A. \& Mauriès J.P. 2018. Contribution à la connaissance des Chamaesomatinae, avec description, ontogenèse et écologie de Xystrosoma santllorence n. sp. (Diplopoda, Chordeumida, Craspedosomatidea, Chamaesomatidae). Bulletin de la Société d'Histoire naturelle de Toulouse 154: 57-70.

Shear W.A. 1988. Systematic position of the milliped species Alloiopus solitarius Attems and the genus Ghilarovia Gulicka (Chordeumatida, Anthroleucosomatidae). Myriapodologica 2: 51-58.

Shear W.A. 1990. On the Central and East Asian milliped family Diplomaragnidae (Diplopoda, Chordeumatida, Diplomaragnoidea). American Museum Novitates 2977: 1- 40. 
Shear W.A. \& Leonard W.P. 2004. The millipede family Anthroleucosomatidae new to North America: Leschius mcallisteri, n.gen., n.sp. (Diplopoda: Chordeumatida: Anthroleucosomatidae). Zootaxa 609: 1-7. https://doi.org/10.11646/zootaxa.609.1.1

Short M., Vahtera V., Wesener T. \& Golovatch S.I. 2020. The millipede family Polyxenidae (Diplopoda, Polyxenida) in the faunas of the Crimean Peninsula and Caucasus, with notes on other European Polyxenidae. Zootaxa 4772 (2): 306-332. https://doi.org/10.11646/zootaxa.4772.2.4

Sierwald P. \& Spelda J. 2020. MilliBase. Available from http://www.millibase.org [accessed 28 May 2020]. https://doi.org/10.14284/370

Silvestri F. 1903. Anamastigona pulchellum Silvestri. In: Berlese A. (ed.) Acari, Myriopoda et Scorpiones hucusque in Italia reperta 98 (2). https://doi.org/10.5962/bhl.title.69269

Spelda J. 1991. Zur Faunistik und Systematik der Tausendfüssler (Myriapoda) Südwestdeutschlands. Jahreshefte der Gesellschaft für Naturkunde Württemberg 146: 211-232.

Spelda J. 1996. Millipedes as aids for the reconstruction of glacial refugia (Myriapoda: Diplopoda). Mémoires du Muséum national d'histoire naturelle, Paris 169: 151-161.

Spelda J. 2001. Review of the millipede genus Pterygophorosoma Verhoeff, 1897 (Diplopoda, Chordeumatida, Craspedosomatidae). Andrias 15: 29-48.

Spelda J. 2005. Improvements in the knowledge of the myriapod fauna of southern Germany between 1988 and 2005 (Myriapoda: Chilopoda, Diplopoda, Pauropoda, Symphyla). Peckiana 4: 101-129.

Spelda J. 2008. The genus Pyrgocyphosoma Verhoeff, 1910 (Diplopoda, Chordeumatida: Craspedosomatidae): New aspects on systematics, distribution and ecology. Schubartiana 3: 5-48.

Spelda J. 2015. Clase Diplopoda Orden Chordeumatida.Revista IDE@-SEA 26A: 1-15.

Spungisis V. 2010. Fauna of millipedes (Diplopoda) in Latvia with notes on occurrence, habitat preference and abundance. Latvijas Entomologs 48: 107-115.

Stoev P. 2008. A new species of Acanthopetalum Verhoeff, 1900 (Diplopoda: Callipodida: Schizopetalidae) from Bulgaria, with a review of the A. richii (Gray, 1832) group. International Journal of Myriapodology 2: 155-170. Available from https://brill.com/view/journals/ijm/1/2/article-p155.xml [accessed 8 Sep. 2021].

Strasser K. 1959. Phänologische Studien an Diplopoden und Bemerkungen über einige nordadriatische Arten. Annalen des naturhistorischen Museum in Wien 63: 461-467.

Strasser K. 1981. Über italienische, besonders cavernicole Diplopoden, III. Bollettino del museo civico di storia naturale di Verona 8: 221-233.

Strasser K. \& Minelli A. 1984. Elenco dei diplopodi d'Italia - A checklist of Italian Diplopods. Societa Veneziana di Scienze Naturali Lavori 9 (2): 193-212.

Sziráki Gy. 1966. Classification Key for Hungarian female Diplopods. PhD thesis, Eötvös Loránd University, Faculty of Science, Department of Systematic Zoology and Ecology. [In Hungarian.]

Tajovský K. 1996. Life-cycle of the millipede Melogona voigti (Verhoeff, 1899) from a suburban forest in South Bohemia. Mémoires du Muséum national d'histoire naturelle, Paris 169: 509-514.

Tajovský K. 2001. Millipedes (Diplopoda) of the Czech Republic. Myriapodologica Czecho-Slovaca 1: $11-24$.

Tajovský K., Mock A. \& Papáč V. 2014. The genus Hylebainosoma Verhoeff, 1899 (Diplopoda, Chordeumatida, Haaseidae): Redescription of Hylebainosoma tatranum, description of a new troglobiont 
KIME R.D. \& ENGHOFF H., Atlas of European millipedes 3: Order Chordeumatida

species and notes to the Hylebainosoma-Romanosoma species group. Zootaxa 3764 (5): 501-523. https://doi.org/10.11646/zootaxa.3764.5.1

Telfer M.G., Gregory S.J., Kime R.D., Owen C. \& Spelda J. 2015: Ceratosphys amoena Ribaut, 1920 and Hylebainosoma nontronensis Mauriès \& Kime, 1999 new to Britain (Diplopoda: Chordeumatida). Bulletin of the British Myriapod and Isopod Group 28: 15-30.

Thaler K. 1984. Fragmenta faunistic tirolensia - VI (Arachnida: Arenei, Opiliones; Myriapoda: Diplopoda, Chilopoda; Insecta: Coleoptera, Carabidae). Berichte des naturhistorischen-medizinischen Vereins Innsbruck 71: 97-118.

Vagalinsky B. \& Golovatch S. I. 2016. Two new species of Anamastigona from Cyprus and an updated key to species of the genus (Diplopoda: Chordeumatida: Anthroleucosomatidae). European Journal of Taxonomy 227: 1-19. https://doi.org/10.5852/ejt.2016.227

Vagalinski B. \& Lazányi E. 2018. Revision of the millipede tribe Brachyiulini Verhoeff, 1909 (Diplopoda: Julida: Julidae), with descriptions of new taxa. Zootaxa 4421 (1): 1-142.

https://doi.org/10.11646/zootaxa.4421.1.1

Vagalinski B., Stoev P. \& Enghoff H. 2015. A review of the millipede genus Typhloiulus Latzel, 1884 (Diplopoda: Julida: Julidae), with a description of three new species from Bulgaria and Greece. Zootaxa 3999 (3): 334-362. https://doi.org/10.11646/zootaxa.3999.3.2

Vagalinski B., Golovatch S.I., Akkari N. \& Stoev P. 2019. Simplogonopus rubellus (Attems, 1902) gen. n., comb. n. (Diplopoda: Polydesmida: Trichopolydesmidae): Revealing the identity of an enigmatic Eastern-Mediterranean millipede. Acta Zoologica Bulgarica 71 (3): 325-334.

Verhoeff K.W. 1900. Beiträge zur Kenntniss paläarktischer Myriopoden. XIII. Aufsatz: Zur vergleichenden Morphologie, Phylogenie, Gruppen- und Art-Systematik der Ascospermophora. Archiv für Naturgeschichte 66 (1): 347-402.

Verhoeff K.W. 1910. Über Diplopoden. 11.-15. (31.-35.) Aufsatz: Beiträge zur Kenntnis der Glomeriden, Juliden, Ascospermorpha und Lysiopetaliden, sowie zur Fauna Siziliens, Untersuchungen über Art- und Gruppensystematik; Morphologie, nachembryonale Entwicklung. Nova acta Academiae Caesareae Leopoldino-Carolinae Germanicae Naturae Curiosorum 92 (2): 139-448.

Verhoeff K.W. 1913. Zwei neue Gattungen der Trachyzona n. superfam. der Ascospermophora. Zoologischer Anzeiger 42: 125-143.

Verhoeff K.W. 1921. Über Diplopoden der Riviera und einige alpenländische Chilognathan (92. Diplopoden-Aufsatz). Archiv für Naturgeschichte 87A (2): 1-110.

Wesener T. 2015. Integrative redescription of a forgotten Italian pill millipede endemic to the Apuan Alps - Glomeris apuana Verhoeff, 1911 (Diplopoda, Glomerida, Glomeridae). Zootaxa 4039 (2): 391400. https://doi.org/10.11646/zootaxa.4039.2.11

Wesener T. \& Conrad C. 2016. Local hotspots of endemism or artifacts of incorrect taxonomy? The Status of microendemic pill millipede species of the genus Glomeris in northern Italy (Diplopoda, Glomerida). PLoS ONE 11 (9): e0162284. https://doi.org/10.1371/journal.pone.0162284

Wytwer J. \& Golovatch S.I. 2004. Redescription of the East Carpathian millipedes Ochogona (Beskidia) jankowskii (Jawłowski, 1938) (Diplopoda, Chordeumatida, Craspedosomatidae). Fragmenta faunistica 47 (1): 39-45. https://doi.org/10.3161/00159301FF2004.47.1.039 
Published on: 22 September 2021

Topic editor: Nesrine Akkari

Desk editor: Kristiaan Hoedemakers

Printed versions of all papers are also deposited in the libraries of the institutes that are members of the EJT consortium: Muséum national d'histoire naturelle, Paris, France; Meise Botanic Garden, Belgium; Royal Museum for Central Africa, Tervuren, Belgium; Royal Belgian Institute of Natural Sciences, Brussels, Belgium; Natural History Museum of Denmark, Copenhagen, Denmark; Naturalis Biodiversity Center, Leiden, the Netherlands; Museo Nacional de Ciencias Naturales-CSIC, Madrid, Spain; Real Jardín Botánico de Madrid CSIC, Spain; Zoological Research Museum Alexander Koenig, Bonn, Germany; National Museum, Prague, Czech Republic. 


\section{Appendix 1: maps}

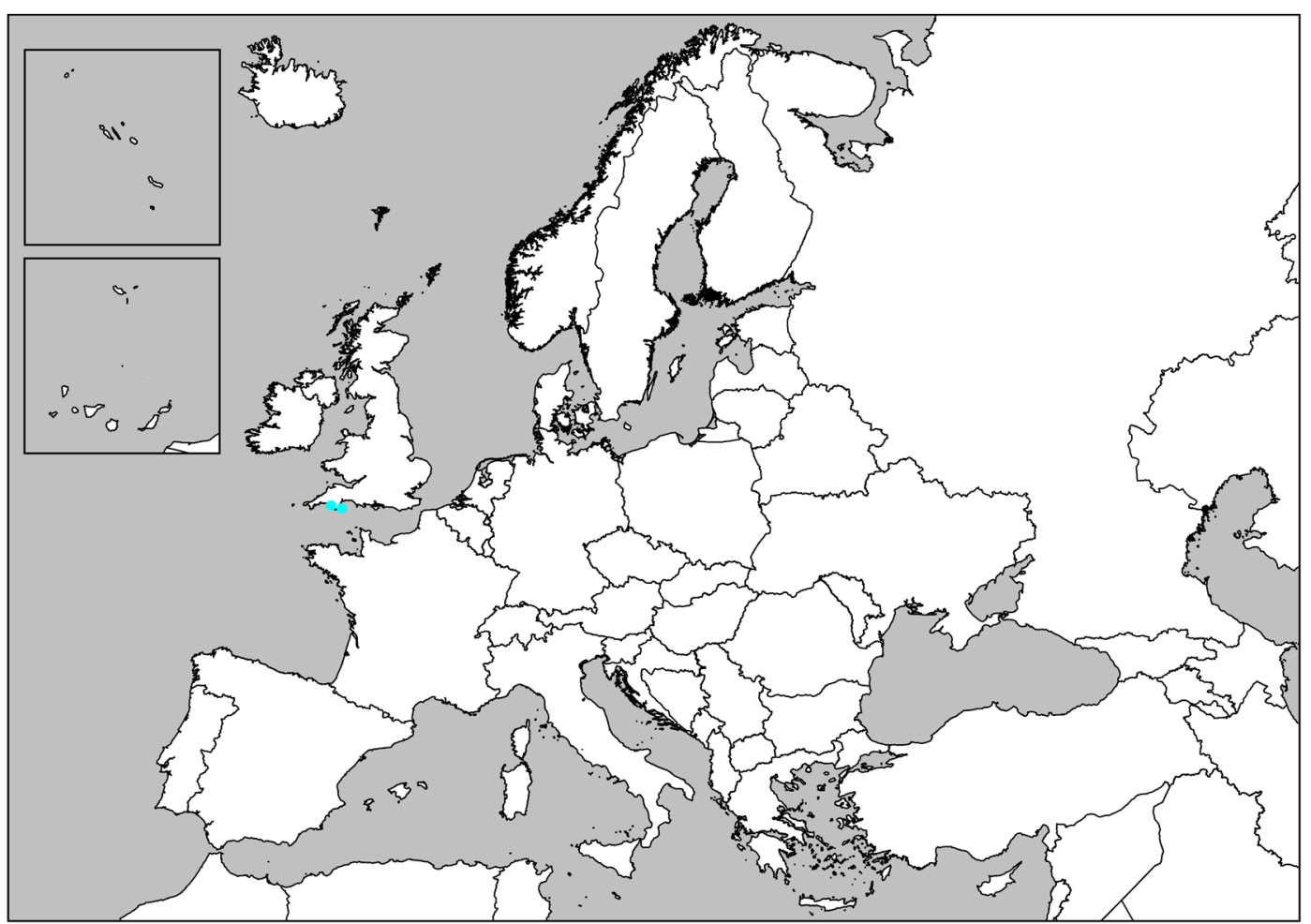

1. Anthogona britannica Gregory, Jones \& Mauriès, 1993

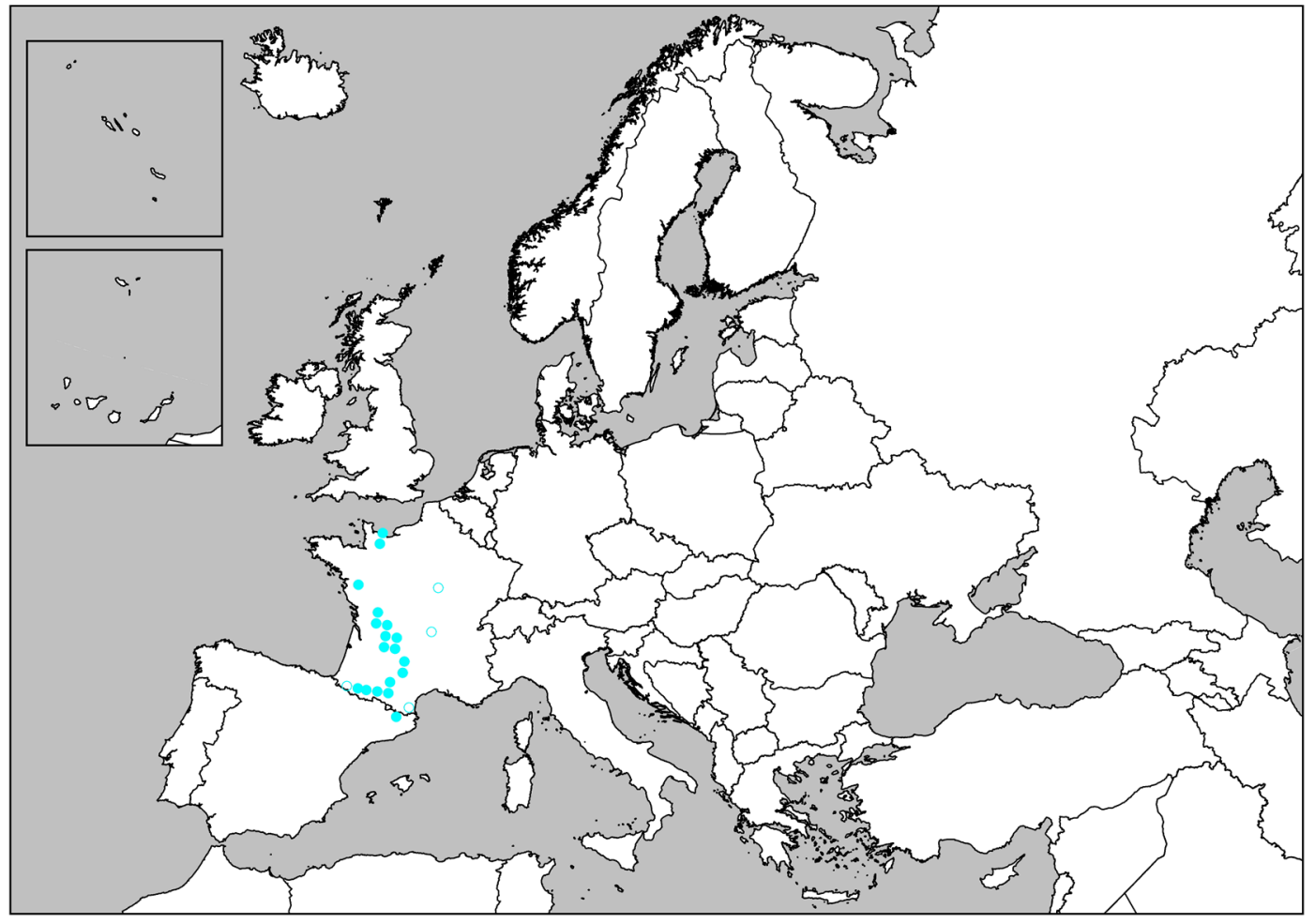

2. Anthogona variegata Ribaut, 1913 


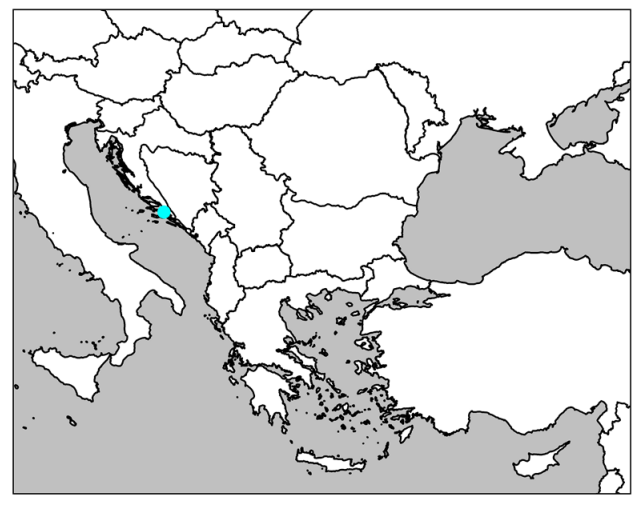

3. Biokoviella mauriesi Mršić, 1992

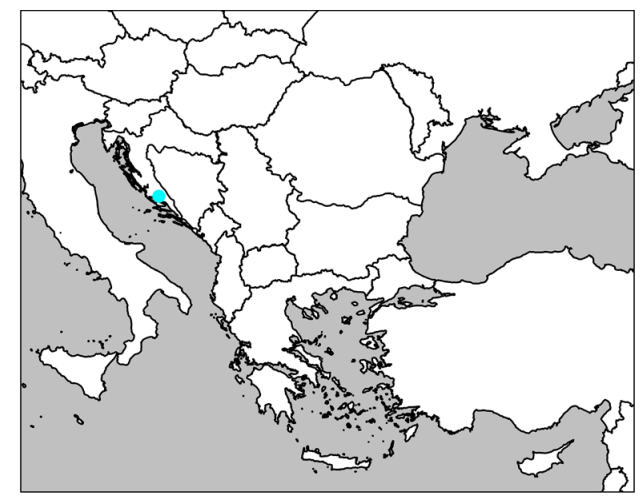

4. Biokoviella mosorensis Antić \& Dražina, 2016

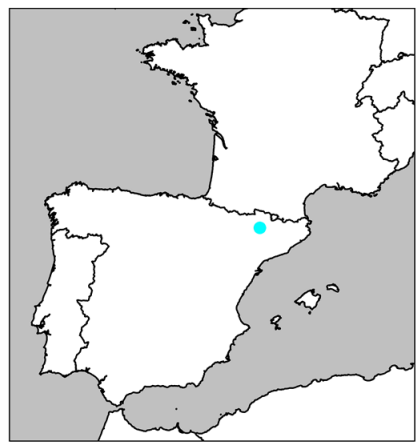

5. Cranogona cornuta Ribaut, 1913

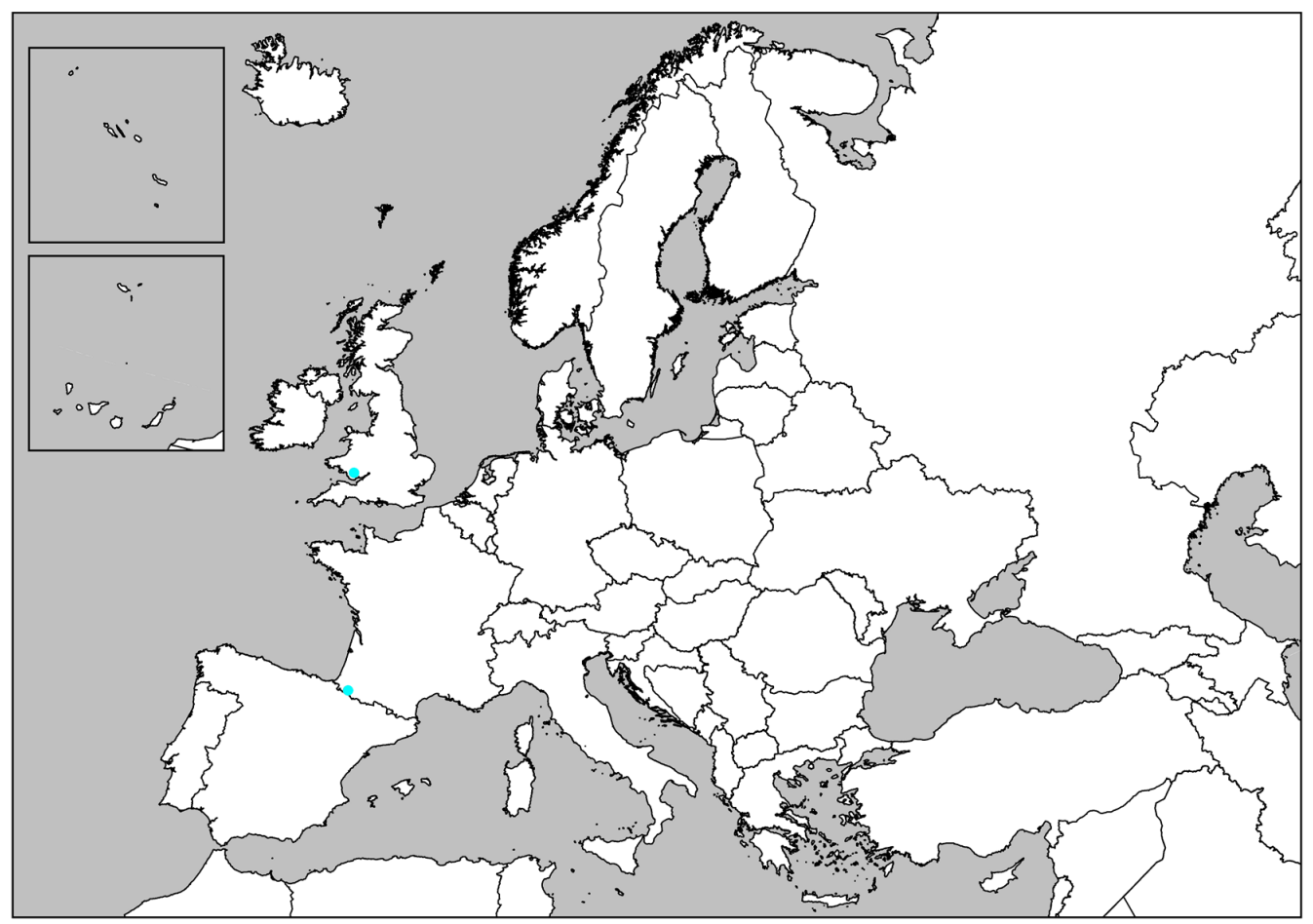

6. Cranogona dalensi Mauriès, 1965 


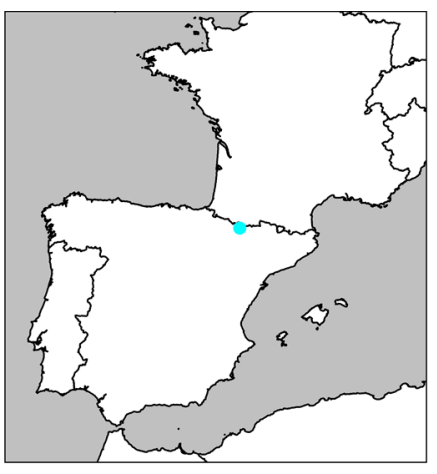

7. Cranogona delicata Mauriès, 1963

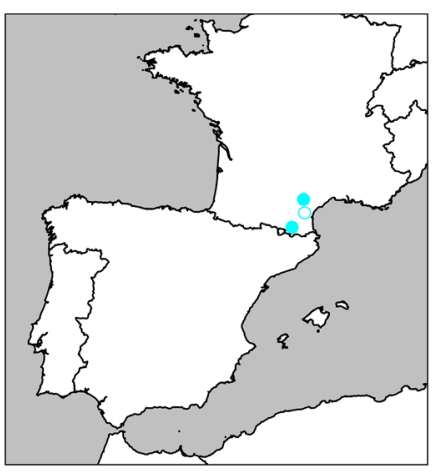

10. Cranogona orientale Ribaut, 1913

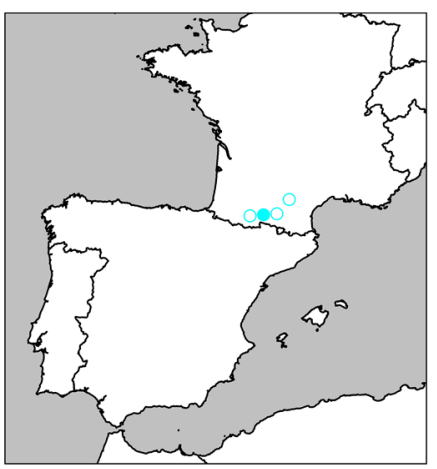

13. Cranogona uncinata Ribaut, 1951

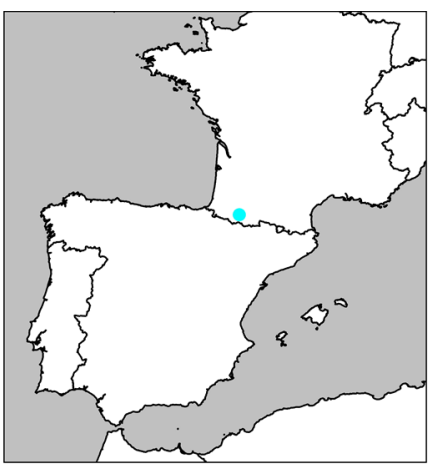

8. Cranogona denticulata Delmas, 1927

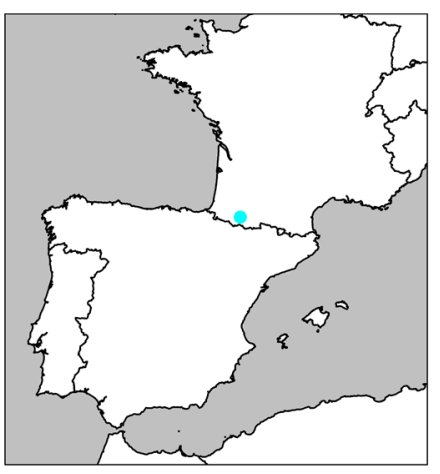

11. Cranogona pavida Ribaut, 1951

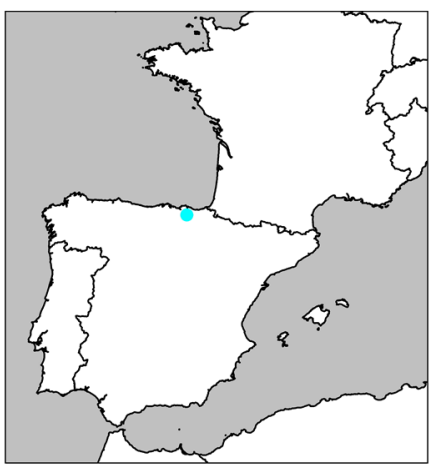

9. Cranogona espagnoli Vicente \& Mauriès, 1980

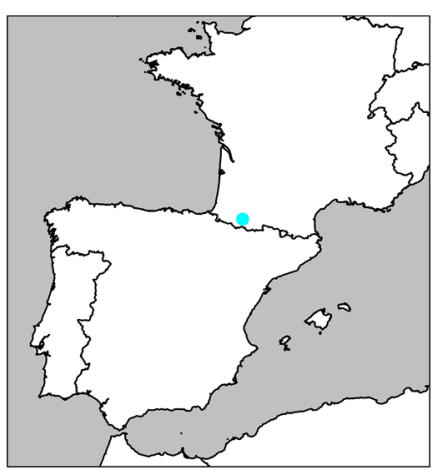

12. Cranogona touyaensis Mauriès, 1975

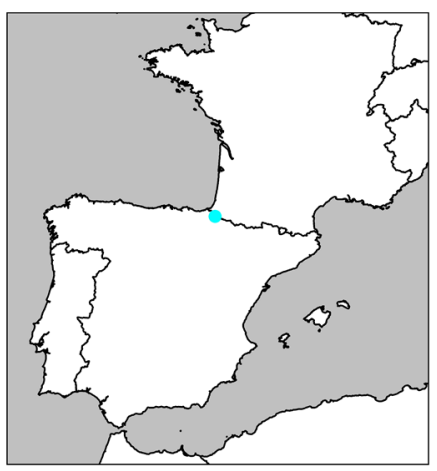

14. Cranogona vasconica Ribaut, 1913 


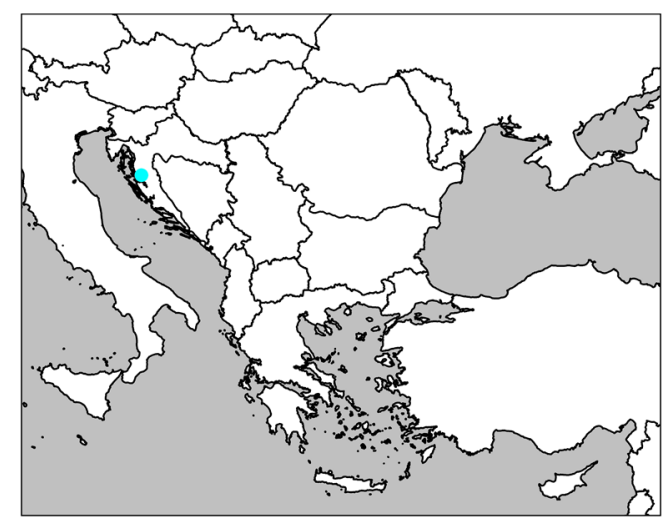

15. Egonpretneria brachychaeta Strasser, 1966

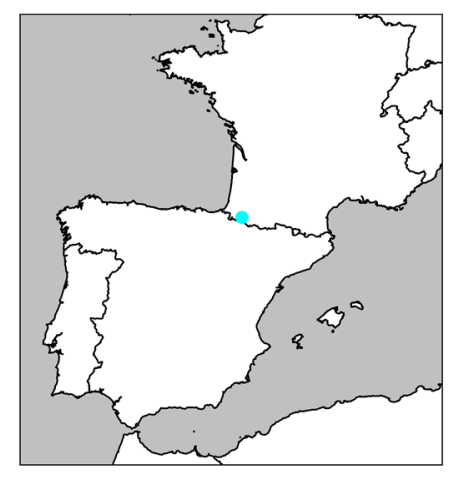

17. Escualdosoma gourbaultae (Mauriès, 1965)

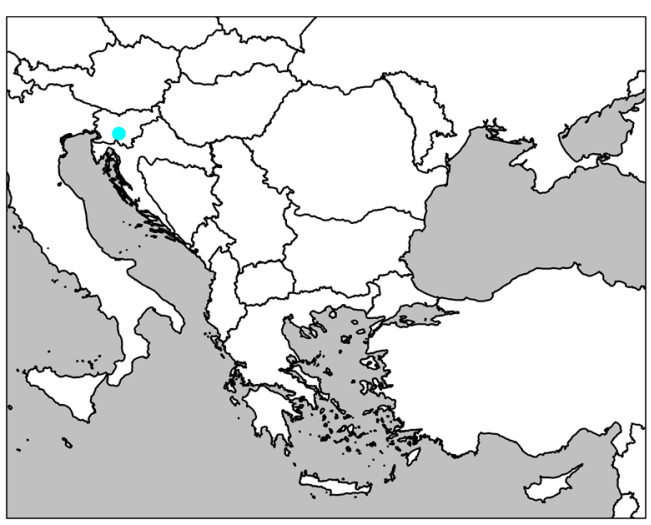

19. Haasia cormuata (Strasser, 1940)

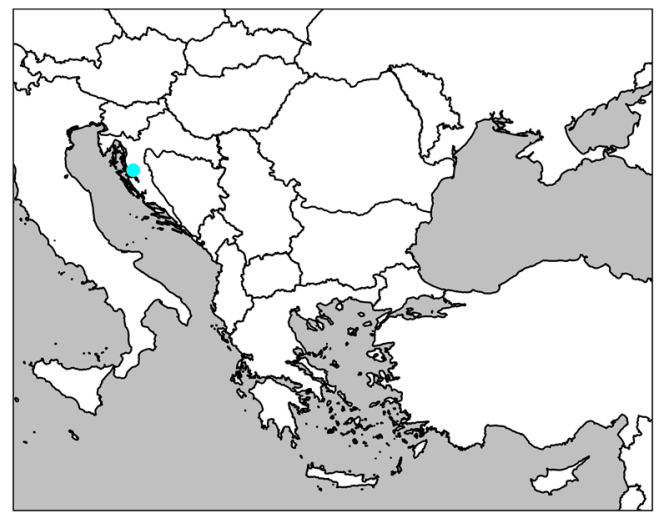

16. Egonpretneria vudutschajldi Antić \& Dražina, 2015

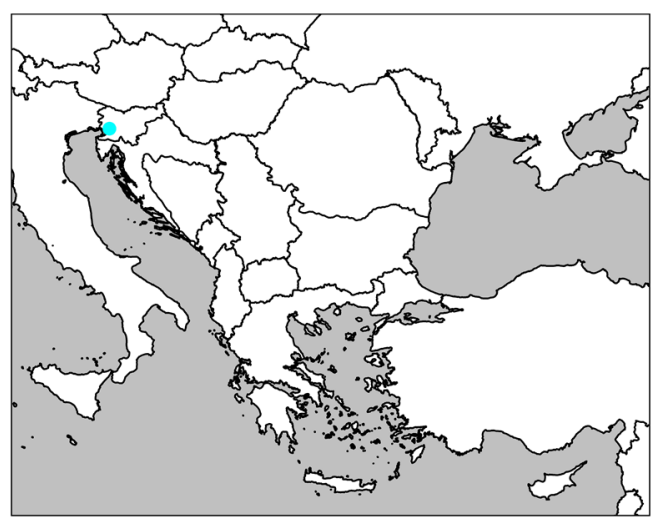

18. Haasia carinifera (Strasser, 1935)

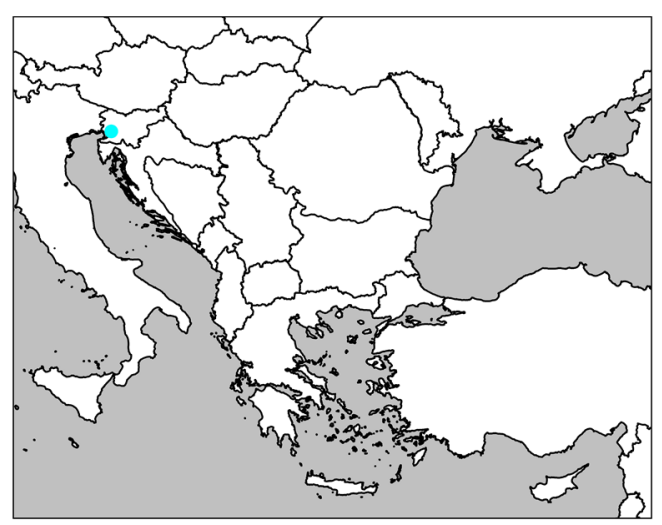

20. Haasia falsa (Strasser, 1971) 


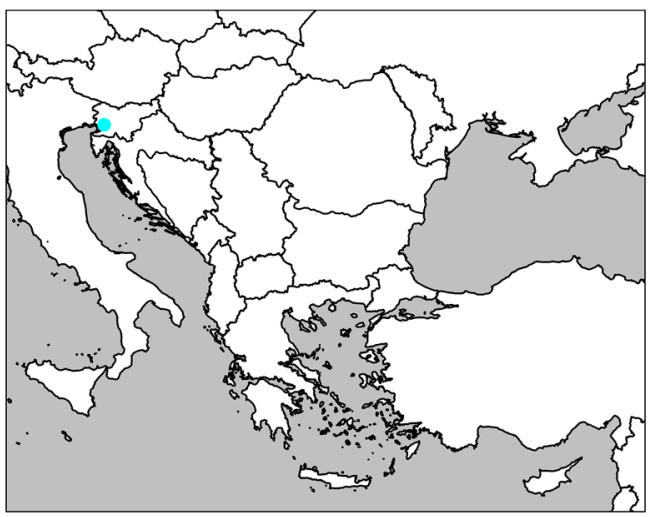

21. Haasia hadzii (Strasser, 1966)

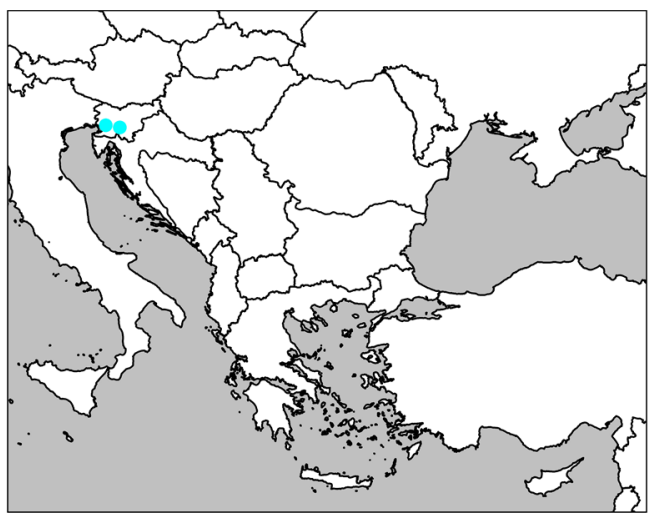

23. Haasia largescutata (Strasser, 1935)

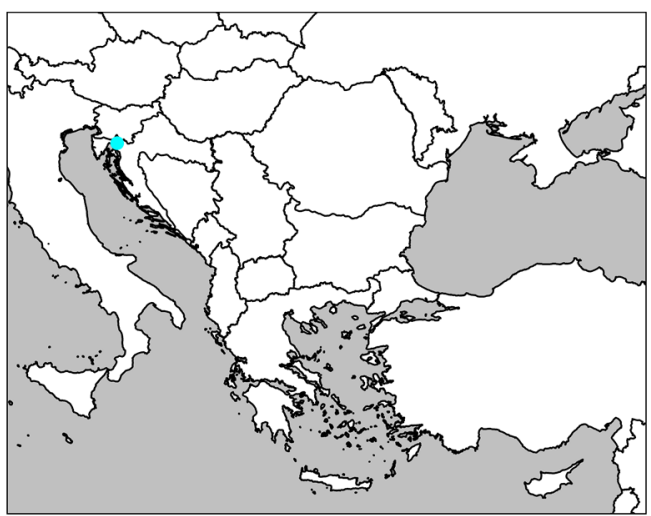

25. Haasia pretneri (Strasser, 1940)

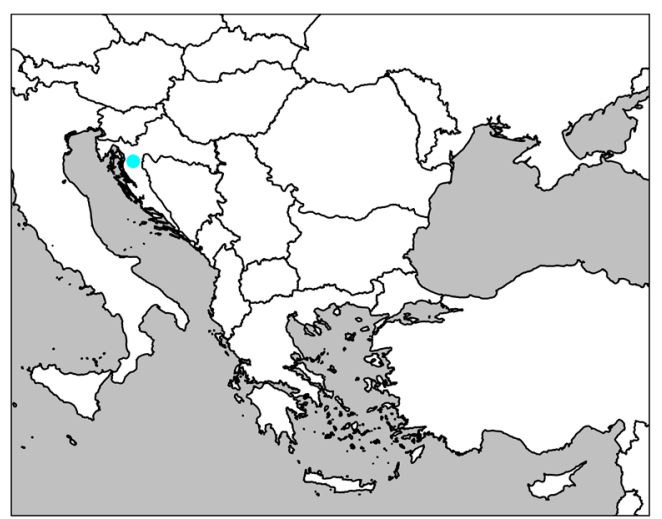

22. Haasia jalzici Antić \& Dražina, 2015

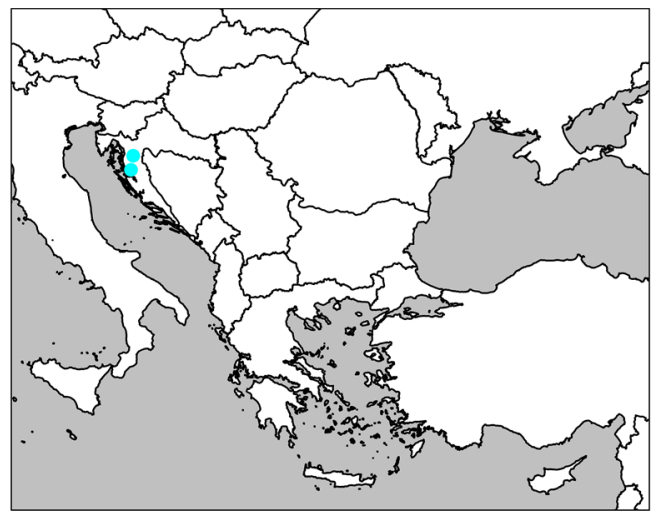

24. Haasia likana (Strasser, 1966)

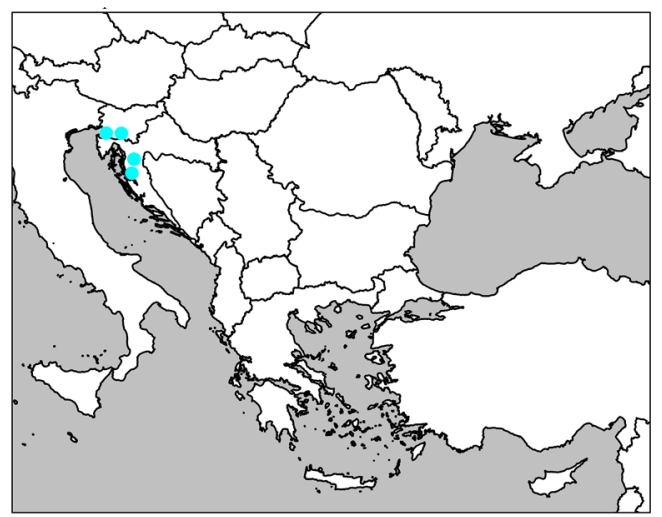

26. Haasia stenopodium (Strasser, 1966) 


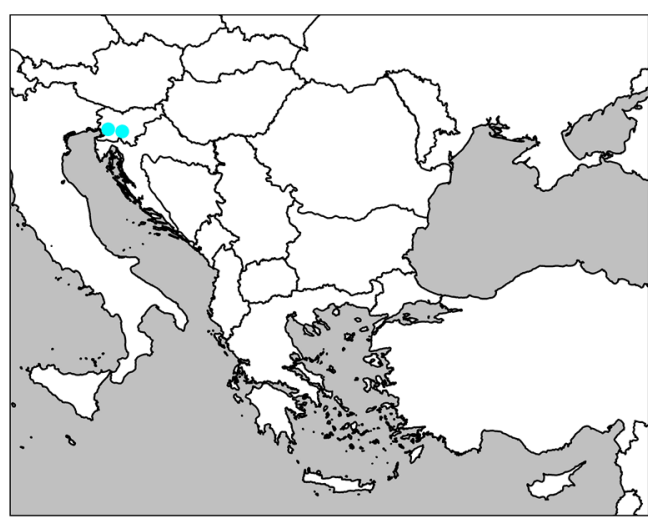

27. Haasia tridentis (Verhoeff, 1931)

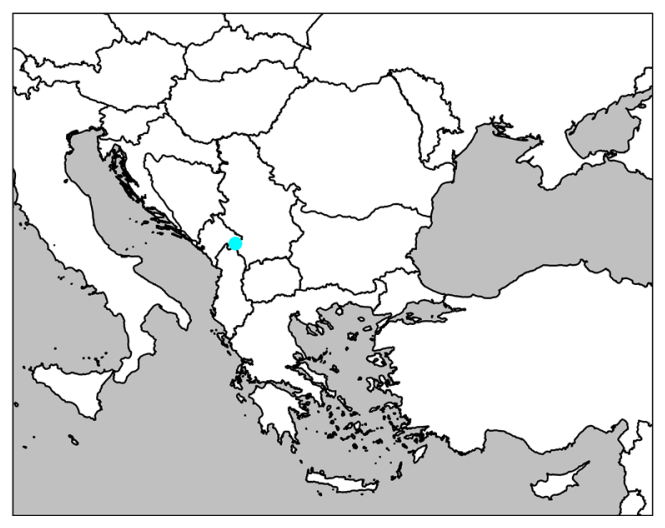

29. Macrochaetosoma bertiscea Antić \& Makarov, 2015

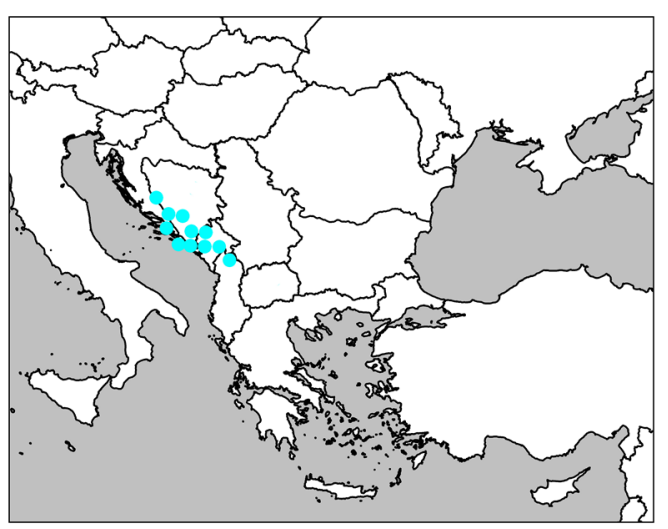

31. Macrochaetosoma troglomontanum Absolon \& Lang, 1933

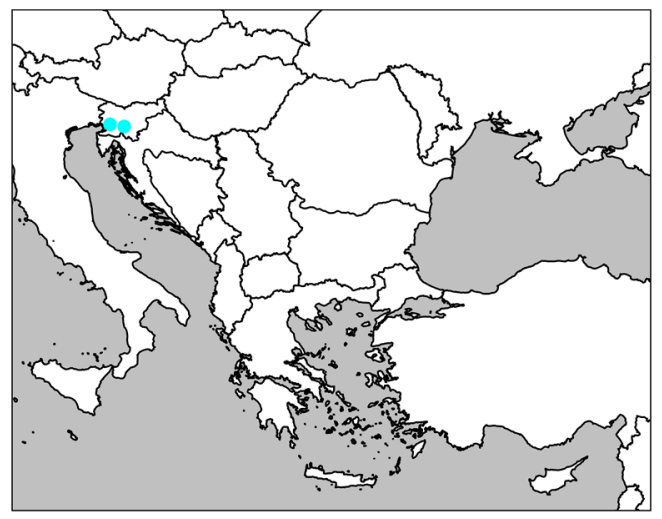

28. Haasia troglodytes (Latzel, 1884)

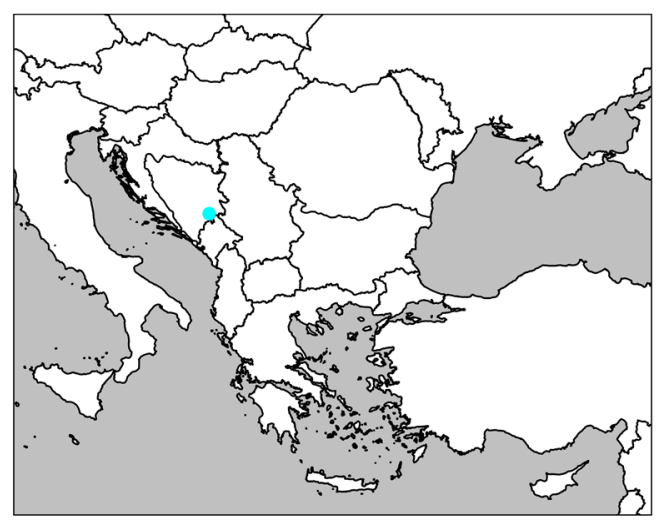

30. Macrochaetosoma drinae Strasser, 1962

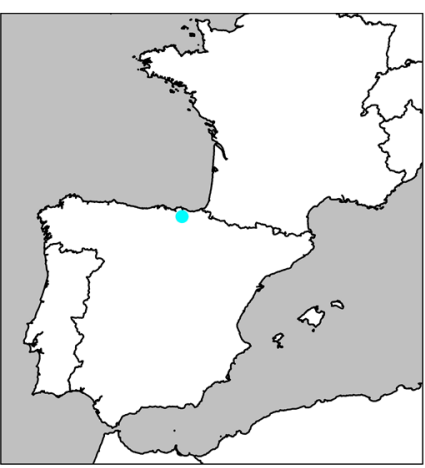

32. Vascanthogona vicenteae Mauriès \& Barraqueta, 1985 


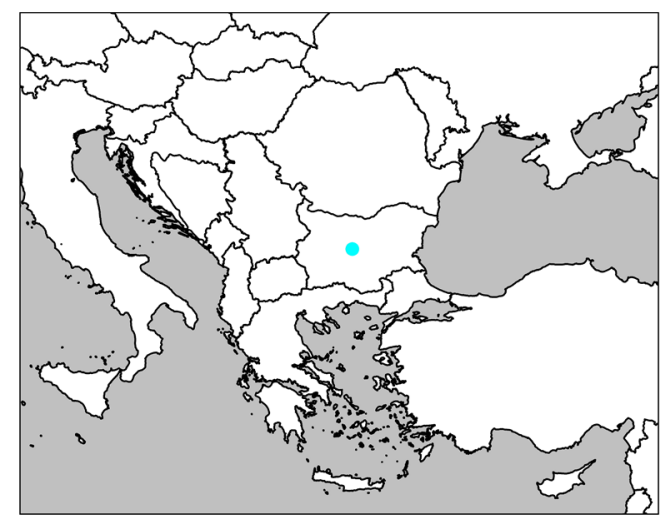

33. Anamastigona alba (Strasser, 1960)

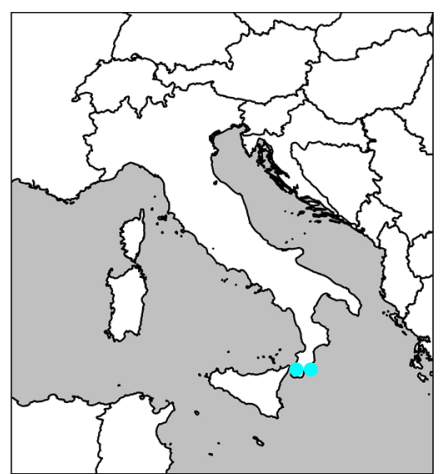

35. Anamastigona aspromontis

(Strasser, 1970)

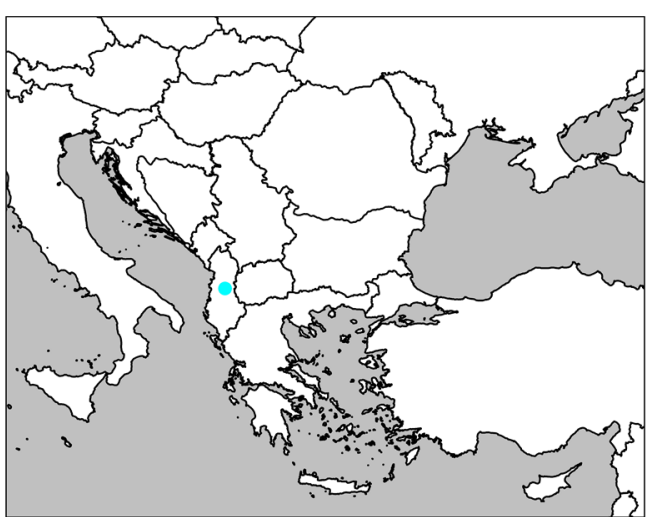

34. Anamastigona albanensis Mauriès, Golovatch \& Stoev, 1997

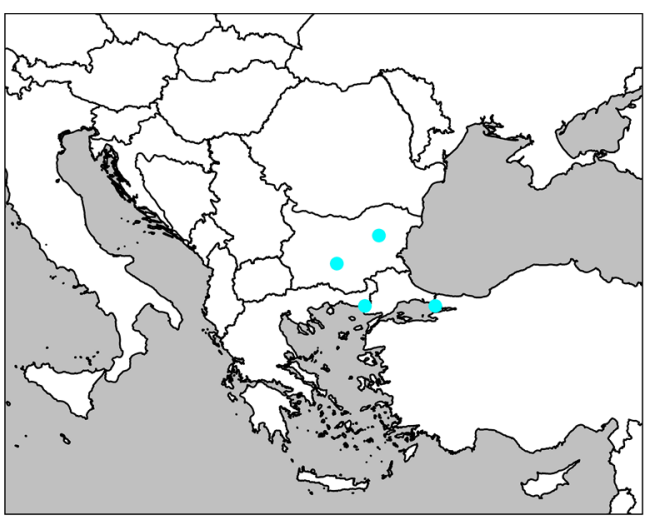

36. Anamastigona bilselii (Verhoeff, 1940)

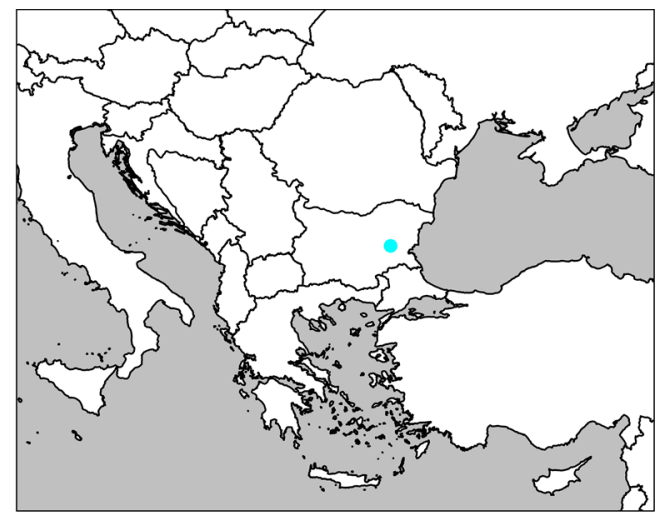

38. Anamastigona falcata (Gulička, 1967) 


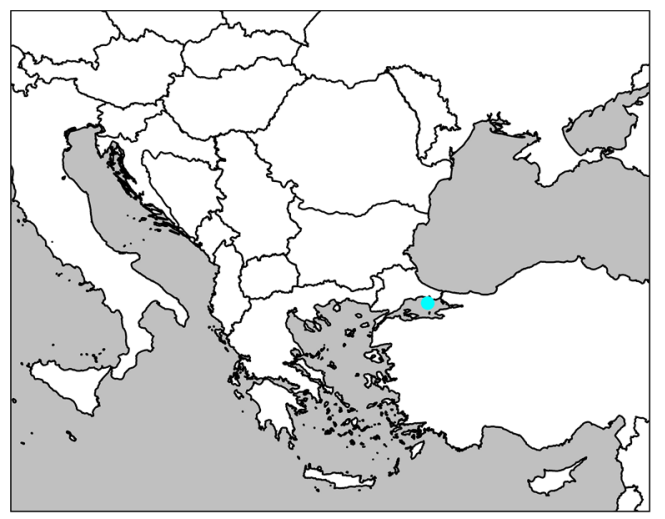

39. Anamastigona halophila (Verhoeff, 1940)

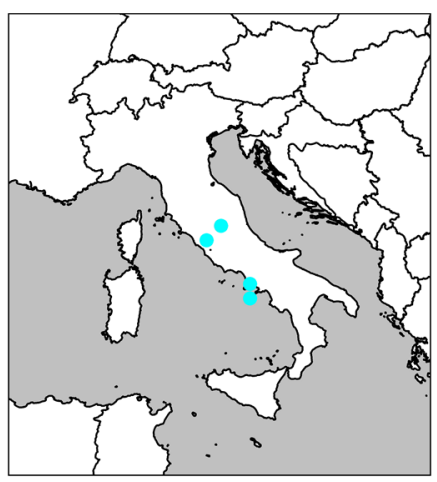

41. Anamastigona hispidula (Silvestri, 1894)

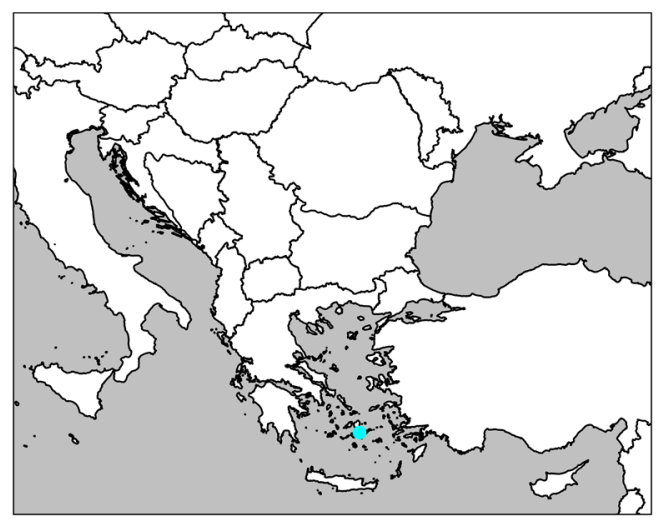

43. Anamastigona matsakisi Mauriès \& Karamouna, 1984

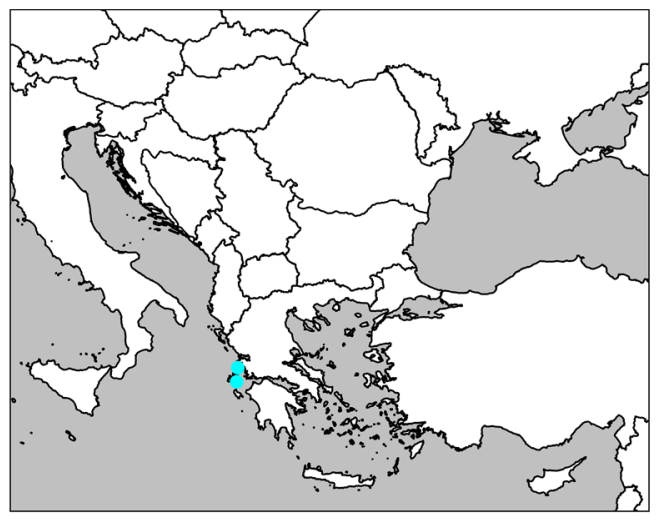

40. Anamastigona hauseri (Strasser,1974)

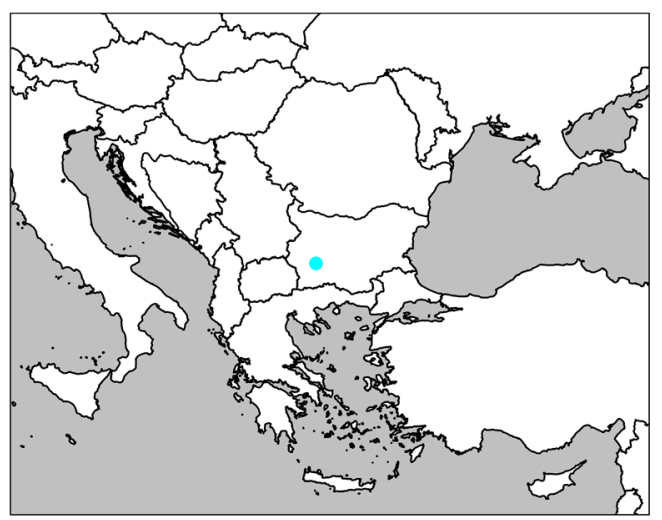

42. Anamastigona lepenicae (Strasser, 1975)

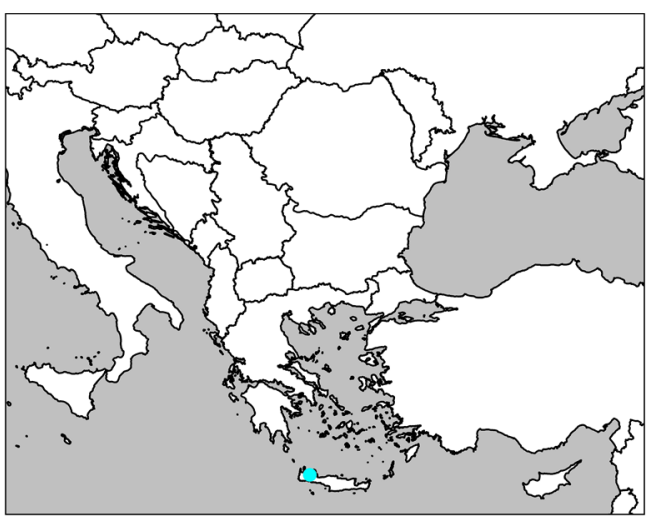

44. Anamastigona mediterranea Ćurčić, Makarov \& Lymberakis, 2001 


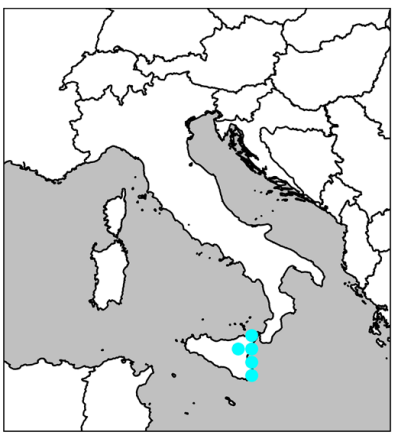

45. Anamastigona meridionalis Silvestri, 1898

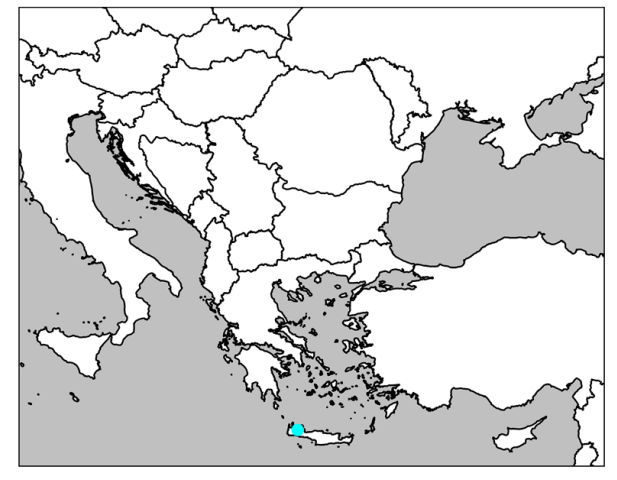

46. Anamastigona penicillata

(Attems, 1902)

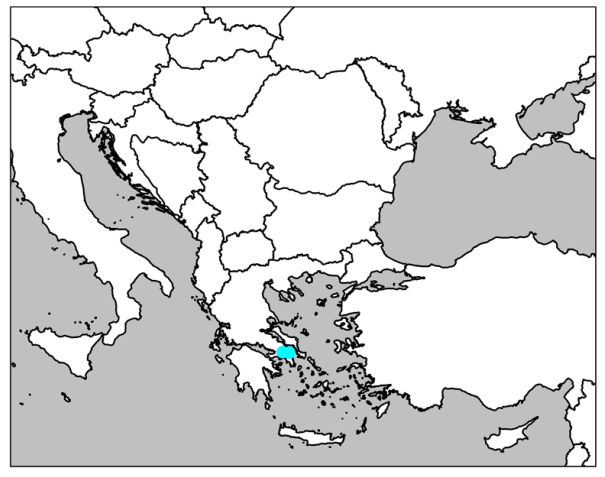

47. Anamastigona pentelicona (Verhoeff, 1925)

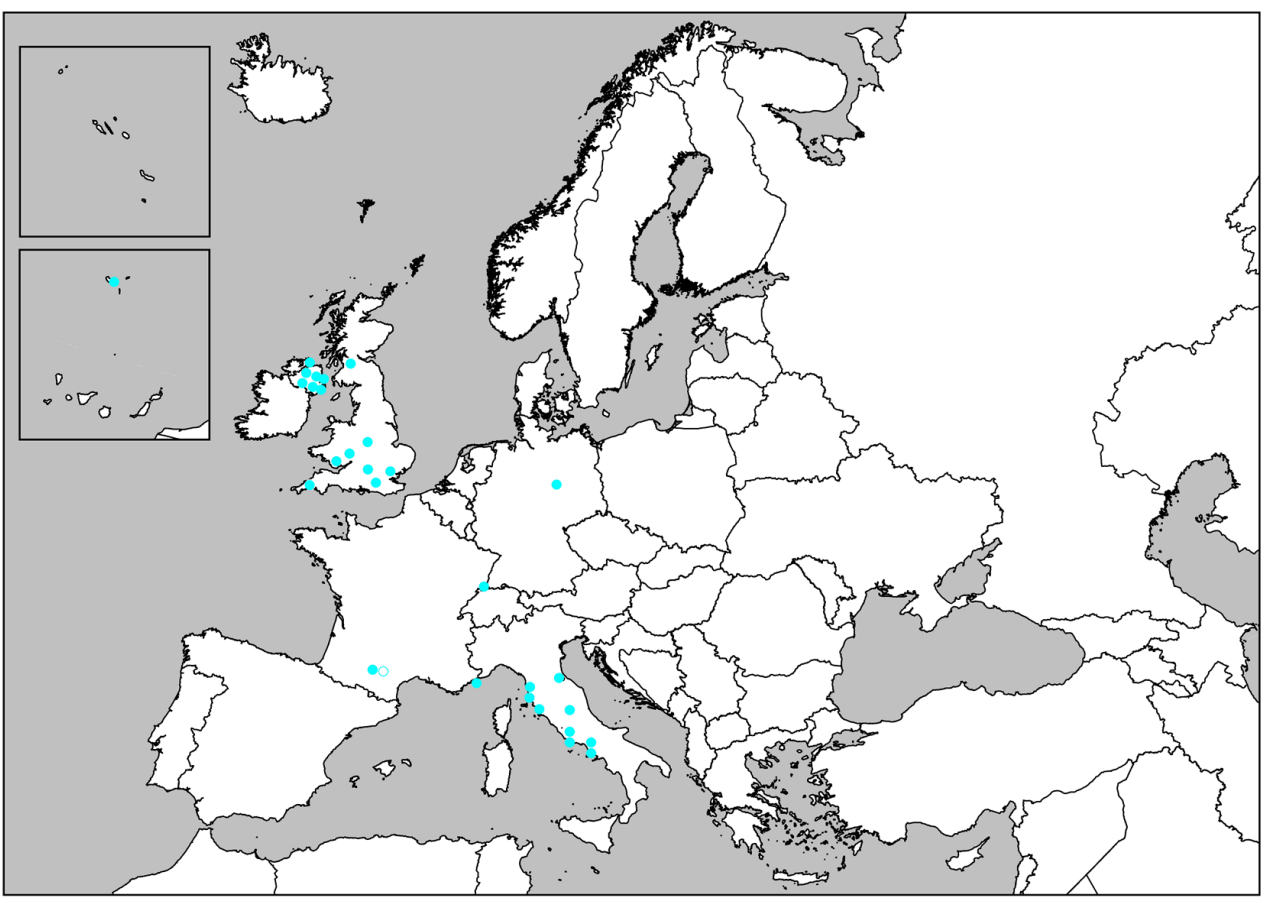

48. Anamastigona pulchella (Silvestri, 1894) 


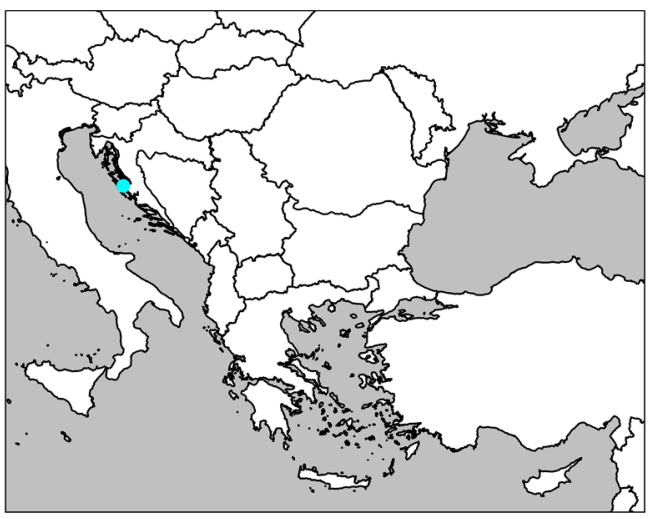

49. Anamastigona radmani Makarov, Rađa, Rađa, Tomić, Mitić \& Curcić, 2007

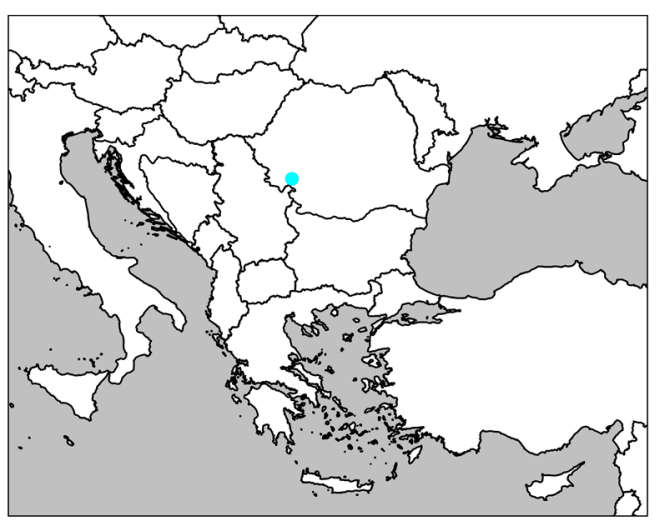

51. Anthroleucosoma spelaeum Ceuca, 1964

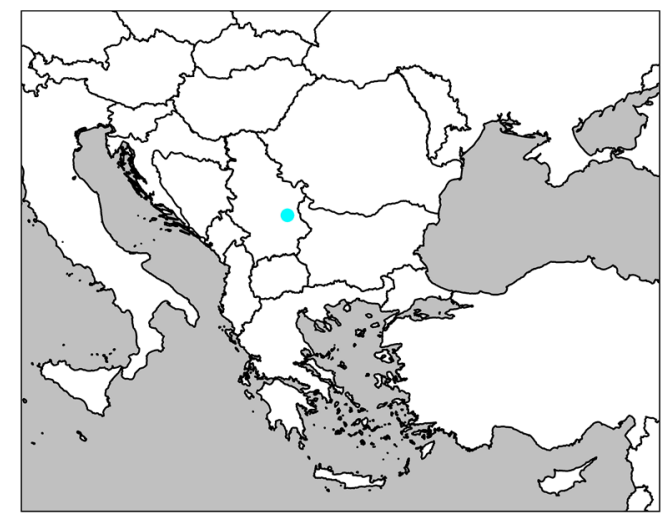

53. Belbogosoma bloweri Ćurčić \& Makarov, 2008

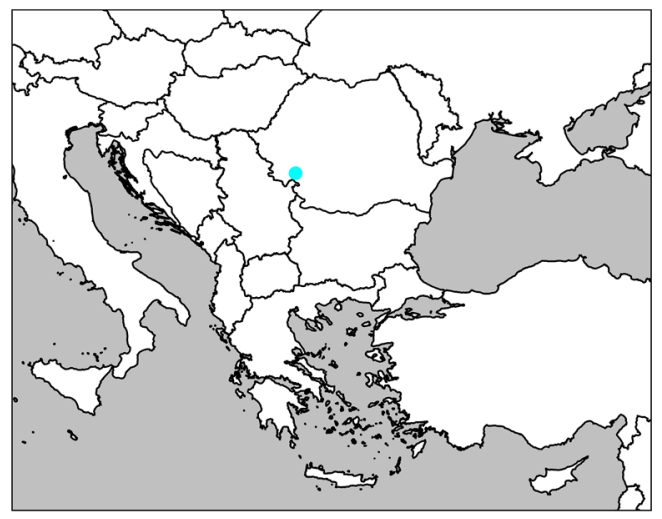

50. Anthroleucosoma banaticum Verhoeff, 1899

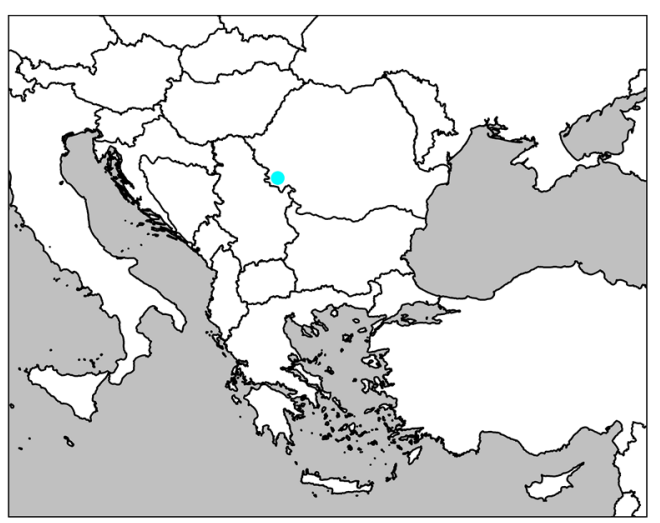

52. Banatosoma ocellatum

(Tabacaru, 1967)

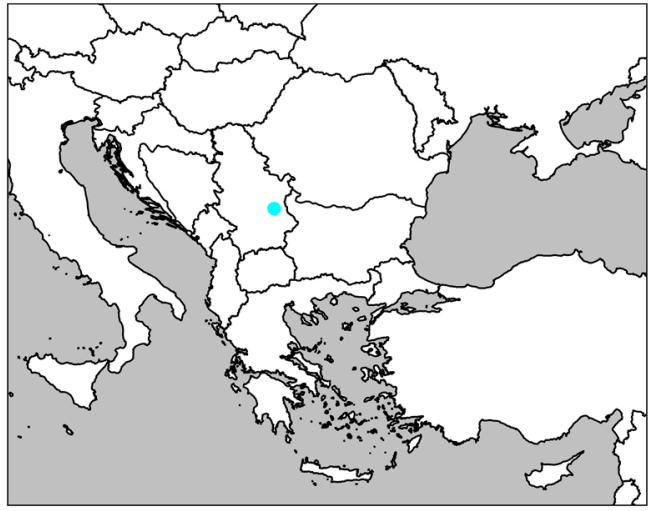

54. Belbogosoma stribogi Antić \& Makarov, 2014 


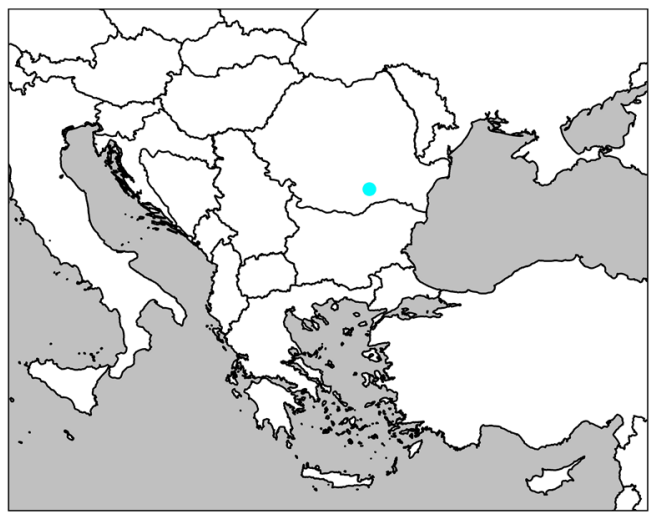

55. Bulgardicus bucarestensis

Tabacaru \& Giurginca, 2006

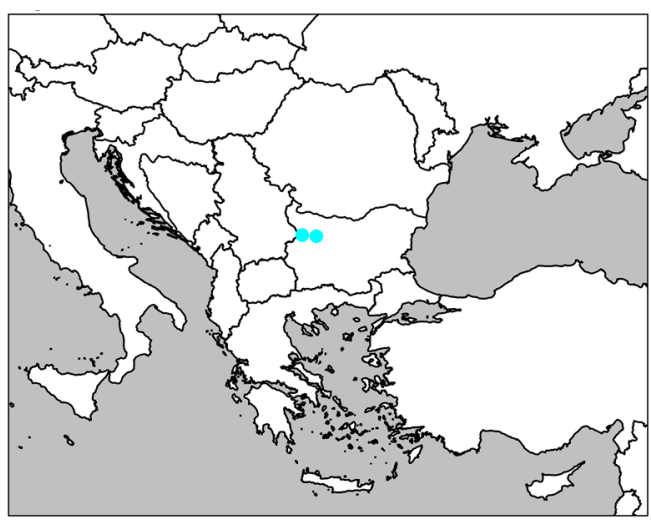

57. Bulgarosoma bureschi Verhoeff, 1926

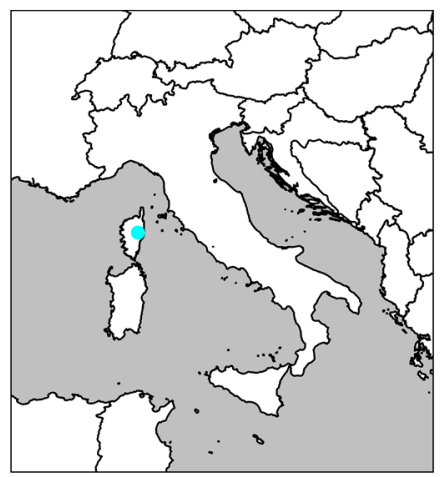

59. Camptogona delamarei Mauriès, 1969

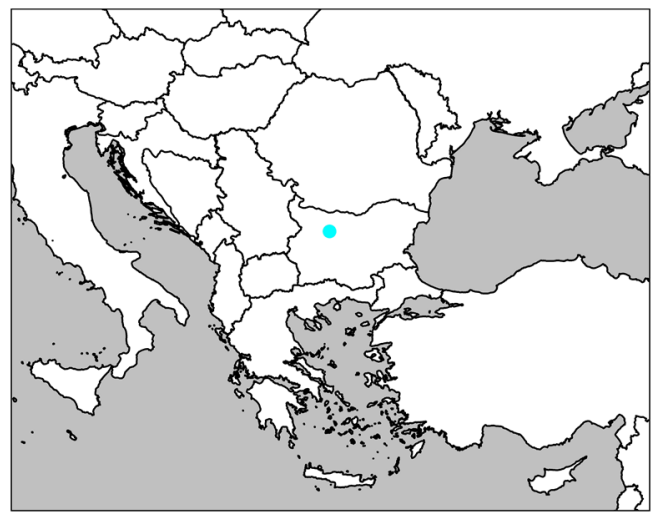

56. Bulgardicus tranteevi Strasser, 1960

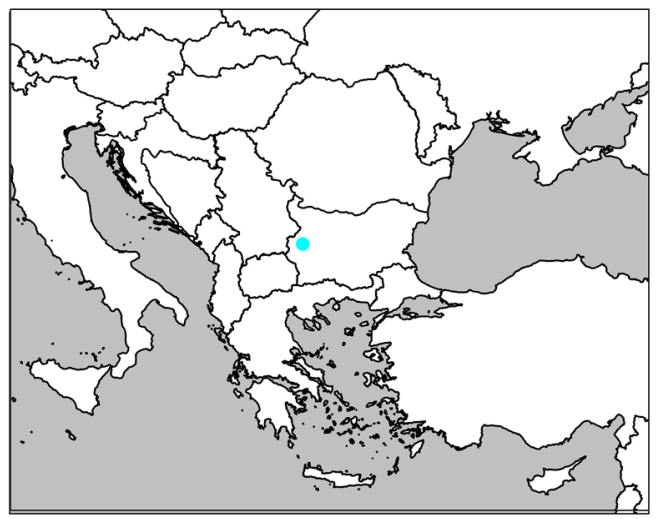

58. Bulgarosoma superficiei Strasser, 1975

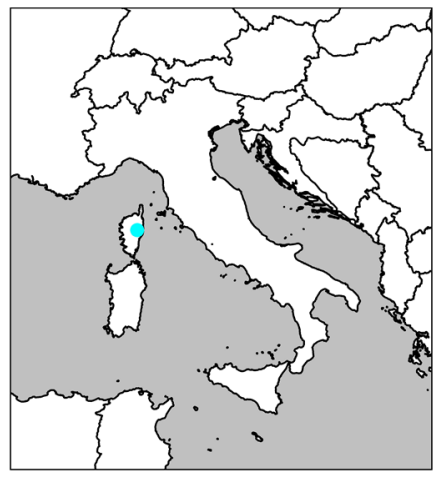

60. Camptogona duboscqui (Brölemann, 1903) 


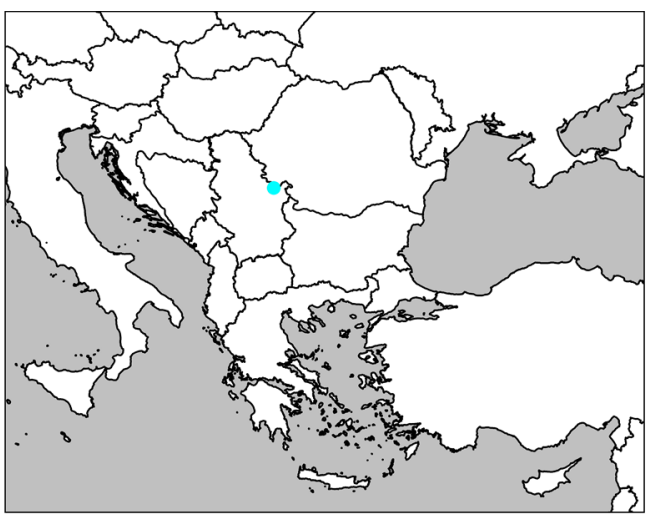

61. Cornogonopus pavicevici Antić, 2020

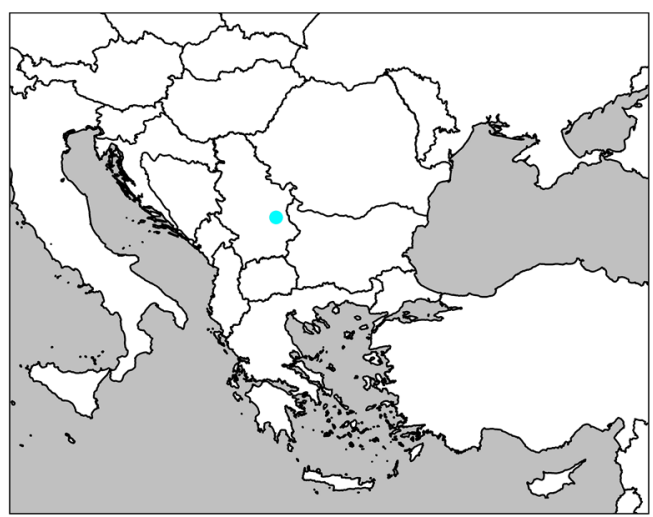

63. Dazbogosoma naissi

Makarov \& Ćurčić, 2012

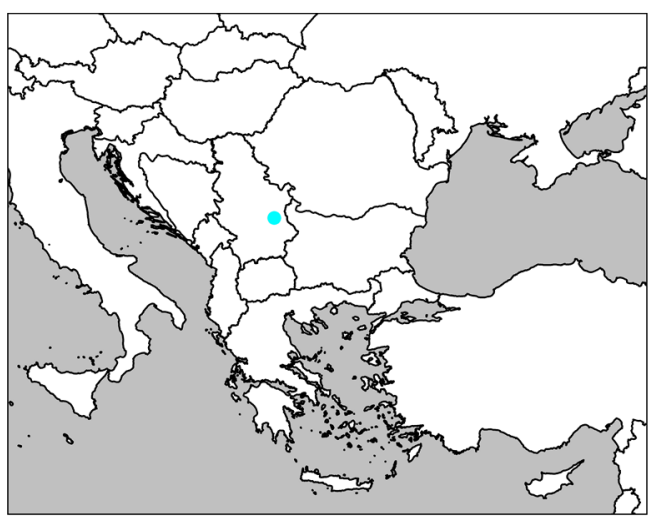

65. Perunosoma trojanicum

Ćurčić \& Makarov, 2007

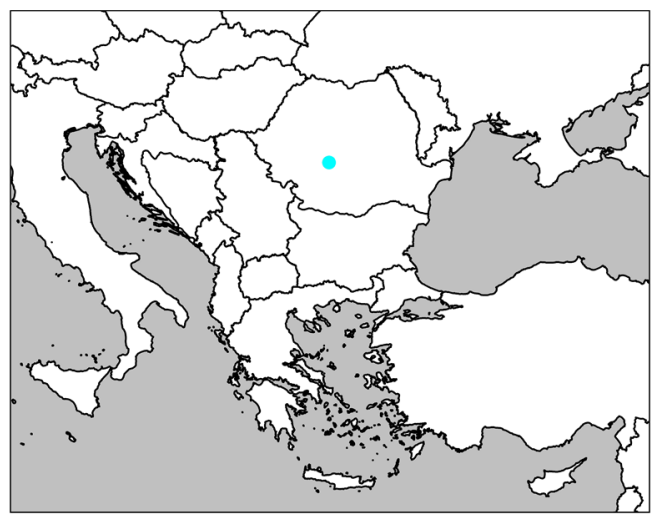

62. Dacosoma motasi Tabacaru, 1967

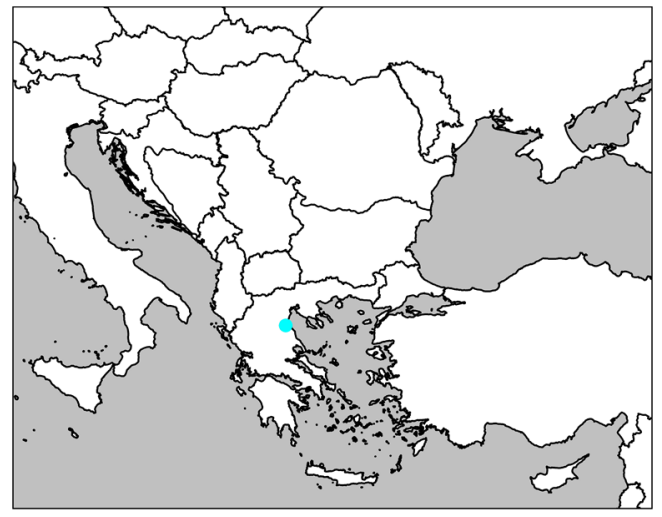

64. Krueperia nivalis Verhoeff, 1900

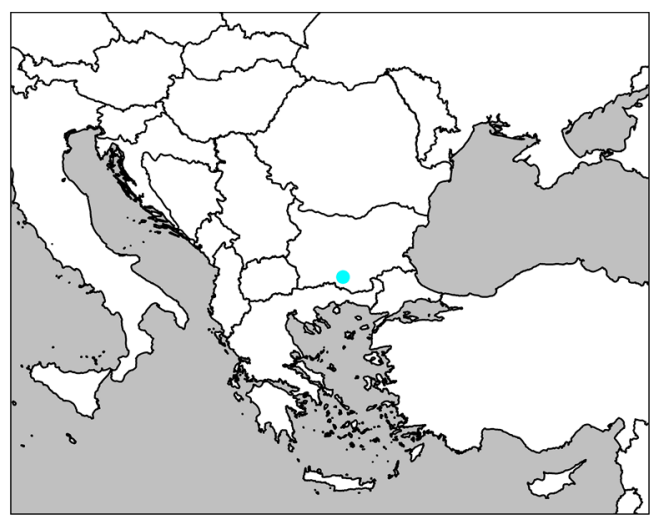

66. Rhodoposoma rhodopinum

(Strasser, 1966) 


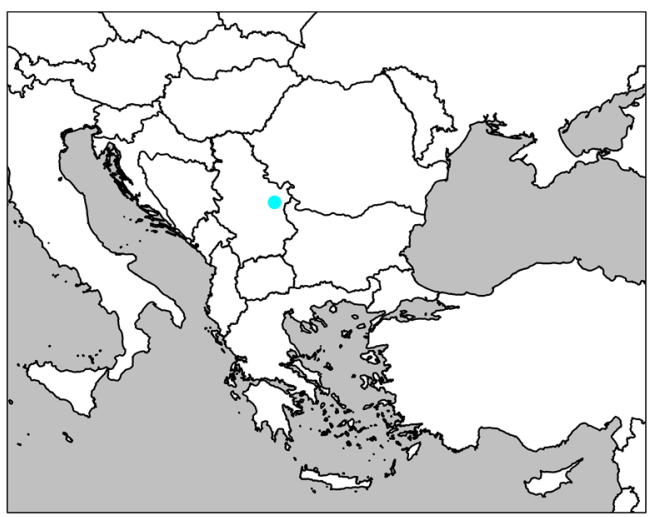

67. Serbosoma beljanicae (Ćurčić \& Makarov, 1998)

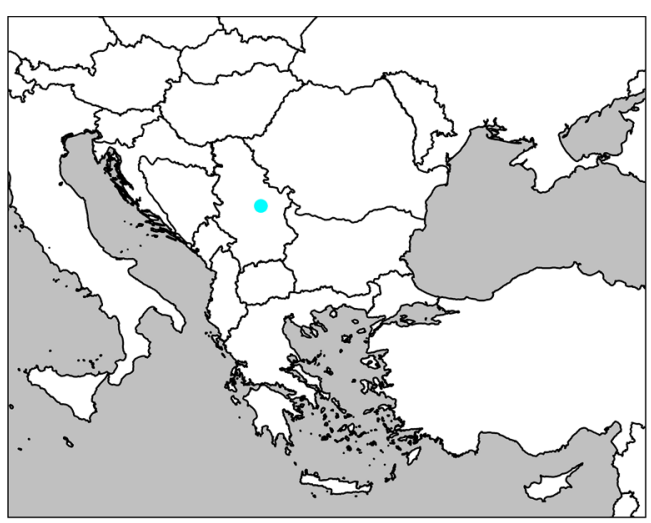

69. Serbosoma kucajense (Ćurčić \& Makarov, 1998))

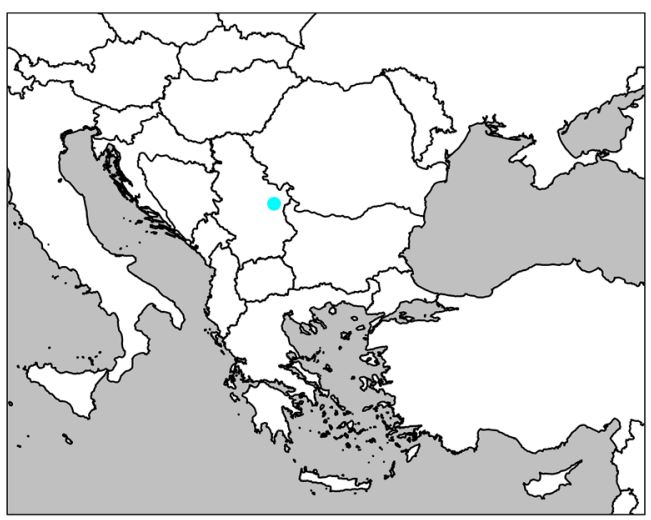

71. Serbosoma zagubicae

(Ćurčić \& Makarov, 1998)

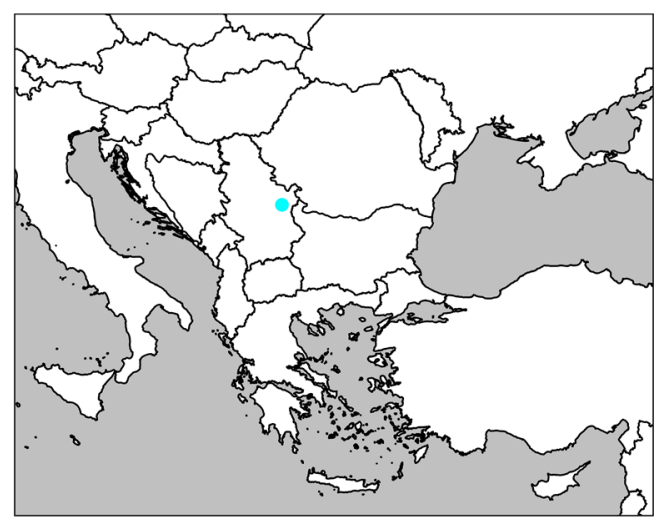

68. Serbosoma crucis (Strasser, 1960)

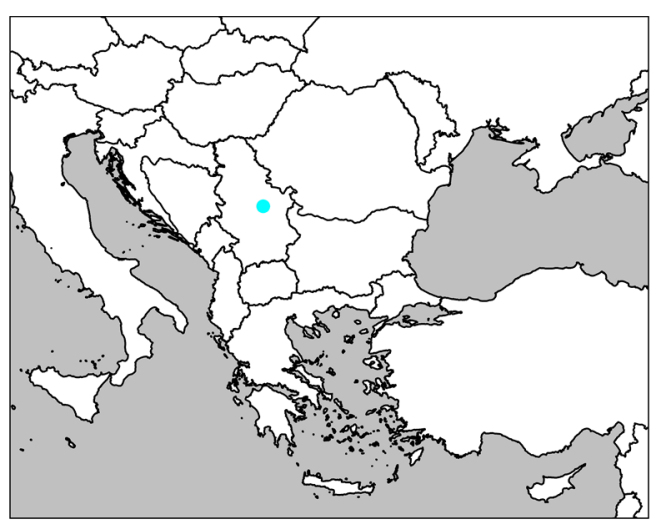

70. Serbosoma lazarevense (Ceuca, 1964)

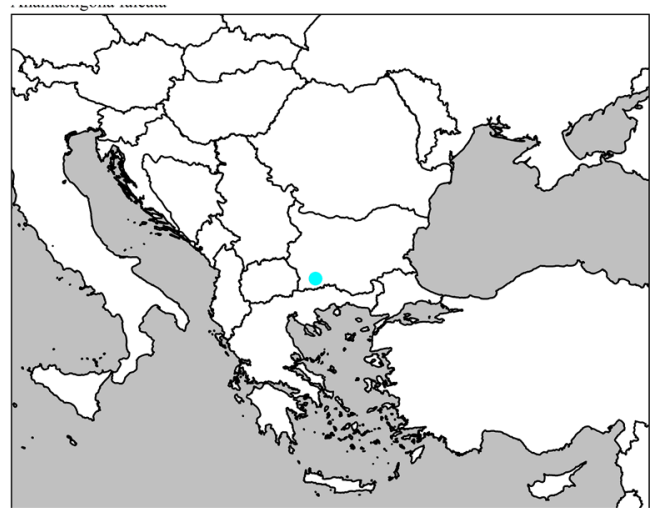

72. Stygiosoma beroni Gulička, 1967 


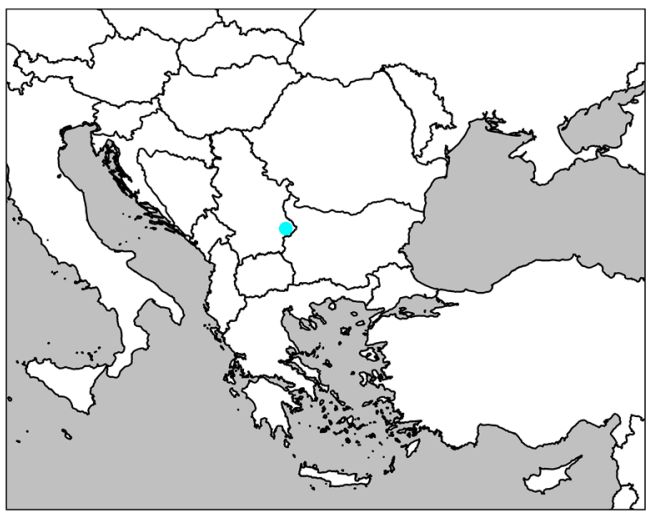

73. Svarogosoma bozidarcurcici Makarov, 2003

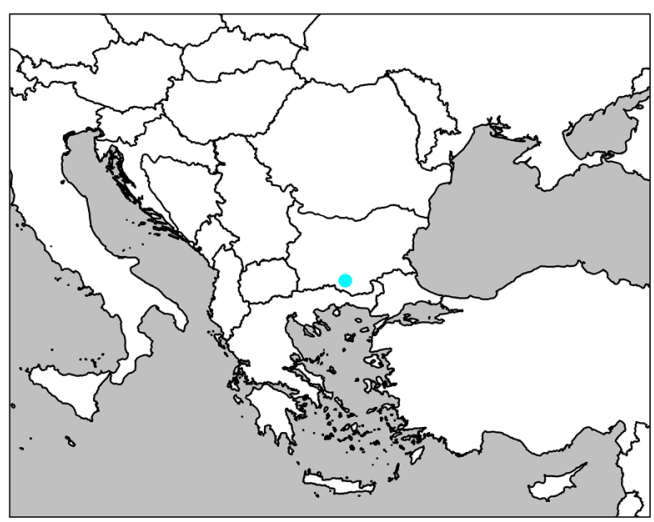

75. Troglodicus tridentifer Gulička, 1967

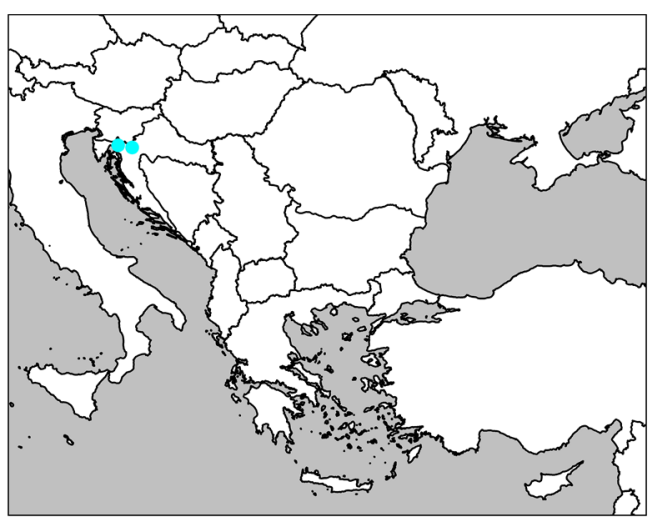

77. Attemsia coniuncta Strasser, 1939

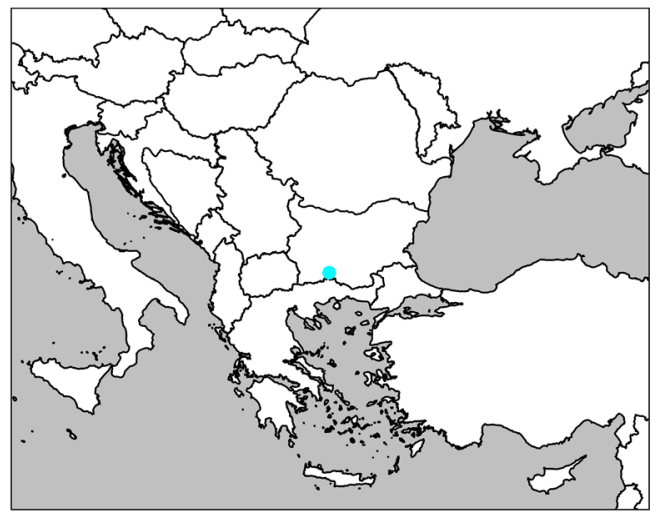

74. Troglodicus meridionalis (Tabacaru, 1967)

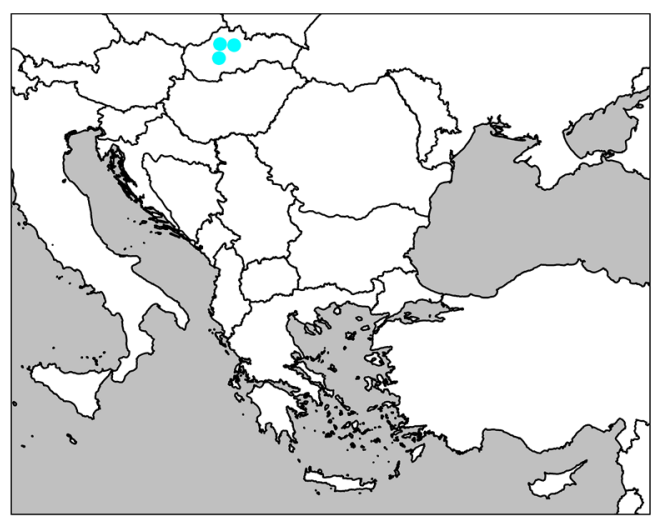

76. Allorhiscosoma sphinx

(Verhoeff, 1907)

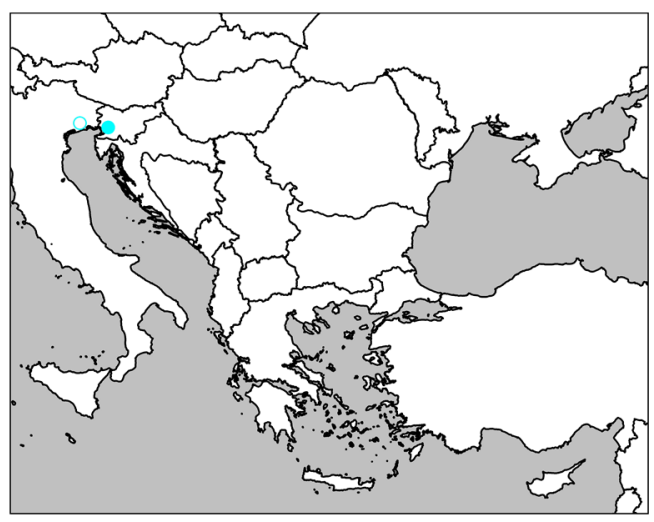

78. Attemsia dolinensis Verhoeff, 1909 


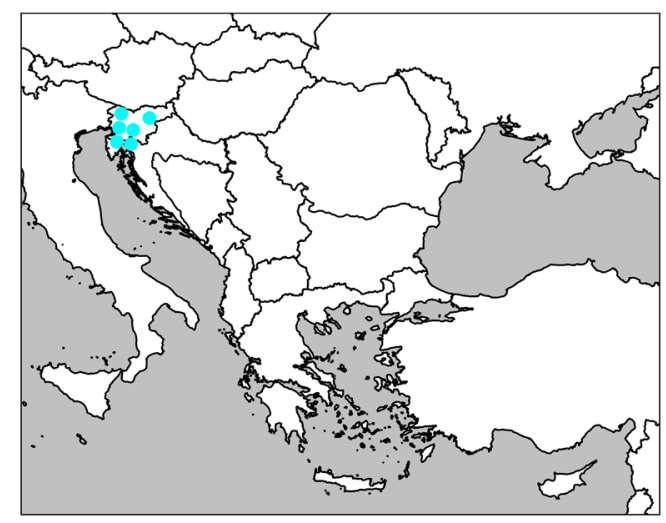

79. Attemsia falcifera Verhoeff, 1899

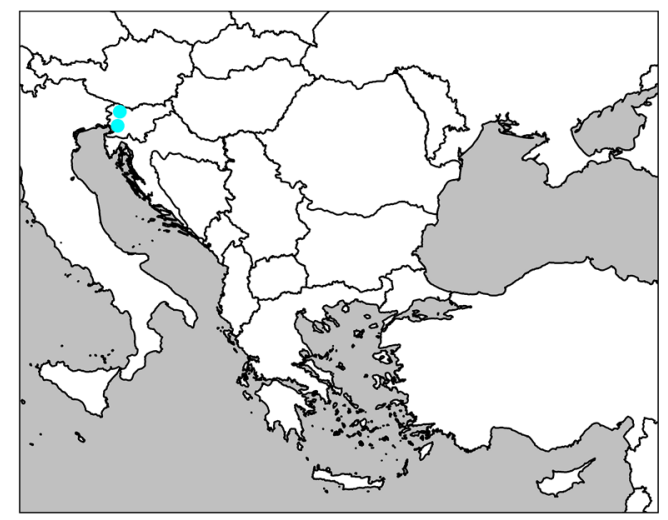

81. Attemsia stygia (Latzel, 1884)

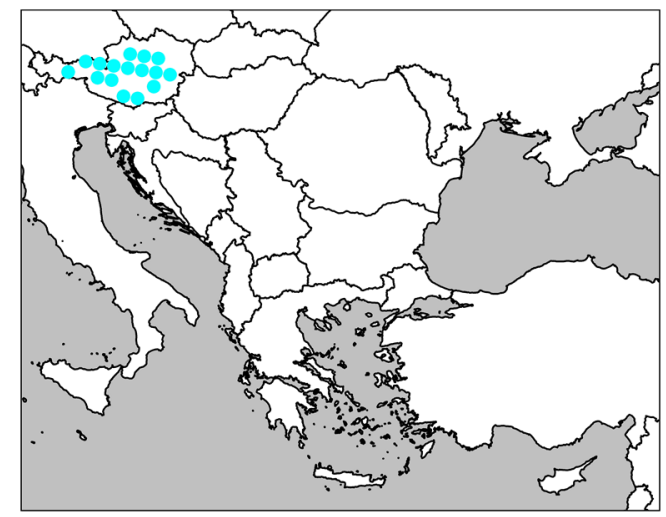

83. Dendromonomeron oribates

(Latzel, 1884)

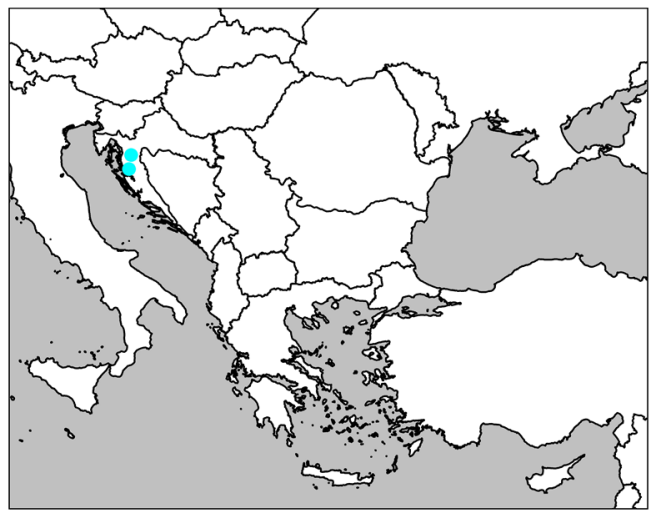

80. Attemsia likana Strasser, 1966

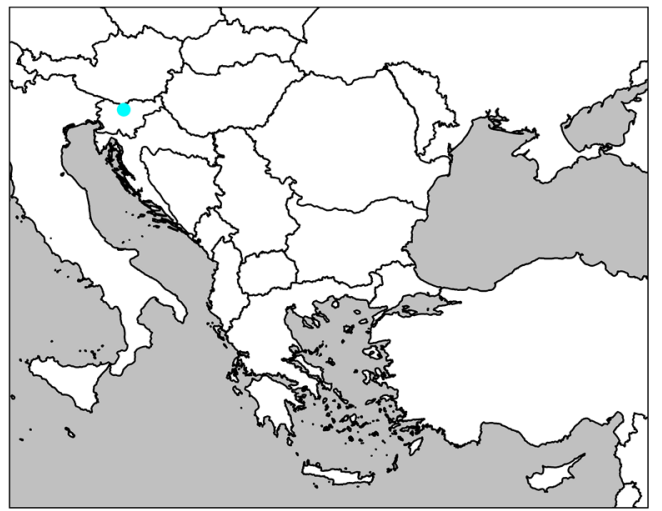

82. Coelogonium cavernarum Strasser, 1937

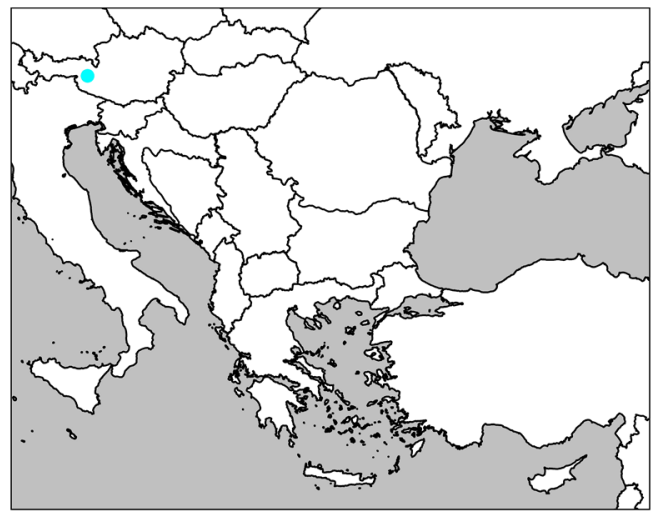

84. Dimastosternum franzi Attems, 1949 


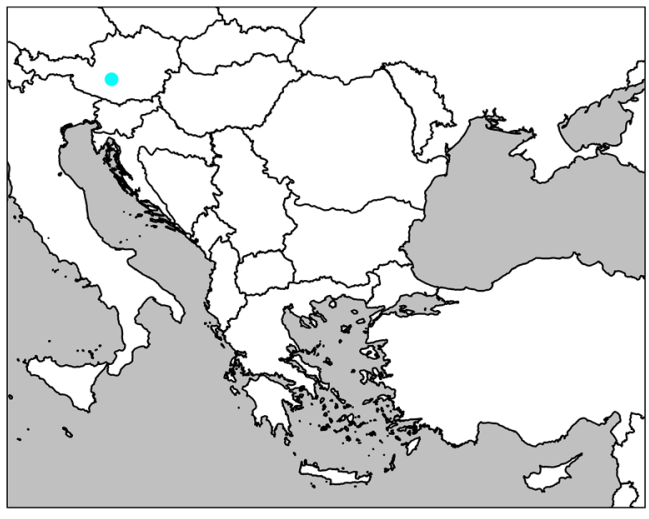

85. Dimastosternum holdhausi Attems, 1927

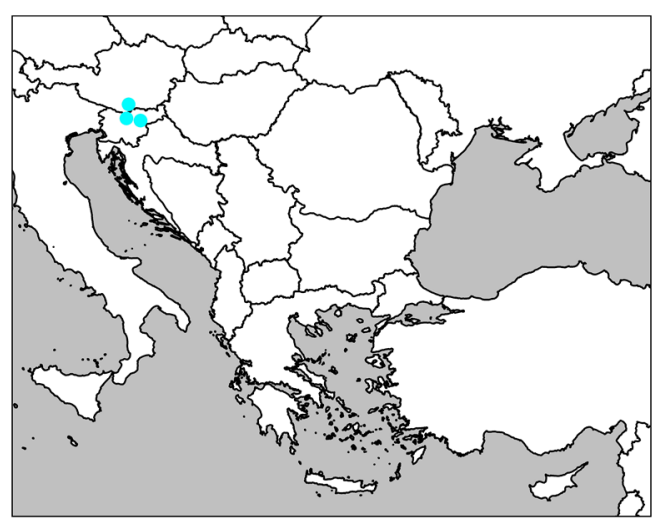

87. Glomogonium karawankarum Strasser, 1965

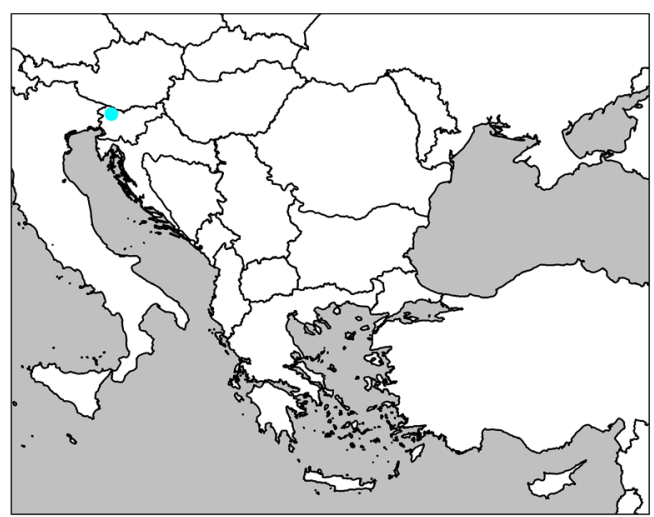

89. Julialpium alabardatum (Strasser, 1937)

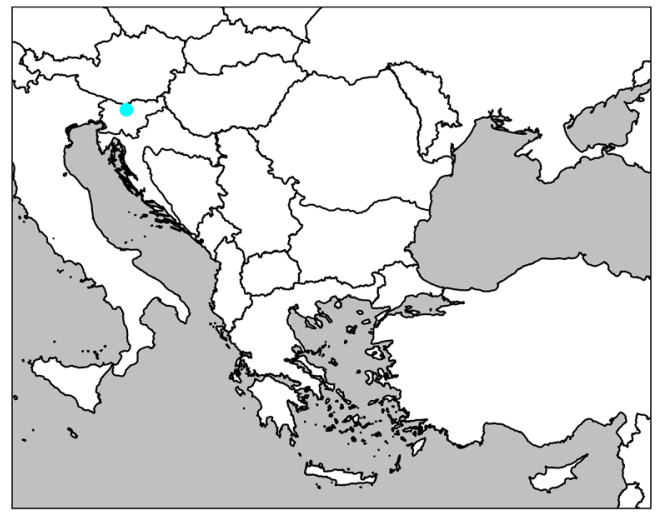

86. Eurygonium alticola (Strasser, 1937)

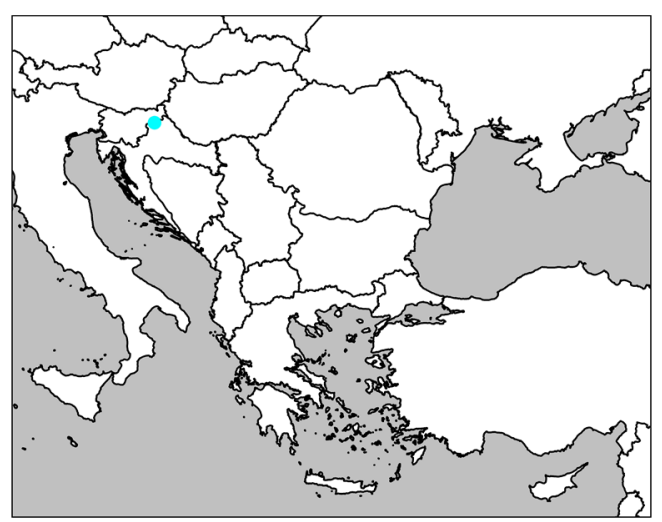

88. Grassographia makolensis Mršić, 1987

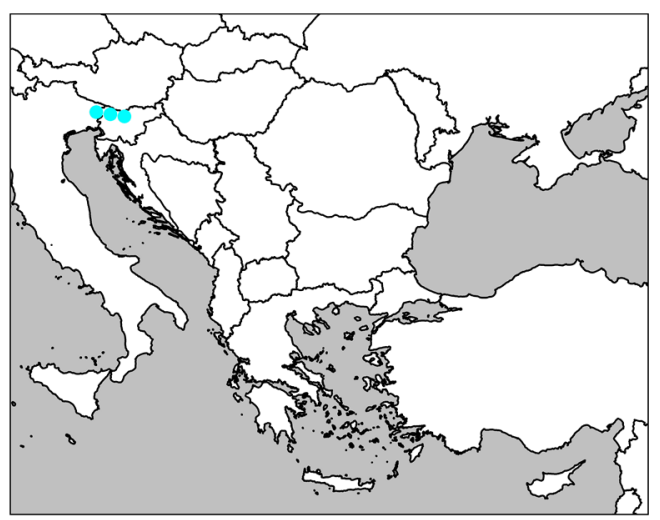

90. Mecogonopodium bohiniense Strasser, 1933 


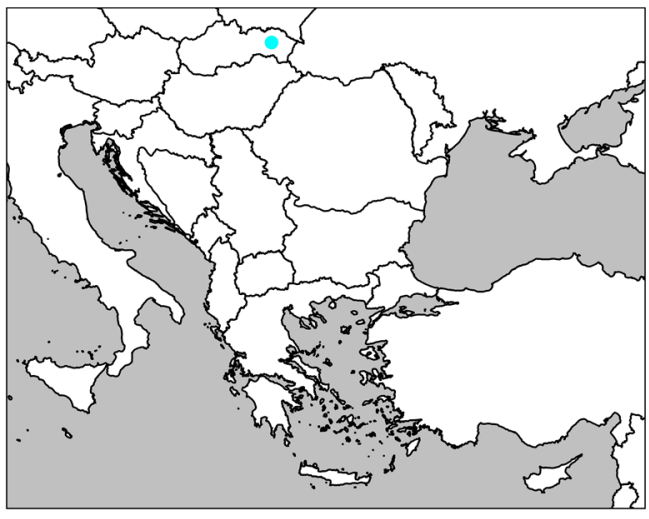

91. Mecogonopodium carpathicum Mock \& Tajovský, 2008

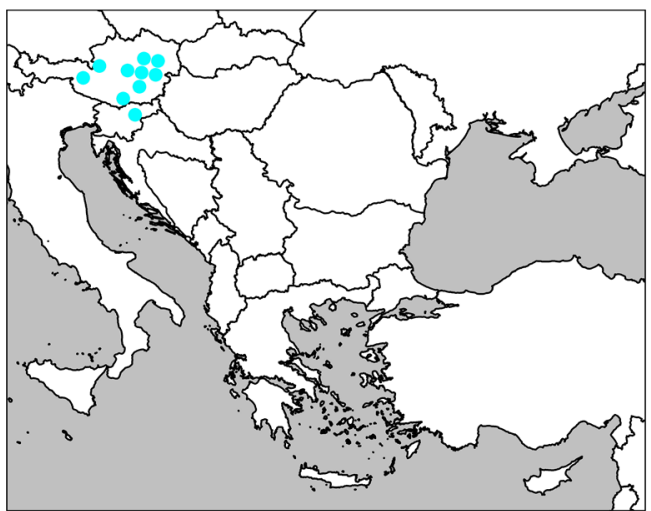

93. Polyphematia moniliformis

(Latzel, 1884)

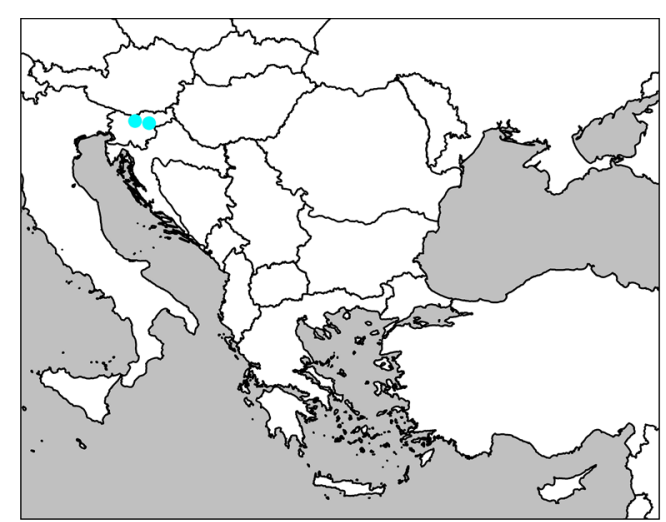

95. Stiphrogonium attemsi

Strasser, 1937

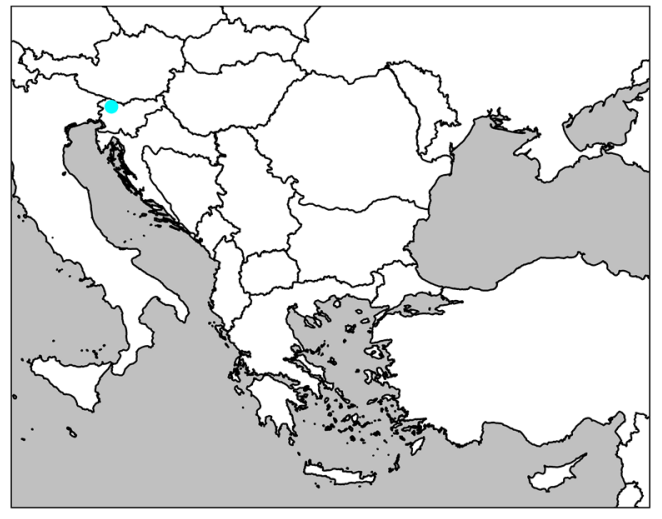

92. Mecogonopodium zirianum Mršić, 1987

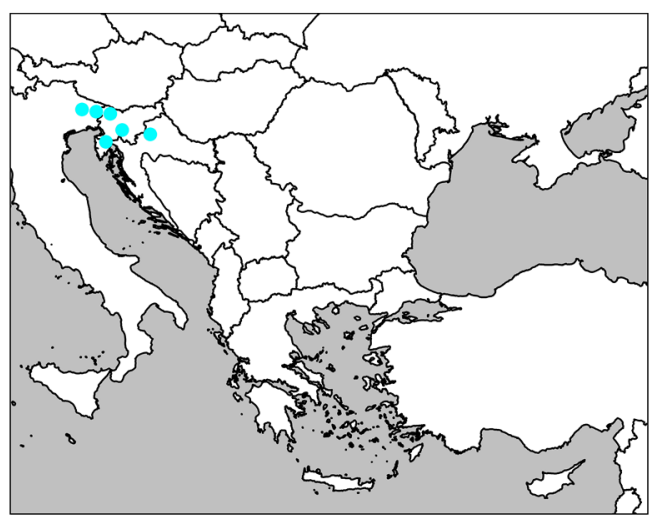

94. Schubartia lohmanderi

Verhoeff, 1927

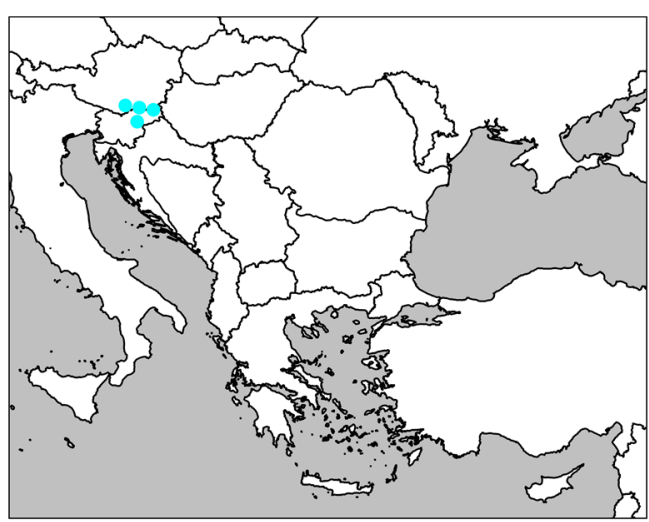

96. Symphyosphys serkoi

Strasser, 1939 


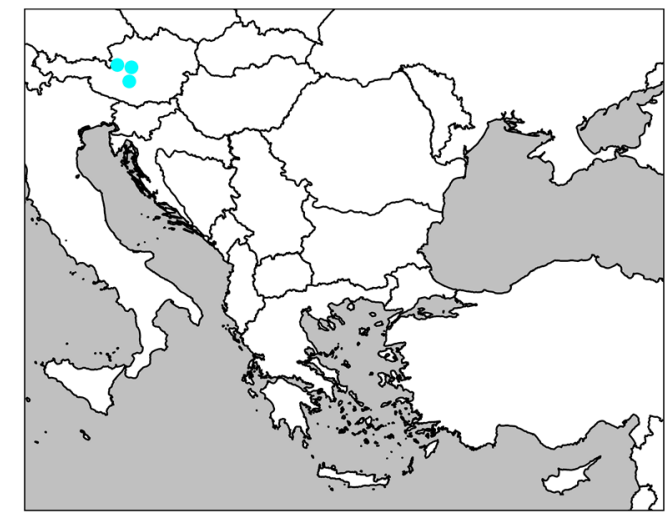

97. Syngonopodium aceris

Verhoeff, 1913

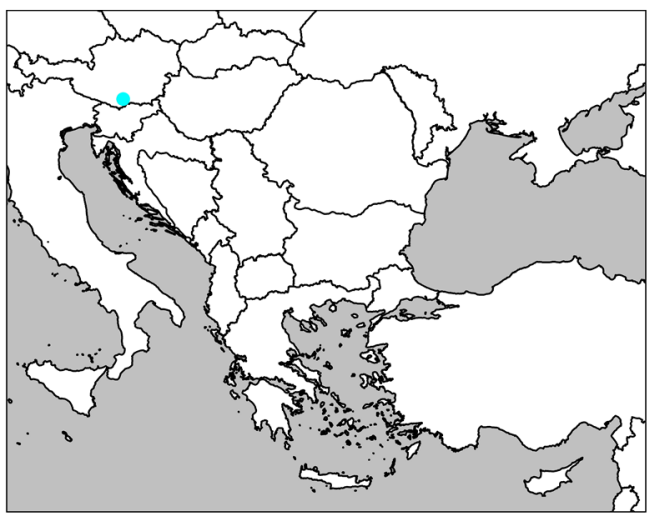

99. Tylogonium hoelzeli

Strasser, 1959

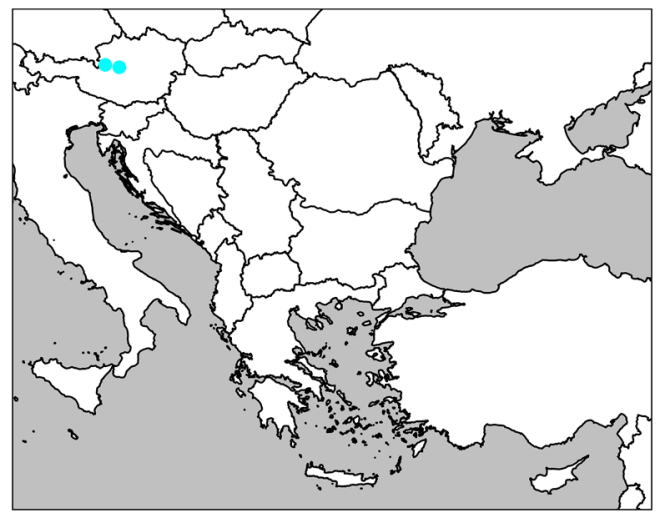

98. Syngonopodium cornutum Verhoeff, 1929

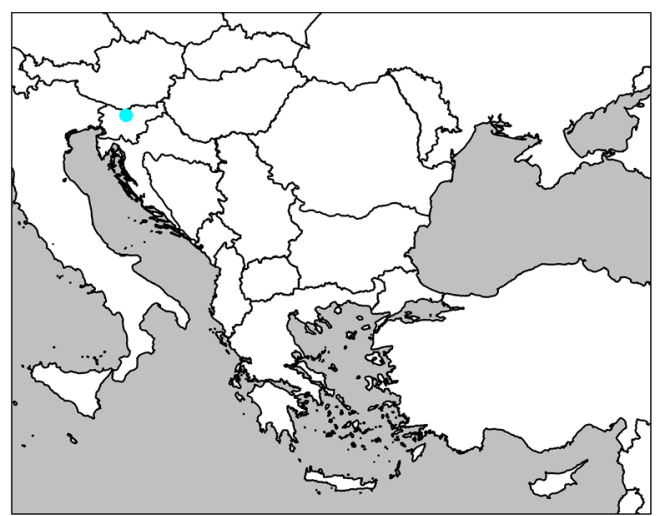

100. Tylogonium nivifidele Strasser, 1937

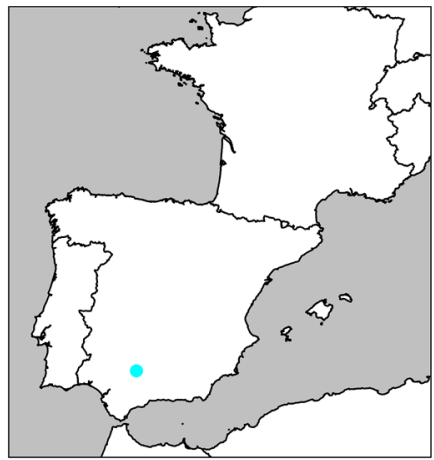

101. Beticosoma longipenis

Mauriès, 1990 


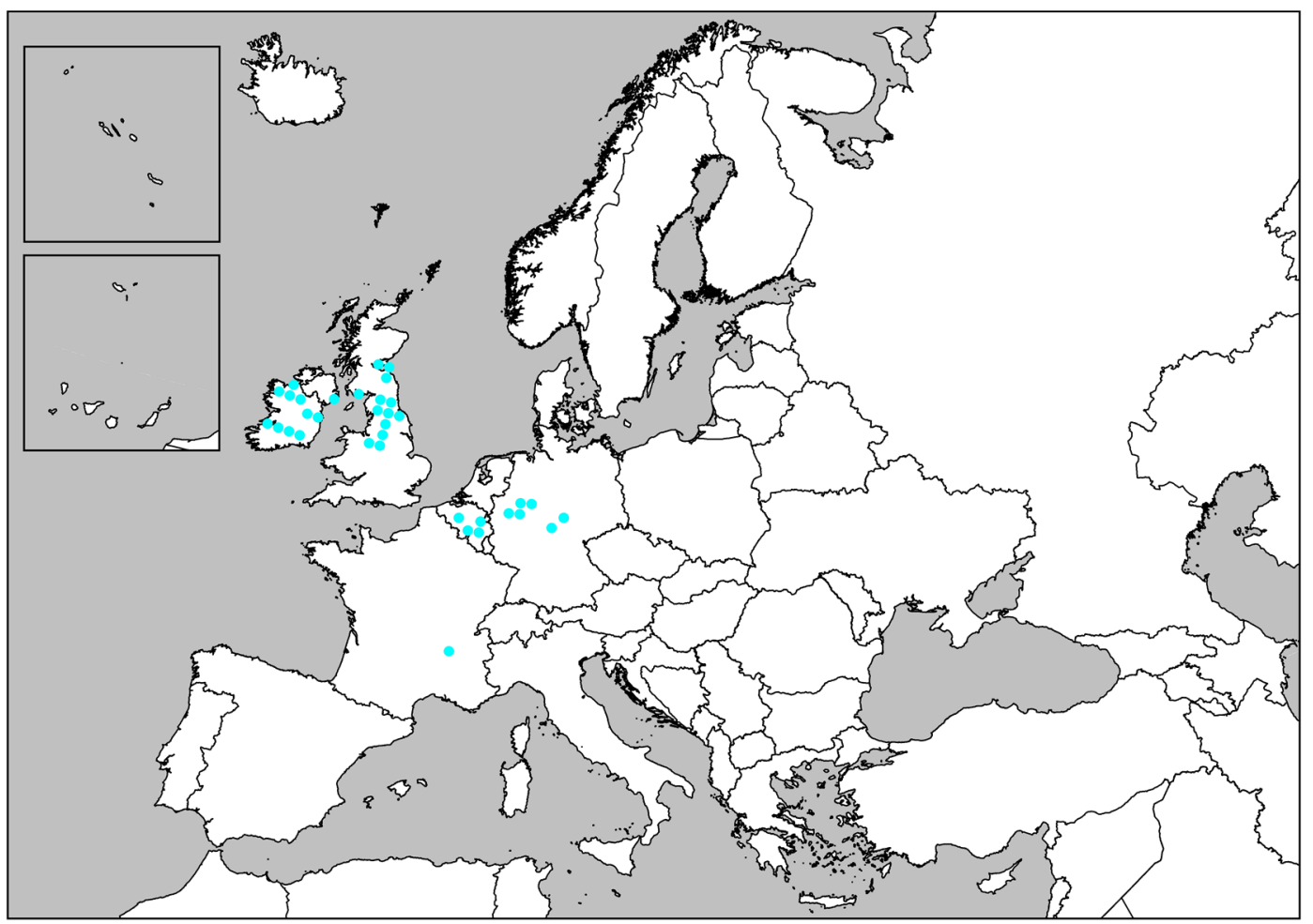

102. Brachychaeteuma bagnalli Verhoeff, 1911

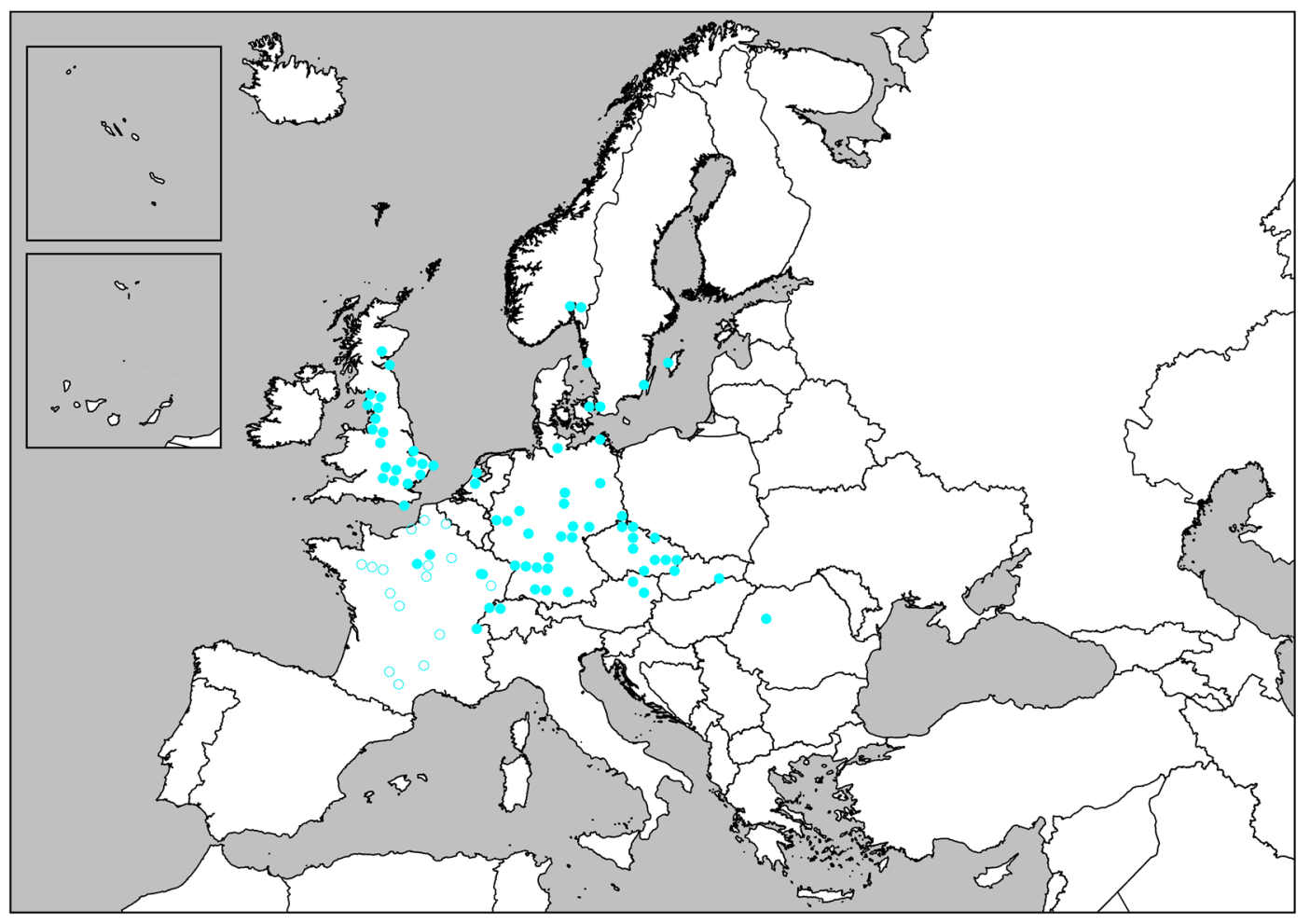

103. Brachychaeteuma bradeae (Brölemann, H.K. Brade-Birks \& S.G. Brade-Birks, 1917) 


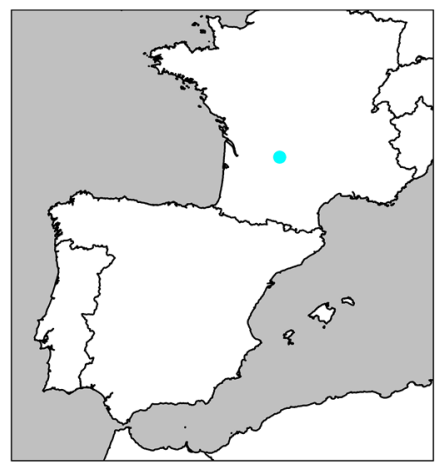

104. Brachychaeteuma cadurcense Mauriès, 1967

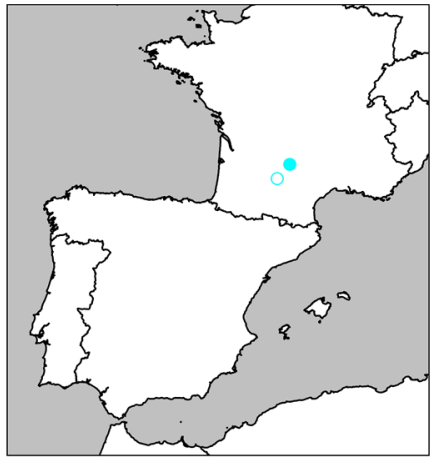

105. Brachychaeteuma furcatum Ribaut, 1956

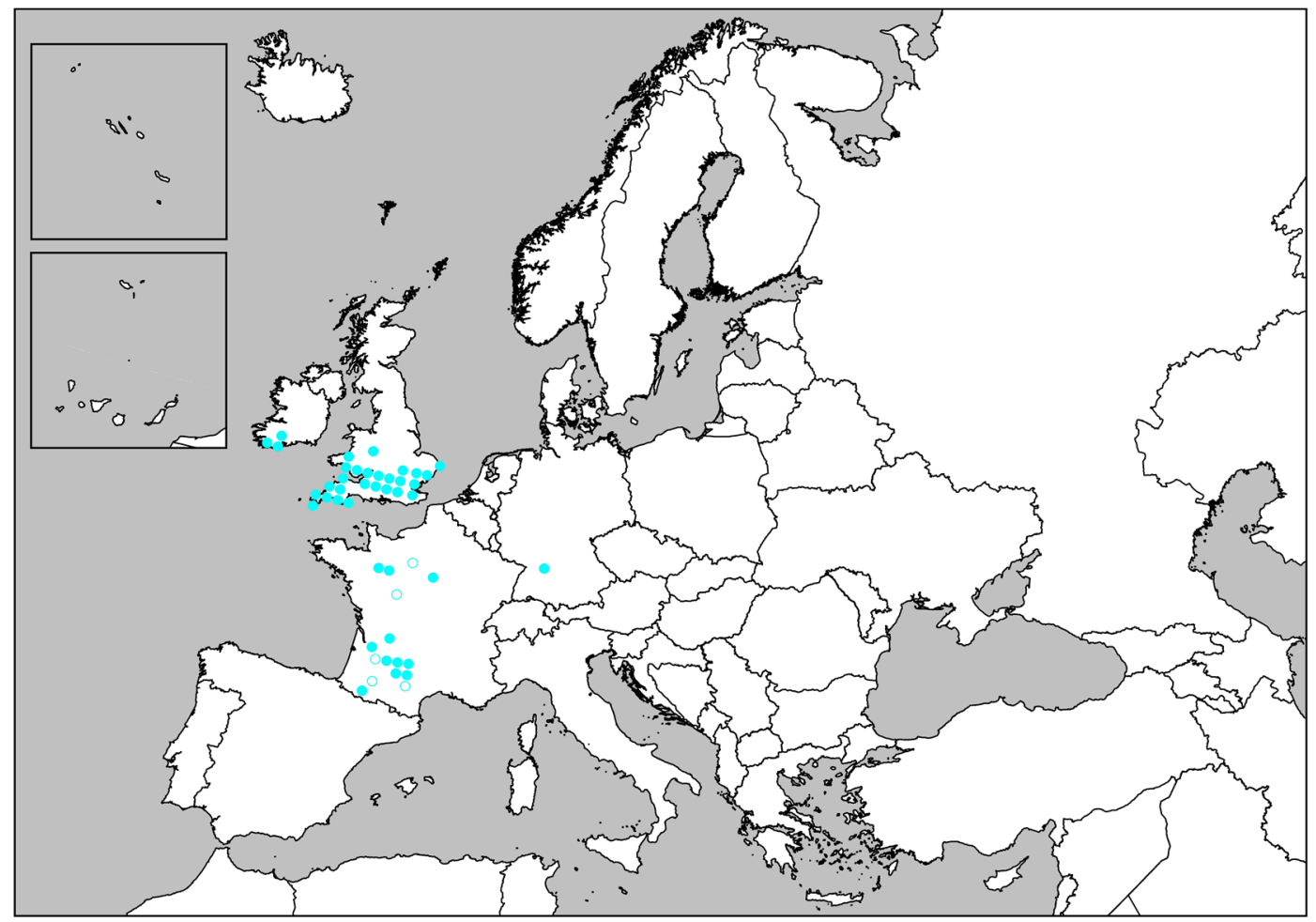

106. Brachychaeteuma melanops H.K. Brade-Birks \& S.G. Brade-Birks, 1918

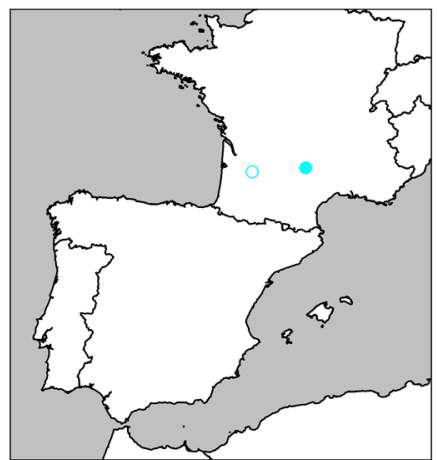

107. Brachychaeteuma peniculatum Ribaut, 1948

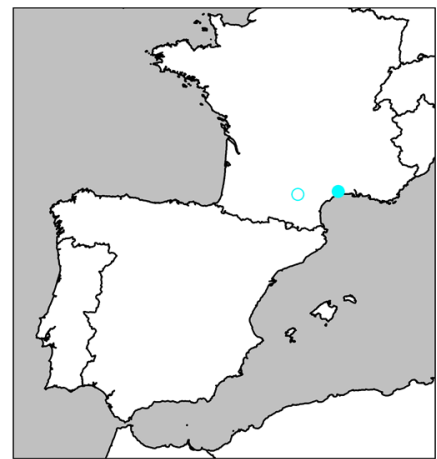

108. Brachychaeteuma plumosum Ribaut, 1947

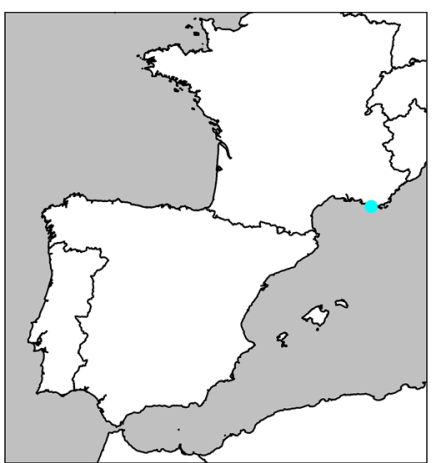

109. Brachychaeteuma provinciale Ribaut, 1956 


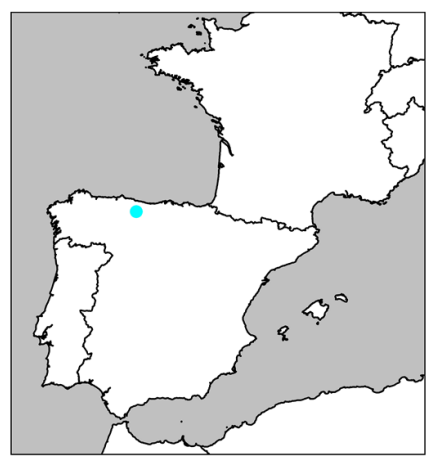

110. Asturasoma chapmani Mauriès, 1981

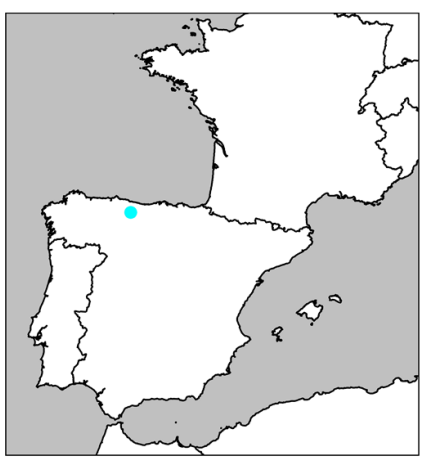

111. Asturasoma fowleri Mauriès, 1981

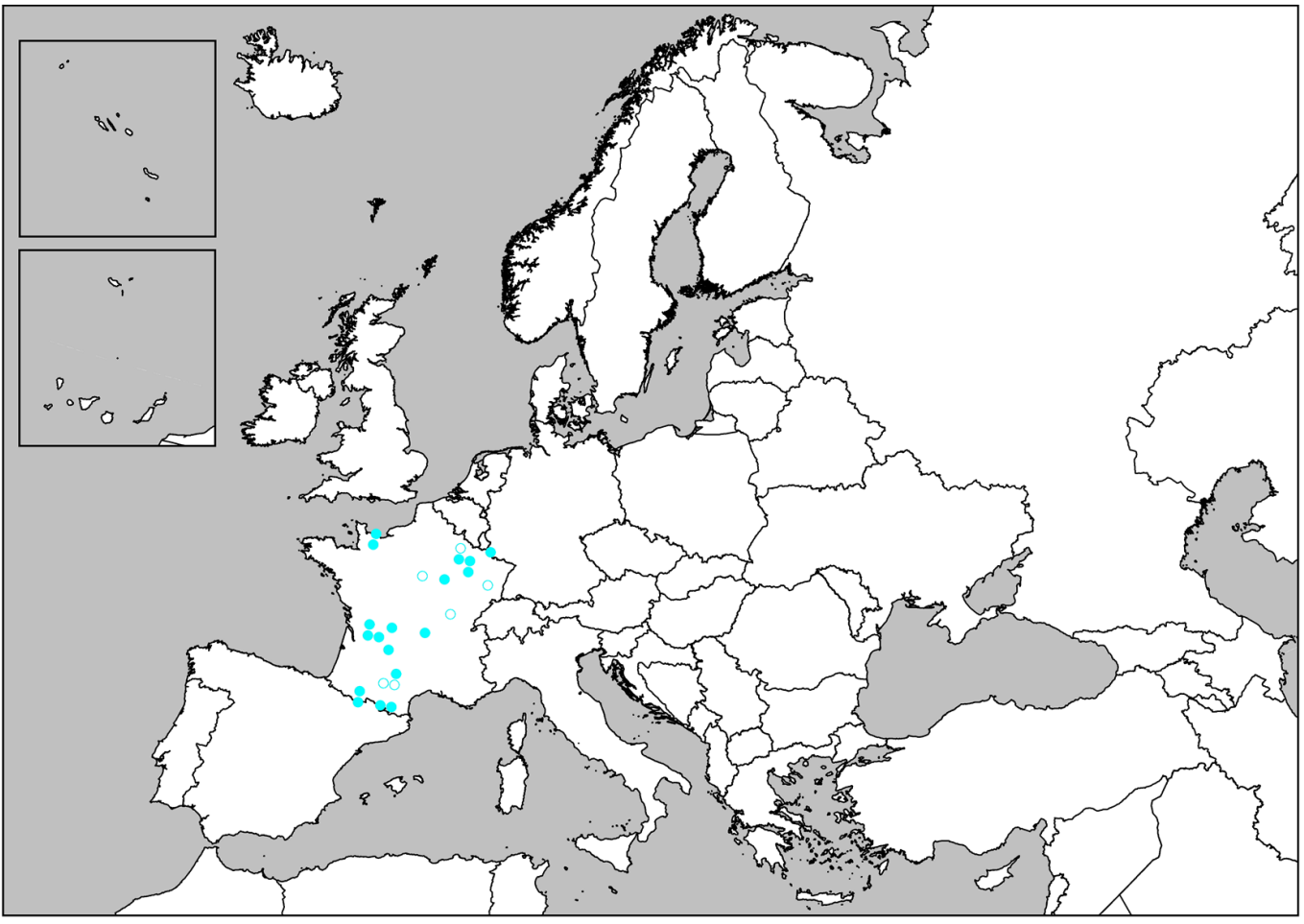

112. Chamaesoma broelemanni Ribaut \& Verhoeff, 1913

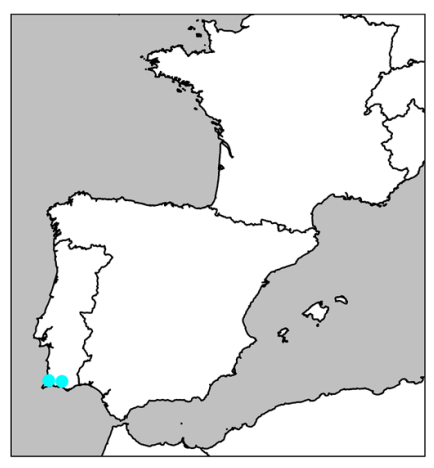

113. Coiffaiteuma turdetanorum Mauriès, 1964

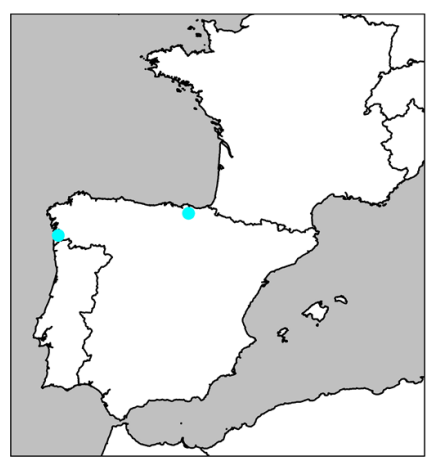

114. Krauseuma viscaiamum Mauriès \& Barraqueta, 1985

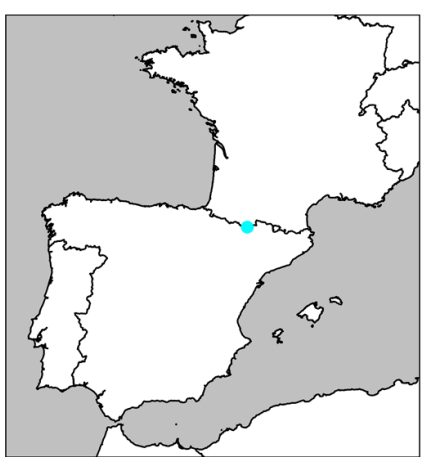

115. Marboreuma brouquissei Mauriès, 1988 


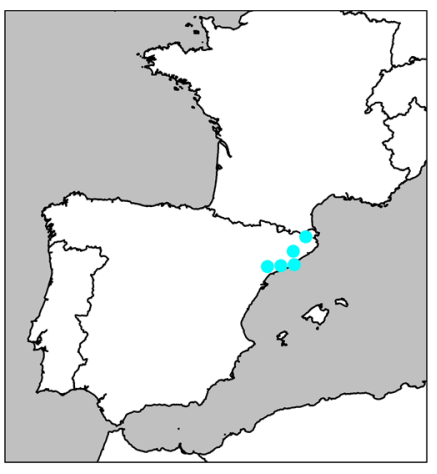

116. Origmatogona catalonica Ribaut, 1923

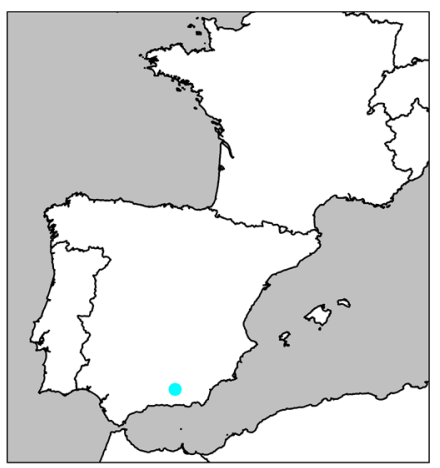

119. Origmatogona tinauti Mauriès, 1990

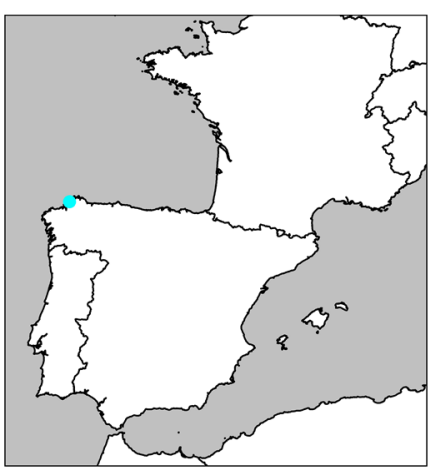

122. Scutogona ferrolensis Mauriès, 2015

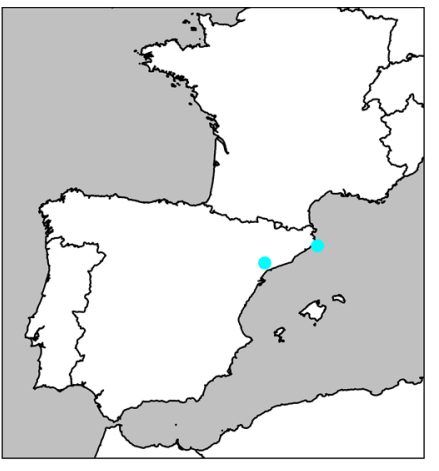

117. Origmatogona jacetanorum Mauriès, 1964

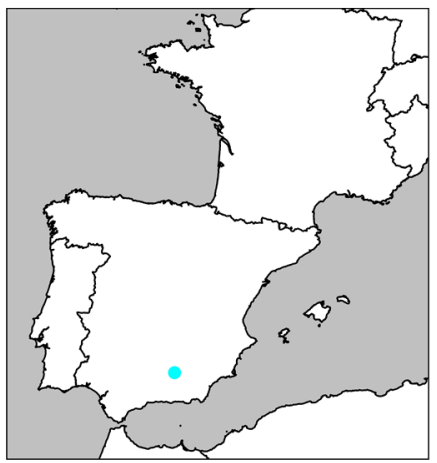

120. Origmatogona toniperezi Mauriès, 2014

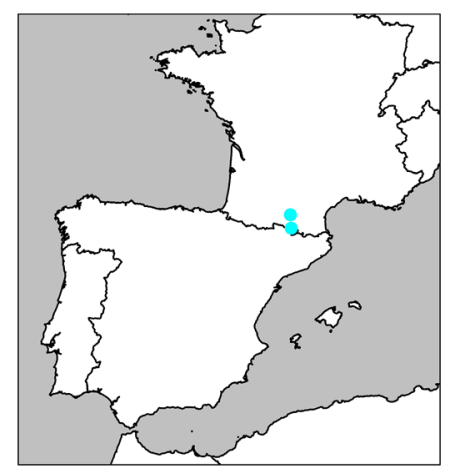

123. Scutogona jeanneli Ribaut, 1913

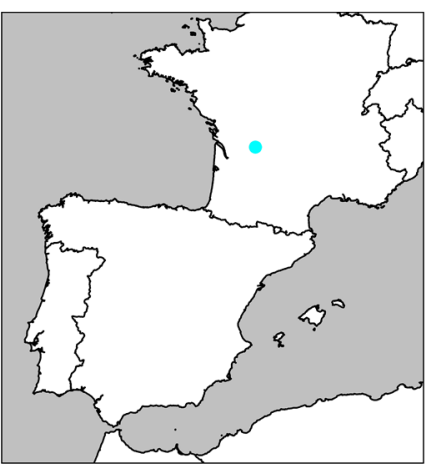

118. Origmatogona kimeorum Mauriès, 1990

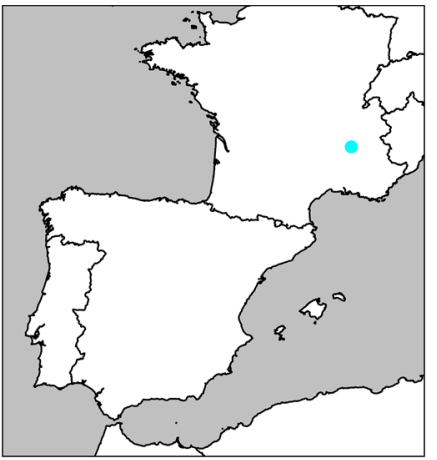

121. Scutogona alba Schubart, 1958

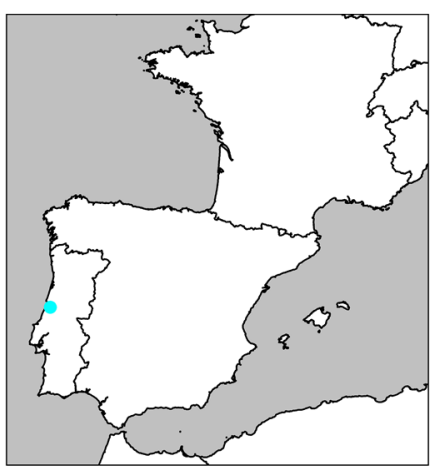

124. Scutogona minor Enghoff \& Reboleira, 2013 


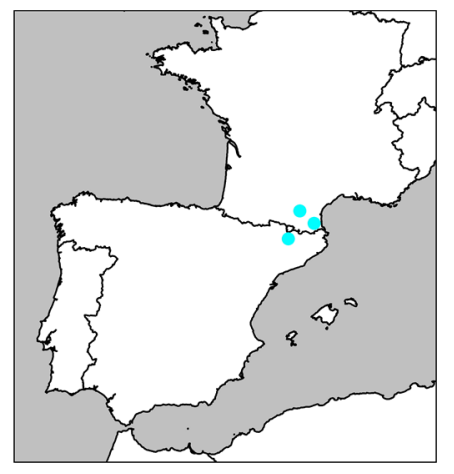

125. Scutogona mutica Ribaut, 1923

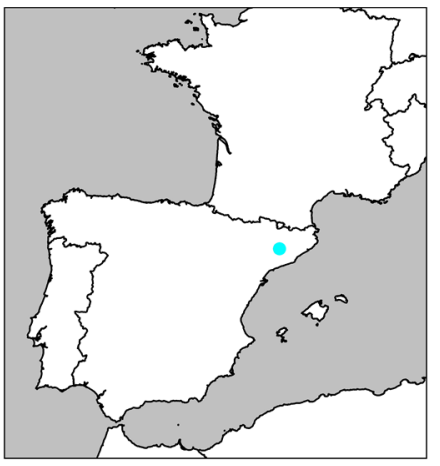

128. Scutogona vivesi Mauriès \& Vicente, 1977

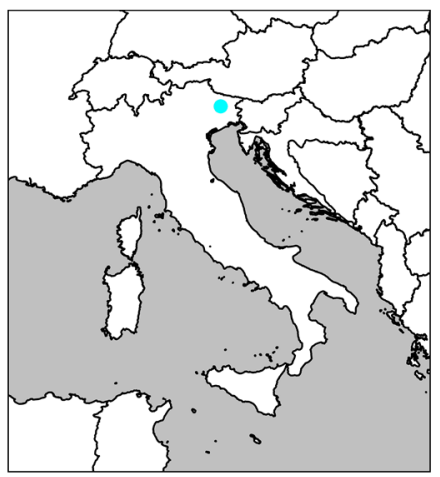

131. Verhoeffeuma minellii Mauriès, 1990

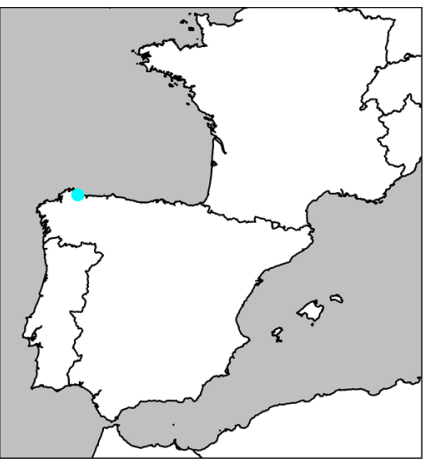

126. Scutogona oculinigra

Mauriès \& Vicente, 1977

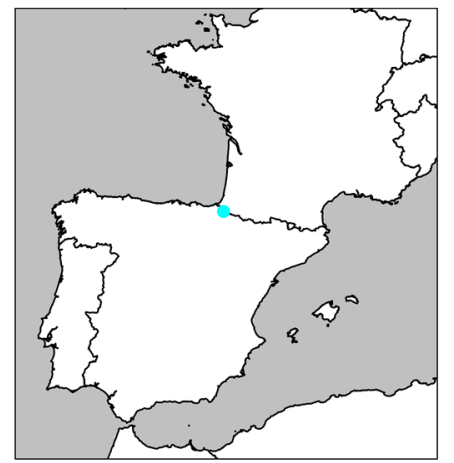

129. Vascosoma coiffaiti Mauriès, 1966

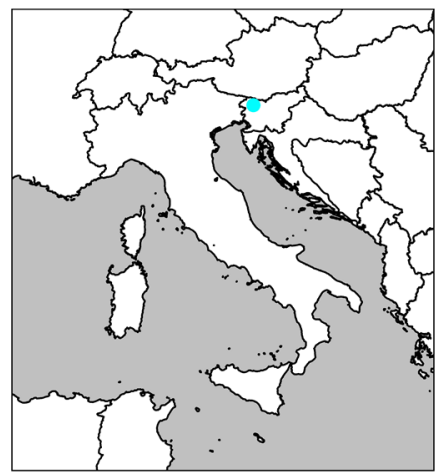

132. Verhoeffeuma spinosum Strasser, 1937

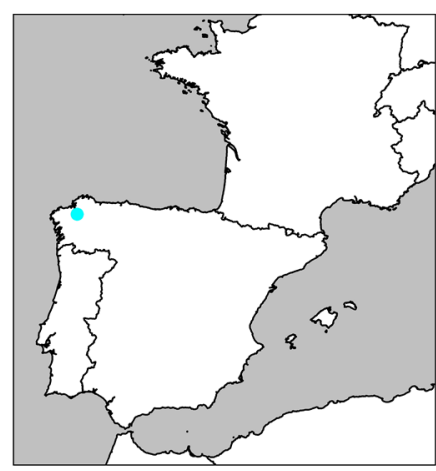

127. Scutogona suboculinigra Mauriès, 2015

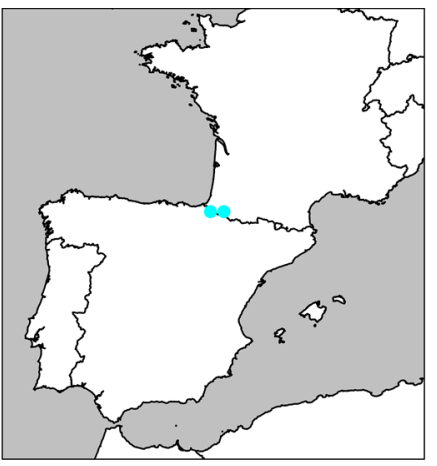

130. Vascosoma duprei

Mauriès, 1990

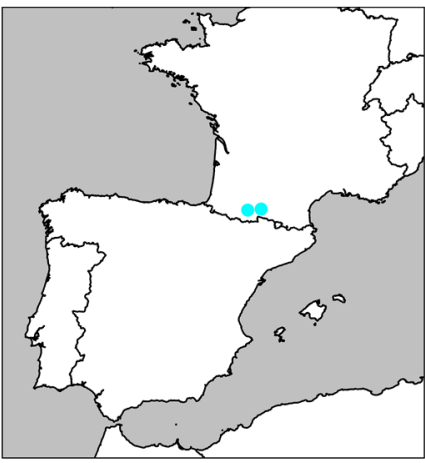

133. Xystrosoma beatense Ribaut, 1927 


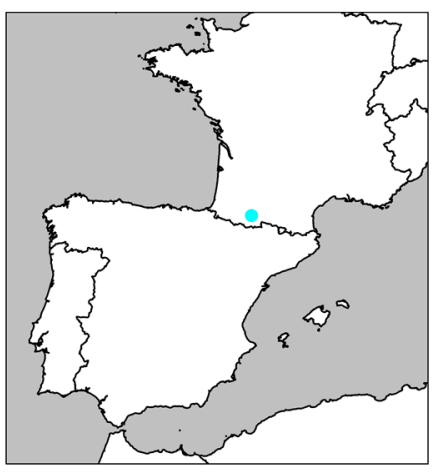

134. Xystrosoma cassagnaui Mauriès, 1965

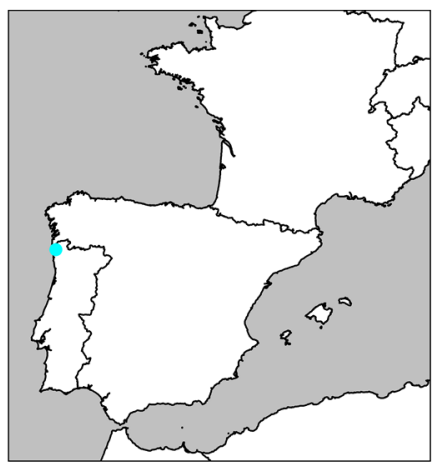

137. Xystrosoma lusitanicum Mauriès, 2015

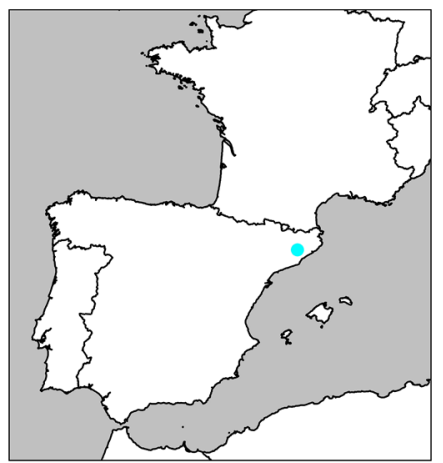

140. Xystrosoma santllorence Serra \& Mauriès, 2018

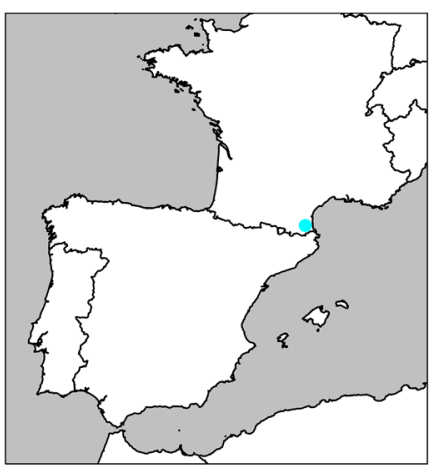

135. Xystrosoma catalonicum Ribaut, 1927

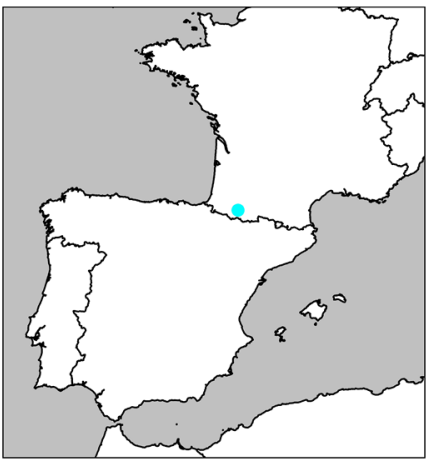

138. Xystrosoma murinum Ribaut, 1927

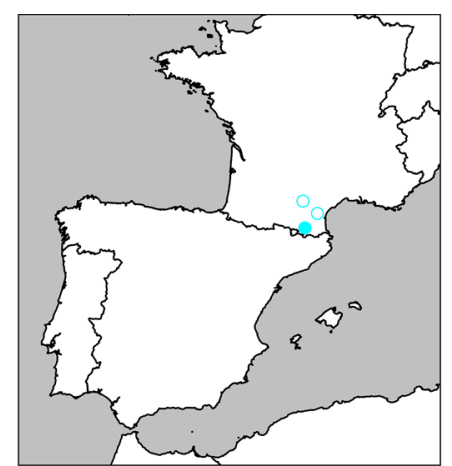

141. Xystrosoma tectosagum Ribaut, 1927

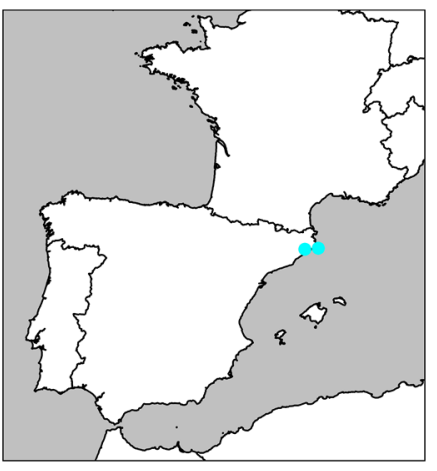

136. Xystrosoma coiffaiti Mauriès, 1964

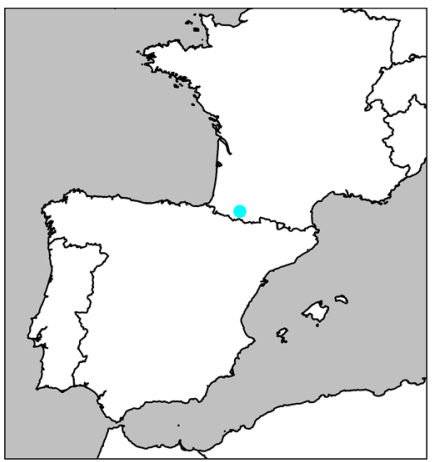

139. Xystrosoma pyrenaicum Ribaut, 1927

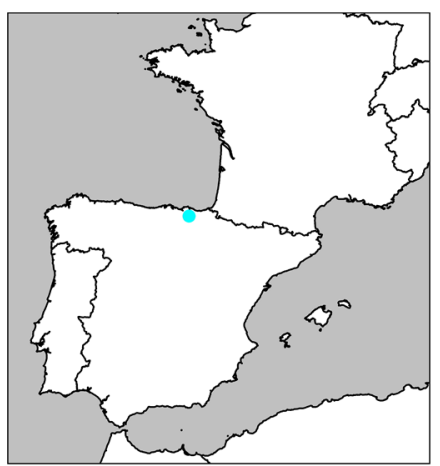

142. Xystrosoma vasconicum Mauriès \& Barraqueta, 1985 


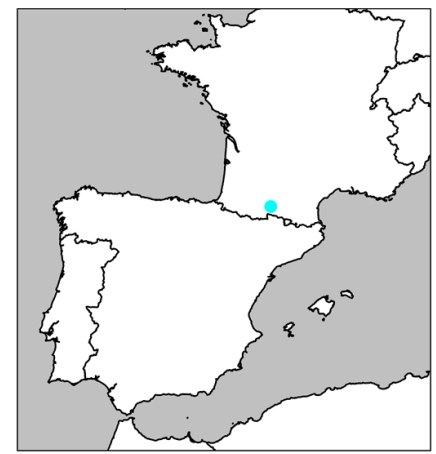

143. Chordeuma consoranense Ribaut, 1956

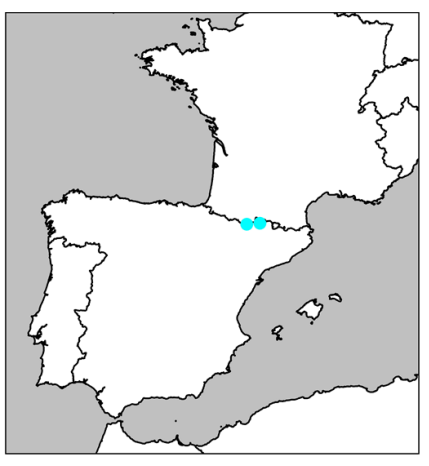

146. Chordeuma intermedium Ribaut, 1913

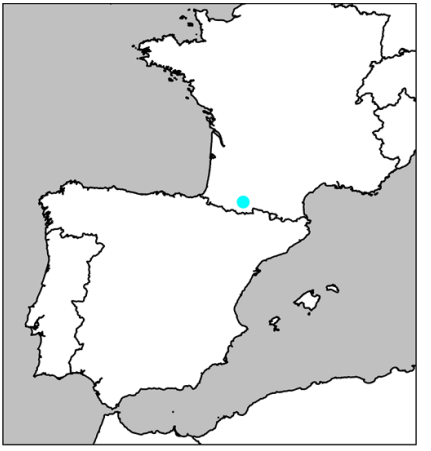

144. Chordeuma iluronense Ribaut, 1913

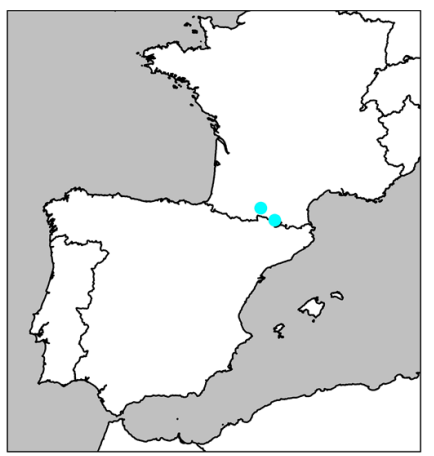

147. Chordeuma montanum Ribaut, 1956

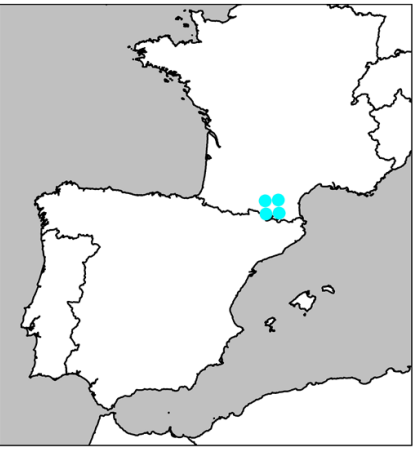

145. Chordeuma inornatum Ribaut, 1913

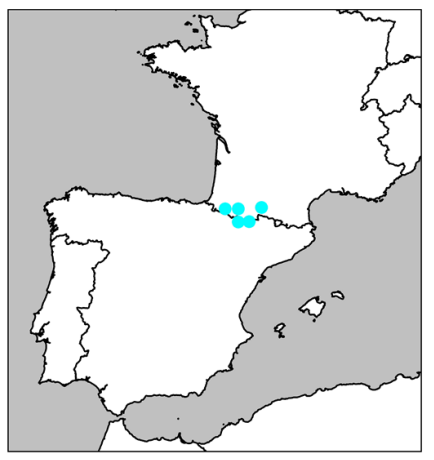

148. Chordeuma muticum Ribaut, 1927

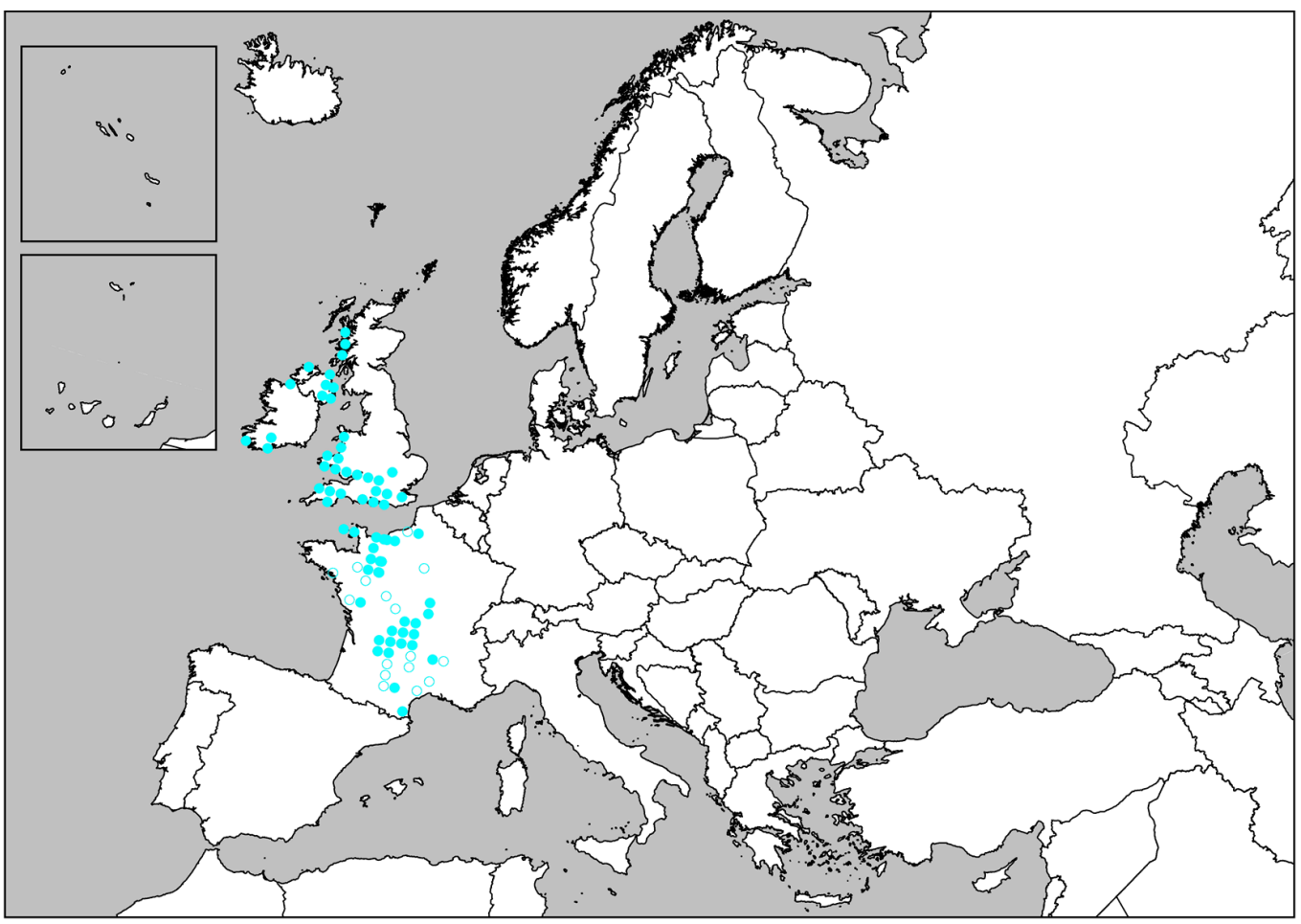

149. Chordeuma proximum Ribaut, 1913 


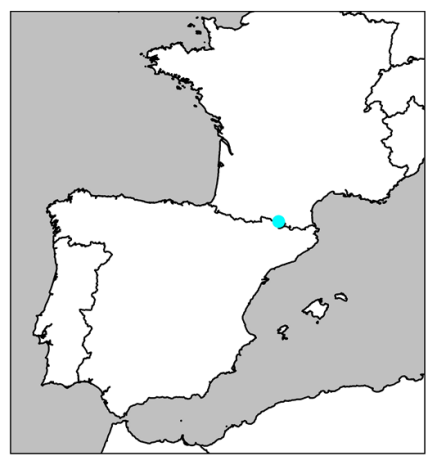

150. Chordeuma reflexum Brolemann, 1927

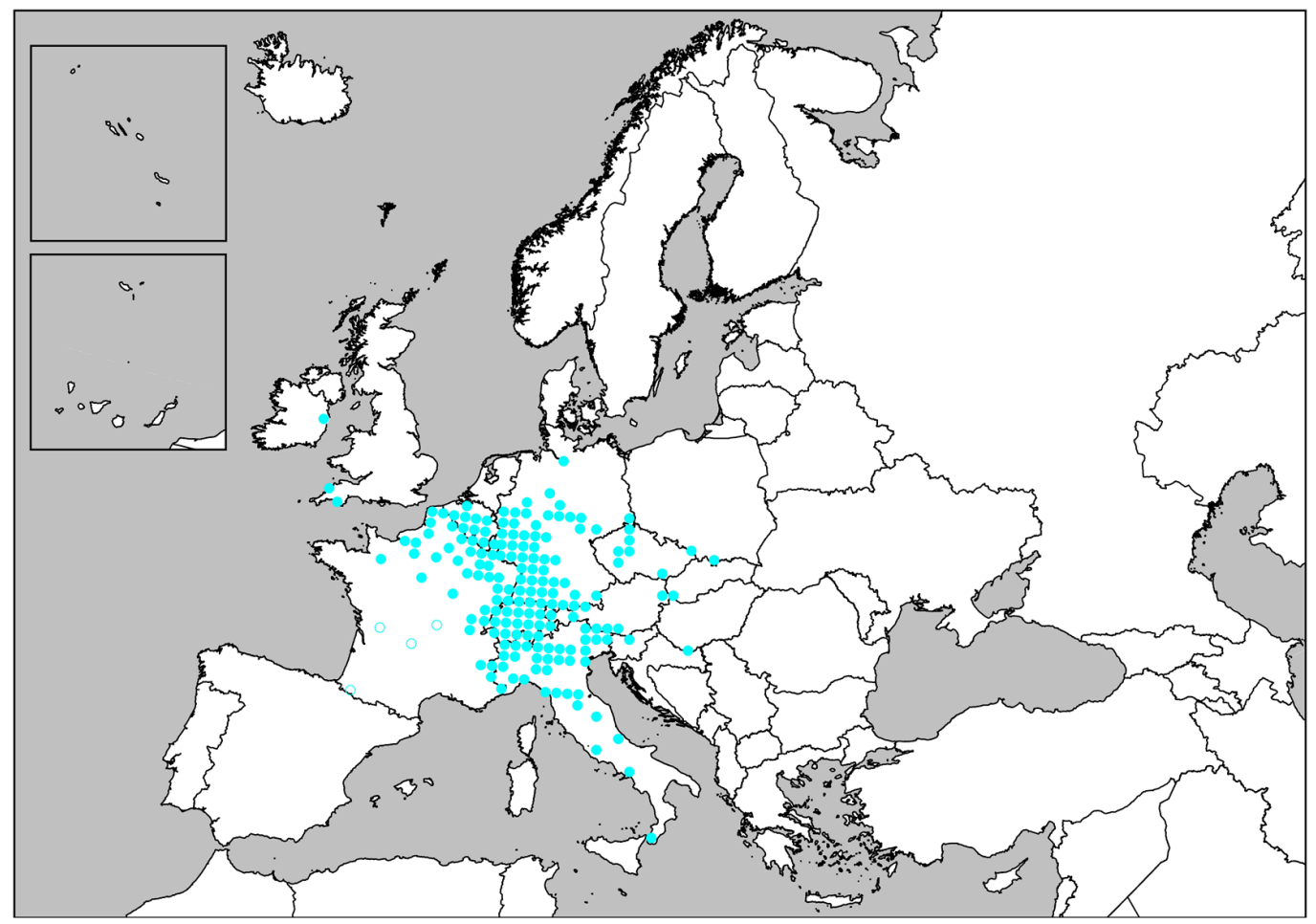

151. Chordeuma sylvestre C.L.Koch, 1847

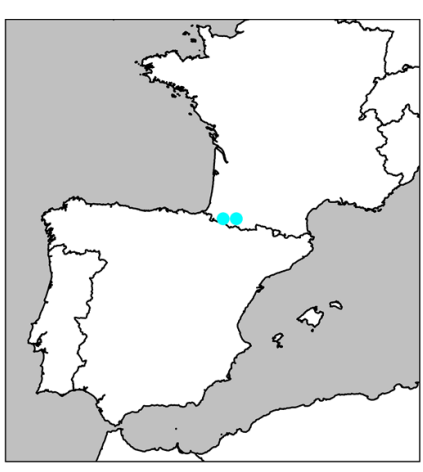

152. Chordeuma trifidum Ribaut, 1913

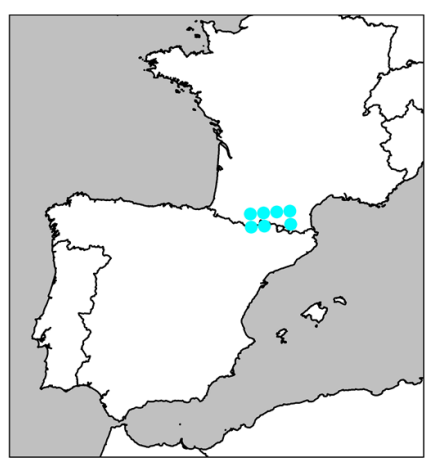

153. Chordeuma utriculosum Ribaut, 1913

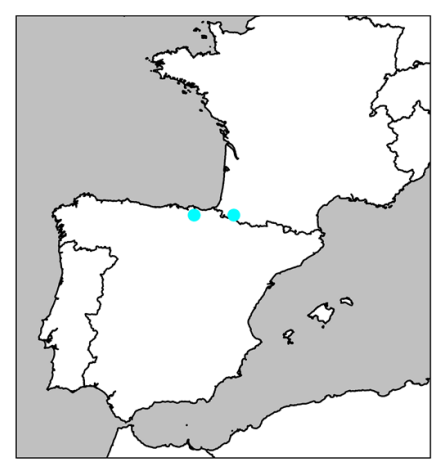

154. Chordeuma vasconicum Ribaut, 1913 


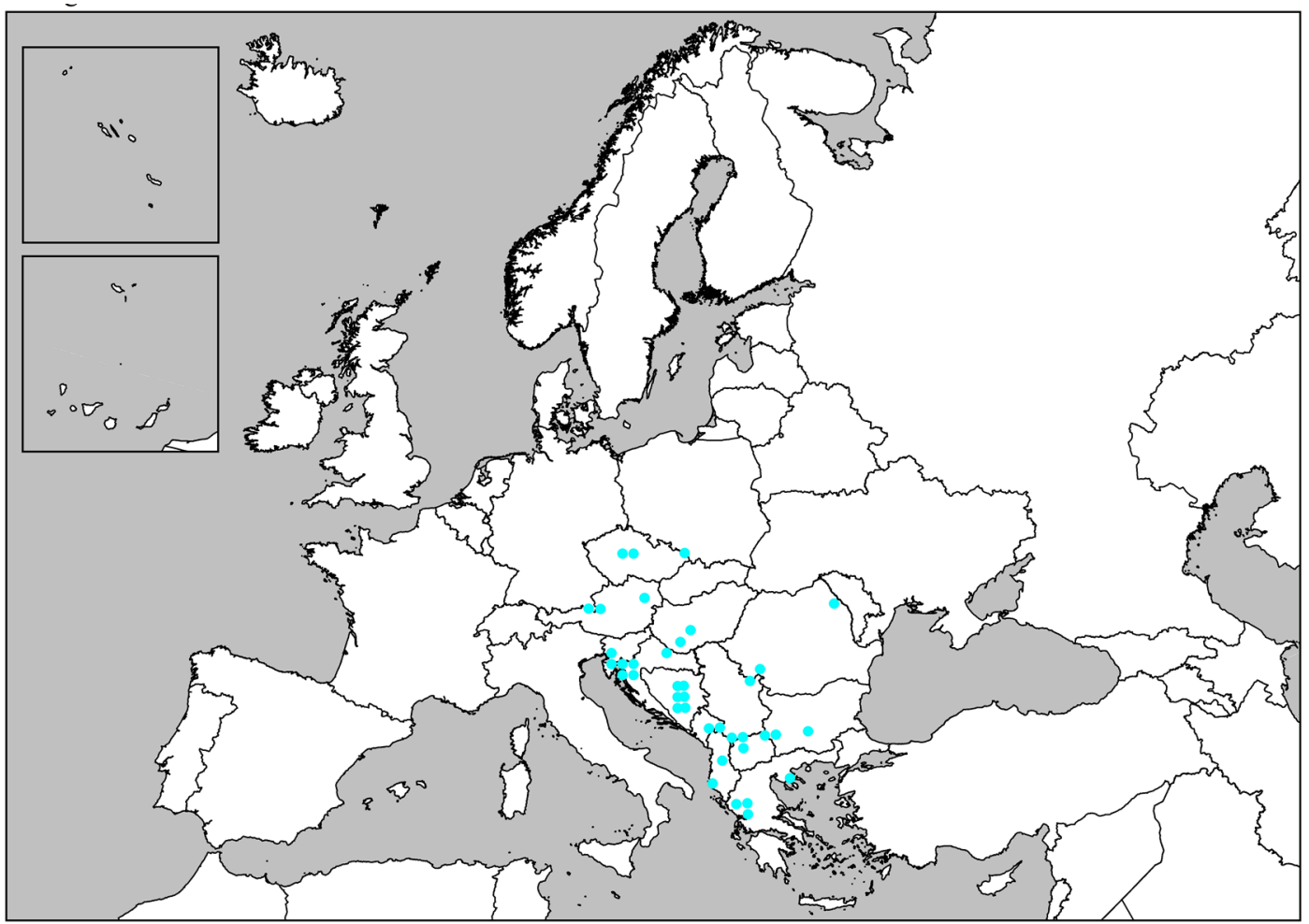

155. Melogona broelemanni (Verhoeff, 1897)

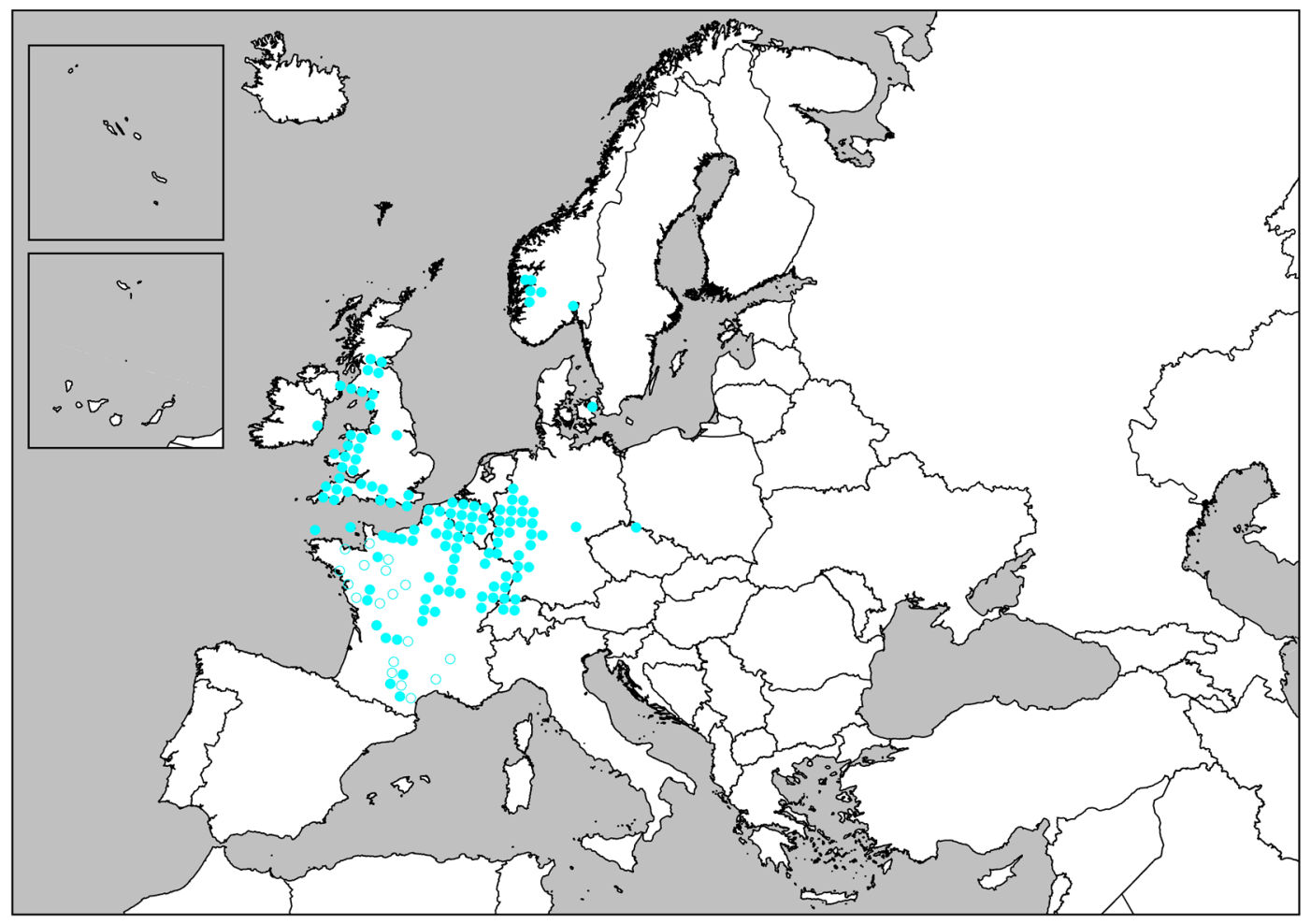

156. Melogona gallica (Latzel, 1884) 


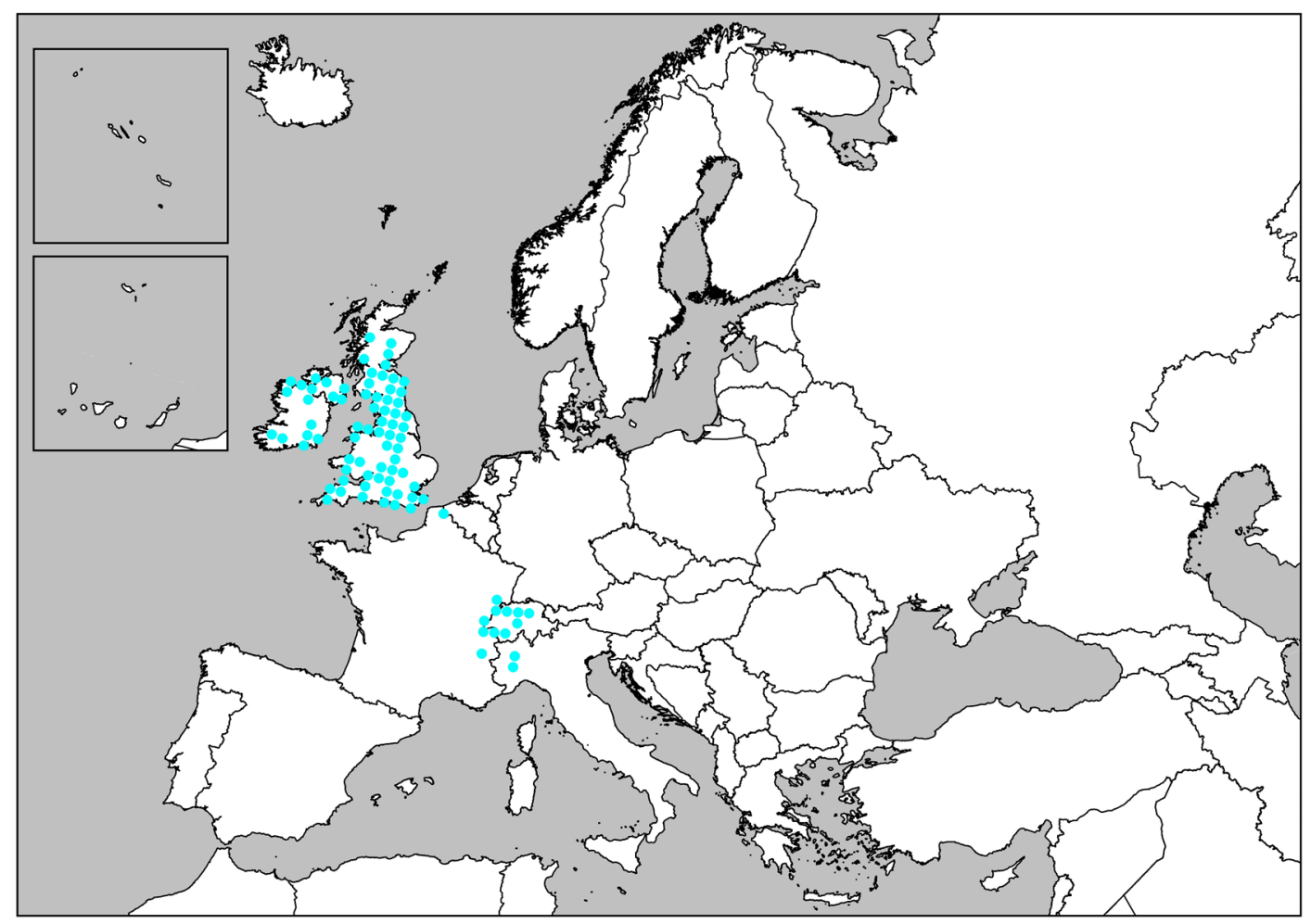

157. Melogona scutellaris (Ribaut, 1913)

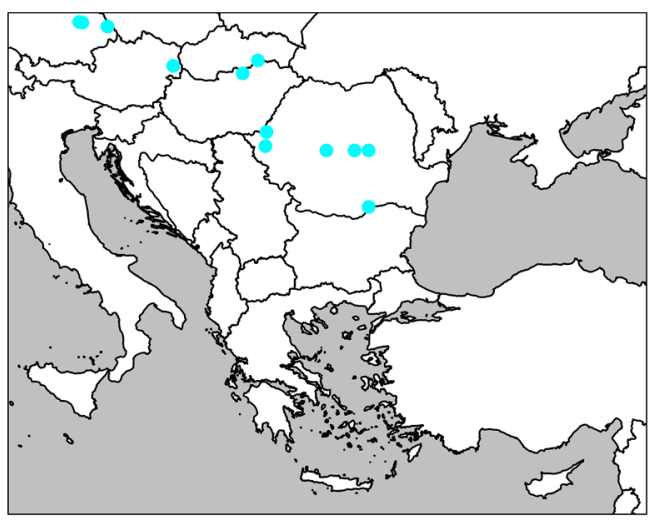

158. Melogona transsilvanica

(Verhoeff, 1897) 


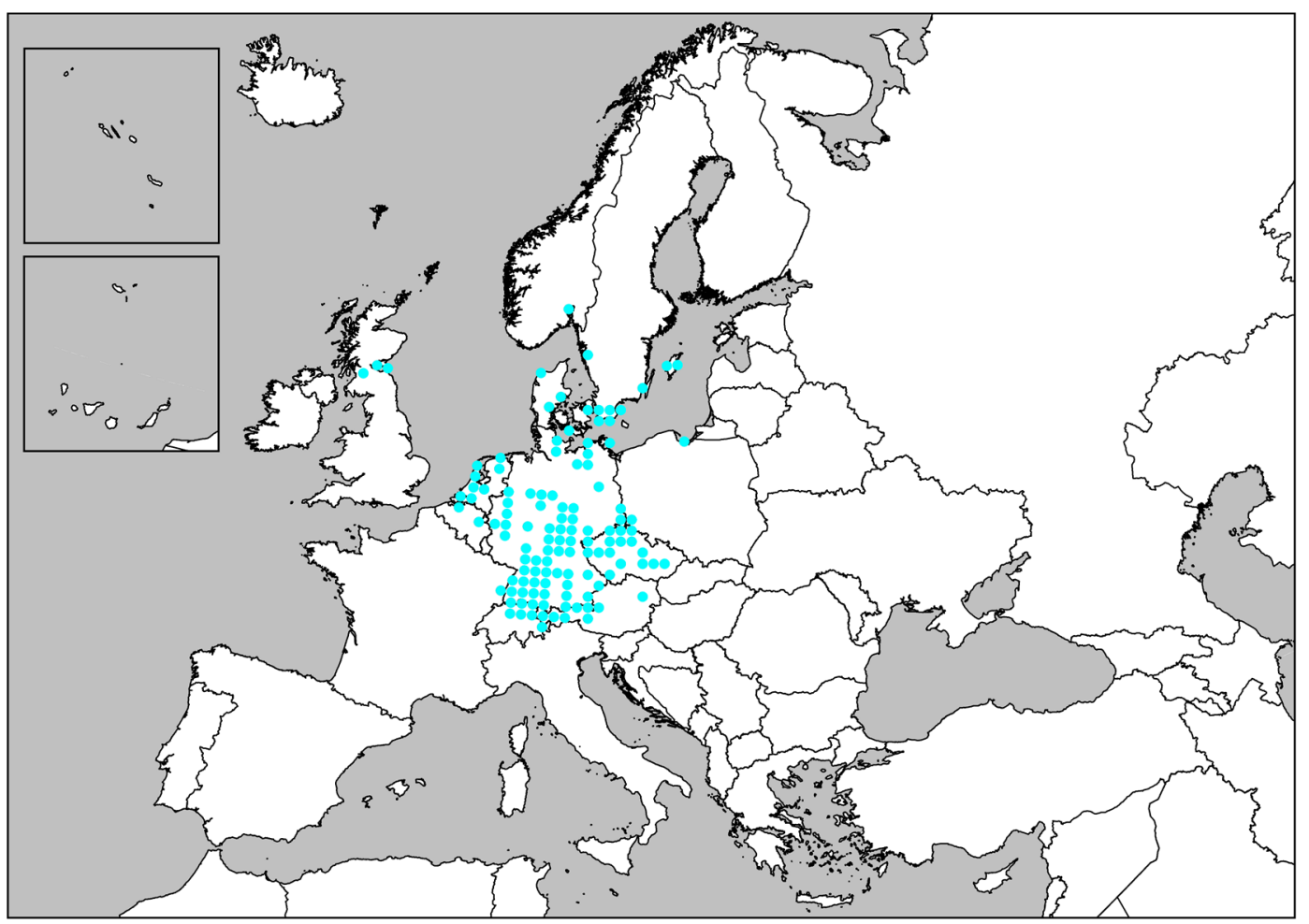

159. Melogona voigti (Verhoeff, 1899)

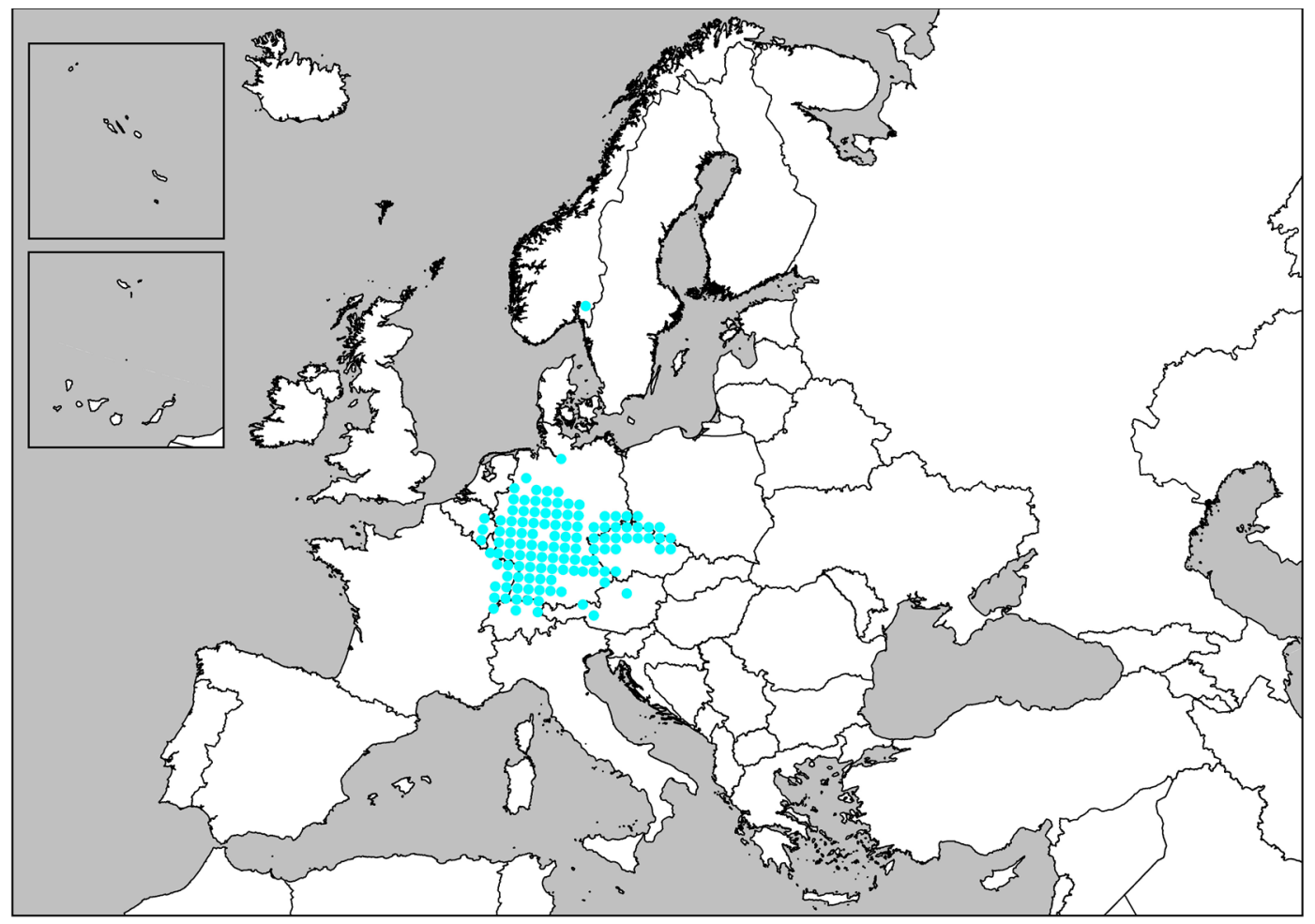

160. Mycogona germanica (Verhoeff, 1892) 


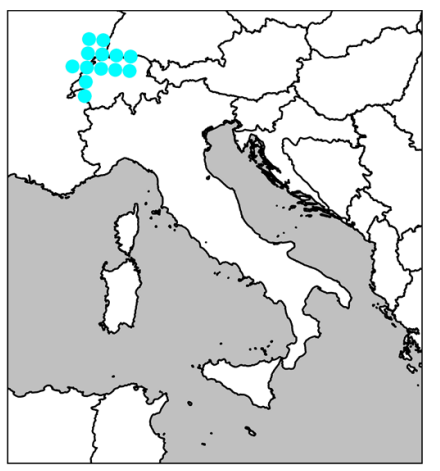

161. Orthochordeumella fulva (Rothenbühler, 1899)

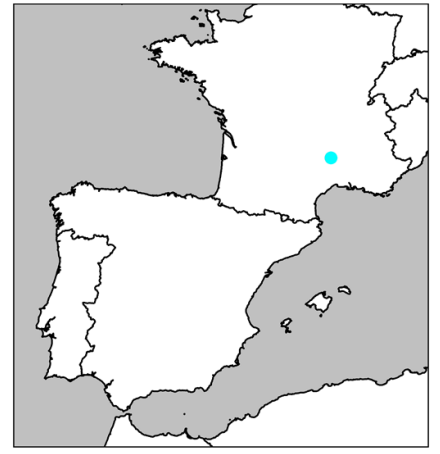

162. Orthochordeumella leclerci (Mauriès, 1985)

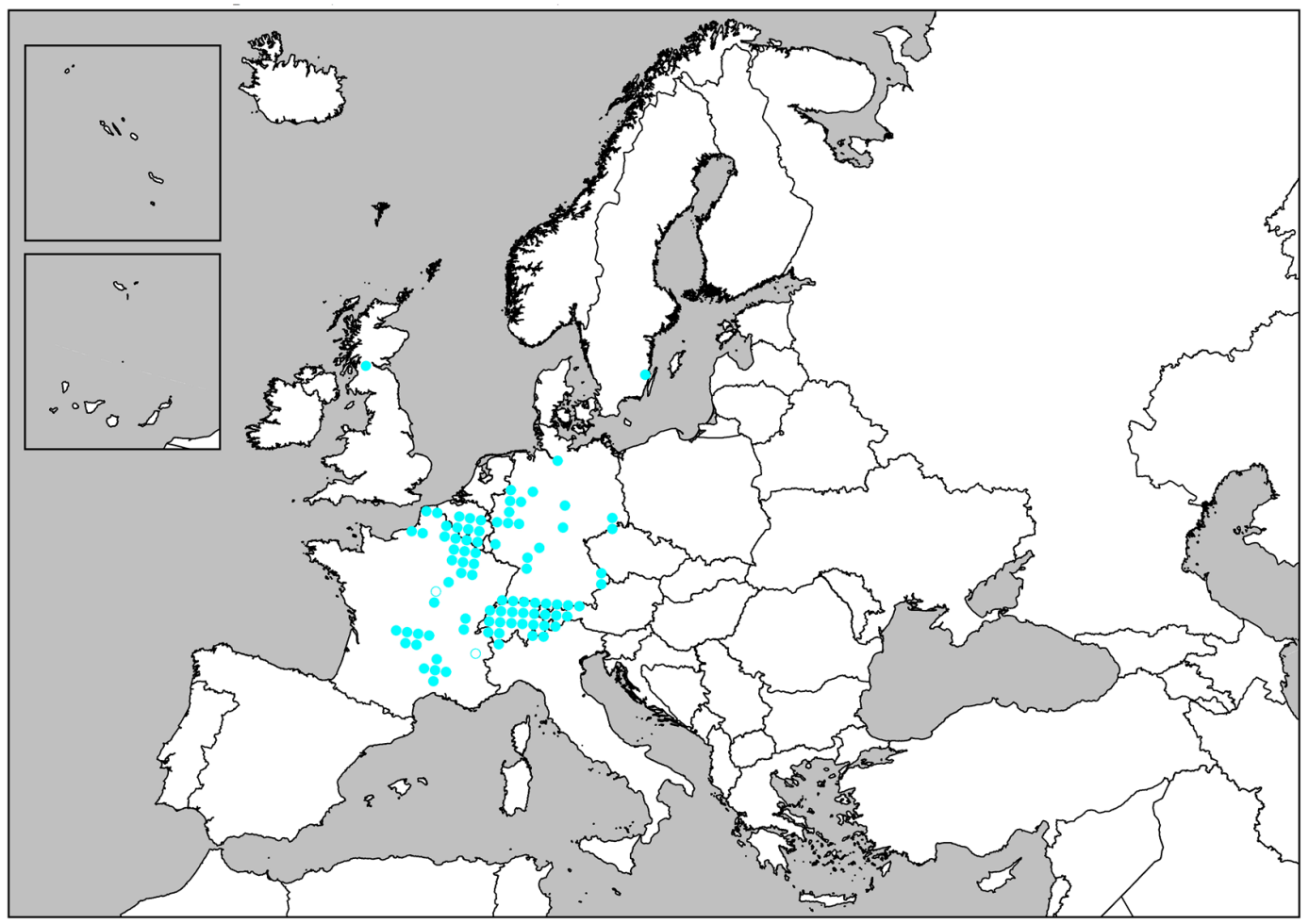

163. Orthochordeumella pallida (Rothenbühler, 1899)

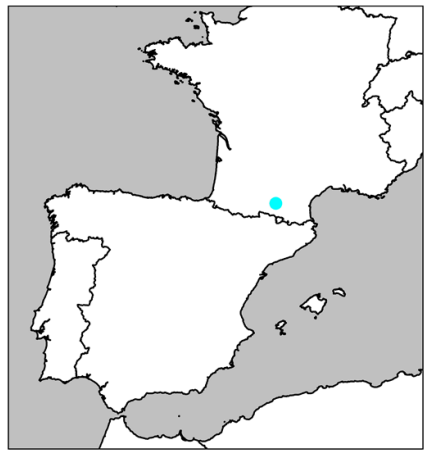

164. Orthochordeumella pyrenaica Mauriès, 1965

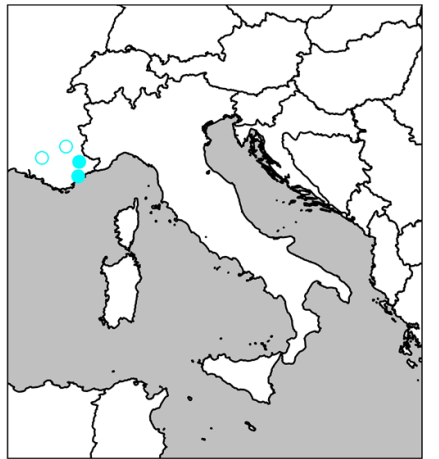

165. Parachordeuma broelemanni Ribaut, 1912

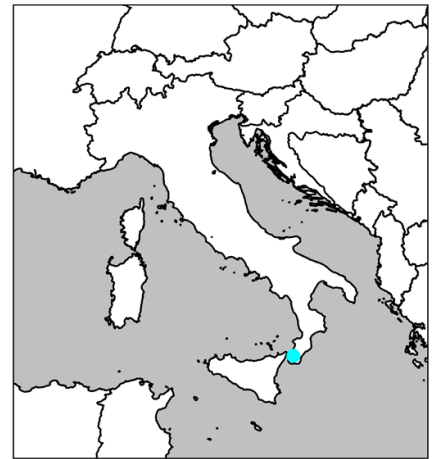

166. Aspromontia ruffoi Strasser, 1970 


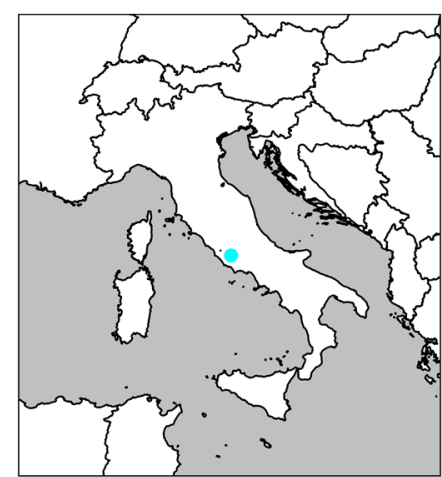

167. Atractosoma abnorme Verhoeff, 1900

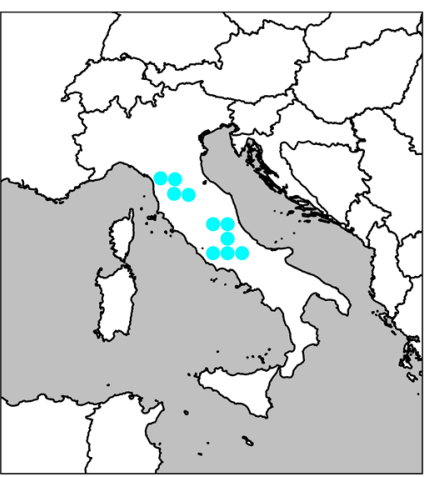

170. Atractosoma cecconii Silvestri, 1898

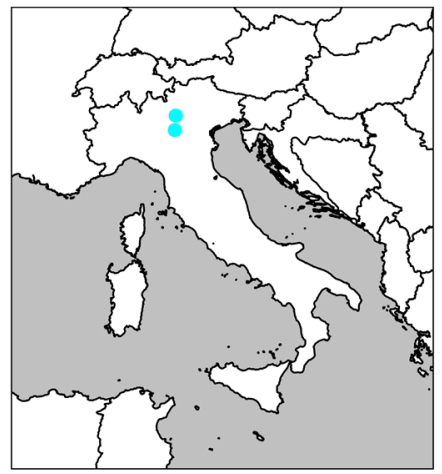

173. Atractosoma ghidinii Manfredi, 1935

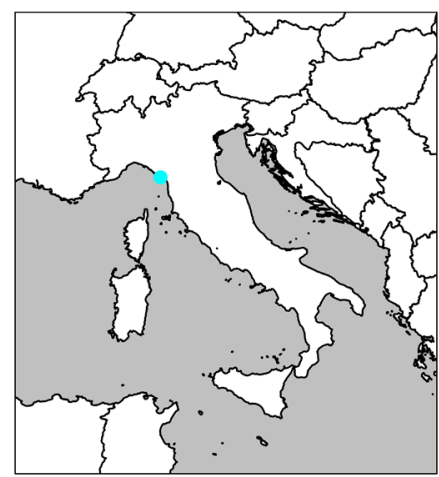

168. Atractosoma blechnorum Verhoeff, 1936

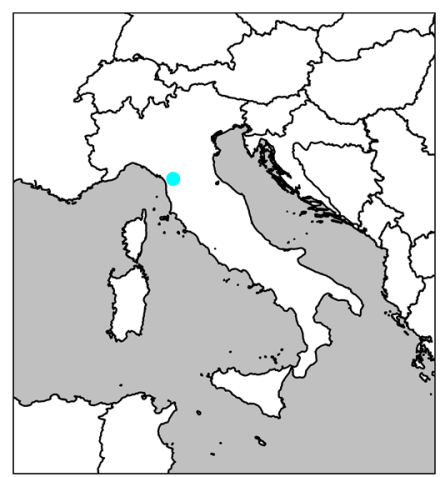

171. Atractosoma confine Berlese, 1895

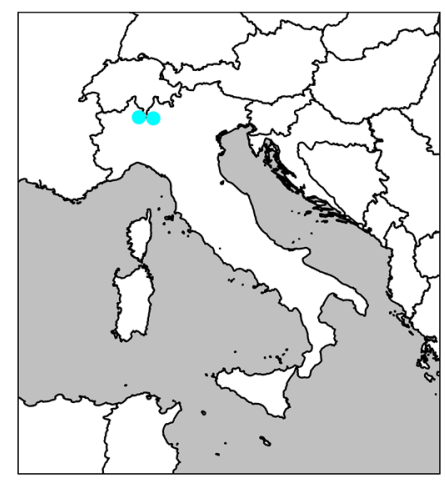

174. Atractosoma gibberosum Verhoeff, 1900

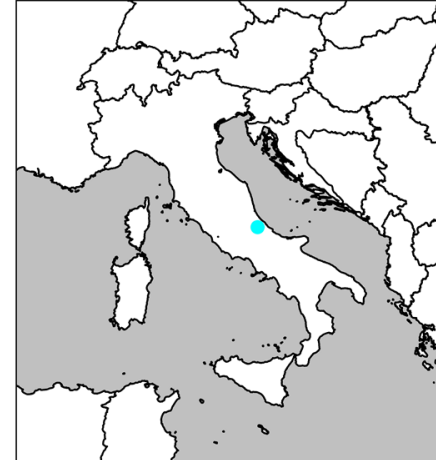

169. Atractosoma cavannae Silvestri, 1898

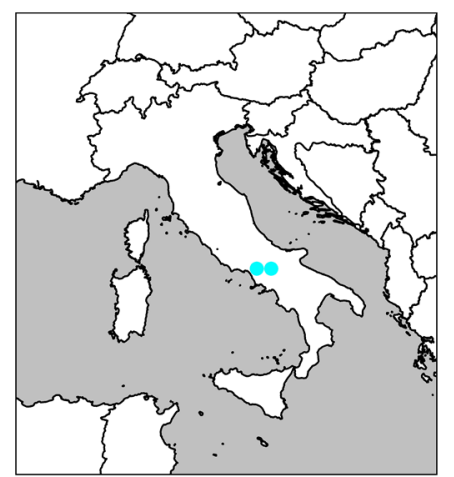

172. Atractosoma divaricatum Strasser, 1981

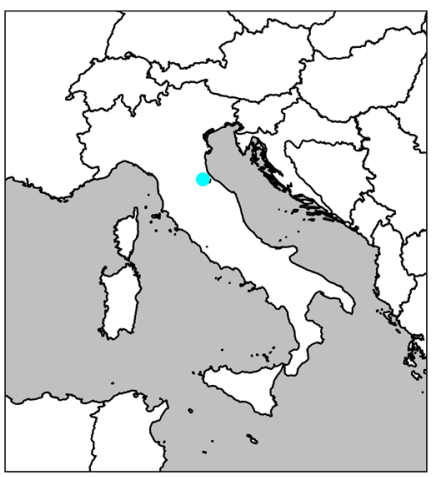

175. Atractosoma marinense Verhoeff, 1932 


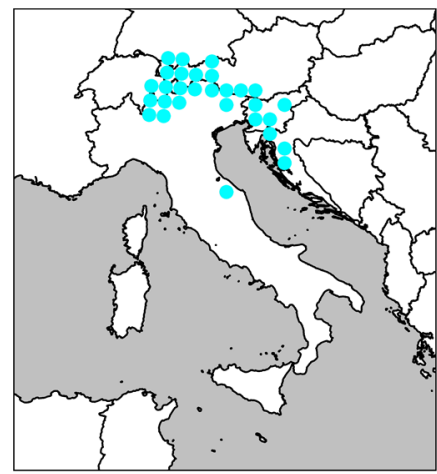

176. Atractosoma meridionale Fanzago, 1876

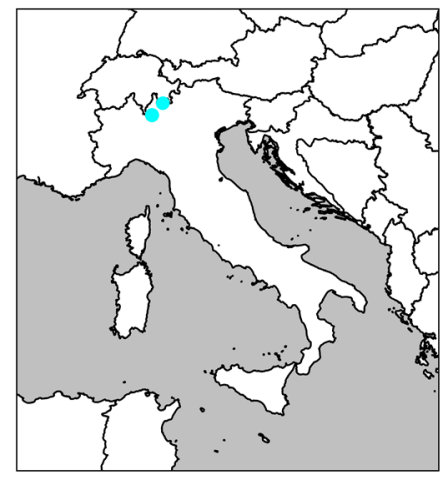

179. Atractosoma tellinense Brölemann, 1892

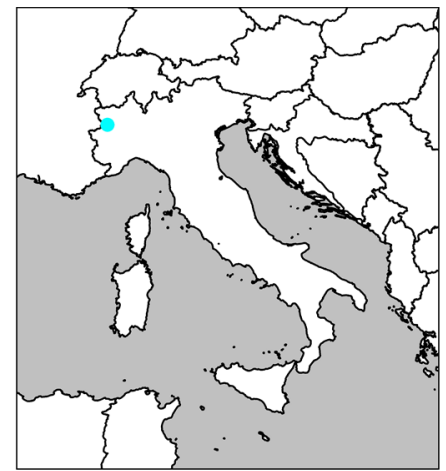

182. Autaretia osellai Strasser, 1978

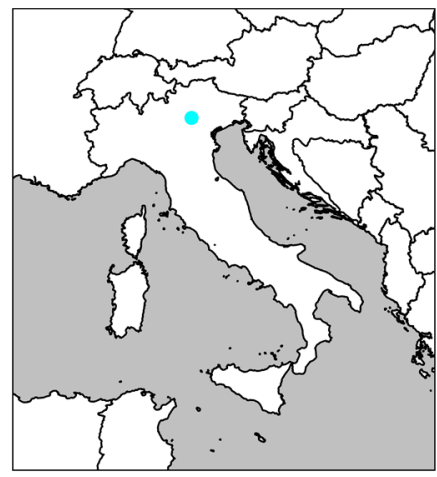

177. Atractosoma paolettii (Strasser, 1977)

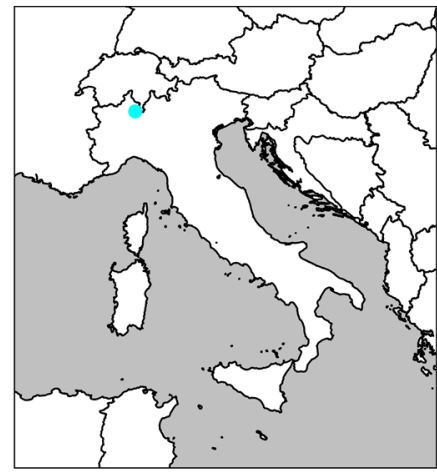

180. Atractosoma troglobium Manfredi, 1930

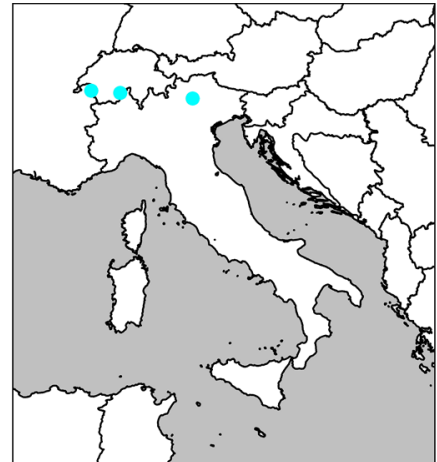

183. Basigona athesina (Fedrizzi, 1877)

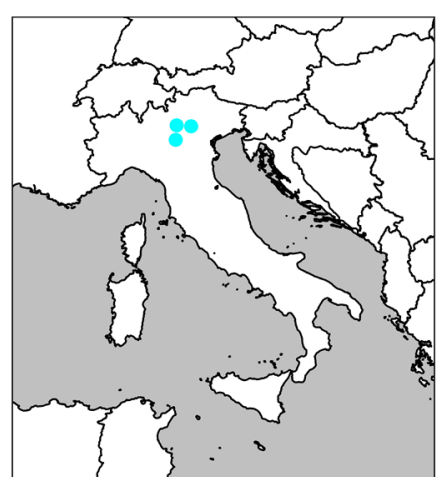

178. Atractosoma ruffoi Manfredi, 1940

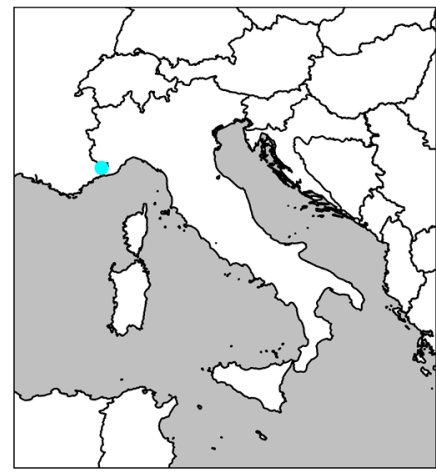

181. Autaretia aliciae Geoffroy \& Mauriès, 2017

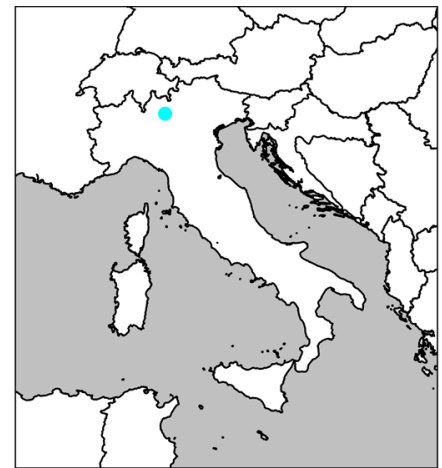

184. Bergamosoma bergomatium (Verhoeff, 1925) 


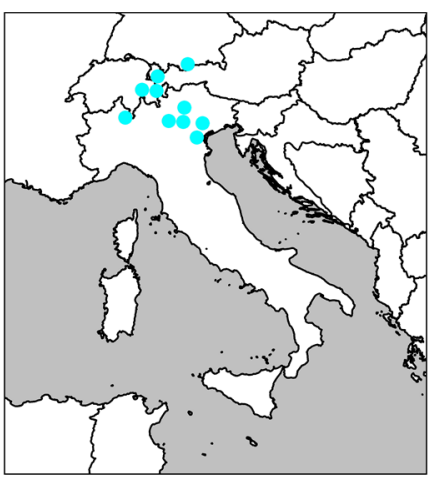

185. Bergamosoma canestrinii (Fedrizzi, 1878)

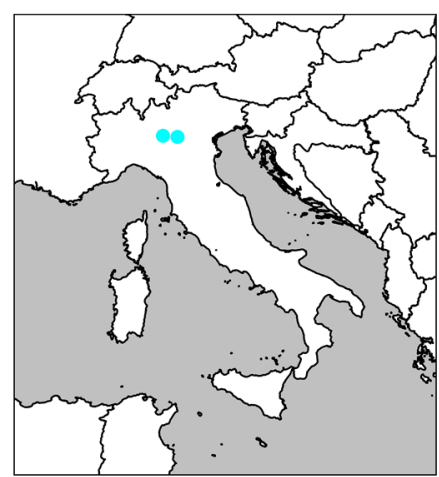

186. Bergamosoma grottoloi (Strasser, 1973)

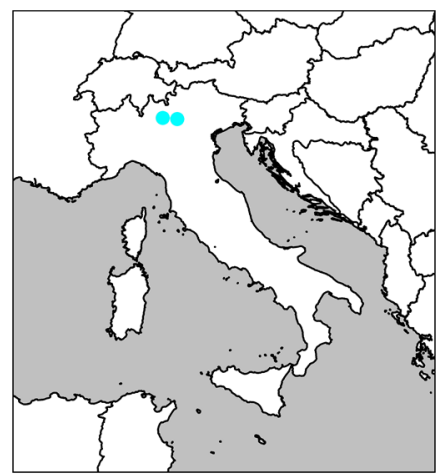

189. Bergamosoma sevini (Verhoeff, 1931)

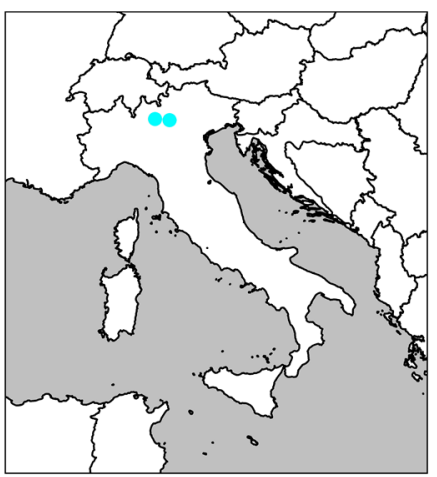

187. Bergamosoma hessei (Verhoeff, 1931)

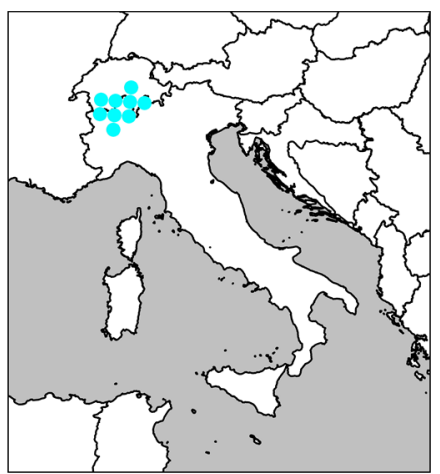

190. Bomogona helvetica (Verhoeff, 1894)

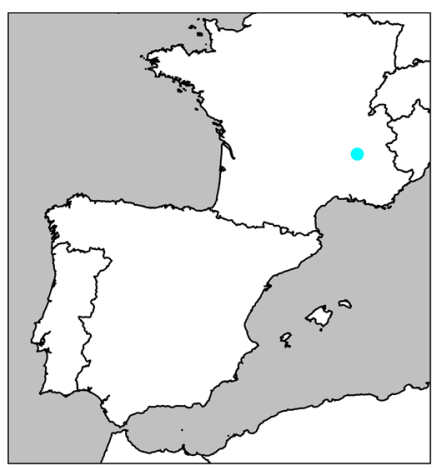

193. Broelemanneuma furcatum Ribaut, 1913 


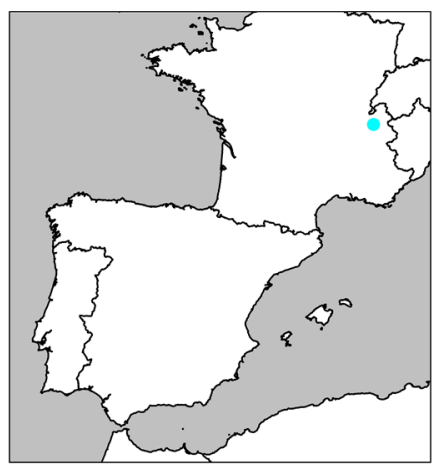

194. Broelemanneuma gayi Demange, 1968

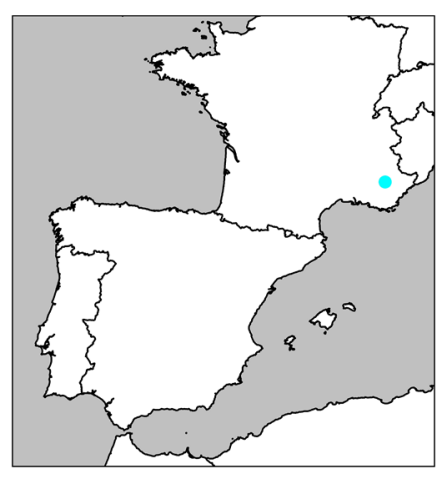

197. Broelemanneuma pectiniger (Brölemann, 1902)

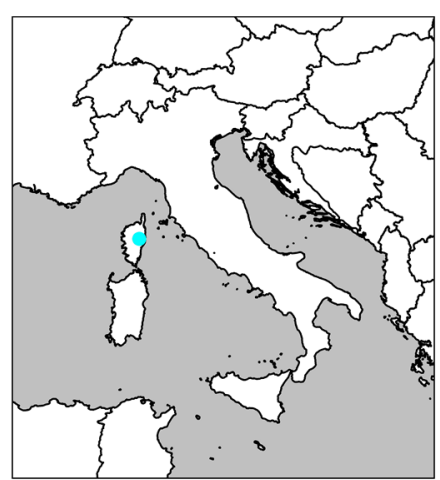

200. Corsicosoma legeri

(Brölemann, 1903)

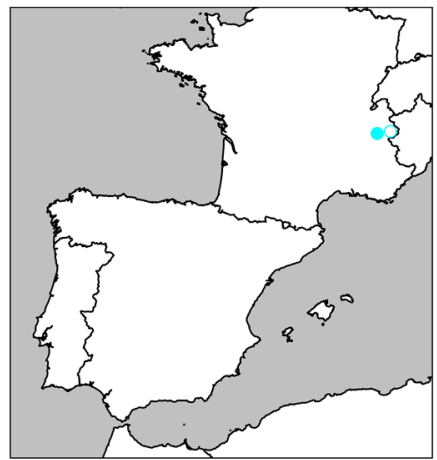

195. Broelemanneuma gineti Ribaut, 1954

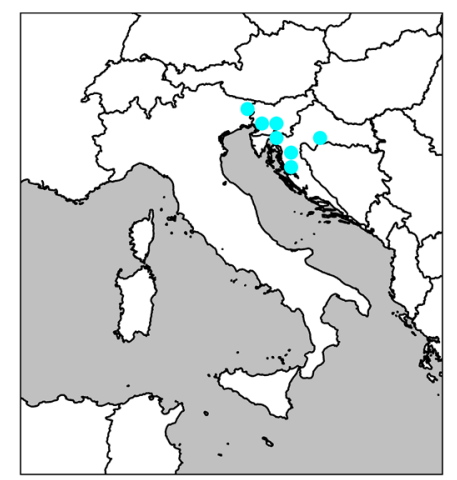

198. Carniosoma verhoeffi (Attems, 1927)

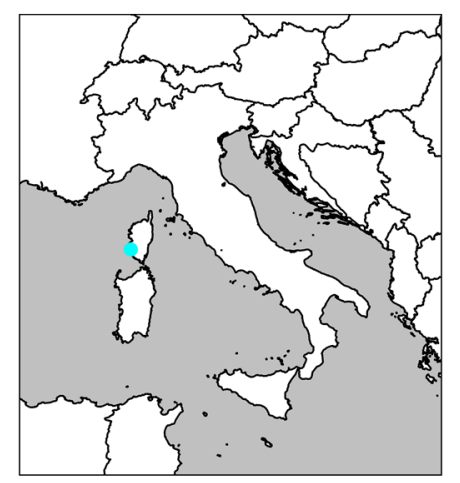

201. Craspedosoma blaniulides Latzel, 1900

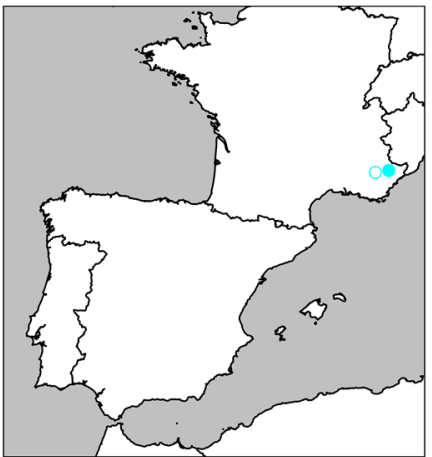

196. Broelemanneuma palmatum (Brölemann, 1902)

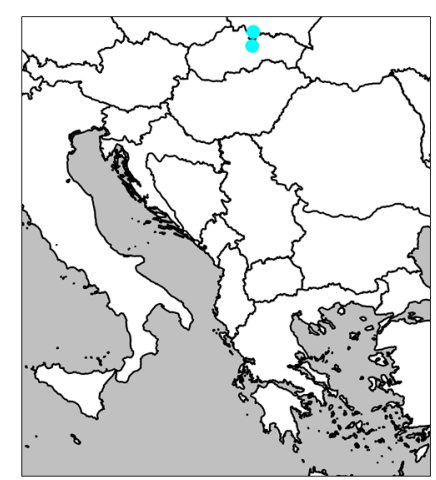

199. Chelogona carpathicum (Latzel, 1882)

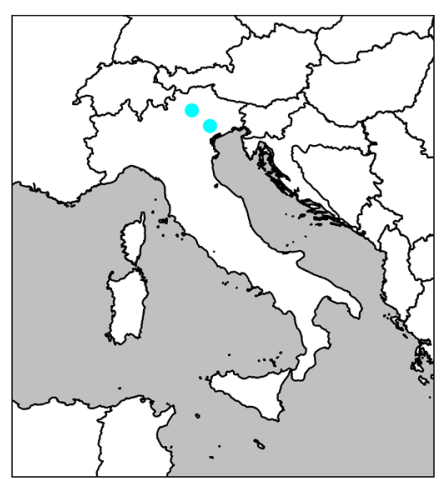

202. Craspedosoma brentanum Verhoeff, 1926 


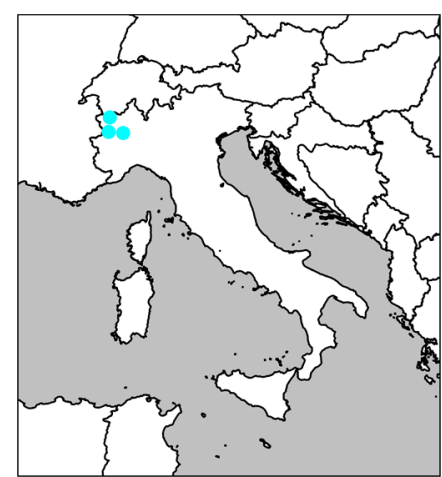

203. Craspedosoma doranum Verhoeff, 1932

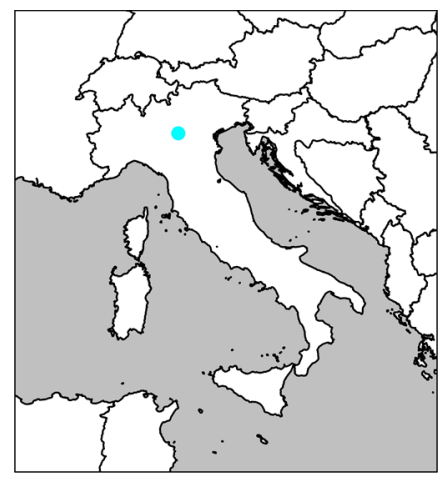

204. Craspedosoma fontanellum Attems, 1927

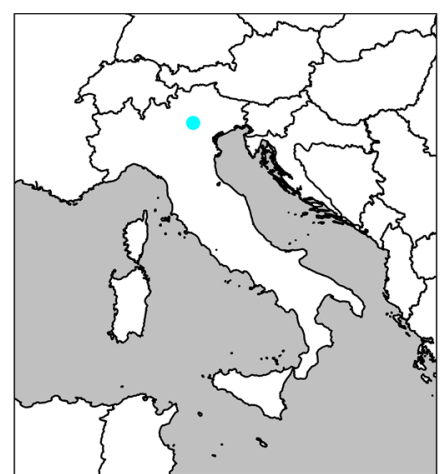

206. Craspedosoma italicum Silvestri, 1903

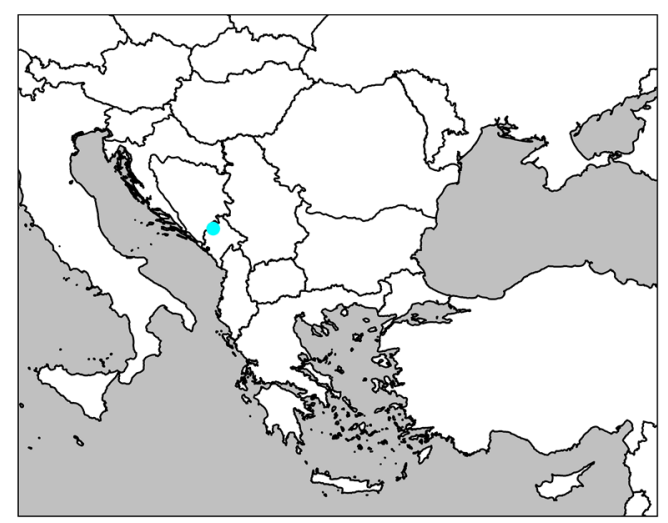

208. Craspedosoma montenigrinum Mršić, 1987

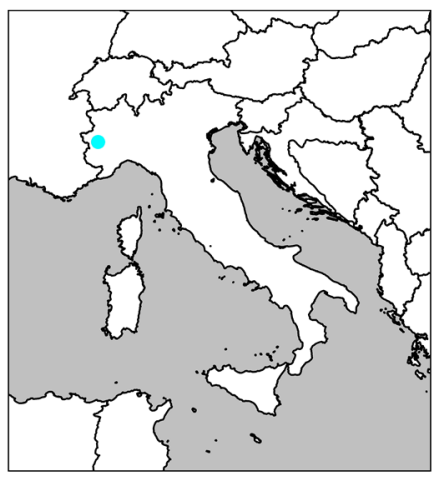

205. Craspedosoma furculigerun Verhoeff, 1936

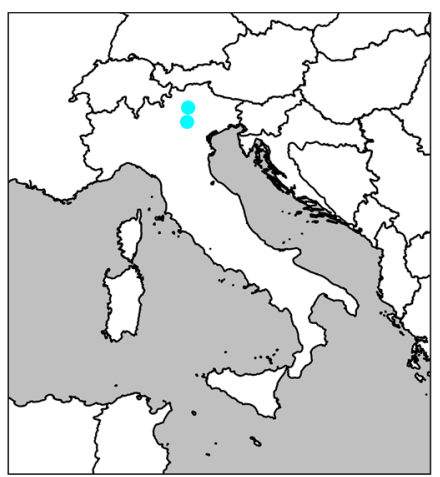

207. Craspedosoma levicanum Fedrizzi, 1876

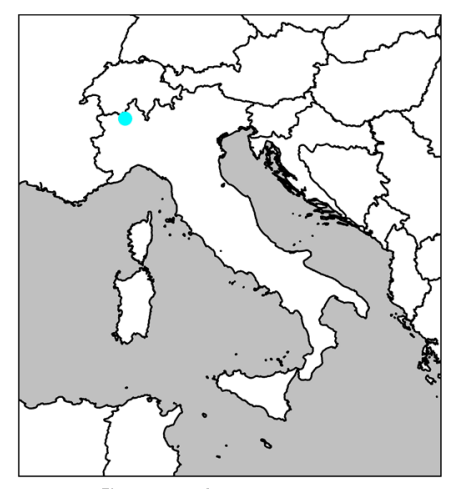

209. Craspedosoma oropense Verhoeff, 1936 


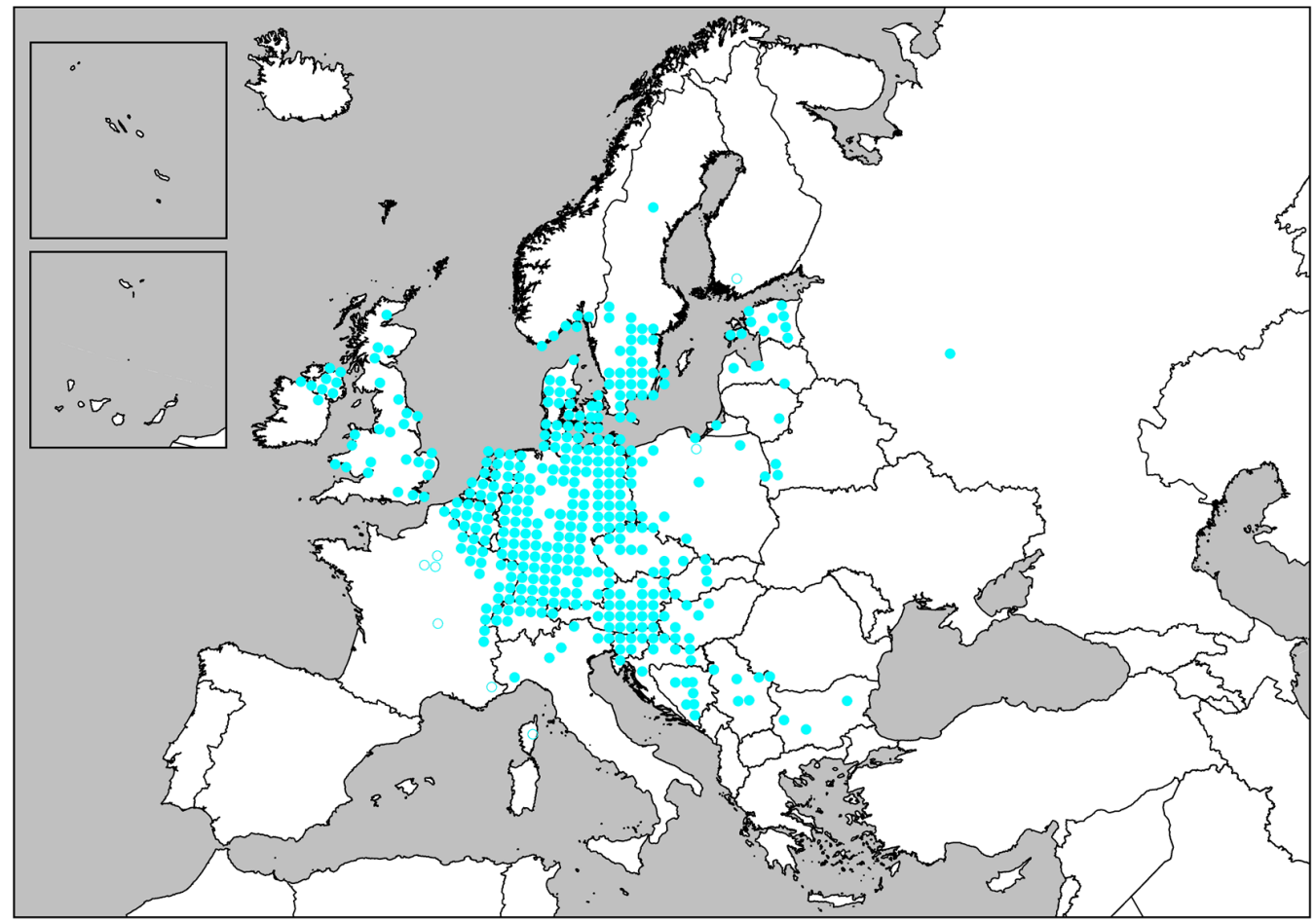

210. Craspedosoma raulinsii Leach, 1814

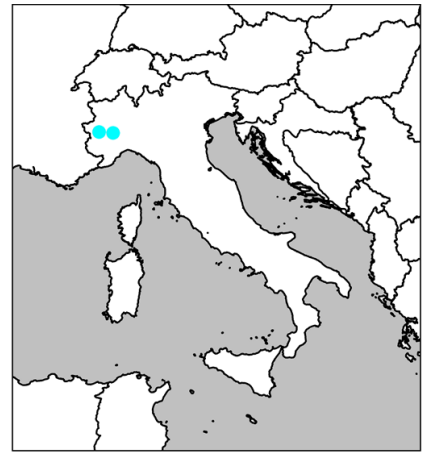

211. Craspedosoma ruborum Verhoeff, 1930

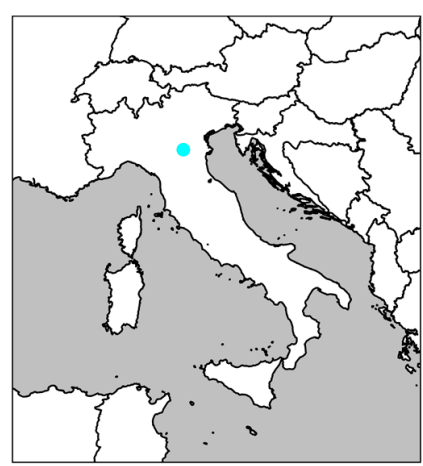

214. Craspedosoma trilobum Silvestri, 1903

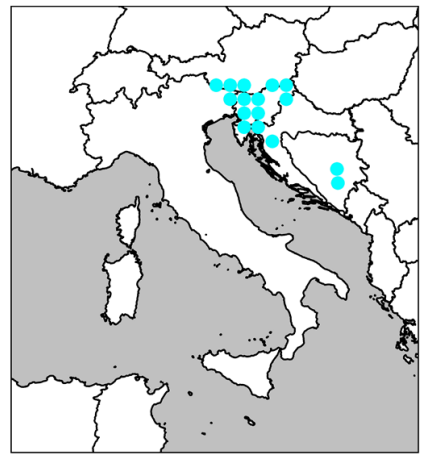

212. Craspedosoma slavum Attems, 1929

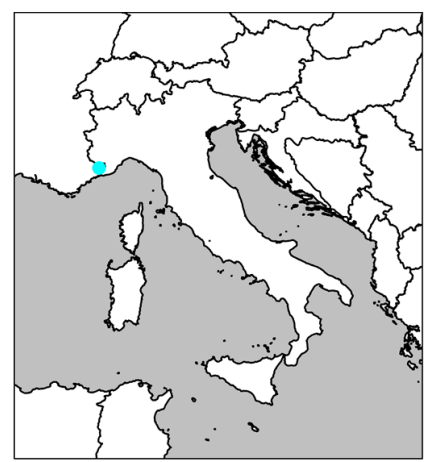

215. Crossosoma brolemanni Strasser, 1975

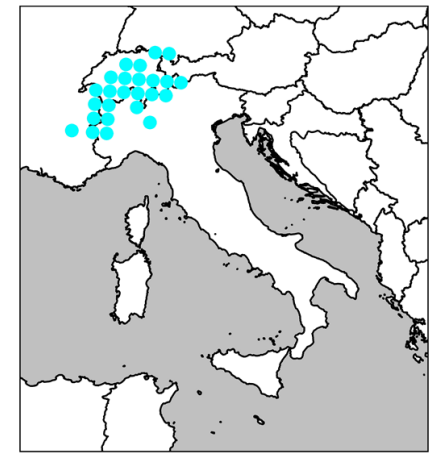

213. Craspedosoma taurinorum Silvestri, 1898

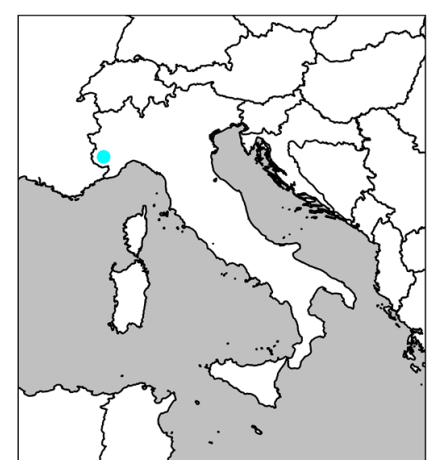

216. Crossosoma casalei Strasser, 1979 


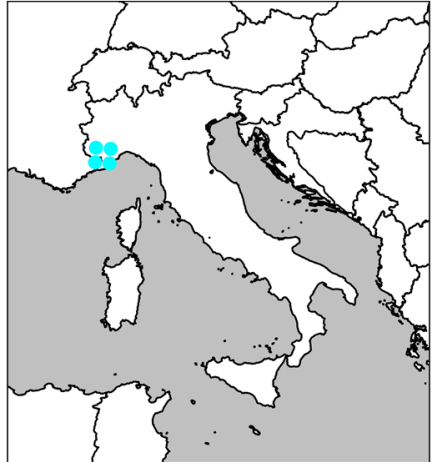

217. Crossosoma cavernicola (Manfredi, 1951)

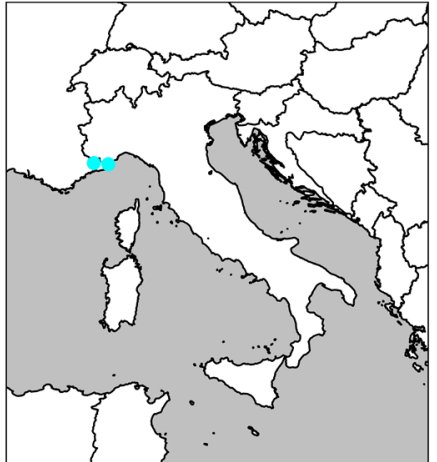

218. Crossosoma falciferum Strasser, 1975

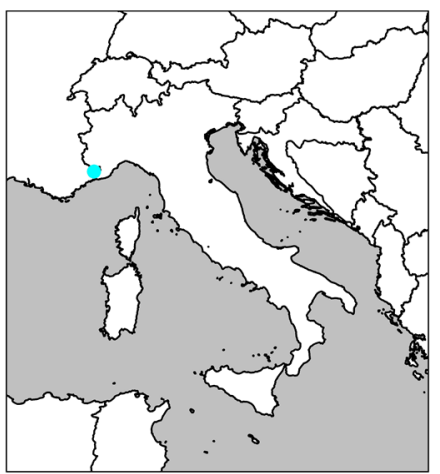

221. Crossosoma parvum Strasser, 1979

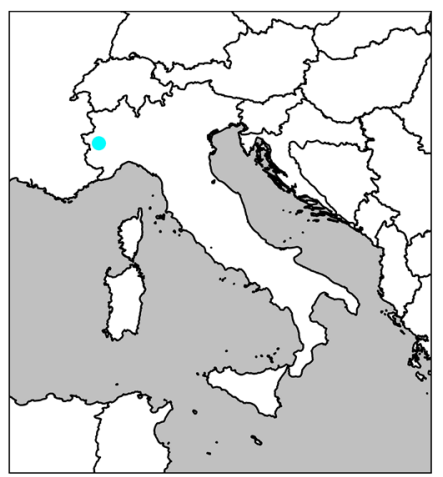

224. Crossosoma semipes (Strasser, 1958)

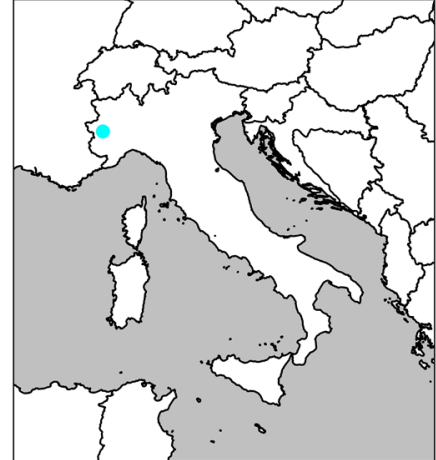

219. Crossosoma fossum Strasser, 1979

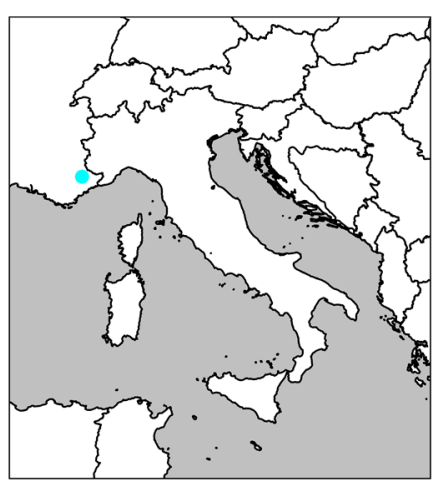

222. Crossosoma peyerimhoff (Brölemann, 1902)

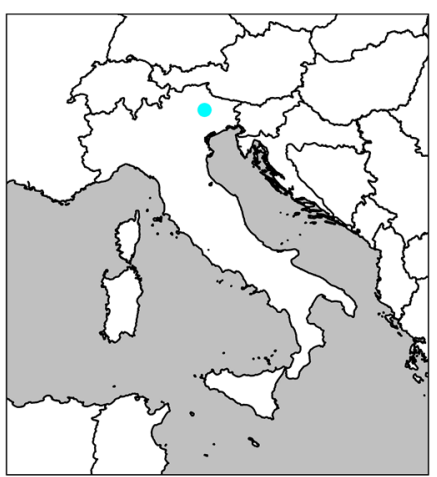

225. Dactylophorosoma albocarinatum

Manfredi, 1940 


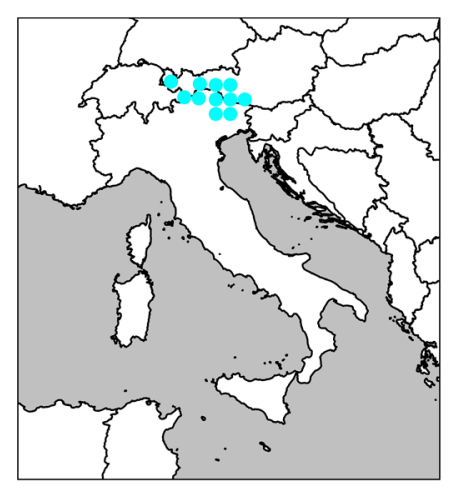

226. Dactylophorosoma nivisatelles Verhoeff, 1900

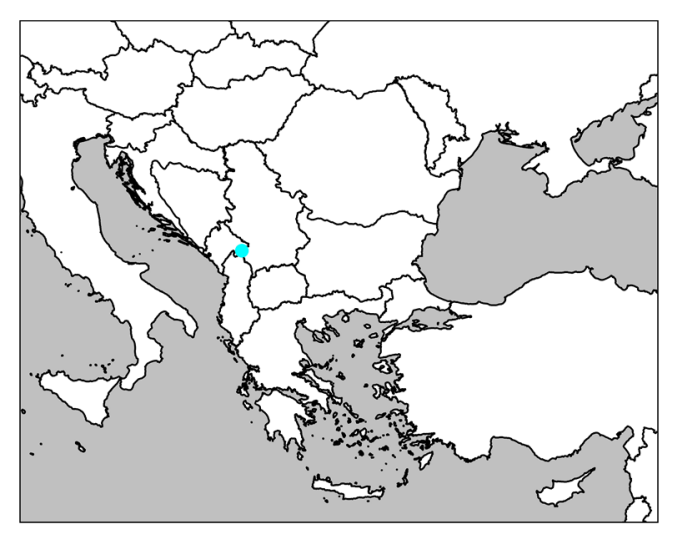

228. Dyocerasoma drimicum Mršić, 1985

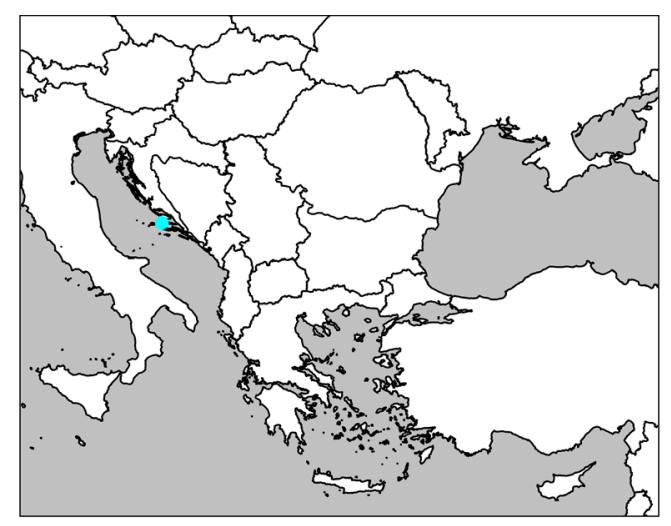

230. Dyocerasoma insulanum Attems, 1951

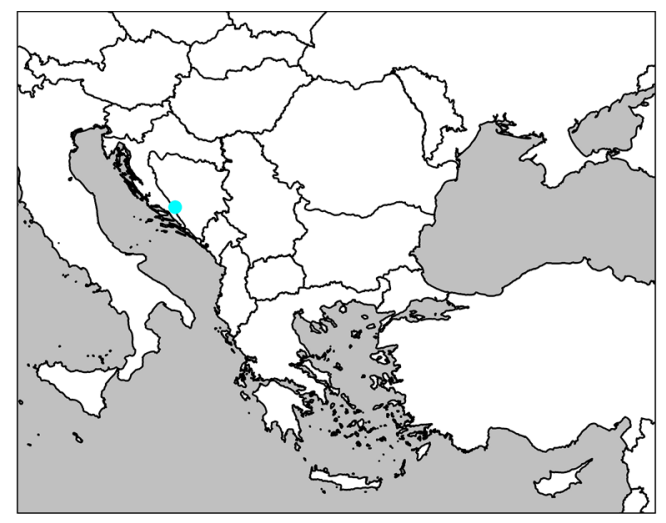

227. Dyocerasoma biokovense Mršić, 1986

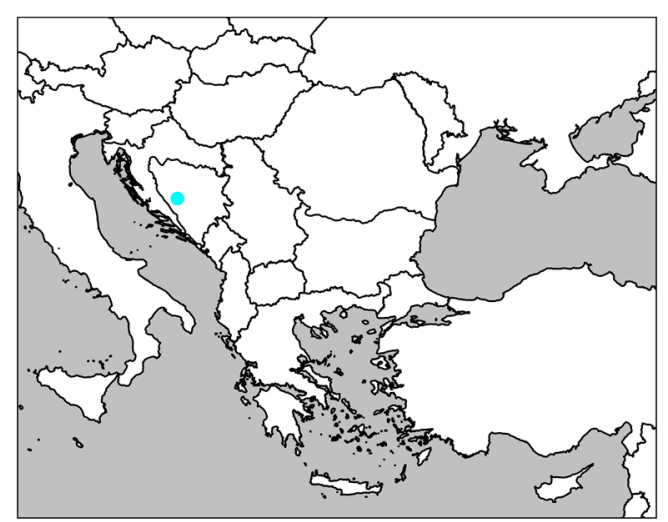

229. Dyocerasoma furcilliferum (Verhoeff, 1897)

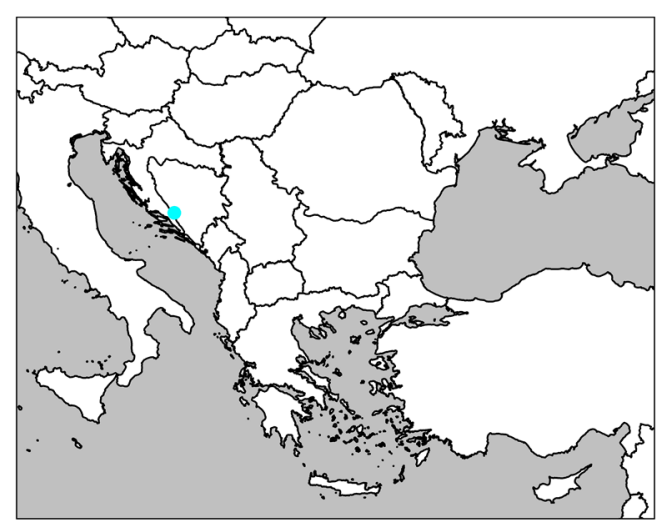

231. Dyocerasoma intermedium Makarov, Lučić, Mitić \& Rađa, 2003 


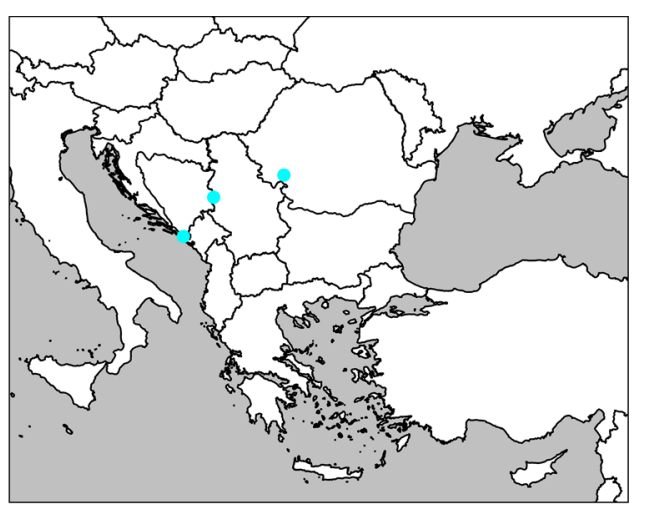

232. Dyocerasoma lignivorum (Verhoeff, 1899)

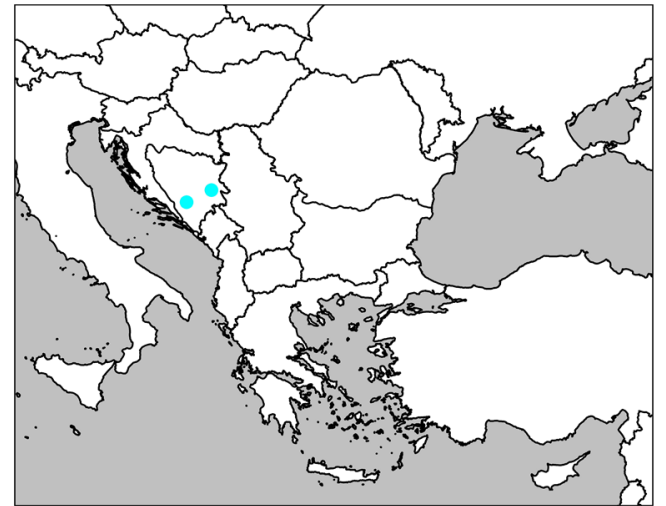

233. Dyocerasoma narentanum (Verhoeff, 1901)

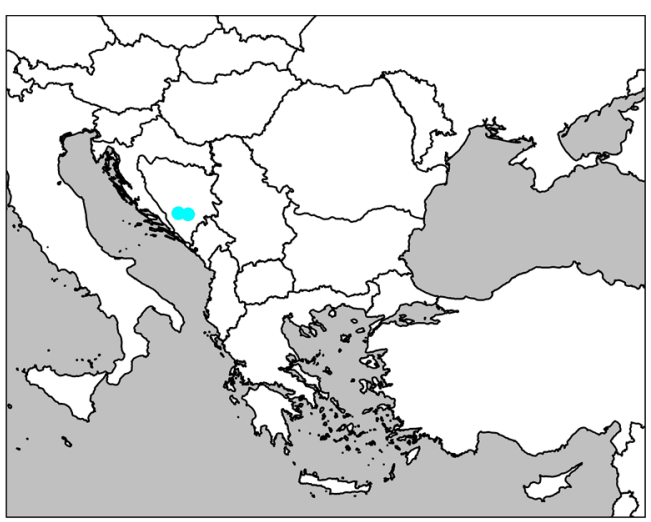

234. Dyocerasoma nivisatelles (Verhoeff, 1897)

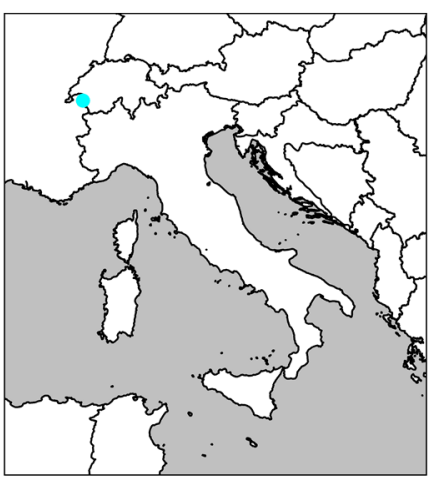

235. Helvetiosoma blanci

(Faës, 1902)

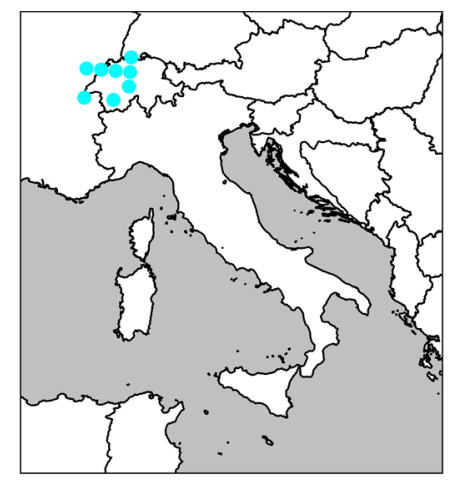

236. Helvetiosoma helveticum (Verhoeff, 1900)

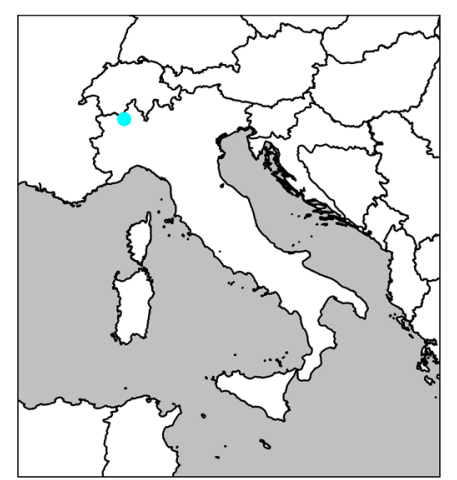

237. Helvetiosoma montemorense (Faës, 1905)

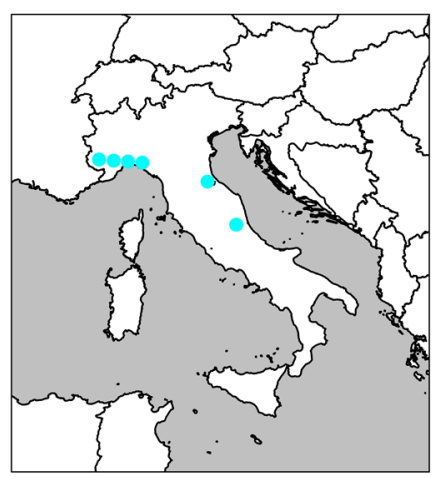

238. Iulogona apenninorum (Verhoeff, 1913) 


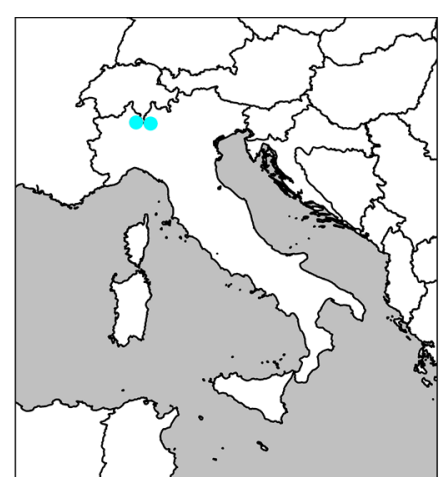

239. Iulogona hamuligera (Verhoeff, 1913)

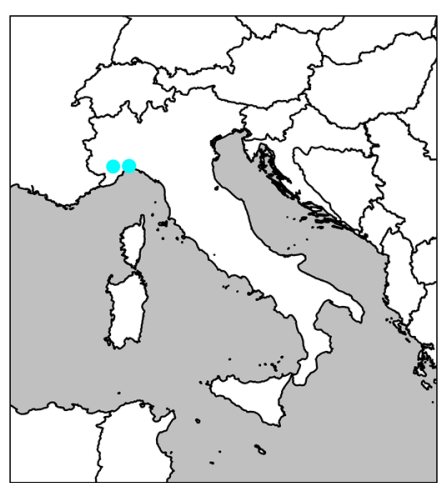

240. Iulogona ligurina (Verhoeff, 1910)

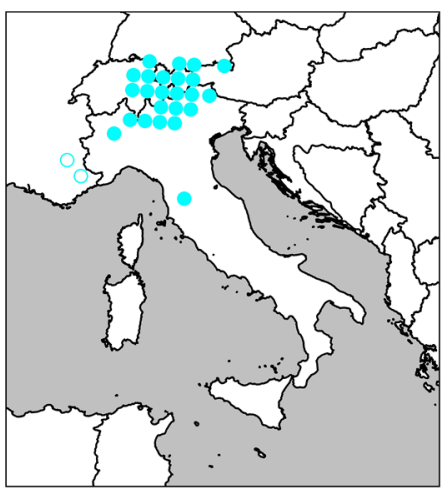

241. Iulogona tirolensis (Verhoeff, 1894)

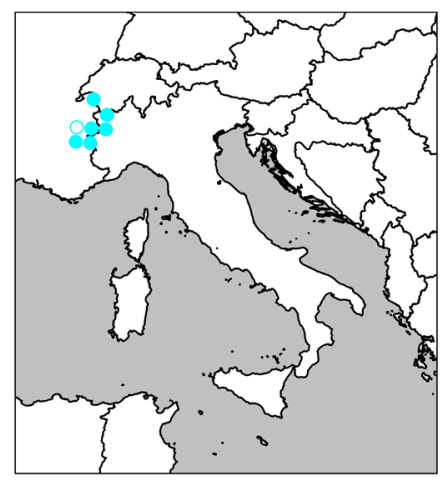

242. Janetschekella valesiaca (Faës, 1902)

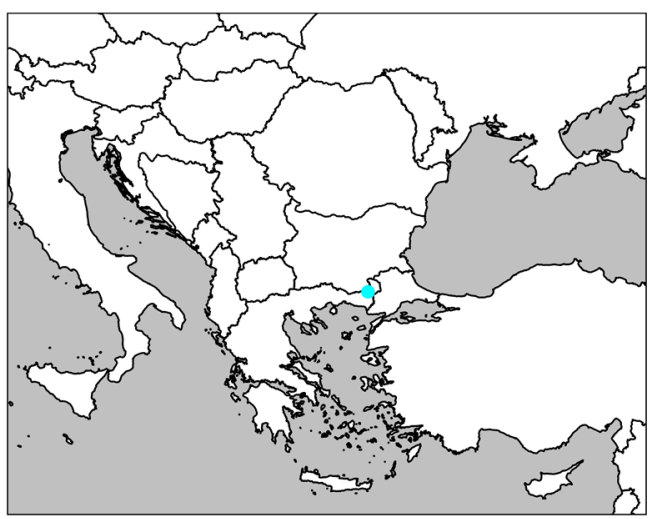

243. Kelempekia martensi Strasser, 1974

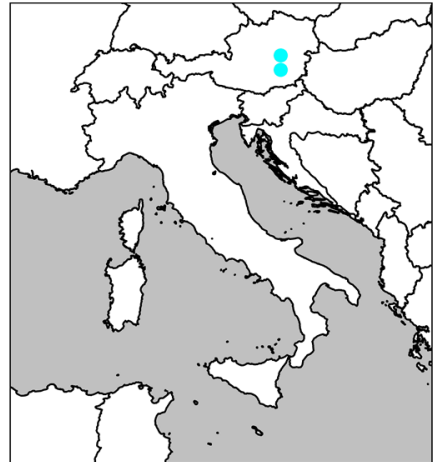

244. Listrocheiritium bohemicum (Rosický, 1876)

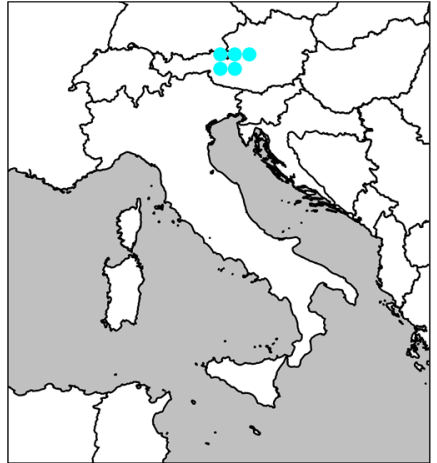

245. Listrocheiritium cervinum Verhoeff, 1925

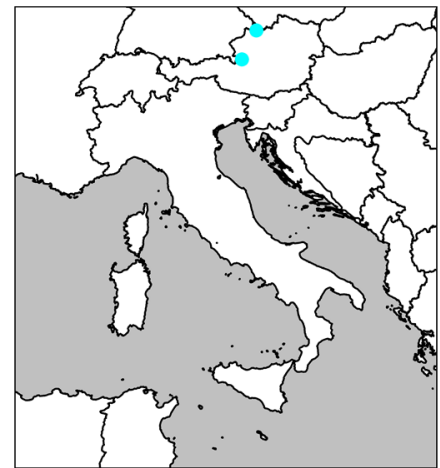

246. Listrocheiritium noricum Verhoeff, 1913 


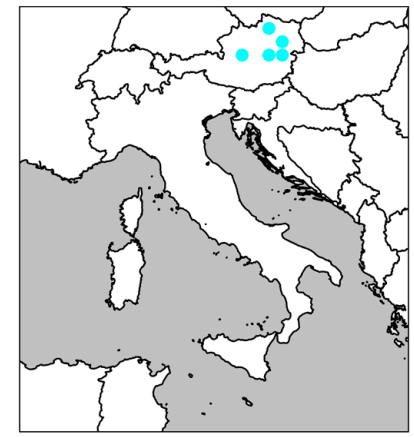

247. Listrocheiritium nubium

Verhoeff, 1915

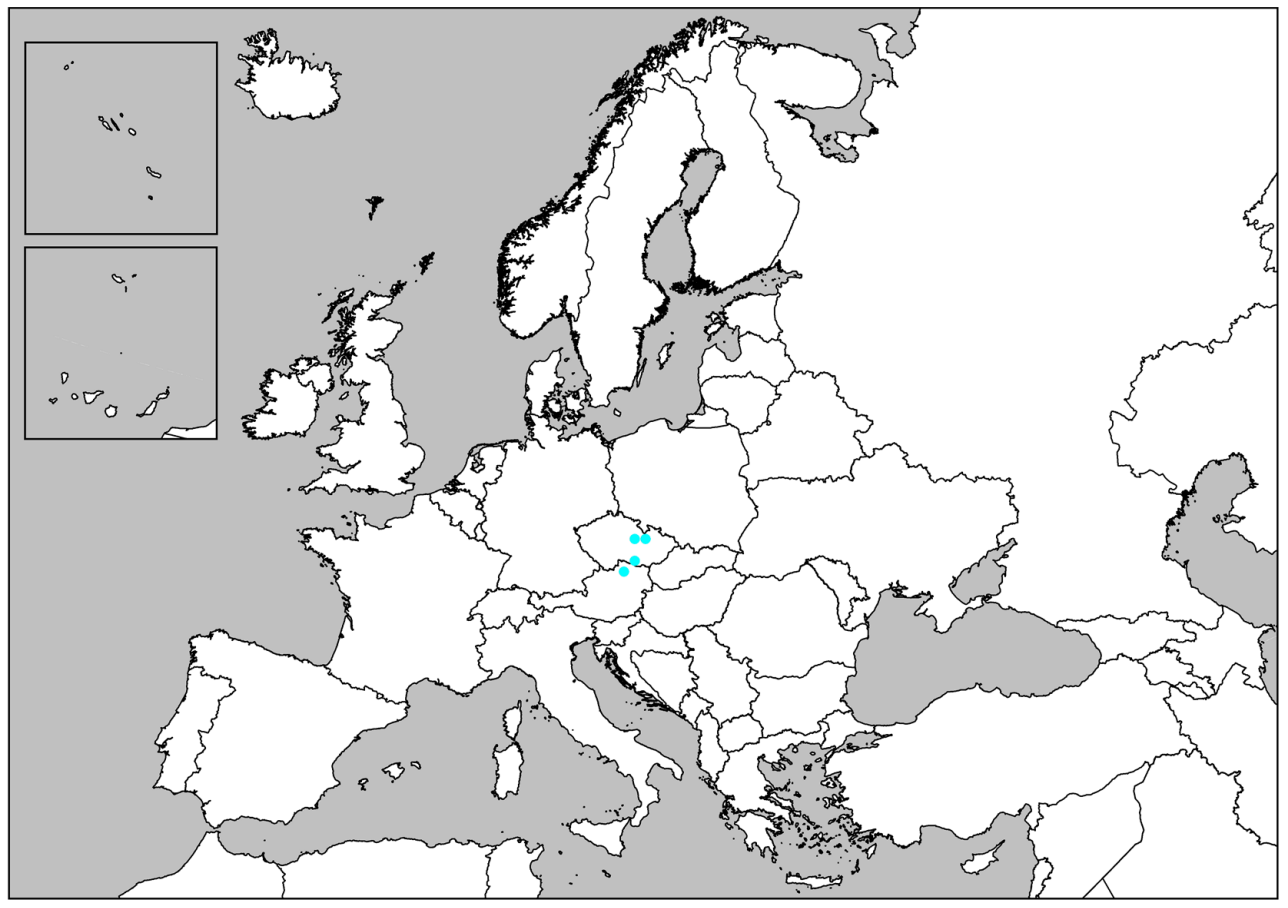

248. Listrocheiritium septentrionale Gulička, 1965

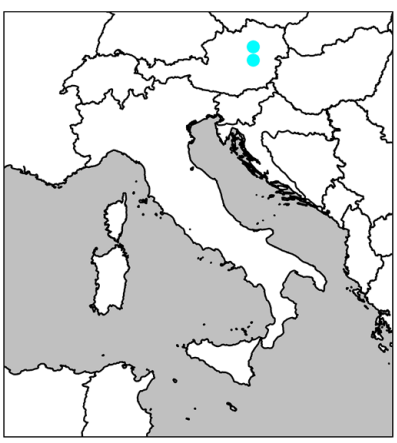

249. Listrocheiritium styricum Verhoeff, 1915

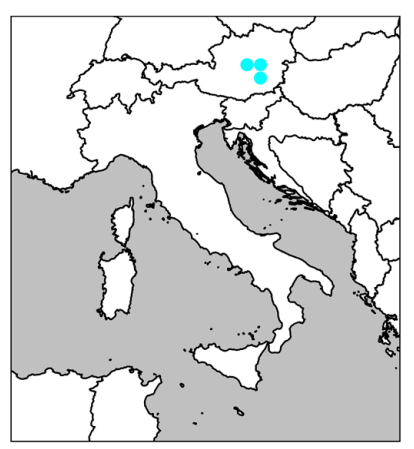

250. Listrocheiritium susurrinum Attems, 1926

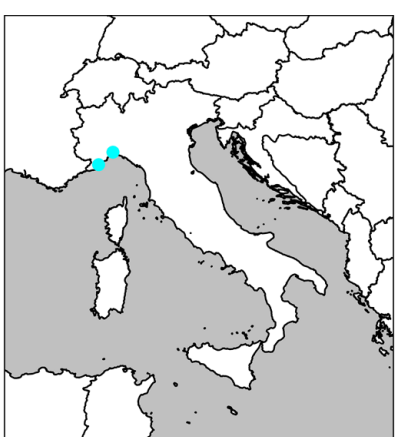

251. Litogona hyalops (Latzel, 1889) 


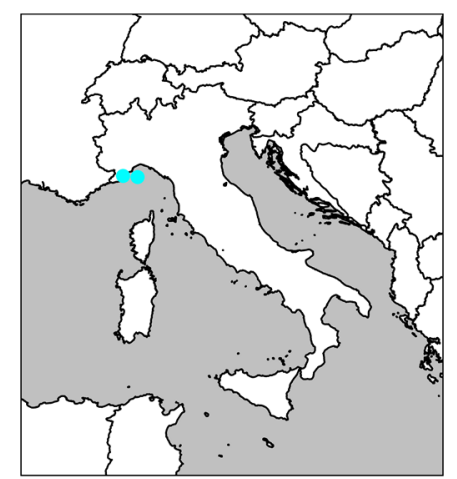

252. Litogona mirabilis

(Manfredi, 1948)

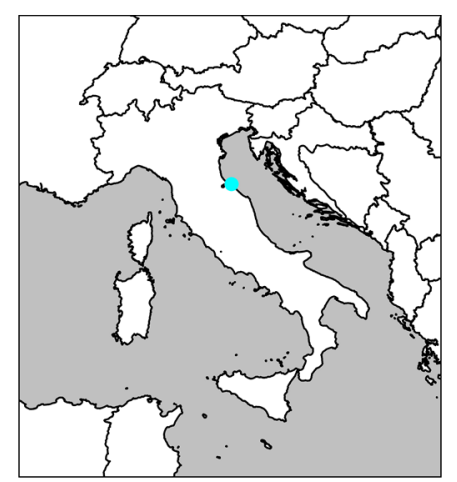

255. Manfredia concii

Manfredi, 1953

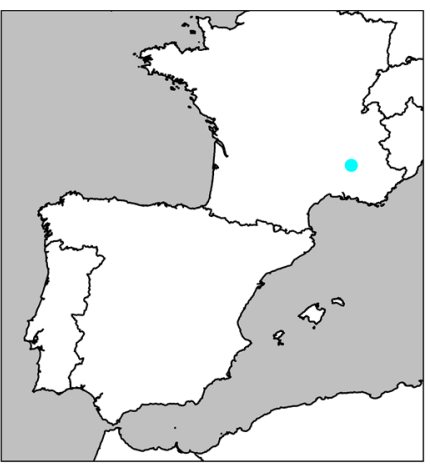

258. Nanogona balazuci

(Schubart, 1958)

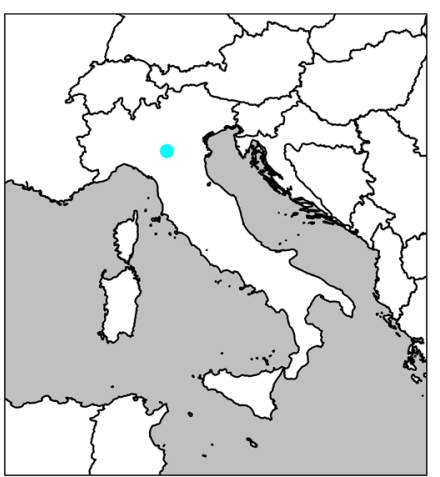

253. Manfredia aemiliana (Manfredi,1932)

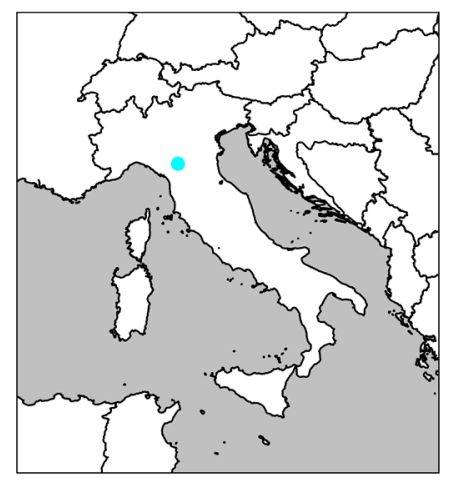

256. Manfredia guareschii Manfredi, 1950

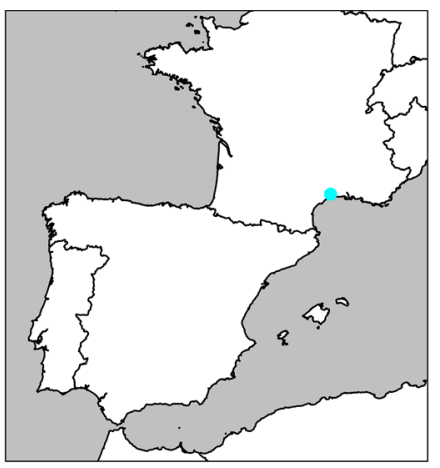

259. Nanogona cebennica (Ribaut, 1947)

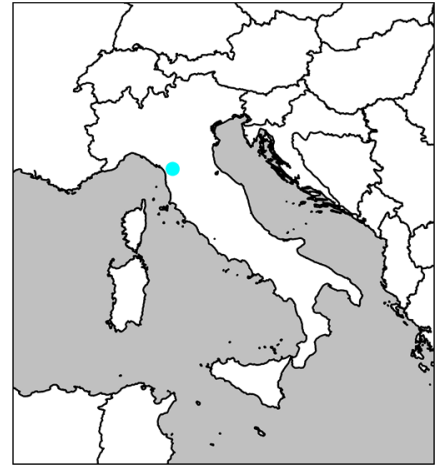

254. Manfredia apuana Strasser, 1971

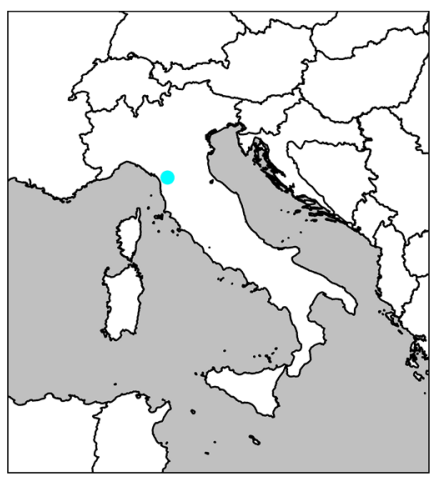

257. Manfredia lanzai

Manfredi, 1948

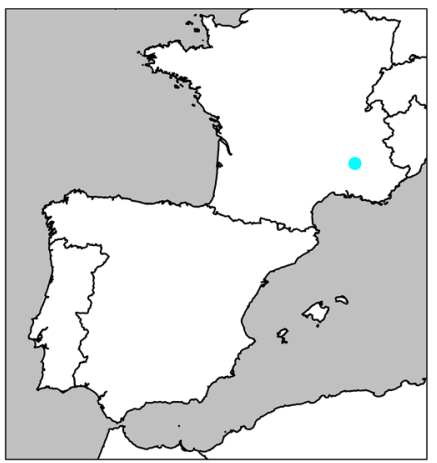

260. Nanogona dovidi

(Demange, 1966) 


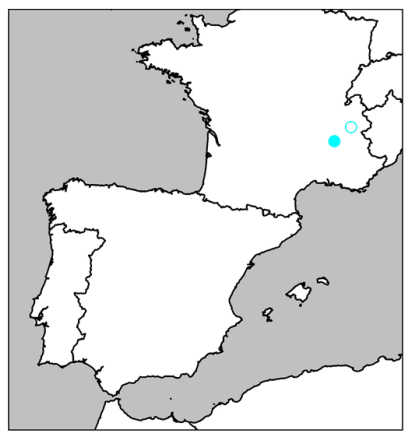

261. Nanogona digitata

(Ribaut, 1913)

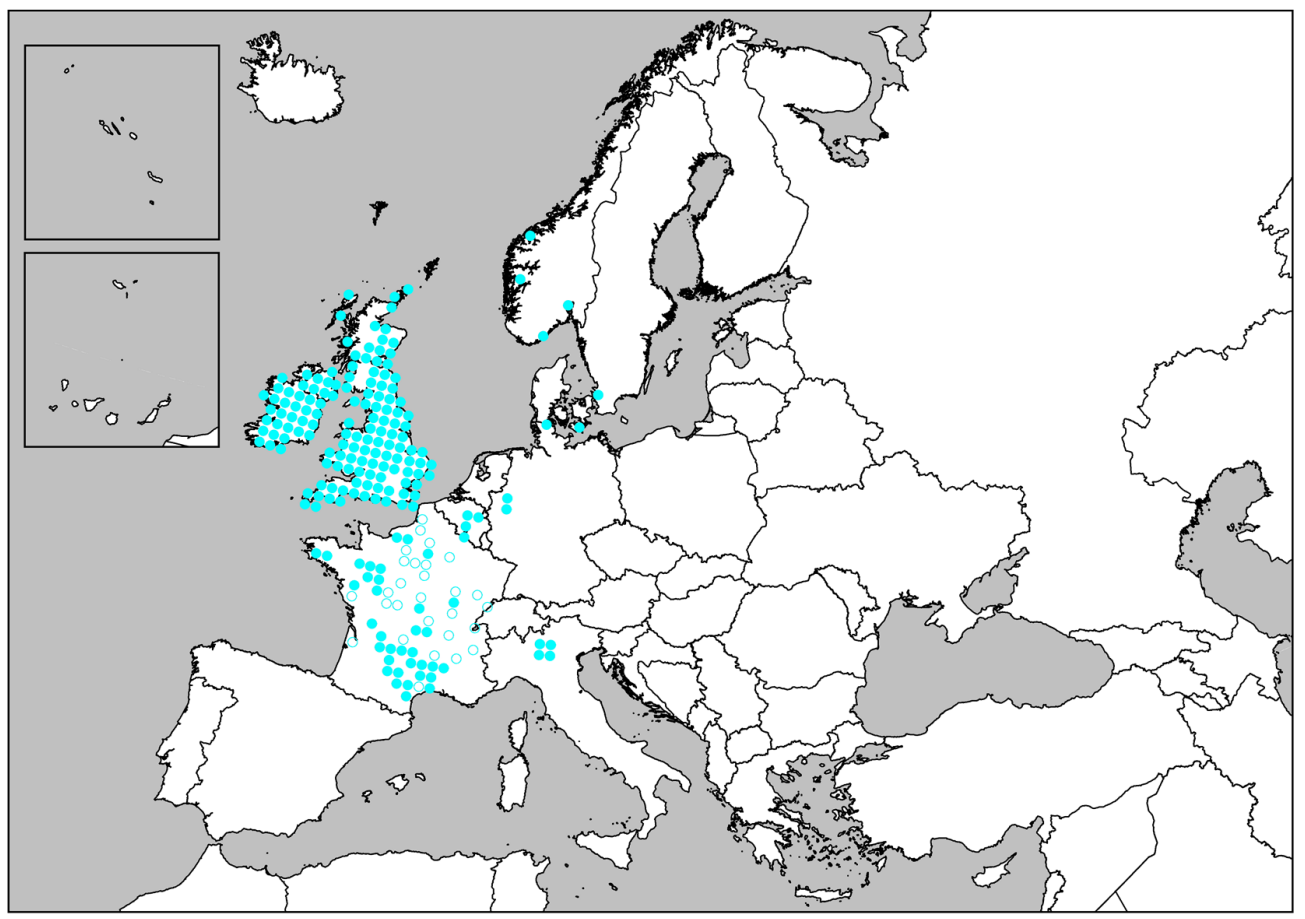

262. Nanogona polydesmoides (Leach, 1814)

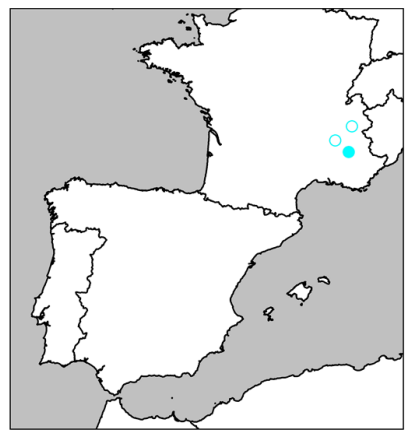

263. Nanogona uncinata (Ribaut, 1913)

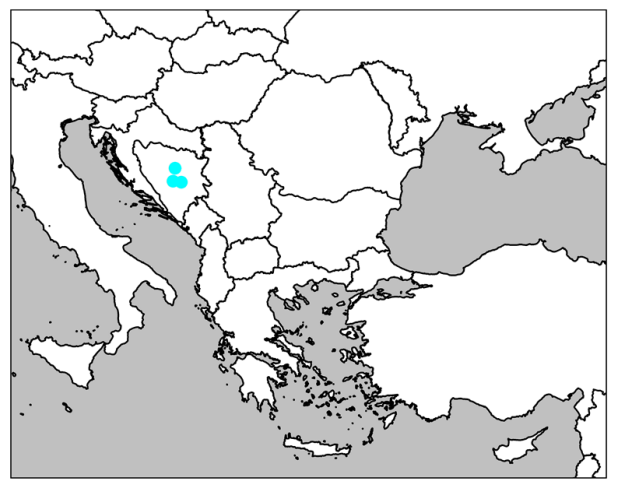

264. Ochogona apfelbecki (Verhoeff, 1897) 


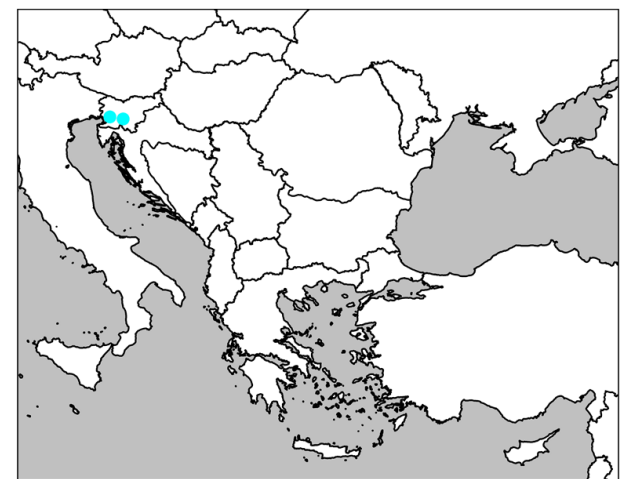

265. Ochogona attemsi (Verhoeff, 1907)

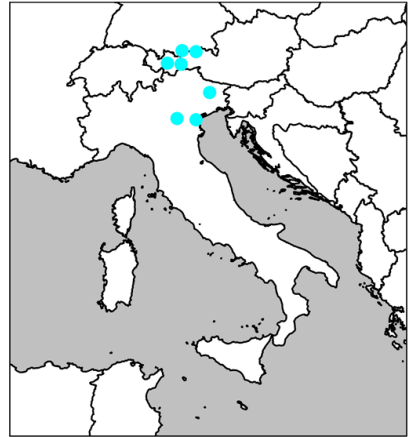

266. Ochogona brentana

(Verhoeff, 1927)

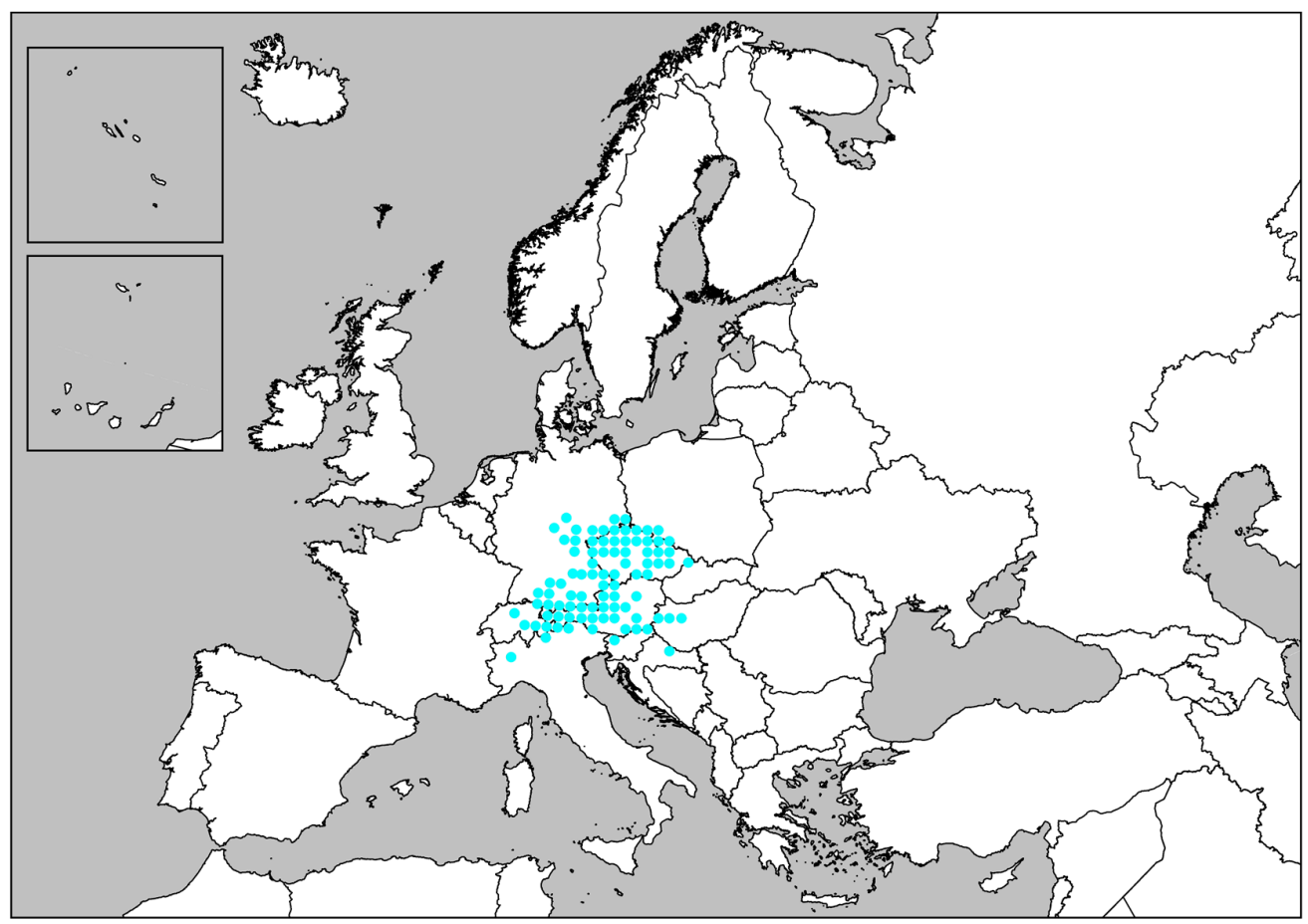

267. Ochogona caroli (Rothenbühler, 1900)

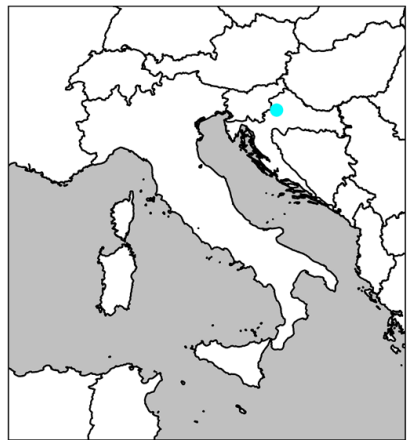

268. Ochogona cervina

(Verhoeff, 1899)

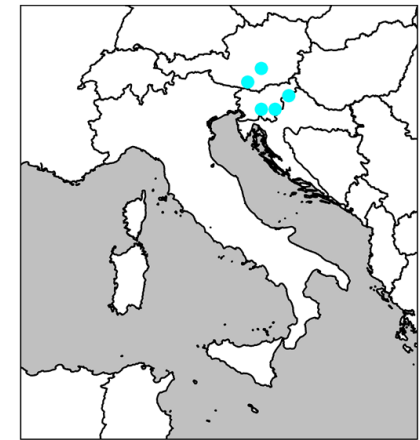

269. Ochogona condylocoxa (Attems, 1899)

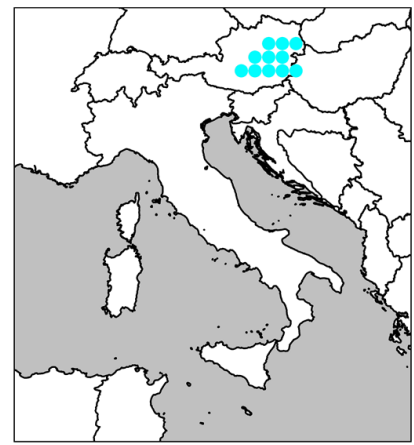

270. Ochogona elaphron (Attems, 1895) 


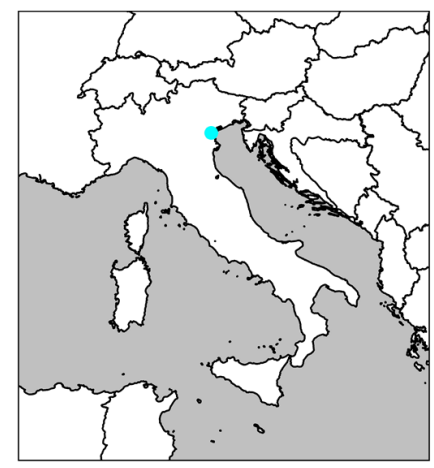

271. Ochogona enganeorum (Verhoeff, 1927)

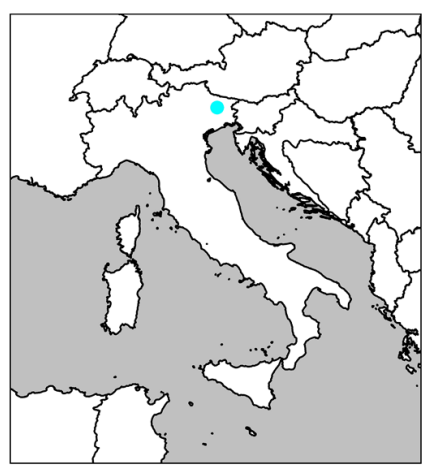

272. Ochogona friulana (Strasser, 1937)

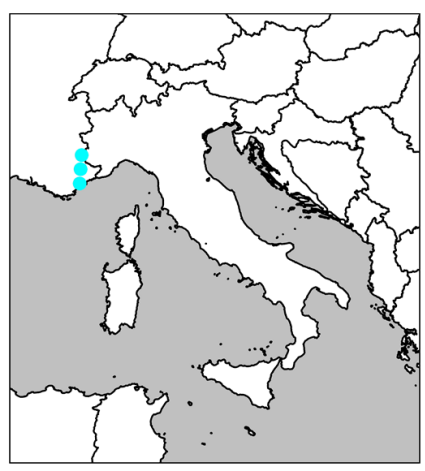

273. Ochogona gallitarum (Brölemann, 1900)

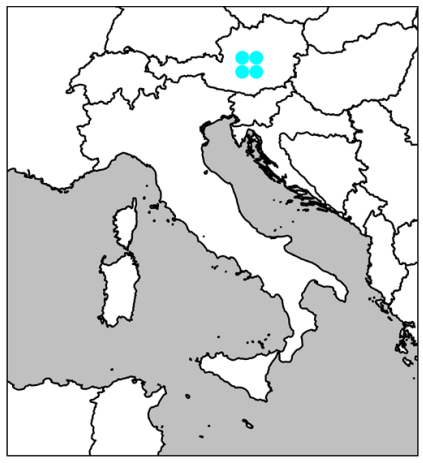

275. Ochogona holdhausi (Attems, 1926)

(Attems, 1926)

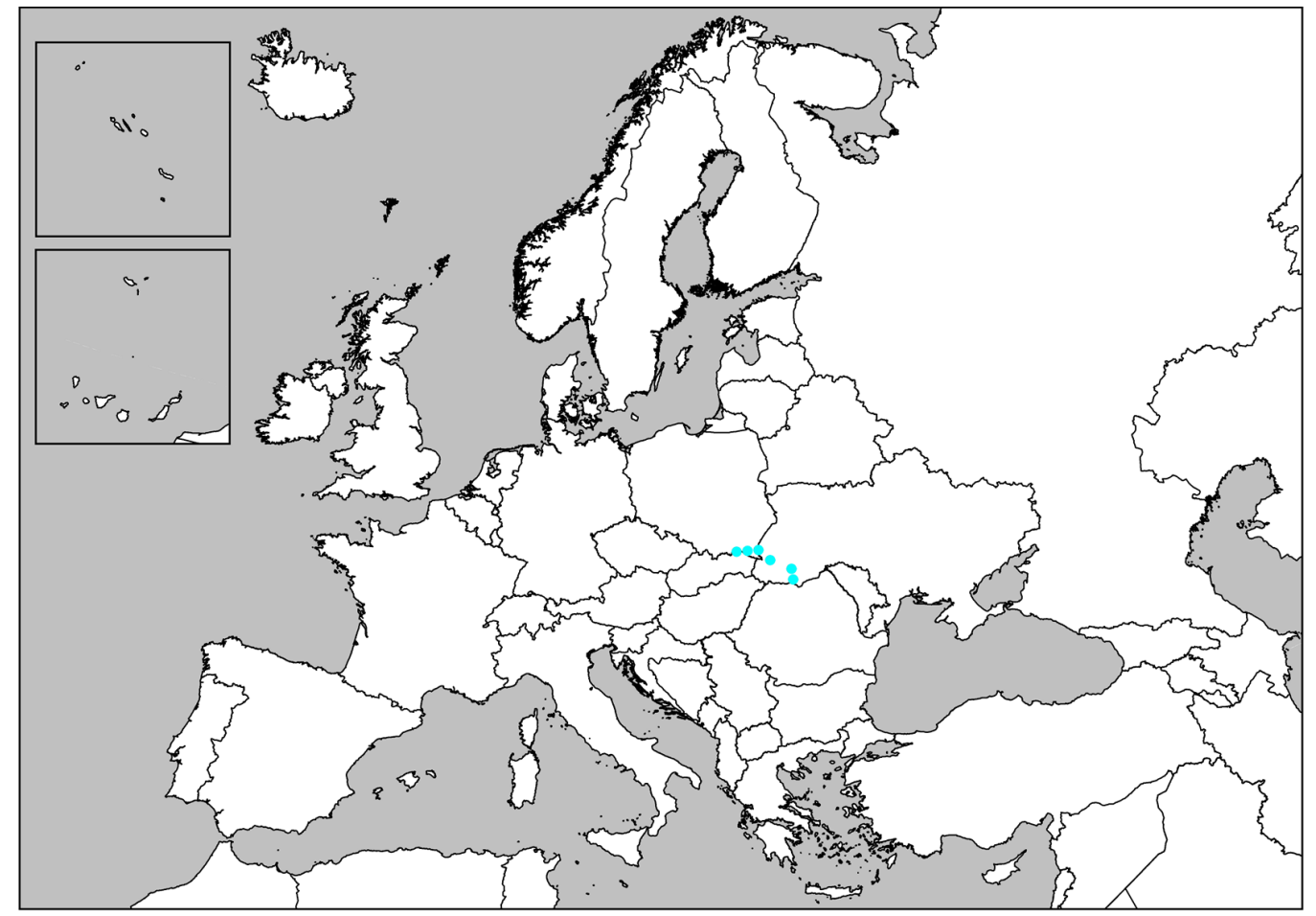

276. Ochogona jankowskii (Jawłowski, 1938) 


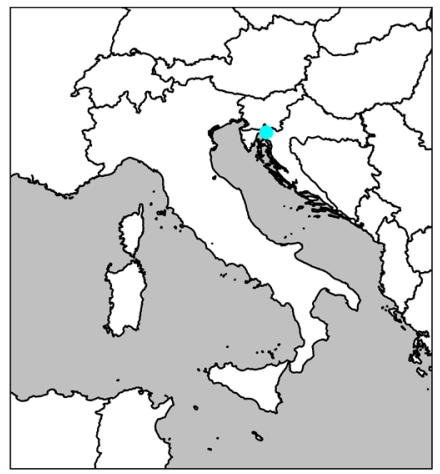

277. Ochogona latzeli

(Attems, 1927)

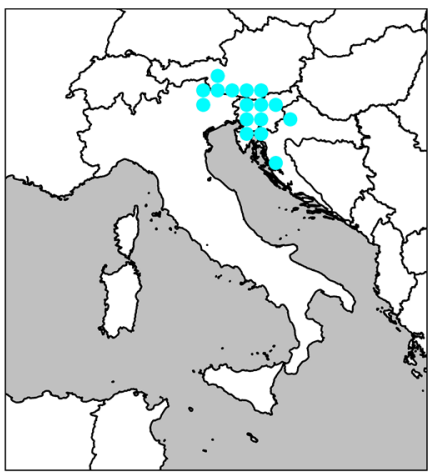

280. Ochogona pusilla

(Verhoeff, 1893)

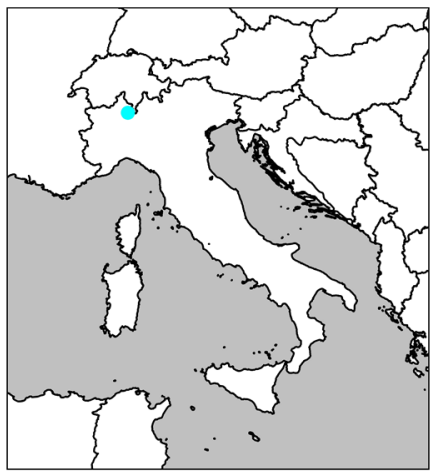

283. Oroposoma catascaphium Verhoeff, 1936

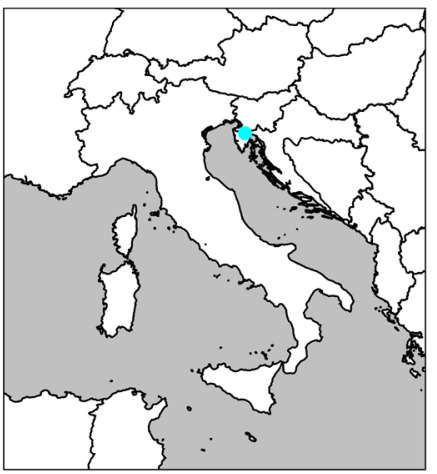

278. Ochogona manfredii (Strasser, 1942)

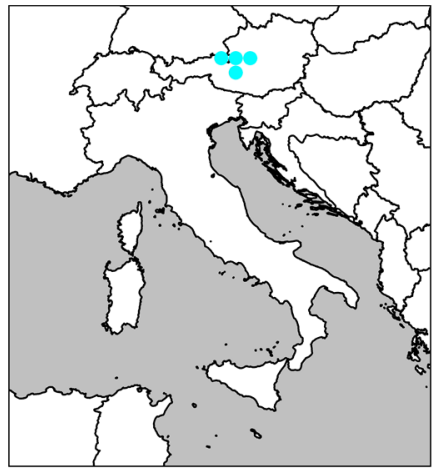

281. Ochogona regalis

(Verhoeff, 1913)

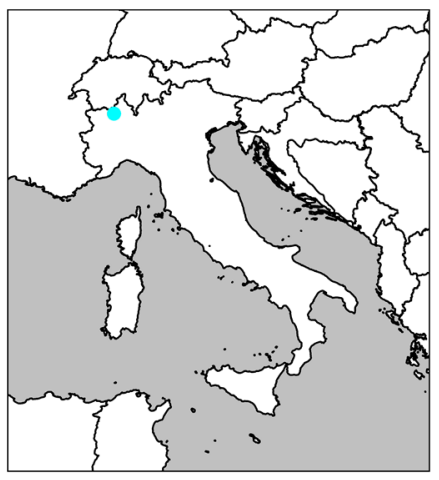

284. Oroposoma emiliae

Manfredi, 1953

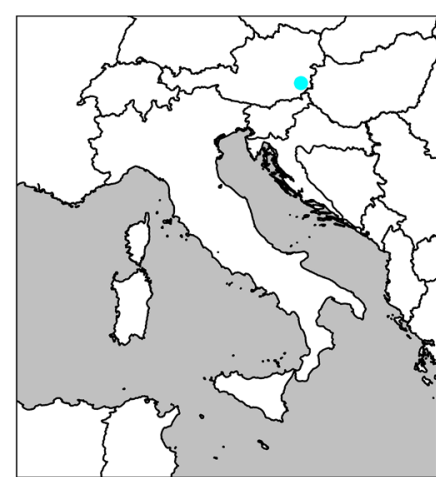

279. Ochogona phyllophaga (Attems, 1899)

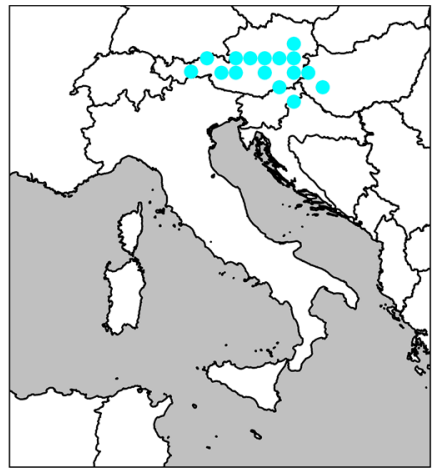

282. Ochogona triaina (Attems, 1895)

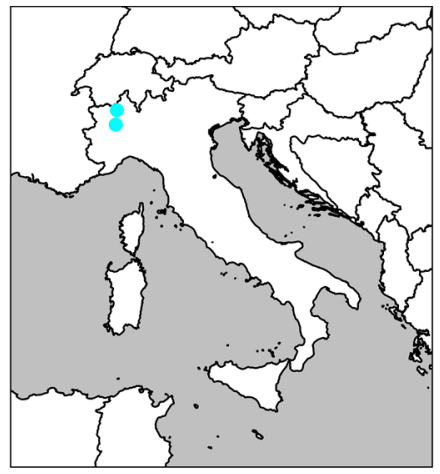

285. Oroposoma fagorum Verhoeff, 1936 


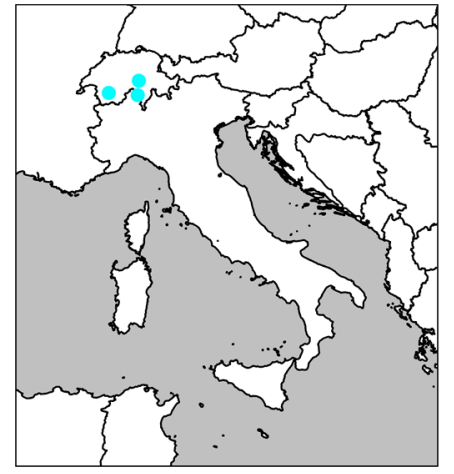

286. Oroposoma granitivagum Verhoeff, 1936

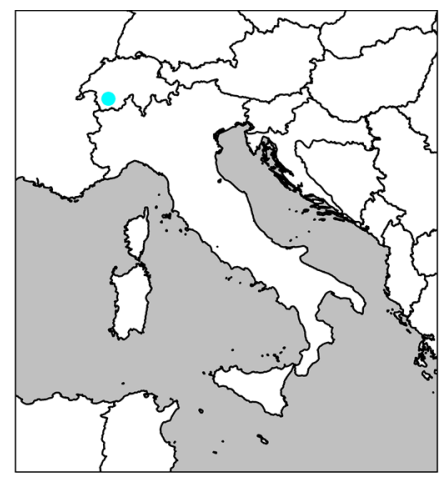

287. Oroposoma nivale (Faës, 1902)

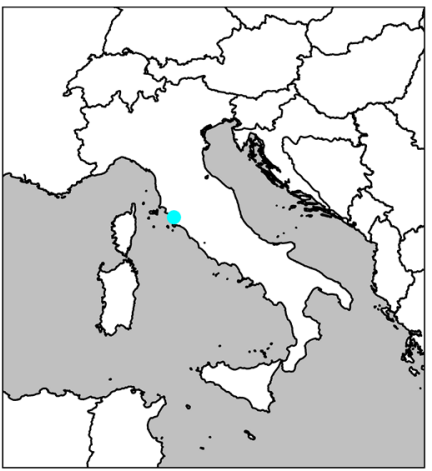

290. Paradactylophorosoma insulanum (Attems, 1908)

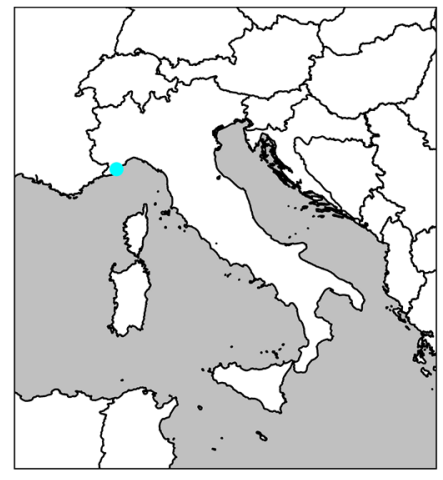

293. Plectogona bonzanoi (Strasser, 1975)

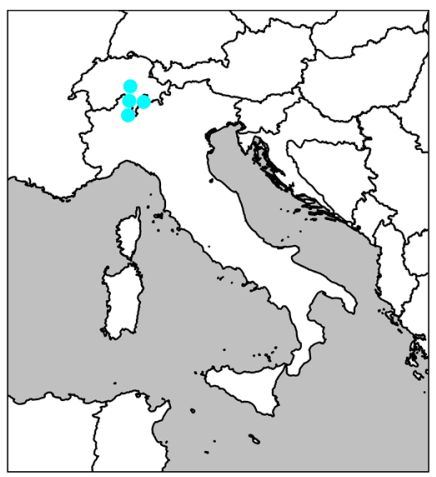

288. Oroposoma ticinense Manfredi, 1957

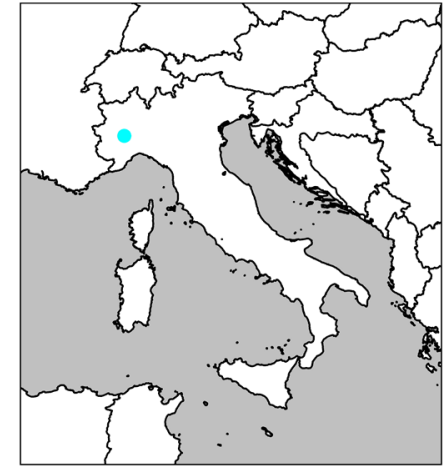

291. Pedemontia delmastroi Mauriès, 1994

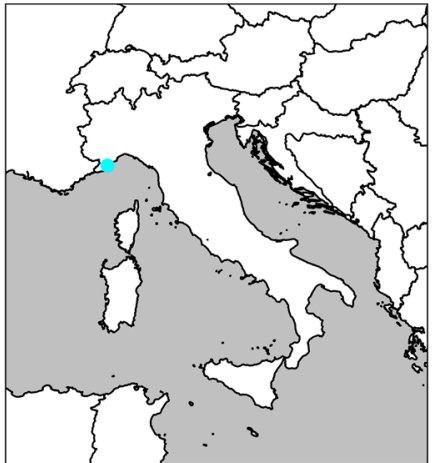

294. Plectogona franciscoloi (Manfredi, 1953) 


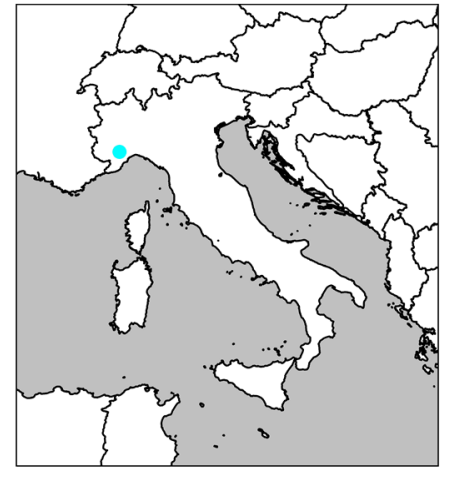

295. Plectogona morisii (Strasser, 1975)

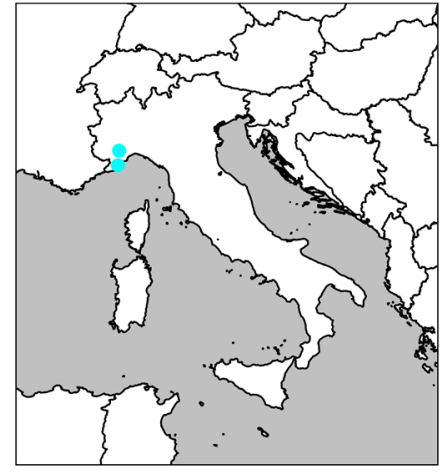

296. Plectogona sanfillipoi (Manfredi, 1956)

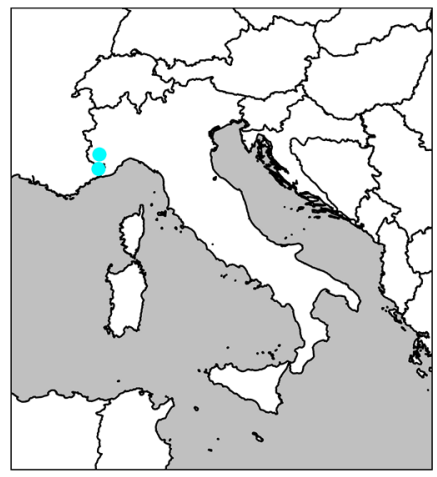

297. Plectogona vignai (Strasser, 1970)

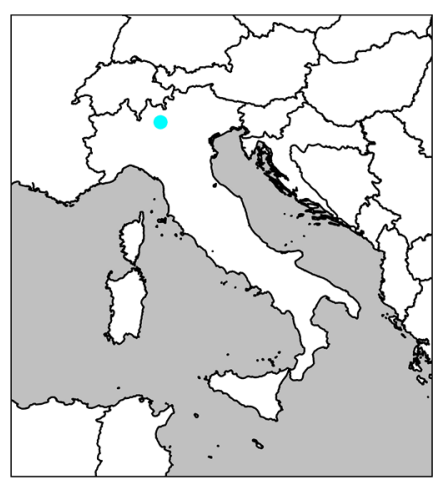

300. Pyrgocyphosoma armigerum Verhoeff, 1925

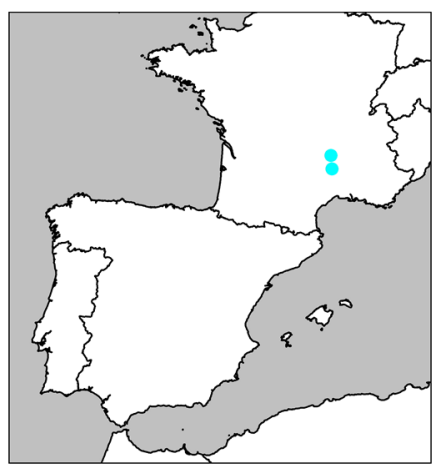

303. Pyrgocyphosoma balazuci Mauriès \& Kime, 1999 (Verhoeff, 1900)

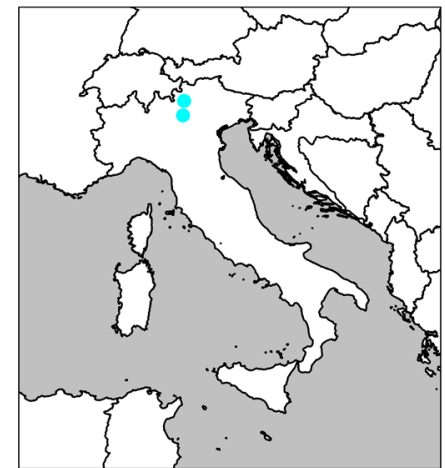

302. Pyrgocyphosoma aspidiorum Verhoeff, 1931

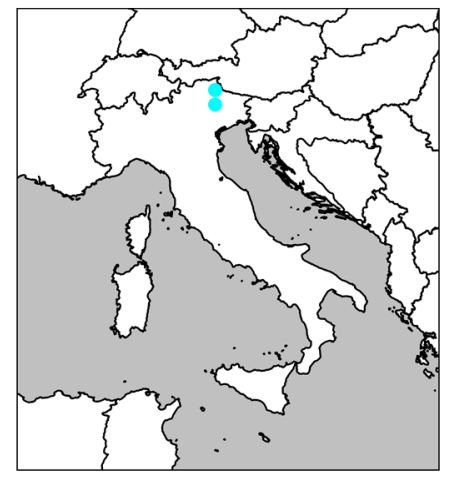

299. Pterygophorosoma

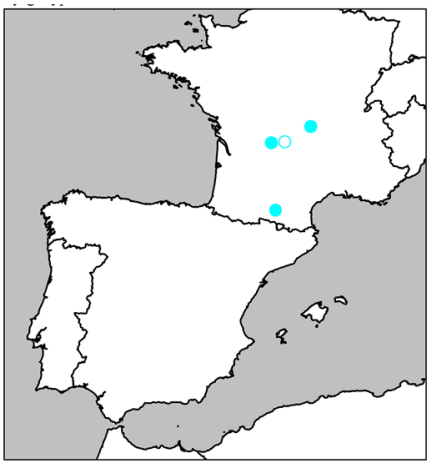

(Ribaut \& Brolemann, 1932) 


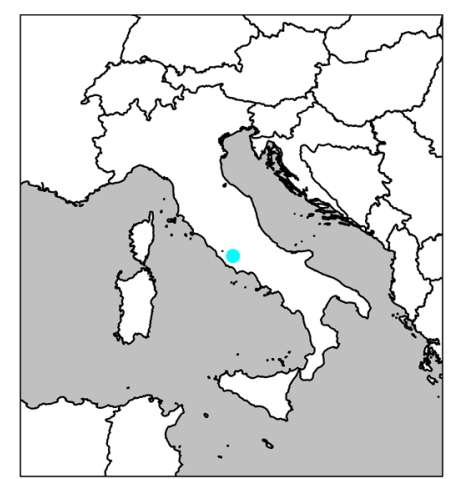

304. Pyrgocyphosoma bidentatum (Verhoeff, 1900)

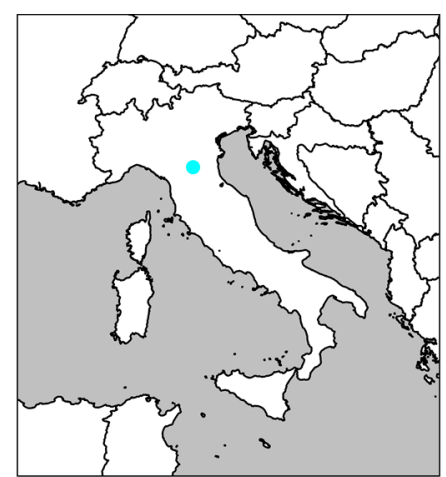

307. Pyrgocyphosoma centrale (Silvestri, 1898)

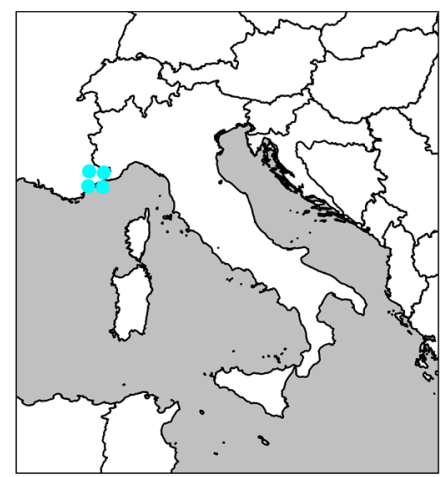

310. Pyrgocyphosoma doriae (Silvestri, 1898)

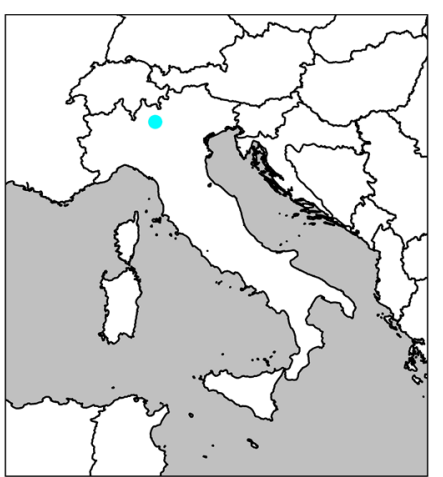

305. Pyrgocyphosoma brembanum Verhoeff, 1931

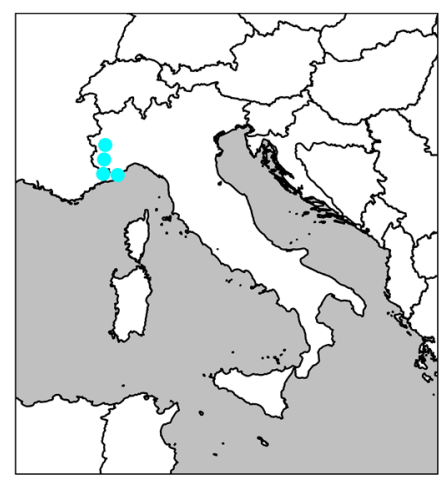

308. Pyrgocyphosoma dalmazzense Verhoeff, 1930

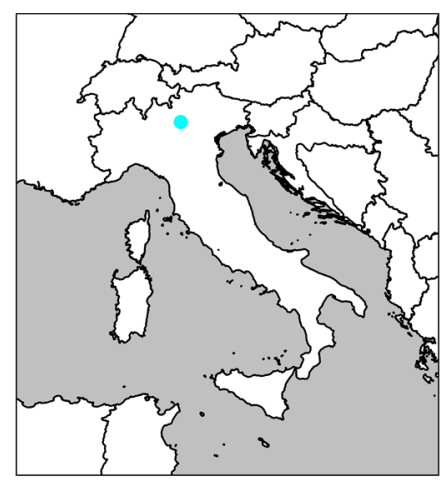

311. Pyrgocyphosoma edrinum Verhoeff, 1934

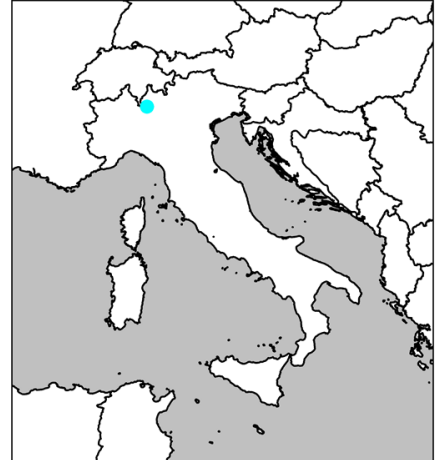

306. Pyrgocyphosoma brunatense (Verhoeff, 1910)

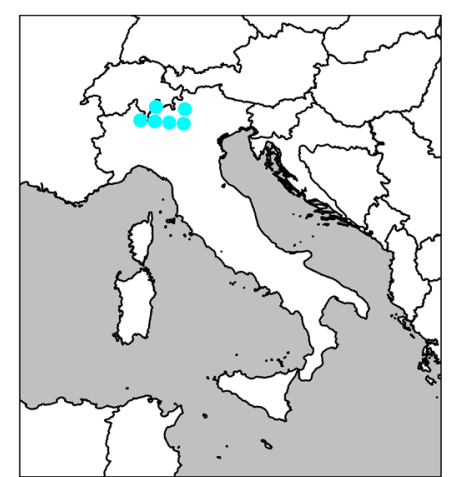

309. Pyrgocyphosoma dentatum (Brölemann, 1892)

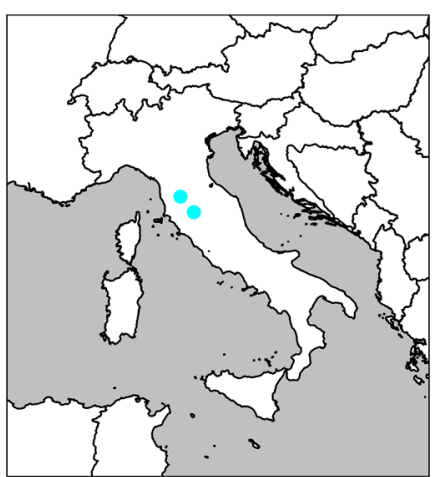

312. Pyrgocyphosoma florentinum (Silvestri, 1903) 


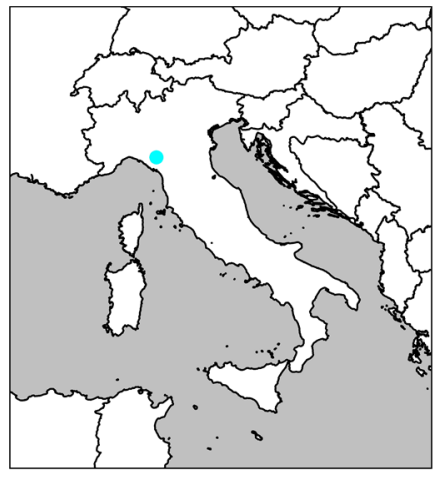

313. Pyrgocyphosoma fonticuli Verhoeff, 1936

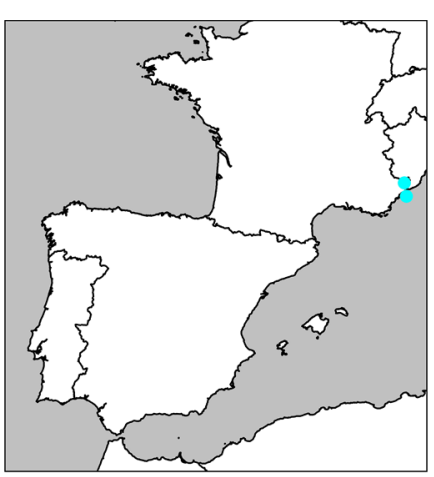

316. Pyrgocyphosoma jucundum (Brolemann, 1935)

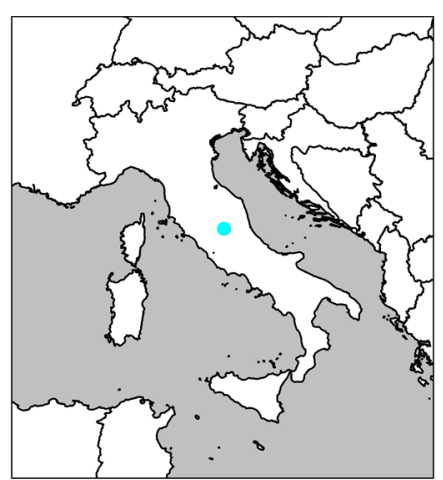

319. Pyrgocyphosoma marmorense Verhoeff, 1932

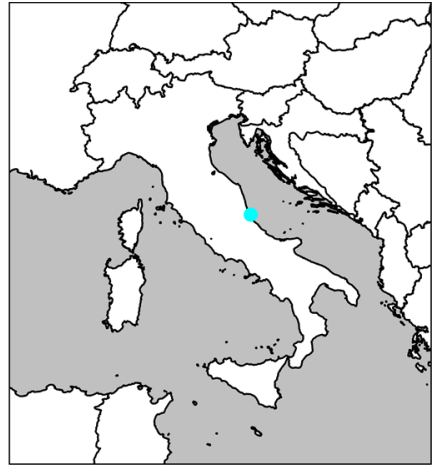

314. Pyrgocyphosoma gattii (Silvestri, 1898)

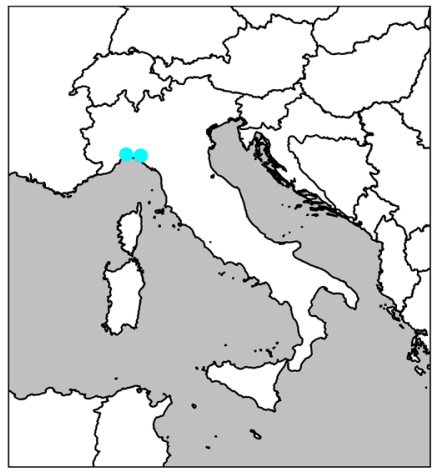

317. Pyrgocyphosoma ligusticum (Silvestri, 1898)

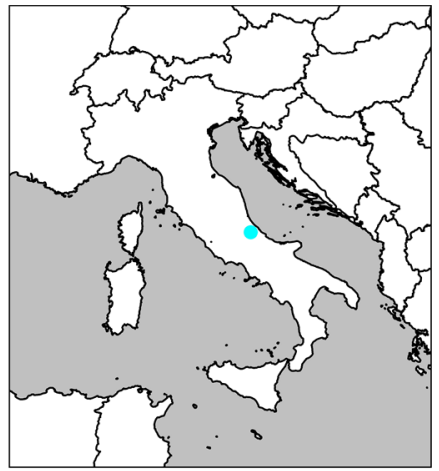

320. Pyrgocyphosoma marrucinum Manfredi, 1950

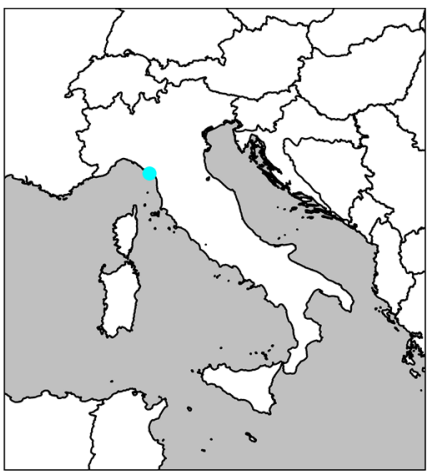

315. Pyrgocyphosoma grassii (Silvestri, 1898)

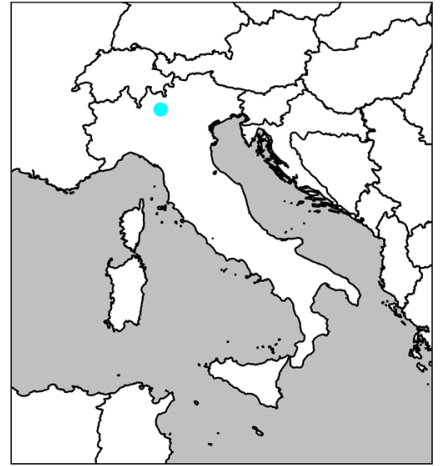

318. Pyrgocyphosoma longilamellatum Verhoeff, 1931

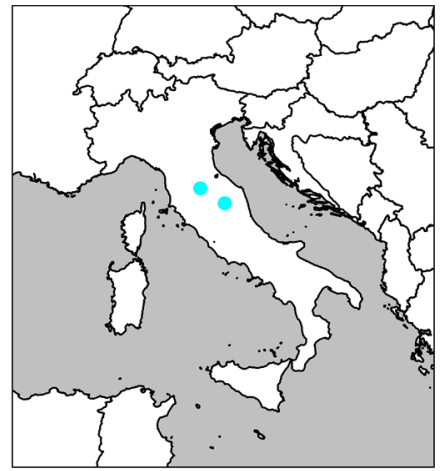

321. Pyrgocyphosoma mevaniense (Silvestri, 1894) 


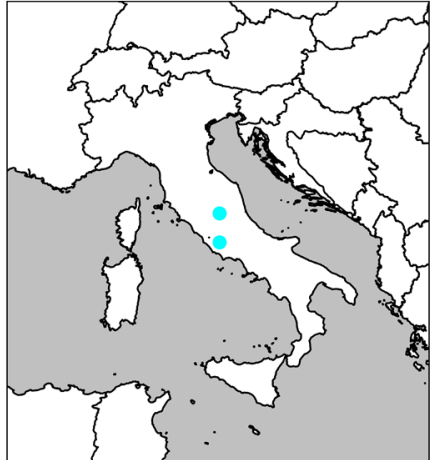

322. Pyrgocyphosoma oppidicola (Silvestri, 1898)

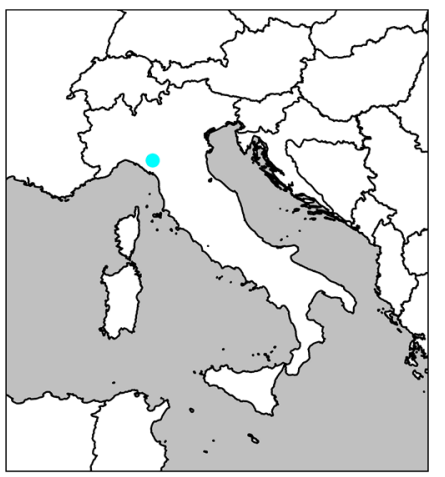

325. Pyrgocyphosoma pontremolense Verhoeff, 1936

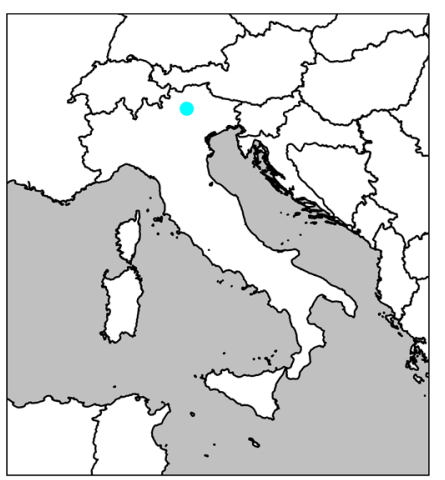

328. Pyrgocyphosoma ravinense Verhoeff, 1936

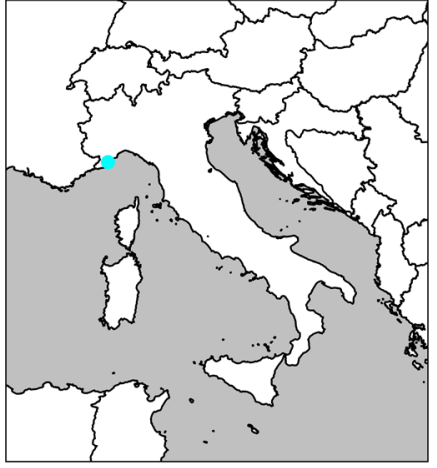

323. Pyrgocyphosoma ormeanum Verhoeff, 1930

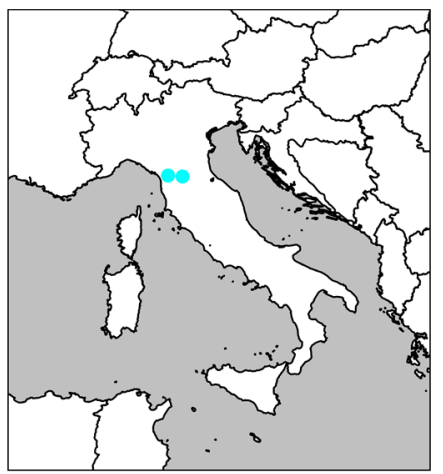

326. Pyrgocyphosoma pracchiense Verhoeff, 1932

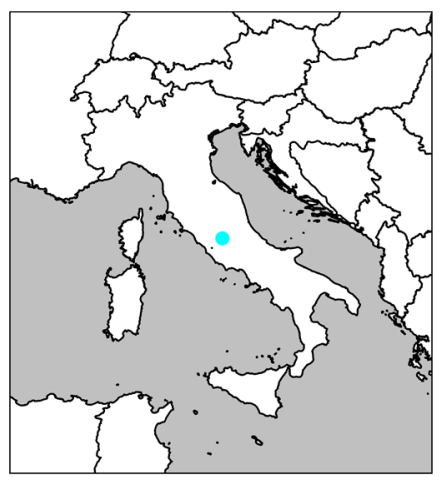

329. Pyrgocyphosoma reatinum Strasser, 1977

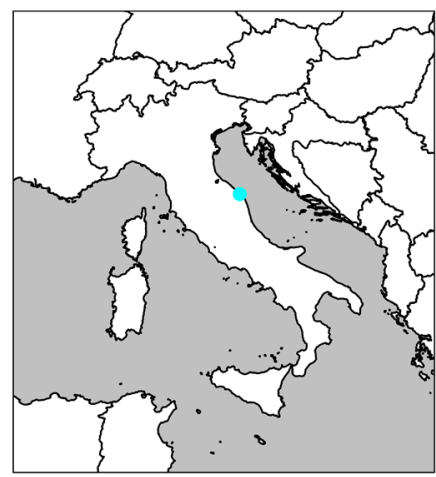

324. Pyrgocyphosoma picenum Manfredi, 1953

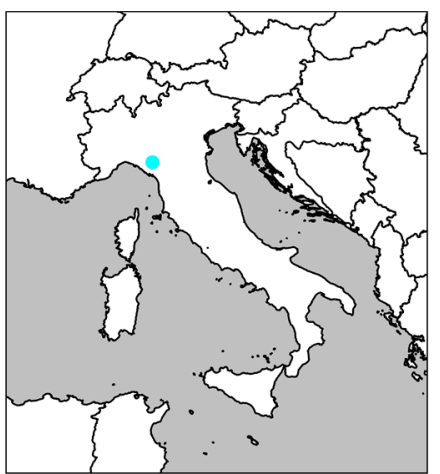

327. Pyrgocyphosoma quercuun Verhoeff, 1936

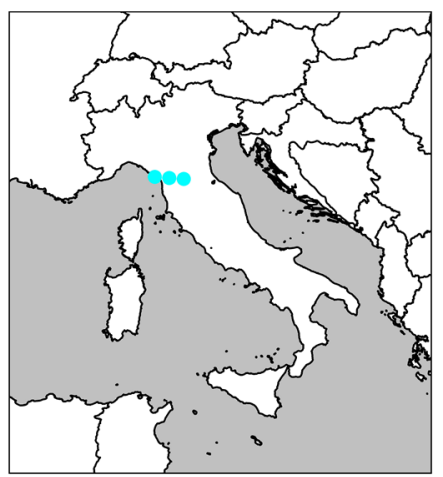

330. Pyrgocyphosoma renanum Verhoeff, 1932 


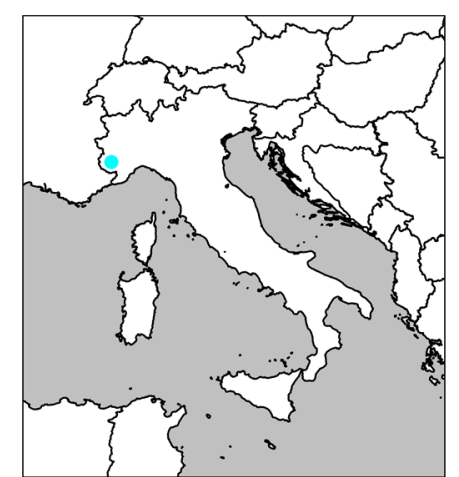

331. Pyrgocyphosoma roccavionense Verhoeff, 1937

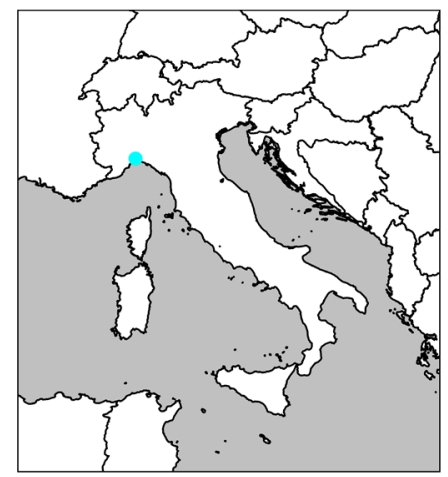

334. Pyrgocyphosoma serpentinum Verhoeff, 1932

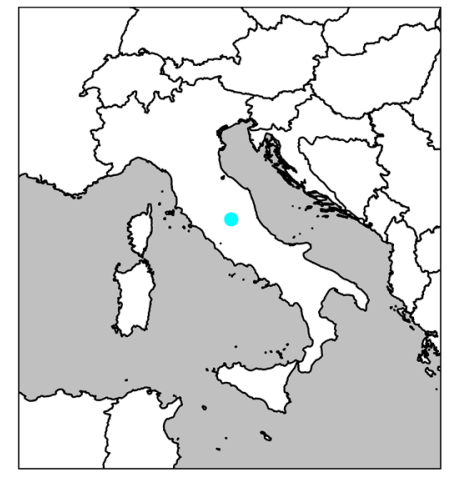

337. Pyrgocyphosoma terminilli Strasser, 1977

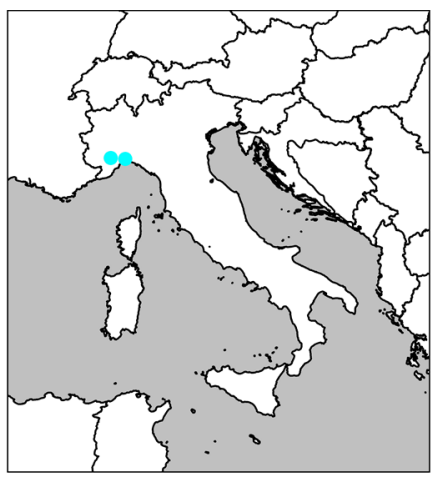

332. Pyrgocyphosoma savonense (Verhoeff, 1910)

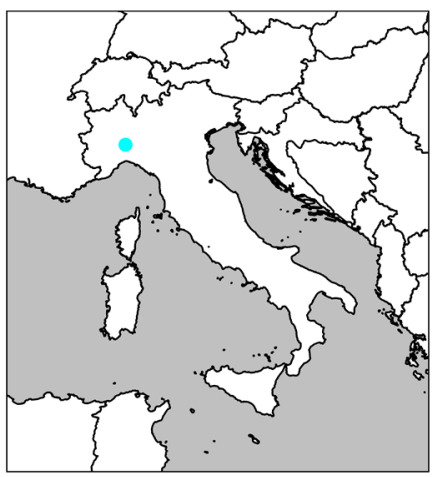

335. Pyrgocyphosoma serravallense Verhoeff, 1936

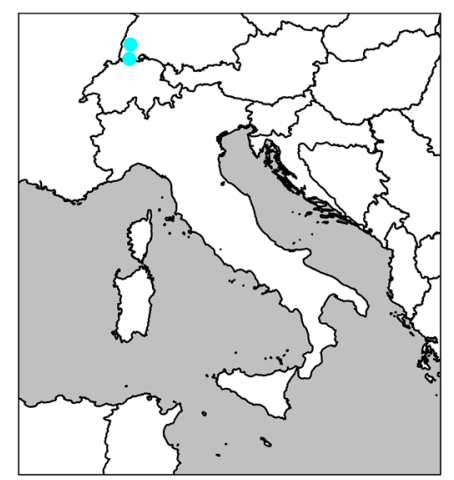

338. Pyrgocyphosoma titianum (Verhoeff, 1910)

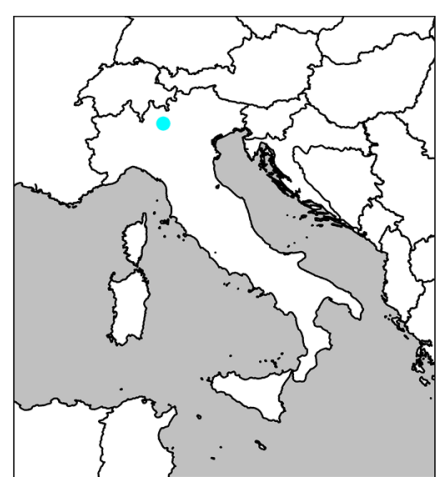

333. Pyrgocyphosoma serianum Verhoeff, 1937

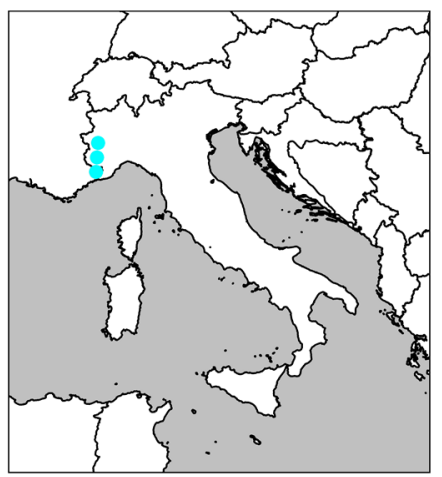

336. Pyrgocyphosoma tendanum Verhoeff, 1930

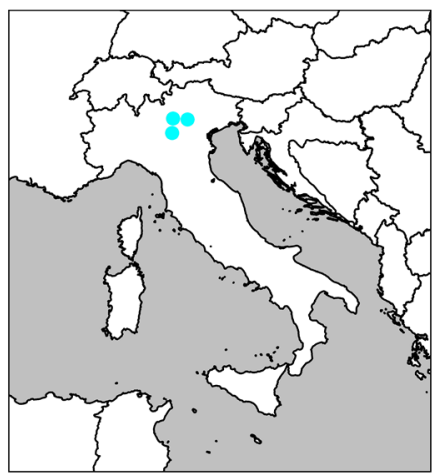

339. Pyrgocyphosoma tridentimum (Silvestri, 1898) 


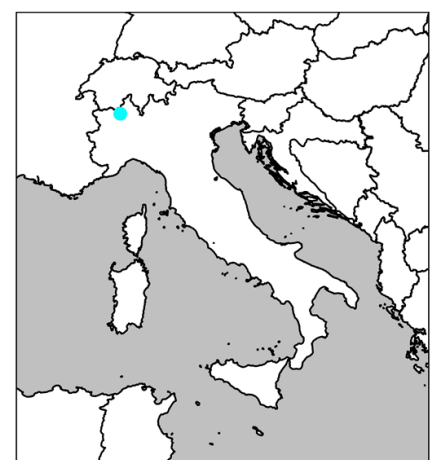

340. Pyrgocyphosoma vallicola (Silvestri, 1898)

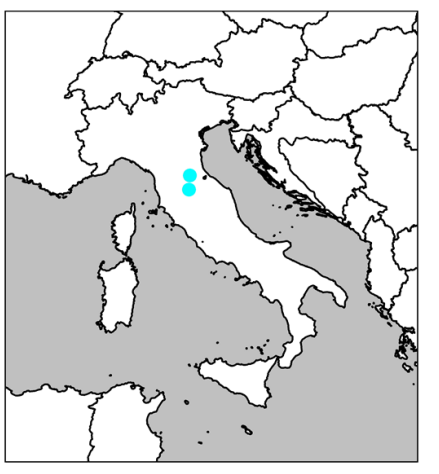

341. Pyrgocyphosoma vallombrosae (Silvestri, 1898)

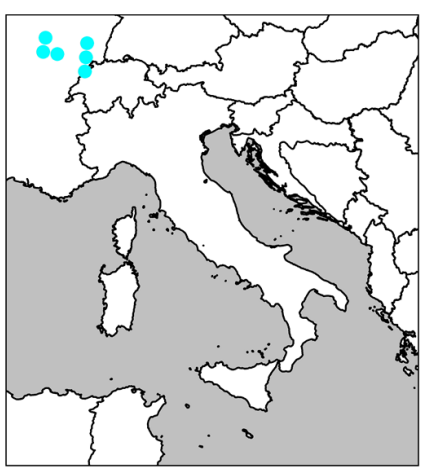

343. Rhymogona hessei (Ravoux, 1935)

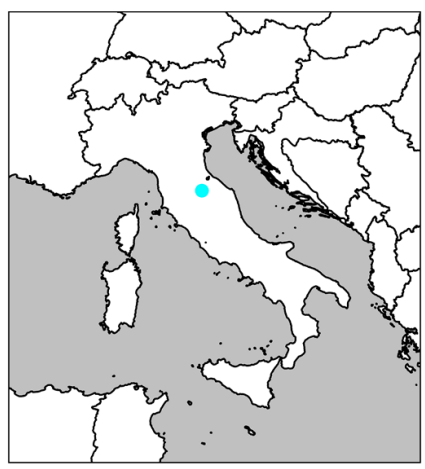

342. Pyrgocyphosoma zangherii Manfredi, 1951

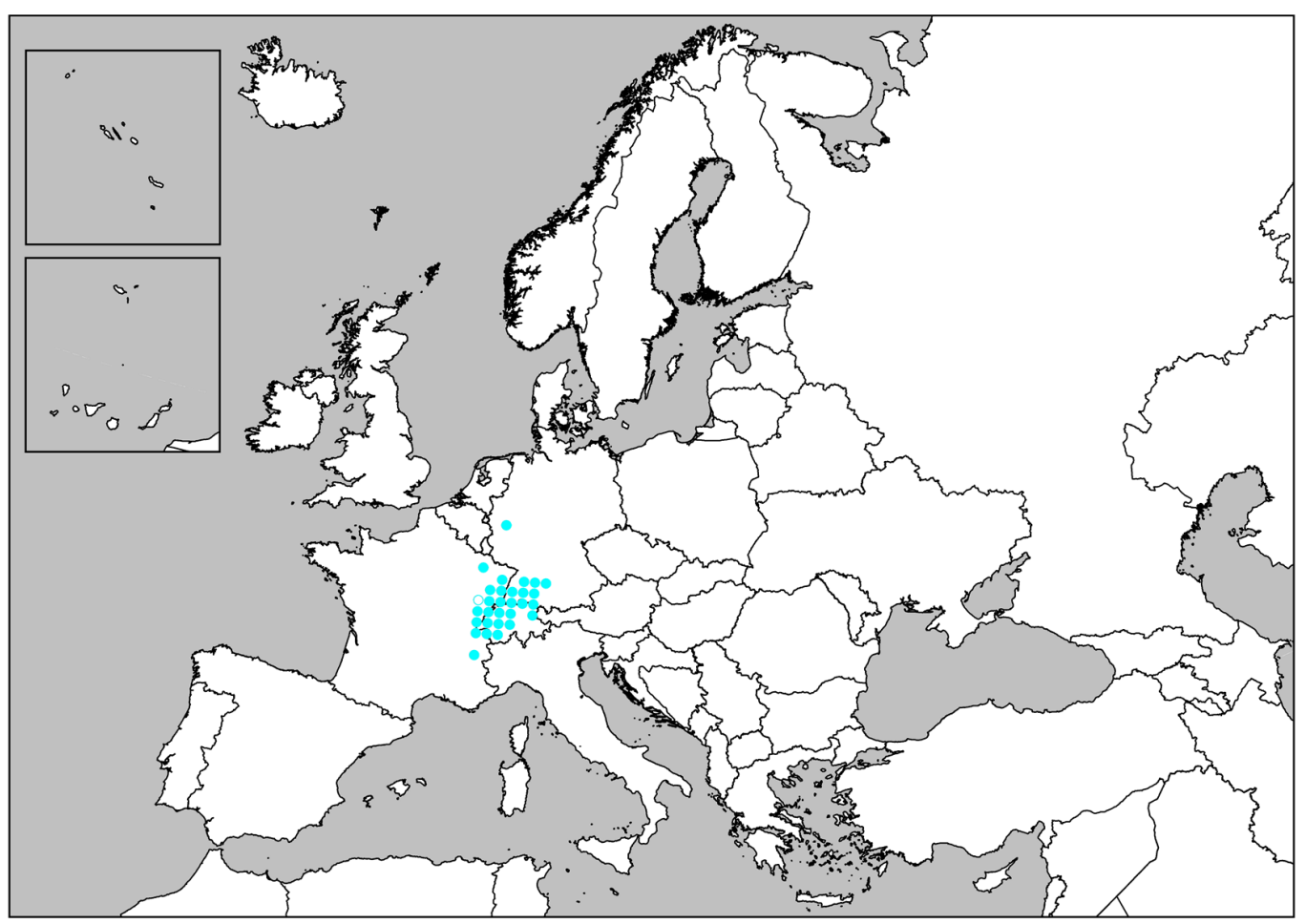

344. Rhymogona montivaga (Verhoeff, 1894) 


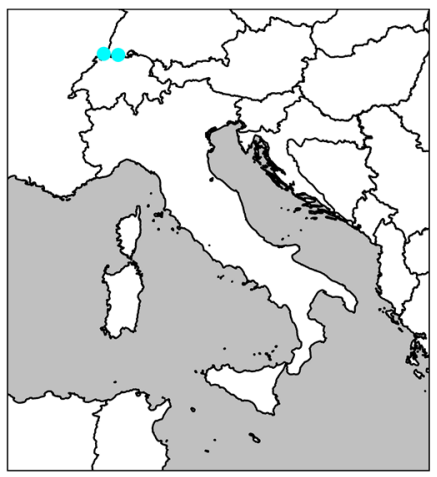

345. Rhymogona serrata (Bigler, 1912)

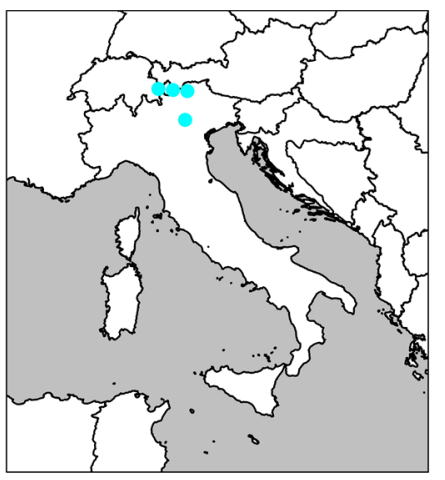

348. Rothenbuehleria minima (Rothenbühler, 1899)

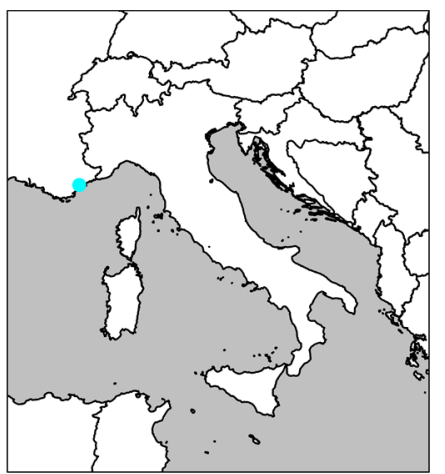

351. Semiosoma bordei Ribaut, 1913

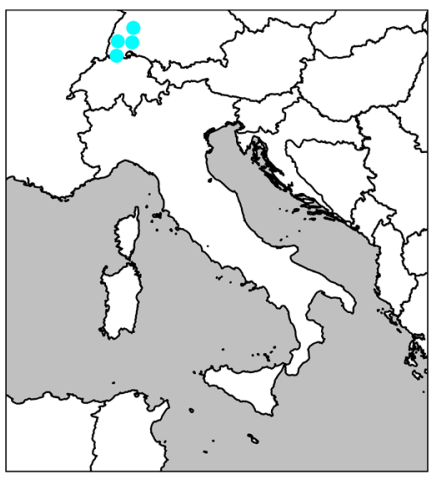

346. Rhymogona verhoeffi (Bigler, 1913)

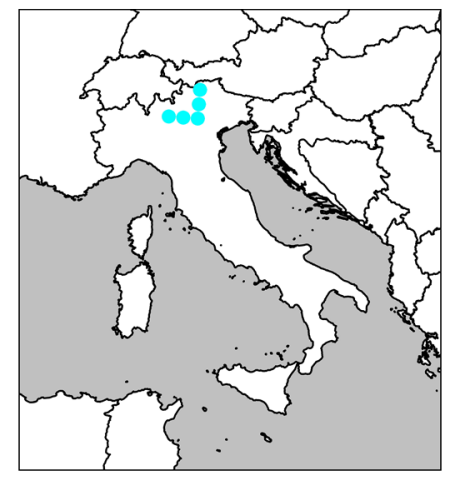

349. Rothenbuehleria tirolensis Verhoeff, 1900

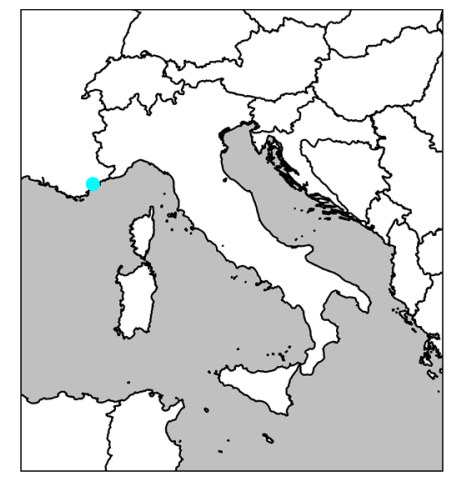

352. Semiosoma devillei (Brölemann, 1901)

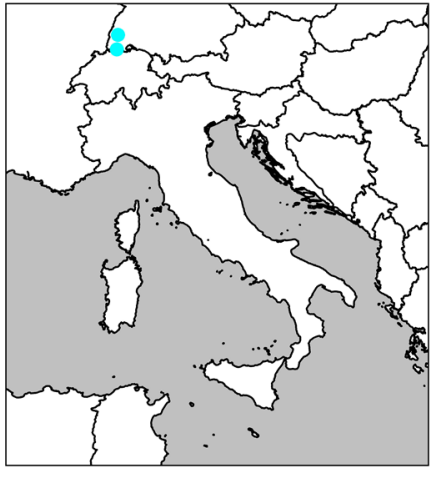

347. Rhymogona wehrana (Verhoeff, 1910)

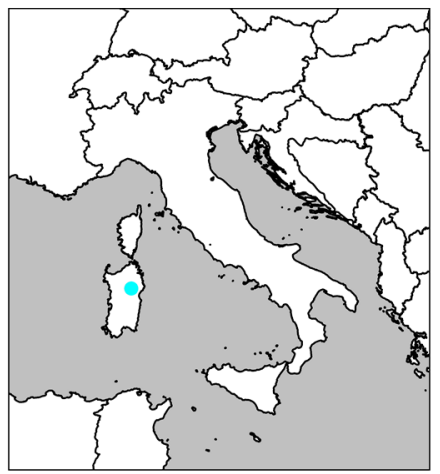

350. Sardosoma franchettii Manfredi, 1956

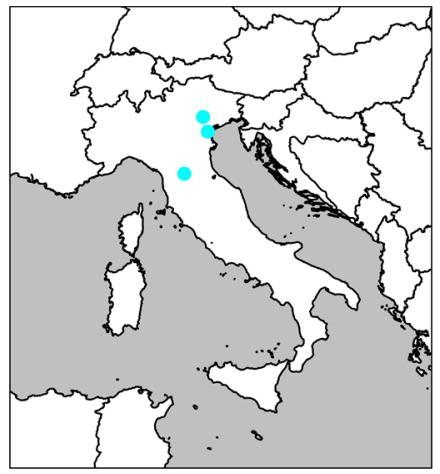

353. Semiosoma minutum (Berlese, 1894) 


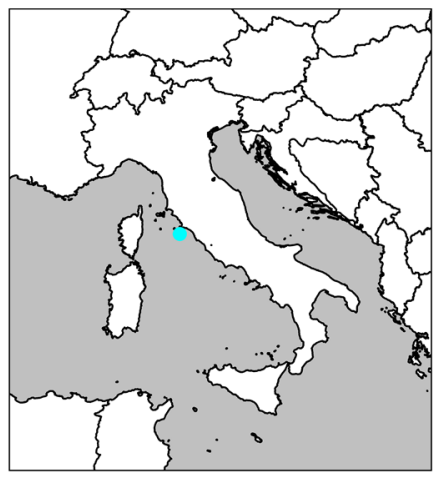

354. Synischiosoma argentarium Attems, 1927

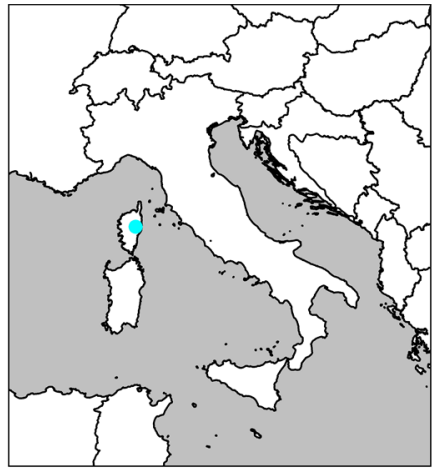

356. Cyrnosoma beroni (Mauriès, 1969)

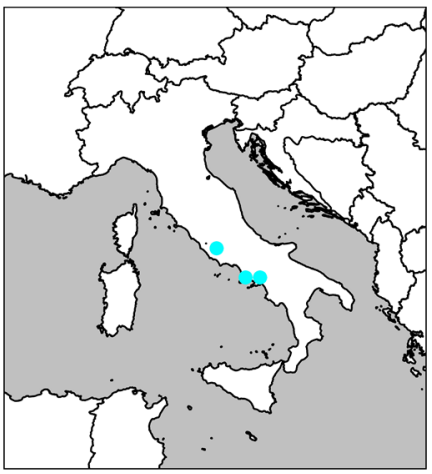

355. Synischiosoma murorum (Silvestri, 1902)

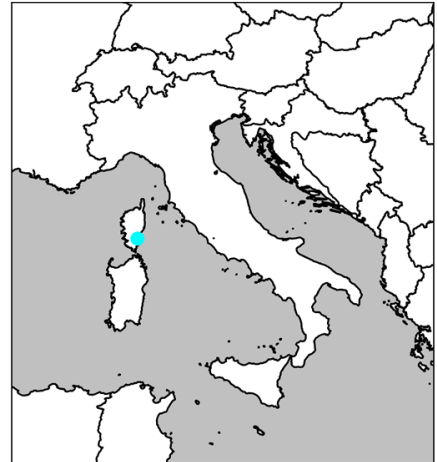

357. Cyrnosoma coineaui (Mauriès, 1969)

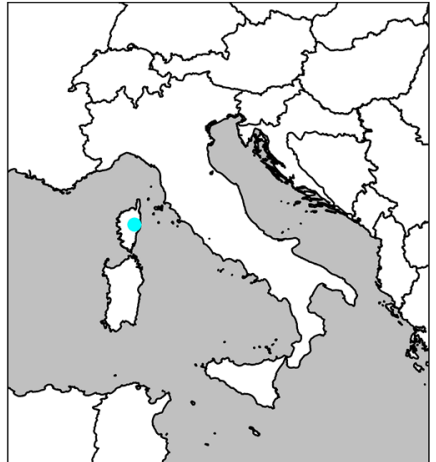

358. Cyrnosoma strasseri (Mauriès, 1969)

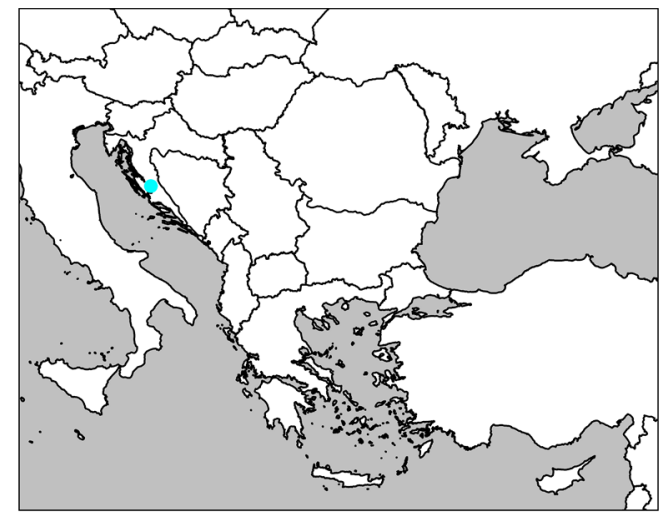

359. Dalmatosoma agaricum Antić \& Makarov, 2018 


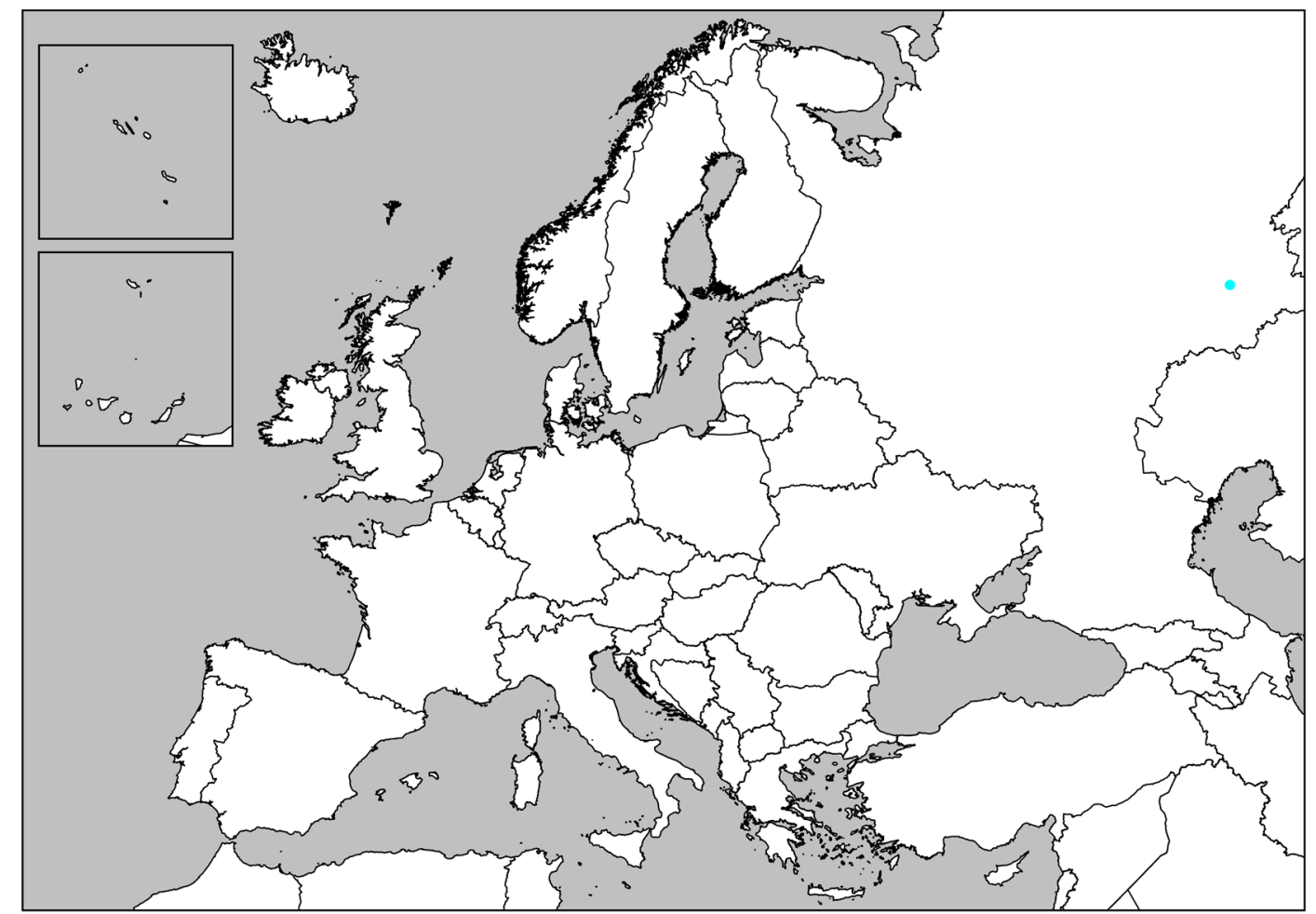

360. Altajosoma golovatchi (Shear, 1990)

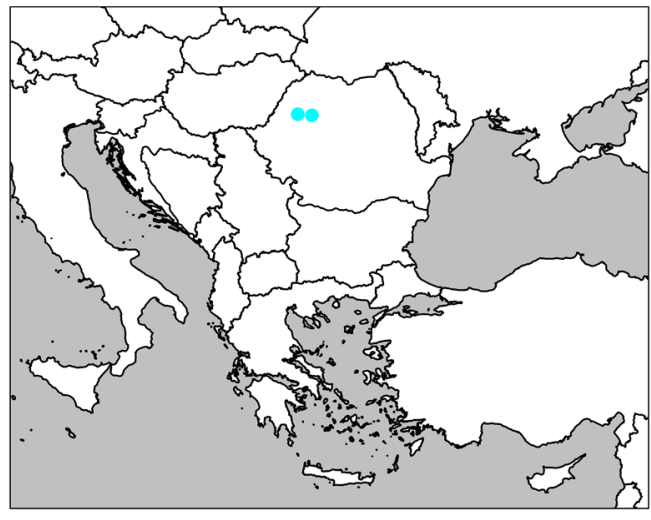

361. Entomobielzia getica Ceuca, 1964

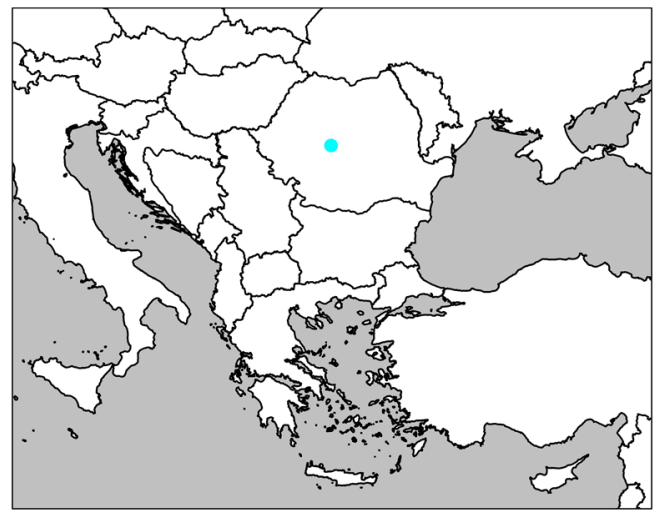

362. Entomobielzia kimakowizii (Verhoeff, 1897) 


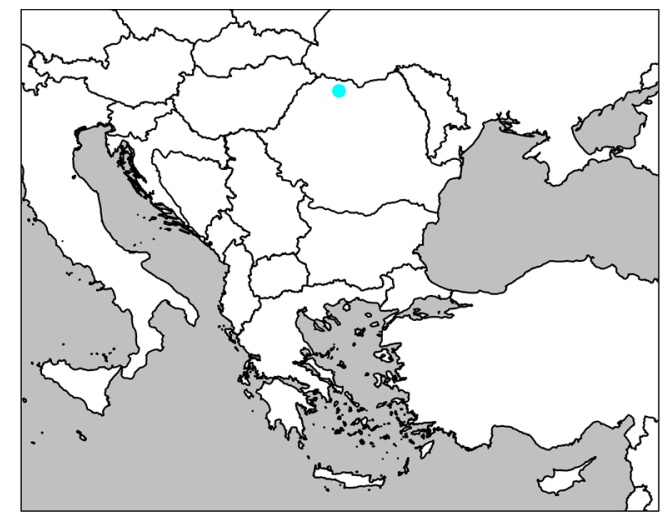

363. Entomobielzia varvarai Ceuca, 1985

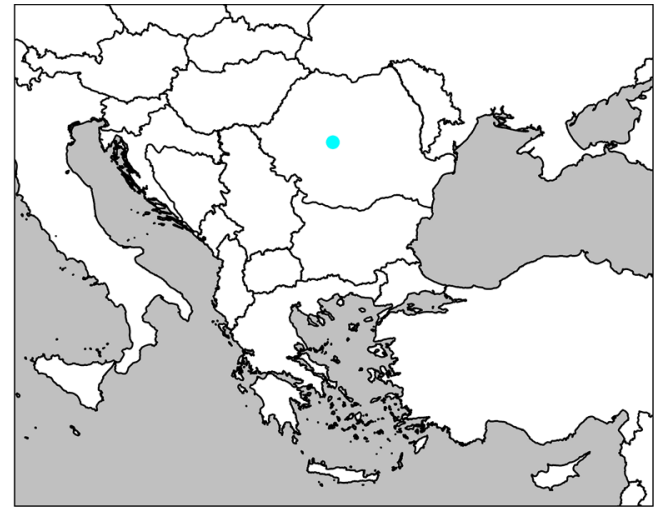

364. Pseudoclis octocera Attems, 1899

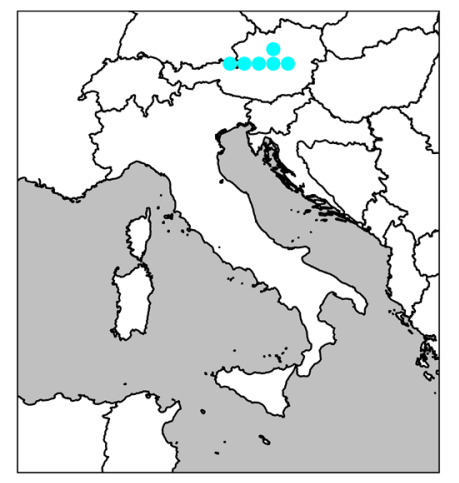

365. Haasea cyanopida (Attems, 1903)

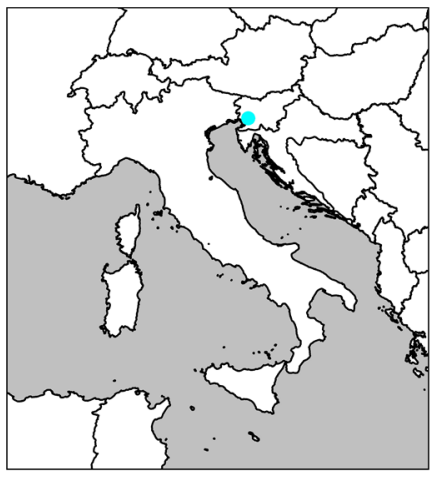

366. Haasea faucium

(Verhoeff, 1931)

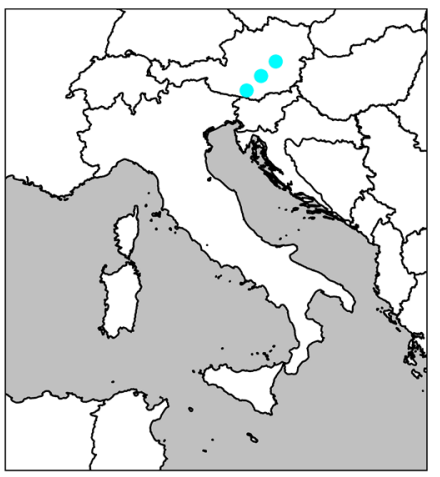

367. Haasea filicis

(Verhoeff, 1929)

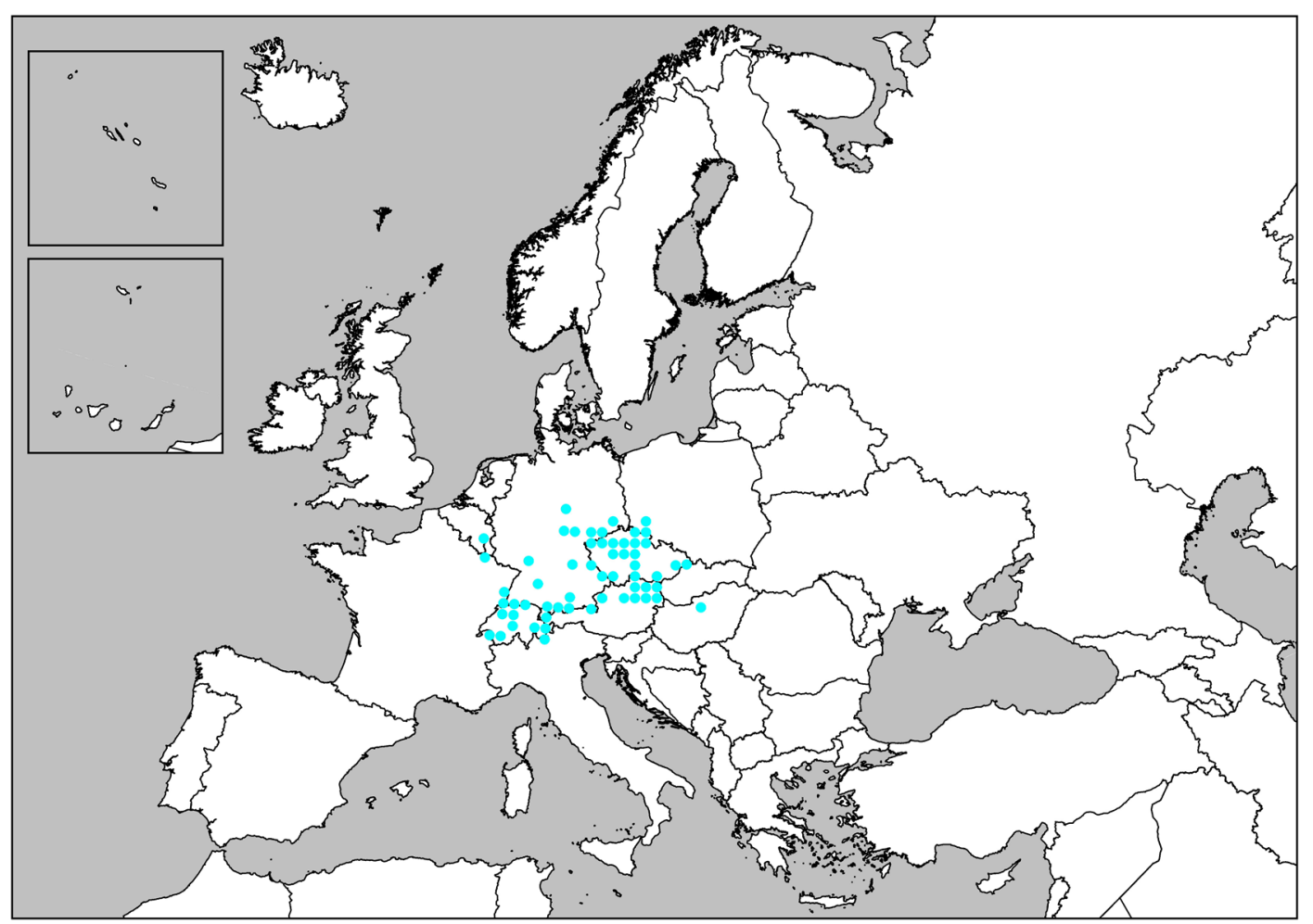

368. Haasea flavescens (Latzel, 1884) 


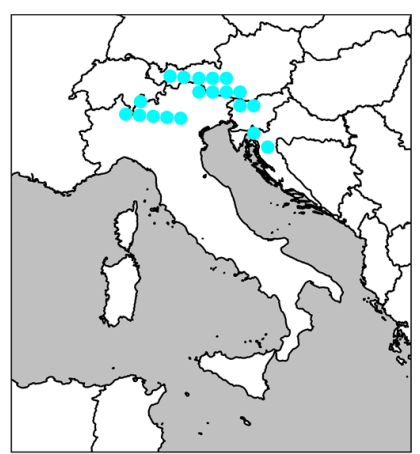

369. Haasea fonticulorum (Verhoeff, 1910)

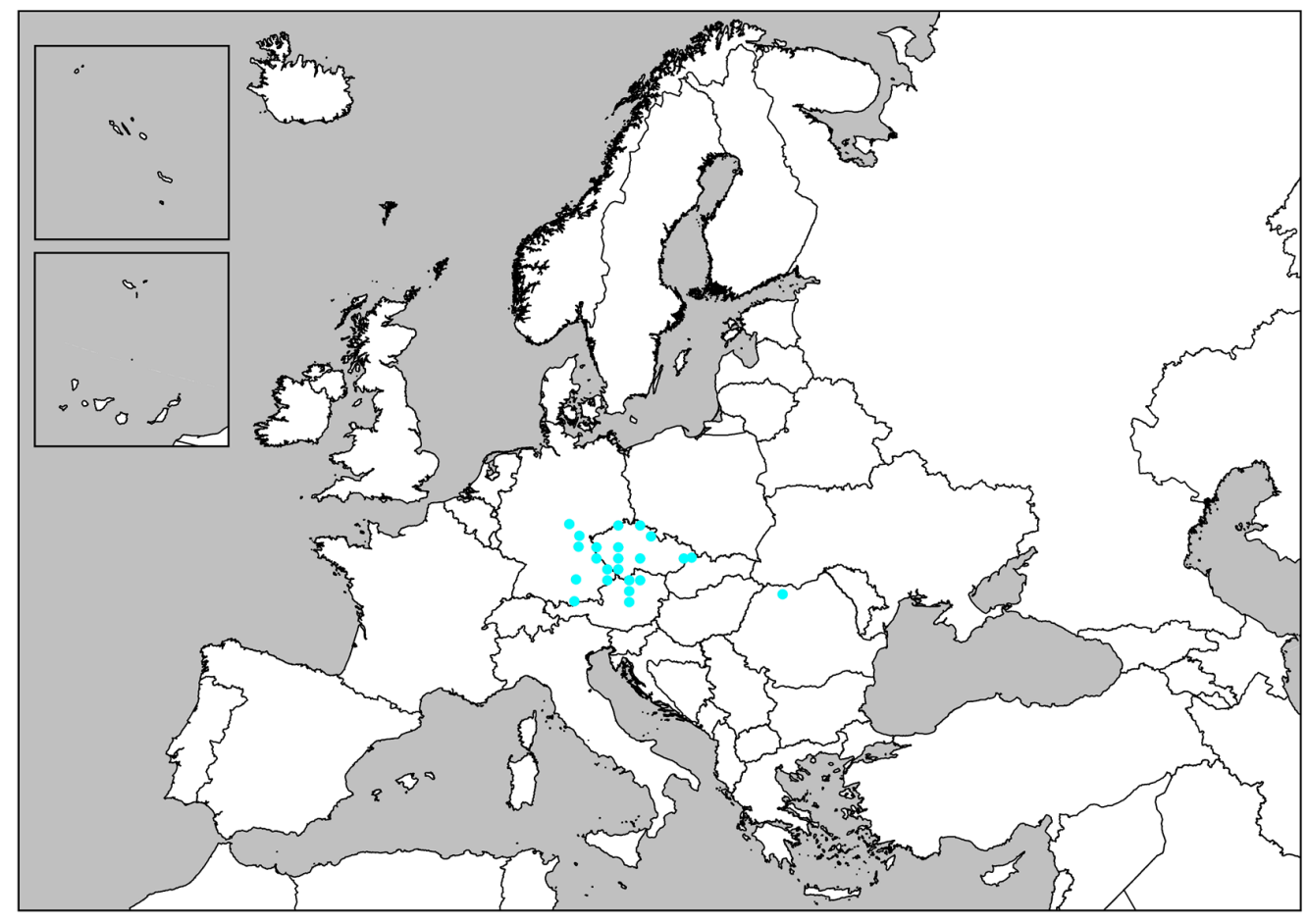

370. Haasea germanica (Verhoeff, 1901)

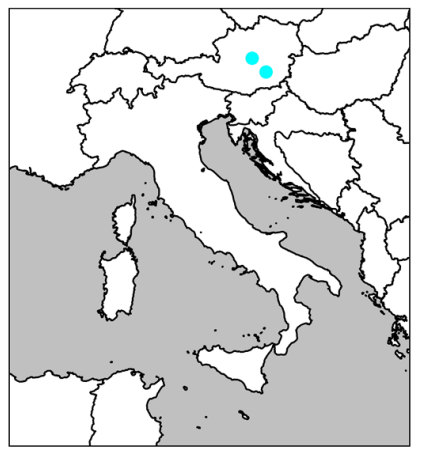

371. Haasea gruberi Antić \& Akkari, 2020

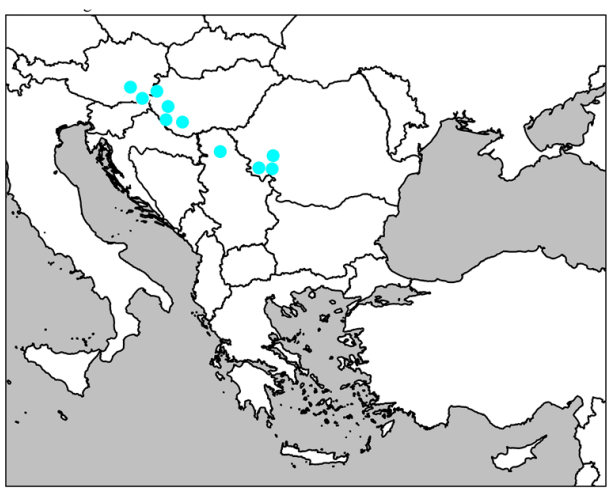

372. Haasea hungarica (Verhoeff, 1928) 


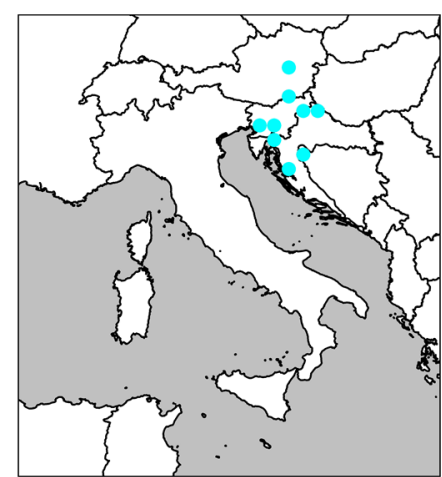

373. Haasea inflata

(Verhoeff, 1907)

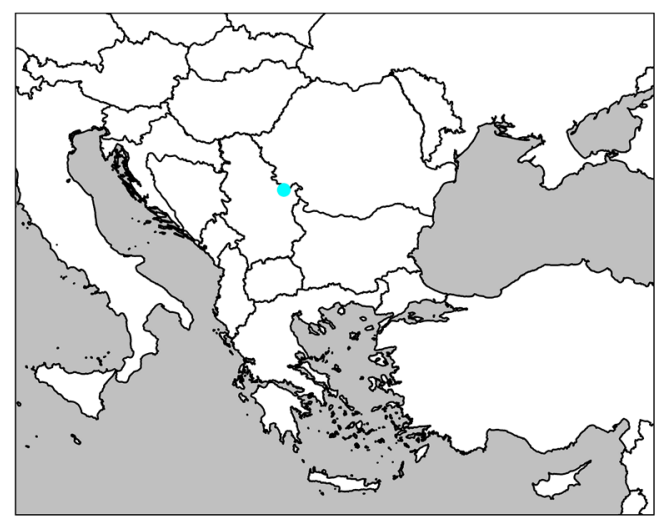

374. Haasea intermedia Mršić, 1985

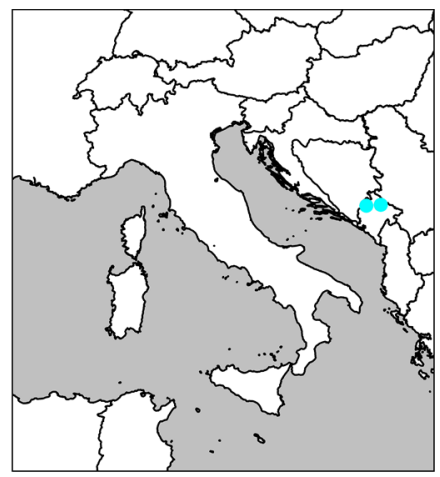

375. Haasea lacusnigri (Gulička, 1968)

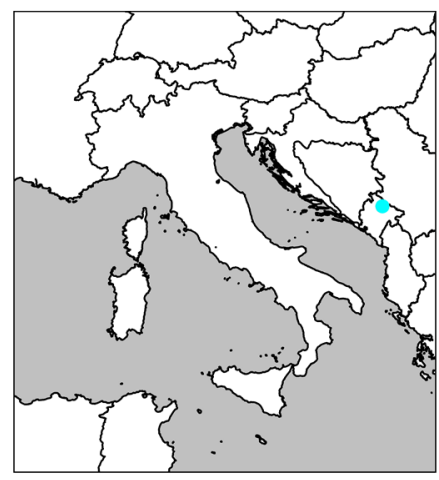

376. Haasea makarovi Antić \& Akkari, 2020

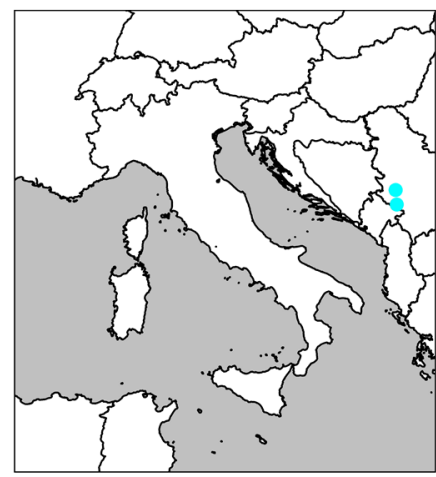

377. Haasea microcorna

(Strasser, 1971)

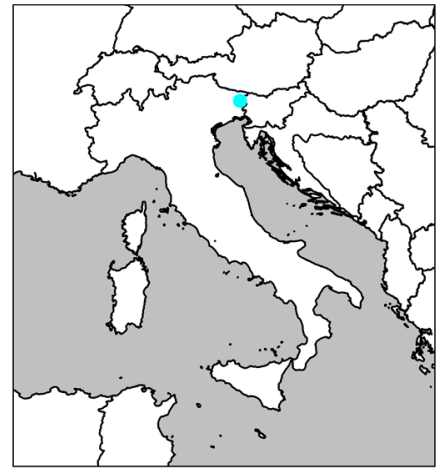

378. Haasea musimontium (Strasser, 1937)

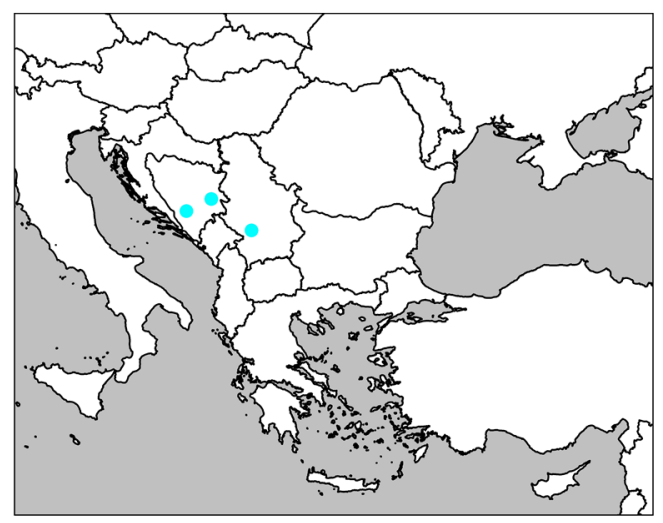

379. Haasea plasana (Verhoeff, 1899) 


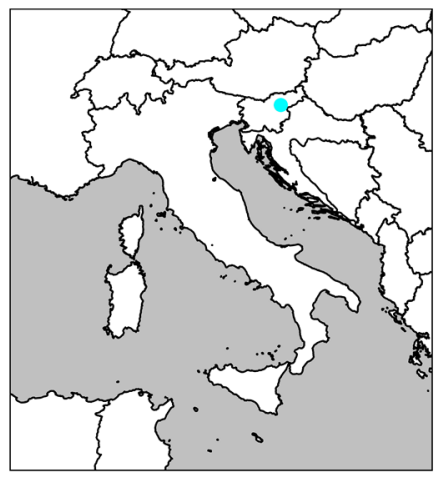

380. Haasea pretneri

(Strasser, 1966)

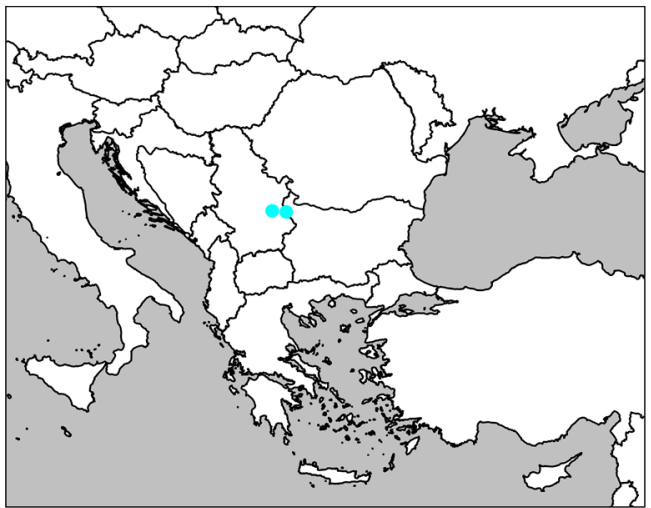

381. Haasea vidinensis (Strasser, 1973)

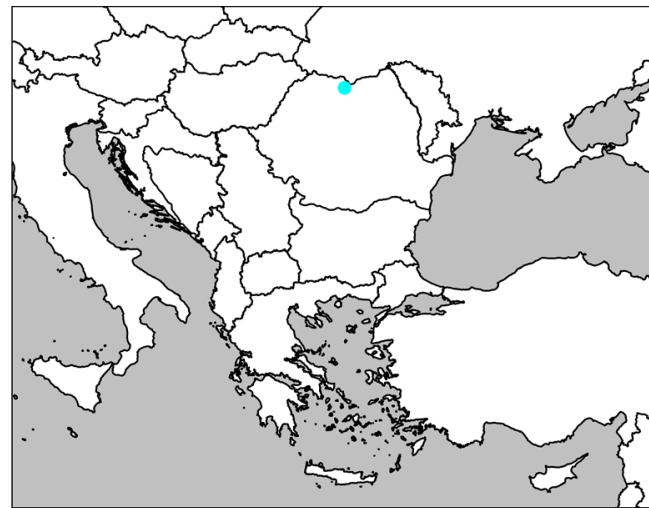

382. Hylebainosoma birtei (Ceuca, 1967)

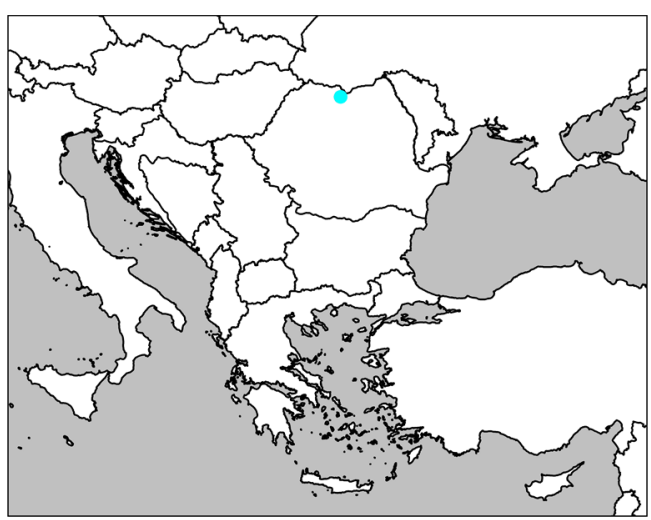

383. Hylebainosoma cavernicola (Ceuca, 1967)

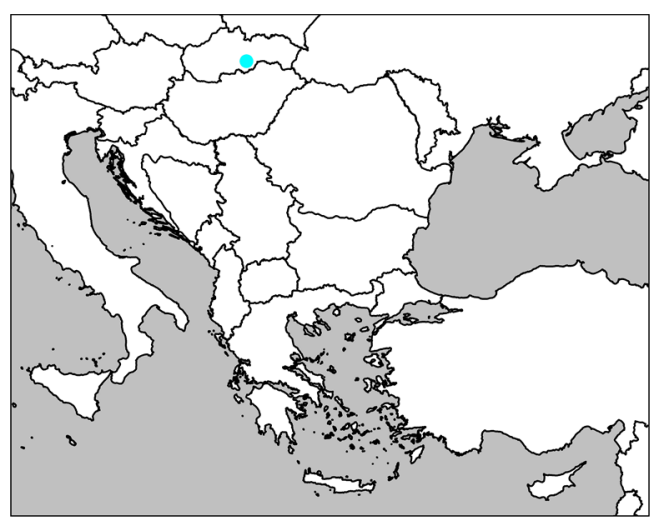

384. Hylebainosoma gulickai

Tajovský, Mock \& Papáč, 2014 


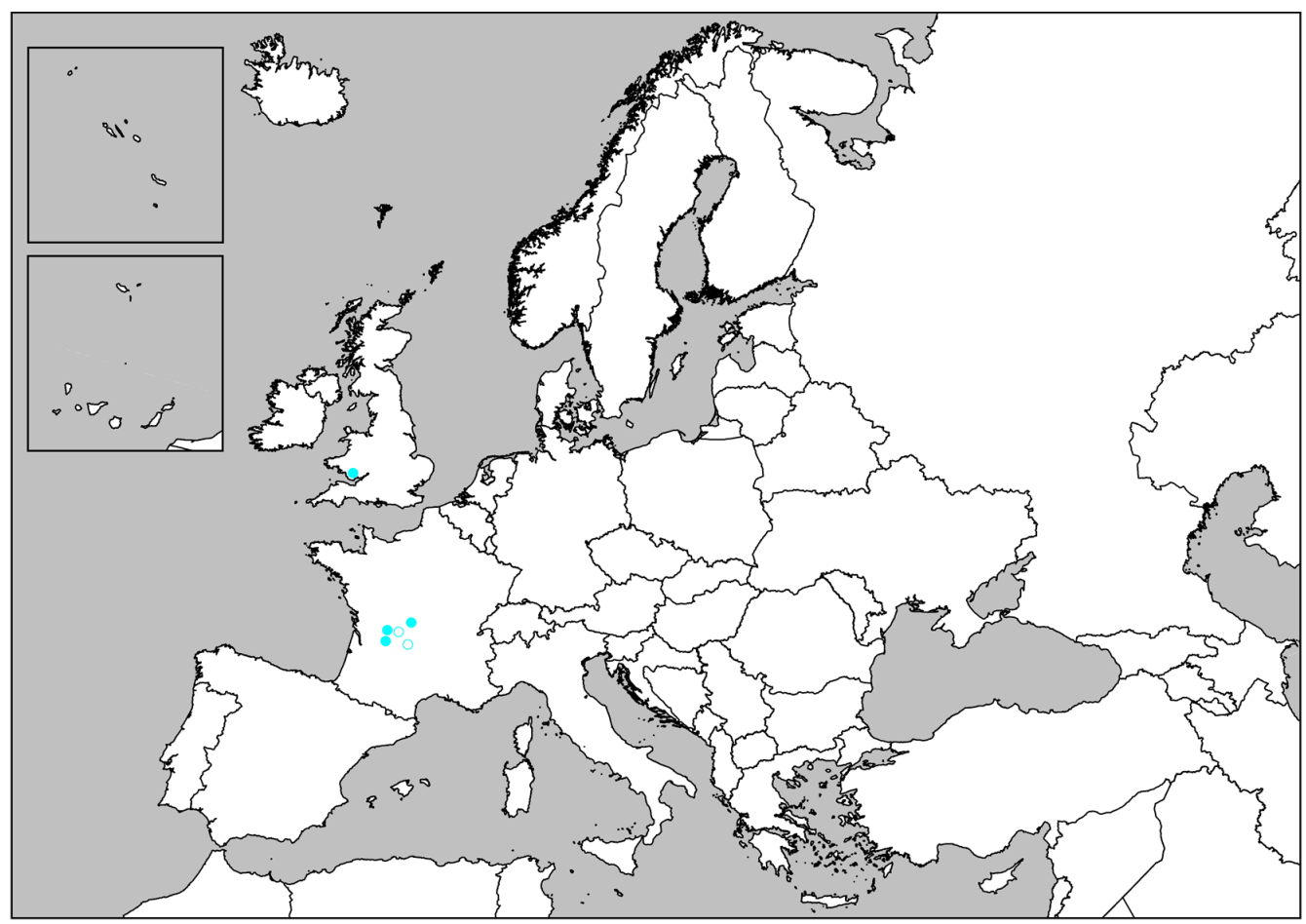

385. Hylebainosoma nontronense Mauriès \& Kime, 1999

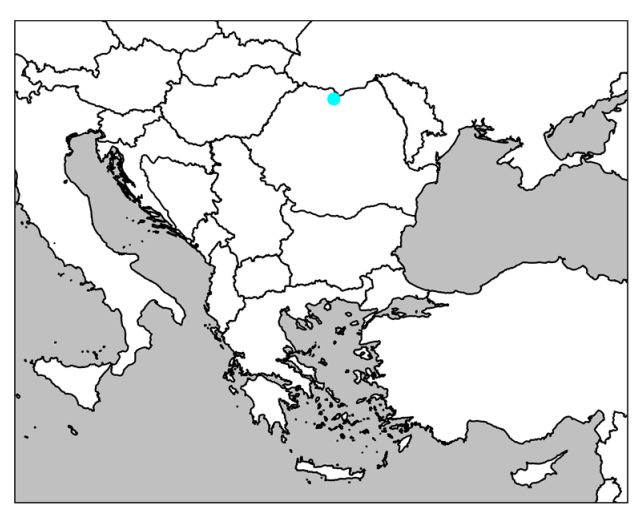

386. Hylebainosoma odici (Ceuca, 1967)

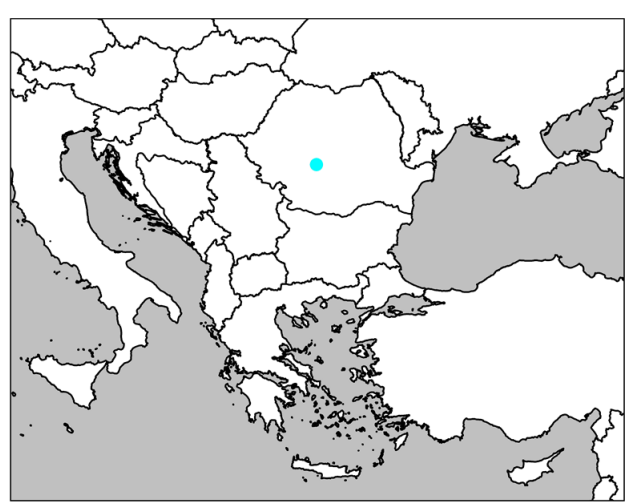

387. Hylebainosoma oltenicum (Ceuca, 1967)

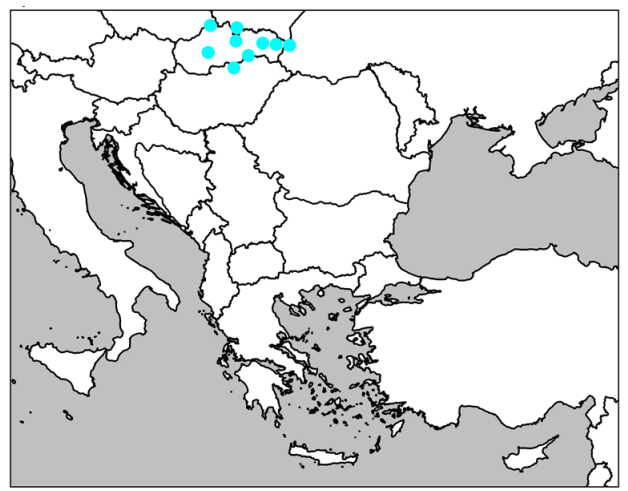

388. Hylebainosoma tatranum Verhoeff, 1899 


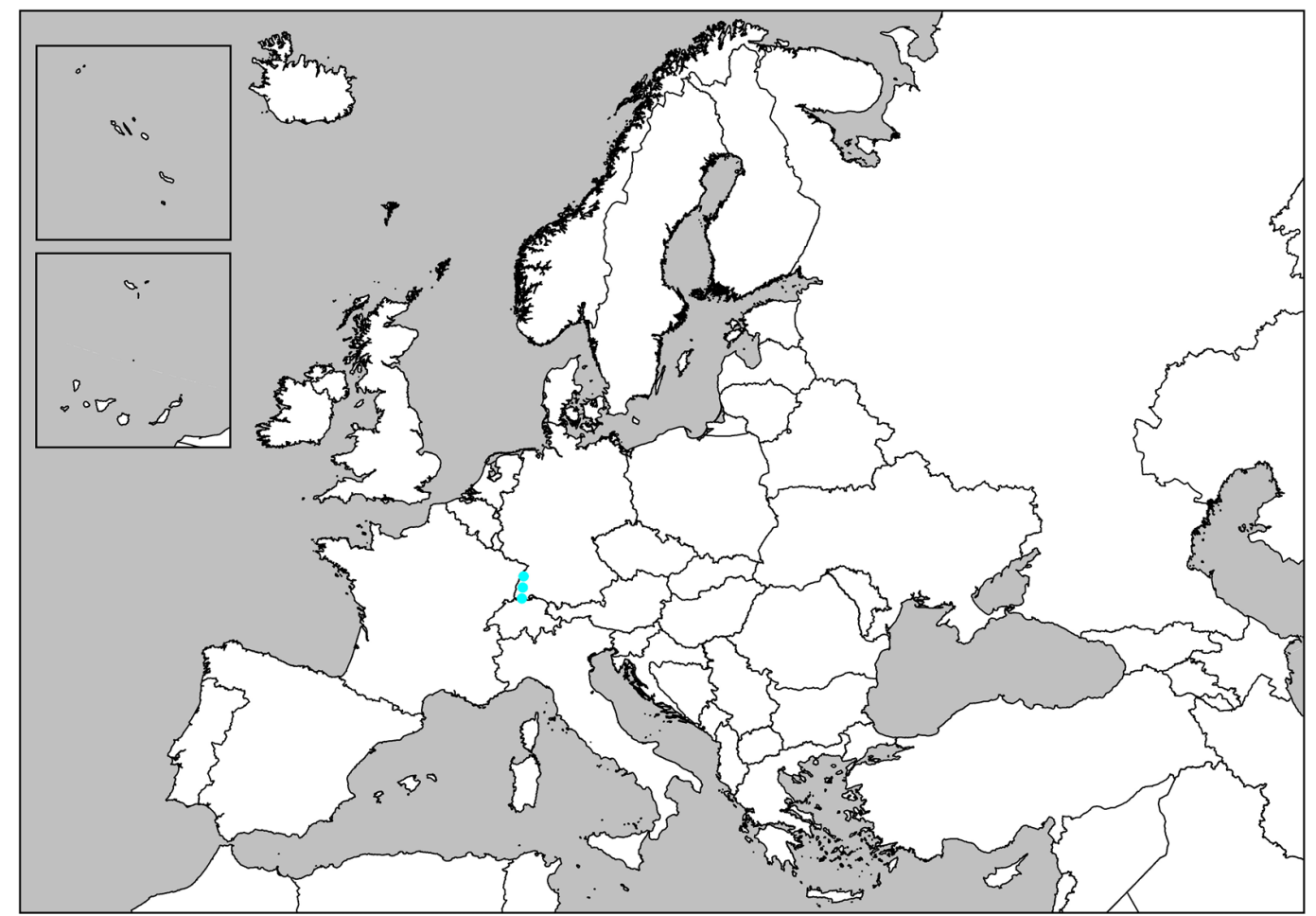

389. Xylophageuma vomrathi Verhoeff, 1911

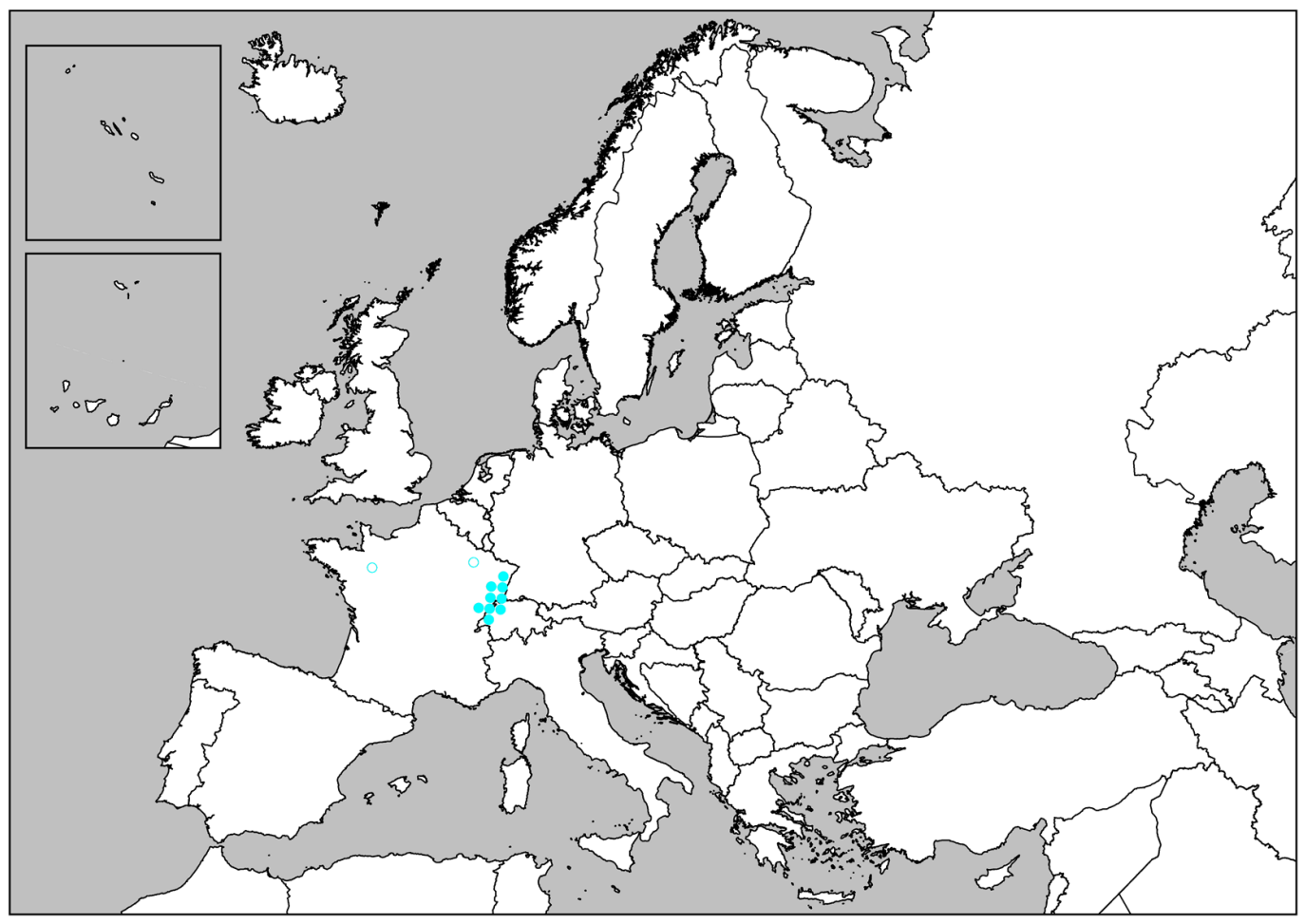

390. Xylophageuma zschokkei Bigler, 1912 


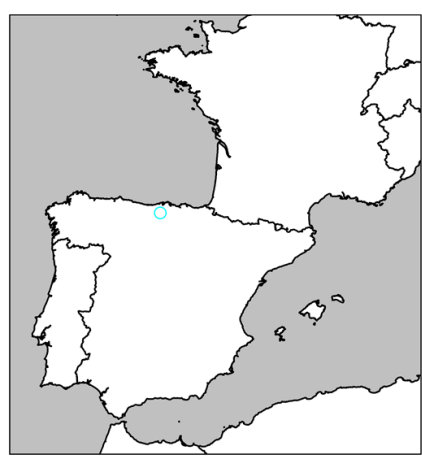

391. Cantabrosoma rogeri Mauriès, 1970

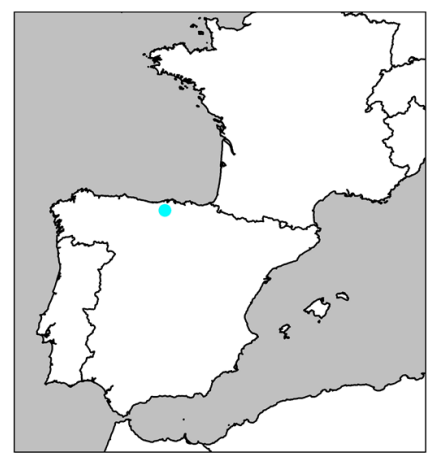

392. Cantabrosoma serrai Mauriès \& Vicente, 1977

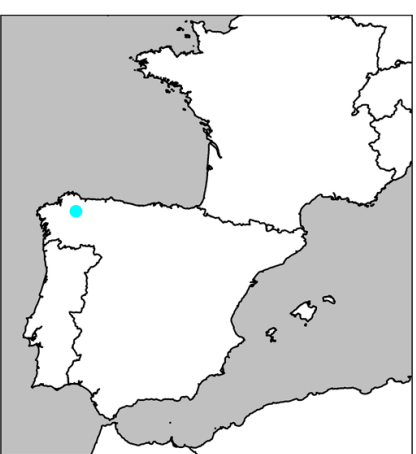

393. Galicisoma biltoni Mauriès, 2015

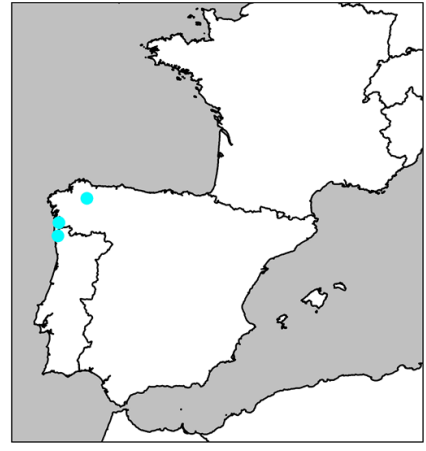

394. Galicisoma desmondkimei Mauriès, 2015

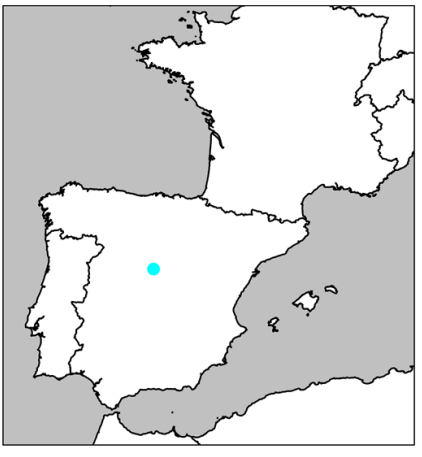

395. Guadarramasoma ramosae

Gilgado, Ledesma, Enghoff \& Mauriès, 2017

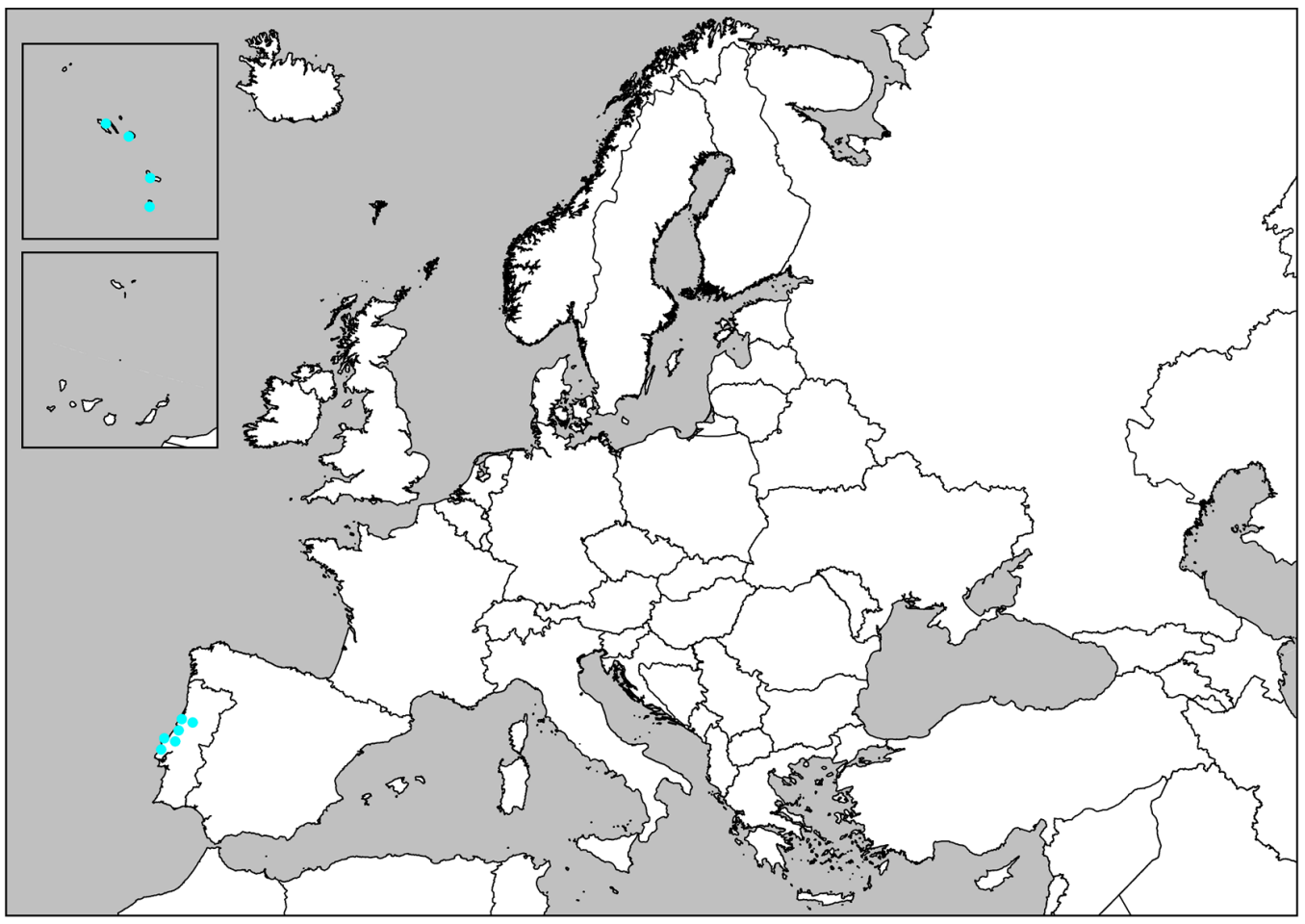

396. Haplobainosoma lusitanum Verhoeff, 1899 


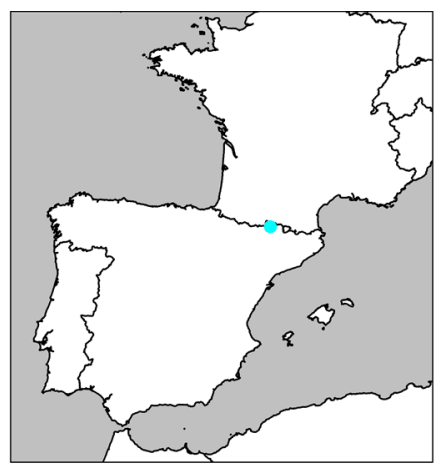

397. Pyreneosoma aranense

Mauriès, 2010

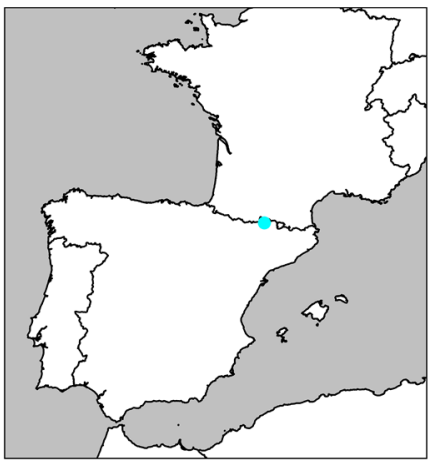

400. Pyreneosoma birosense

Mauriès, 2010

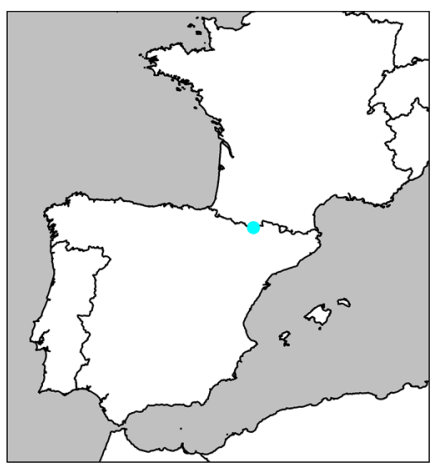

403. Pyreneosoma digitatum

Mauriès, 1959

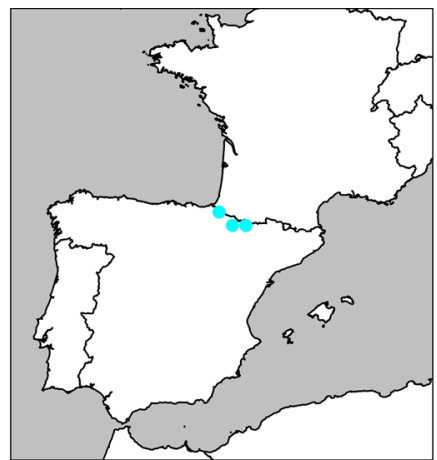

398. Pyreneosoma barbieri

(Mauriès, 1971)

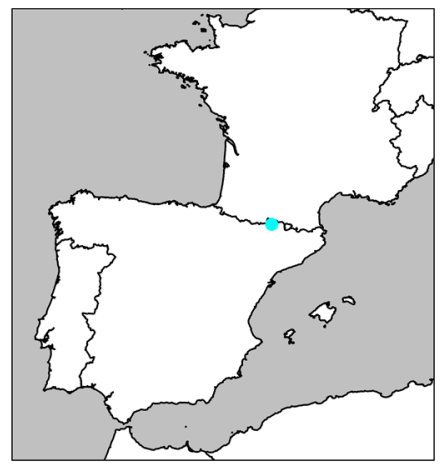

401. Pyreneosoma consoranense Mauriès, 2010

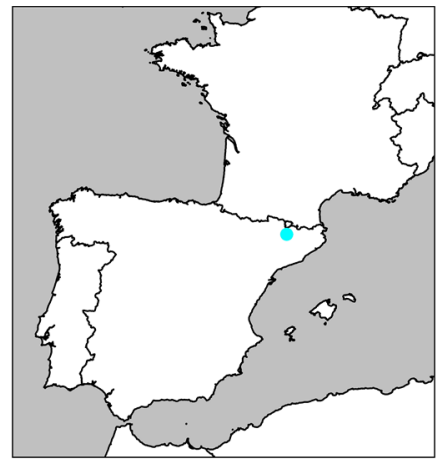

404. Pyreneosoma grandicoxae Mauriès, 2010

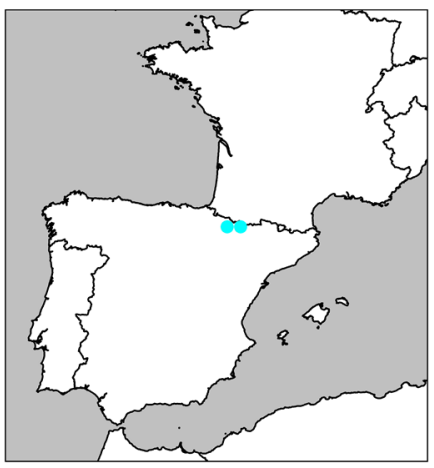

399. Pyreneosoma bessoni Mauriès, 1974

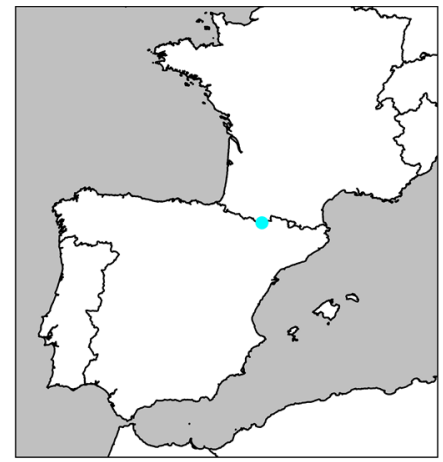

402. Pyreneosoma convenarense Mauriès, 2010

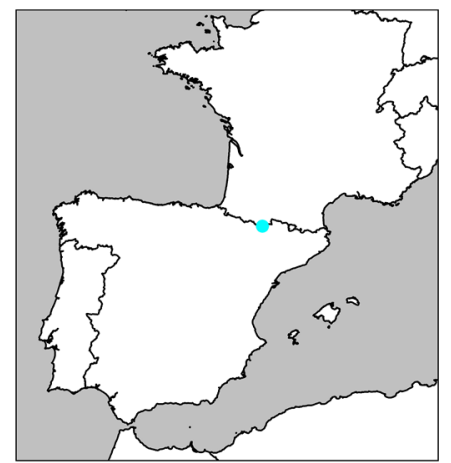

405. Pyreneosoma ribauti Mauriès, 1959 


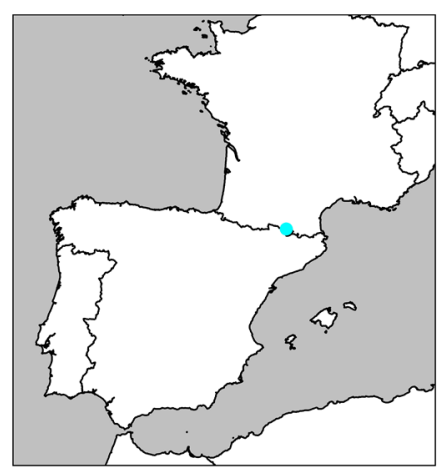

406. Pyreneosoma vicdessosense Mauriès, 2010

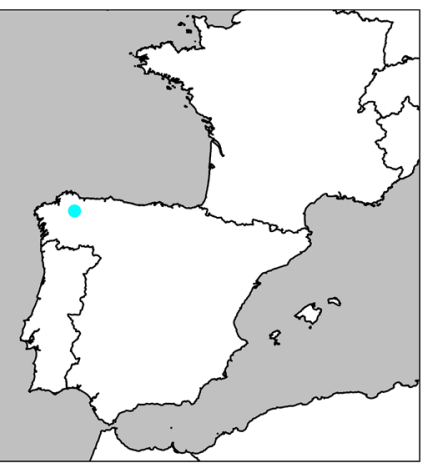

407. Turdulisoma galiciense Mauriès, 2015

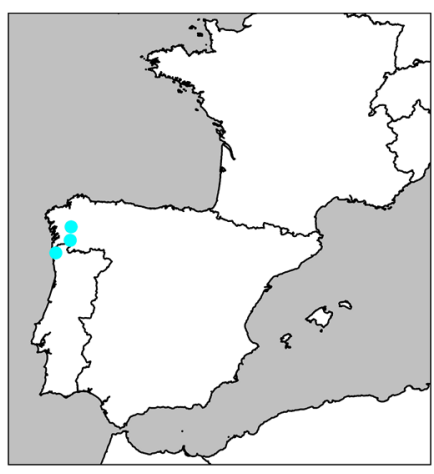

408. Turdulisoma helenreadae Mauriès, 2015

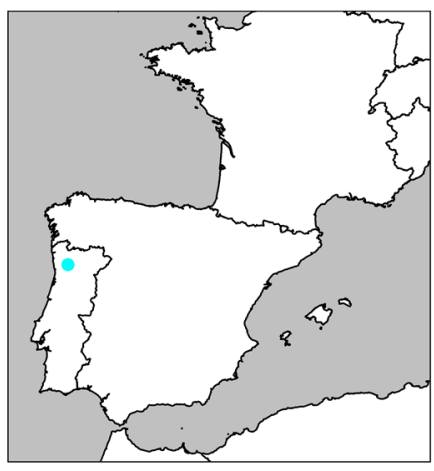

409. Turdulisoma turdulorum Mauriès, 1964

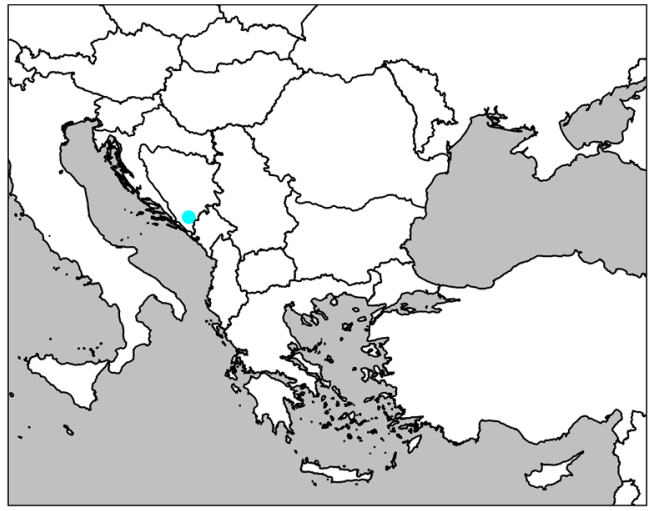

411. Heterolatzelia karlstrasseri Antić \& Makarov, 2015

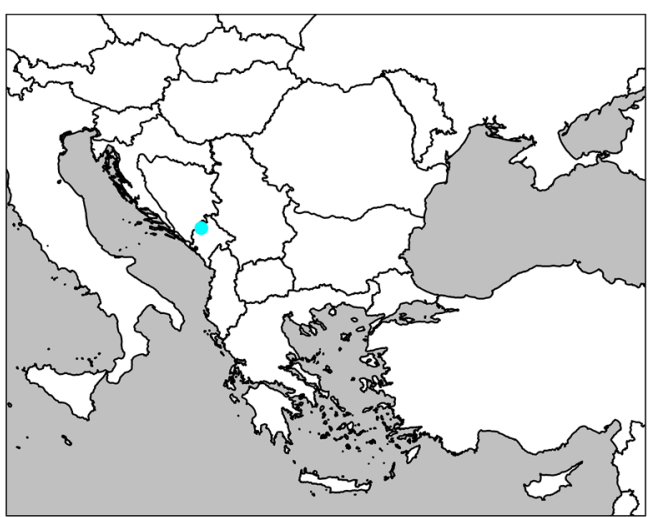

410. Heterolatzelia durmitorensis Gulička, 1968

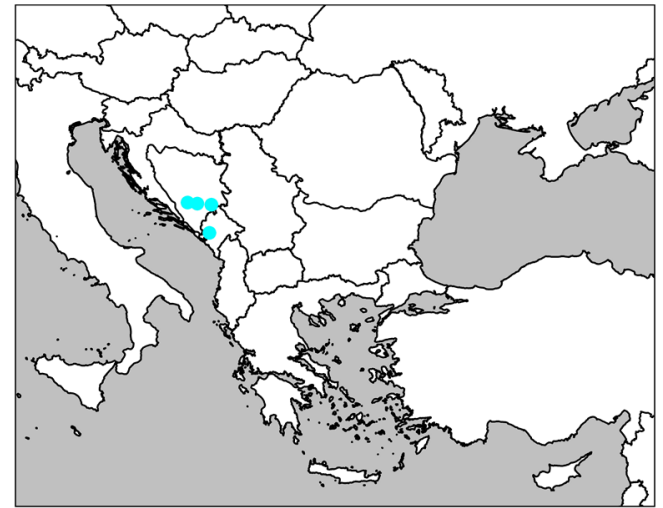

412. Heterolatzelia nivalis Verhoeff, 1897 


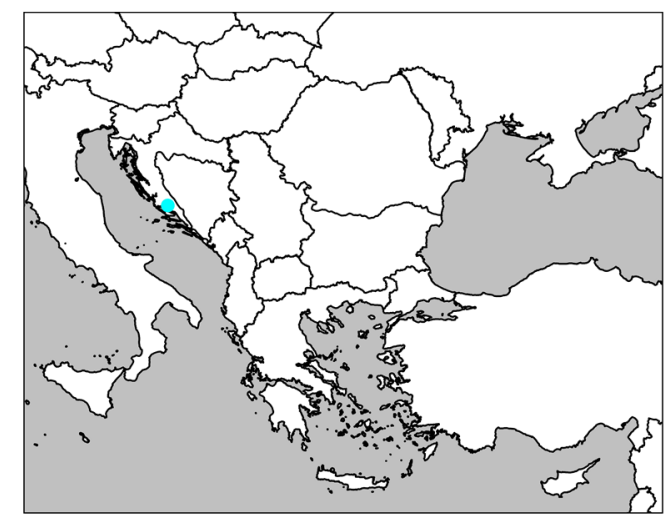

413. Massarilatzelia dugopoljica Makarov \& Rada, 2011

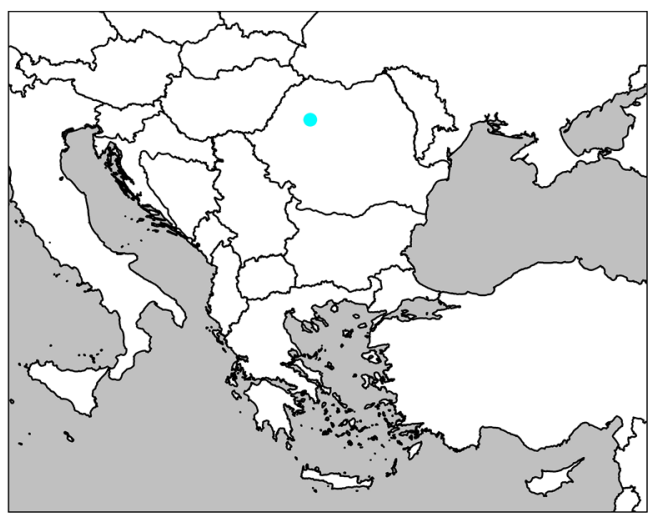

415. Hungarosoma inexpectatum Ceuca, 1967

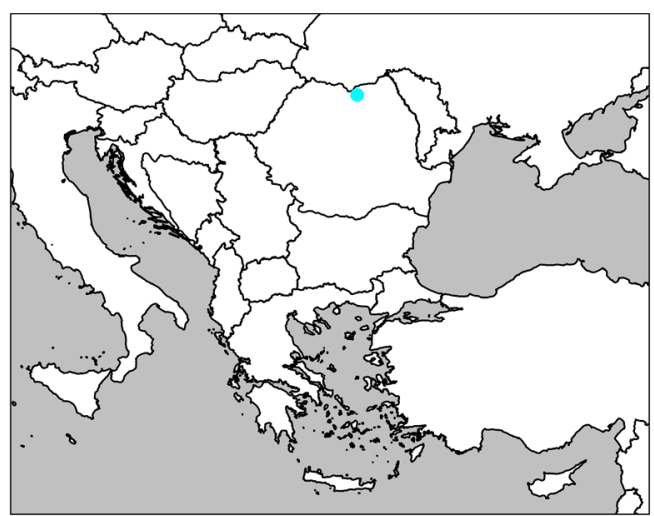

417. Bucovinosoma capusei Tabacaru, 1978

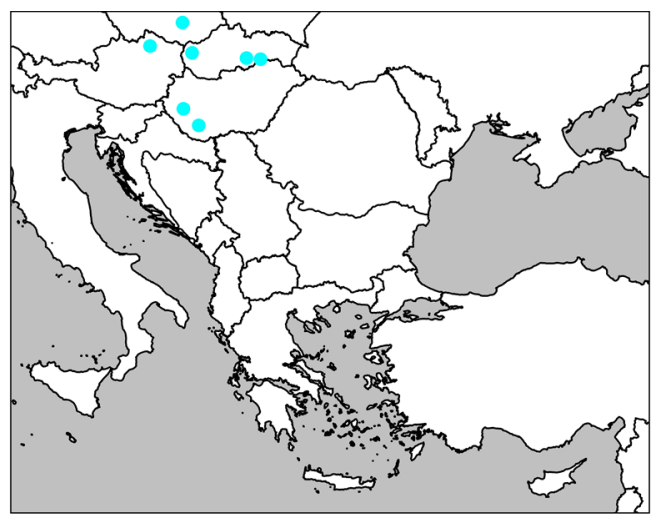

414. Hungarosoma bokori Verhoeff, 1928

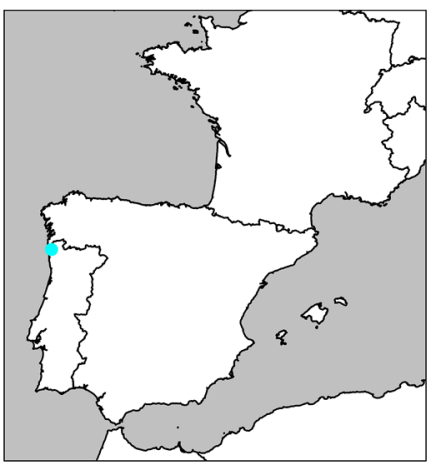

416. Lusitaniosoma machadoi Schubart, 1953

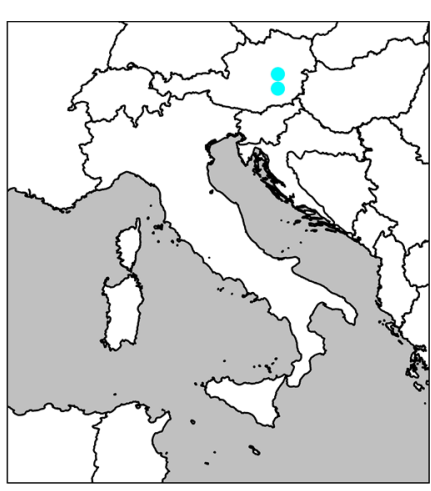

418. Haploporatia cervina Verhoeff, 1929 


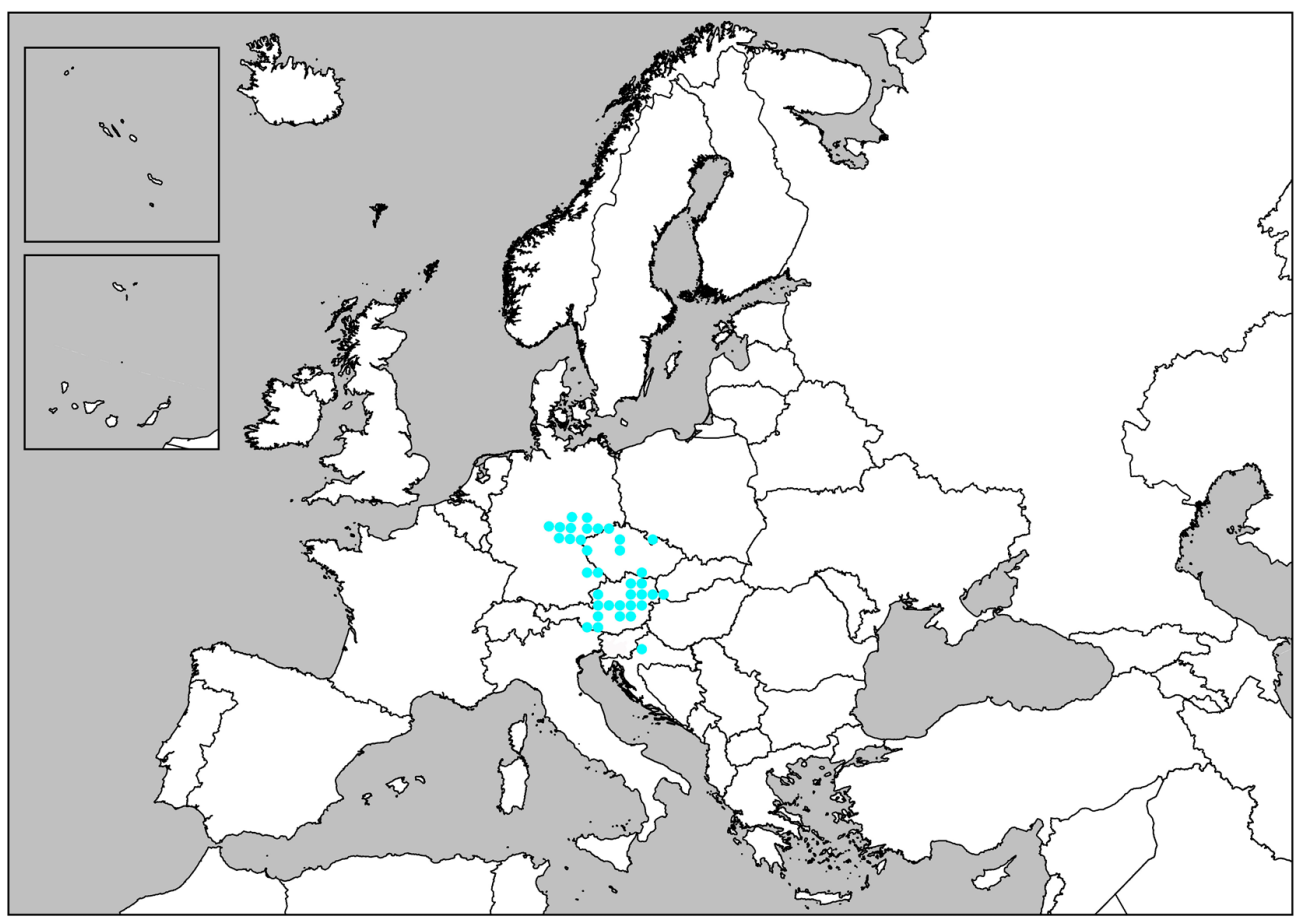

419. Haploporatia eremita Verhoeff, 1909

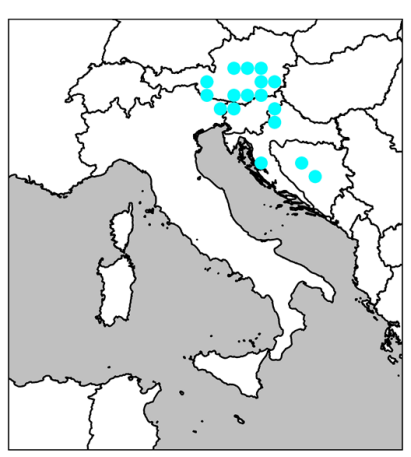

420. Haploporatia similis (Attems, 1895)

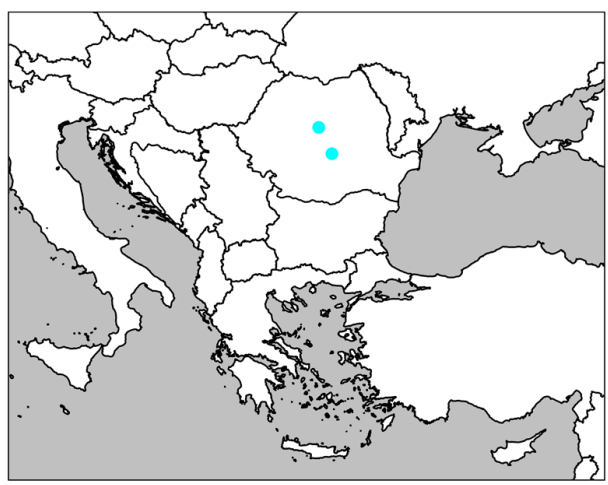

422. Heterobraueria scopifera Verhoeff, 1898

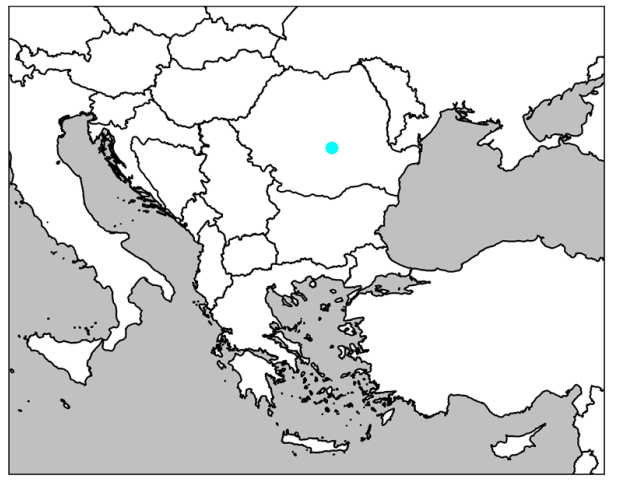

421. Heterobraueria karoli Verhoeff, 1897

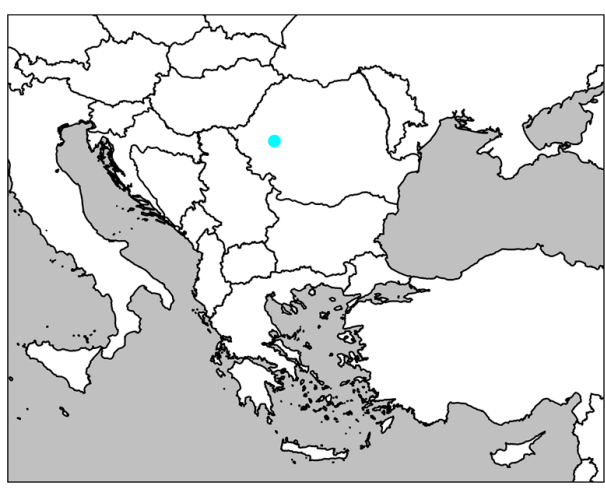

423. Karpatophyllon banaticum Ceuca, 1989 


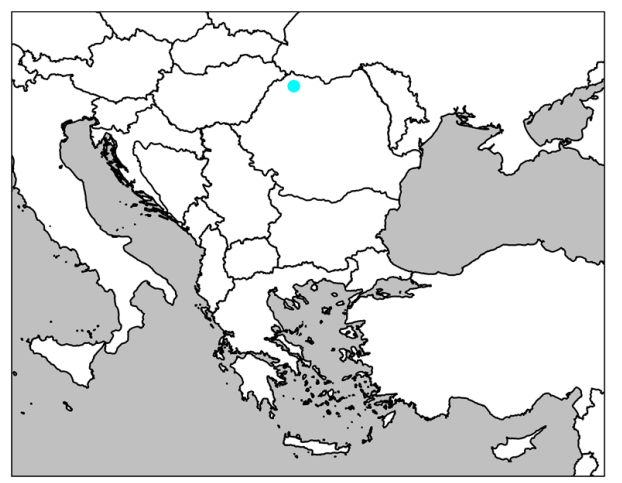

424. Karpatophyllon carpaticum Ceuca, 1985

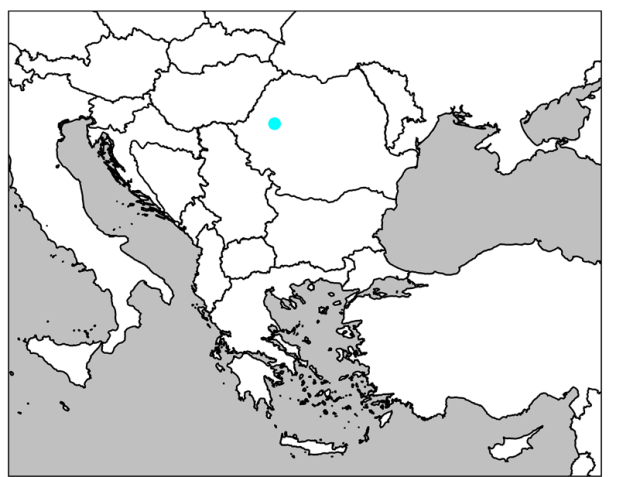

425. Karpatophyllon dacicum Ceuca, 1964

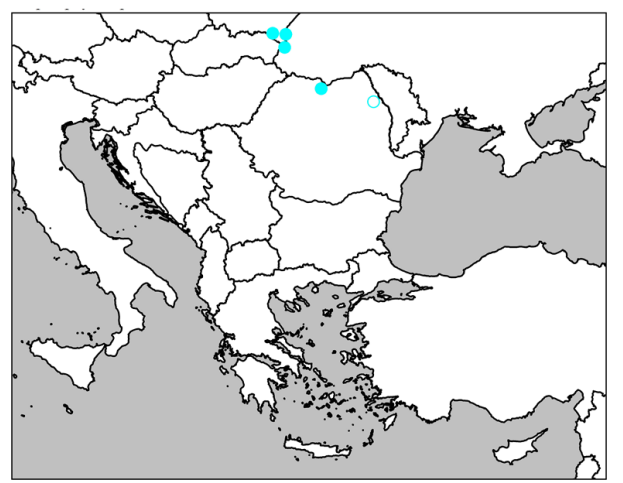

426. Karpatophyllon polinskii Jawłowski, 1928

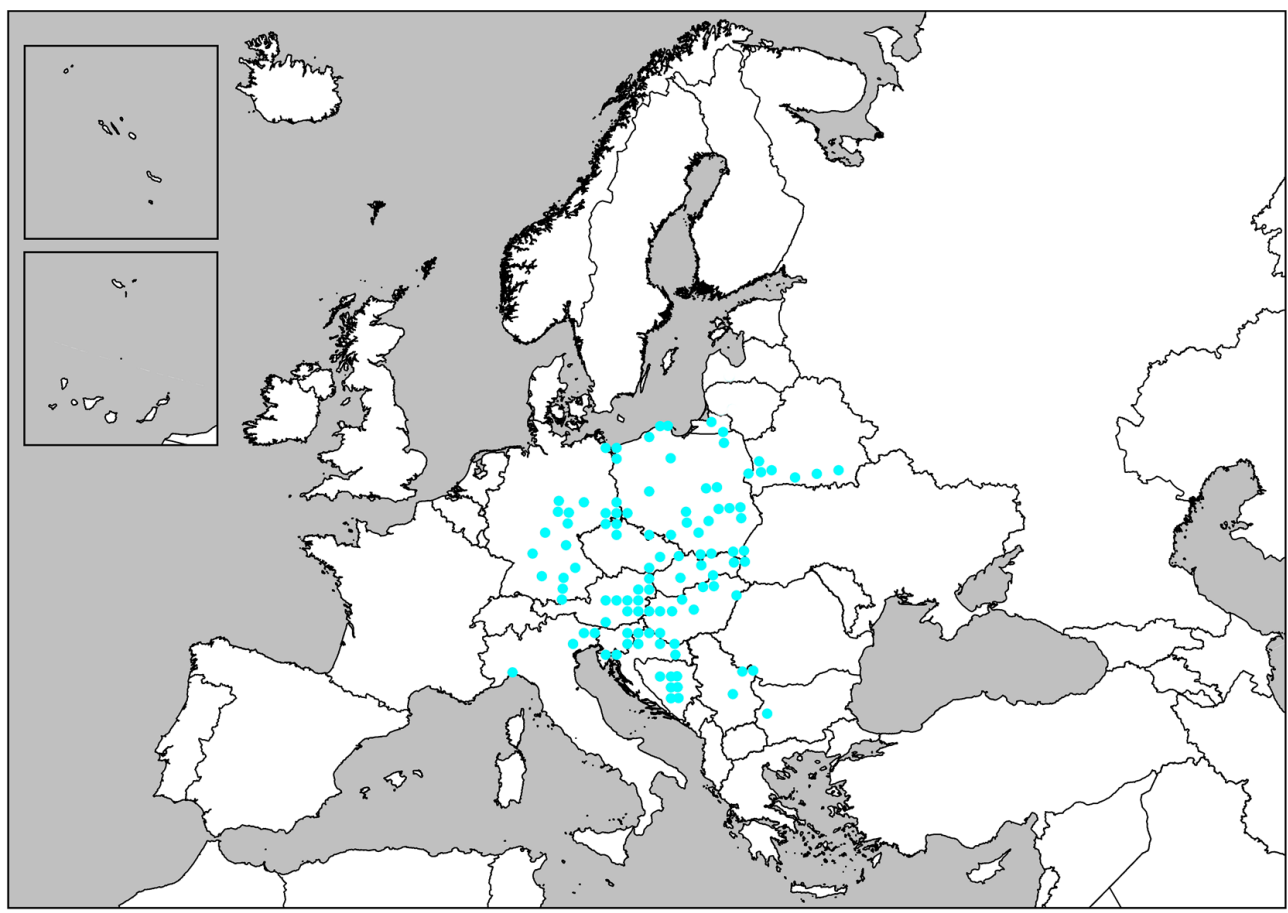

427. Mastigona bosniensis (Verhoeff, 1897) 


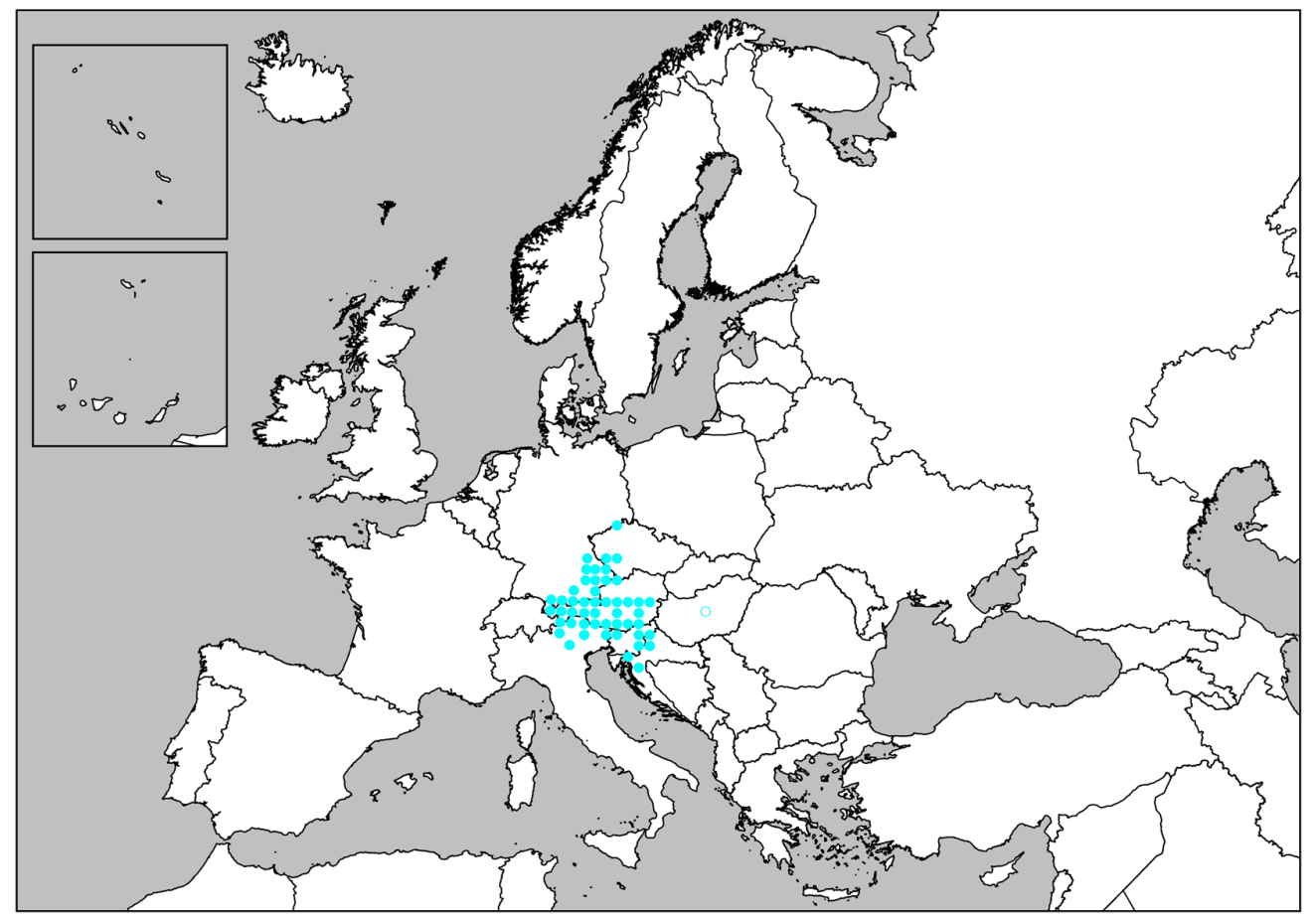

428. Mastigona mutabilis (Latzel, 1884)

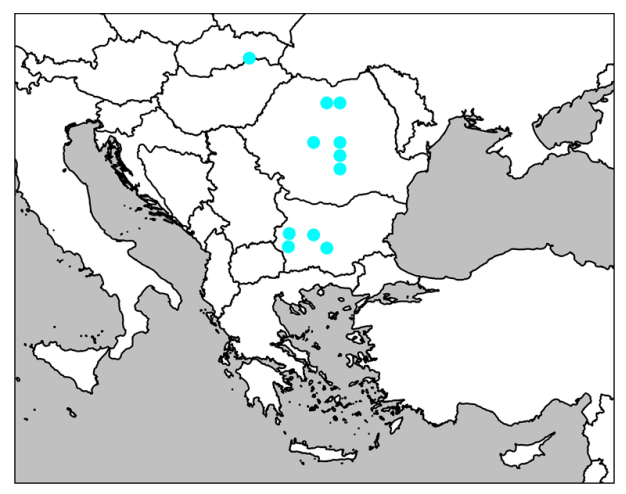

429. Mastigona transsylvanica (Verhoeff, 1897)

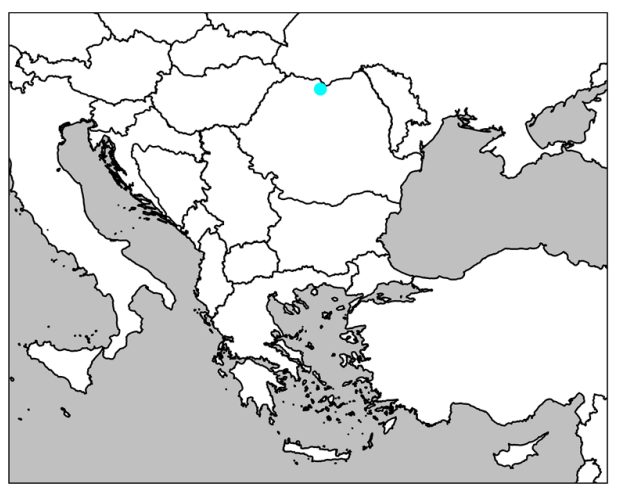

430. Mastigophorophyllon aberratum Ceuca, 1985

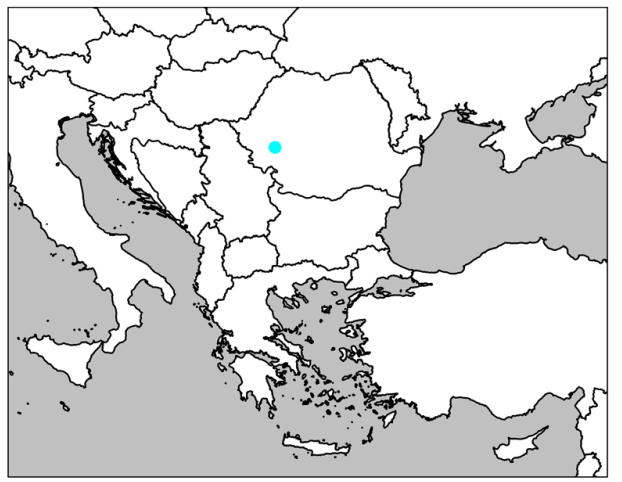

432. Mastigophorophyllon banarescui Ceuca, 1976 


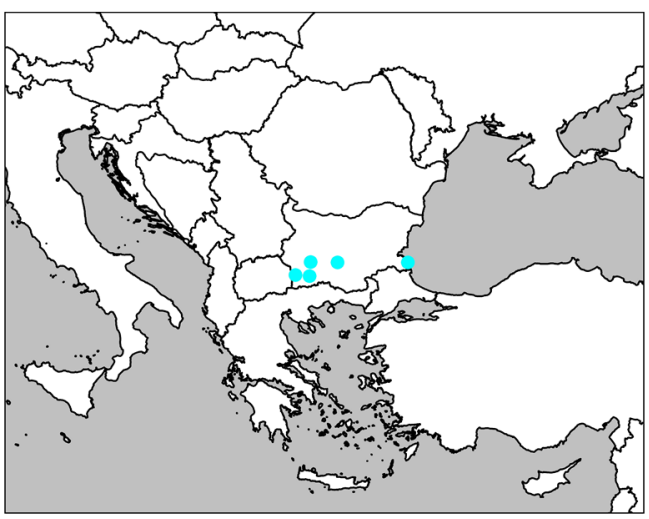

433. Mastigophorophyllon bulgaricum Schubart, 1934

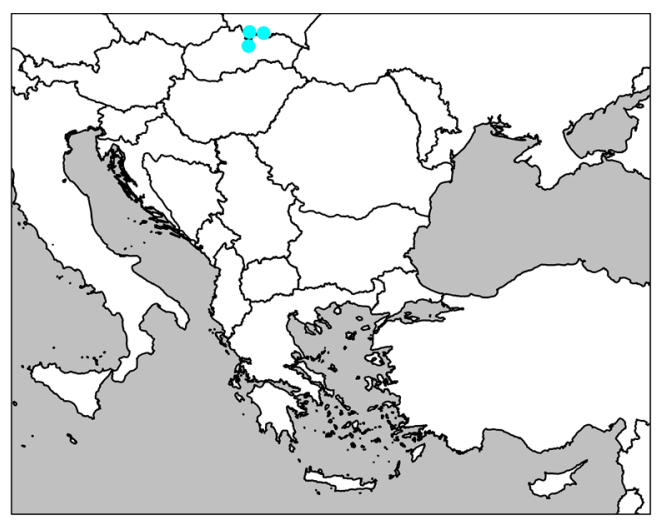

435. Mastigophorophyllon cirriferum Verhoeff, 1899

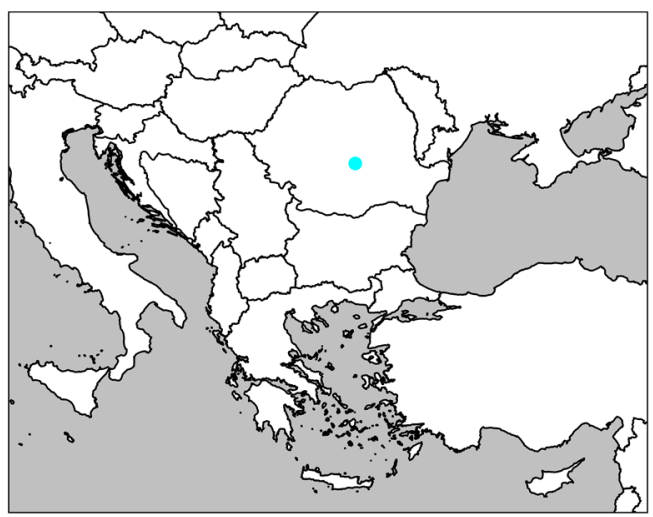

437. Mastigophorophyllon deubeli Verhoeff, 1898

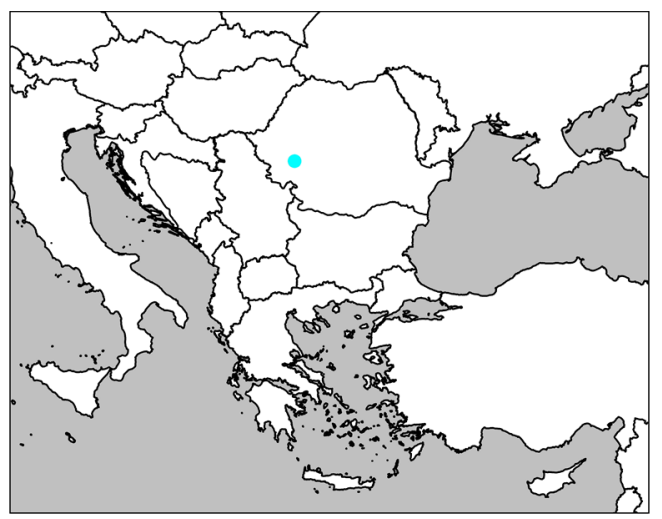

434. Mastigophorophyllon carpaticum Ceuca, 1976

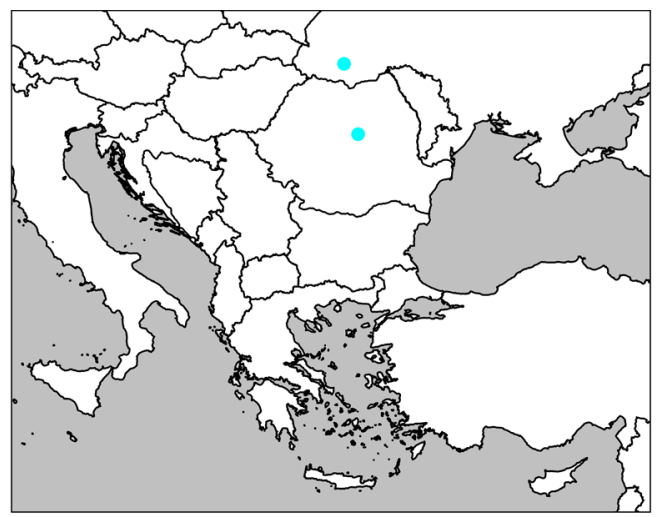

436. Mastigophorophyllon crinitum Attems, 1926

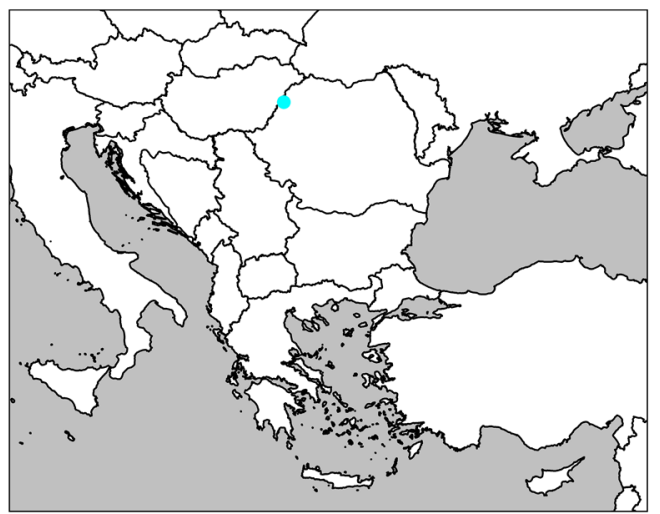

438. Mastigophorophyllon jickelii Verhoeff, 1900 


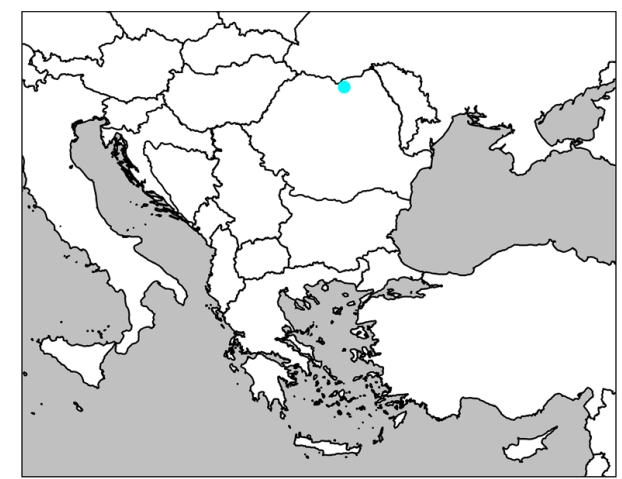

439. Mastigophorophyllon moldavicum Ceuca, Crisan \& Olaru, 1996

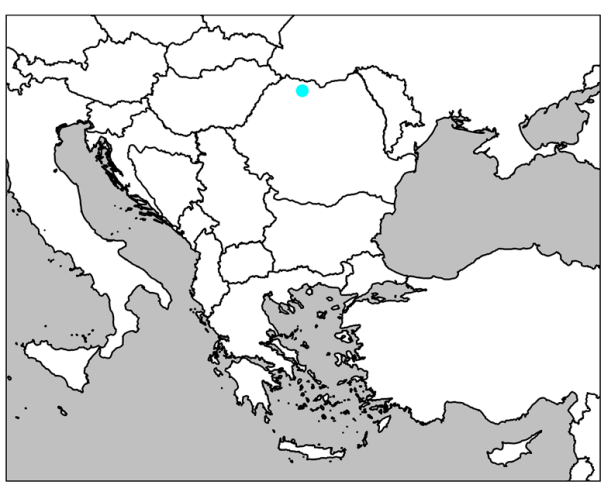

440. Mastigophorophyllon parapenicilligerum Crisan \& Ceuca, 1998

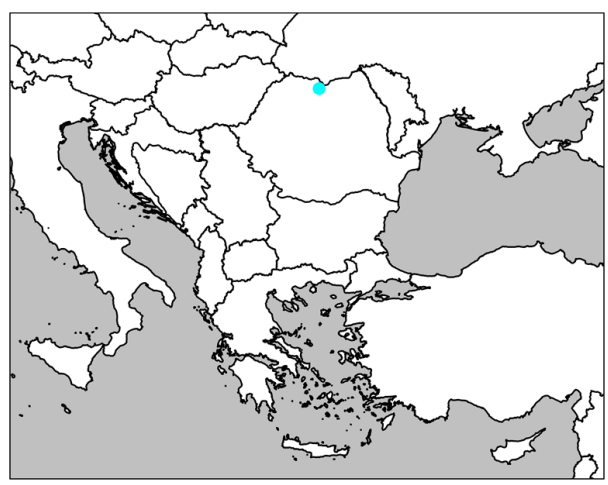

441. Mastigophorophyllon penicilligerum Verhoeff, 1899

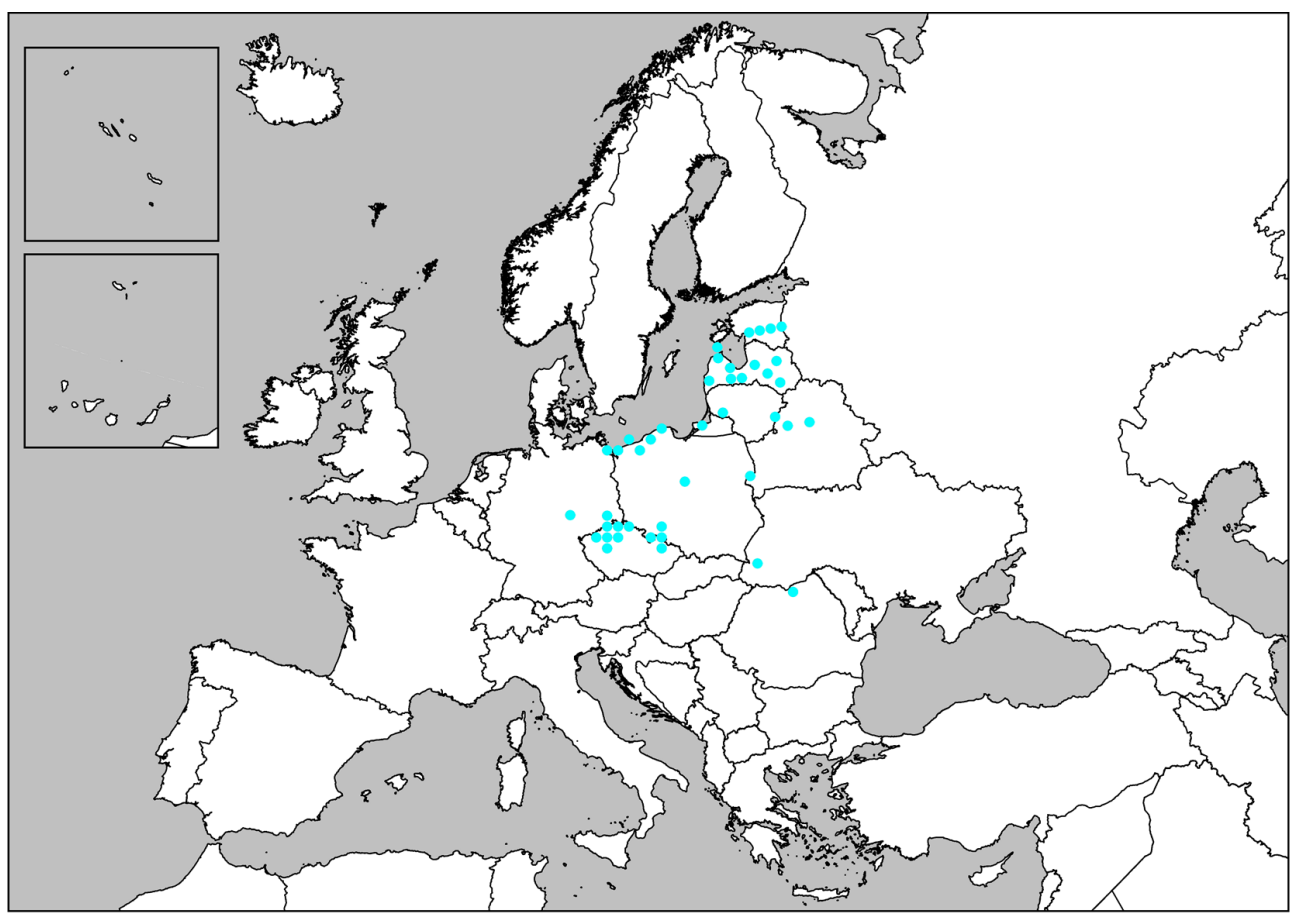

442. Mastigophorophyllon saxonicum Verhoeff, 1910 


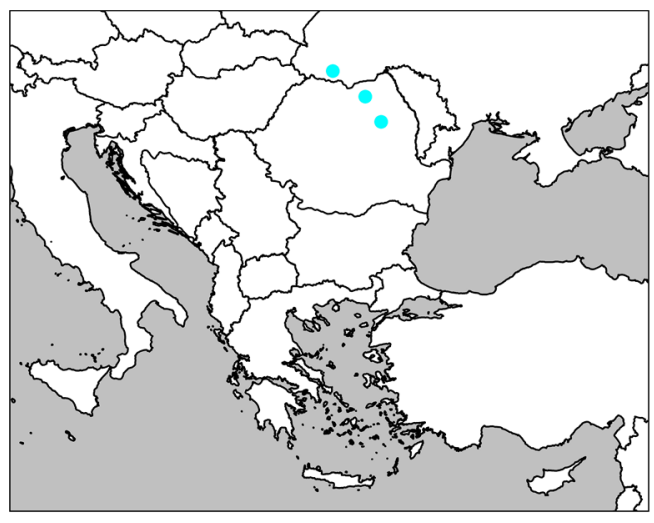

443. Mastigophorophyllon serrulatum Attems, 1926

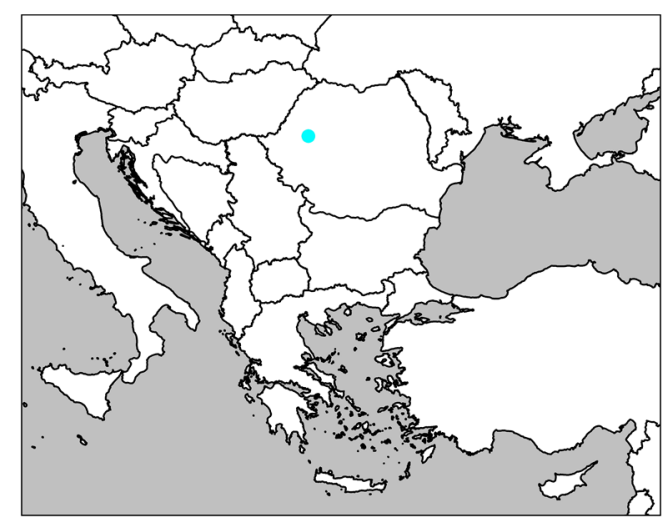

444. Paraporatia racovitzai Ceuca, 1967

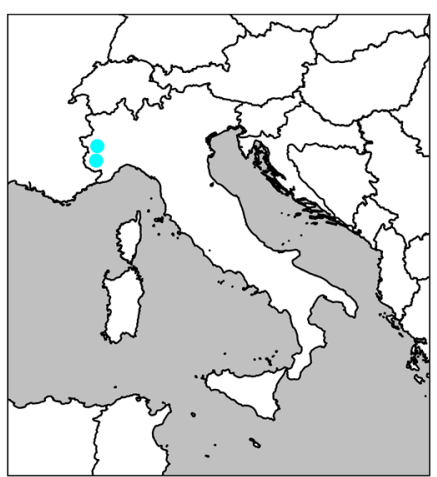

445. Taurinosoma graniticola Verhoeff, 1932

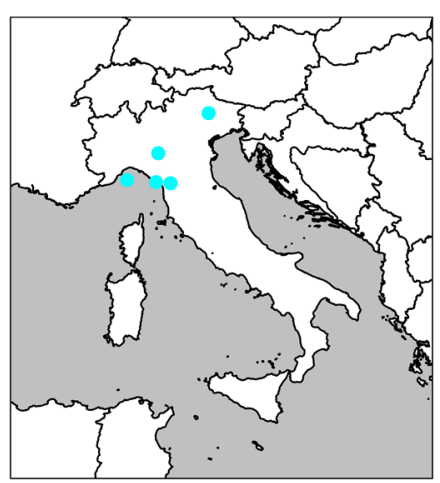

448. Thaumaporatia apuana Verhoeff, 1909

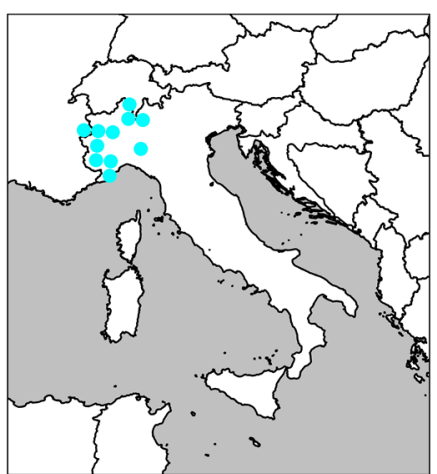

446. Tessinosoma caelebs Verhoeff, 1911

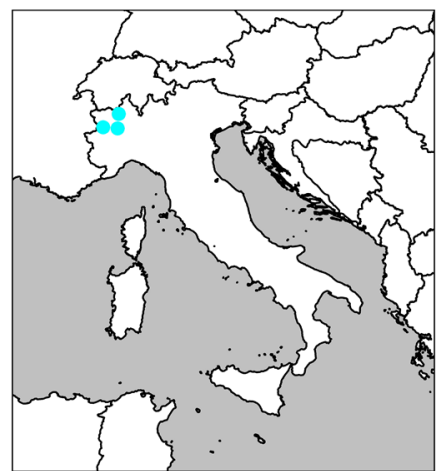

449. Thaumaporatia oropensis Verhoeff, 1936

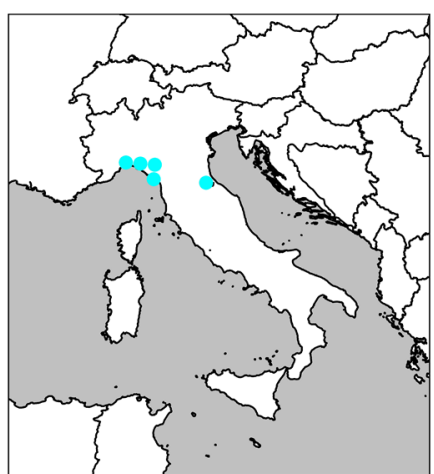

447. Thaumaporatia apenninorum Verhoeff, 1909

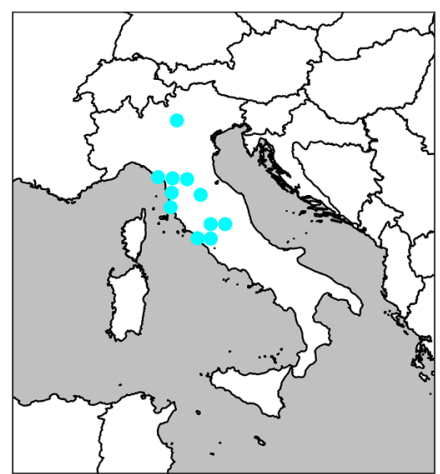

450. Thaumaporatia plumigera (Verhoeff, 1900) 


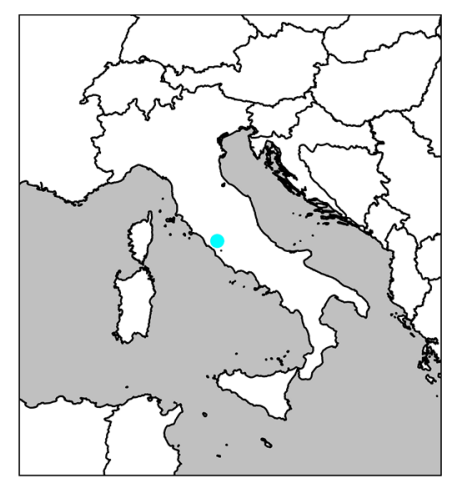

451. Thaumaporatia sorattina Verhoeff, 1951

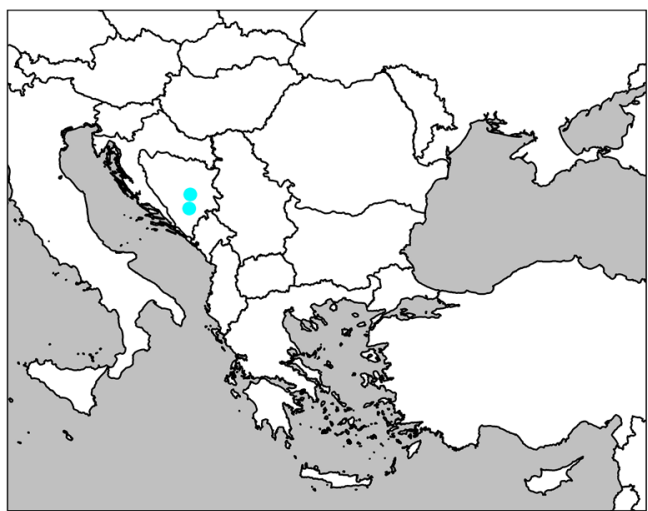

453. Fagina silvatica (Attems, 1904)

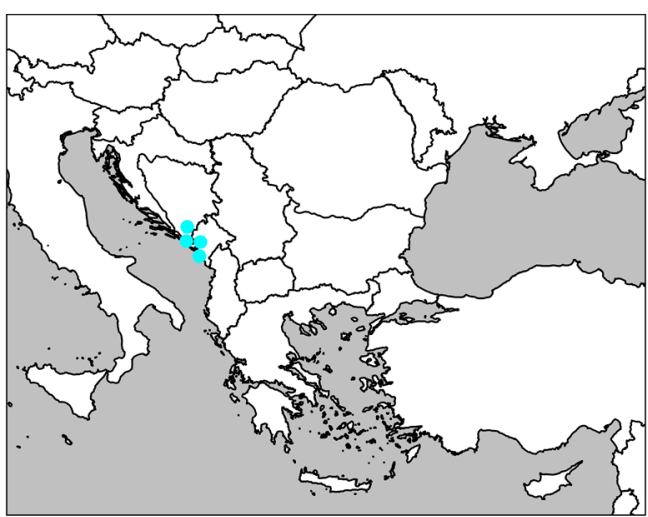

455. Neoatractosoma herzegowinense Verhoeff, 1901

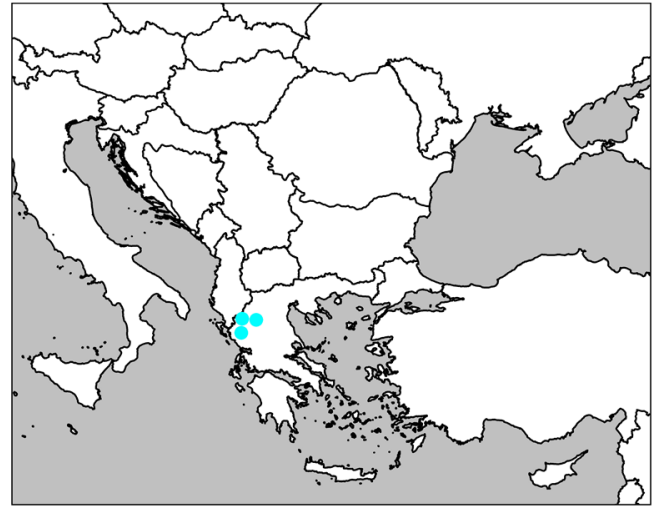

452. Epirosomella loebli Strasser, 1976

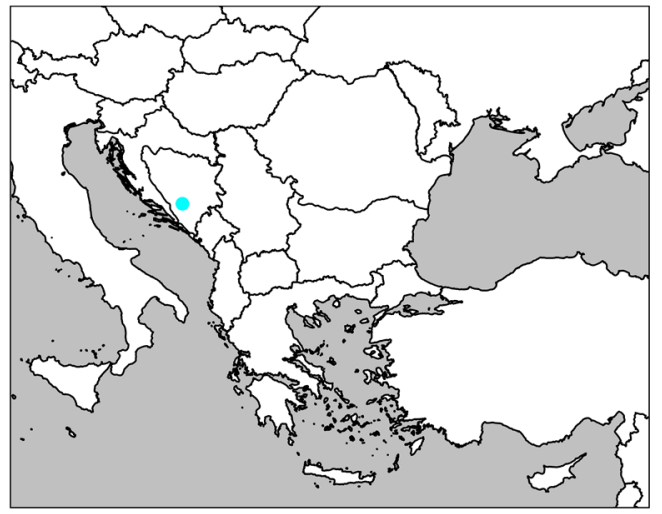

454. Microbrachysoma alpestre Verhoeff, 1897

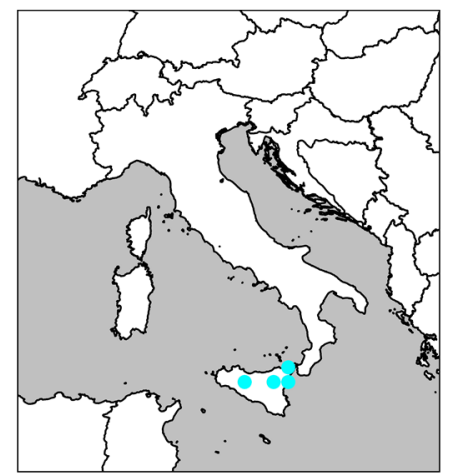

456. Neoatractosoma kleinenbergi Silvestri, 1898 


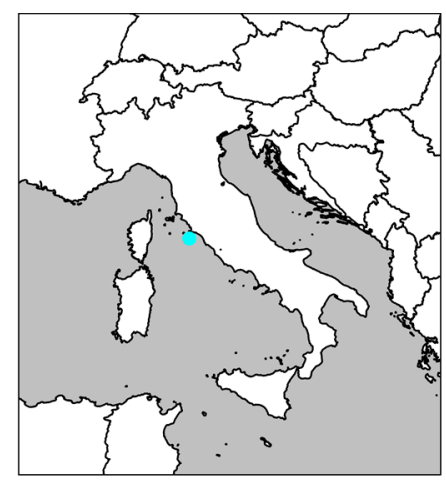

457. Neoatractosoma strandi

Attems, 1927

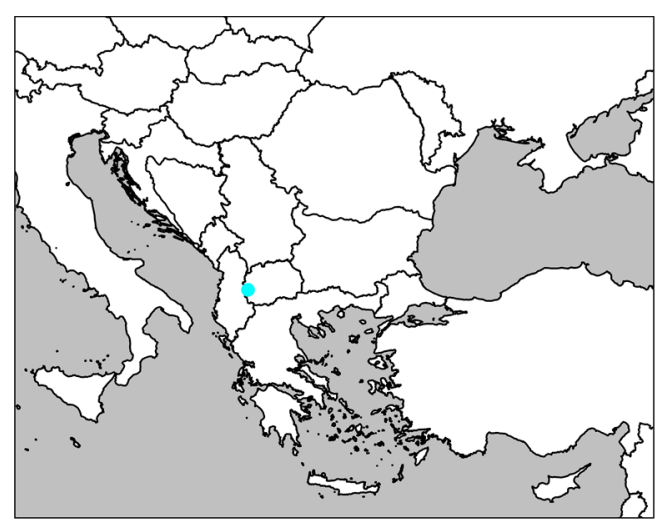

459. Paeonisoma faucium Verhoeff, 1932

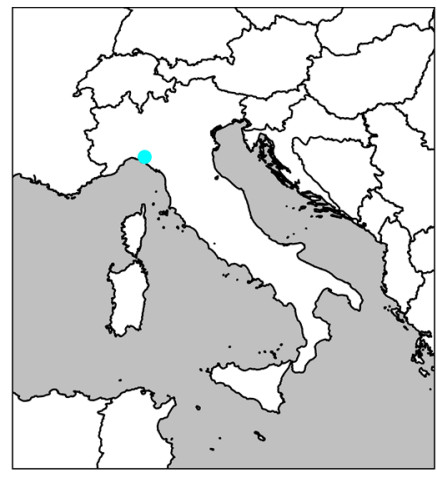

461. Pseudocraspedosoma bensai (Manfredi, 1935)

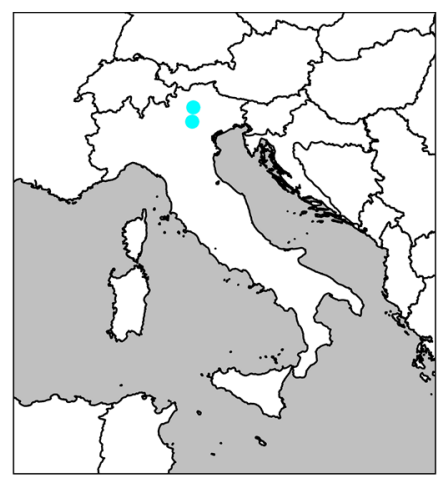

462. Pseudocraspedosoma brentanum (Verhoeff, 1930)

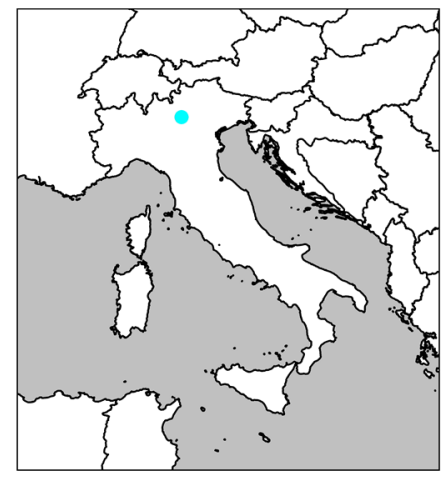

458. Osellasoma caoduroi Mauriès, 1984

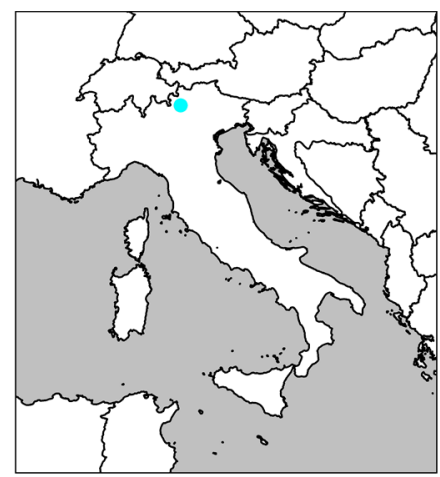

460. Pseudocraspedosoma alpivagum (Verhoeff, 1901)

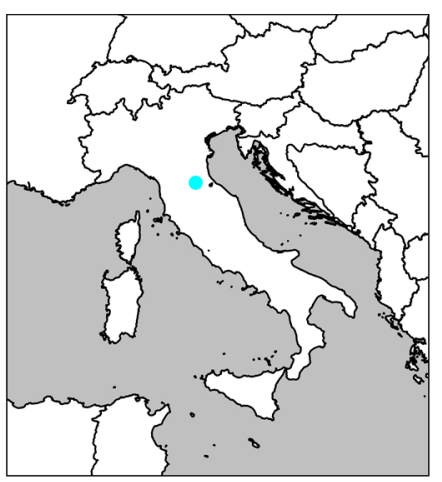

463. Pseudocraspedosoma falteronense (Manfredi, 1951) 


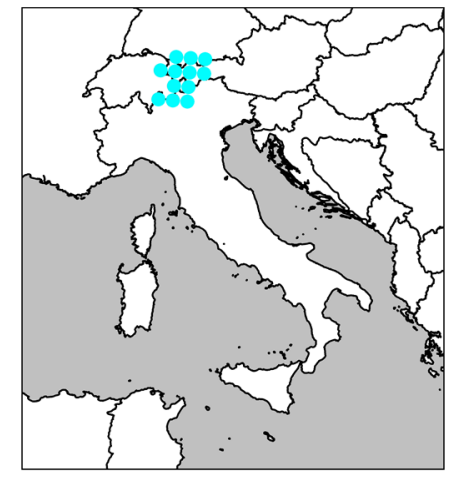

464. Pseudocraspedosoma grypischium (Rothenbühler, 1900)

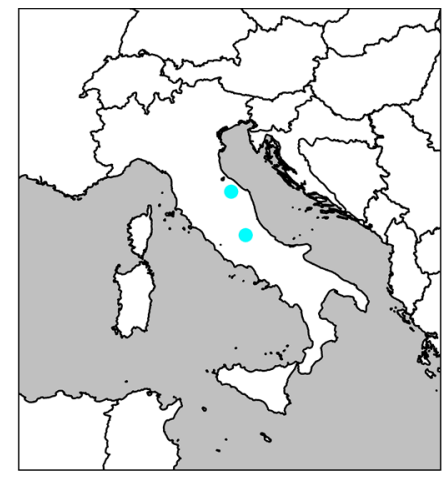

465. Pseudocraspedosoma nemorense Silvestri, 1898

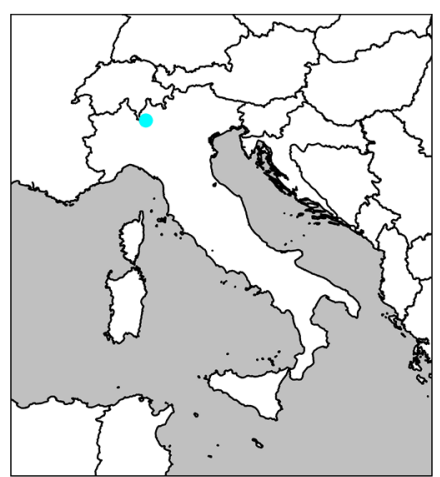

466. Pseudocraspedosoma peniculorum (Verhoeff, 1910)

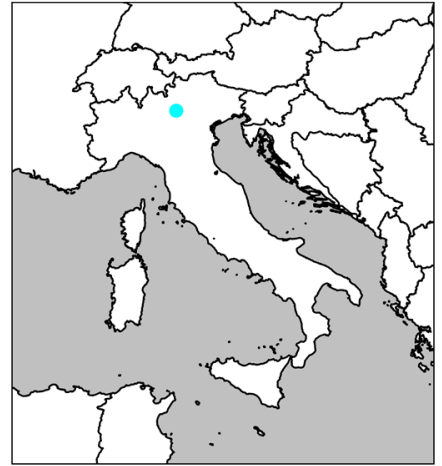

467. Pseudocraspedosoma vestonense (Verhoeff, 1934)

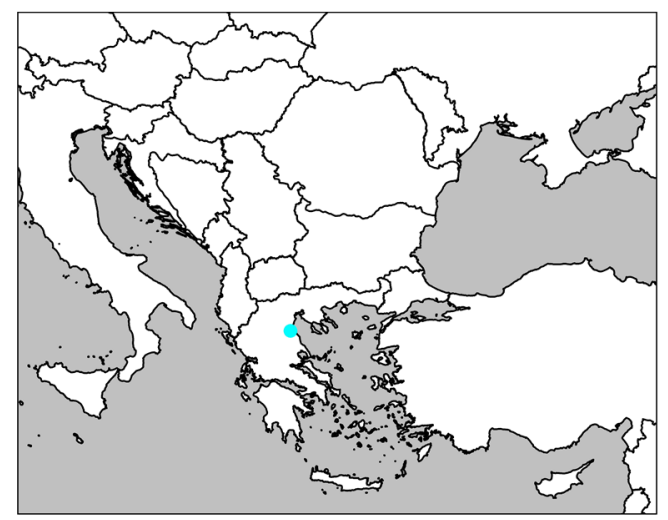

469. Schizmohetera olympica Mauriès, 2003

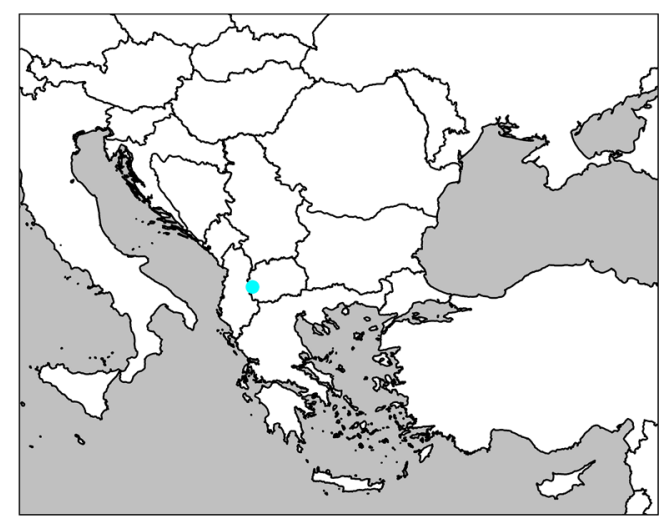

468. Schizmohetera curcici Makarov, 2001

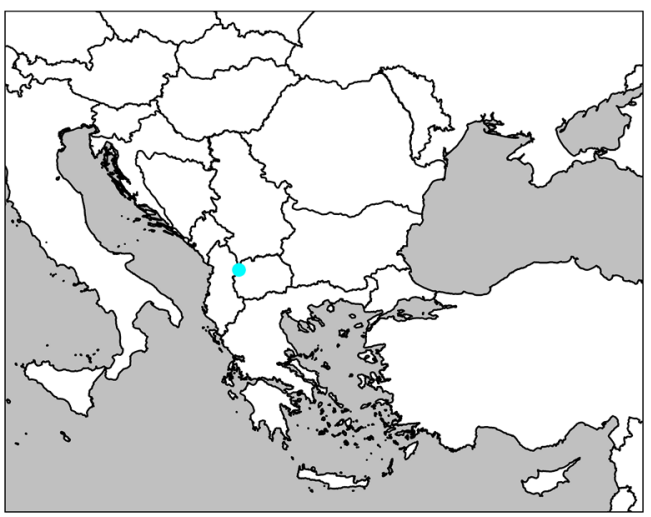

470. Schizmohetera sketi Mršić, 1987 


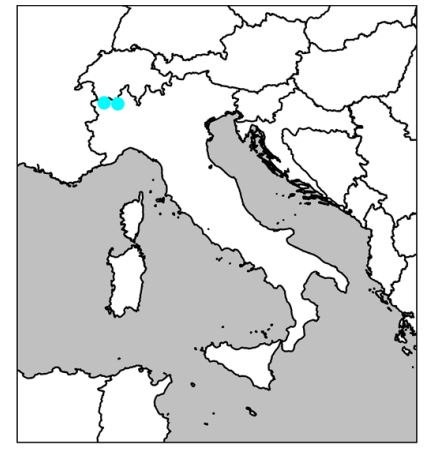

471. Trimerophorella ornata (Faës, 1902)

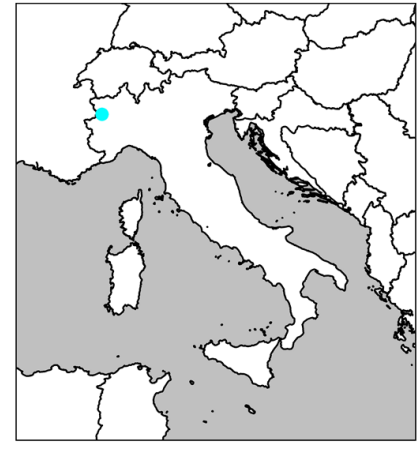

472. Trimerophorella paradisia Meyer, 1983

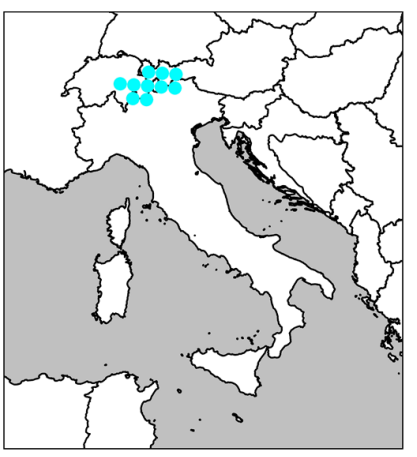

473. Trimerophorella rhaetica (Rothenbühler, 1901)

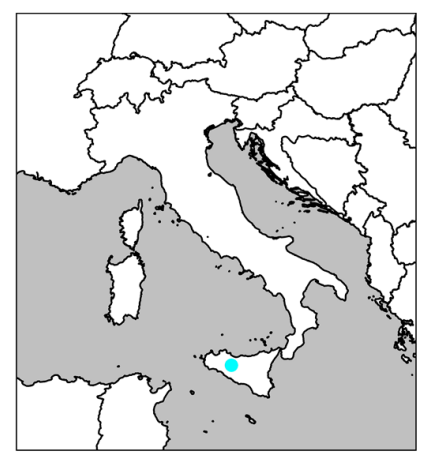

474. Brachytropisoma giardinae Silvestri, 1898

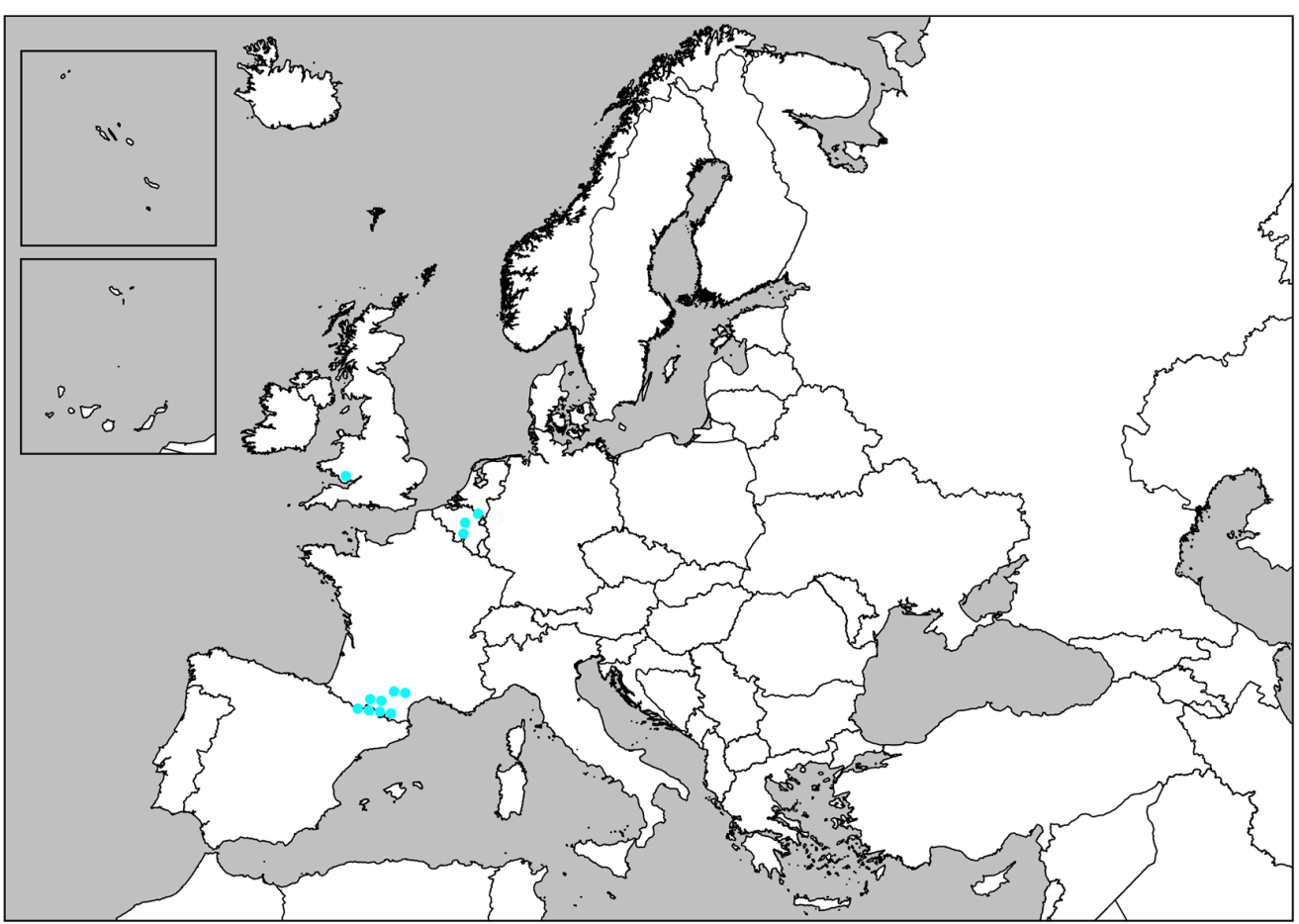

475. Ceratosphys amoena Ribaut, 1920 


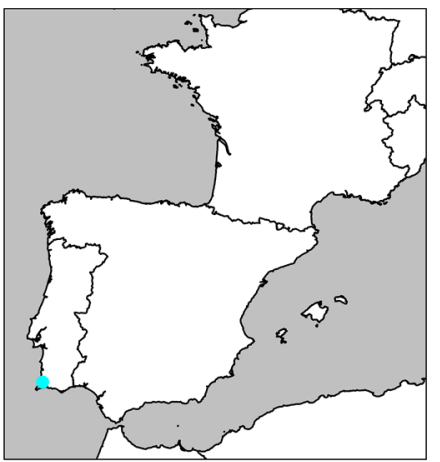

476. Ceratosphys angelieri Mauriès, 1964

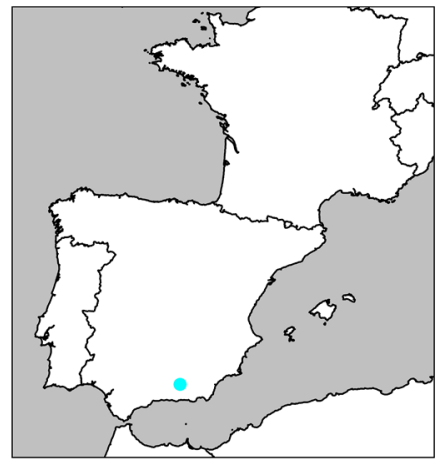

479. Ceratosphys cryodeserti Gilgado, Mauriès \& Enghoff, 2015

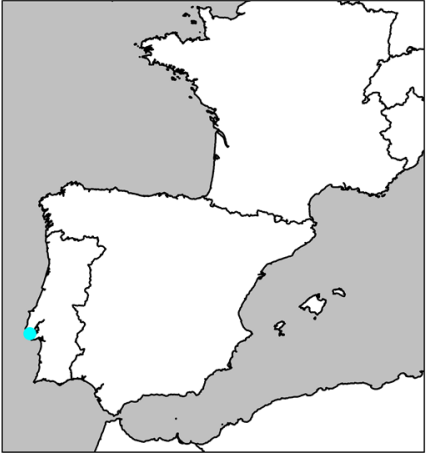

477. Ceratosphys bakeri Mauriès, 1990

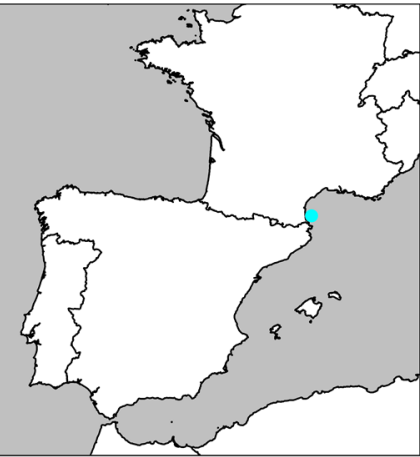

478. Ceratosphys banyulensis Brolemann, 1926

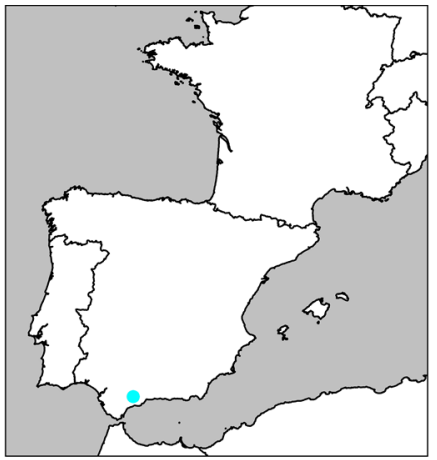

480. Ceratosphys deharvengi Mauriès, 1978

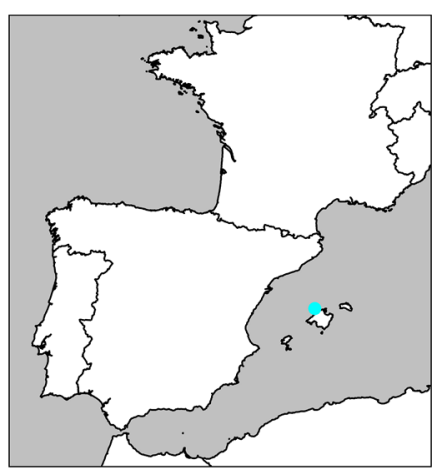

481. Ceratosphys escolai Mauriès, 2013

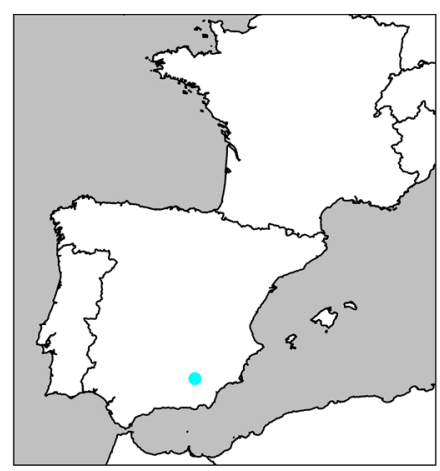

482. Ceratosphys fernandoi Mauriès, 2014

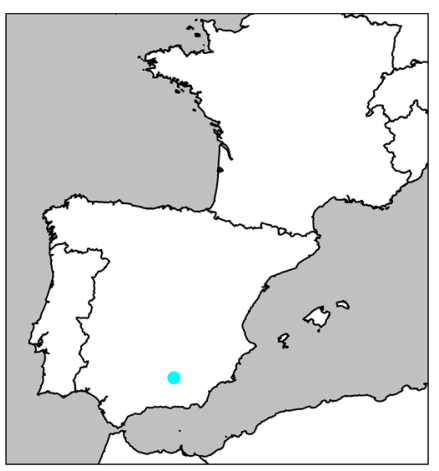

483. Ceratosphys flammeola Mauriès, 2014

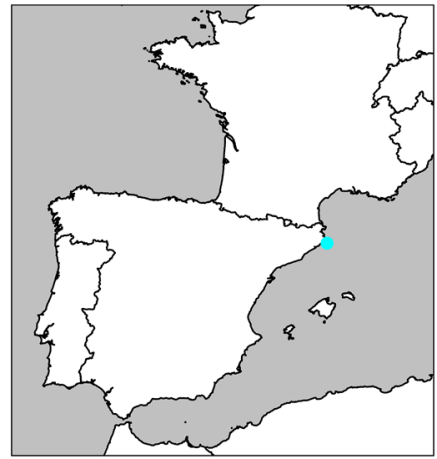

484. Ceratosphys geronensis Mauriès, 1963 


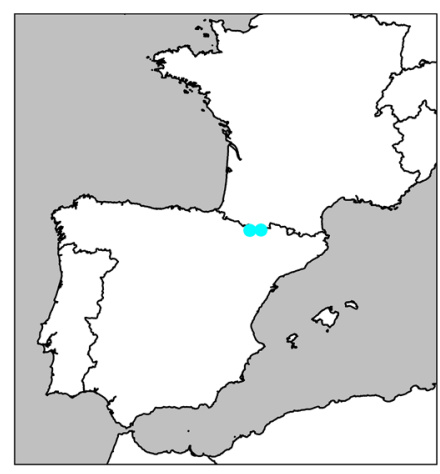

485. Ceratosphys guttata

Ribaut, 1956

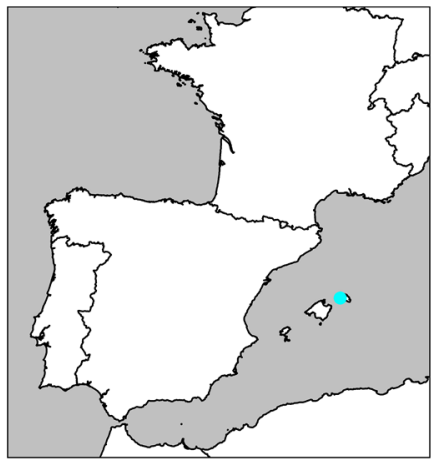

488. Ceratosphys mariacristinae Mauriès, 2013

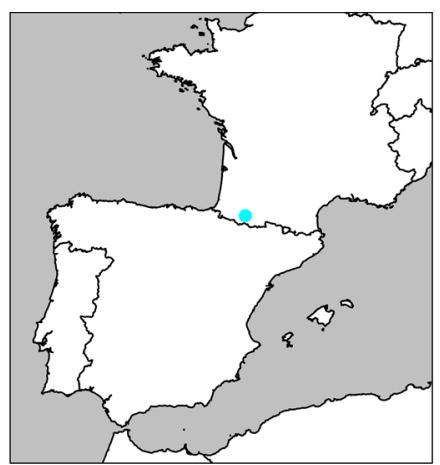

491. Ceratosphys occidentalis Mauriès, 1976

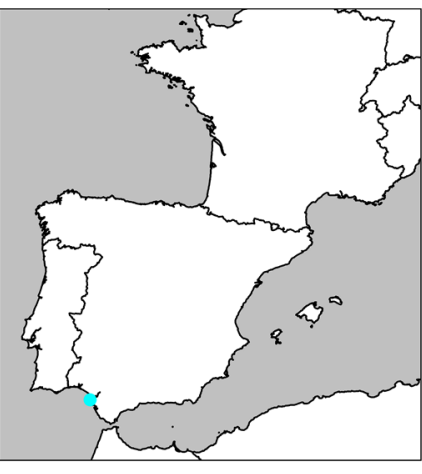

486. Ceratosphys hispanica Ribaut, 1920

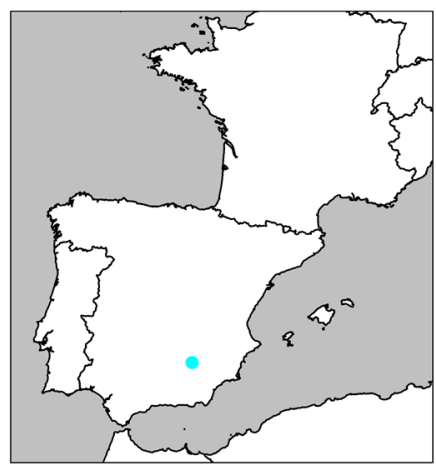

487. Ceratosphys jabaliensis Mauriès, 2013

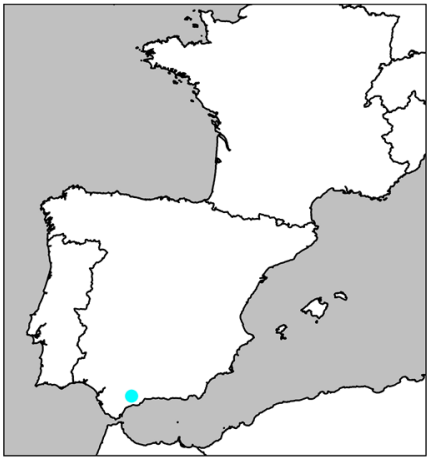

490. Ceratosphys nodipes (Attems, 1952)

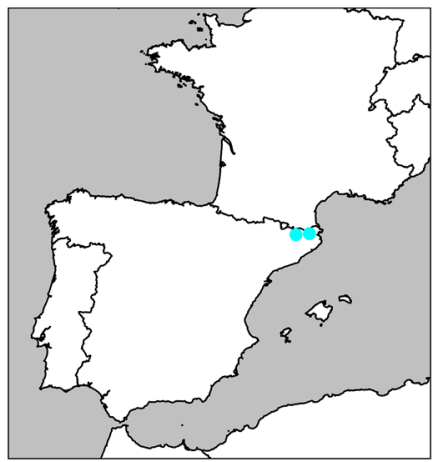

492. Ceratosphys picta Ribaut, 1951 


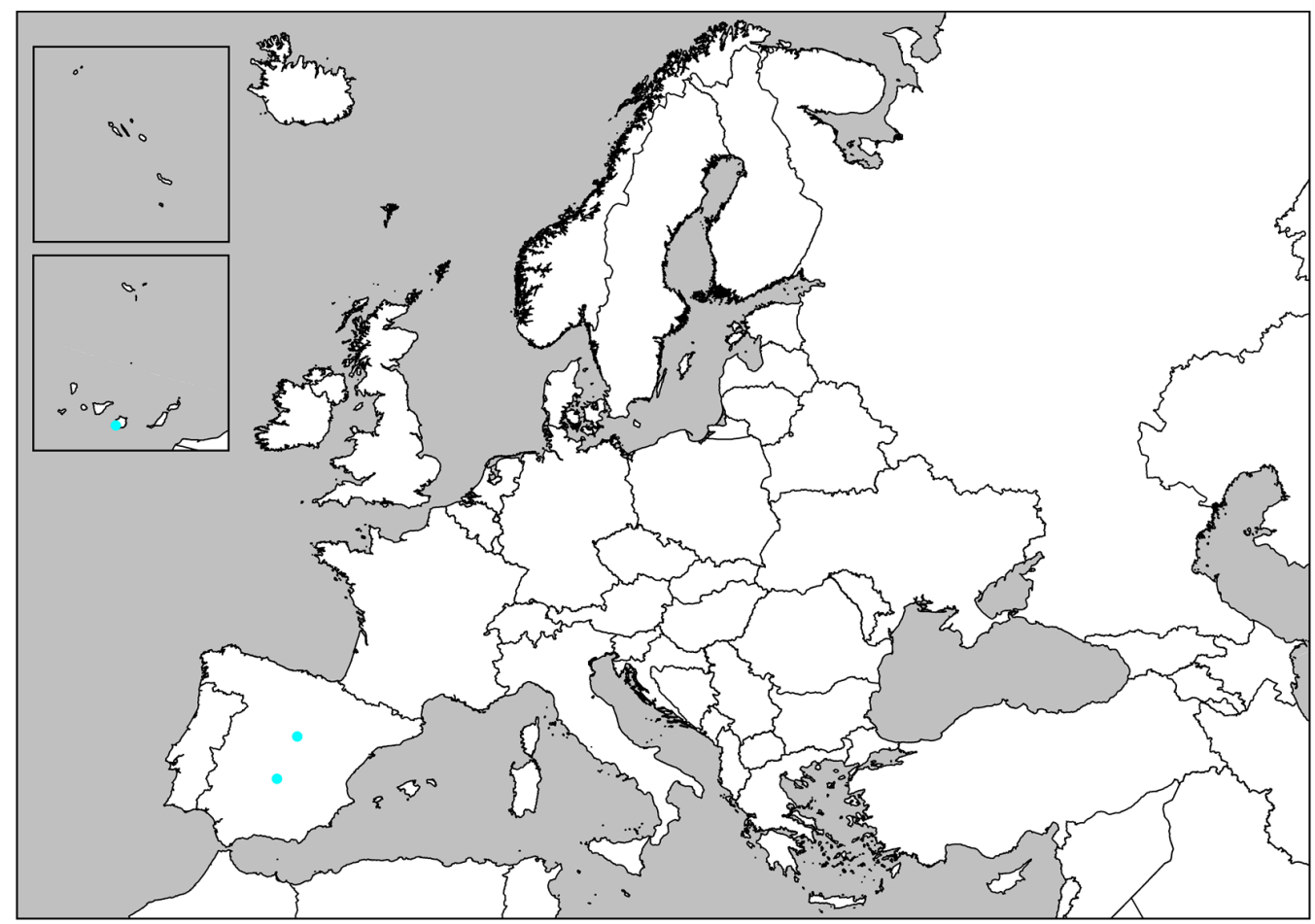

493. Ceratosphys poculifer (Brolemann, 1920)

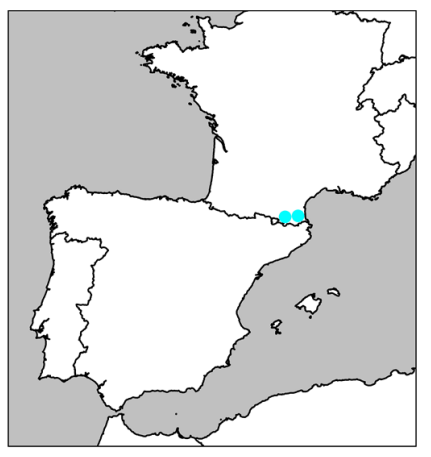

494. Ceratosphys simoni Ribaut, 1920

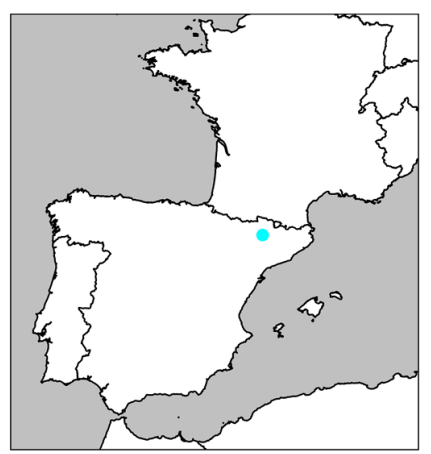

497. Ceratosphys toniserrai Mauriès, 2013

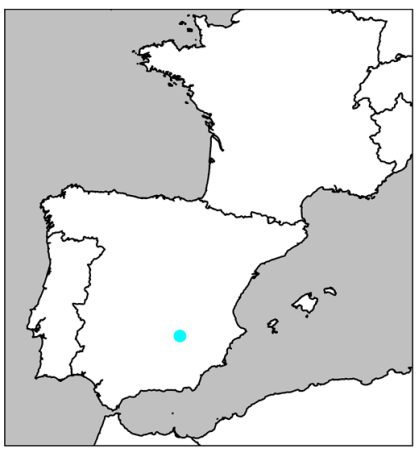

495. Ceratosphys solanasi (Mauriès \& Vicente, 1978)

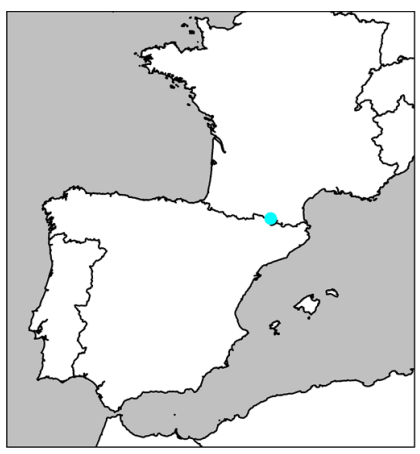

498. Ceratosphys vandeli Mauriès, 1963

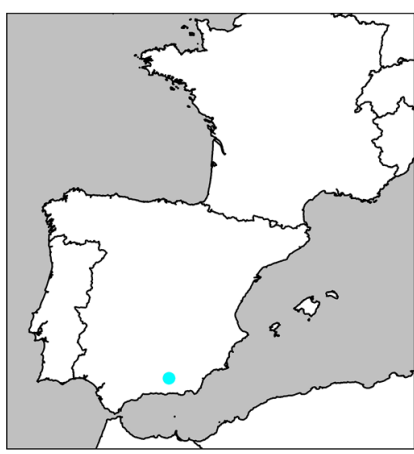

496. Ceratosphys soutadei Mauriès, 1969

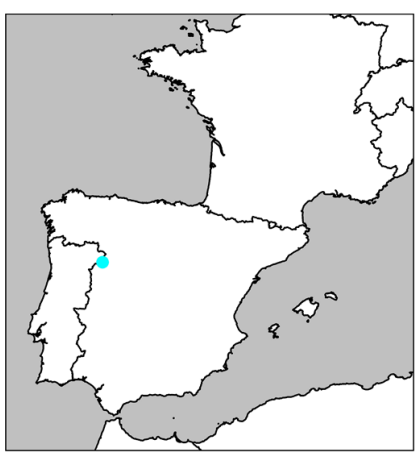

499. Ceratosphys vicenteae Mauriès, 1990 


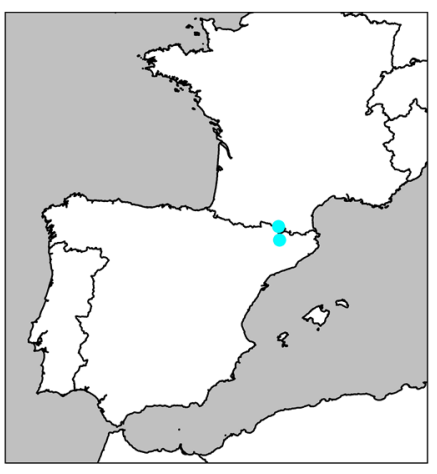

500. Hispaniosoma racovitzai

Ribaut, 1913

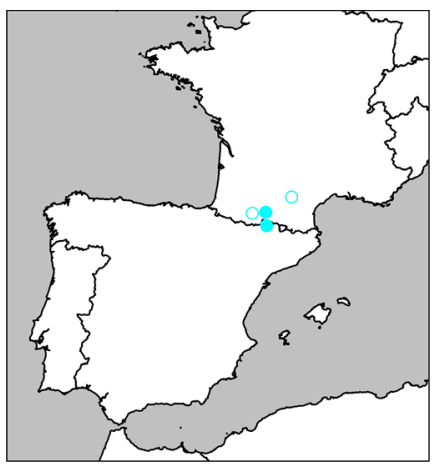

503. Marquetiella pyrenaica ( Ribaut, 1905)

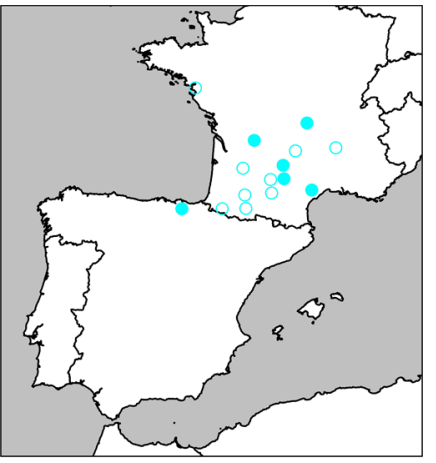

506. Opisthocheiron elegans Ribaut, 1922

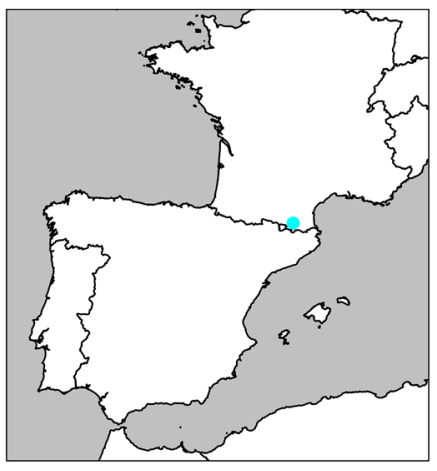

501. Marquetiella auriculata (Ribaut, 1920)

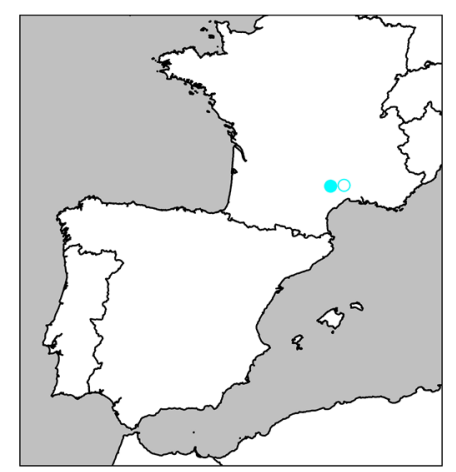

504. Opisthocheiron canayerensis Mauriès \& Geoffroy, 1982

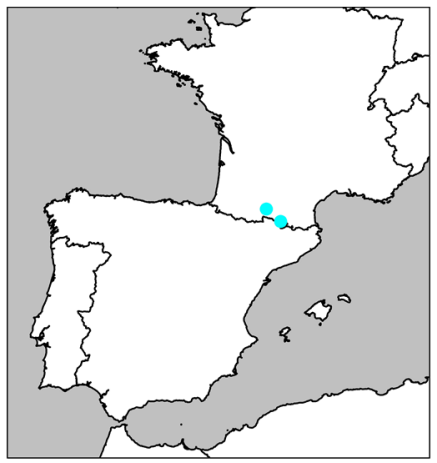

507. Opisthocheiron fallax Ribaut, 1922

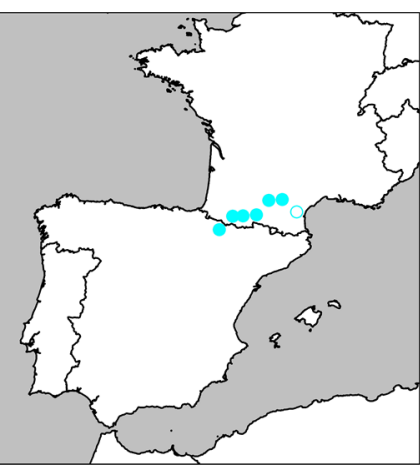

502. Marquetiella lunata (Ribaut, 1920)

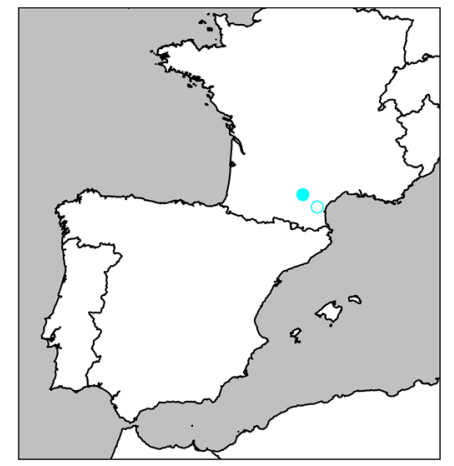

505. Opisthocheiron cornutum Ribaut, 1922

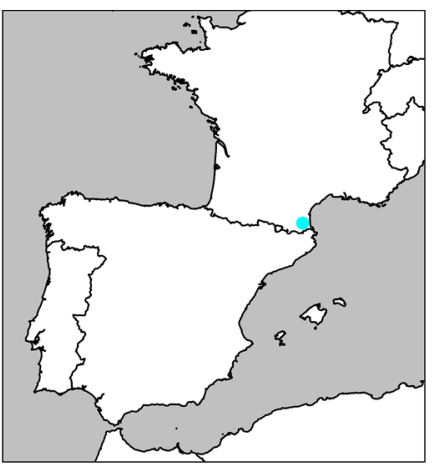

508. Opisthocheiron lacazei Brolemann, 1932 


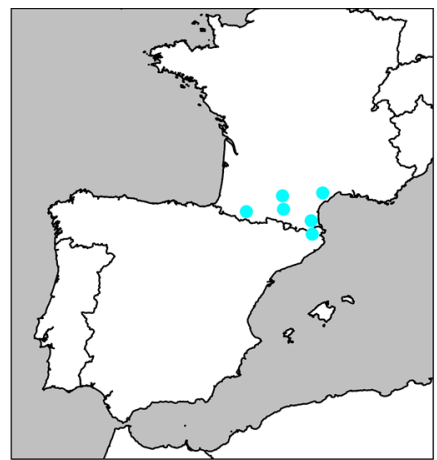

509. Opisthocheiron penicillatum Ribaut, 1913

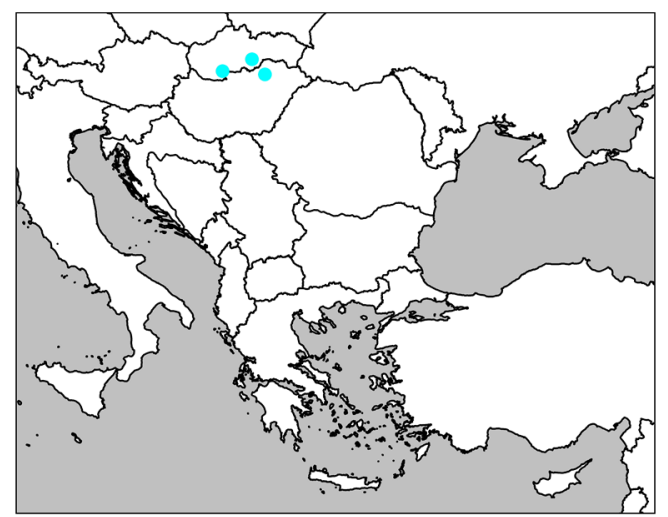

511. Acrochordum evae Loksa, 1960

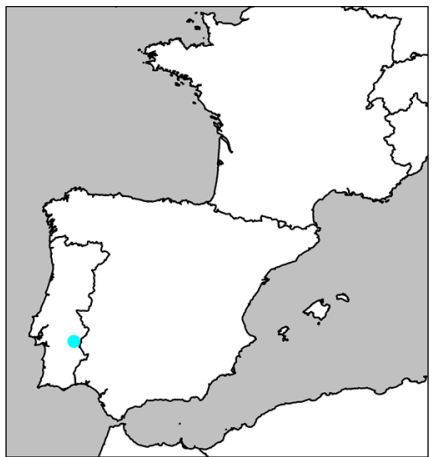

510. Sireuma nobile

Reboleira \& Enghoff, 2014

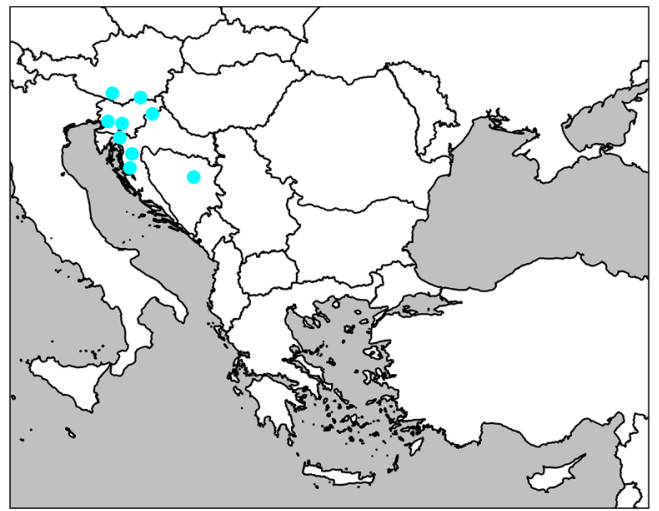

512. Acrochordum flagellatum Attems, 1899

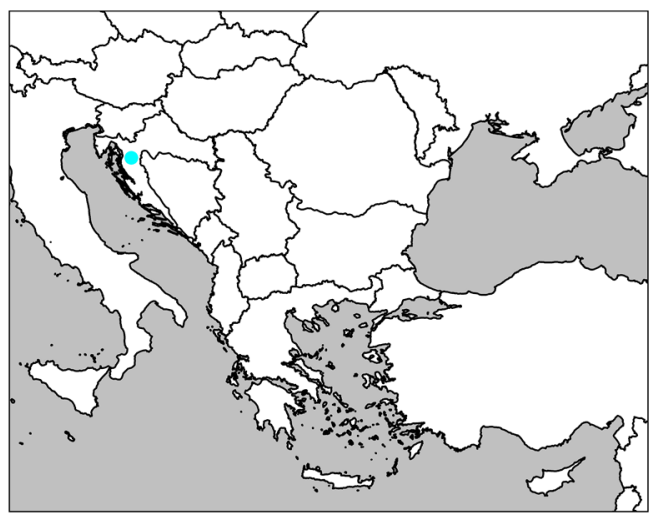

513. Acrochordum plitvicense (Verhoeff, 1929)

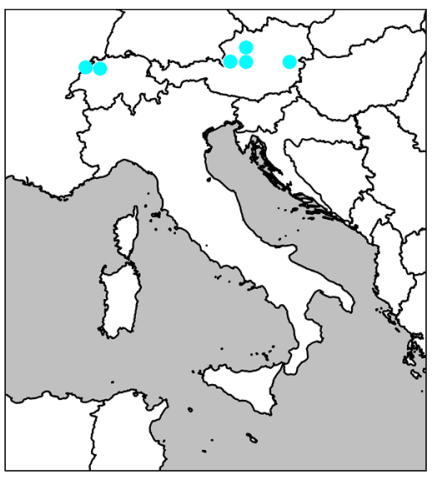

514. Halleinosoma noricum Verhoeff, 1913 


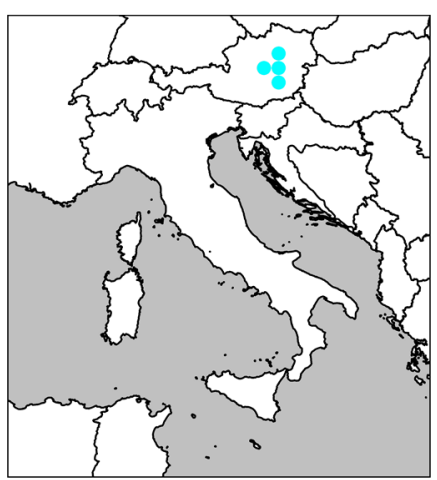

515. Trachygona capito (Attems, 1894)

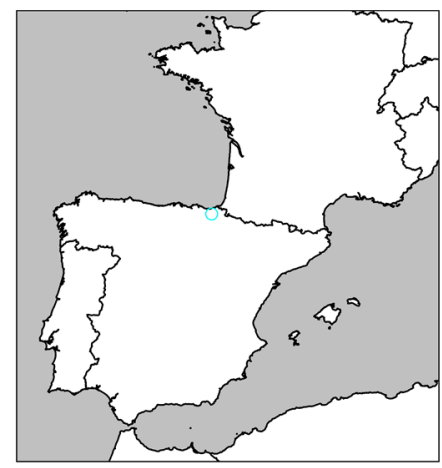

518. Guipuzcosoma comasi Vicente \& Mauriès, 1980

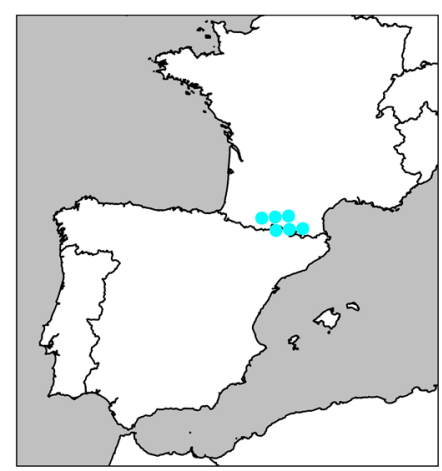

521. Hypnosoma pallidum Ribaut, 1952

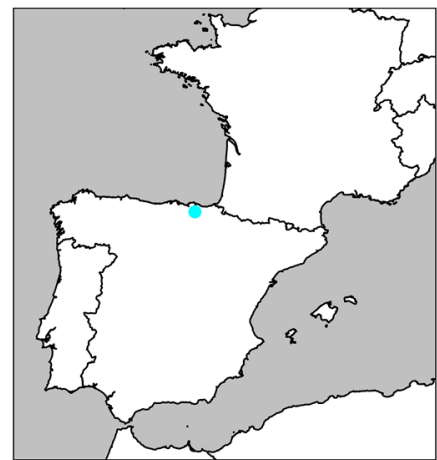

516. Alavasoma muniesai Mauriès \& Vicente, 1977

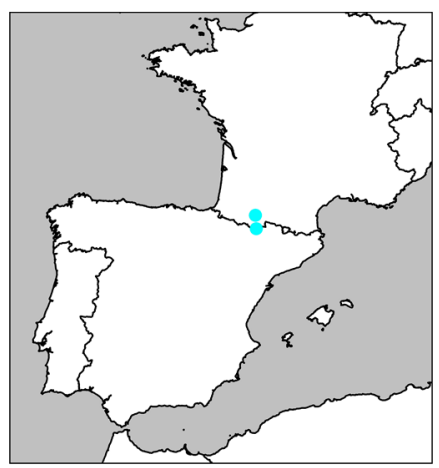

519. Hypnosoma exornatum Ribaut, 1952

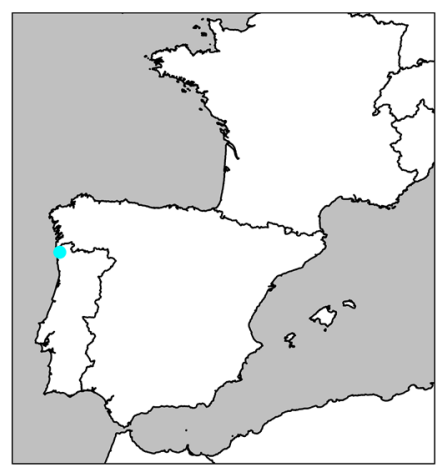

522. Miniusoma litorea Mauriès, 2015

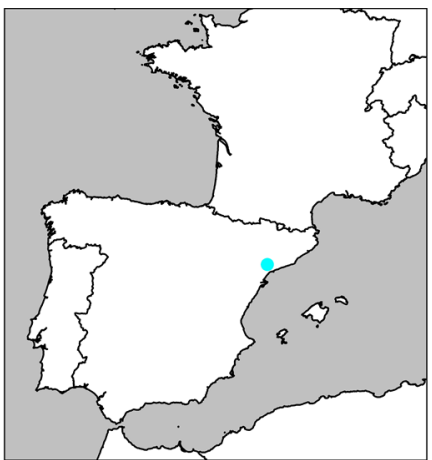

517. Eopsychrosoma serrapradense Serra \& Mauriès, 2015

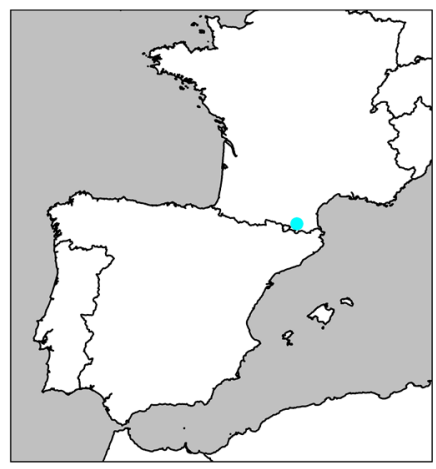

520. Hypnosoma juberthieorum Mauriès, 1968

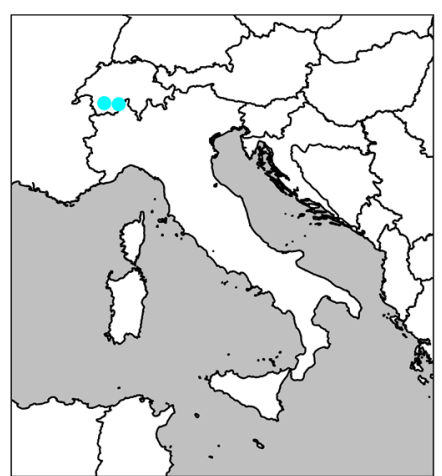

523. Niphatrogleuma wildbergeri Mauriès, 1986 


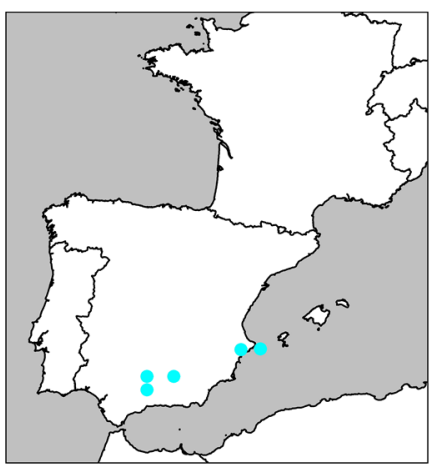

524. Psichrosoma baeticaense Mauriès, 2013

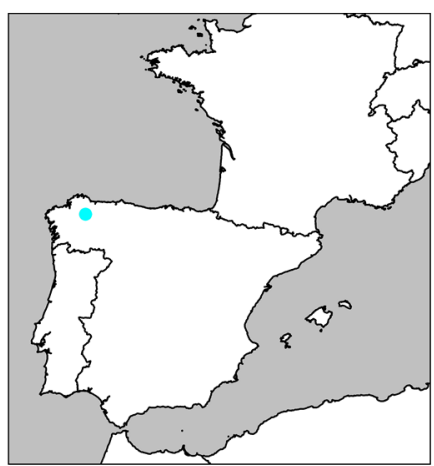

527. Strangulogona lugoensis Mauriès, 2015

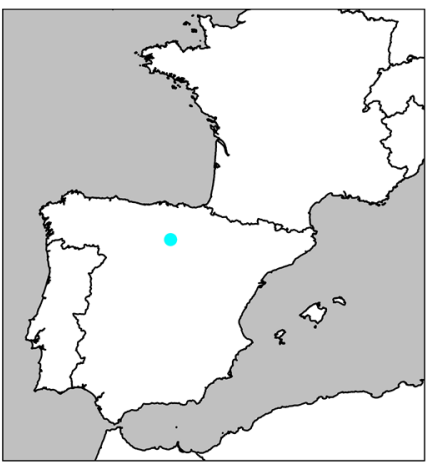

525. Psichrosoma breuili (Mauriès, 1970)

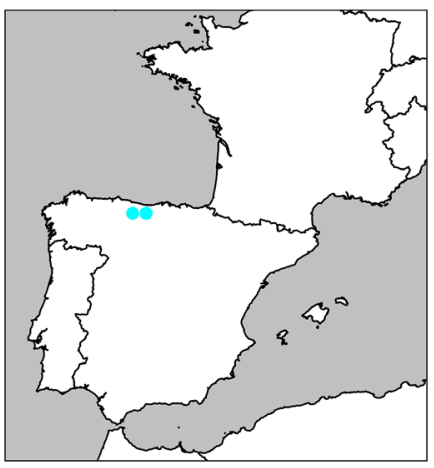

528. Typhlopsychrosoma fadriquei (Mauriès \& Vicente, 1977)

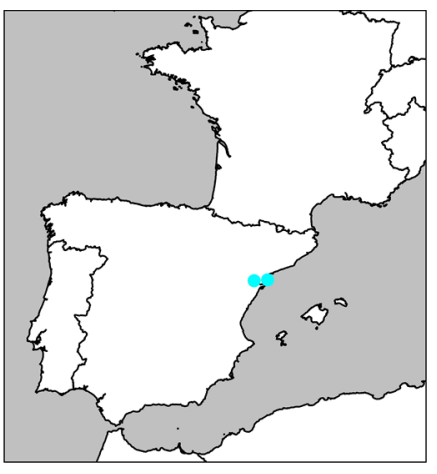

526. Psichrosoma tarraconense (Mauriès, 1970)

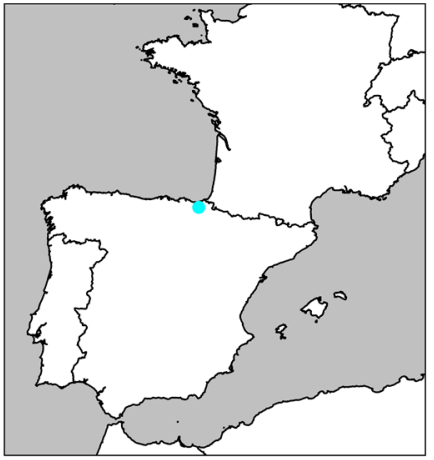

529. Vandeleuma hispanicum Ceuca, 1967

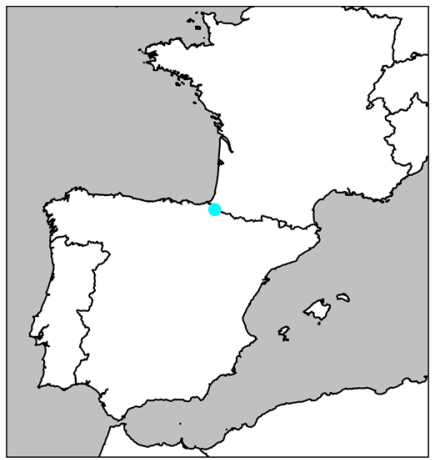

530. Vandeleuma vasconicum Mauriès, 1966 


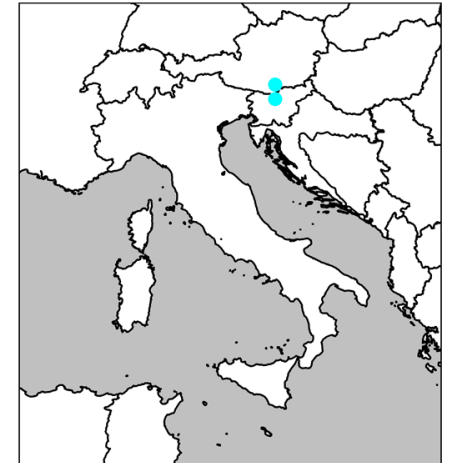

531. Haplogona carynthiaca (Strasser, 1967)

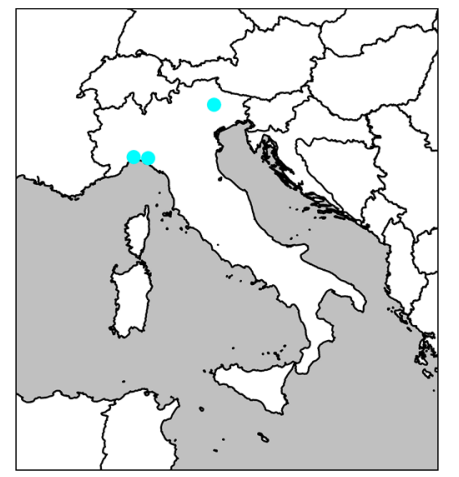

532. Haplogona gestri (Silvestri, 1898)

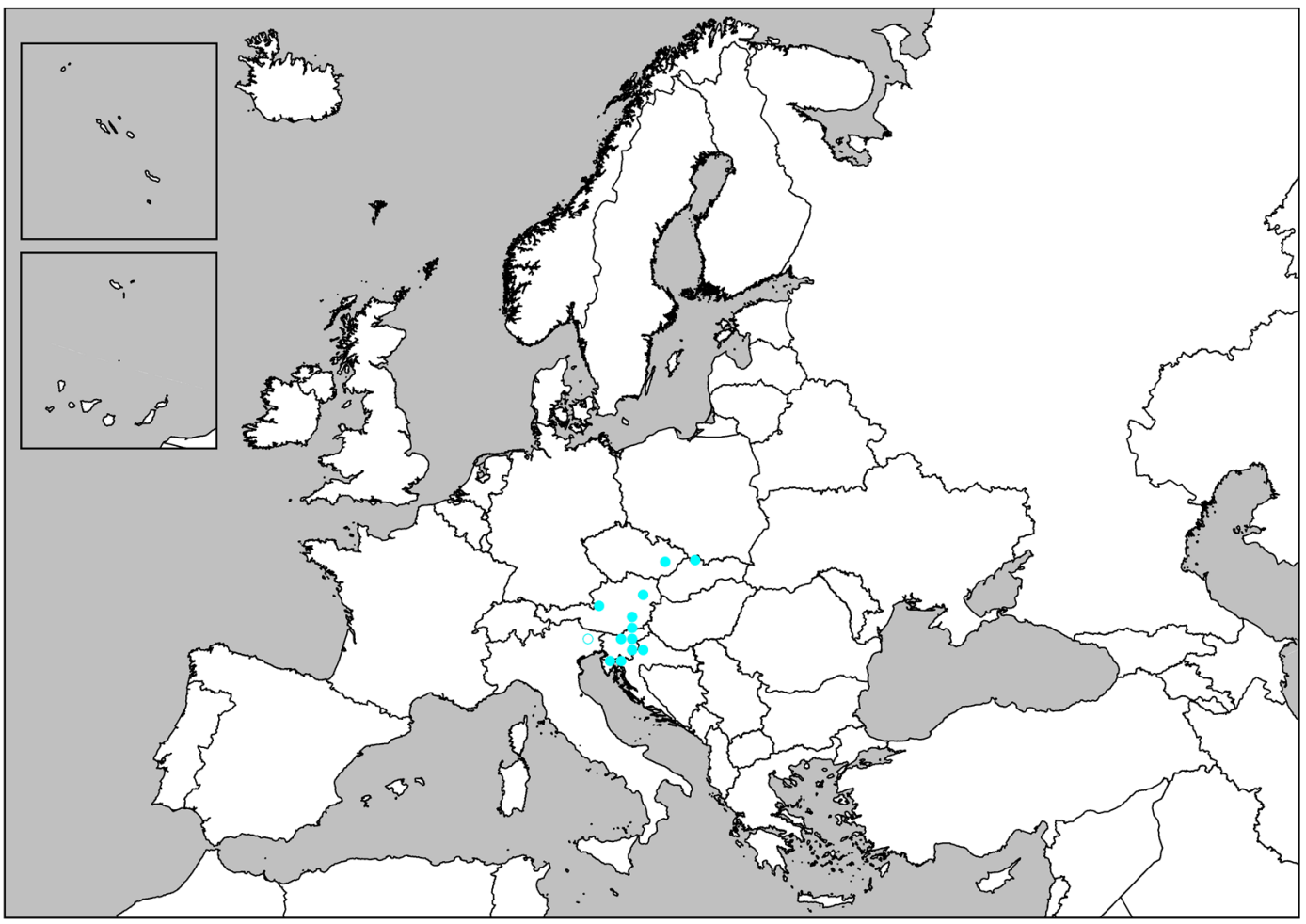

533. Haplogona oculodistincta (Verhoeff, 1893)

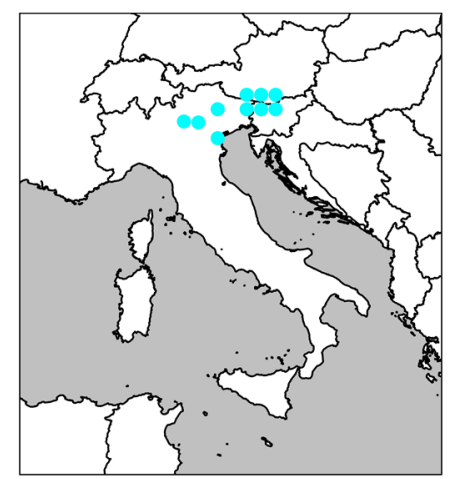

534. Haplogona rothenbuehleri (Verhoeff, 1900) 